RPP-RPT-54911, Rev. 0

\title{
Hanford Single-Shell Tank Leak Causes and Locations - 241-BY and 241-TY Farm
}

\author{
C.L. Girardot, D.G. Harlow
}

Washington River Protection Solutions

Richland, WA 99352

U.S. Department of Energy Contract DE-AC27-08RV14800

$\begin{array}{lll}\text { EDT/ECN: } & \text { DRF } & \text { UC: N/A } \\ \text { Cost Center: } & \text { 2KE00 } & \text { Charge Code: } 201334 \\ \text { B\&R Code: } & \text { N/A } & \text { Total Pages: } 272 \text { JDA 11/19/13 }\end{array}$

Key Words: leak location, leak cause, tank, integrity assessment, BY Farm, TY Farm, BY-103, TY-103, TY-104, TY-105, TY-106, leak assessment

Abstract: This document identifies 241-BY Tank Farm (BY Farm) and 241-TY Tank Farm (TY Farm) leak causes and locations for the 100 series leaking tanks (241-BY-103, 241-TY-103, 241-TY-104, 241TY-105, and 241-TY-106) identified in RPP-RPT-43704, Hanford BY Farm Leak Assessments Report, and in RPP-RPT-42296, Hanford TY Farm Leak Assessments Report. This document satisfies the BY and TY Farm portion of the target (T04) in the Hanford Federal Facility Agreement and Consent Order milestone M-045-91F.

TRADEMARK DISCLAIMER. Reference herein to any specific commercial product, process, or service by trade name, trademark, manufacturer, or otherwise, does not necessarily constitute or imply its endorsement, recommendation, or favoring by the United States Government or any agency thereof or its contractors or subcontractors.

$\frac{\begin{array}{l}\text { APPROVED } \\ \text { By Janis D. Aardal at 9:04 am, Nov 19, } 2013\end{array}}{\text { Release Approval }}$

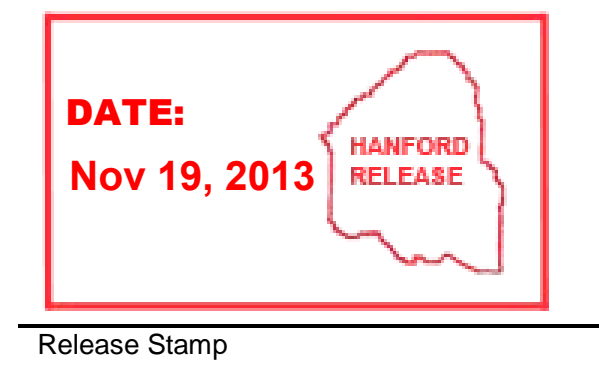

Approved For Public Release 


\section{RPP-RPT-54911, Rev. 0}

\section{EXECUTIVE SUMMARY}

This document identifies 241-BY Tank Farm (BY Farm) and 241-TY Tank Farm (TY Farm) leak causes and locations for the 100-series leaking tanks in BY and TY Farm. The leak causes and locations report for all of the 100-series single-shell leaking tanks is one of the targets (M045-91-T04) in the Hanford Federal Facility Agreement and Consent Order milestone M-04591F. The T04 target requires that the Department of Energy provide to State of Washington, Department of Ecology (Ecology) a report on the 100-series single-shell tanks which have been or will be identified as having leaked in RPP-32681, Rev. 0, Process to Assess Tank Farm Leaks in Support of Retrieval and Closure Planning, leak assessment reports.

The leak assessment report for BY Farm, RPP-RPT-43704, Rev. 0A, Hanford BY-Farm Leak Assessments Report, lists one 100-series tank that either continues to be or is recommended to be classified as a leaking tank in BY Farm, 241-BY-103 (BY-103). The leak assessment report for TY Farm, RPP-RPT-42296, Rev, 0, Hanford TY-Farm Leak Assessments Report, lists four 100series tanks that either continue to be or are recommended to be classified leaking tanks in TY Farm, 241-TY-103 (TY-103), 241-TY-104 (TY-104), 241-TY-105 (TY-105), and 241-TY-106 (TY-106).

All of the other eleven 100-series tanks in BY Farm and two 100-series tanks in TY Farm are classified as "sound" or are identified in RPP-RPT-43704, Rev. 0A, and RPP-RPT-42296, Rev. 0 , respectively, as requiring re-assessment of their classification per TFC-ENG-CHEM-D-42, Tank Leak Assessment Process. The TFC-ENG-CHEM-D-42 assessments are not part of the M045-91-T04 target.

This BY and TY Farm leak causes and locations document is part of a series of tank farm reports that identify leak causes and locations for 100-series leaking tanks. A summary and conclusions document will be issued, RPP-RPT-54909, Hanford Single-Shell Tank Leak Causes and Locations - Summary, that compiles the results from all of the leak causes and locations tank farm reports when they have been issued which will fulfill the T04 target requirements.

The identification of four of the five leaking BY and TY Farm tank leak locations focused on the first indication of radiation detected in drywells as well as liquid level decreases as appropriate, with the exception of tank TY-104 which could not be identified. Leak detection laterals were not installed underneath the BY and TY Farm tanks. For TY Farm, direct pushes were installed in 2005 to further characterize the tank leaks which were used to collaborate with the drywell data.

The BY and TY Farm leaking tanks were likely due to chemistry-corrosion from the storage of Tri-butyl phosphate (TBP) waste which is conducive to stress corrosion cracking and pitting. Thermal shock (rate of temperature rise) and tank construction conditions (cold weather ductileto-brittle transition temperature impact effects) were also likely to have affected tank integrity. There appears to be very little contribution from tank design and liner bulging. However, some or all of the factors can act serially or together to contribute to tank liner failure. The leaking BY and TY Farm tanks all stored undiluted TBP waste whereas the sound tanks stored diluted TBP waste as well as other waste types. 


\section{RPP-RPT-54911, Rev. 0}

\section{TABLE OF CONTENTS}

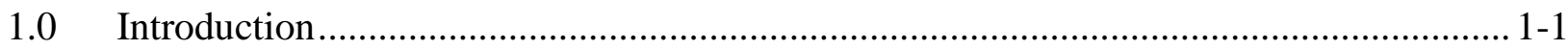

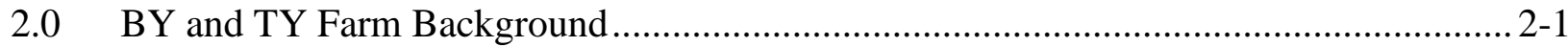

3.0 BY and TY Farm Commonalities ....................................................................... 3-1

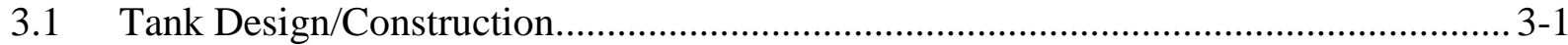

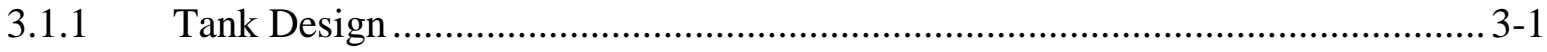

3.1.2 Tank Construction Conditions ............................................................... 3-4

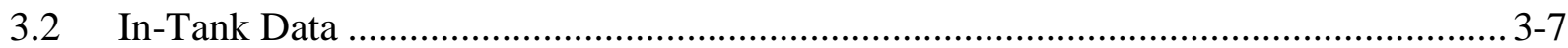

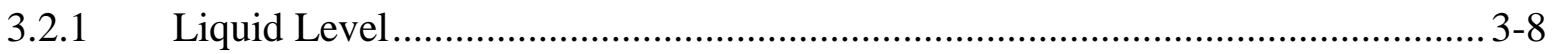

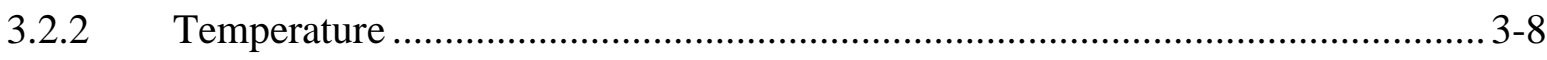

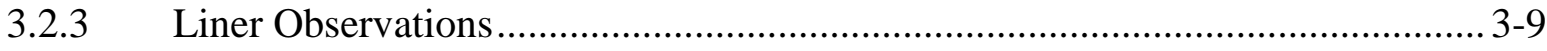

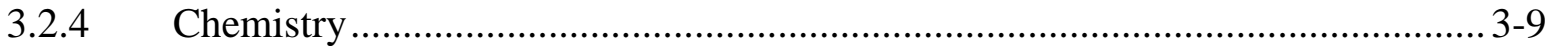

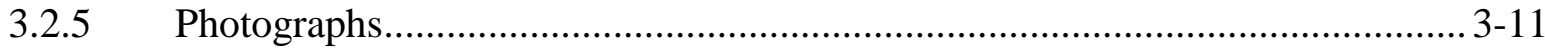

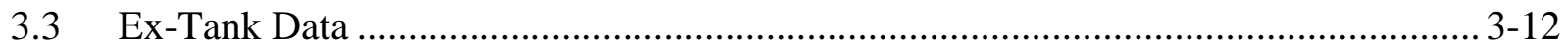

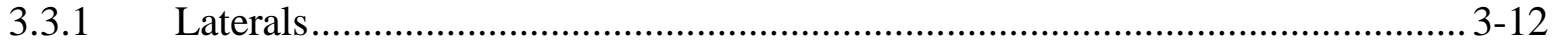

3.3.2 Drywells ................................................................................... $3-12$

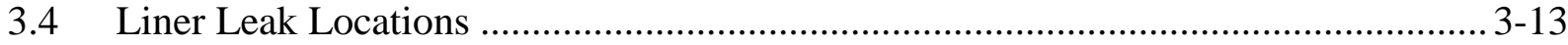

3.5 Possible Liner Leak Cause(s) .................................................................... 3-14

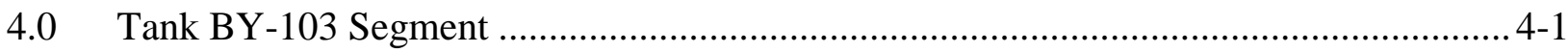

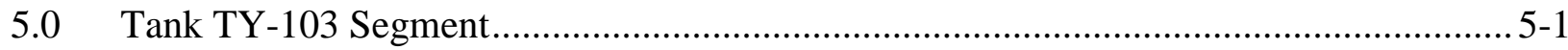

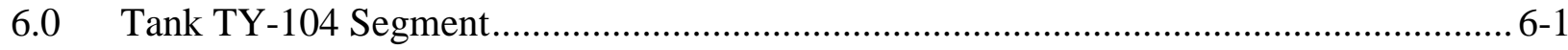

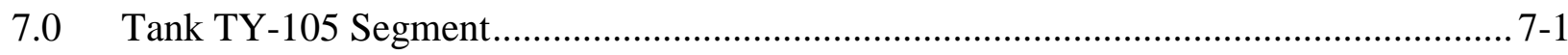

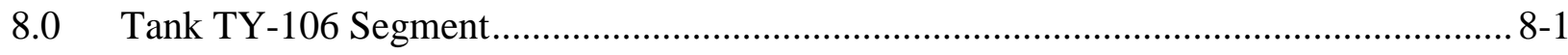

9.0 Conclusions ................................................................................................

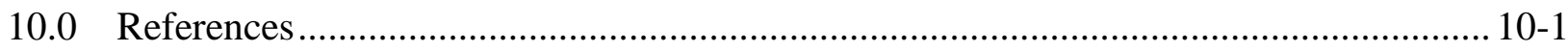


RPP-RPT-54911, Rev. 0

\section{APPENDICES}

Appendix A Meeting Minutes ................................................................................... A-1

Appendix B Comments Resolutions for BY and TY Farm Reports ................................... B-1

\section{LIST OF FIGURES}

Figure 2-1. BY Farm Leaking Tank and Associated Drywells ......................................... 2-2

Figure 2-2. TY Farm Leaking Tanks and Associated Drywells ...........................................2-3

Figure 3-1. BY and TY Farm Base Footing and Wall Reinforcing ................................... 3-2

Figure 3-2. BY and TY Farm Tank Bottom Liner to Sidewall Transition Design Detail .......... 3-3

Figure 3-3. B C T U Tank Farm Knuckle Configuration with Three Ply Waterproofing .......... 3-4

Figure 3-4. TY Farm Construction Photograph .......................................................... 3-6

\section{LIST OF TABLES}

Table 2-1. Leaking BY and TY Farm Tanks with Waste Type........................................... 2-4

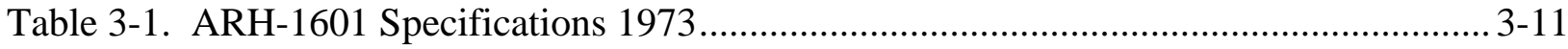

Table 9-1. BY and TY Farm Leaking Tanks ............................................................ 9-2

Table 9-2. BY and TY Farm Sound Tanks ................................................................ 9-3 


\section{Acronyms and Abbreviations}

\begin{tabular}{|c|c|}
\hline $221-\mathrm{U}$ & U Plant \\
\hline 241-BY & BY \\
\hline 241-TY & TY \\
\hline 241-BY Farm & BY Farm \\
\hline 241-TY Farm & TY Farm \\
\hline ARHCO & Atlantic Richfield Hanford Company \\
\hline ASME & American Society of Mechanical Engineers \\
\hline ASTM & American Society for Testing and Materials \\
\hline BGS & below grade surface \\
\hline $\mathrm{BiPO}_{4}$ & bismuth phosphate \\
\hline BPF & Blueprint File \\
\hline B \& PVC & Boiler \& Pressure Vessel Code \\
\hline $\mathrm{c} / \mathrm{m}, \mathrm{cpm}$ & counts per minute \\
\hline $\mathrm{c} / \mathrm{s}, \mathrm{cps}$ & counts per second \\
\hline DOE & Department of Energy \\
\hline DST(s) & double-shell tank(s) \\
\hline Ecology & State of Washington, Department of Ecology \\
\hline GM & gamma \\
\hline GMP & gamma probe \\
\hline ITS & in tank solidification \\
\hline LL & liquid level \\
\hline $\mathrm{NaI}$ & sodium-iodide \\
\hline $\mathrm{NO}_{2}^{-}$ & nitrite \\
\hline $\mathrm{NO}_{3}^{-}$ & nitrate \\
\hline OCP & open circuit potential \\
\hline $\mathrm{OH}^{-}$ & hydroxide \\
\hline ORP & Office of River Protection \\
\hline PCSACS & PC Surveillance Analysis Computer System \\
\hline PUREX & Plutonium Uranium Extraction (fuels reprocessing) Plant \\
\hline REDOX & Reduction Oxidation (fuels reprocessing) Plant \\
\hline SCC & stress corrosion cracking \\
\hline SGLS & Spectral Gamma Logging System \\
\hline SP & scintillation probe \\
\hline SRS & Savannah River Site \\
\hline SSP & shielded scintillation probe \\
\hline SST(s) & single-shell tank(s) \\
\hline UNH & uranyl nitrate hexahydrate \\
\hline WRPS & Washington River Protection Solutions, LLC \\
\hline \multicolumn{2}{|l|}{ Units } \\
\hline $\mathrm{Ci}$ & curie \\
\hline${ }^{\circ} \mathrm{F}$ & degrees Fahrenheit \\
\hline $\mathrm{ft}$ & feet \\
\hline gal & gallon \\
\hline
\end{tabular}




$\begin{array}{ll}\mathrm{K} & 1000 \\ \mathrm{kgal} & \text { kilogallon }\left(10^{3} \text { gallons }\right) \\ \mathrm{in} & \text { inches } \\ \mathrm{L} & \text { liter } \\ \mathrm{lb} & \text { pound } \\ \mathrm{M} & \text { moles per liter } \\ \mathrm{pCi} & \text { picocurie }\left(10^{-12} \text { curies }\right) \\ \mathrm{pCi} / \mathrm{g} & \text { picocurie per gram } \\ \mathrm{yr} & \text { year }\end{array}$

\section{Waste Type Abbreviations}

$\begin{array}{ll}\text { 1C } & \text { first cycle waste } \\ \text { 1C-FeCN } & \text { treated 1C (ferrocyanide) waste } \\ \text { B } & \text { B Plant HLW } \\ \text { BL } & \text { B Plant Low Level Waste } \\ \text { CPLX } & \text { complexant concentration } \\ \text { CW } & \text { coating waste } \\ \text { CWP } & \text { PUREX coating waste } \\ \text { DW } & \text { decontamination waste } \\ \text { EB } & \text { Evaporator Bottoms } \\ \text { Evap. } & \text { Evaporator Feed (post 1976) } \\ \text { HLW } & \text { high-level waste } \\ \text { IX } & \text { Ion Exchange waste } \\ \text { MW } & \text { Metal waste } \\ \text { NCPLX } & \text { non-complexed waste } \\ \text { OWW } & \text { organic wash waste } \\ \text { P } & \text { PUREX HLW supernatant } \\ \text { R } & \text { REDOX High Level Waste } \\ \text { RIX } & \text { REDOX ion exchange waste } \\ \text { TBP } & \text { Tri-butyl phosphate waste }\end{array}$


RPP-RPT-54911, Rev. 0

\subsection{INTRODUCTION}

The Hanford Federal Facility Agreement and Consent Order target M-045-91F-T04 indicated that part of the RPP-32681, Process to Assess Tank Farm Leaks in Support of Retrieval and Closure Planning, reporting would include leak causes and locations reports for all of the 100-series single-shell leaking tanks. This document is part of a series of documents that identifies leak causes and locations of 100-series single-shell leaking tanks that have been identified in the individual RPP-32681 tank farm leak assessments. An overall leak causes and locations summary and conclusions document will be prepared along with background and common tank farm information when all of the 100-series single-shell leaking tanks have been addressed (RPP-RPT-54909, Hanford Single-Shell Tank Leak Causes and Locations - Summary and Conclusion, to be issued). The information from this document will be incorporated into the summary conclusions report on leak integrity for the Hanford Federal Facility Agreement and Consent Order milestone M-045-91F.

The 241-BY Tank Farm (BY Farm) and 241-TY Tank Farm (TY Farm) tanks with leak losses are addressed in this document on an individual tank basis. The leak location and cause analysis for BY Farm and TY Farm have been combined as both farms were built to Type III tank requirements at about the same time and therefore have some common features.

The BY Farm assessment in RPP-RPT-43704, Rev. 0A, Hanford BY-Farm Leak Assessments Report, reported a leak loss for only the 241-BY-103 (BY-103) tank but recommended that all of the other eleven BY Farm tanks be further assessed using TFC-ENG-CHEM-D-42, Rev B-2, Tank Leak Assessment Process. The sound tanks as well as the assumed leaker category of tanks were included because of the uncertainty of the liner integrity for In Tank Solidification (ITS) and ITS bottoms tanks. ITS involved concentration/solidification of tank waste including metal waste (MW), tri-butyl phosphate waste (TBP), PUREX wastes, etc.

The TY Farm assessment in RPP-RPT-42296, Rev. 0, Hanford TY-Farm Leak Assessments Report, reported leak losses for the following tanks: 241-TY-103, 241-TY-104, 241-TY-105, and 241-TY-106. There was insufficient data to establish a leak inventory estimate for tank 241TY-101 and the tank was recommended to be reassessed per TFC-ENG-CHEM-D-42.

The identification of BY and TY Farm tank leak locations focused on the first indication of radiation detected in drywells as well as liquid level decreases as appropriate. Laterals were not installed underneath the BY and TY Farm tanks. For TY Farm, direct pushes were installed in 2005 to further characterize the tank leaks which were used to collaborate with the drywell data.

The BY and TY Farm leaks were likely due to chemistry-corrosion, thermal shock, and tank construction conditions. Tank design and liner bulging do not seem to have contributed to liner failures in BY and TY Farm.

Five meetings between July 2011 through September 2011 were held to review status of the assumed leakers in BY and TY Farm with the Office of River Protection (ORP) and the State of Washington, Department of Ecology (Ecology) personnel (see Appendix A). Comments were received, responses developed, and additions/revisions were made to the document (see Appendix B). 


\subsection{BY AND TY FARM BACKGROUND}

The BY Farm is comprised of twelve Single-Shell Tanks (SSTs) built in 1948 to 1949. The TY Farm is comprised of six SSTs built in 1951 to 1952. The BY Farm tanks are arranged in rows of three tanks each, forming a cascade. The TY Farm tanks are arranged in rows of two tanks each, forming a cascade with the second tank cascade tie line outlet blanked off $\sim 55-\mathrm{ft}$ from the tank. The intended route from the capped tie line is not defined. Tanks in each of the farms have a 758,000-gal storage capacity. The tanks consist of a carbon steel liner inside a reinforced concrete shell. The concrete shell is a domed structure approximately $46-\mathrm{ft}$ in height and $\sim 84-\mathrm{ft}$ in diameter at the footing. The steel tank liner covers the 75-ft inner diameter tank bottom and sidewalls to a height of $\sim 23$-ft as measured from the tank center. The tank footing is dish shaped and slopes from the sidewall to the tank center (i.e., 12-in. elevation drop from the knuckle). The tank footing connects to the sidewall over a 4-ft radius knuckle (see Section 3.1.1).

The method used to identify leak location consisted of reviewing in-tank and ex-tank leak detection information. This provided the basic data identifying where and when the first leaks were detected. In-tank leak detection consists of liquid level measurement that can be augmented with photographs which in some cases can provide an indication of the vertical leak location on the sidewall. Ex-tank leak detection for the leaking tanks consists of drywells (Figure 2-1 and Figure 2-2). The in-tank and ex-tank leak detection can provide an indication of the possible leak location radially and vertically around the tank.

Similarly, potential leak causes can be determined with in-tank and ex-tank information that is not directly related to leak detection. These other in-tank parameters can include temperature of the supernatant and sludge, types of waste, and chemical determination by either transfer or samples analysis. Ex-tank leak cause information can be assembled from many sources including design media, construction conditions, technical specifications, and other sources.

Data sources have included data sheets, plots of data, internal letters, documents, and monthly-quarterly-semi-annual-annual reports. The preferred source was the actual data sheets but they were not available for all cases which resulted in using the best data source available. 
Figure 2-1. BY Farm 100-Series Leaking Tank and Associated Drywells Tank inner ring is steel liner, outer ring is outer edge of tank footing

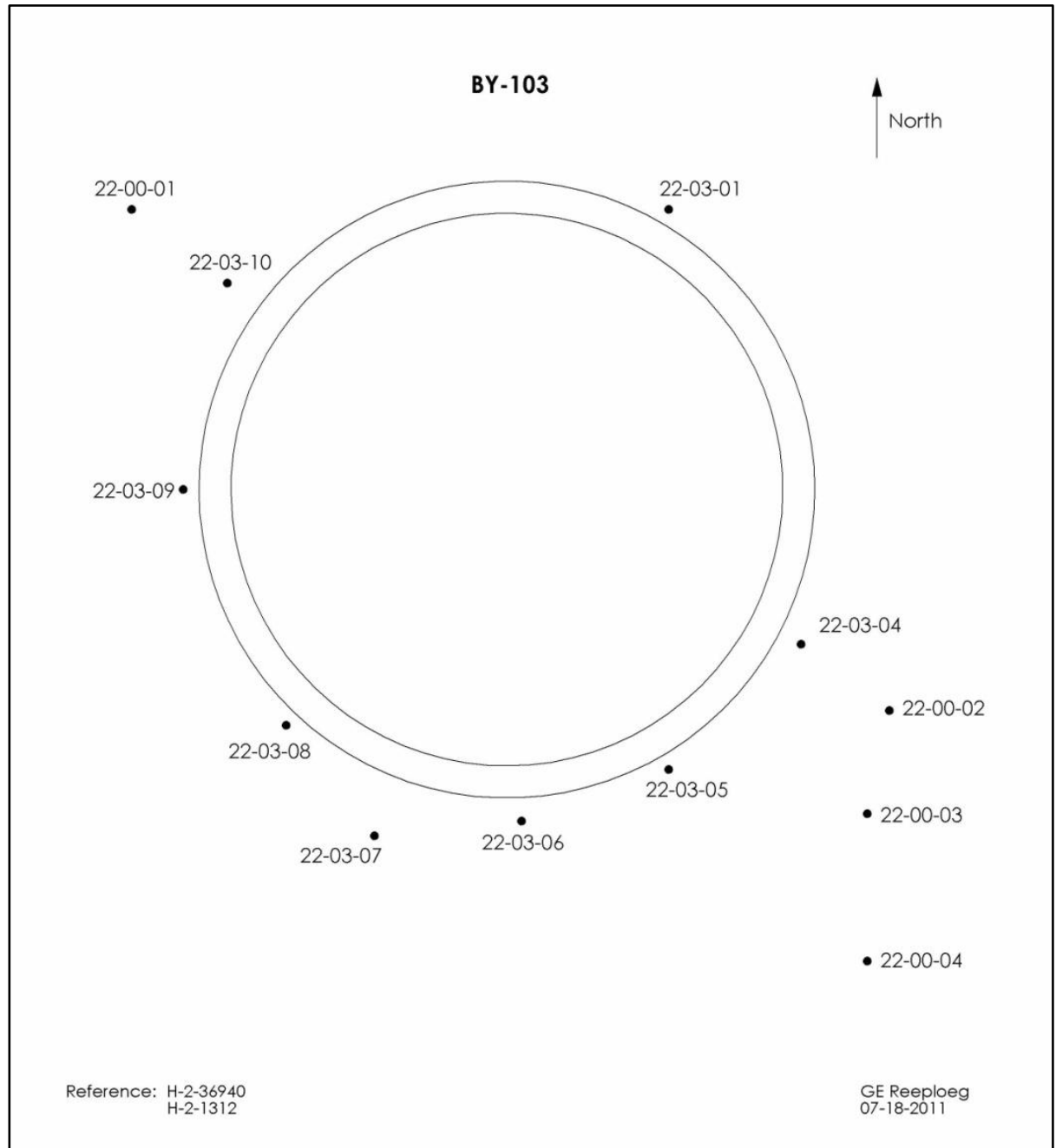

Note: The only leaking BY Farm tank (BY-103) is located in the Northeast corner of the farm (see Figure 3-3) 
Figure 2-2. TY Farm Leaking 100-Series Tanks and Associated Drywells Tank inner ring is steel liner, outer ring is outer edge of tank footing

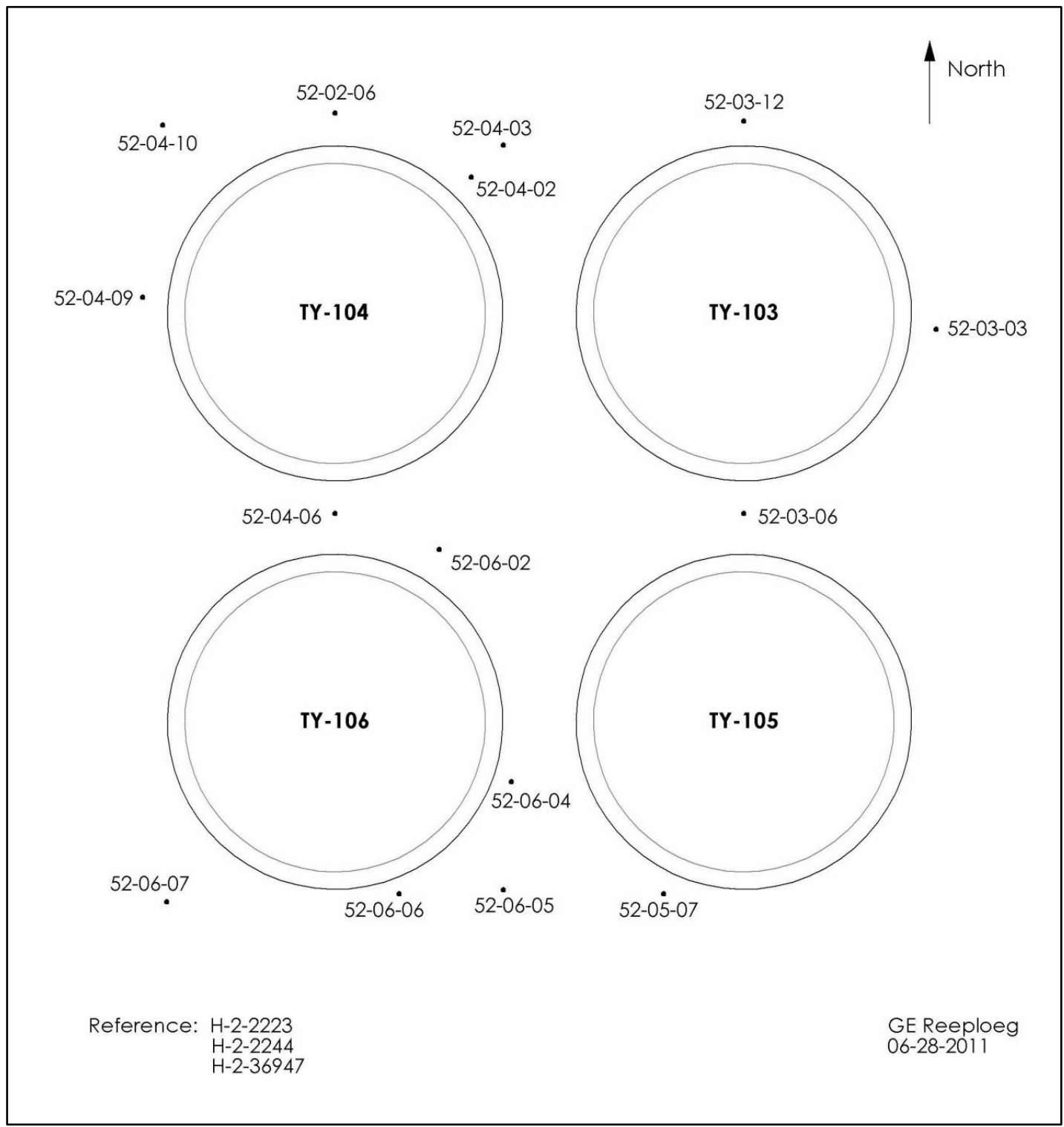

Note: Tanks TY-101 to TY-102 lie to the north of tanks TY-103, and TY-104 (see Figure 3-4)

In 2005, direct pushes were constructed around TY Farm to further characterize the tank leaks. The location of the direct pushes is addressed in the individual TY Farm segments.

All of the leaking tanks in the BY and TY Farms contained Tri-butyl phosphate (TBP) waste (see Table 2-1). TBP waste was the only waste type stored in tanks TY-105 and TY-106. Tanks BY103 and TY-103 stored undiluted TBP waste for approximately two years, and tank TY-104 stored undiluted TBP waste for approximately 19 months. 
RPP-RPT-54911, Rev. 0

\section{Table 2-1. Leaking BY and TY Farm Tanks with Waste Type}

\begin{tabular}{|c|c|}
\hline Tank & Waste Types \\
\hline BY-103 & MW, TBP, P, CW, OWW, EB \\
\hline TY-103 & $\begin{array}{r}\text { TBP, 1C-FeCN, DW, CW, OWW, 1C/CW, } \\
\text { EB, R, B, BL, RIX }\end{array}$ \\
\hline TY-104 & TBP, 1C-FeCN, DW, OWW, BL \\
\hline TY-105 & TBP \\
\hline TY-106 & TBP \\
\hline
\end{tabular}

The following sections describe some of the important common tank features and conditions that could affect tank leak location and cause. Affected tanks are noted where the features and conditions do not apply to all. This is followed by a BY and TY Farm tank-by-tank analysis of the possible leak location(s) and causes for each of the leaking tanks and a comparison of leaking and sound tanks in the conclusion section. The BY Farm tank segment contains excerpts from RPP-RPT-43704 and TY Farm tank segments contain excerpts from RPP-RPT-42296. 
RPP-RPT-54911, Rev. 0

\subsection{BY AND TY FARM COMMONALITIES}

\subsection{TANK DESIGN/CONSTRUCTION}

\subsubsection{Tank Design}

The steel bottom of the BY and TY Farm tanks intersect the sidewall on a 4-ft radius similar to the knuckle transitions in earlier designed tank farms (BPF-73550, Drawings D-2 and D-3, Specification for Construction of Composite Storage Tanks (B, C, T, and U Tank Farms)). The BY and TY Farm tank base footing is shown on Figure 3-1 from the bottom of the footing to the first keyed wall construction joint (H-2-1314 and H-2-2246, 75- Foot Tank Base Footing and Wall Reinforcing, Waste Disposal Facility, 241-BY and TY, respectively). This figure shows the concrete foundation and sidewall intersection between the dished bottom and the sidewall, water stop at the base footing to wall construction joint, the first wall construction joint, and other details. The 7-in wide water stop at the footing construction joint is $3 \frac{1}{2}-$-in above the joint and 31/2-in below the joint. The earlier 241-BCTU tanks notched footing construction joint does not incorporate a water stop.

Figure 3-2 shows the detail of the knuckle liner to the 55-lb roll roofing, grout/fabric, and three ply asphaltic waterproof membranes between the bottom and sidewall intersection (H-2-1312, BY Farm and H-2-2244, TY Farm 75-Foot Tank Sections, Section "D-D”, respectively). The three ply asphaltic membrane waterproofing between the wall liner and the concrete shell continued the design from 241-BCTU Farms shown in Figure 3-3 which shows the rounded knuckle configuration and the bottom and sidewall three ply water proofing. BY and TY Farm both used full penetration butt welds similar to 241-BCTU tank farms which are shown on BPF73550 . 
RPP-RPT-54911, Rev. 0

Figure 3-1. BY and TY Farm Base Footing and Wall Reinforcing Drawing H-2-2246

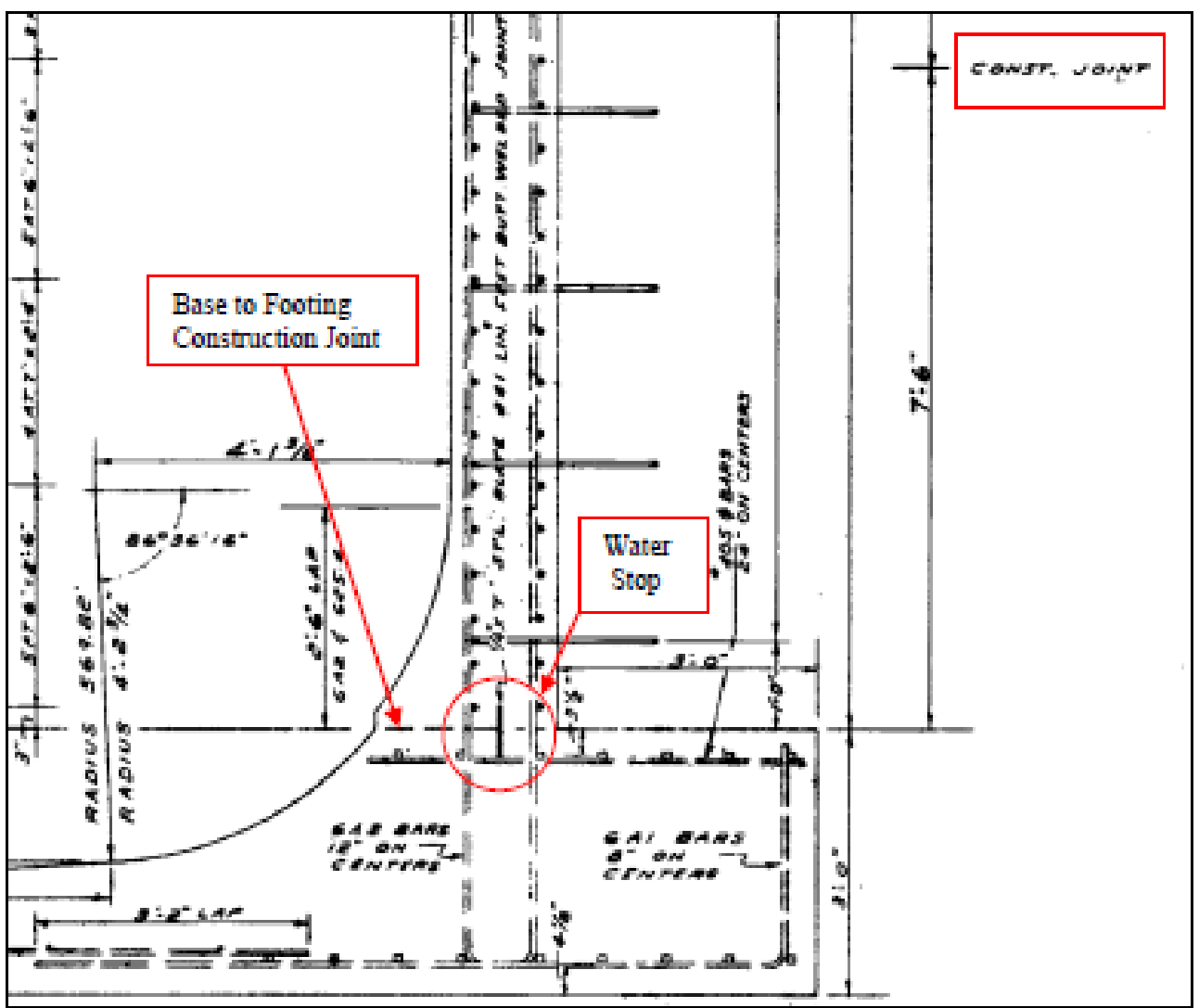

The bottom of the outer footing is 45 - $\mathrm{ft} 101 / 2$-in BGS and the top of the outer footing is 42 - $\mathrm{ft} 10 \frac{1}{2}-$-in BGS 
Figure 3-2. BY and TY Farm Tank Bottom Liner to Sidewall Transition Design Detail Drawings H-2-1312 and H-2-2244

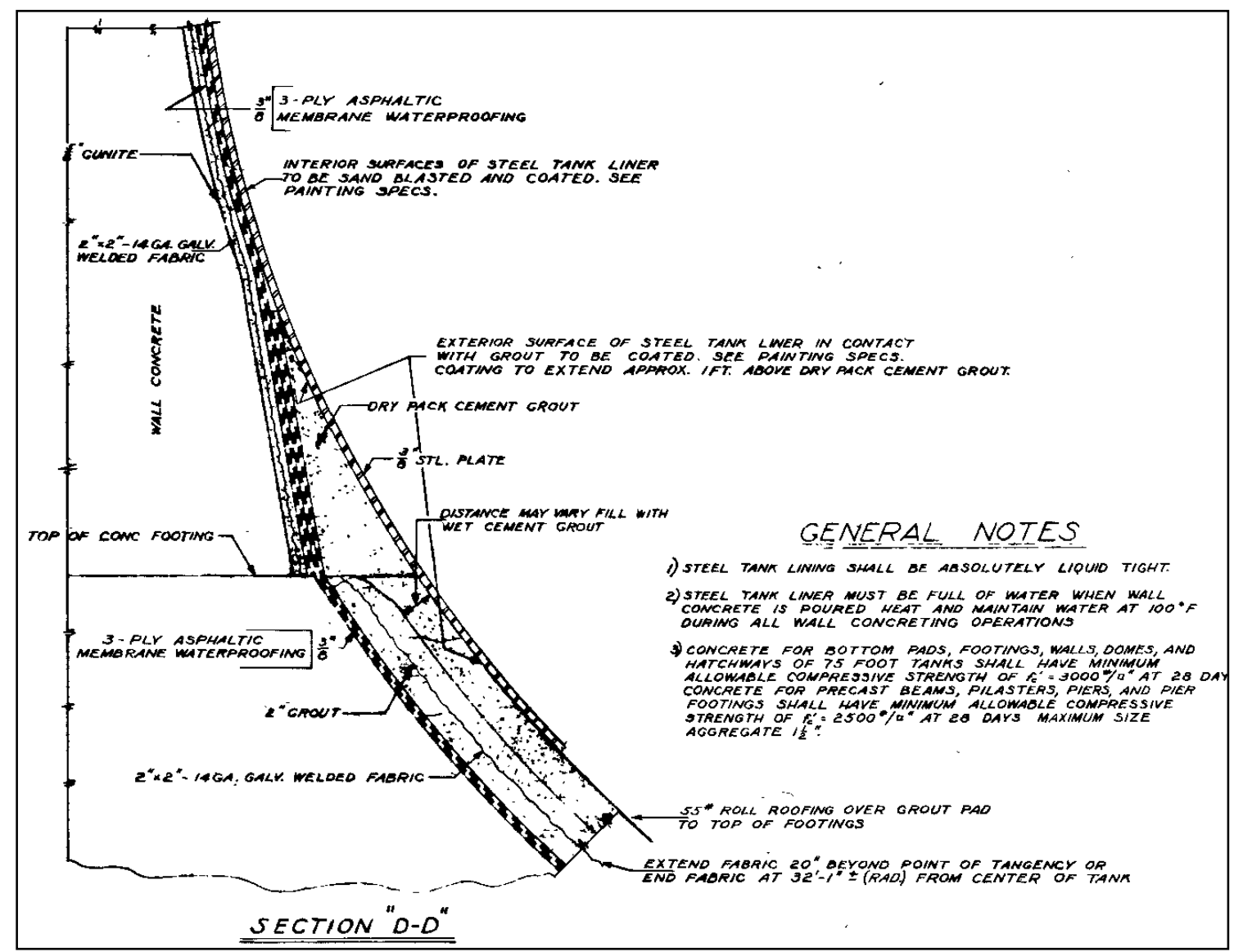


Figure 3-3. B C T U Tank Farm Knuckle Configuration with Three Ply Waterproofing (BPF-73550, Drawings D-2 and D-3)

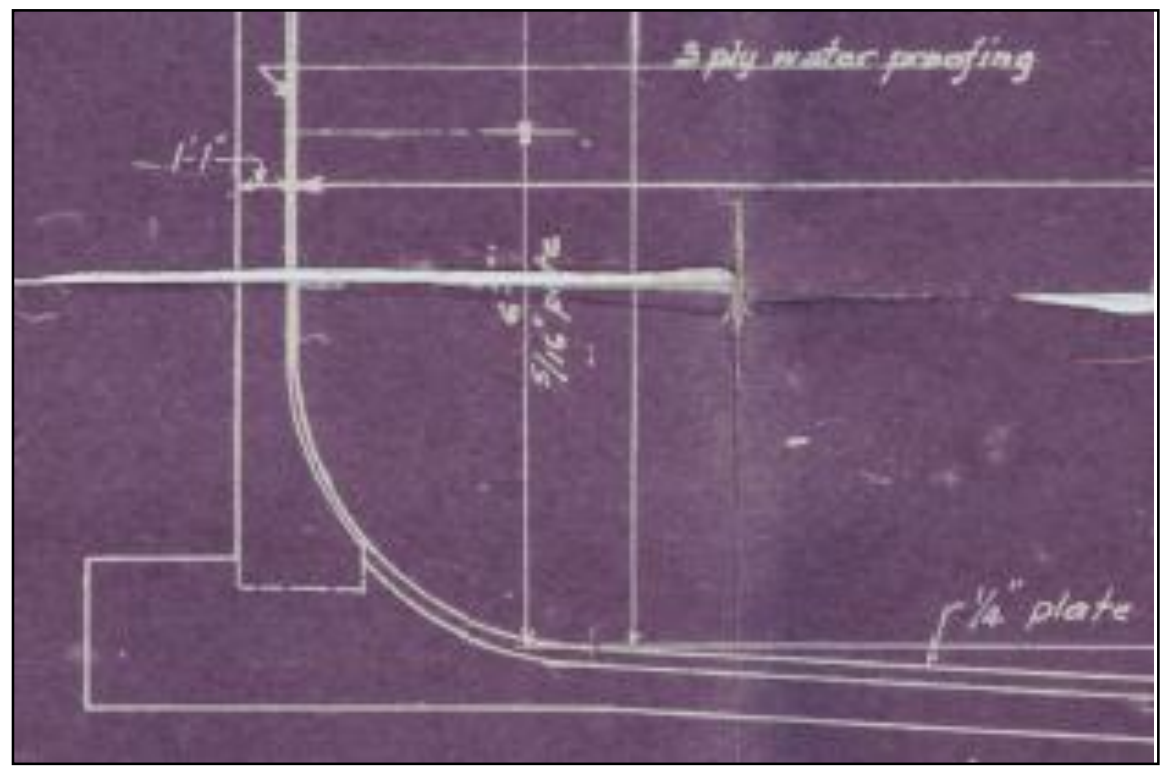

A BY Farm and TY Farm improvement in tank liner plate layout, over the 241-TX Farm tanks, was made which called for rolled plates of larger dimensions. The improved layout and use of larger plates reduced the amount of joint preparation, fit-up, and radiographic examination of welded joints (HW-24800-30, Design and Construction History Project C-271 241-BY Tank Farm). The re-weld on BY Farm tanks and TY Farm was $2.52 \%$ and $4.19 \%$, respectively. The subcontractor for BY Farm was Morrison-Knudsen Co. who had just finished 241-BX Farm and 241-TX Farm (HW-24800-30). The subcontractor for TY Farm was Fred J. Early, Jr. Co. Inc. X-Ray Products Corp. was responsible for the radiographic (X-Ray) inspection of all welded joints for the tank liners for BY Farm and TY Farm tanks in accordance with HW-4590, NonDestructive Inspection of Welded Joints in Steel Tank Liners for Waste Disposal Facilities.

\subsubsection{Tank Construction Conditions}

The following is from BY Farm construction history, HW-24800-30:

The last base of the BY Farm tanks was poured on November 22, 1948. All of the bases were finished before the occurrence of cold weather. Cold weather, snow, and rain affected the steel construction during the months of December and January as specifications did not allow welding when the material to be welded was extremely cold $\left(<0^{\circ} \mathrm{F}\right)(\mathrm{HW} 3783$, Specification Additional Waste Storage Facilities). The surface within 3-in was required to be preheated to a temperature warm to the hand before welding between $0^{\circ} \mathrm{F}$ and $32^{\circ} \mathrm{F}$. The temperature for December 1948 and January 1949 averaged $26.9^{\circ} \mathrm{F}$ and $13.9^{\circ} \mathrm{F}$, respectively (PNNL-15160, Hanford Site Climatological Summary 2004 with Historical Data). An access hole was cut in one of the knuckle plates to allow welders and X-ray inspectors to move equipment directly into the tank as opposed to elevating and lowering the equipment over the sides of the tank. Water was added to the tank and heated to $100^{\circ} \mathrm{F}$ before application of the tank liner waterproof 
membrane. The redesign of knuckle plate forming part of the steel tank liners and the introduction of specific techniques for shop fabrication of these plates improved field fit up, reduced weld repair and practically eliminated distortion of the welded assembly. No concrete was poured during freezing weather.

\section{Figure 3-4 BY Farm Construction Photograph (N1D0000725)}

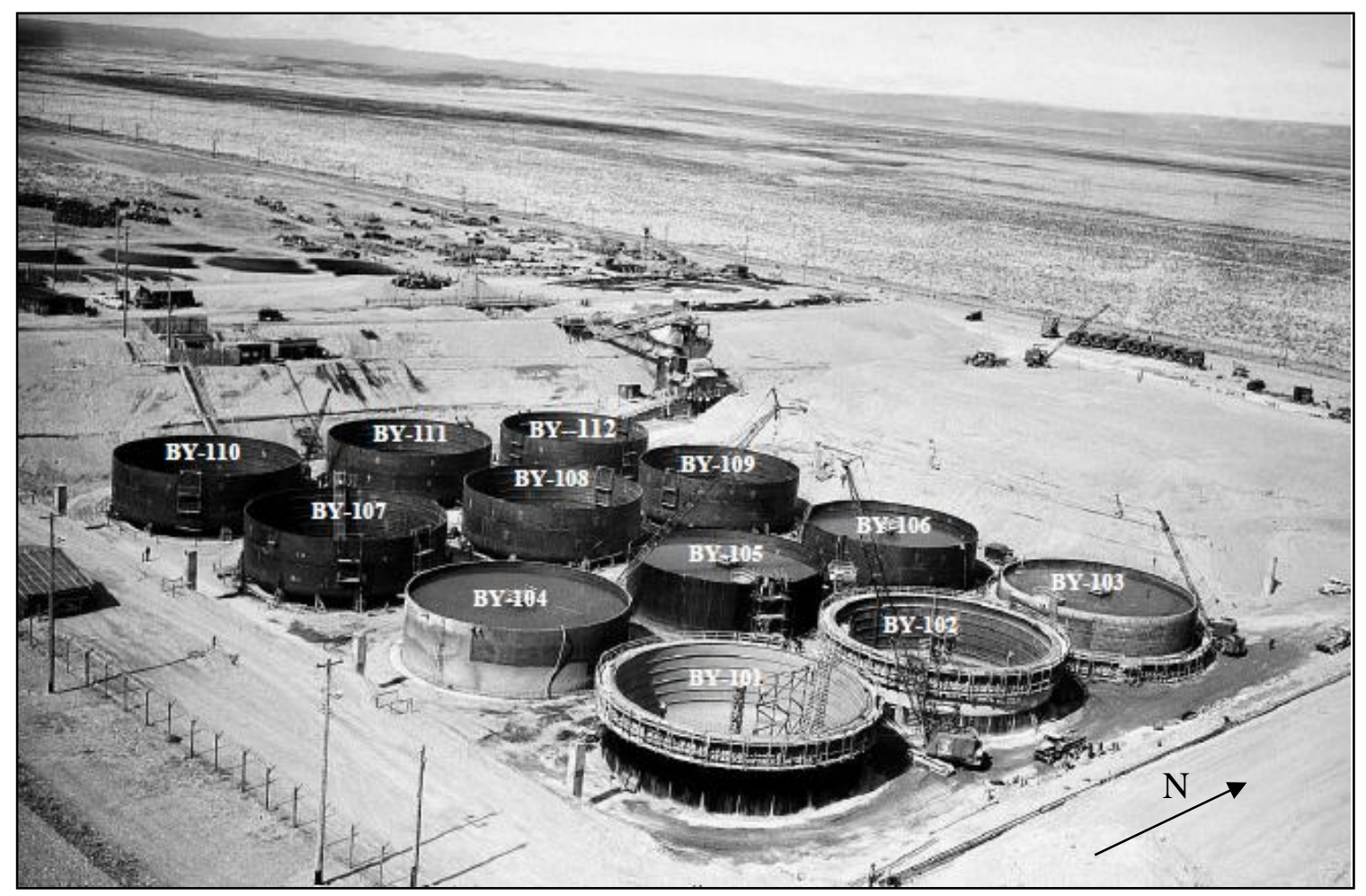

Details for the construction of BY Farm can be found in RPP-RPT-50840, Rev. 0, 241-BY Tank Farm Construction History.

The following is from TY Farm construction history, HW-24800-86, Design and Construction History Project C-418 241-TY Tank Farm:

The last base of the TY Farm tanks was completed on August 9, 1951. Cold weather, snow, and rain affected the steel construction during the months of December and January as specifications did not allow welding when the material to be welded was extremely cold with the same restrictions as BY Farm (HW 4696, Specifications Waste Disposal Facilities 241-BZ and TY Tank Farm). The surface within 3-in was required to be preheated to a temperature warm to the hand before welding between $0^{\circ} \mathrm{F}$ and $32^{\circ} \mathrm{F}$. The temperature for December 1951 and January 1952 averaged $27.4^{\circ} \mathrm{F}$ and $25.2^{\circ} \mathrm{F}$, respectively (PNNL-15160). An access hole was cut in one of the knuckle plates to allow welders and X-ray inspectors to move equipment directly into the tank as opposed to elevating and lowering the equipment over the sides of the tank. Water was added to the tank and heated to $100^{\circ} \mathrm{F}$ before application of the tank liner waterproof membrane. Fabrication and installation of the knuckle sub-assemblies included: cold pressed 
forming, stress relieved, then assembled into five plate sections with seams welded and then stress relieved. These sub-assemblies were delivered to the job site from the manufacturer as opposed to fabrication on site. Knuckle sections were then fitted in place and machine welded to the bottom plate. Concrete cold weather specifications are in HW-4696.

\section{Figure 3-4. TY Farm Construction Photograph (N1D0057718)}

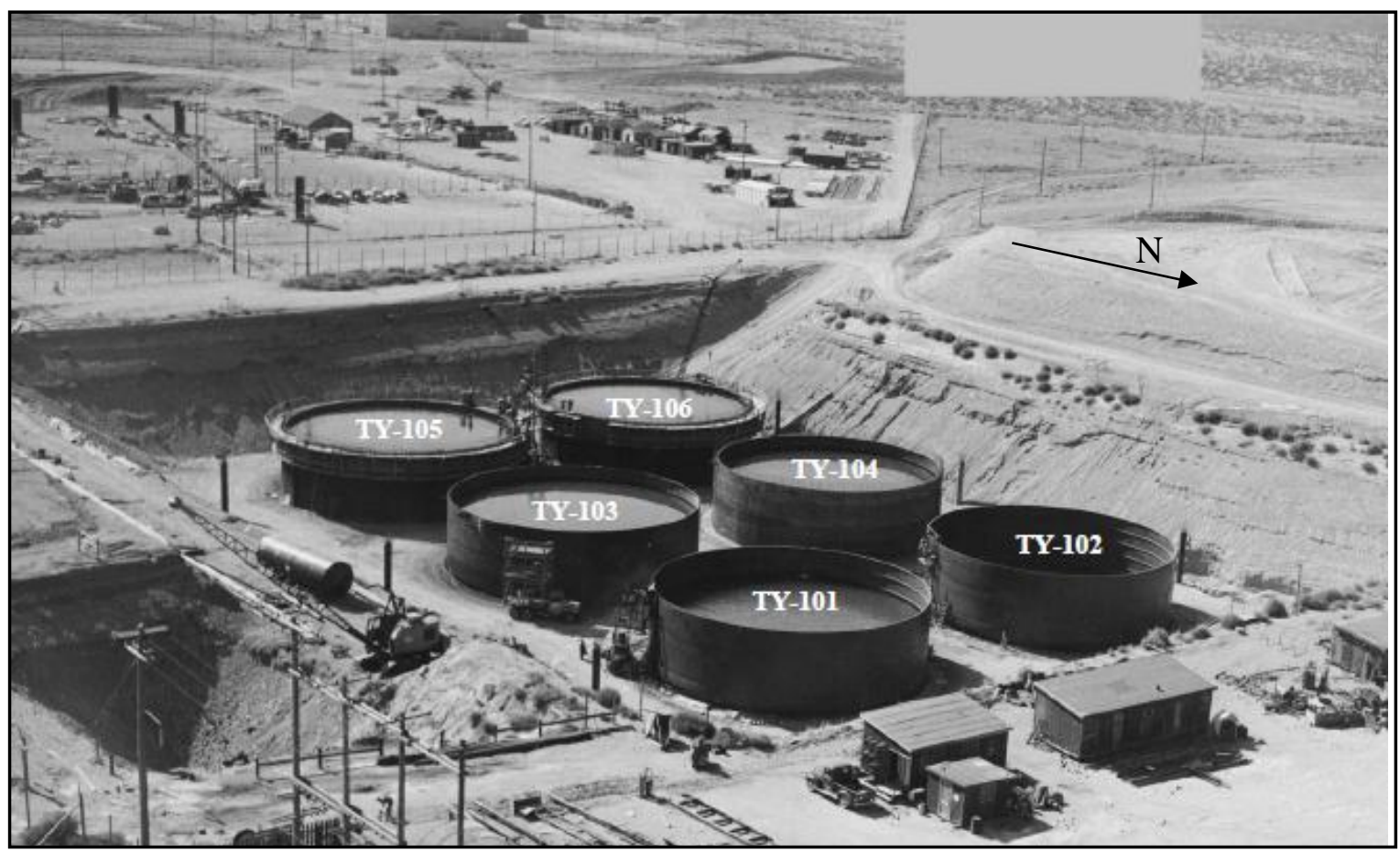

The metallurgical factors that limited carbon steel's ability to resist impact at low temperature were perhaps not well understood when BY and TY Farm were constructed and were not specified for the 0.375-in thick ASTM A 283, Standard Specifications for Intermediate Tensile Strength Carbon Steel Plates, mild carbon steel liner at the time. Current standards for construction of pressure vessels ASME Boiler \& Pressure Vessel Code (B\&PVC), Section VIII, Rules for Construction of Pressure Vessels, provides for a minimum design metal temperature of $18^{\circ} \mathrm{F}$ for vessels constructed of carbon and low alloy steels of nominal thickness $\leq 10-\mathrm{mm}$ (0.394-in). For the purposes of this report, it will be assumed that the $18^{\circ} \mathrm{F}$ design temperature is applicable to the fabrication of ASTM A 283 carbon steel at the time of BY and TY Farm construction.

Boxes from the list of Vendor Information Reports for the BY and TY Farms were searched for any Chemical and Physical Test Reports for the tank steel plates used in the two farms but none were found. No other quality information for either farm was found during the search. 
A review of toughness and the ductile-to-brittle transition temperature for carbon steels (designated as "impact transition temperature") in Mark's Standard Handbook for Mechanical Engineers, Tenth Edition, indicates that carbon content can have a significant effect. Decreased carbon content not only raises the propagation energy needed for crack growth but also lowers the temperature for transition from ductile-to-brittle behavior (reference Fig 6.2.11 in Marks), suggesting that the B\&PVC Section VIII low temperature service limit may be lower than what could be expected for steel of the vintage used in BY and TY Farm construction. The concentrations of carbon and trace impurities and their effect on this property is not specifically known, and low temperature impact resistance could only be determined reliably by impact testing of actual tank specimens.

Only five tanks are leakers among the total eighteen tanks located in BY Farm and TY Farm with four of the leaking tanks in TY Farm. The leaking tanks in the TY Farm experienced a less severe winter than BY Farm. This would tend to indicate that the ductile-to-brittle transition temperature did not play a large part in the BY Farm and TY Farm tank liner leaks.

Below the transition temperature, the metal loses its ability to absorb forces such as induced loads, or the impact of falling objects without fracturing. In this circumstance it is possible for micro-fissures or hairline cracks to be created. Later, when the metal is subjected to high stress, it might be possible for the cracks to propagate through the metal, or possibly subject the weakened areas to increased corrosion.

Any low temperatures experienced during construction at or less than the $18^{\circ} \mathrm{F}$ allowable temperature where impact loading (e.g. a dropped tool or piece of equipment from scaffolding) had the potential for creating micro-fissures may have triggered fissures in the steel liner.

The BY Farm tank liners are A 283 or A 285 carbon steel. The specifications for ASTM A 283 and A 285 carbon steel have changed over time. BY Farm tank construction specification, HW3783, stated that the storage tank liner shall conform to ASTM A-283-46T (Tentative Specifications for Low and Intermediate Tensile Strength Carbon-Steel Plates of Structural Quality) grade A, B, or C or ASTM A-285-46 grade A, B, or C, with additional specific requirements on carbon composition of " $0.25 \%$ maximum." TY Farm tank construction specification, HW-4696, stated that the storage tank liner shall conform to ASTM A-283-49-T grade $\mathrm{B}$, with specific requirements on carbon composition ranging from $0.08 \%$ to $0.16 \%$ (ASTM A-283-49-T, Tentative Specifications for Low and Intermediate Tensile Strength Carbon-Steel Plates of Structural Quality; HW-4696, Specifications Waste Disposal Facilities 241-BZ and TY Tank Farm). Later revisions to ASTM A-283 specifications lowered the allowable carbon content to 0.14 from $0.17 \%$.

The possible variability of liner steel from either different runs from the same supplier, or because of multiple suppliers could affect the resistance to low temperatures.

\subsection{IN-TANK DATA}

The general information in this section is further developed and applied to the leaking tanks in Sections 4.4, 5.4, 6.4, 7.4, and 8.4 to understand implications of the conditions that could affect liner leaks and identify possible liner leak locations. 
RPP-RPT-54911, Rev. 0

\subsubsection{Liquid Level}

The following is an excerpt from RPP-ENV-39658 (Hanford SX-Farm Leak Assessments Report):

"Originally liquid levels were measured using pneumatic dip tubes (HW-10475-C, Hanford Technical Manual Section C, page 908). This practice was later replaced and a manual tape with a conductivity electrode was used to detect the liquid surface $(\mathrm{H}-2$ 2257, Conductor Reel for Liquid Level Measurement). The biggest limitations of the manual tape measurements were failures of the electrodes, solids forming on the electrode and measurement precision. The statistical accuracy of the manual tape and electrode measurement technique was 0.75 in. ( 2,060 gal), as determined in July 1955 (HW-51026, Leak Detection - Underground Storage Tanks, page 4). Later, liquid-level determinations were automated in many of the SSTs to provide more accurate and reliable measurements".

It was stated in RPP-RPT-43704 that the accuracy for the manual tape can vary from 0.25 -in to 2-in for different tanks depending on surface conditions (liquid/solids), boiling, ALC operation, and conductivity.

The in-tank repeatability limits for FIC liquid level gauges are \pm 0.25-in (Letter 72730-80-097, "Review of Classification of Six Hanford Single-Shell "Questionable Integrity (QI)" Tanks").

Transfer discrepancies of greater than 1.5-in (4125 gal) measured at the first hour and every two hours thereafter with an FIC, manual tape, or flowmeter required an orderly and immediate shutdown, investigation, and notification. The 1.5-in discrepancy requirement was a specification limit in ARH-1601, Section D, Specifications and Standards for the Operation of Radioactive Waste Tank Farms and Associated Facilities.

Liquid level measured by manual tape (MT) is calculated for BY and TY Farm tanks with the formula: volume $=($ MT Reading -12 -in $)$ X $(2750$ gal/in $)+12,500$ gal $($ LET-082172, H.N . Raymond to C.J. Francis, August 21, 1972, Maximum Operating Levels and Cascade Levels in 200-West area Tank Farms [IDMS Accession D196208887]). Even though the letter title indicates only West area, the above formula for both the BY and TY tanks is found in the letter. The formula was confirmed to have been used as late as 1980 in RHO-CD-896, page 76, for the then current tank T-111 volume (488 kgal) and MT reading (173-in) which verified use of the formula. All half yearly and quarterly report ending volumes in this document were calculated with this formula. Original MT readings and the MT readings in PCSACS are all measured to the bottom inside center of the tanks. The ENRAF liquid level readings in PCSACS also read to the bottom inside center of the tank.

\subsubsection{Temperature}

Individual tank segments will address tank specific temperatures in relation to the applicable temperature requirements (ARH-951, Limitations for Use of Underground Waste Tanks). The ARH-951 document was issued December 18, 1969 and indicated that tank temperatures for BY and TY Farm should be held below $230^{\circ} \mathrm{F}$ with a $5^{\circ} \mathrm{F}$ per day rise for liquid temperatures below $180^{\circ} \mathrm{F}$ and a $3^{\circ} \mathrm{F}$ per day rise for liquid temperatures above $180^{\circ} \mathrm{F}$ during waste addition to the 
RPP-RPT-54911, Rev. 0

tank. This was the earliest operation limitations found for these tank farms. The boiling waste tank farms had higher temperature limitations associated with the tank liners as well as the tank exhaust system (H-2-36933, Limitations for Existing Storage Tanks for Radioactive Wastes from Separations Plants).

Document HW-20742, Loss of Depleted Metal Waste Supernate to Soil, reports metal waste (MW) was cascaded into a series of tanks with tank BY-103 being the last in the series of six tanks (including BX-101, BX-102, and BX-103) and temperatures were recorded to be approximately $70^{\circ} \mathrm{F}$ in tank BY-103. Tank BY-103 also received PUREX HLW supernatant which would probably be at or slightly above $180^{\circ} \mathrm{F}$.

Tri-Butyl Phosphate (TBP) wastes were concentrated and cooled to $\sim 180^{\circ} \mathrm{F}$ within the plant and were estimated to be $110-180^{\circ} \mathrm{F}$ after pumping to the storage tanks (HW-19140, Uranium Recovery Technical Manual, p. 1209).

No temperature data were recovered for the leaking BY and TY Farm tanks when the tanks first received waste until approximately 1970 (see PCSACS).

\subsubsection{Liner Observations}

A bulge in a tank liner may result in the direct failure of the liner or cause enough stress or thinning on the steel liner plates and welds that they become more susceptible to the effects of corrosion. Experience indicates that bulging tends to be a dynamic phenomenon, and it is possible that a tank with no measured bulge at one point in time may actually have had a displaced liner that was not detected at another time.

No liner bulges were reported for the BY Farm and TY Farm tanks.

\subsubsection{Chemistry}

The types of corrosion that may occur in the Hanford Site SSTs include uniform corrosion, stress corrosion cracking (SCC), pitting, crevice, and liquid-air interface corrosion which were identified in HNF-3018, Rev. 0, Single-Shell Tank Sluicing History and Failure Frequency.

Uniform corrosion rates for SSTs are reported to be generally less than 1 mil/year (HNF-3018, Rev. 0) for the SSTs. Carbon steel exposed to alkaline solutions has a low general corrosion rate (PNL-5488, Prediction Equations for Corrosion Rates of A-537 and A-516 Steels in Double Shell Slurry). However, the presence of the nitrate ion may induce various forms of localized attack (i.e., SCC, pitting, etc.).

\section{Nitrate Ion-Induced Stress Corrosion Cracking}

Stress corrosion cracking is the growth of cracks in a corrosive environment. It can lead to unexpected sudden failure of normally ductile metals subjected to a tensile stress, especially at elevated temperatures. The chemical environment that causes SCC for a given alloy is often one which is only mildly corrosive to the metal but the condition for SCC is highly dependent on specific waste chemistries. 
Nitrate ion-induced SCC is the predominant threat to the integrity of the steel liners in the SSTs and double-shell tanks (DSTs) at the Hanford Site and many investigations have been performed to establish the parameters under which the tanks can be protected from this threat. This work, together with the efforts of many others, led to the adoption of the waste chemistry control limits for SCC prevention in 1983 (OSD-T-151-00017, Operating Specifications for the Aging Waste Operations in Tank Farms 241-AY and 241-AZ).

The factors governing the rates of nitrate ion-induced SCC cracking by Hanford Site DST wastes were recently reviewed (RPP-RPT-47337, Specifications for the Minimization of the Stress Corrosion Cracking Threat in Double-Shell Tank Wastes). In brief, the test results led to the conclusion that the rates of nitrate ion-induced SCC depended on the properties of the steel, the applied potential versus the open circuit potential (OCP), the temperature and the concentrations of aggressive substances such as nitrate ion, and the potential inhibitors such as hydroxide and nitrite ion.

The technical work has shown that SCC is promoted by high temperatures, high nitrate ion concentrations, low hydroxide ion concentrations, low nitrite ion concentrations, and low nitrite ion/nitrate ion concentration ratios. Tanks with maximum temperatures less than $122^{\circ} \mathrm{F}$ would not be expected to experience significant SCC damage regardless of waste types (HNF-3018, Rev. 0). Tanks with the maximum temperatures above $122^{\circ} \mathrm{F}$ and a ratio of nitrate concentration to the sum of nitrite and hydroxide concentrations greater than 2.5 would be expected to suffer SCC-related damage (HNF-3018, Rev. 0). The concentration of nitrate and temperature are parameters that have the most effect on SCC. However, the $\mathrm{pH}$ (hydroxide) and nitrite can inhibit SCC. The current DST operating specifications for chemistry are reported in OSD-T151-00007, Rev. 10, Operating Specifications for the Double-Shell Storage Tanks. While the chemistry specifications stated in this document were prepared for the DSTs, corrosion mechanisms and corrosion protection mechanisms applicable to DST primary tank metal liners are equally applicable to the older SST metal liners.

\section{Localized Corrosion: Crevice, Pitting, and Liquid-Air Interface Corrosion}

Crevice corrosion can occur in regions where a small volume of solution cannot readily mix with the bulk solution such as under deposits, between metal flanges, and other confined areas. Once initiated, crevice corrosion proceeds by the same mechanism as pitting corrosion (RPP-RPT33306, IQRPE Integrity Assessment Report for the 242-A Evaporator Tank System).

Pitting corrosion is the localized corrosion of a metal surface confined to a point or small area that takes the form of cavities. Pitting corrosion in dilute solutions $\left(\mathrm{NO}_{3}{ }^{-}<1 \mathrm{M}\right)$ of waste has been studied at the Savannah River Site (SRS). Pitting has been determined to not be a problem at hydroxide concentrations greater than $1 \mathrm{M}$ for any of the diluted waste solutions tested (WSRC-TR-90-512, Effect of Temperature on the Nitrite Requirement to Inhibit Washed Sludge, Oblath and Congdon 1987, Inhibiting Localized Corrosion during Storage of Dilute Waste). Nitrate ion was determined to be the usual controlling aggressive species when its concentrations ranged between $0.01 \mathrm{M}$ and 1M (WSRC-TR-90-512). The presence of hydroxide ion and nitrite ion has shown to inhibit pitting corrosion due to the aggressive nitrate ion. This work led to the conservative recommendation that the concentration of nitrite ion be greater than 
$0.033 \mathrm{M}$ for the avoidance of pitting in dilute solutions of nitrate ion at $\mathrm{pH} 10$ and $40^{\circ} \mathrm{C}\left(104^{\circ} \mathrm{F}\right)$ (RPP-ASMT-53793, Rev. 0).

The chemical compositions required for prevention of pitting corrosion can also be applied as limits for prevention of liquid-air interface corrosion at the surface of the supernatant.

Crevice, pitting, and liquid-air interface corrosion are types of localized corrosion possible in the SSTs; however, historically SCC is the more predominant type of corrosion of concern.

\section{Historical Corrosion Control}

The earliest chemical specifications for SSTs addressing $\mathrm{pH}$, nitrite, nitrate, and hydroxide are listed in Table 3-1 (ARH-1601, Section D, Specifications and Standards for the Operation of Radioactive Waste Tank Farms and Associated Facilities, 1973).

Table 3-1. ARH-1601 Specifications 1973

\begin{tabular}{|c|c|}
\hline \multicolumn{2}{|c|}{ Waste Tank Farms and Associated Facilities Specifications } \\
\hline Variable & Specification \\
\hline $\mathrm{pH}$ & Minimum 8.0 \\
\hline $\mathrm{NO}_{2}^{-}$ & $500 \mathrm{ppm}$ \\
\hline $\mathrm{NO}_{3}^{-}$ & $<6 \mathrm{M}$ \\
\hline $\mathrm{OH}^{-}$ & $<7 \mathrm{M}$ \\
\hline
\end{tabular}

There was no similar specification found that addressed all of these parameters during the operation of BY and TY Farm prior to 1973. However, if the ARH-601 specifications were in effect during BY and TY Farm waste storage, the storage of undesirable concentrations of $\mathrm{NO}_{2}{ }^{-}$, $\mathrm{NO}_{3}{ }^{-}$, and $\mathrm{OH}^{-}$would result in vulnerability to $\mathrm{SCC}$ and/or localized corrosion if temperatures were elevated.

Historical waste sample data as well as temperatures are typically not available for the SSTs and none were recovered for the leaking BY and TY Farm tanks. Thus, the concentrations of $\mathrm{NO}_{2}{ }^{-}$, $\mathrm{NO}_{3}{ }^{-}$, and $\mathrm{OH}^{-}$listed in Sections 4.4.4, 5.4.4, 6.4.4, 7.4.4, and 8.4.4 are typical concentrations that were reported for the waste types listed that could be based on limited data and/or were values obtained from process flowsheets. Therefore, waste chemistry conditions are speculative when sample and temperature data is unavailable especially when multiple waste types are present in the tank.

\subsubsection{Photographs}

Available photographs of the leaking tanks in BY and TY Farm were reviewed. Photographs were reviewed to identify beachlines possibly indicating previous operations of overfilling the tank, damaged equipment, possible liner bulges, and any other anomalies that could be indicative of a tank liner leak, and/or possible leak location. See the photograph sections in the individual tank segments for details. The photographs do not appear to indicate any anomalies such as over fill "beach lines" except for tank TY-104 or indications of liner bulging. No other documentation was found indicating a liner bulge for the leaking BY and TY Farm tanks. 
RPP-RPT-54911, Rev. 0

\subsection{EX-TANK DATA}

The general information in this section is further developed and applied to the leaking tanks in Sections 4.5, 5.5, 6.5, 7.5, and 8.5 to understand implications of the conditions that could affect liner leaks and identify possible liner leak locations.

\subsubsection{Laterals}

Leak detection laterals were installed approximately 10-ft underneath some of the tanks containing self-boiling waste in 241-A and 241-SX Farms. Lateral leak detection systems were not installed under the BY and TY Farm tanks.

\subsubsection{Drywells}

Seven original BY Farm drywells were drilled around the tanks in 1949 (H-2-36933, Well Information As-Built 200 E Area; RPP-RPT-43704, Hanford BY-Farm Leak Assessments Report). One BY Farm drywell, 22-11-08, was reported being drilled in 1944 (GJ-HAN-28, Tank Summary Data Report for Tank BY-111). The remainder of the BY Farm drywells were drilled between 1967 and 1974 (H-2-36933). Three original TY Farm drywells were drilled around the tanks in 1952 (H-2-36942). The remainder of the TY Farm drywells were drilled between 1971 and 1977 (H-2-36942). Drywells were drilled vertically from the surface and drywell coordinates and detailed drywell information, e.g. pipe dimensions and configuration, for the leaking BY and TY Farm tanks are addressed in references sited in the individual tank segments. Drywells will not be useful to detect releases that enter the soil from the tank unless the volume released is sufficiently large to facilitate lateral transport to a drywell typically to within $\sim 1$-ft of the drywell. The vertical height of a tank liner leak may not be directly related to the point of detection in the drywell. This is especially true for small leaks that may flow downward some distance before encountering a drywell.

The "00" series drywells (drywell 22-00-01, BY Farm) were installed shortly after tank construction, usually around the periphery of the farm and most extend to 150-ft BGS. Others with tank numbers embedded in the drywell number (22-03-01, tank BY-103) were constructed later, sometimes after tank operations had ceased and generally to 100-ft BGS, with a few deeper than 100-ft BGS. The usual number of drywells surrounding a tank is one to four. If there are more, then there likely was some concern regarding a release which was being investigated. The third number corresponds to the clocked position of the drywell with respect to due north.

Four gamma ray probe types were used to monitor gamma in drywells to detect leaks (HNF3136, Analysis Techniques and Monitoring Results, 241-SX Drywell Surveillance Logs). The most widely used probe was the unshielded gross gamma sodium-iodide (NaI) probe (or probe 04 and the shielded NaI probe was referred to as probe 14). The NaI probe (04) is very sensitive and able to record gamma ray activity from 30 counts per second (cps) up to about 40,000 cps $(15 \mathrm{mR} / \mathrm{hr})$ before the data becomes unreliable (RHO-RE-EV-4, Supporting Information for the Scientific Basis for Establishing Dry Well Monitoring Frequencies. The next most commonly used probe was the Red-GM (or probe 02 ) which is less sensitive but can reliably record gross gamma at much higher levels of activity (up to $\sim 500 \mathrm{R} / \mathrm{hr}$ ). Operation of these and other probes are discussed in HNF-3136. A scintillation probe (SP) was also used to measure low levels of 
radiation in the drywells. Leak location identification is primarily focused on the first indication of a leak and is therefore typically concerned with the lower levels of gross gamma detection and initial migration.

Drywell sections in the individual tank segments contain gross gamma figures taken from HNF3532, Analysis of Historical Gross Gamma Logging Data from BY Tank Farm, and HNF-3831, Analysis of Historical Gross Gamma Logging Data from TY Tank Farm, showing continuing or new contamination in the drywells based on BGS depth from 1975 to 1995 . Some of these gross gamma figures show anomalous data that appear to be unexplained detections that do not reflect radioactivity in the soil. In 1997 and 1998, a baseline characterization of the gamma-rayemitting radionuclides distributed in the vadose zone sediments beneath and around BY and TY Farm was performed using spectral gamma logs (SGLS) and documented in GJO-HAN-6, Vadose Zone Characterization Project at the Hanford Tank Farms BY Tank Farm Report, and GJO-HAN-16, Vadose Zone Characterization Project at the Hanford Tank Farms TY Tank Farm Report, respectively. The gross gamma figure detection sensitivity is lower than SGLS ( 10 $\mathrm{pCi} / \mathrm{g}$ versus $\sim 0.1 \mathrm{pCi} / \mathrm{g}$ equivalent Cs-137). Therefore, radioactivity $\leq 10 \mathrm{pCi} / \mathrm{g}$ does not appear on the gross gamma figures (GJO-HAN-6; GJO-HAN-16). SGLS logging can confirm both Cs137 and/or Co-60 radioactivity which can assist in the leak location analysis, and the SGLS data is weighted more heavily on interpreting drywells. The criteria for drywell monitoring are defined in RHO-ST-34 (A Scientific Basis for Establishing Drywell-Monitoring Frequencies) with the monitoring frequency found in SD-WM-TI-356 (Waste Storage Tank Status and Leak Detection Criteria).

All of the radiation readings in drywells are assumed to be maximum or peak readings unless otherwise noted and are from the Red-GM probe unless otherwise indicated. The individual tank segments report the available drywell data in the drywell section and in some cases the more recent direct push drywells installed to locate detailed soil radioactivity. The drywell summary section provides the analyses of the associated drywells and any direct pushes with the tank that is of concern.

In 2005, additional direct pushes were installed around tanks TY-103, TY-105, and TY-106 to further characterize the leaks from these tanks due to the lack of drywells located in these areas (RPP-RPT-34870, Small Diameter Geophysical Logging in the 241-TY Tank Farm). Results of the direct pushes are addressed in the individual tank segments.

\subsection{LINER LEAK LOCATIONS}

Drywell radioactivity when first detected can indicate a radial or depth location of a tank leak, migration of the tank leak, or the possible migration of an adjacent tank leak. The radial drywell radioactivity is also dependent on any possible flow paths from the actual tank liner leak location to the drywell itself as well as the waste viscosity and distance to the drywell. Drywells can also be an indication of the tank liner sidewall leak depending on the vertical location but need to be analyzed relative to non-tank liner leaks associated with pipe lines or other sources.

Liquid level decreases can be used for sidewall as well as bottom liner leaks but need to be analyzed in relationship with the vertical level of the tank drywell radioactivity, evaporation and drywell contamination from pipe line leaks and other non-tank sources. 
A liner leak may have penetrated the waterproof membrane at any location and followed concrete cracks or construction joints to a different location including the top of the tank footing. Therefore, the point of waste egress from the tank liner may not be the point of entry of the leaking waste to the soil. Later indications of radioactivity in the drywells with improved detector capabilities could indicate additional leakage but the location of the leak could not be pinpointed without some additional information.

The lack of radioactivity above background in a drywell indicates that if there was a liner leak it either occurred at another location and/or the leak flow was insufficient to be detected with the probes used in the drywell. When there is no radioactivity detected in a drywell or no recoverable data for a drywell it is not included as part of the leak location analysis.

Later 2005 direct pushes can help further characterize the leak location by supporting earlier data and/or providing additional information.

\subsection{POSSIBLE LINER LEAK CAUSE(S)}

Analysis of the BY and TY Farm commonalities which centered on tank design/construction, intank data, and ex-tank data indicates that there were up to three BY and TY Farm tank conditions that could contribute to a failed liner: thermal shock, chemistry-corrosion, and tank construction temperature. There appears to be very little contribution from tank design (no inherent flaws have been documented in the literature reviewed) and liner bulging. Some or all of the factors can act serially or together to contribute to tank liner failure. The following sections provide a tank-by-tank review of these conditions as they relate to liner leak causes.

Other general tank construction factors such as the quality of materials and fabrication could also contribute to tank liner failure. Because no evidence has been found to substantiate quality defects, these are not included as a leak cause. 


\subsection{TANK BY-103 SEGMENT}

\section{TABLE OF CONTENTS}

4.1 Tank BY-103 Background History ...................................................................... 4-4

4.2 Tank BY-103 Operations Summary ...................................................................... 4-5

4.3 Tank Design/Construction.................................................................................... 4-7

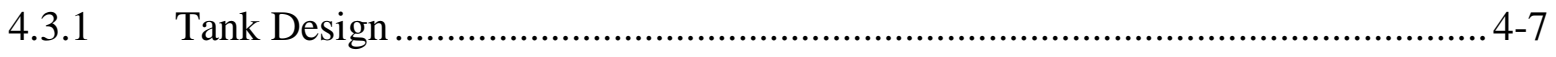

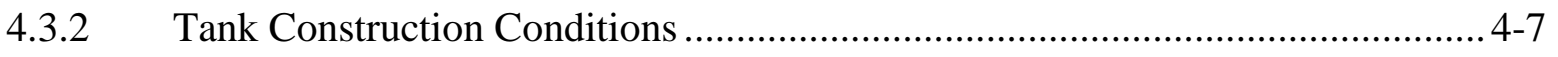

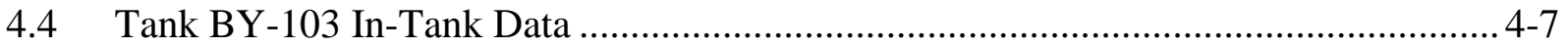

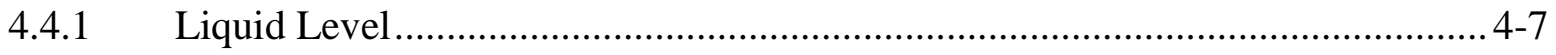

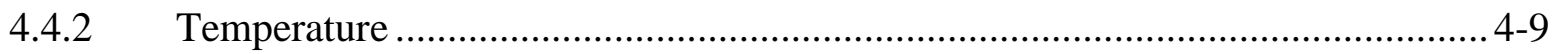

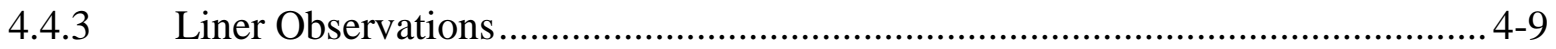

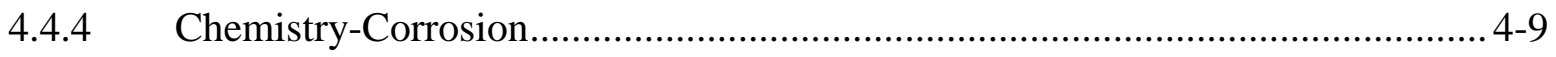

4.4.5 Photographs............................................................................................... 4-11

4.5 Tank BY-103 Ex-Tank Data ………………................................................... 4-12

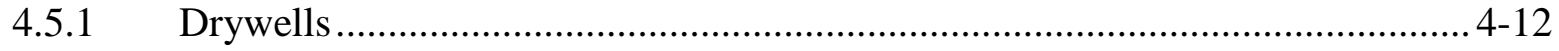

4.5.1.1 Drywell 22-00-01 (N53 $\left.{ }^{\circ} \mathrm{W}\right)$............................................................... 4-13

4.5.1.2 Drywell 22-00-02 (S60 $\left.{ }^{\circ} \mathrm{E}\right)$.................................................................... 4-14

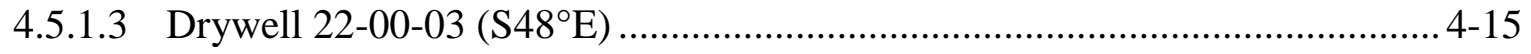

4.5.1.4 Drywell 22-00-04 (S37 $\mathrm{E})$................................................................. 4-16

4.5.1.5 Drywell 22-03-01 (N30 $\left.{ }^{\circ} \mathrm{E}\right)$................................................................. 4-17

4.5.1.6 Drywell 22-03-04 (S62 ${ }^{\circ}$ ) ……………............................................ 4-18

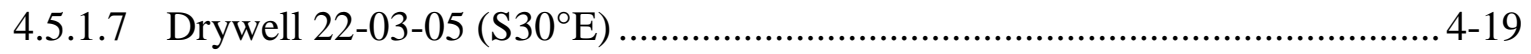

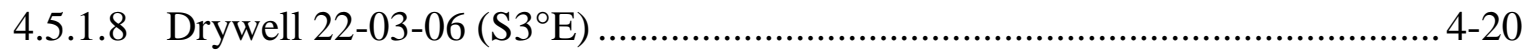

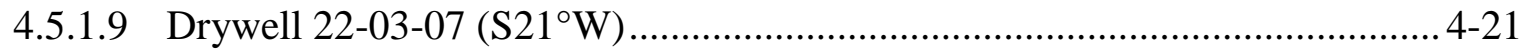

4.5.1.10 Drywell 22-03-08 (S43ํ) W) .............................................................. 4-22

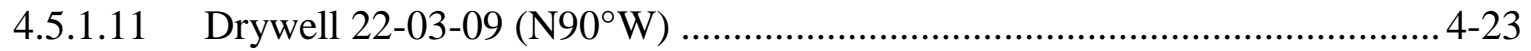

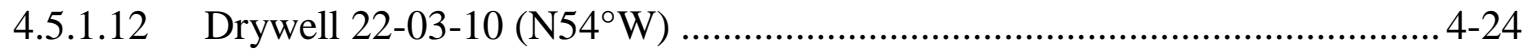

4.5.2 Drywell Summary ……………………................................................ 4-25

4.6 Possible Tank BY-103 Liner Leak Location(s) ……................................................ 4-26

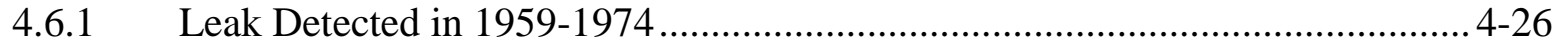

4.6.2 Leak Detected in February 1973 ................................................................... 4-28

4.6.3 Leak Detected in 1972-1974 .......................................................................... 4-29 
4.6.4 Leak Location Summary ..................................................................... 4-30

4.7 Possible Tank BY-103 Liner Leak Cause(s) ………........................................... 4-32

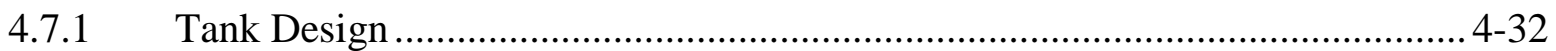

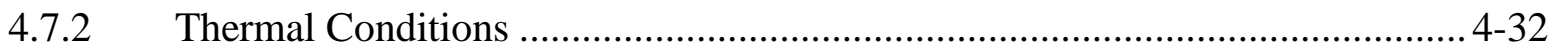

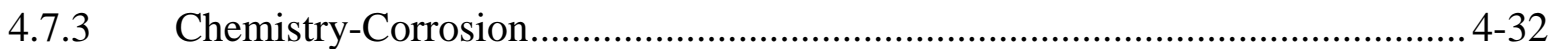

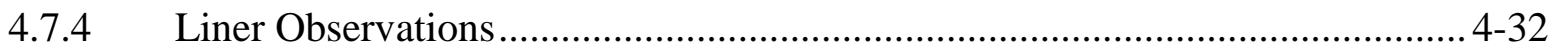

4.7.5 Tank Construction Temperature ................................................................ 4-33

4.8 Tank BY-103 Conclusions ................................................................................... 4-33

\section{APPENDICES}

Appendix A1 Tank BY-103 Gross Gamma Drywell Data ..................................................... A1-1

\section{LIST OF FIGURES}

Figure 4-1. Tank BY-103 Associated Drywells .................................................................. 4-4

Figure 4-2. Operational Leak History of Tank BY-103 …………………………................... 4-6

Figure 4-3. Tank BY-103 End of Quarter Surface Level ...................................................... 4-8

Figure 4-4. Tank BY-103 Tar Seepage March 5, 1981 .................................................... 4-12

Figure 4-5. Tank BY-103 Drywell 22-00-01 (HNF-3532) ................................................... 4-13

Figure 4-6. Tank BY-103 Drywell 22-00-02 (HNF-3532) ……………………................... 4-14

Figure 4-7. Tank BY-103 Drywell 22-00-03 (HNF-3532) ……………………………....... 4-15

Figure 4-8. Tank BY-103 Drywell 22-00-04 (HNF-3532) .................................................... 4-16

Figure 4-9. Tank BY-103 Drywell 22-03-01 (HNF-3532) ................................................... 4-17

Figure 4-10. Tank BY-103 Drywell 22-03-04 (HNF-3532) ................................................... 4-18

Figure 4-11. Tank BY-103 Drywell 22-03-05 (HNF-3532) ……………………………….... 4-19

Figure 4-12. Tank BY-103 Drywell 22-03-06 (HNF-3532) ………………………….......... 4-20

Figure 4-13. Tank BY-103 Drywell 22-03-07 (HNF-3532) ................................................... 4-21

Figure 4-14. Tank BY-103 Drywell 22-03-08 (HNF-3532) ................................................... 4-22 
Figure 4-15. Tank BY-103 Drywell 22-03-09 (HNF-3532) ................................................... 4-23

Figure 4-16. Tank BY-103 Drywell 22-03-10 (HNF-3532) .................................................... 4-24

Figure 4-17. Tank BY-103 Possible Leak Location (1959-1974) ........................................... 4-27

Figure 4-18. Tank BY-103 Possible Leak Location (February 1973) ...................................... 4-28

Figure 4-19. Tank BY-103 Possible Leak Location (1972-1974) ............................................ 4-29

Figure 4-20. Tank BY-103 Possible Radial Leak Locations .................................................... 4-31

\section{LIST OF TABLES}

Table 4-1. Tank BY-103 Waste Storage Chronology ………….............................................. 4-10

Table 4-2. Waste Chemistries for Waste Types Stored in Tank BY-103 ................................. 4-10

Table A1-1. Tank BY-103 Drywell Radioactivity (K counts per minute) ..............................A1-2

Table A1-2. Tank BY-103 Associated Drywell Radioactivity (K counts per minute)............ A1-6 


\subsection{TANK BY-103 BACKGROUND HISTORY}

This section provides information on the historical waste loss event associated with Single-Shell Tank (SST) 241-BY-103 (BY-103). There are twelve drywells located around tank BY-103 with specified distances from the drywell to the tank footing shown in Figure 4-1: 22-00-01 and 2200-03 installed in July 1949; 22-03-01, 22-03-05, and 22-03-09 installed in August 1970; 22-0304 and 22-03-06 installed in December 1972; and 22-00-02, 22-00-04, 22-03-07, 22-03-08, and 22-03-10 installed in December 1973.

The bottom of the tank footing is $\sim 48$ - $\mathrm{ft} 7$-in Below Grade Surface (BGS) with $\sim 8$ - $\mathrm{ft}$ soil cover over the dome (WHC-SD-WM-TI-665, Soil Load above Hanford Waste Storage Tanks; H-21312, 75 Foot Tank Sections).

Figure 4-1. Tank BY-103 Associated Drywells

Tank inner ring is steel liner, outer ring is outer edge of tank footing

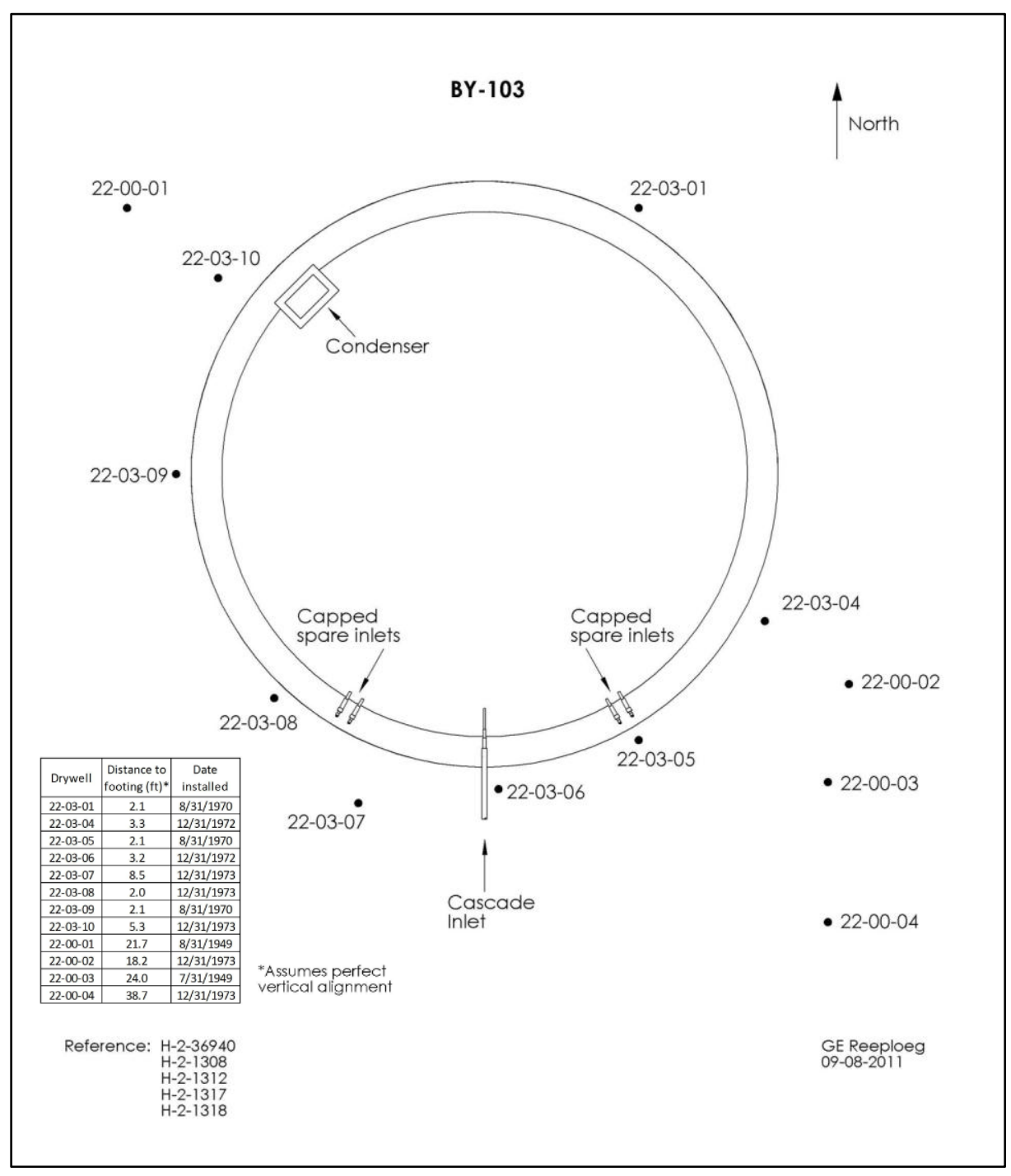


RPP-RPT-54911, Rev. 0

\subsection{TANK BY-103 OPERATIONS SUMMARY}

Tank BY-103 was constructed from 1948 to 1949 and started receiving metal waste (MW) in October 1950 from the cascade line from tank BY-102 and was filled full by March 1951. The MW in tank BY-103 was from the bismuth phosphate plutonium separations process that contained all of the uranium, approximately 90 percent of the original fission product activity, and approximately one percent of the plutonium. In May 1954, tank BY-103 underwent MW removal operations to provide feed to the 221-U Tri-Butyl Phosphate (TBP) plant (WHC-MR0132, A History of the 200 Area Tank Farms).

Tank BY-103 was emptied to tanks C-104 and C-105, and was declared empty on June 3, 1954. Tank BY-103 then received $\sim 739 \mathrm{kgal}$ of TBP waste from the 221-U TBP Plant in 1955 and the volume was reported at approximately $739 \mathrm{kgal}$ through the second quarter of 1957 . Beginning in the third quarter 1957, approximately $700 \mathrm{kgal}$ of TBP waste was transferred out of the tank leaving $\sim 26 \mathrm{kgal}$ of TBP waste in tank BY-103. In the fourth quarter of 1957, approximately $421 \mathrm{kgal}$ of PUREX high level waste (HLW) supernatant was received from tank C-106. The volume of tank BY-103 was reported at $736 \mathrm{kgal}$ at the end of the first quarter 1957. In 1957, ferrocyanide scavenging operations took place in tank BY-103 to recover Cs-137. It appears no other transfers took place in tank BY-103 from the first quarter of 1958 to the second quarter of 1965 (WHC-MR-0132).

On March 19, 1965 the in-tank solidification (ITS) unit \#1 (ITS-1) was started up and was operated until August 1971, when it was converted from an evaporator to a cooler for ITS unit \#2 (ITS-2). Both units were shut down June 30, 1974 (RPP-RPT-43704). During operation of the ITS units, tank BY-103 served as a feed tank for ITS-1 and ITS-2 for coating waste (CW) from various tanks. Waste was also transferred to and from other BY Farm tanks, and tank BY-103 received evaporator bottoms (EB) waste generated by the ITS program. See document RPPRPT-55804, Common Factors Relating to Liner Failures in Single-Shell Tanks, for additional information on the ITS process.

In July 1969, high radiation counts were obtained in drywell 22-00-03 and tank BY-103 was classified as a "borderline leaker" in 1970 (Internal letter "Integrity of Tanks 241-BY-103 and 241-TY-103"). However, data were not recovered for drywell 22-00-03 during this time. In 1971, high count rates in the newly drilled drywell 22-03-05 were obtained, and the liquid operating level in tank BY-103 was lowered to 13-ft to permit continued operation of the tank as a bottoms receiver in the ITS program (see Section 4.4.1). Radioactivity continued to be detected in the surrounding drywells and tank BY-103 was classified as a confirmed leaker in 1973. Tank BY-103 could have started leaking as early as 1959 based on radioactivity that was detected in drywell 22-00-03 (see Appendix A1).

Tank BY-103 was declared stabilized in November 1997 by use of a saltwell jet pump. Tank BY-103 is estimated to contain $9 \mathrm{kgal}$ of sludge, $405 \mathrm{kgal}$ of saltcake, $55 \mathrm{kgal}$ of drainable interstitial liquid, and no supernatant (HNF-EP-0182, Rev. 301, Waste Tank Summary Report for Month Ending April 30, 2013).

The operational history of tank BY-103 leak related details including liquid level is charted in Figure 4-2. 


\section{Figure 4-2. Operational Leak History of Tank BY-103}

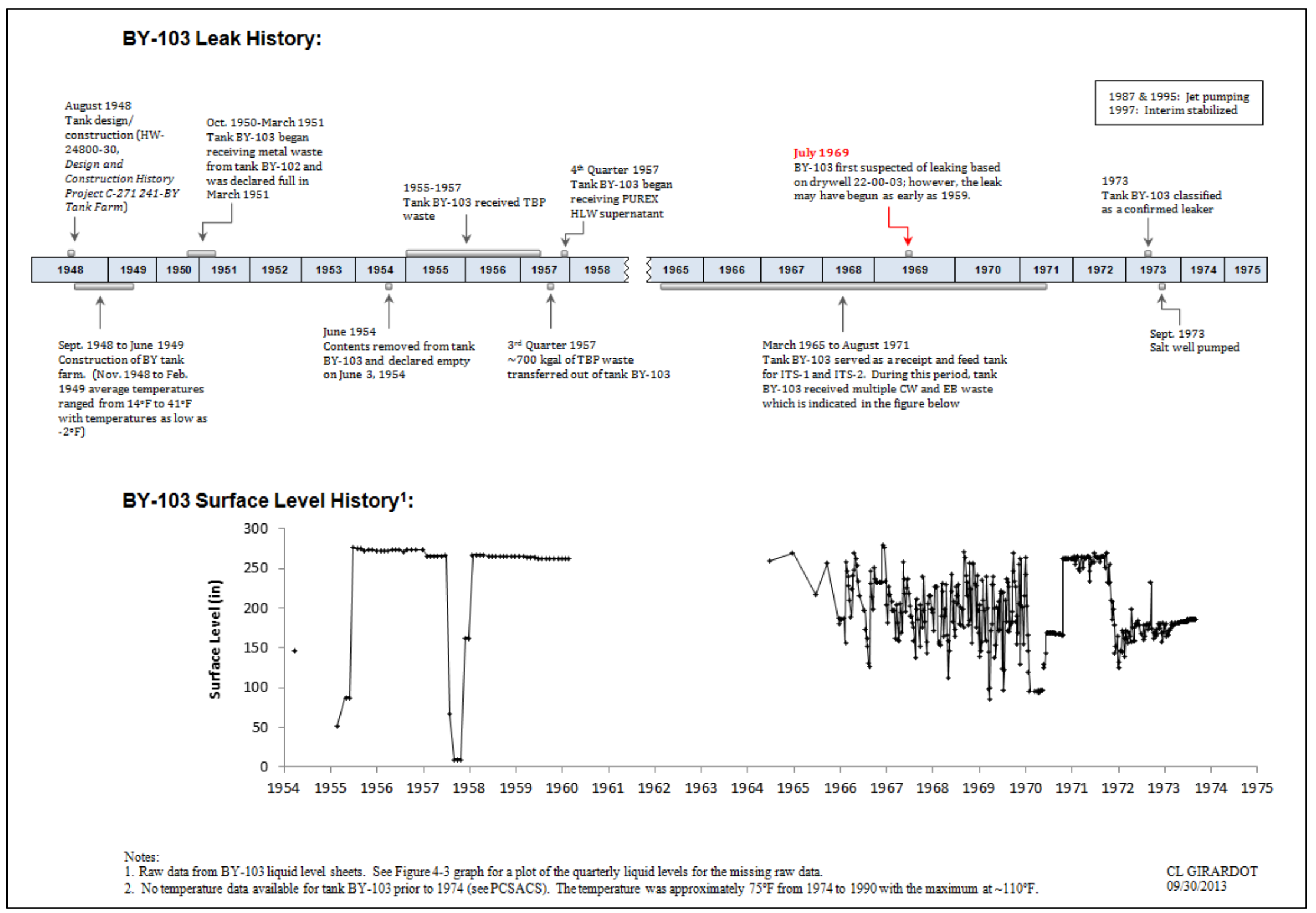


RPP-RPT-54911, Rev. 0

\subsection{TANK DESIGN/CONSTRUCTION}

\subsubsection{Tank Design}

The BY Farm tank design continued important features of the earlier 241-BCTU tanks (BPF73550). The steel bottom intersects the sidewall on a 4-ft radius. Full penetration butt welds with $\mathrm{x}$-ray inspection and three ply asphaltic membrane waterproofing between the wall liner and the concrete shell continued those design features found to be important for liner integrity during the 241-SX Farm leak assessment (RPP-RPT-54910, Hanford Single-Shell Tank Leak Causes and Locations - 241-SX Tank Farm). The top of the tank footing is 45-ft 7-in BGS and is 3 - $\mathrm{ft}$ thick with the bottom of the footing at $\sim 48$ - $\mathrm{ft} 7$-in BGS.

\subsubsection{Tank Construction Conditions}

The BY Farm was constructed from September 1948 to June 1949. Cold weather can affect the steel construction during the months of December and January. Temperatures for December 1948 and January 1949 averaged $26.9^{\circ} \mathrm{F}$ and $13.9^{\circ} \mathrm{F}$, respectively, with temperatures as low as $-2^{\circ} \mathrm{F}$ (PNNL-15160). Weather precautions for welding and concrete were specified in HW-3783 (see Section 3.1.2).

As described in Section 3.1.2, cold weather affects the ductile-to-brittle steel transition temperature, with $18^{\circ} \mathrm{F}$ being the assumed unrestricted low temperature construction limitation for the carbon steel liner, which could result in a fracture upon impact. Low temperatures experienced during construction at or less than the $18^{\circ} \mathrm{F}$ allowable temperature could cause impact loading (e.g. a dropped tool or piece of equipment from scaffolding) and result in the potential for creating micro-fissures weakening the steel liner.

\subsection{TANK BY-103 IN-TANK DATA}

\subsubsection{Liquid Level}

The tank BY-103 liquid level was apparently not reviewed for a decrease when radioactivity was first detected in the drywells in 1969 as no mention was found in any of the monthly reports, individual letters, or previous leak assessment reports examined for this report. No liquid level data sheets were found for tank BY-103. In 1971, after high counts of radioactivity were found in drywell 22-03-05, the liquid level was lowered to $13-\mathrm{ft}$ solids level in tank BY-103. The liquid level was lowered to permit continued use of the tank in the ITS-1 and 2 bottoms loop (Internal letter "Integrity of Tanks 241-BY-103 and 241-TY-103"). It is unclear whether the lowering of the liquid level was related to the drywell radioactivity. No liquid level data were recovered for tank BY-103 except for quarterly liquid level data (WHCMR-0132).

In September 1973 a P-10 salt well system was installed in tank BY-103. Three pumping events in October 1976 yielded only 1,200 gal of supernatant (SD-WM-TI-356). Jet pumping was initiated on March 31, 1987 and total pump production was 78,500 gal before the pump failed on September 8, 1987. Pumping resumed on August 5, 1995 responding to an increased liquid level and an additional $17 \mathrm{kgal}$ was pumped out before the pump was shut 
down on October 20, 1995. On November 24, 1997 interim stabilization was determined to be completed with less than $38 \mathrm{kgal}$ of pumpable liquid remaining in the tank (HNF-SD-RETI-178, Single-Shell Tank Interim Stabilization Record).

The leak volume for tank BY-103 was estimated to be $<5 \mathrm{kgal}$, roughly equivalent to a 2 -in liquid level drop, and may be based on a 2-in uncertainty for manual tape measurements (RPP-RPT-43704). No documented evaluation for the leak volume was recovered.

The liquid level plot in Figure 4-3 indicates the transfer activity into and out of tank BY-103. The liquid levels are end of quarter levels so this figure may not reflect all transfers into and out of the tank that occurred during the operational history. See Figure 4-2 for historical monthly liquid level readings.

Figure 4-3. Tank BY-103 End of Quarter Surface Level

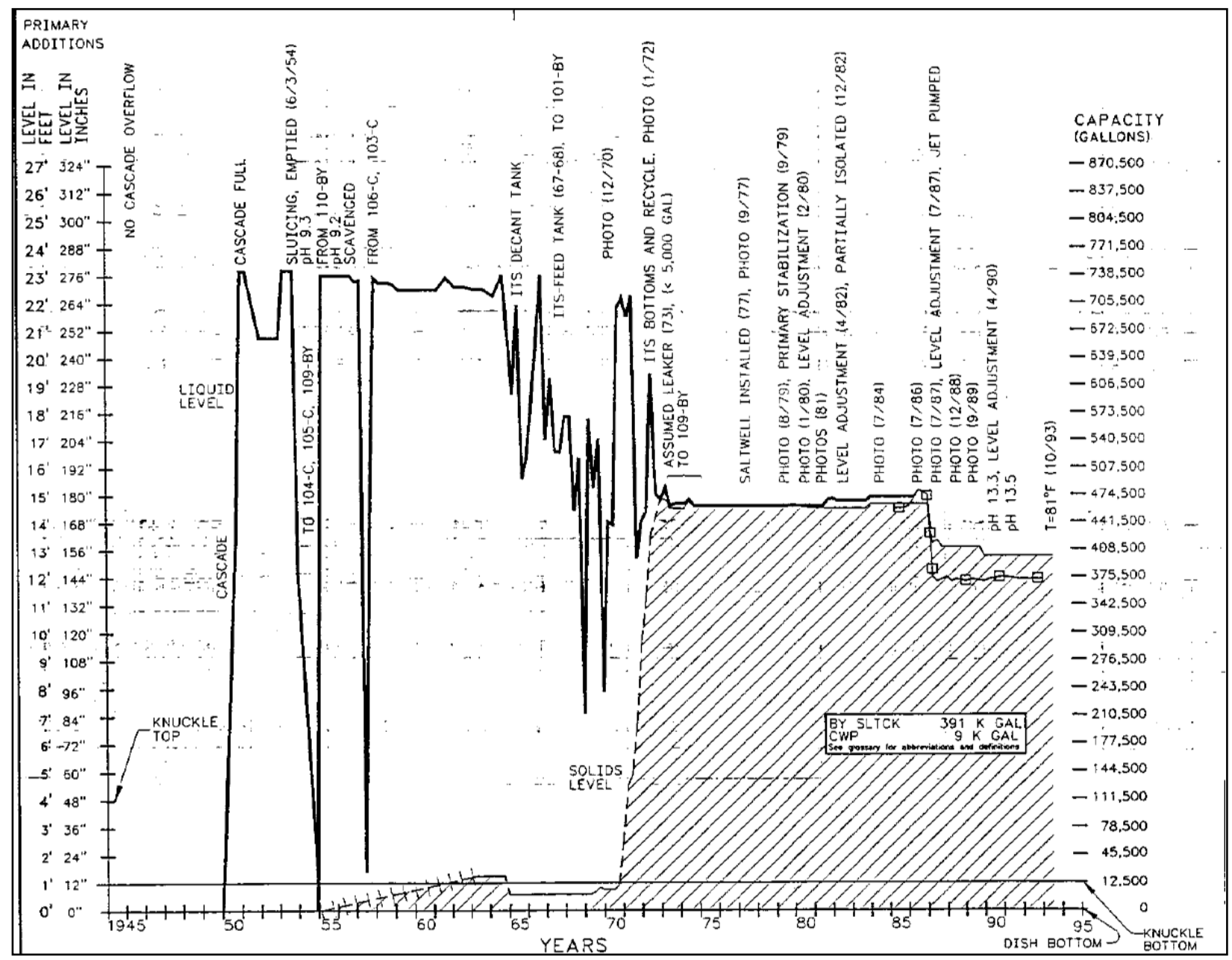

WHC-SD-WM-ER-349, Rev 0, June 1994, Historical Tank Content Estimate for the Northeast Quadrant of the Hanford 200 East Area 


\section{RPP-RPT-54911, Rev. 0}

\subsubsection{Temperature}

No temperature data were recovered for tank BY-103 from October 1950 when the tank was first put into service until 1974. However, it was reported in document HW-20742 (Loss of Depleted Metal Waste Supernatant to Soil) that MW was routed to 241-BX Farm tanks that cascaded into BY Farm tanks. Also stated in this document, tank BY-103 was the last tank in this series and temperatures ranged from $180^{\circ} \mathrm{F}$ in the first cascade tank (tank BX-101) to $70^{\circ} \mathrm{F}$ in tank BY-103 in February 1951.

Tank BY-103 received TBP waste from 221-U Plant from 1955 to 1957 . TBP wastes were concentrated and cooled to $\sim 180^{\circ} \mathrm{F}$ within the plant and were estimated to be $110-180^{\circ} \mathrm{F}$ after routing to the storage tanks.

Tank BY-103 received PUREX waste supernatant between 1957 and 1965 from tank C-106. Temperatures of PUREX supernatant from PUREX plant would probably be at or slightly above $180^{\circ} \mathrm{F}$, so temperatures of this waste type would likely be less in tank BY-103. However, the temperature rates of rise are unknown.

Tank BY-103 received ITS evaporator bottoms (EB) waste periodically from 1965-1972 which would have likely been at boiling temperatures. However, this period was after radioactivity was first detected in the drywells (see Section 4.5.1). Temperature data were recovered from 1972-1974, towards the end of the ITS program, and average temperatures were recorded to be about $150^{\circ} \mathrm{F}$ with the maximum temperature at approximately $160^{\circ} \mathrm{F}$ (WHC-SD-WM-DP-207, Rev. 0, Operating Data to In-Tank Solidification (ITS)-2 for January 1 to October 10, 1974; WHC-SD-WM-DP-210, Rev. 0, Operating Data to In-Tank Solidification (ITS)-2 for July 1 to December 31, 1972; WHC-SD-WM-DP-231, Rev. 0, Operating Data to In-Tank Solidification (ITS)-2 for January 1 to October 10, 1974).

Average temperatures were approximately $75^{\circ} \mathrm{F}$ from 1974 to 1990 with the maximum approximately at $110^{\circ} \mathrm{F}$ in 1974 (see PCSACS).

\subsubsection{Liner Observations}

No liner observations relating to a tank BY-103 leak have been found.

\subsubsection{Chemistry-Corrosion}

Tank BY-103 began receiving MW in October 1950 and received various waste types throughout operation as shown in Table 4-1. The typical concentrations for nitrite, nitrate, and hydroxide for these waste types are shown in Table 4-2. Nitrite and hydroxide are known as nitrate-induced SCC inhibitors. One key characteristic for inhibiting SCC is to maintain a high nitrite concentration to nitrate concentration ratio (see Section 3.2.4). 
RPP-RPT-54911, Rev. 0

Table 4-1. Tank BY-103 Waste Storage Chronology

\begin{tabular}{|c|c|c|}
\hline Date & Waste Type & Length of Storage \\
\hline $\begin{array}{c}\text { October 1950 - June 1954 } \\
\text { (declared empty June 3, 1954) }\end{array}$ & MW & $\sim 4$ years \\
\hline $\begin{array}{c}\text { 1955-1957 } \\
\text { (leaving a TBP heel of } \sim 26 \mathrm{kgal} \text { ) }\end{array}$ & TBP & $\sim 2$ years \\
\hline 1957- 1965 & PUREX HLW supernatant & $\sim 8$ years \\
\hline 1965-1972 & CWP, OWW, EB & $\sim 7$ years \\
\hline
\end{tabular}

Table 4-2. Waste Chemistries for Waste Types Stored in Tank BY-103

\begin{tabular}{|c|c|c|c|c|}
\hline Waste Type & {$\left[\mathrm{NO}_{3}{ }^{-}\right]^{1}$} & {$\left[\mathrm{NO}_{2}^{-}\right]^{1}$} & {$\left[\mathrm{OH}^{-}\right]^{1}$} & $\begin{array}{c}\text { Meets Current } \\
\text { DST Specification }\end{array}$ \\
\hline $\mathrm{MW}^{1}$ & 0.59 & Not reported & 1.16 & Yes $^{5}$ \\
\hline $\mathrm{TBP}^{1}$ & 7.35 & Not reported & 0.09 & $\mathrm{No}^{3}$ \\
\hline PUREX HLW $^{1}$ & 1.3 & $3.37^{4}$ & Not reported & Yes \\
\hline $\mathrm{CW}^{1}$ & 0.6 & 0.9 & 1.0 & Yes \\
\hline $\mathrm{OWW}^{1}$ & 0.06 & Not reported & Not reported & $\mathrm{Yes}^{6}$ \\
\hline
\end{tabular}

1. Reference WHC-EP-0449, 1991, The Sort on Radioactive Waste Type Model: A Method to Sort Single-Shell Tanks into Characteristic Groups.

2. Reference OSD-T-151-00007, Rev. 12, (2013), Operating Specifications for the Double-Shell Storage Tanks.

3. To be within the current DST specification limit, $\left[\mathrm{NO}_{3}{ }^{-}\right] \leq 5.5 \mathrm{M}$

4. Average of three A Farm PUREX waste samples, RL-SEP-183 RD, PUREX Tank Farm Supernatant Solution Composition.

5. Even with no reported value for nitrite, the ratio of nitrate to nitrite and hydroxide would still be less than 2.5 as stated in the current DST specification.

6. According to the assumption from reference WHC-EP-0772, Characterization of the Corrosion Behavior of the Carbon Steel Liner in Hanford Site Single-Shell Tanks.

The first waste type stored in tank BY-103 was MW cascaded from tank BY-102 and was stored in the tank for approximately four years. Metal waste should not be a concern for either pitting or SCC under the tank BY-103 conditions.

Tank BY-103 stored only TBP waste from 221-U Plant from 1955 to 1957. Samples of TBP waste indicate hydroxide concentrations below $0.1 \mathrm{M}$ and nitrate concentrations above $6 \mathrm{M}$. The high nitrate concentration violates the current DST specification for waste chemistry as nitrate must be below $5.5 \mathrm{M}$. These conditions of the TBP waste would likely create an environment conducive to SCC and/or pitting.

Tank BY-103 stored PUREX HLW for approximately eight years on top of an approximate $26 \mathrm{kgal}$ TBP heel. The approximately $710 \mathrm{kgal}$ of PUREX HLW added on top of the TBP heel which would have reduced the corrosiveness of the TBP waste. Tank BY-103 could have started leaking as early as 1959 which at this time contained the mixture of PUREX HLW and TBP wastes. The TBP waste stored from 1955 to 1957, along with the residual TBP heel stored for an additional two years before the tank started leaking, could have set up a pitting situation along with SCC. 
Tank BY-103 also stored CW, OWW, and EB during the ITS program for approximately seven years until the supernatant was pumped out of the tank. PUREX coating waste, PUREX HLW, and OWW should not be a concern for either pitting or SCC under the tank BY-103 conditions. Also, it was determined that the chemistry of the EB generated during the ITS process and stored in tank BY-103 would not be expected to increase the propensity for SCC or pitting (RPP-RPT-55804).

\subsubsection{Photographs}

Tar seepage (asphalt) was visible below the lead flashing at the top of the liner in 1980 photographs of tank BY-103 (see Figure 4-4). In this case only a small portion of the tank circumference was affected. Several other BY and TY tanks experienced tar seepage at the top of the liner and in the side walls (ARH-1496, Review of Storage Tank Integrity). The report indicated the mastic compound (three-ply asphaltic membrane waterproofing) placed between the steel liner and the concrete wall during construction could cause the asphalt to flow through a breach in the liner and lead flashing connection at non-boiling waste temperatures. It is unknown if any other asphalt seepage was experience in tank BY-103 as photographs were not available of the tank wall below the level of the waste indicated in Figure 4-4.

No other anomalies were indicated from a review of available tank BY-103 photographs (see Section 3.2.5). 


\section{Figure 4-4. Tank BY-103 Tar Seepage March 5, 1981 (Photograph 9511211 11-CN)}

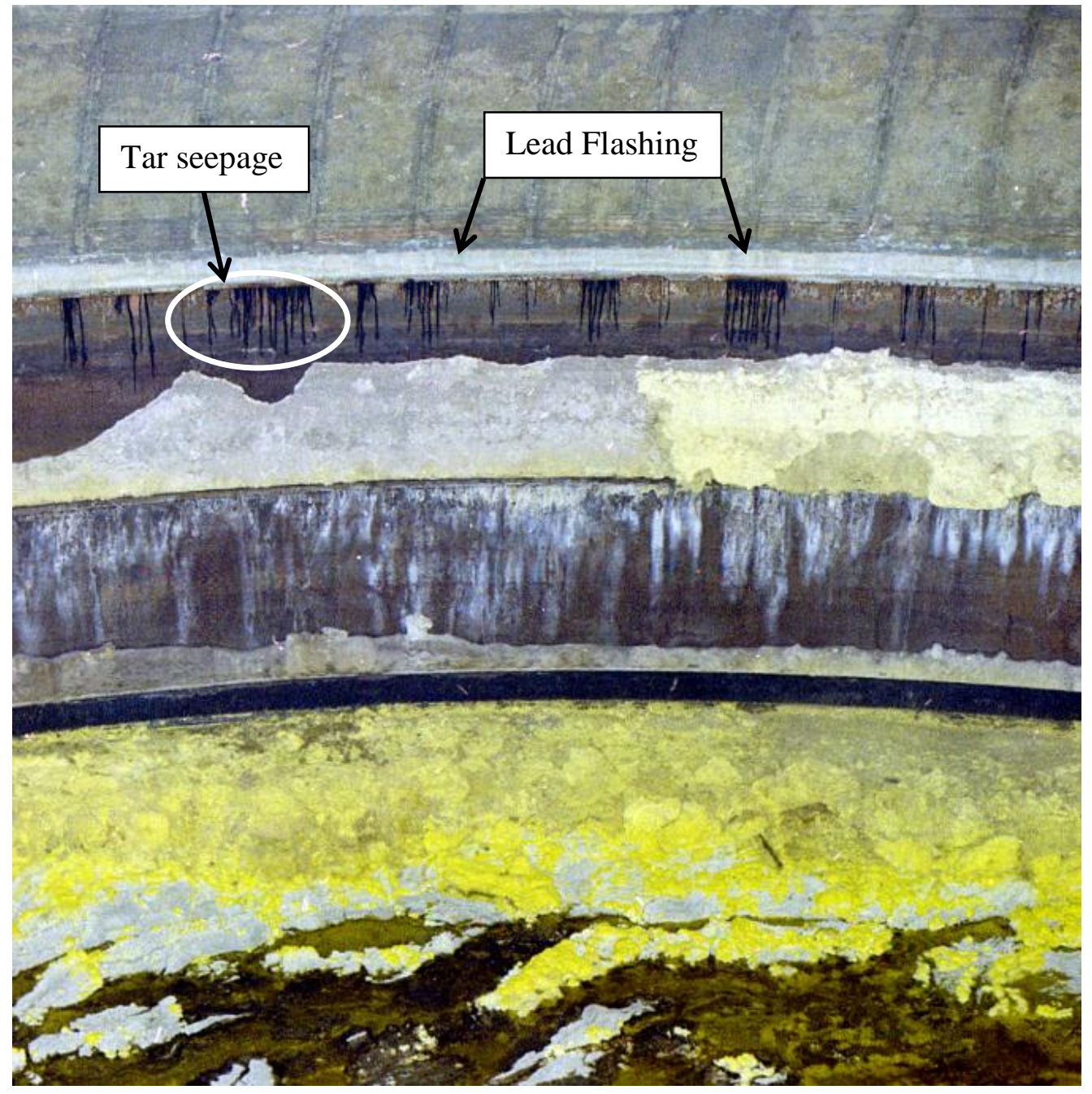

\subsection{TANK BY-103 EX-TANK DATA}

\subsubsection{Drywells}

There are twelve drywells located around tank BY-103: 22-00-01 and 22-00-03 installed in July 1949; 22-03-01, 22-03-05, and 22-03-09 installed in August 1970; 22-03-04 and 22-0306 installed in December 1972; and 22-00-02, 22-00-04, 22-03-07, 22-03-08, and 22-03-10 installed in December 1973. All of the radiation readings in drywells are assumed to be maximum or peak readings unless otherwise noted (see Section 3.3.2). The following subsections report the available drywell information and the drywell summary section provides the analyses of the associated drywells with tank BY-103. 
RPP-RPT-54911, Rev. 0

\subsubsection{Drywell 22-00-01 $\left(\mathrm{N53}^{\circ} \mathrm{W}\right)$}

Drywell 22-00-01 was drilled on August 31, 1949 with the first recoverable readings on September 9, 1959 and September 21, 1960 at less than 0.1K cpm. The next recoverable reading was on December 21, 1965 with a peak of $8 \mathrm{~K}$ cpm at $50-\mathrm{ft}$ BGS. Radioactivity levels then slowly declined from 1965 and were reported at 3.4K cpm at 60-ft BGS in January 1973 (see Appendix A1).

In February 1996, Cs-137 was the only man-made contaminant detected reported in this drywell from the surface to 6-ft, from 38 to $60-\mathrm{ft}$, from 76 to $82-\mathrm{ft}$, and from 86 to $97-\mathrm{ft}$ BGS (GJ-HAN-20). It appears radioactivity detected in drywell 22-00-01 may not be associated with tank BY-103 as drywell 22-03-10 (installed December 1973), located between tank BY103 and drywell 22-00-01, first reported radioactivity in early 1975 at a much higher BGS level (see Section 4.5.1.12). GJ-HAN-20 reports, "Contamination below $38 \mathrm{ft}$ probably originated from a subsurface source, because the contamination does not extend to the surface at significant concentrations. Tank BY-103 or BY-106 is the likely source for this contamination." It remains inconclusive the source of radioactivity detected in this drywell, therefore, drywell 22-00-01 is not included as part of the leak location for tank BY-103. Figure 4-5 shows the depths of radioactivity from 1975 to 1994 (HNF-3532).

Figure 4-5. Tank BY-103 Drywell 22-00-01 (HNF-3532)

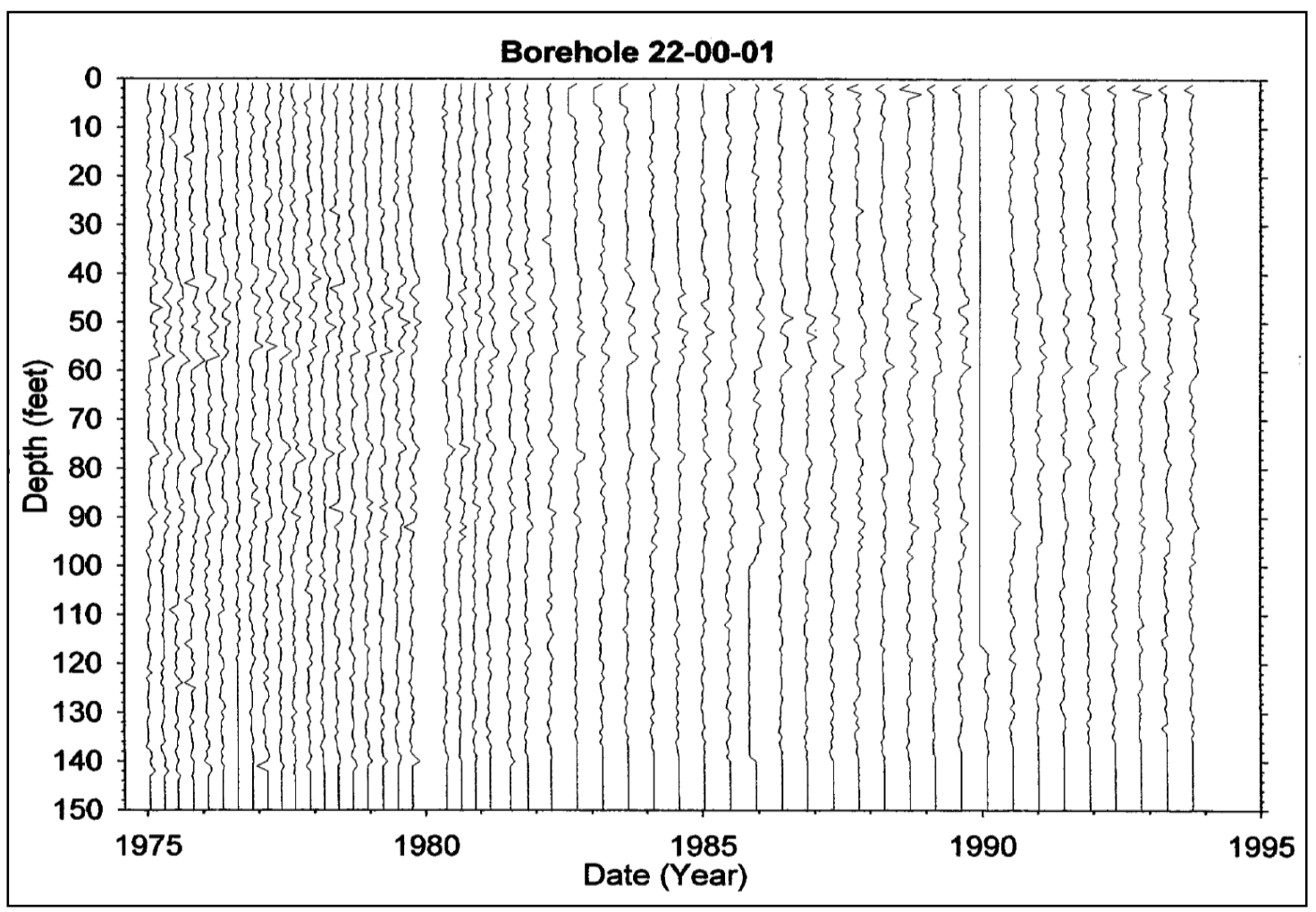

Note: Bottom of the tank footing is $\sim 48$-ft 7 -in BGS 


\subsubsection{Drywell 22-00-02 $\left(\mathrm{S60}^{\circ} \mathrm{E}\right)$}

Drywell 22-00-02 was drilled on December 31, 1973 with the first recoverable reading on April 16, 1974 at $27.1 \mathrm{~K} \mathrm{cpm}$ at $60-\mathrm{ft}$ BGS (see Appendix A1). Radiation levels remained relatively stable through January 1, 1975 at depths ranging from 53 to 60 -ft BGS. No other raw data were recovered after 1975 for drywell 22-00-02.

In February 1996, GJ-HAN-20 states Cs-137, Co-60, and Sb-125 were the only man-made contaminants detected in this drywell. Cs-137 was detected continuously from the surface to about 17-ft BGS, and discontinuously at lower depths which was likely from surface contamination that was carried down by the drilling process or migration (GJ-HAN-20). Co60 was detected near 50-ft BGS near the minimum detection limit and from 74 to $100-\mathrm{ft}$ BGS with concentrations up to $2 \mathrm{pCi} / \mathrm{g}$. A small concentration (about $0.7 \mathrm{pCi} / \mathrm{g}$ ) of Sb-125 was also detected near 52 -ft BGS.

Document GJ-HAN-20 states that tank BY-103 is most likely the source of the Co-60 and Sb125 that was detected at approximately 50-ft BGS. Figure 4-6 shows the depths of radioactivity from 1975 to 1994 (HNF-3532).

Figure 4-6. Tank BY-103 Drywell 22-00-02 (HNF-3532)

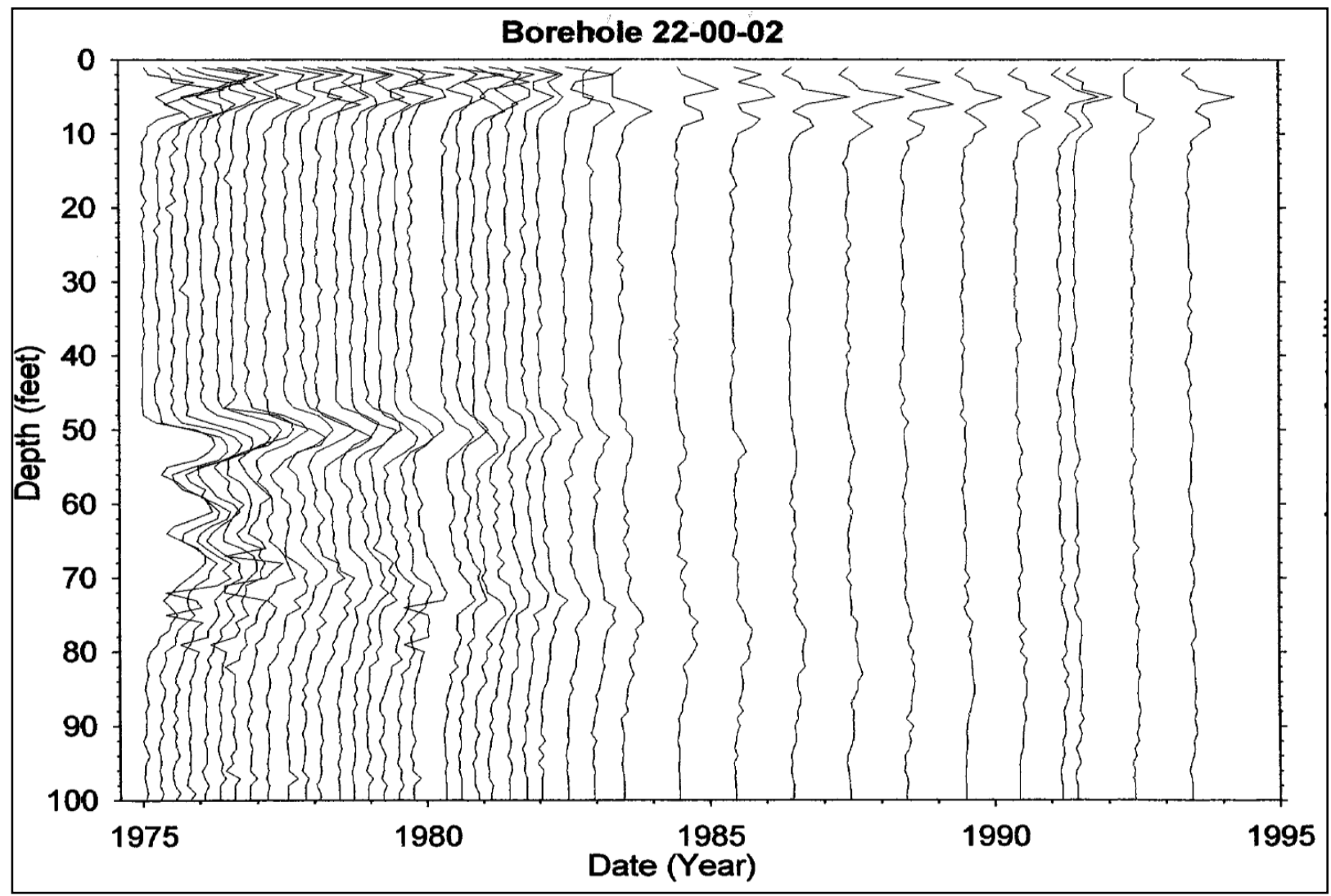

Note: Bottom of the tank footing is $\sim 48$-ft 7 -in BGS 
RPP-RPT-54911, Rev. 0

\subsubsection{Drywell 22-00-03 $\left(\mathrm{S}^{\circ} 8^{\circ} \mathrm{E}\right)$}

Drywell 22-00-03 was drilled July 31, 1949 with the first recoverable reading on September 9, 1959 as greater than $100 \mathrm{~K}$ cpm from 42 to $150-\mathrm{ft}$ BGS. Radioactivity continued to be detected in this drywell and was reported at > 1,000K cpm in December 1965 from 50-92-ft BGS which was indicated to be mostly Co-60. No data were recovered from December 1965 until August 22, 1972 with a peak reported at 212.3K cpm at 60-ft BGS (see Appendix A1). Radioactivity then slowly declined and on June 6, 1986 was reported at $14.1 \mathrm{~K}$ cpm at $74-\mathrm{ft}$ BGS.

In February 1996, Cs-137, Cs-60, and Sb-125 were the only man-made contaminants detected in this drywell (GJ-HAN-20). From the ground surface to about 102-ft BGS, Cs-137 was detected continuously at concentrations up to about $20 \mathrm{pCi} / \mathrm{g}$. Co-60 was detected almost everywhere below 46-ft BGS at concentrations up to $13 \mathrm{pCi} / \mathrm{g}$ and $\mathrm{Sb}-125$ was detected from 54 to $72-\mathrm{ft} \mathrm{BGS}$ at concentrations of about $2 \mathrm{pCi} / \mathrm{g}$. Document GJ-HAN-20 states the Cs-137, Co-60, and Sb-125 detected in this drywell likely originated from tank BY-103. Tank BY103 was first suspected of leaking in 1969 based on radioactivity being detected in drywell 22-00-03 (Internal letter "Integrity of tanks 241-BY-103 and 241-TY-103"). Figure 4-7 shows the depths of radioactivity from 1975 to 1994 (HNF-3532).

Figure 4-7. Tank BY-103 Drywell 22-00-03 (HNF-3532)

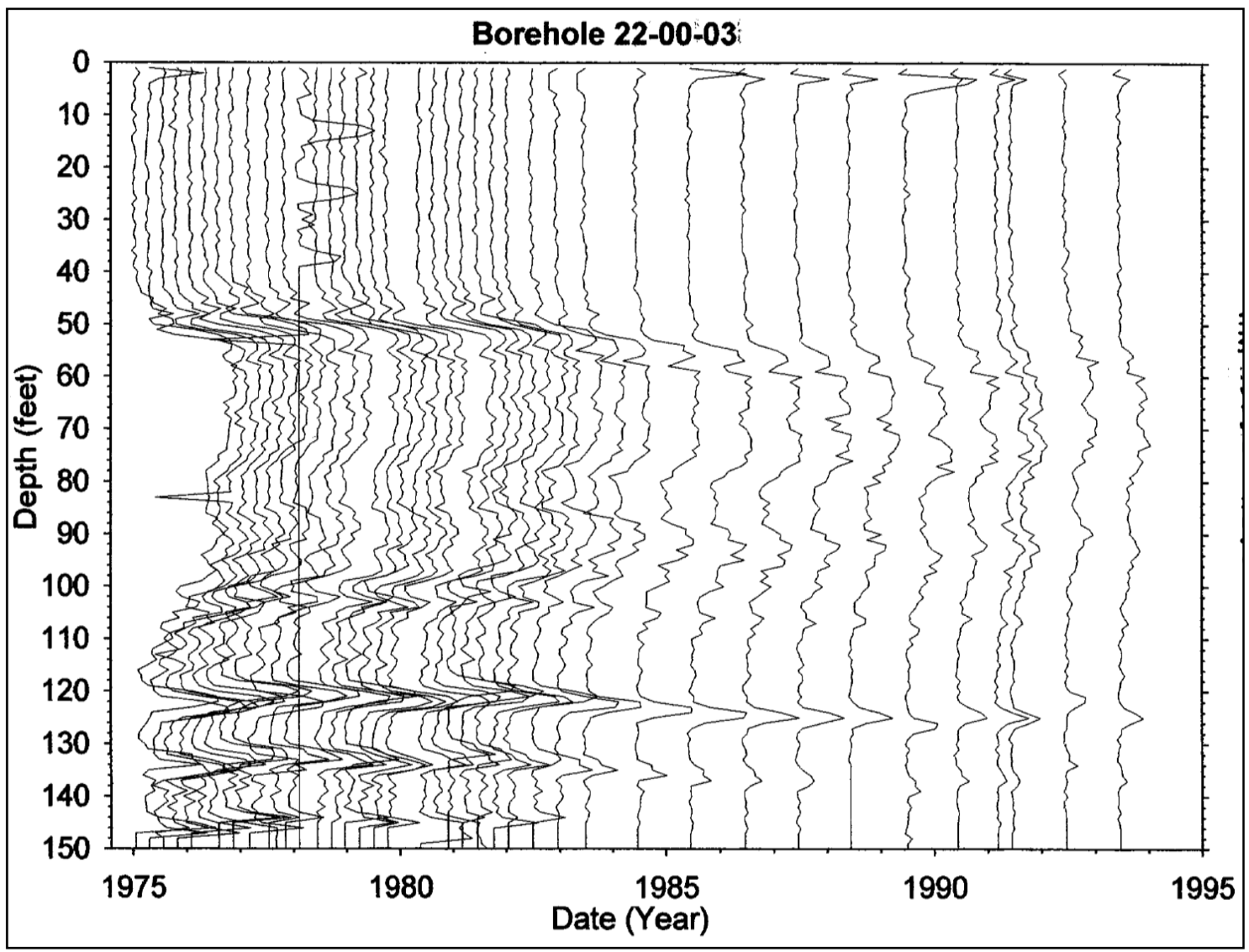

Note: Bottom of the tank footing is $\sim 48$-ft 7 -in BGS 
RPP-RPT-54911, Rev. 0

\subsubsection{Drywell 22-00-04 $\left(\mathrm{S37}^{\circ} \mathrm{E}\right)$}

Drywell 22-00-04 was drilled on December 31, 1973 with the first recoverable reading on April 16, 1974 at $17.9 \mathrm{~K} \mathrm{cpm}$ at $79-\mathrm{ft}$ BGS. Radioactivity remained relatively stable to January 1975 at this BGS depth (see Appendix A1). No other raw data were recovered after 1975 for drywell 22-00-04.

In February 1996, Cs-137 and Co-60 were the only man-made contaminants detected in this drywell (GJ-HAN-19, Vadose Zone Monitoring Project at the Hanford Tank Farms Tank Summary Data Report for Tank BY-102). From the ground surface to 33-ft BGS and from 95 to 99-ft BGS, Cs-137 was detected continuously and intermittently from 33 to 95-ft BGS. Concentrations of Cs-137 were measured at levels below $1 \mathrm{pCi} / \mathrm{g}$. Co-60 was detected below 56-ft BGS at concentrations less than $1 \mathrm{pCi} / \mathrm{g}$. Document GJ-HAN-19 states, "The Co-60 may have originated from a more distant subsurface source such as tank BY-103." Figure 4-8 shows the depths of radioactivity from 1975 to 1994 (HNF-3532).

Figure 4-8. Tank BY-103 Drywell 22-00-04 (HNF-3532)

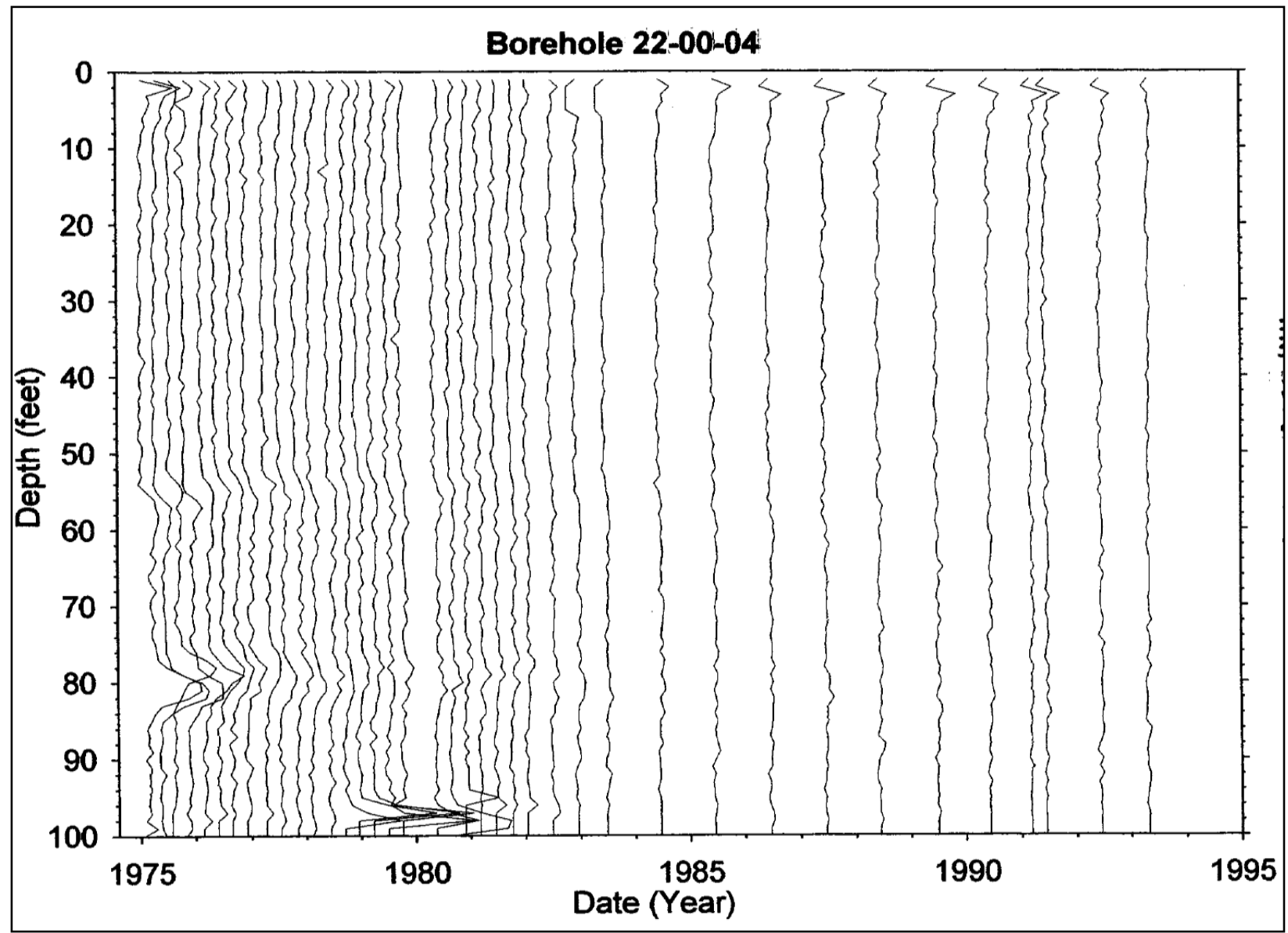

Note: Bottom of the tank footing is $\sim 48$-ft 7 -in BGS 
RPP-RPT-54911, Rev. 0

\subsubsection{Drywell 22-03-01 $\left(\mathrm{N30}^{\circ} \mathrm{E}\right)$}

Drywell 22-03-01 was drilled on August 31, 1970 with the first recoverable reading in March 1971 as less than 50K cpm. No data were recovered from 1971 until August 1972 and radioactivity was reported as less than values through March 1987 (see Appendix A1).

In February 1996, Cs-137 was the only man-made contaminant detected almost continuously in this drywell at concentrations of $2 \mathrm{pCi} / \mathrm{g}$ or less (GJ-HAN-20). Document GJ-HAN-20 states, "No evidence exists for a subsurface source of contamination near this borehole." Since historical records report little radioactivity in this drywell, drywell 22-03-01 is not included as part of the leak location for tank BY-103. Figure 4-9 shows the depths of radioactivity from 1975 to 1994 (HNF-3831).

Figure 4-9. Tank BY-103 Drywell 22-03-01 (HNF-3532)

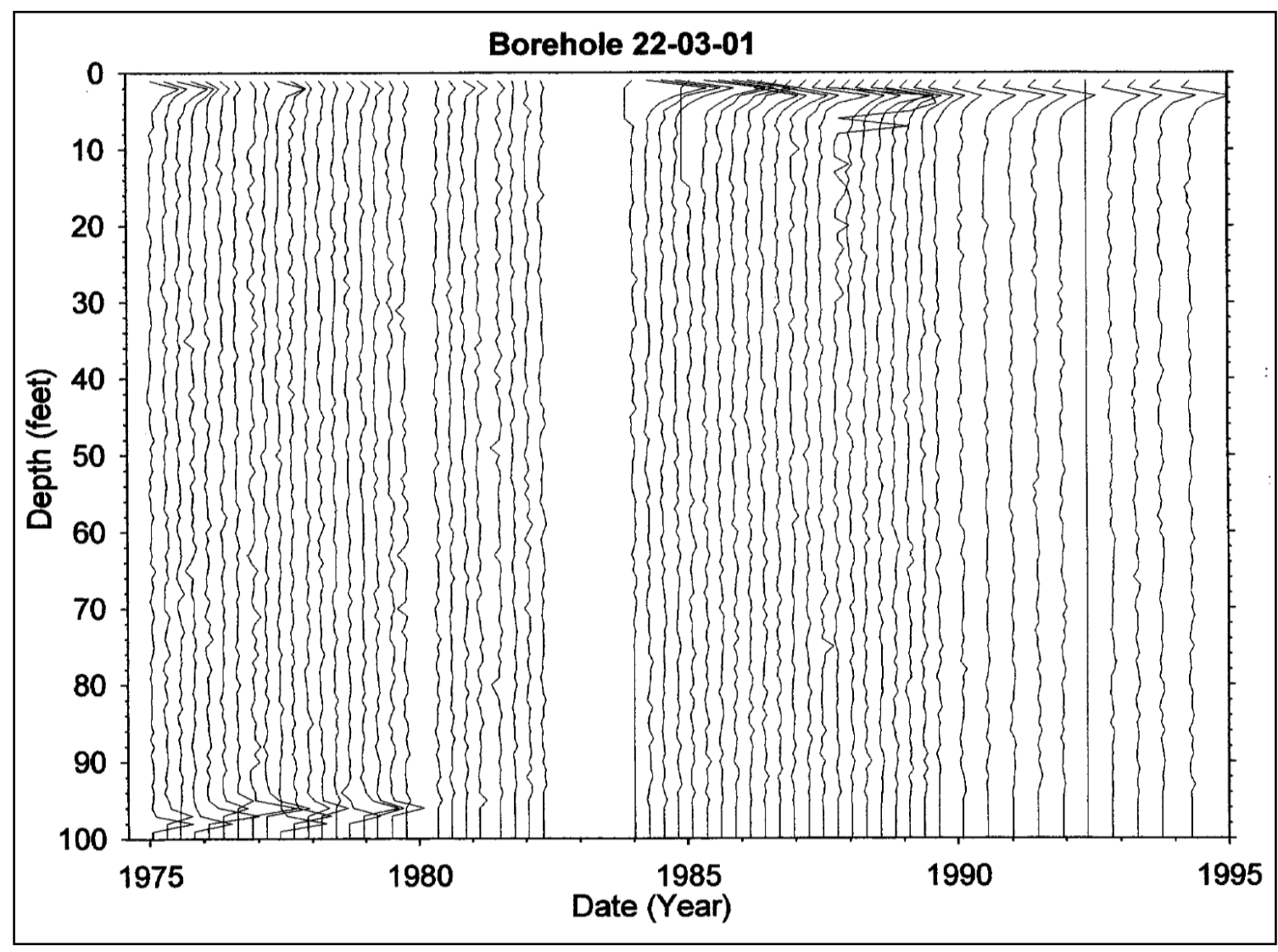

Note: Bottom of the tank footing is $\sim 48$ - $\mathrm{ft} 7$-in BGS 
RPP-RPT-54911, Rev. 0

\subsubsection{Drywell 22-03-04 $\left(\mathrm{S62}^{\circ} \mathrm{E}\right)$}

Drywell 22-03-04 was drilled on December 31, 1972 and was not initially read until February 1973 with no significant radiation detected in this drywell (Internal letter "Integrity of Tanks 241-BY-103 and 241-TY-103"). However, raw data were recovered for drywell 22-03-04 during this period and on February 6, 1973 radioactivity was reported at $70 \mathrm{~K} \mathrm{cpm}$ at $52-\mathrm{ft}$ BGS. Radiation levels gradually declined from 1973 and were reported at $3.6 \mathrm{~K} \mathrm{cpm}$ at $51-\mathrm{ft}$ BGS in 1987 (see Appendix A1).

In February 1996, Cs-137, Co-60, and Sb-125 were the only man-made contaminants detected in this drywell (GJ-HAN-20). Cs-137 was detected continuously from the surface to about 32-ft BGS and discontinuously below 32-ft BGS with a maximum concentration of $\sim 30 \mathrm{pCi} / \mathrm{g}$. Co-60 was detected in two intervals: one near 49-ft BGS and the other near 75-ft BGS with a maximum concentration of about $1 \mathrm{pCi} / \mathrm{g}$. Sb- 125 was detected from 50 to $52-\mathrm{ft} \mathrm{BGS}$ at a maximum concentration of about $1 \mathrm{pCi} / \mathrm{g}$. Document GJ-HAN-20 states, "The zone of Co-60 contamination from 45.5 to $58 \mathrm{ft}$ probably originated from a leak of tank BY-103, at or near its bottom at $48 \mathrm{ft}$ in depth. The lower zone of Co-60 contamination near $75 \mathrm{ft}$ also originated from the subsurface, apparently from tank BY-103" and "Sb-125 originated from the subsurface, most likely from the same leak that produced the Co-60 contamination near $50 \mathrm{ft}$. The source was most certainly tank BY-103, which is the only tank near borehole 22-03-04." This statement seems appropriate as there is no other nearby sources that would explain the Co-60 contamination. Figure 4-10 shows the depths of radioactivity from 1975 to 1994 (HNF-3532).

Figure 4-10. Tank BY-103 Drywell 22-03-04 (HNF-3532)

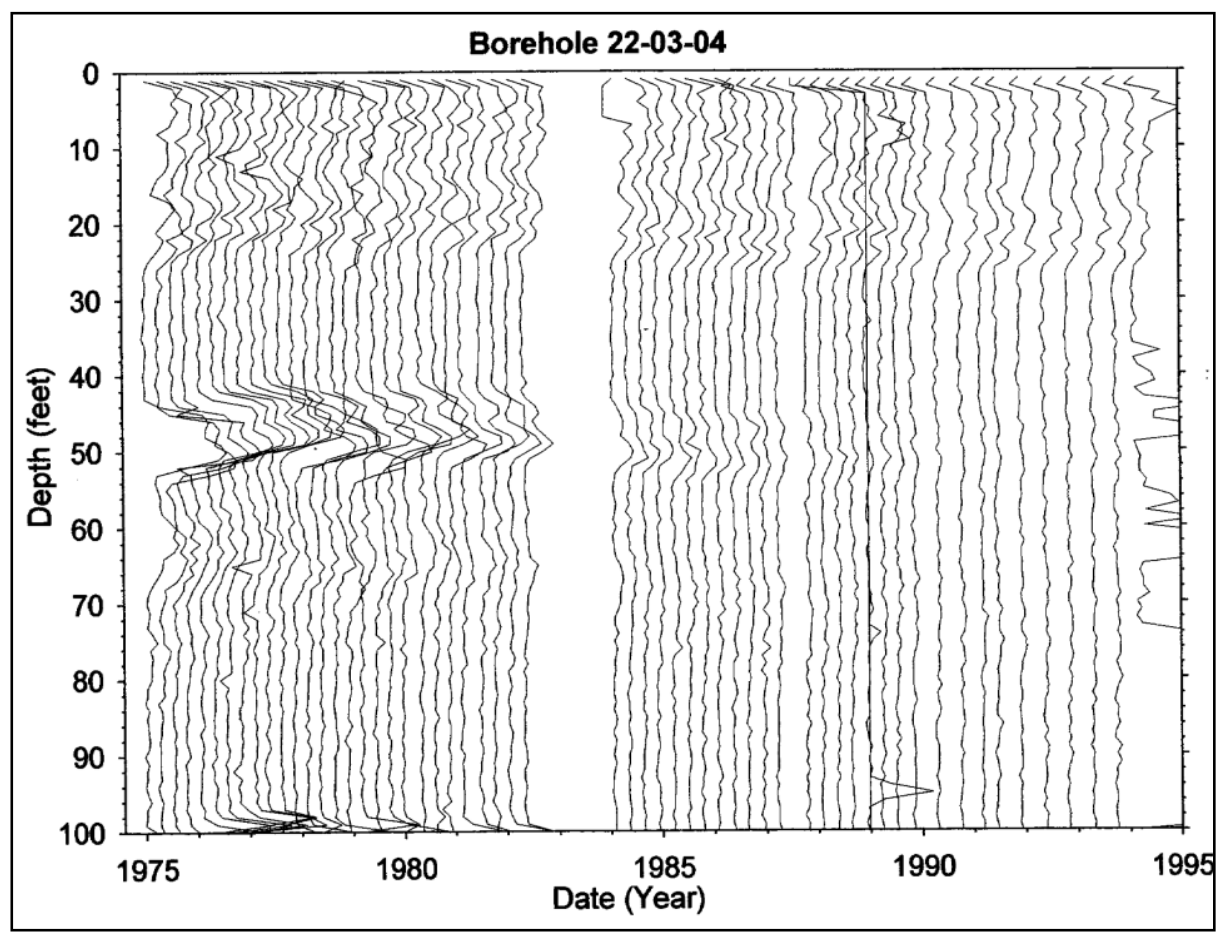

Note: Bottom of the tank footing is $\sim 48$ - $\mathrm{ft} 7$-in BGS 
RPP-RPT-54911, Rev. 0

\subsubsection{Drywell 22-03-05 $\left(\mathrm{S30}^{\circ} \mathrm{E}\right)$}

Drywell 22-03-05 was drilled on August 31, 1970 with the first recoverable reading in March 1971. It was reported in Internal letter "Integrity of Tanks 241-BY-103 and 241-TY-103" that "...high readings were noted in drywell 22-03-05..." in March 1971 and ..."continued to increase until December 1971 when the liquid in the tank was lowered to a maximum operating level of 13 feet to permit continued use of the tank in the ITS- 1 and 2 bottoms loop."

No actual data were recovered until August 22, 1972 when radioactivity was reported at $3811.9 \mathrm{~K} \mathrm{cpm}$ at 36-ft BGS. Radiation levels remained relatively stable through December 1973 and then were reported at $763.8 \mathrm{~K} \mathrm{cpm}$ at 35-ft BGS on January 1, 1974. Radioactivity continued to be detected at this BGS depth through March 1987 (see Appendix A1).

In February 1996, Cs-137 and Co-60 were the only man-made contaminants detected in this drywell (GJ-HAN-20). High concentrations of Cs-137 along the entire length of this drywell were reported with the maximum concentration 2,300 pCi/g at 45-ft BGS (GJ-HAN-20). Co60 was detected near 50-ft BGS with concentrations at $\sim 7 \mathrm{pCi} / \mathrm{g}$. Document GJ-HAN-20 states, "When the tank was filled, the waste level was about $25 \mathrm{ft}$ below ground level. Tank BY-103 or a subsurface pipe (such as the cascade line near depth $25 \mathrm{ft}$ ) for that tank is the most likely source of this contamination." Figure 4-11 shows the depths of radioactivity from 1980 to 1990 (HNF-3532) (see Section 4.5.2). It was not clear why data was not plotted from 1975 to 1980 .

Figure 4-11. Tank BY-103 Drywell 22-03-05 (HNF-3532)

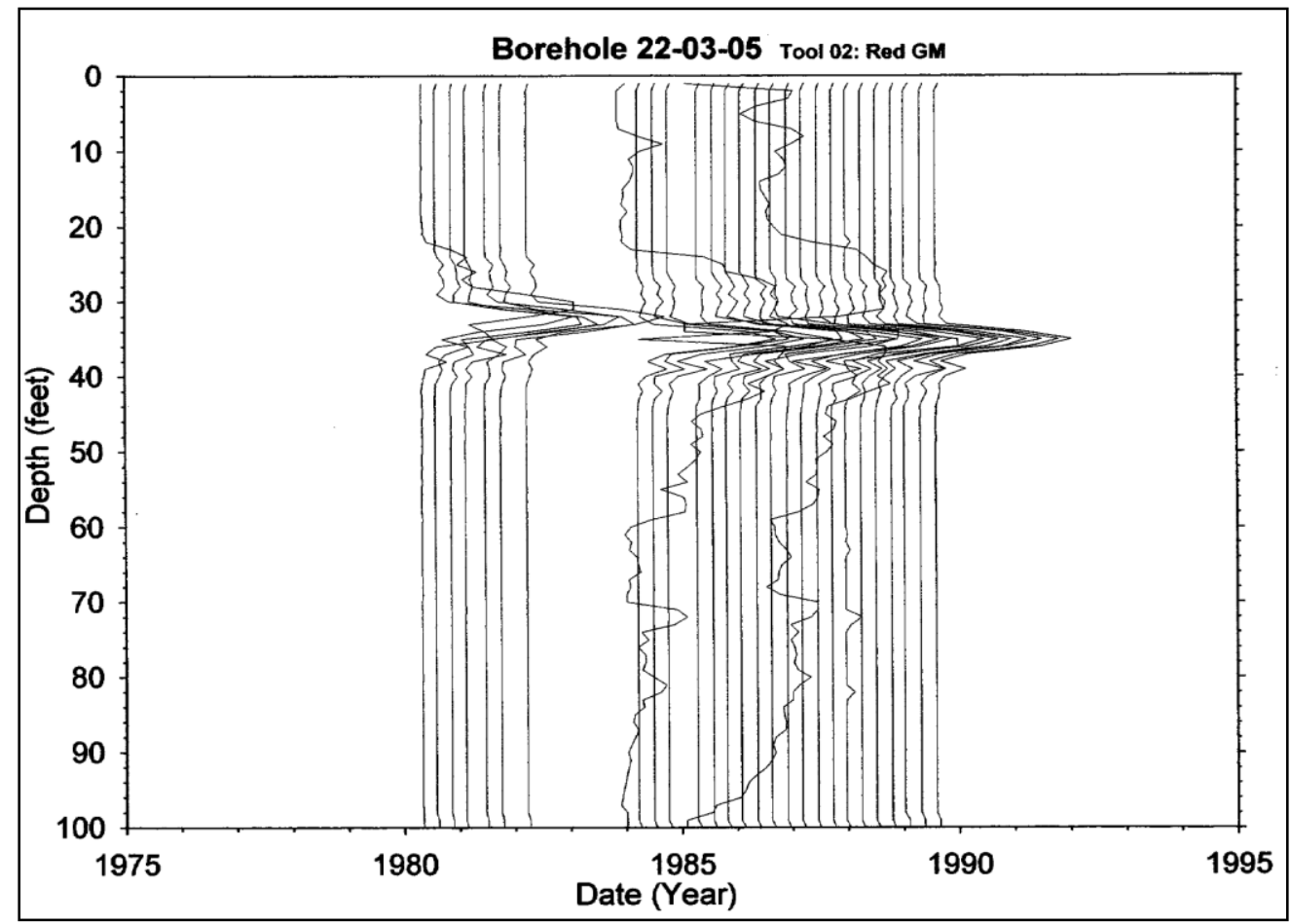

Note: Bottom of the tank footing is $\sim 48$-ft 7-in BGS 
RPP-RPT-54911, Rev. 0

\subsubsection{Drywell 22-03-06 $\left(\mathrm{S3}^{\circ} \mathrm{E}\right)$}

Drywell 22-03-06 was drilled on December 31, 1972 and was not initially read until February 1973 which reported a moderately high count rate but exhibited a stable peak at 45-ft BGS (Internal letter "Integrity of Tanks 241-BY-103 and 241-TY-103"). Readings were reported to be $226.4 \mathrm{~K}$ cpm at $45-\mathrm{ft}$ BGS on February 6, 1973. A second peak was first recorded on January 1, 1974 at $60 \mathrm{~K} \mathrm{cpm}$ at 25-ft BGS. Both peaks continued to be detected through 1981 and the peak at 25-ft BGS continued through March 1987 (see Appendix A1).

In February 1996, Cs-137, Co-60, and Sb-125 were the man-made contaminants detected in this drywell and the most likely source of this contamination is tank BY-103 (GJ-HAN-20). Also stated in this document, "The total gamma-ray peak near $24 \mathrm{ft}$ might be due to contamination in or near the cascade line or other subsurface piping for tank BY-103, or the peak might be due to a leak from tank BY-103" (see Section 4.5.2). Figure 4-12 shows the depths of radioactivity from 1975 to 1994 (HNF-3532).

Figure 4-12. Tank BY-103 Drywell 22-03-06 (HNF-3532)

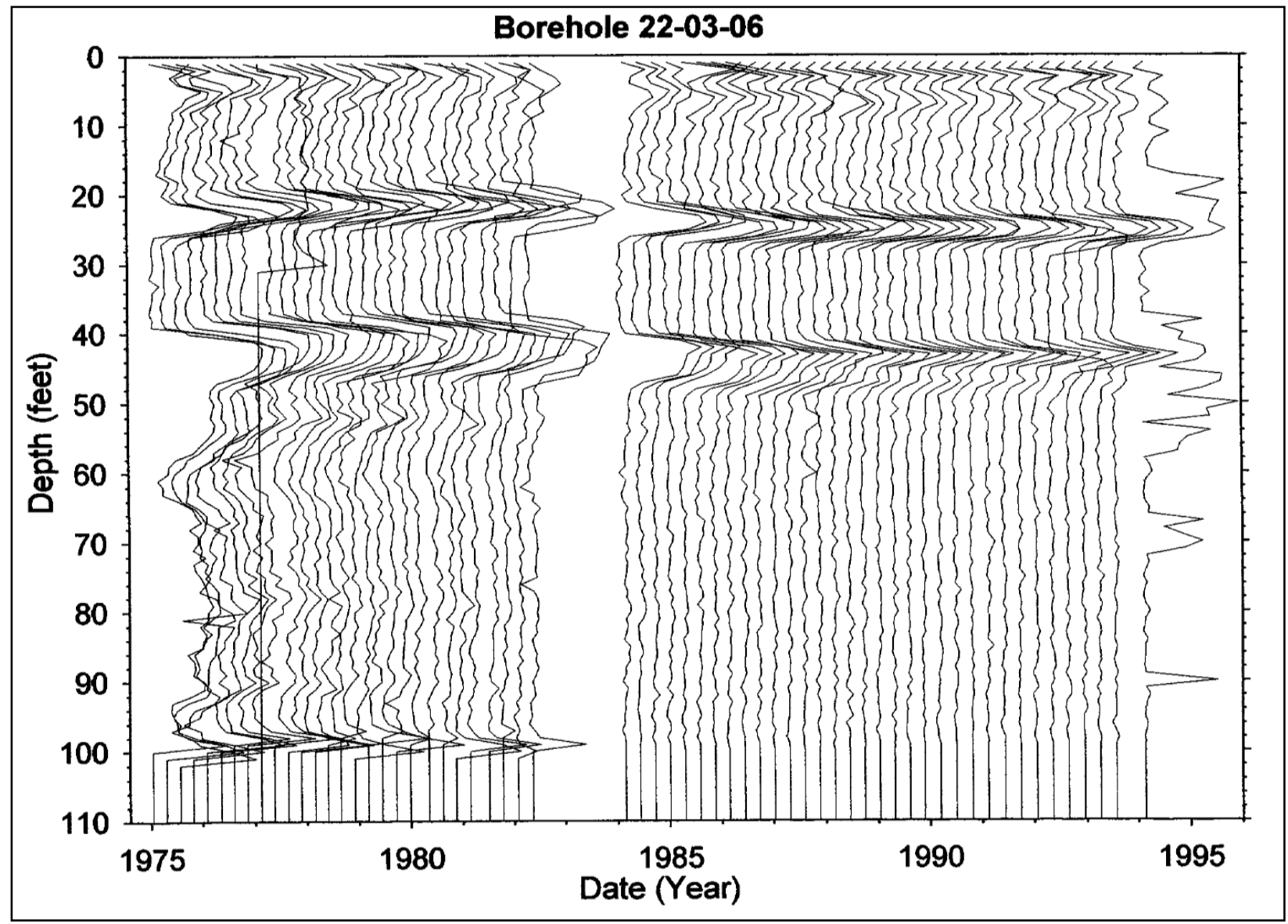

Note: Bottom of the tank footing is $\sim 48$-ft 7 -in BGS 
RPP-RPT-54911, Rev. 0

\subsubsection{Drywell 22-03-07 $\left(\mathrm{S} 21^{\circ} \mathrm{W}\right)$}

Drywell 22-03-07 was drilled on December 31, 1973 with the first recoverable reading on April 8, 1974 at $11.1 \mathrm{~K} \mathrm{cpm}$ at 58-ft BGS. Radiation levels remained relatively stable through February 1976 and then slowly declined from March 1977 to less than values by 1986 (see Appendix A1).

In February 1996, Cs-137 and Co-60 were the only man-made radionuclides detected in this drywell (GJ-HAN-20). From the ground surface to about 24-ft BGS, Cs-137 was reported at concentrations less than $10 \mathrm{pCi} / \mathrm{g}$; however, no evidence exists for a subsurface source of Cs137 at this drywell. From 53 to $94-\mathrm{ft}$ BGS, Co-60 was detected at concentrations less than 0.5 pCi/g. Document GJ-HAN-20 states, "Tank BY-103 or its piping is the most likely source of the Co-60 contamination." However, a tank BY-103 piping leak seems unrealistic at the depth of radioactivity with no radioactivity above 50-ft BGS. Figure 4-13 shows the depths of radioactivity from 1975 to 1994 (HNF-3532).

Figure 4-13. Tank BY-103 Drywell 22-03-07 (HNF-3532)

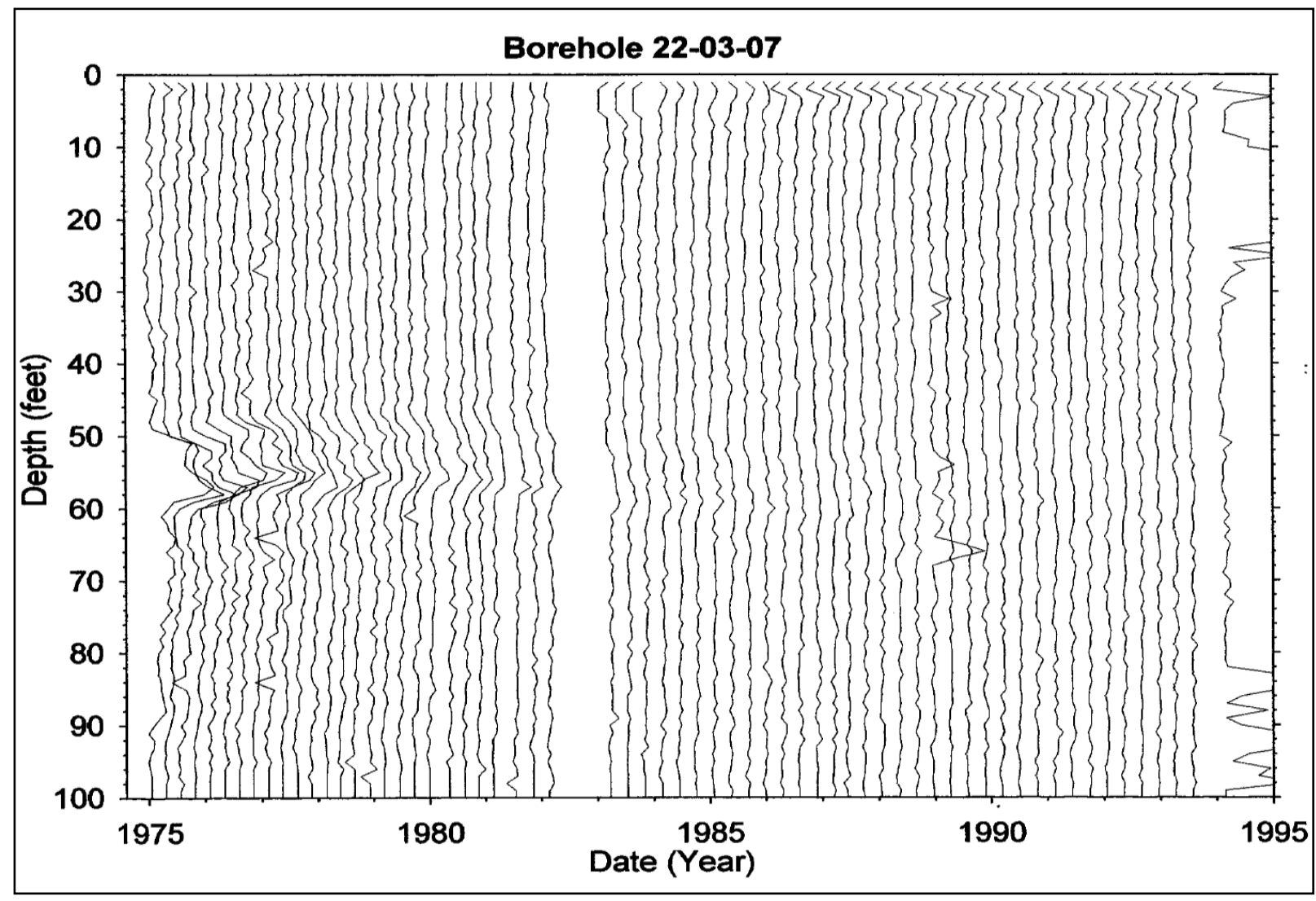

Note: Bottom of the tank footing is $\sim 48$ - $\mathrm{ft} 7$-in BGS 
RPP-RPT-54911, Rev. 0

\subsubsection{Drywell 22-03-08 $\left(\mathrm{S43}^{\circ} \mathrm{W}\right)$}

Drywell 22-03-08 was drilled on December 31, 1973 with the first recoverable reading on April 8, 1974 at 30.7K cpm at 46-ft BGS. Radiation levels gradually declined and were reported as less than values beginning March 1985 (see Appendix A1).

In February 1996, Cs-137 and Co-60 were the only man-made contaminants found in this drywell (GJ-HAN-20). From the ground surface to about 12-ft BGS, Cs-137 was detected continuously at concentrations less than $40 \mathrm{pCi} / \mathrm{g}$. Co-60 was detected near 48 and $92-\mathrm{ft} \mathrm{BGS}$ at concentrations less than $0.4 \mathrm{pCi} / \mathrm{g}$. Document GJ-HAN-20 reports, "No evidence for a deeper subsurface source, such as a tank leak, exists for Cs-137 at this borehole" and, "The Co-60 contamination near $48 \mathrm{ft}$ most likely originated from tank BY-103, whose bottom is at that depth. The deeper Co-60 contamination probably also originated from tank BY-103, either migrating down through the sediments or following the casing." Figure 4-14 shows the depths of radioactivity from 1975 to 1994 (HNF-3831).

Figure 4-14. Tank BY-103 Drywell 22-03-08 (HNF-3532)

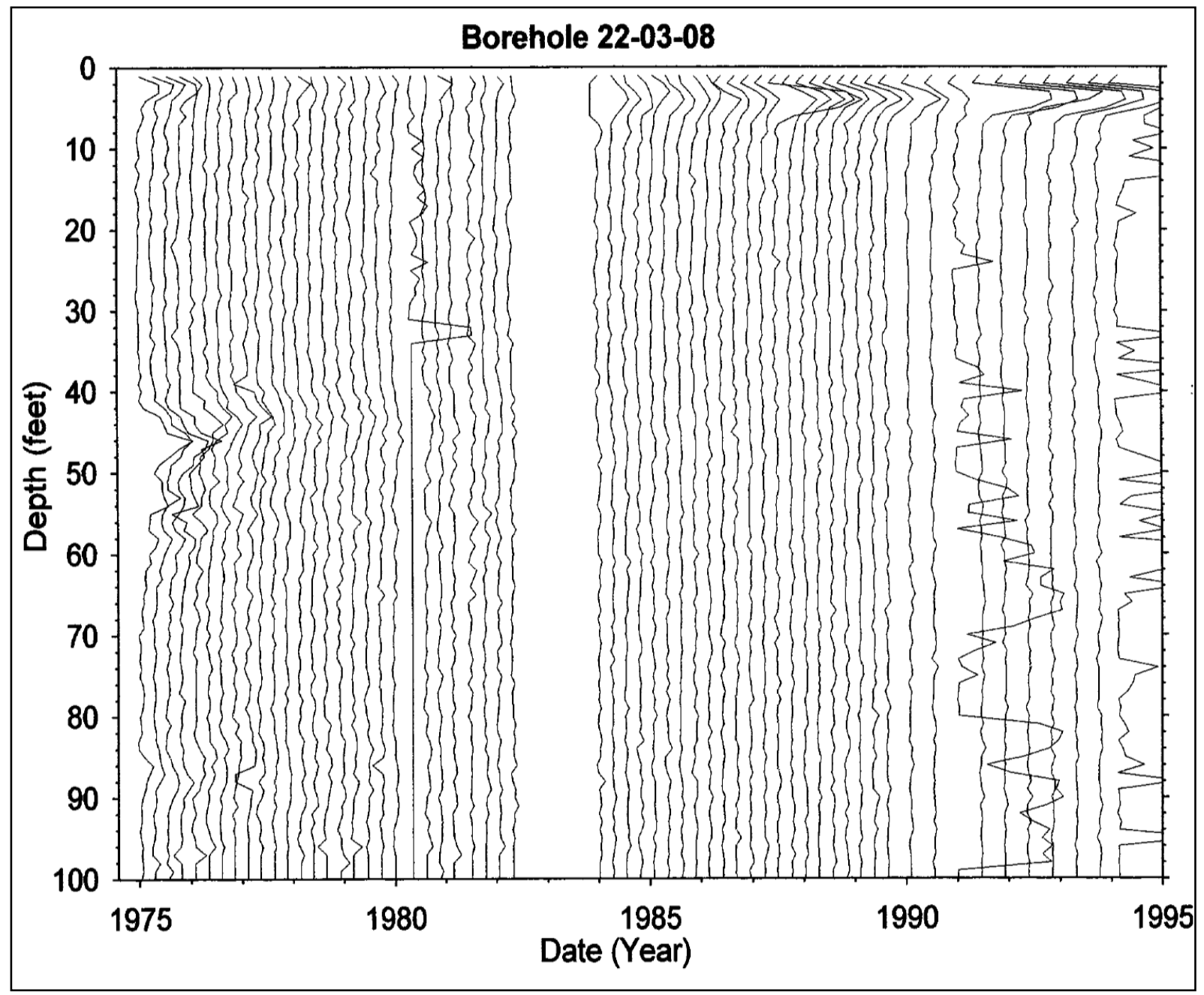

Note: Bottom of the tank footing is 48-ft 7-in BGS 
RPP-RPT-54911, Rev. 0

\subsubsection{Drywell 22-03-09 $\left(\mathrm{N90}^{\circ} \mathrm{W}\right)$}

Drywell 22-03-09 was drilled on August 31, 1970 with the first recoverable reading in March 1971 which was reported to be less than $50 \mathrm{~K}$ cpm (Internal letter "Integrity of Tanks 241-BY103 and 241-TY-103"). The next available reading was on August 22, 1972 and radioactivity was reported to be $27.6 \mathrm{~K}$ cpm from 57-62-ft BGS. Radiation readings were reported to be low and stable in drywell 22-03-09 until December 1972 when an increase in the spread of the readings was noted (from 58-71-ft BGS to 56-77-ft BGS) (Internal letter "Integrity of Tanks 241-BY-103 and 241-TY-103"). Additional peaks were reported on July 17, 1973 at 62.6K cpm at 18-ft BGS and on December 24, 1973 at 15K cpm at 34-ft BGS and continued through March 1987 (see Appendix A1). The source of the peak reported at 18-ft BGS remains unclear but is probably associated with buried piping and not the tank liner as 18-ft BGS is located above the tank liner.

Document HNF-3532 reports two broad peaks of radioactivity near 34-ft BGS and 68-ft BGS. In February 1996, Cs-137, Co-60, and Eu-154 were the only man-made contaminants detected in this drywell (GJ-HAN-20). From the ground surface to about 48-ft BGS, Cs-137 was detected; however, GJ-HAN-20 reports, "No evidence of a subsurface source of Cs-137 exists at this borehole." Co-60 was detected near 6 and 49-ft BGS and Eu-154 was reported near 6$\mathrm{ft}$ BGS. Document GJ-HAN-20 states, "The Co-60 near $49 \mathrm{ft}$ probably originated from tank BY-103" and "Tank BY-106, (to be published), which is also an assumed leaker and is located about $16 \mathrm{ft}$ west of borehole 22-03-09, is also a possible source for this contamination. Figure 4-15 shows the depths of radioactivity from 1975 to 1994 (HNF-3535).

Figure 4-15. Tank BY-103 Drywell 22-03-09 (HNF-3532)

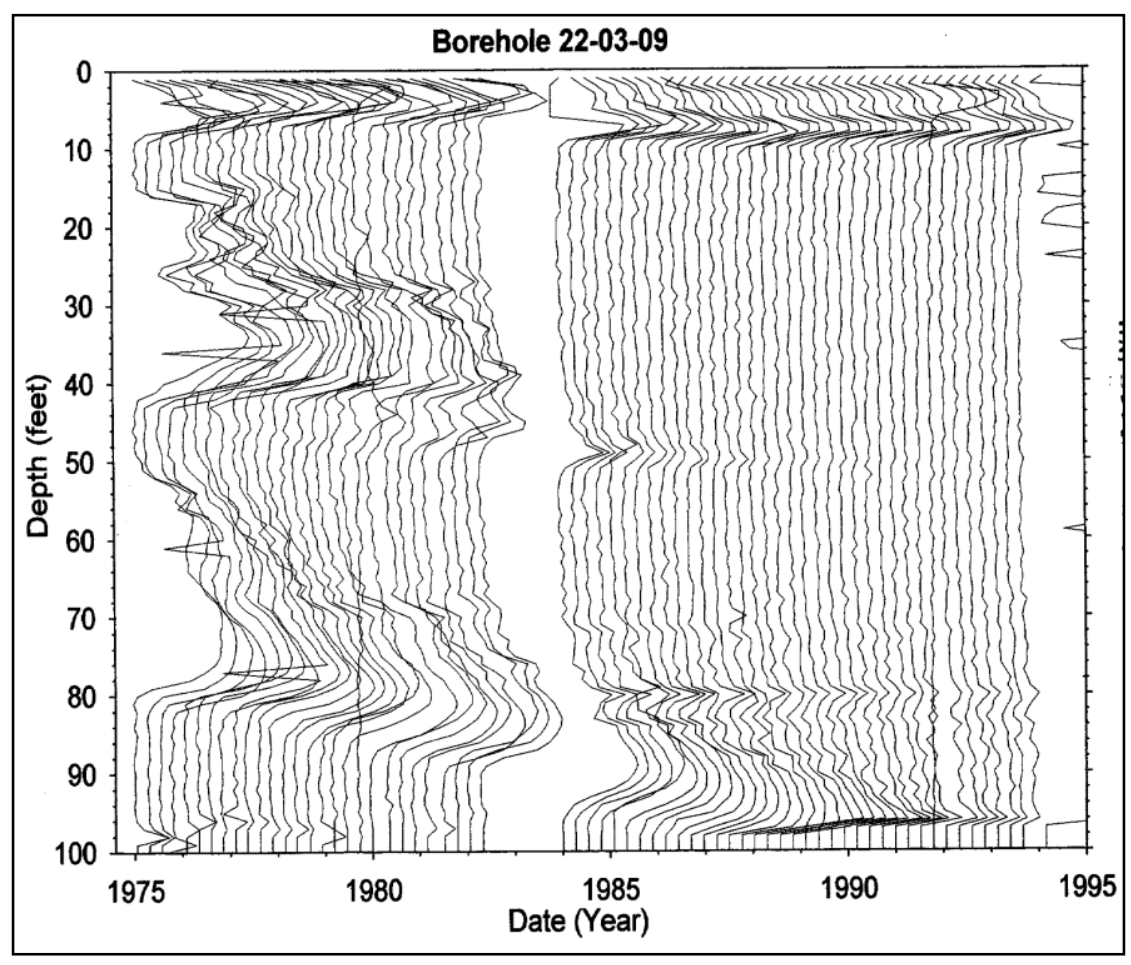

Note: Bottom of the tank footing is $\sim 48$-ft 7 -in BGS 
RPP-RPT-54911, Rev. 0

\subsubsection{Drywell 22-03-10 $\left(\mathrm{N54}^{\circ} \mathrm{W}\right)$}

Drywell 22-03-10 was drilled on December 31, 1973 with the first recoverable reading on April 24, 1974 as less than values through September 24, 1974. On January 24, 1975 radioactivity was reported at $3.2 \mathrm{~K} \mathrm{cpm}$ at $21-\mathrm{ft}$ BGS and remained relatively stable through March 1987 (see Appendix A1).

In February 1996, Cs-137 was the only man-made contaminant detected in this drywell, occurring continuously from the surface to 50-ft BGS at concentrations less than $50 \mathrm{pCi} / \mathrm{g}$ (GJ-HAN-20). Document GJ-HAN-20 reports, "Tank BY-103 (including its ancillary piping) is the most likely source of the subsurface contamination at this borehole. Tank BY-106 (to be published), which is located about $29 \mathrm{ft}$ southwest of borehole 22-03-10, is also a possible source of the contamination." Figure 4-16 shows the depths of radioactivity from 1975 to 1994 (HNF-3831).

Figure 4-16. Tank BY-103 Drywell 22-03-10 (HNF-3532)

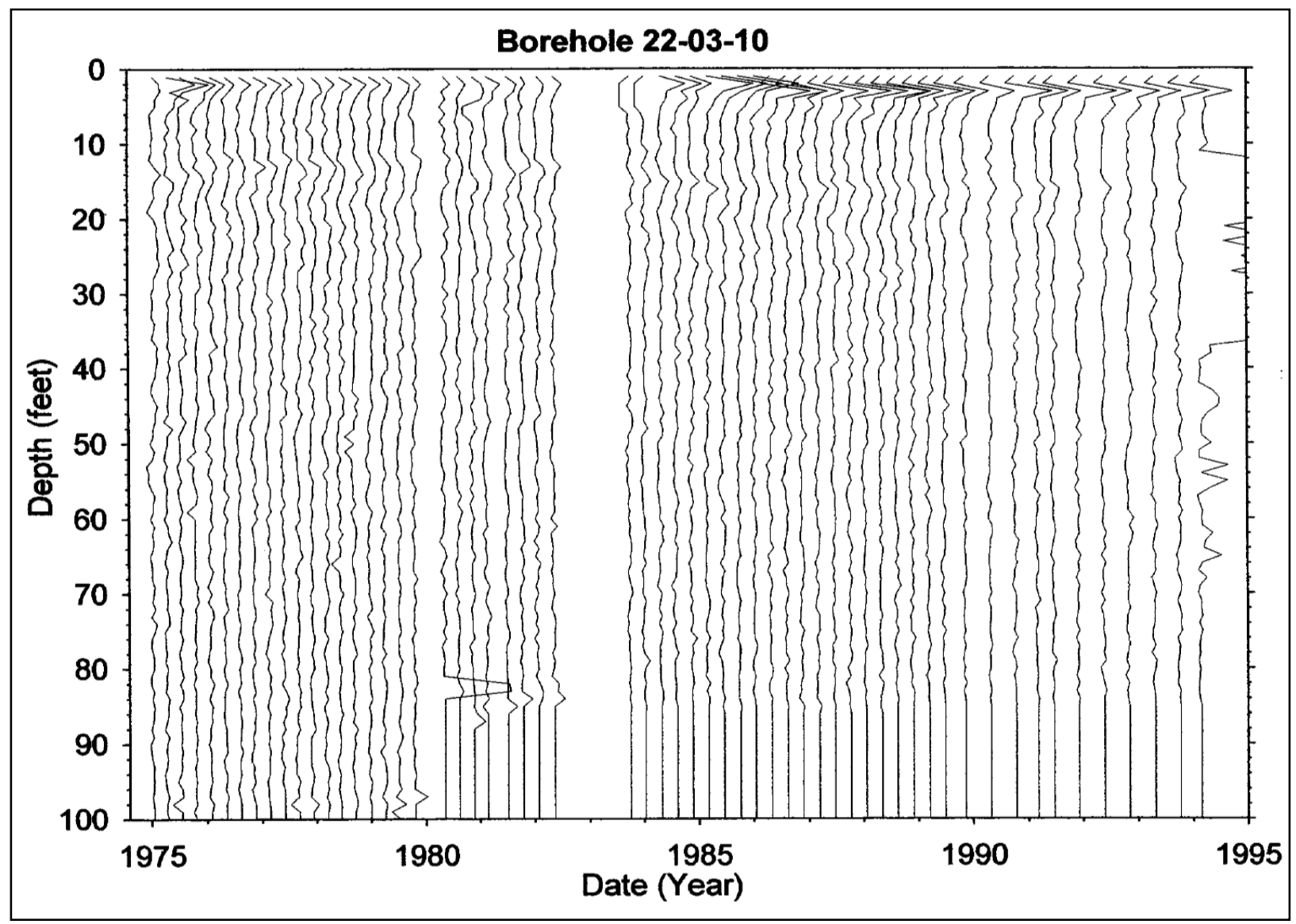

Note: Bottom of the tank footing is $\sim 48$-ft 7 -in BGS 
RPP-RPT-54911, Rev. 0

\subsubsection{Drywell Summary}

Tank BY-103 was first suspected of leaking in 1969 due to radioactivity being detected in drywell 22-00-03; however, a leak may have begun as early as 1959 based on radioactivity present in drywell 22-00-03 during this time either in tank BY-103 or tank BY-106.

Tank BY-103 drywell 22-03-01 does not indicate any contamination associated with a tank leak. The source of radioactivity detected in drywell 22-00-01 remains inconclusive with the possible source being tank BY-103 and/or BY-106 and it is located farther from the tank than the intervening drywell 22-03-10. Therefore, drywells 22-03-01 and 22-00-01 are not included in the leak location for tank BY-103.

The remaining ten drywells all detect radioactivity that could be associated with a tank leak. The first recoverable reading for drywell 22-03-05 reported high levels of radioactivity August 1972 at $~ 35$-ft BGS indicating a possible sidewall leak with the remote possibility of a spare inlet line seal leak. The first recoverable reading for drywell 22-00-03 reported radioactivity in September 1959 from 42 to $150-\mathrm{ft}$ BGS and continued to increase indicating a leak from tank BY-103. Nearby drywells 22-00-02, 22-00-04, and 22-03-04 report lower levels of radioactivity and were likely the result of migration from the tank leak.

Radioactivity detected at the higher BGS levels in drywell 22-03-05 does not appear to be the result of leakage from the cascade inlet pipe nor the capped spare inlet lines (see Figure 4-1). The spare inlet nozzles and the cascade inlet pipe are located at approximately 22-ft 1-in BGS and radioactivity was detected at a much lower BGS level in drywell 22-03-05. It appears radioactivity detected at the higher BGS level in drywell 22-03-05 is likely the result of sidewall leak from tank BY-103.

The first recoverable reading for drywell 22-03-06 reported radioactivity February 1973 at 45 $\mathrm{ft}$ BGS with a second peak reported later in 1974 at 27-ft BGS. The peak in drywell 22-03-06 was only 5-ft lower than the cascade inlet line and the drywell is located approximately 3.2-ft from the tank. Therefore, the source of radioactivity for drywell 22-03-05 remains unclear as a leak from the cascade inlet line is possible and/or a tank sidewall leak. The peak located at 45-ft BGS in drywell 22-03-06 is likely from tank BY-103 near the tank footing and appears to be separate from the radioactivity detected in drywell 22-03-05 or the radioactivity at the 27-ft BGS level due to timing, radioactivity level, and distance.

The first recoverable reading for drywell 22-03-07 detected radioactivity April 1974 at 58-ft BGS. Radioactivity detected in this drywell was at a much lower BGS level compared to nearby drywells 22-03-06 and 22-03-08 likely indicating radioactivity detected in drywell 2203-07 was due to migration from a nearby leak site.

Three peaks were reported for drywell 22-03-09 in 1972-1973 at 18-ft BGS, 34-ft BGS, and from 57 to 62-ft BGS. Radioactivity in drywell 22-03-10 was reported as less than values in April 1974; however, lower levels of radioactivity were reported beginning in January 1975 at 21-ft BGS. Radioactivity was also reported for drywell 22-03-08 in April 1974 at 46-ft BGS. It appears tank BY-103 leaked near drywell 22-03-09 as levels of radioactivity were much higher compared to the nearby drywells 22-03-08 and 22-03-10. It appears the peak detected 
in drywell 22-03-09 at 34-ft BGS indicate a possible sidewall leak and the source of radioactivity at the 18 -ft $\mathrm{BGS}$ level remains unclear since no pipes that were reported to have leaked are present in this area at these BGS levels (see Figure 4-1). Also, the nearby spare inlet lines at $22-\mathrm{ft} 1$-in BGS do not appear to be a likely source location. Radioactivity detected in drywells 22-03-08 and 22-03-10 is likely the result of migration from the leak site that appears to be near drywell 22-03-09.

There is a remote possibility that the drywell 22-03-10 radioactivity came from a leak at the tank condenser but no direct indication has been found. Indirectly, the storage of TBP waste between $110^{\circ} \mathrm{F}$ and $180^{\circ} \mathrm{F}$ for two years or PUREX HLW supernatant at a projected $180+^{\circ} \mathrm{F}$ for eight years could have produced condensate from the condenser which may have leaked out the gasket.

The first three sidewall construction joints are at 23 -ft 6-in BGS, 28 -ft 9-in BGS, and $~ 36-\mathrm{ft}$ 3-in BGS (H-2-1312). It is possible construction joints in the sidewall could be leak paths through the concrete wall should the liner fail above or at the construction joints. The top construction joint dimensions from tank TY-103 (H-2-2244) were used to calculate the tank BY-103 BGS level as the equivalent BY-103 dimensions are not available.

There are no drywells present on the north or northeastern side of tank BY-103 except for drywell 22-03-01, so it remains unclear whether radioactivity is present in this area. No direct pushes or laterals were installed near tank BY-103.

\subsection{POSSIBLE TANK BY-103 LINER LEAK LOCATION(S)}

A liner leak may have penetrated the waterproof membrane at any location or pooled on the waterproof membrane and followed concrete cracks or construction joints to a different location for egress to the soil, including the top of the tank footing. Tank BY-103 had multiple leak sites that possibly consisted of multiple sidewall leaks and leaks that were located near the tank footing based on ten drywells that report radioactivity. There is also a remote possibility of a leak from the cascade inlet line, capped spare inlets, and/or the condenser; however, it remains inconclusive.

There were no drywells or direct pushes located on the northern or northeastern side of tank BY-103, with the exception of drywell 22-03-01. Therefore, it is possible leaks could have occurred and not been detected in this vicinity of tank BY-103.

\subsubsection{Leak Detected in 1959-1974}

Tank BY-103 was first suspected of leaking in 1969 due to radioactivity being detected in drywell 22-00-03; however, the leak may have begun as early as 1959 (see site A in Figure 4-17). It appears a sidewall leak is present near drywell 22-03-05 as radioactivity was the highest in this drywell with a peak detected at 36-ft BGS. The peak at 36- $\mathrm{ft}$ BGS is 14- $\mathrm{ft}$ below the capped spare inlets and $\sim 10$-ft above the top of the tank footing with drywell 22-0305 located 2.1-ft from the tank footing. The probability favors a tank sidewall leak versus a leak from the capped spare inlets. Radioactivity detected in drywells 22-03-04, 22-00-02, 2200-03, and 22-00-04 were likely from migration of the leak located near drywell 22-03-05 as 
radioactivity levels were lower and peaks were detected at lower BGS levels (ranging from 42 to 79-ft BGS). Radioactivity continued to be detected in the drywells surrounding the tank and tank BY-103 was classified as a confirmed leaker in 1973.

Figure 4-17. Tank BY-103 Possible Leak Location (1959-1974)

Tank inner ring is steel liner, outer ring is outer edge of tank footing

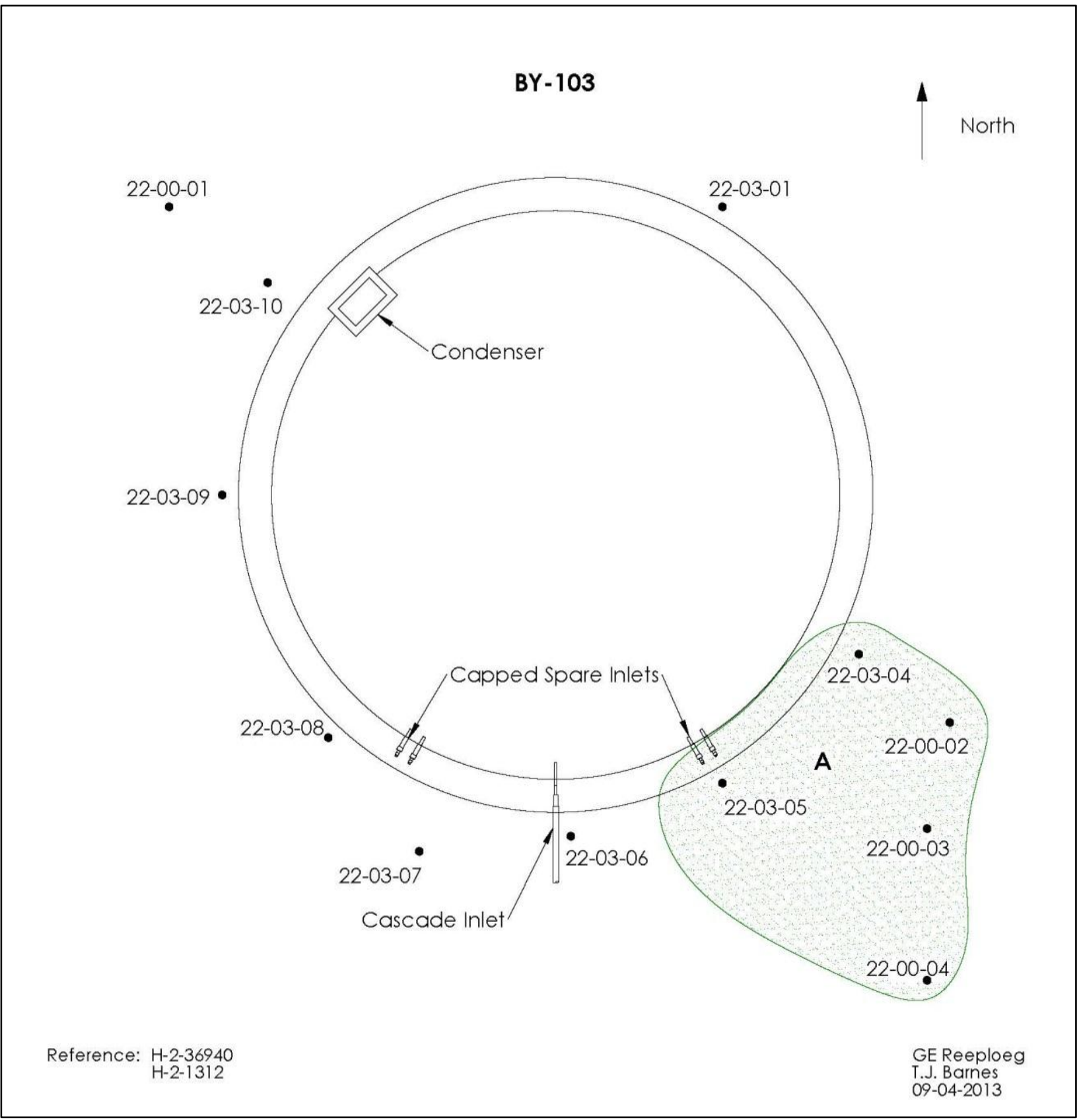

Tank BY-103 was first suspected of leaking in 1969 due to radioactivity being detected in drywell 22-00-03; however, the leak may have begun as early as 1959. 


\subsubsection{Leak Detected in February 1973}

The first recoverable reading for drywell 22-03-06 reported radioactivity at 45-ft BGS in February 1973 with a second peak at 27-ft BGS later detected in July 1974 (see site B in Figure 4-18). The peak detected at 45-ft BGS appears to be a separate leak site from site A (see Figure 4-17) near the tank footing. However, there may be migration from site A (see Figure 4-20). The peak detected at 27-ft BGS could be the result of sidewall leak and/or a leak from the cascade inlet line. Radioactivity was also reported in drywell 22-03-07 at 58-ft BGS in April 1974. It appears radioactivity detected in drywell 22-03-07 is likely from migration from site B and/or site C (see Figure 4-19).

Figure 4-18. Tank BY-103 Possible Leak Location (February 1973)

Tank inner ring is steel liner, outer ring is outer edge of tank footing

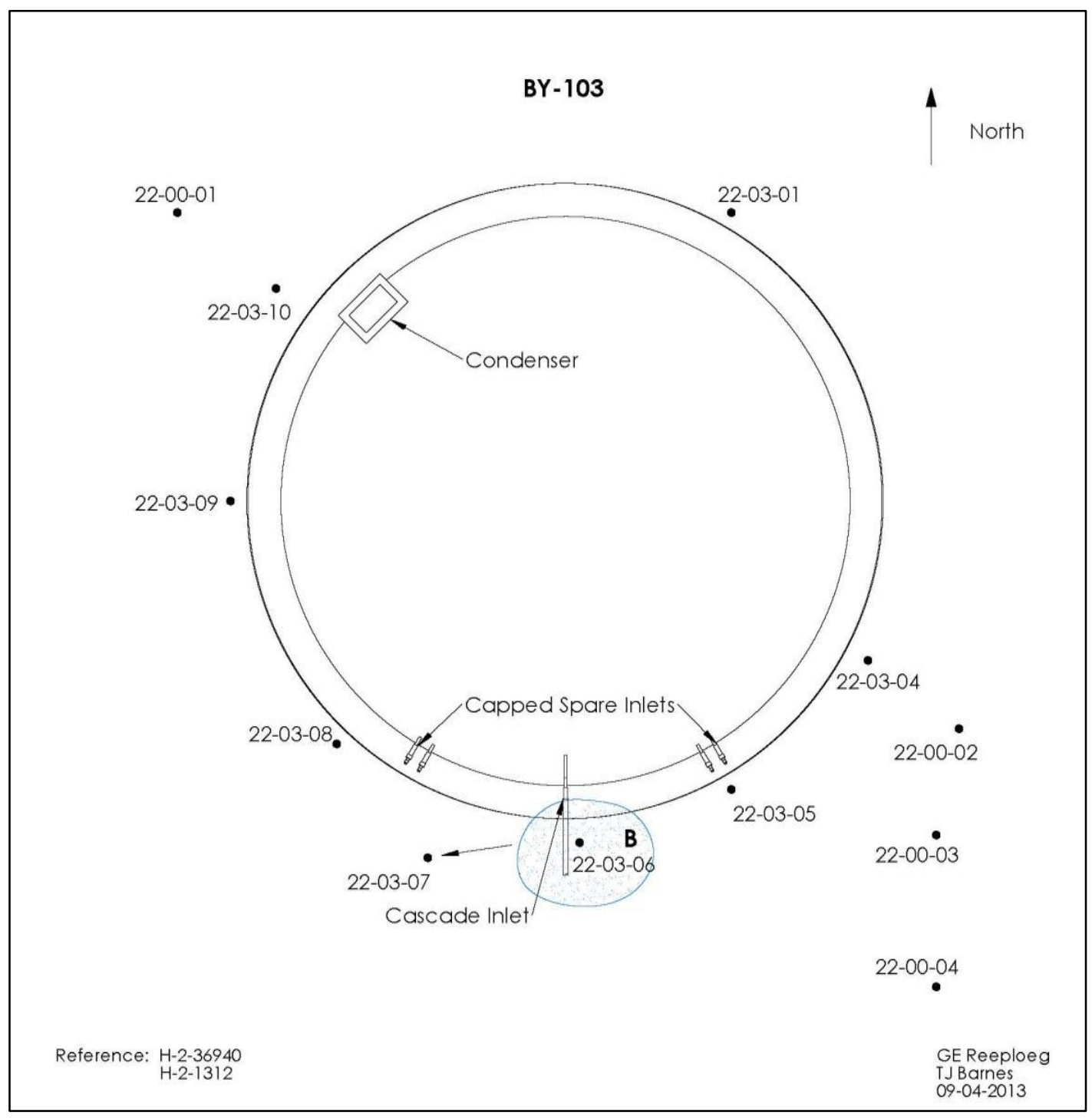

Tank BY-103 was first suspected of leaking in 1969 due to radioactivity being detected in drywell 22-00-03; however, the leak may have begun as early as 1959. 


\subsubsection{Leak Detected in 1972-1974}

The first recoverable reading for drywell 22-03-09 reported radioactivity in August 1972 from 57 to $62-\mathrm{ft}$ BGS. Two additional peaks were recorded July 1973 at 18-ft BGS and December 1973 at 34-ft BGS. Radioactivity was first detected January 1975 in drywell 22-03-10 at 21-ft BGS. Drywell 22-03-08 reported a peak at 46-ft BGS in April 1974. It appears tank BY-103 leaked near drywell 22-03-09 (see site $C$ in Figure 4-19) as radioactivity levels were the highest compared to the surrounding drywells. It appears the peak detected in drywell 22-0309 at 34-ft BGS indicate a possible sidewall leak since no pipes that were reported to have leaked are present in this area at this BGS level. The source of the 18-ft peak (located above the tank liner) detected in drywell 22-03-09 remains uncertain. There is a remote possibility that the drywell 22-03-10 radioactivity came from a leak at the tank condenser (see Section 4.5.2). Radioactivity was also reported in drywell 22-03-07 at 58-ft BGS in April 1974. It appears radioactivity detected in this drywell is likely from migration from site B (see Figure 4-18) and/or site $C$.

Figure 4-19. Tank BY-103 Possible Leak Location (1972-1974)

Tank inner ring is steel liner, outer ring is outer edge of tank footing

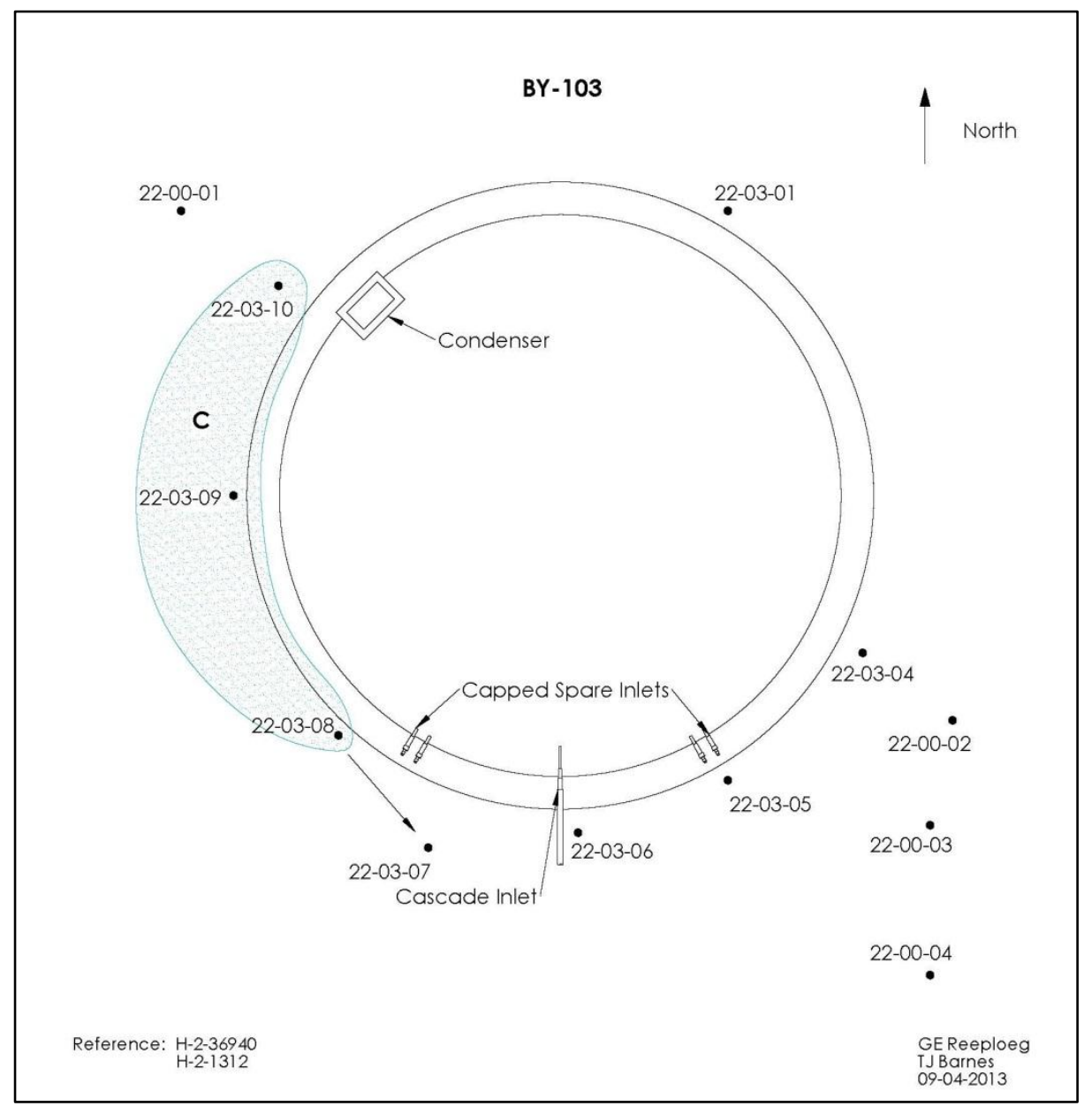

Tank BY-103 was first suspected of leaking in 1969 due to radioactivity being detected in drywell 22-00-03; however, the leak may have begun as early as 1959 . 


\section{RPP-RPT-54911, Rev. 0}

\subsubsection{Leak Location Summary}

Tank BY-103 was first suspected of leaking in 1969 due to radioactivity being detected in drywell 22-00-03; however, the leak may have begun as early as 1959. Radioactivity was reported in drywells 22-03-04, 22-03-05, 22-00-02, 22-00-03, and 22-00-04 in 1959-1974. It appears a sidewall leak is present near drywell 22-03-05 due to radioactivity being the highest in this drywell as shown in site A (see Figure 4-20). It appears radioactivity detected in drywells 22-03-04, 22-00-02, 22-00-03, and 22-00-04 is due to migration from the leak near drywell 22-03-05 due to lower levels of radioactivity and BGS levels.

It appears radioactivity detected in drywell 22-03-06 (see site B in Figure 4-20) could be from a sidewall leak from tank BY-103 and/or a leak from the cascade inlet line. The source remains uncertain; however, it appears this is a separate leak site from sites A and C.

The first recoverable reading for drywell 22-03-09 reported radioactivity in August 1972 from 57 to $62-\mathrm{ft}$ BGS. Two additional peaks were recorded July 1973 at 18 -ft BGS and December 1973 at 34-ft BGS. Radioactivity was first detected January 1975 in drywell 22-03-10 at 21-ft BGS. Drywell 22-03-08 reported a peak at 46-ft BGS in April 1974. It appears tank BY-103 leaked near drywell 22-03-09 (see site C in Figure 4-20) as radioactivity levels were the highest compared to the surrounding drywells. It appears the peak detected in drywell 22-0309 at 34-ft BGS indicate a possible sidewall leak since no pipes that were reported to have leaked are present in this area at this BGS level. The source of the 18-ft peak (located above the tank liner) detected in drywell 22-03-09 remains uncertain. There is a remote possibility that the drywell 22-03-10 radioactivity came from a leak at the tank condenser (see Section 4.5.2).

It appears radioactivity detected in drywell 22-03-07 is likely from migration from site B and/or site C (see Figure 4-20).

Tank BY-103 had multiple leak sites that possibly consisted of multiple sidewalls leaks and leaks that were located near the tank footing based on ten drywells that report radioactivity. There is also a possibility of a leak from the cascade inlet line and/or the condenser; however, it remains inconclusive. It is possible contamination exists underneath the tank and in the north and northeastern portion of the tank; however, no drywells (with the exception of drywell 22-03-01) or laterals are present in this area so it remains inconclusive.

Leak locations in Figure 4-20 are based on peak readings and are a representation of possible initial boundaries of radioactivity.

No evidence was found for a liner bulge occurring in tank BY-103, and it remains unclear if a liner bulge once existed in the tank during its operation. However, tank BY-103 non-boiling temperatures are not likely to be a factor in causing a liner bulge. 
Figure 4-20. Tank BY-103 Possible Radial Leak Locations Tank inner ring is steel liner, outer ring is outer edge of tank footing

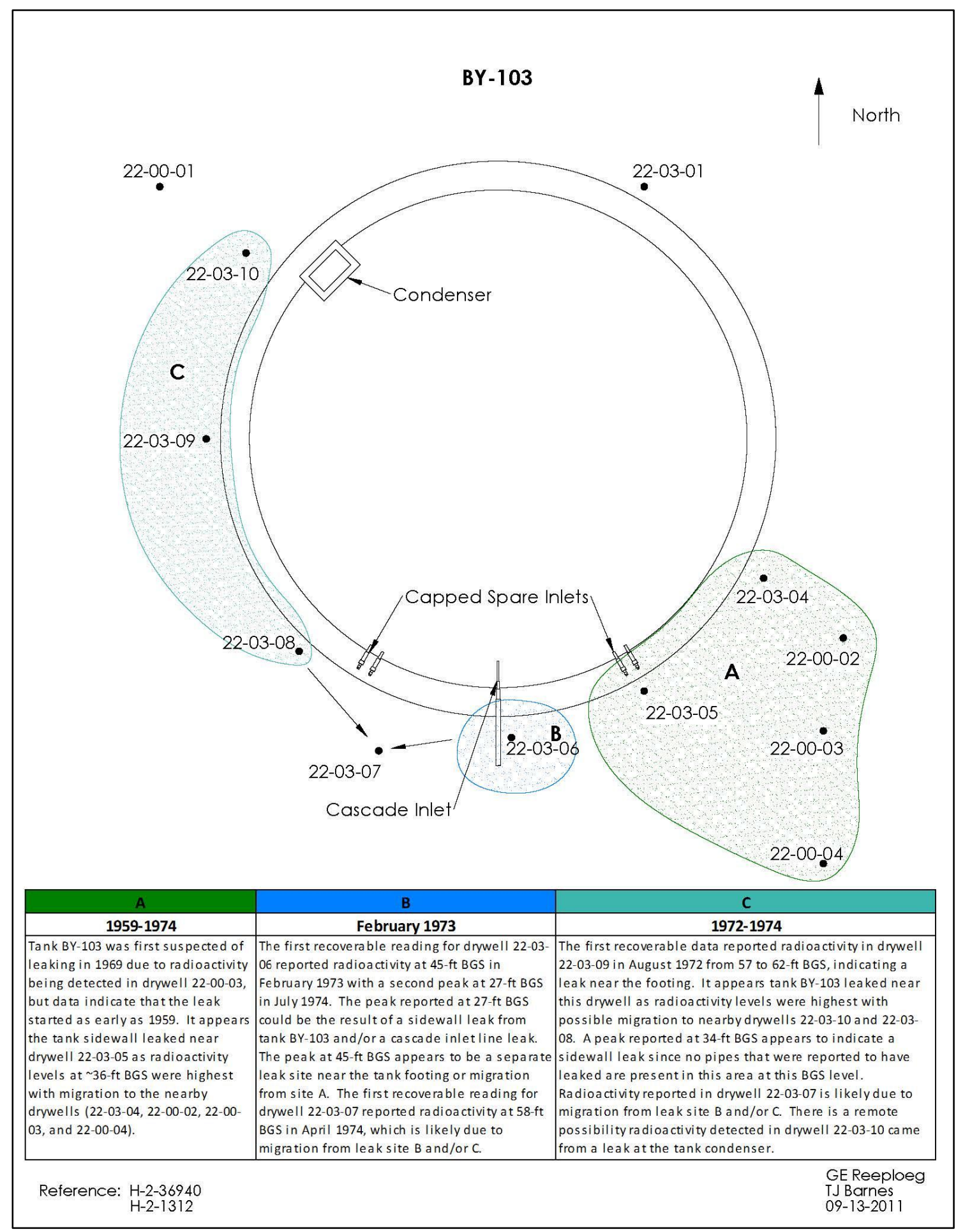


RPP-RPT-54911, Rev. 0

\subsection{POSSIBLE TANK BY-103 LINER LEAK CAUSE(S)}

Tank BY-103 was examined against five conditions that could contribute to a failed liner.

\subsubsection{Tank Design}

The BY Farm tank design does not appear to be a factor contributing to a failed liner (see Section 3.1.1).

\subsubsection{Thermal Conditions}

No temperature data are available for tank BY-103 prior to 1974 although tank BY-103 held non-boiling waste before 1959, when drywell data suggested a tank leak. Since no records are available, it is uncertain what the maximum temperature was in tank BY-103 during operation as well as the rate of temperature rise when waste was initially added. However, the thermal attributes of the waste would indicate that thermal stresses were minimal especially before the very first indications of a tank leak.

Thermal shock creates stress both from rapid temperature rise as well as waste-induced high temperatures which were thought to be minimal and should not have challenged the tank storage limits.

Temperature requirements in ARH-951 (Limitations for Use of Underground Waste Tanks) issued December 18, 1969 indicated that tank temperatures should be held below $230^{\circ} \mathrm{F}$.

\subsubsection{Chemistry-Corrosion}

Tank BY-103 was first filled with MW (1950) which was stored for $\sim 4$ years and then transferred. TBP waste was added to the tank and stored for $\sim 2$ years and then transferred leaving a $26 \mathrm{kgal}$ heel (1957). PUREX HLW supernatant was added to the TBP heel which was stored for $\sim 8$ years and transferred leaving a $143 \mathrm{kgal}$ heel (1965). Tank BY-103 was first suspected of leaking in 1969 due to radioactivity being detected in drywell 22-00-03 but the leak may have begun as early as 1959 based on high level radioactivity present in drywell 22-00-03. There were only three waste types received and stored in tank BY-103 up to 1959.

The TBP waste consisting of low hydroxide, high nitrate, and unknown nitrite concentrations created an environment conducive to pitting and SCC. The TBP waste appears to be the most probable waste environment to have affected the tank liner before 1959. Other leaks may have occurred after 1959 and been affected by subsequent waste types but an exact chronology is not available to pinpoint other subsequent causes if any.

\subsubsection{Liner Observations}

A review of the available photographs taken December 10, 1973 does not contain any evidence of a tank bottom liner bulge. There is no documentation available indicating a liner bulge was present in tank BY-103. 


\subsubsection{Tank Construction Temperature}

Average winter temperatures were $26.9^{\circ} \mathrm{F}$ and $13.9^{\circ} \mathrm{F}$ in December 1948 and January 1949, respectively, with temperatures as low as $-2^{\circ} \mathrm{F}$ during the period when the BY Farm tank liners were being constructed. The low temperatures experienced during construction were less than the current $18^{\circ} \mathrm{F}$ allowable temperature where impact loading had the potential for creating micro-fissures. Impact occurrences could have occurred during cold temperatures that may have triggered fissures in the steel liner. The BY Farm construction specifications (HW-3783, Specifications Additional Waste Storage Facilities 200 East Area, 241-BY) contained specific detailed requirements for cold weather construction and it appears these

low temperatures had less of an impact during the construction of BY Farm compared to 241SX Farm construction (see Section 4.3.2).

\subsection{TANK BY-103 CONCLUSIONS}

Evidence indicates that the tank BY-103 liner leaked at or near the tank footing and at sidewall locations. Based on engineering judgment and available information the following conditions listed in the order of importance lead to the tank BY-103 failed liner.

1. TBP waste storage chemistry-corrosion -pitting and SCC

2. Thermal conditions

3. Construction conditions-ductile-to-brittle transition temperature

There are several liner leak cause conditions that were examined but the most likely cause of the tank BY-103 leak is TBP waste storage chemistry-corrosion. TBP waste storage could have created a chemical environment susceptible to nitrate-induced SCC or pitting on a potentially stressed metal liner.

Thermal and construction conditions could have also contributed to the liner failure but to a lesser degree. Tank design doesn't seem to have contributed to liner failure and there is no evidence of liner bulging. However, some or all of the factors can act serially or together to contribute to tank liner failure. 
RPP-RPT-54911, Rev. 0

APPENDIX A1

TANK BY-103 GROSS GAMMA DRYWELL DATA

A1-1 
Table A1-1. Tank BY-103 Drywell Radioactivity (K counts per minute)

(August 1972 to March 1987) (Sheet 1 of 4)

(Drywell Data Sheets* Retrieved on August 31, 2011 and SD-WM-TI-356)

\begin{tabular}{|c|c|c|c|c|c|c|c|c|c|c|c|c|c|c|c|c|c|c|c|c|}
\hline \multirow{2}{*}{\multicolumn{2}{|c|}{$\begin{array}{c}\text { 22-03-01 } \\
\text { Drilled 8/1970 }\end{array}$}} & \multicolumn{3}{|c|}{ 22-03-04 } & \multicolumn{4}{|c|}{ 22-03-05 } & \multicolumn{3}{|c|}{ 22-03-06 } & \multicolumn{3}{|c|}{ 22-03-07 } & \multicolumn{3}{|c|}{ 22-03-08 } & \multicolumn{3}{|c|}{ 22-03-09 } \\
\hline & & \multicolumn{3}{|c|}{ Drilled 12/31/1972 } & \multicolumn{4}{|c|}{ Drilled 8/31/1970 } & \multicolumn{3}{|c|}{ Drilled 12/31/1972 } & \multicolumn{3}{|c|}{ Drilled 12/31/1973 } & \multicolumn{3}{|c|}{ Drilled 12/31/1973 } & \multicolumn{3}{|c|}{ Drilled 8/31/1970 } \\
\hline Date & $\begin{array}{l}\text { Peak } \\
(\mathbf{K} \\
\text { cpm) }\end{array}$ & Date & $\begin{array}{c}\text { Peak } \\
(\mathbf{K} \\
\text { cpm) }\end{array}$ & $\begin{array}{l}\text { Depth } \\
\text { (ft } \\
\text { BGS) }\end{array}$ & Date & Probe & $\begin{array}{l}\text { Peak } \\
(\mathbf{K} \\
\mathbf{c p m})\end{array}$ & $\begin{array}{l}\text { Depth } \\
\text { (ft } \\
\text { BGS) }\end{array}$ & Date & $\begin{array}{c}\text { Peak } \\
(\mathbf{K} \\
\mathbf{c p m})\end{array}$ & $\begin{array}{l}\text { Depth } \\
\text { (ft } \\
\text { BGS) }\end{array}$ & Date & $\begin{array}{c}\text { Peak } \\
(\mathbf{K} \\
\text { cpm) }\end{array}$ & $\begin{array}{l}\text { Depth } \\
\text { (ft } \\
\text { BGS) }\end{array}$ & Date & $\begin{array}{c}\text { Peak } \\
(\mathbf{K} \\
\mathbf{c p m})\end{array}$ & $\begin{array}{l}\text { Depth } \\
\text { (ft } \\
\text { BGS) }\end{array}$ & Date & $\begin{array}{c}\text { Peak } \\
(\mathbf{K} \\
\text { cpm) }\end{array}$ & $\begin{array}{l}\text { Depth } \\
\text { (ft } \\
\text { BGS) }\end{array}$ \\
\hline $3 / 71^{2}$ & $<50$ & \multirow{7}{*}{\multicolumn{3}{|c|}{$\begin{array}{l}\text { Drywell 22-03-04 initially } \\
\text { read February } 1973^{2}\end{array}$}} & $3 / 71-12 / 71$ & $\mathrm{NA}^{1}$ & $\mathrm{High}^{2}$ & $\mathrm{NA}^{1}$ & \multirow{7}{*}{\multicolumn{3}{|c|}{$\begin{array}{l}\text { Drywell 22-03-06 initially } \\
\quad \text { read February } 1973^{2}\end{array}$}} & \multicolumn{3}{|c|}{-} & \multicolumn{3}{|c|}{-} & $3 / 71^{2}$ & $<50$ & $\mathrm{NA}^{1}$ \\
\hline $8 / 22 / 72$ & $<12$ & & & & $8 / 22 / 72$ & $\mathrm{NA}^{1}$ & 3811.9 & 36 & & & & \multicolumn{3}{|c|}{-} & \multicolumn{3}{|c|}{-} & $8 / 22 / 72$ & 27.6 & $57-62$ \\
\hline \multicolumn{2}{|c|}{$\mathrm{NA}^{1}$} & & & & $9 / 26 / 72$ & $\mathrm{NA}^{1}$ & 3800.6 & 35 & & & & \multicolumn{3}{|c|}{-} & \multicolumn{3}{|c|}{-} & \multicolumn{3}{|c|}{$\mathrm{NA}^{1}$} \\
\hline \multicolumn{2}{|c|}{$\mathrm{NA}^{1}$} & & & & $10 / 3 / 72$ & $\mathrm{NA}^{1}$ & 3535.3 & 35 & & & & & - & & & - & & & $\mathrm{NA}^{1}$ & \\
\hline \multicolumn{2}{|c|}{$\mathrm{NA}^{1}$} & & & & $11 / 7 / 72$ & $\mathrm{NA}^{1}$ & 3979.4 & 35 & & & & & - & & & - & & & $\mathrm{NA}^{1}$ & \\
\hline \multicolumn{2}{|c|}{$\mathrm{NA}^{1}$} & & & & $12 / 5 / 72$ & $\mathrm{NA}^{1}$ & 3884.7 & 35 & & & & & - & & & - & & & $\mathrm{NA}^{1}$ & \\
\hline $1 / 25 / 73$ & none & & & & $1 / 3 / 73$ & $\mathrm{NA}^{1}$ & 3665.2 & 35 & & & & & - & & & - & & $1 / 25 / 73$ & 45 & $58-71$ \\
\hline \multicolumn{2}{|c|}{$\mathrm{NA}^{1}$} & $2 / 6 / 73$ & 70 & 52 & $2 / 6 / 73$ & $\mathrm{NA}^{1}$ & 3173.6 & 37 & $2 / 6 / 73$ & 226.4 & 45 & & - & & & - & & & $\mathrm{NA}^{1}$ & \\
\hline & & $3 / 30 / 73$ & 60.8 & 51 & $3 / 20 / 73$ & $\mathrm{NA}^{1}$ & 2208.4 & 40 & & $\mathrm{NA}^{1}$ & & & - & & & - & & $3 / 30 / 73$ & 60 & $56-75$ \\
\hline & & & $\mathrm{NA}^{1}$ & & $4 / 3 / 73$ & $\mathrm{NA}^{1}$ & 2137.2 & 41 & & $\mathrm{NA}^{1}$ & & & - & & & - & & & $\mathrm{NA}^{1}$ & \\
\hline & & $5 / 23 / 73$ & 41.3 & 52 & $5 / 2 / 73$ & $\mathrm{NA}^{1}$ & 4185.3 & 37 & & $\mathrm{NA}^{1}$ & & & - & & & - & & $5 / 2 / 73$ & 75 & $56-77$ \\
\hline $6 / 25 / 73$ & $<12$ & $6 / 25 / 73$ & 57.9 & 50 & $6 / 22 / 73$ & $\mathrm{NA}^{1}$ & 2488.9 & 35 & $6 / 25 / 73$ & 194.9 & 42 & & - & & & - & & $6 / 29 / 73$ & 75 & $55-74$ \\
\hline & & & $\mathrm{NA}^{1}$ & & $7 / 2 / 73$ & $\mathrm{NA}^{1}$ & 2372.6 & 36 & & $\mathrm{NA}^{1}$ & & & - & & & - & & 7/17/73 & 62.6 & 18 \\
\hline & & $8 / 3 / 73$ & 19.9 & 49 & $8 / 6 / 73$ & $\mathrm{NA}^{1}$ & 2843.6 & 35 & & $\mathrm{NA}^{1}$ & & & - & & & - & & $8 / 6 / 73$ & 49.3 & 18 \\
\hline & & $9 / 4 / 73$ & 15.3 & 51 & $9 / 4 / 73$ & $\mathrm{NA}^{1}$ & 2906.6 & 35 & & $\mathrm{NA}^{1}$ & & & - & & & - & & $9 / 4 / 73$ & 45.6 & 18 \\
\hline & & $10 / 2 / 73$ & 28.3 & 51 & $10 / 2 / 73$ & $\mathrm{NA}^{1}$ & 1798.8 & 35 & & $\mathrm{NA}^{1}$ & & & - & & & - & & $10 / 2 / 73$ & 70.3 & 19 \\
\hline & & $11 / 6 / 73$ & 18.4 & 50 & $11 / 6 / 73$ & $\mathrm{NA}^{1}$ & 3611.5 & 36 & & $\mathrm{NA}^{1}$ & & & - & & & - & & $11 / 6 / 73$ & 38.1 & 18 \\
\hline & & $12 / 5 / 73$ & 58.7 & 52 & $12 / 5 / 73$ & $\mathrm{NA}^{1}$ & 5452.1 & 37 & $12 / 31 / 73$ & 206.2 & 40 & & $\mathrm{NA}^{1}$ & & & $\mathrm{NA}^{1}$ & & $12 / 24 / 73$ & 82.5 & 56-78 \\
\hline & & & & & & & & & & 60.0 & 25 & & & & & & & & 120 & 19 \\
\hline $1 / 1 / 74$ & $<6$ & $1 / 9 / 74$ & 54.0 & 51 & $1 / 7 / 74$ & $\mathrm{NA}^{1}$ & 763.8 & 35 & $1 / 1 / 74$ & 204.0 & 44 & & $\mathrm{NA}^{1}$ & & & $\mathrm{NA}^{1}$ & & $1 / 1 / 74$ & 18 & 32 \\
\hline & & & & & & & & & & & & & & & & & & & 105 & 63 \\
\hline
\end{tabular}


RPP-RPT-54911, Rev. 0

Table A1-1. Tank BY-103 Drywell Radioactivity (K counts per minute)

(August 1972 to March 1987) (Sheet 2 of 4)

(Drywell Data Sheets* Retrieved on August 31, 2011 and SD-WM-TI-356)

\begin{tabular}{|c|c|c|c|c|c|c|c|c|c|c|c|c|c|c|c|c|c|c|c|c|}
\hline \multicolumn{2}{|c|}{ 22-03-01 } & \multicolumn{3}{|c|}{ 22-03-04 } & \multicolumn{4}{|c|}{ 22-03-05 } & \multicolumn{3}{|c|}{ 22-03-06 } & \multicolumn{3}{|c|}{ 22-03-07 } & \multicolumn{3}{|c|}{ 22-03-08 } & \multicolumn{3}{|c|}{ 22-03-09 } \\
\hline Date & $\begin{array}{c}\text { Peak } \\
(\mathbf{K} \\
\text { cpm) }\end{array}$ & Date & $\begin{array}{c}\text { Peak } \\
(\mathbf{K} \\
\text { cpm) }\end{array}$ & $\begin{array}{c}\text { Depth } \\
\text { (ft } \\
\text { BGS) }\end{array}$ & Date & Probe & $\begin{array}{c}\text { Peak } \\
(\mathbf{K} \\
\text { cpm) }\end{array}$ & $\begin{array}{c}\text { Depth } \\
\text { (ft } \\
\text { BGS) }\end{array}$ & Date & $\begin{array}{c}\text { Peak } \\
(\mathbf{K} \\
\text { cpm) } \\
\end{array}$ & $\begin{array}{c}\text { Depth } \\
\text { (ft } \\
\text { BGS) }\end{array}$ & Date & $\begin{array}{c}\text { Peak } \\
(\mathrm{K} \\
\text { cpm) }\end{array}$ & $\begin{array}{c}\text { Depth } \\
\text { (ft } \\
\text { BGS) }\end{array}$ & Date & $\begin{array}{c}\text { Peak } \\
(\mathbf{K} \\
\text { cpm) }\end{array}$ & $\begin{array}{c}\text { Depth } \\
\text { (ft } \\
\text { BGS) }\end{array}$ & Date & $\begin{array}{c}\text { Peak } \\
(\mathbf{K} \\
\text { cpm) }\end{array}$ & $\begin{array}{c}\text { Depth } \\
\text { (ft } \\
\text { BGS) }\end{array}$ \\
\hline \multicolumn{2}{|c|}{$\mathrm{NA}^{1}$} & \multicolumn{3}{|c|}{$\mathrm{NA}^{1}$} & $2 / 28 / 74$ & GMP & 440.0 & 32 & $2 / 1 / 74$ & 198.0 & 44 & \multicolumn{3}{|c|}{$\mathrm{NA}^{1}$} & \multicolumn{3}{|c|}{$\mathrm{NA}^{1}$} & $2 / 1 / 74$ & 42 & 32 \\
\hline $3 / 29 / 74$ & $<12$ & $3 / 26 / 74$ & 48.5 & 52 & $3 / 1 / 74$ & GMP & 624 & 32 & $3 / 1 / 74$ & 195.0 & 42 & \multicolumn{3}{|c|}{$\mathrm{NA}^{1}$} & \multicolumn{3}{|c|}{$\mathrm{NA}^{1}$} & $3 / 1 / 74$ & 48 & 33 \\
\hline \multirow{2}{*}{\multicolumn{2}{|c|}{$\mathrm{NA}^{1}$}} & \multirow{2}{*}{$4 / 30 / 74$} & \multirow{2}{*}{48.6} & \multirow{2}{*}{53} & \multirow{2}{*}{$4 / 1 / 74$} & \multirow{2}{*}{ GMP } & \multirow{2}{*}{585} & \multirow[b]{2}{*}{32} & \multirow{2}{*}{$4 / 1 / 74$} & \multirow{2}{*}{168.0} & \multirow{2}{*}{42} & \multirow{2}{*}{$4 / 8 / 74$} & \multirow{2}{*}{11.1} & & & & & & 60 & 34 \\
\hline & & & & & & & & & & & & & & 58 & 4/8/74 & 30.7 & 46 & 4/9/74 & 87.6 & 68 \\
\hline $\mathrm{NA}$ & & $5 / 20 / 74$ & 47.0 & 52 & $5 / 3 / 74$ & $\mathrm{NA}^{1}$ & 710.0 & 34 & $5 / 7 / 74$ & 185.6 & 45 & $5 / 23 / 74$ & 15.1 & 58 & $5 / 1 / 74$ & 30.6 & 47 & $5 / 1 / 74$ & 114 & 33 \\
\hline $\mathrm{NA}$ & & $6 / 3 / 74$ & 47.0 & 52 & $6 / 1 / 74$ & GMP & 450 & 31 & $6 / 1 / 74$ & 186.0 & 46 & $6 / 1 / 74$ & 24 & 58 & $6 / 27 / 74$ & 23.4 & 45 & $6 / 1 / 74$ & 177 & 34 \\
\hline & & & & & & & & & & 60.0 & 27 & & & & & & & & 45 & 18 \\
\hline $7 / 1 / 74$ & $<6$ & $7 / 3 / 74$ & 45.7 & 52 & $7 / 1 / 74$ & GMP & 639 & 31 & $7 / 1 / 74$ & 174.0 & 45 & $7 / 1 / 74$ & 13.8 & 59 & $7 / 1 / 74$ & 18 & 46 & $7 / 1 / 74$ & 201 & 34 \\
\hline & & & & & & & & & & & & & & & & & & & 84 & 67 \\
\hline NA & & $8 / 7 / 74$ & 494 & 49 & $8 / 15 / 74$ & $N A^{1}$ & 8167 & 33 & $8 / 22 / 74$ & 61.5 & 23 & $8 / 20 / 74$ & 150 & 57 & $8 / 22 / 74$ & 105 & 43 & $8 / 22 / 74$ & 276 & 33 \\
\hline N & & $8 / / 1 / 4$ & 49.4 & 49 & $8 / 13 / / 4$ & $\mathrm{NA}$ & 810.1 & 33 & $8 / 227 / 4$ & 195.0 & 45 & $8 / 20 / / 4$ & 15.9 & 31 & $8 / 227 / 4$ & 19.5 & 43 & $8 / 221 / 4$ & 210 & 33 \\
\hline & & & & & & & & & & 75.0 & 23 & & & & & & & & 54 & 18 \\
\hline $\mathrm{N} A$ & & $9 / 6 / 74$ & 42.2 & 50 & $9 / 26 / 74$ & GMP & 480 & 32 & $9 / 25 / 74$ & 201.0 & 45 & $9 / 25 / 74$ & 17.7 & 57 & $9 / 25 / 74$ & 20.1 & 43 & $9 / 25 / 74$ & 288 & 35 \\
\hline & & & & & & & & & & & & & & & & & & & 93 & 68 \\
\hline $\mathrm{N}$ & & $10 / 11 / 74$ & 42.8 & 51 & $10 / 11 / 74$ & $\mathrm{NA}^{1}$ & 652.1 & 34 & & $N A^{1}$ & & & $N A^{1}$ & & & $\mathrm{NA}^{1}$ & & $10 / 11 / 74$ & 279.84 & 34 \\
\hline $\mathrm{N} A$ & & $11 / 7 / 74$ & 42.8 & 53 & $11 / 6 / 74$ & $\mathrm{NA}^{1}$ & 956.6 & 34 & & $\mathrm{NA}^{-}$ & & & $\mathrm{NA}^{2}$ & & & $\mathrm{NA}^{2}$ & & $10 / 11 / / 4$ & 78 & 68 \\
\hline $\mathrm{N}$ & & $12 / 3 / 74$ & 42.9 & 51 & $12 / 3 / 74$ & $\mathrm{NA}^{1}$ & 3838 & 36 & & $\mathrm{NA}^{1}$ & & & $\mathrm{NA}^{1}$ & & & $\mathrm{NA}^{1}$ & & & $\mathrm{NA}^{1}$ & \\
\hline $\mathrm{N} A$ & & $1 / 1 / 75$ & 36.6 & 52 & $1 / 1 / 75$ & $\mathrm{NA}^{1}$ & 4217 & 37 & $1 / 24 / 75$ & 158.3 & 44 & $1 / 24 / 75$ & 13.0 & 58 & $10 / 11 / 74$ & 17.2 & 46 & & 321.24 & 35 \\
\hline $1 / 24 / 75$ & $<3$ & $1 / 24 / 75$ & 39.6 & 50 & $1 / 29 / 75$ & $\mathrm{NA}^{1}$ & 782.2 & 34 & & $\mathrm{NA}^{1}$ & & & $\mathrm{NA}^{1}$ & & & $\mathrm{NA}^{1}$ & & $1 / 24 / / 3$ & 75 & 74 \\
\hline $7 / 11 / 75$ & $<3$ & $7 / 11 / 75$ & 340 & 50 & $7 / 2 / 75$ & $N A^{1}$ & 8378 & 34 & $7 / 11 / 75$ & 1439 & 42 & $7 / 11 / 75$ & 124 & 57 & $1 / 24 / 75$ & 11.3 & 46 & $7 / 11 / 75$ & 316.62 & 35 \\
\hline /11/10 & 3 & 1/111/2 & 34.9 & 50 & $1 / 21 / 3$ & NA & 831.8 & 34 & $1 / 11 / 18$ & 143.9 & 42 & //11//5 & 12.4 & 51 & $7 / 11 / 75$ & 10.0 & 45 & $1 / 11 / 15$ & 99 & 74 \\
\hline $1 / 29 / 76$ & $<3$ & $2 / 5 / 76$ & 296 & 48 & $2 / 3 / 76$ & $N A^{1}$ & 7613 & 32 & $2 / 5 / 76$ & 1302 & 40 & $2 / 4 / 76$ & 100 & 55 & $2 / 4 / 76$ & 76 & 43 & $2 / 4 / 76$ & 239.4 & 35 \\
\hline $1 / 291 / 0$ & 3 & $2 / 3 / 10$ & 29.0 & 48 & 273710 & NA & 101.3 & 32 & $2 / 3 / / 0$ & 150.2 & 40 & $2 / 4 / / 0$ & 10.0 & 53 & $2 / 4 / 10$ & 1.0 & 43 & $2 / 4 / / 0$ & 105 & 75 \\
\hline
\end{tabular}


RPP-RPT-54911, Rev. 0

Table A1-1. Tank BY-103 Drywell Radioactivity (K counts per minute)

(August 1972 to March 1987) (Sheet 3 of 4)

(Drywell Data Sheets* Retrieved on August 31, 2011 and SD-WM-TI-356)

\begin{tabular}{|c|c|c|c|c|c|c|c|c|c|c|c|c|c|c|c|c|c|c|c|c|}
\hline \multicolumn{2}{|c|}{ 22-03-01 } & \multicolumn{3}{|c|}{ 22-03-04 } & \multicolumn{4}{|c|}{ 22-03-05 } & \multicolumn{3}{|c|}{ 22-03-06 } & \multicolumn{3}{|c|}{ 22-03-07 } & \multicolumn{3}{|c|}{ 22-03-08 } & \multicolumn{3}{|c|}{ 22-03-09 } \\
\hline Date & $\begin{array}{c}\text { Peak } \\
(\mathbf{K} \\
\text { cpm) }\end{array}$ & Date & $\begin{array}{c}\text { Peak } \\
\text { (K } \\
\text { cpm) }\end{array}$ & $\begin{array}{c}\text { Depth } \\
\text { (ft } \\
\text { BGS) }\end{array}$ & Date & Probe & $\begin{array}{c}\text { Peak } \\
(\mathbf{K} \\
\text { cpm) }\end{array}$ & $\begin{array}{c}\text { Depth } \\
\text { (ft } \\
\text { BGS) }\end{array}$ & Date & $\begin{array}{c}\text { Peak } \\
(\mathbf{K} \\
\text { cpm) }\end{array}$ & $\begin{array}{c}\text { Depth } \\
\text { (ft } \\
\text { BGS) }\end{array}$ & Date & $\begin{array}{c}\text { Peak } \\
(\mathbf{K} \\
\text { cpm) }\end{array}$ & $\begin{array}{c}\text { Depth } \\
\text { (ft } \\
\text { BGS) }\end{array}$ & Date & $\begin{array}{c}\text { Peak } \\
(\mathbf{K} \\
\text { cpm) }\end{array}$ & $\begin{array}{c}\text { Depth } \\
\text { (ft } \\
\text { BGS) }\end{array}$ & Date & $\begin{array}{c}\text { Peak } \\
(\mathbf{K} \\
\text { cpm) }\end{array}$ & 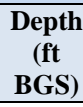 \\
\hline \multirow{2}{*}{$3 / 3 / 77$} & \multirow{2}{*}{$<3$} & \multirow{2}{*}{$3 / 3 / 77$} & \multirow{2}{*}{22.9} & \multirow[b]{2}{*}{49} & \multirow{2}{*}{$3 / 2 / 77$} & \multirow{2}{*}{$\mathrm{NA}^{1}$} & \multirow{2}{*}{746.7} & \multirow{2}{*}{32} & \multirow{2}{*}{$2 / 24 / 77$} & \multirow{2}{*}{107.8} & \multirow{2}{*}{40} & \multirow{2}{*}{$3 / 3 / 77$} & \multirow{2}{*}{7.9} & \multirow{2}{*}{56} & \multirow{2}{*}{$3 / 3 / 77$} & \multirow{2}{*}{6.5} & \multirow{2}{*}{45} & \multirow{2}{*}{$3 / 3 / 77$} & 125.88 & 34 \\
\hline & & & & & & & & & & & & & & & & & & & 114 & 78 \\
\hline \multirow{2}{*}{\multicolumn{2}{|c|}{$\mathrm{NA}^{1}$}} & $9 / 8 / 77$ & 203 & 49 & $9 / 13 / 77$ & $\mathrm{NA}^{1}$ & 6040 & 34 & $7 / 1477$ & 1004 & 40 & $9 / 8 / 77$ & 71 & 56 & $7 / 14 / 77$ & 46 & 46 & $7 / 14 / 77$ & 102 & 34 \\
\hline & & $9 / 8 / 1 / 1$ & 20.3 & 49 & प्रा13/1/ & $\mathrm{NA}$ & 604.0 & 34 & $1 / 14 / / / 1$ & 100.4 & 40 & $9 / 8 / 1 / 1$ & 1.1 & 56 & $1 / 14 / / / 1$ & 4.6 & 46 & $1 / 14 / / / 1$ & 101.4 & 77 \\
\hline & & & & & & & & & & & & & & & & & & & 66 & 35 \\
\hline $3 / 3 / 78$ & $<3$ & $3 / 22 / 78$ & 16.7 & 49 & $3 / 14 / 78$ & $\mathrm{NA}^{\mathrm{T}}$ & 685.0 & 31 & $3 / 17 / 78$ & 89.5 & 39 & $3 / 2 / 78$ & 6.4 & 56 & $3 / 9 / 78$ & 3.8 & 45 & $3 / 16 / 78$ & 92.34 & 78 \\
\hline $\mathrm{N}$ & & $0 / 14 / 78$ & 162 & 48 & $9 / 26178$ & $\mathrm{NA}^{1}$ & 6417 & 33 & $0 / 14 / 78$ & 781 & 41 & $9 / 1 / 78$ & 61 & 55 & $0 / 7 / 78$ & 37 & 46 & & 51 & 35 \\
\hline & & $9 / 14 / 18$ & 16.2 & 48 & $9 / 20 / / 8$ & & 041.1 & 33 & $9 / 14 / / 8$ & $/ 8.1$ & 41 & $9 / 21 / / 8$ & 0.1 & & ס & & & प्रा/ा & 85.38 & 79 \\
\hline $3 / 7 / 70$ & 3 & & & & & $\mathrm{NS}^{1}$ & & & & & & $3 / 21 / 70$ & 58 & 55 & $3 / 7 / 10$ & 30 & 13 & 316170 & 41.4 & 33 \\
\hline $3 / / 7 / 9$ & $<3$ & $3 / 7 / 79$ & 14 & 48 & $3 / 12 / 79$ & $\mathrm{NA}^{1}$ & 633.1 & 33 & $3 / 7 / 79$ & 68.2 & 40 & $3 / 21 / 79$ & 5.8 & 55 & $3 / 1 / 19$ & 3.9 & 43 & $3 / 0 / / 9$ & 77.34 & 79 \\
\hline $2 / 10100$ & 2 & $2 / 10 / 80$ & 12 & 17 & $3 / 10 / 80$ & $\mathrm{NA}^{1}$ & 600.9 & 33 & $2118 / 00$ & 60 & (1) & $2 / 21 / 80$ & 51 & 55 & $2 / 10100$ & 24 & 12 & $2 / 21 / 10$ & 21.6 & 39 \\
\hline 3/18/80 & $<3$ & $3 / 18 / 80$ & 12.1 & $4 /$ & $6 / 2 / 80$ & $\mathrm{NA}^{1}$ & 866.0 & 31 & $3 / 18 / 80$ & 60.6 & 42 & $3 / 31 / 80$ & 5.1 & 55 & $3 / 18 / 80$ & 3.4 & 43 & $3 / 31 / 80$ & 73.44 & 81 \\
\hline $\mathrm{N}$ & & & $\mathrm{NA}^{1}$ & & $6 / 9 / 80$ & $\begin{array}{l}\text { New GMP } \\
\text { No. } 2\end{array}$ & 238.0 & 31 & & $\mathrm{NA}^{1}$ & & & $\mathrm{NA}^{1}$ & & & $\mathrm{NA}^{1}$ & & & $\mathrm{NA}^{1}$ & \\
\hline $\mathrm{N}$ & & & $\mathrm{NA}^{1}$ & & $6 / 16 / 80$ & $\mathrm{NA}^{1}$ & 356.5 & 32 & & $\mathrm{NA}^{1}$ & & & $\mathrm{NA}^{1}$ & & & $\mathrm{NA}^{1}$ & & & $\mathrm{NA}^{1}$ & \\
\hline & & & & & & & & & & & & & & & & & & & 19.2 & 40 \\
\hline $3 / 25 / 81$ & $<3$ & $3 / 24 / 81$ & 9.1 & 48 & $3 / 30 / 81$ & $\mathrm{NA}^{1}$ & 279.3 & 31 & $4 / 1 / 81$ & 51.7 & 41 & $3 / 24 / 81$ & 4.3 & 57 & $3 / 24 / 81$ & 3.5 & 43 & $3 / 24 / 81$ & 62.7 & 82 \\
\hline $3 / 15 / 82$ & $<3$ & $3 / 0 / 82$ & 70 & 40 & $1 / 11 / 82$ & $\mathrm{NA}^{1}$ & 2763 & 32 & $3 / 0 / 82$ & 518 & 22 & $2 / 23 / 82$ & 37 & 56 & $3 / 15 / 82$ & 28 & 44 & $3 / 15 / 82$ & 12.6 & 39 \\
\hline $3 / 15 / 82$ & $<3$ & $3 / 9 / 82$ & 1.9 & 49 & $1 / 11 / 82$ & & 210.3 & 32 & 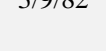 & ग1.0 & & $2123 / 02$ & 5.1 & 30 & $3 / 10 / 02$ & 2.0 & 44 & J/IJ/OL & 55.32 & 83 \\
\hline $3 / 14 / 83$ & $<3$ & $3 / 2 / 83$ & 75 & 50 & $3 / 16 / 83$ & $N A^{1}$ & 3113 & 33 & $3 / 2 / 83$ & 514 & 23 & $2 / 23 / 83$ & 39 & 57 & $3 / 14 / 83$ & 30 & 48 & $3 / 14 / 83$ & 7.38 & 42 \\
\hline & & & & & & & & 33 & $3 / 2 / 83$ & & & & & & & & 48 & $3 / 14 / 83$ & 47.4 & 87 \\
\hline $208 / 84$ & $<3$ & $203 / 84$ & 63 & 50 & $32 / 84$ & 1 & 2372 & 34 & $2 / 23 / 84$ & 164 & 24 & $2 / 2318$ & 34 & 60 & $3 / 12 / 80$ & 2 & 15 & ה & 9.48 & 48 \\
\hline $2 / 28 / 84$ & $<3$ & $2 / 23 / 84$ & 6.3 & 50 & $3 / 2 / 84$ & $\mathrm{NA}^{2}$ & 337.2 & 34 & $2 / 23 / 84$ & 46.4 & 24 & $2 / 23 / 84$ & 3.4 & 60 & $3 / 13 / 84$ & 2.6 & 45 & $2 / 28 / 84$ & 42.9 & 88 \\
\hline
\end{tabular}


RPP-RPT-54911, Rev. 0

Table A1-1. Tank BY-103 Drywell Radioactivity (K counts per minute)

(August 1972 to March 1987) (Sheet 4 of 4)

(Drywell Data Sheets* Retrieved on August 31, 2011 and SD-WM-TI-356)

\begin{tabular}{|c|c|c|c|c|c|c|c|c|c|c|c|c|c|c|c|c|c|c|c|c|}
\hline \multicolumn{2}{|c|}{ 22-03-01 } & \multicolumn{3}{|c|}{ 22-03-04 } & \multicolumn{4}{|c|}{ 22-03-05 } & \multicolumn{3}{|c|}{ 22-03-06 } & \multicolumn{3}{|c|}{ 22-03-07 } & \multicolumn{3}{|c|}{ 22-03-08 } & \multicolumn{3}{|c|}{ 22-03-09 } \\
\hline Date & $\begin{array}{c}\text { Peak } \\
(\mathbf{K} \\
\text { cpm) }\end{array}$ & Date & $\begin{array}{c}\text { Peak } \\
(\mathrm{K} \\
\text { cpm) }\end{array}$ & $\begin{array}{c}\text { Depth } \\
\text { (ft } \\
\text { BGS) }\end{array}$ & Date & Probe & $\begin{array}{c}\text { Peak } \\
(\mathrm{K} \\
\text { cpm) }\end{array}$ & $\begin{array}{c}\text { Depth } \\
\text { (ft } \\
\text { BGS) }\end{array}$ & Date & $\begin{array}{c}\text { Peak } \\
(\mathbf{K} \\
\text { cpm) }\end{array}$ & $\begin{array}{c}\text { Depth } \\
\text { (ft } \\
\text { BGS) }\end{array}$ & Date & $\begin{array}{c}\text { Peak } \\
(\mathbf{K} \\
\text { cpm) }\end{array}$ & $\begin{array}{c}\text { Depth } \\
\text { (ft } \\
\text { BGS) }\end{array}$ & Date & $\begin{array}{c}\text { Peak } \\
(\mathbf{K} \\
\text { cpm) }\end{array}$ & $\begin{array}{c}\text { Depth } \\
\text { (ft } \\
\text { BGS) }\end{array}$ & Date & $\begin{array}{c}\text { Peak } \\
(\mathbf{K} \\
\text { cpm) }\end{array}$ & $\begin{array}{c}\text { Depth } \\
\text { (ft } \\
\text { BGS) }\end{array}$ \\
\hline \multirow{2}{*}{$3 / 18 / 85$} & \multirow{2}{*}{$<3$} & \multirow{2}{*}{$3 / 5 / 85$} & \multirow{2}{*}{4.7} & \multirow{2}{*}{50} & \multirow{2}{*}{$3 / 12 / 85$} & \multirow{2}{*}{$\mathrm{NA}^{1}$} & \multirow{2}{*}{286.1} & \multirow{2}{*}{35} & \multirow{2}{*}{$3 / 5 / 85$} & \multirow{2}{*}{45.3} & \multirow{2}{*}{25} & \multirow{2}{*}{$3 / 20 / 85$} & \multirow{2}{*}{2.8} & \multirow{2}{*}{60} & \multirow[b]{2}{*}{$2 / 13 / 85$} & \multirow{2}{*}{2.5} & \multirow[b]{2}{*}{46} & \multirow{2}{*}{$3 / 11 / 85$} & 6.6 & 49 \\
\hline & & & & & & & & & & & & & & & & & & & 33.36 & 90 \\
\hline \multirow{2}{*}{$3 / 12 / 86$} & \multirow{2}{*}{$<3$} & \multirow{2}{*}{$3 / 17 / 86$} & \multirow{2}{*}{4.3} & \multirow{2}{*}{52} & \multirow{2}{*}{$3 / 10 / 86$} & \multirow{2}{*}{$\mathrm{NA}^{1}$} & \multirow{2}{*}{337.5} & \multirow{2}{*}{35} & $3 / 18 / 86$ & 135 & 4 & $2 / 18 / 86$ & 3.2 & 59 & $3 / 11 / 85$ & $<3$ & $\mathrm{NA}^{1}$ & & 4.62 & 49 \\
\hline & & & & & & & & & 极 & 45.0 & 24 & $3 / 17 / 86$ & $<3$ & $\mathrm{NA}^{1}$ & $3 / 10 / 86$ & $<3$ & $\mathrm{NA}^{1}$ & Jitiolov & 28.74 & 91 \\
\hline 21107 & & $8 / 1297$ & 2 & 51 & & 1 & 2004 & 25 & 7 & $10 ?$ & 25 & 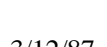 & & 1 & $2 / 1107$ & & 1 & & 3.3 & 50 \\
\hline $3 / 12 / 8 /$ & $<3$ & $3 / 12 / 8 /$ & 3.6 & 51 & $3 / 12 / 8 /$ & $\mathrm{NA}^{\circ}$ & 300.4 & 35 & $3 / 12 / 8 /$ & 48.2 & 25 & $3 / 12 / 81$ & $<3$ & NA & $3 / 11 / 8 /$ & 23 & NA & $5 / 11 / 8 /$ & 24.66 & 93 \\
\hline
\end{tabular}

Note: ${ }^{1} \mathrm{NA}$ : Data not available

${ }^{2}$ Referenced from Internal Letter "Integrity of tanks 241-BY-103 and 241-TY-103" from G.L. Borsheim

*Drywell data sheets located in the Historical Records folder in IDMS/Managed Information 
RPP-RPT-54911, Rev. 0

Table A1-2. Tank BY-103 Associated Drywell Radioactivity (K counts per minute)

(September 1959 to March 1987) (Sheet 1 of 2)

(Drywell Data Sheets* Retrieved on August 31, 2011 and SD-WM-TI-356)

\begin{tabular}{|c|c|c|c|c|c|c|c|c|c|c|c|c|c|c|}
\hline \multicolumn{3}{|c|}{ 22-03-10 } & \multicolumn{3}{|c|}{ 22-00-01 } & \multicolumn{3}{|c|}{ 22-00-02 } & \multicolumn{3}{|c|}{ 22-00-03 } & \multicolumn{3}{|c|}{ 22-00-04 } \\
\hline \multicolumn{3}{|c|}{ Drilled 12/31/1973 } & \multicolumn{3}{|c|}{ Drilled 8/31/1949 } & \multicolumn{3}{|c|}{ Drilled 12/31/1973 } & \multicolumn{3}{|c|}{ Drilled 7/31/1949 } & \multicolumn{3}{|c|}{ Drilled 12/31/1973 } \\
\hline Date & $\begin{array}{c}\text { Peak } \\
(\mathrm{K} \text { cpm })\end{array}$ & $\begin{array}{c}\text { Depth } \\
\text { (ft } \\
\text { BGS) }\end{array}$ & Date & $\begin{array}{c}\text { Peak } \\
\text { (K } \\
\text { cpm) }\end{array}$ & $\begin{array}{c}\text { Depth } \\
\text { (ft } \\
\text { BGS) }\end{array}$ & Date & $\begin{array}{l}\text { Peak } \\
(\mathbf{K} \\
\text { cpm) }\end{array}$ & $\begin{array}{c}\text { Depth } \\
\text { (ft } \\
\text { BGS) }\end{array}$ & Date & $\begin{array}{l}\text { Peak } \\
(\mathrm{K} \\
\text { cpm })\end{array}$ & $\begin{array}{c}\text { Depth } \\
\text { (ft BGS) }\end{array}$ & Date & $\begin{array}{l}\text { Peak } \\
(\mathbf{K} \\
\text { cpm })\end{array}$ & $\begin{array}{c}\text { Depth } \\
\text { (ft } \\
\text { BGS) }\end{array}$ \\
\hline \multirow{20}{*}{\multicolumn{3}{|c|}{$\begin{array}{l}\text { Drywell 22-03-10 not } \\
\text { drilled until December } \\
\quad 31,1973\end{array}$}} & $9 / 9 / 59$ & $<0.1$ & $\mathrm{NA}^{1}$ & \multirow{20}{*}{\multicolumn{3}{|c|}{$\begin{array}{l}\text { Drywell 22-00-02 not } \\
\text { drilled until December } \\
31,1973\end{array}$}} & $9 / 9 / 59$ & $>100$ & $42-150$ & \multirow{20}{*}{\multicolumn{3}{|c|}{$\begin{array}{c}\text { Drywell 22-00-04 } \\
\text { not drilled until } \\
\text { December 31, } 1973\end{array}$}} \\
\hline & & & $9 / 21 / 60$ & $<0.1$ & $\mathrm{NA}^{1}$ & & & & $9 / 21 / 60$ & $40-100$ & $45-50$ & & & \\
\hline & & & & & & & & & & 10 & 42 & & & \\
\hline & & & $12 / 21 / 65$ & 8 & 50 & & & & $11 / 12 / 65$ & $\begin{array}{c}\text { off } \\
\text { scale }\end{array}$ & $52-87$ & & & \\
\hline & & & \multirow{2}{*}{\multicolumn{3}{|c|}{$\mathrm{NA}^{1}$}} & & & & \multirow{2}{*}{$11 / 17 / 65$} & 9 & 42 & & & \\
\hline & & & & & & & & & & $>1000$ & $52-87$ & & & \\
\hline & & & \multicolumn{3}{|c|}{$\mathrm{NA}^{1}$} & & & & $12 / 21 / 65$ & $>1000$ & $50-92$ & & & \\
\hline & & & $8 / 22 / 72$ & $<6$ & $\mathrm{NA}^{1}$ & & & & $8 / 22 / 72$ & 212.3 & 60 & & & \\
\hline & & & \multicolumn{3}{|c|}{$\mathrm{NA}^{1}$} & & & & $1 / 3 / 73$ & 169.1 & 70 & & & \\
\hline & & & \multicolumn{3}{|c|}{$\mathrm{NA}^{1}$} & & & & $2 / 5 / 73$ & 167.5 & 68 & & & \\
\hline & & & $3 / 30 / 73$ & 4.2 & 57 & & & & $3 / 27 / 73$ & 148.6 & 70 & & & \\
\hline & & & \multicolumn{3}{|c|}{$\mathrm{NA}^{1}$} & & & & $4 / 11 / 73$ & 139.6 & 70 & & & \\
\hline & & & $5 / 23 / 73$ & 4.8 & 60 & & & & $5 / 23 / 73$ & 94.7 & 65 & & & \\
\hline & & & $6 / 25 / 73$ & 4.1 & 49 & & & & $6 / 22 / 73$ & 236.2 & 24 & & & \\
\hline & & & \multicolumn{3}{|c|}{$\mathrm{NA}^{1}$} & & & & $7 / 2 / 73$ & 137.8 & 60 & & & \\
\hline & & & $8 / 30 / 73$ & 1.7 & 78 & & & & $8 / 6 / 73$ & 55.4 & 65 & & & \\
\hline & & & $9 / 27 / 73$ & 6.1 & 86 & & & & $9 / 4 / 73$ & 56.2 & 61 & & & \\
\hline & & & \multicolumn{3}{|c|}{$\mathrm{NA}^{1}$} & & & & $10 / 2 / 73$ & 76.9 & 59 & & & \\
\hline & & & $11 / 27 / 73$ & 7.1 & 90 & & & & $11 / 7 / 73$ & 120.9 & 65 & & & \\
\hline & & & $12 / 27 / 73$ & 3.9 & 49 & & & & $12 / 18 / 73$ & 134.5 & 62 & & & \\
\hline \multicolumn{3}{|c|}{$\mathrm{NA}^{1}$} & \multicolumn{3}{|c|}{$\mathrm{NA}^{1}$} & \multicolumn{3}{|c|}{$\mathrm{NA}^{1}$} & $1 / 9 / 74$ & 138 & 61 & \multicolumn{3}{|c|}{$\mathrm{NA}^{1}$} \\
\hline & $\mathrm{NA}^{1}$ & & $2 / 27 / 74$ & 4.1 & 93 & & $\mathrm{NA}^{1}$ & & $2 / 6 / 74$ & 128.5 & 60 & & $\mathrm{NA}^{1}$ & \\
\hline & $\mathrm{NA}^{1}$ & & $3 / 27 / 74$ & 3.8 & 60 & & $\mathrm{NA}^{1}$ & & $3 / 25 / 74$ & 118.4 & 61 & & $\mathrm{NA}^{1}$ & \\
\hline $4 / 24 / 74$ & $<6$ & $\mathrm{NA}^{1}$ & & $\mathrm{NA}^{1}$ & & $4 / 16 / 74$ & 27.1 & 60 & $4 / 30 / 74$ & 116.5 & 62 & $4 / 16 / 74$ & 17.9 & 79 \\
\hline & $\mathrm{NA}^{1}$ & & $5 / 23 / 74$ & 3.9 & 90 & $5 / 22 / 74$ & 26.6 & 59 & $5 / 7 / 74$ & 109.1 & 63 & $5 / 22 / 74$ & 18.5 & 81 \\
\hline & $\mathrm{NA}^{1}$ & & $6 / 27 / 74$ & 3.7 & 57 & $6 / 27 / 74$ & 25.1 & 52 & $6 / 3 / 74$ & 116.6 & 63 & $6 / 27 / 74$ & 18.3 & 79 \\
\hline $7 / 1 / 74$ & $<6$ & $\mathrm{NA}^{1}$ & $7 / 3 / 74$ & 3.7 & 91 & $7 / 17 / 74$ & 23.1 & 60 & $7 / 3 / 74$ & 114 & 61 & $7 / 4 / 74$ & 17.3 & 80 \\
\hline $8 / 22 / 74$ & $<6$ & $\mathrm{NA}^{1}$ & $8 / 7 / 74$ & 4.7 & 58 & $8 / 13 / 73$ & 25.6 & 53 & $8 / 7 / 74$ & 114.9 & 58 & $8 / 7 / 74$ & 20.5 & 79 \\
\hline $9 / 25 / 74$ & $<6$ & $\mathrm{NA}^{1}$ & $9 / 5 / 74$ & 4.1 & 61 & $9 / 12 / 74$ & 21.8 & 53 & $9 / 12 / 74$ & 98.0 & 59 & $9 / 12 / 74$ & 16.1 & 82 \\
\hline & $\mathrm{NA}^{1}$ & & $10 / 11 / 74$ & 3.7 & 59 & $10 / 11 / 74$ & 23.1 & 53 & $10 / 11 / 74$ & 102.8 & 62 & $10 / 11 / 74$ & 15.5 & 80 \\
\hline & $\mathrm{NA}^{1}$ & & $11 / 7 / 74$ & 4.3 & 57 & $11 / 9 / 74$ & 21.3 & 54 & $11 / 7 / 74$ & 95.1 & 59 & $11 / 7 / 74$ & 16.8 & 81 \\
\hline
\end{tabular}


RPP-RPT-54911, Rev. 0

Table A1-2. Tank BY-103 Associated Drywell Radioactivity (K counts per minute) (August 1972 to March 1987) (Sheet 2 of 2)

(Drywell Data Sheets* Retrieved on August 31, 2011 and SD-WM-TI-356)

\begin{tabular}{|c|c|c|c|c|c|c|c|c|c|c|c|c|c|c|}
\hline \multicolumn{3}{|c|}{ 22-03-10 } & \multicolumn{3}{|c|}{ 22-00-01 } & \multicolumn{3}{|c|}{ 22-00-02 } & \multicolumn{3}{|c|}{ 22-00-03 } & \multicolumn{3}{|c|}{ 22-00-04 } \\
\hline Date & $\begin{array}{c}\text { Peak } \\
(\mathrm{K} \text { cpm) }\end{array}$ & $\begin{array}{c}\text { Depth } \\
\text { (ft } \\
\text { BGS) } \\
\end{array}$ & Date & $\begin{array}{c}\text { Peak } \\
\text { (K } \\
\text { cpm) } \\
\end{array}$ & $\begin{array}{c}\text { Depth } \\
\text { (ft } \\
\text { BGS) } \\
\end{array}$ & Date & $\begin{array}{c}\text { Peak } \\
(\mathrm{K} \\
\text { cpm) } \\
\end{array}$ & $\begin{array}{c}\text { Depth } \\
\text { (ft } \\
\text { BGS) } \\
\end{array}$ & Date & $\begin{array}{c}\text { Peak } \\
(\mathrm{K} \\
\text { cpm) } \\
\end{array}$ & $\begin{array}{c}\text { Depth } \\
\text { (ft } \\
\text { BGS) }\end{array}$ & Date & $\begin{array}{c}\text { Peak } \\
(\mathrm{K} \\
\text { cpm) } \\
\end{array}$ & $\begin{array}{c}\text { Depth } \\
\text { (ft } \\
\text { BGS) }\end{array}$ \\
\hline \multicolumn{3}{|c|}{$\mathrm{NA}^{1}$} & $12 / 3 / 74$ & 4.0 & 59 & $12 / 3 / 74$ & 19.7 & 54 & $12 / 3 / 74$ & 103.4 & 62 & $12 / 3 / 74$ & 15.1 & 82 \\
\hline $1 / 24 / 75$ & 3.2 & 21 & $1 / 1 / 75$ & 3.4 & 60 & $1 / 1 / 75$ & 20.2 & 53 & $1 / 1 / 75$ & 93.2 & 63 & $1 / 1 / 75$ & 14.9 & 82 \\
\hline \multicolumn{3}{|c|}{$\mathrm{NA}^{1}$} & \multicolumn{3}{|c|}{$\mathrm{NA}^{1}$} & \multicolumn{3}{|c|}{$\mathrm{NA}^{1}$} & $1 / 24 / 75$ & 97.0 & 60 & \multicolumn{3}{|c|}{$\mathrm{NA}^{1}$} \\
\hline $7 / 11 / 75$ & 3.4 & 27 & \multicolumn{3}{|c|}{$\mathrm{NA}^{1}$} & \multicolumn{3}{|c|}{$\mathrm{NA}^{1}$} & $7 / 11 / 75$ & 88.1 & 60 & \multicolumn{3}{|c|}{$\mathrm{NA}^{1}$} \\
\hline $1 / 22 / 76$ & 3.7 & 24 & \multicolumn{3}{|c|}{$\mathrm{NA}^{1}$} & \multicolumn{3}{|c|}{$\mathrm{NA}^{1}$} & $2 / 4 / 76$ & 78.8 & 57 & \multicolumn{3}{|c|}{$\mathrm{NA}^{1}$} \\
\hline $3 / 3 / 77$ & 3.2 & 22 & \multicolumn{3}{|c|}{$\mathrm{NA}^{1}$} & \multicolumn{3}{|c|}{$\mathrm{NA}^{1}$} & $3 / 3 / 77$ & 59.5 & 58 & \multicolumn{3}{|c|}{$\mathrm{NA}^{1}$} \\
\hline \multicolumn{3}{|c|}{$\mathrm{NA}^{1}$} & \multicolumn{3}{|c|}{$\mathrm{NA}^{1}$} & \multicolumn{3}{|c|}{$\mathrm{NA}^{1}$} & $7 / 14 / 77$ & 56.0 & 59 & \multicolumn{3}{|c|}{$\mathrm{NA}^{1}$} \\
\hline $3 / 16 / 78$ & 3.0 & 21 & \multicolumn{3}{|c|}{$\mathrm{NA}^{1}$} & \multicolumn{3}{|c|}{$\mathrm{NA}^{1}$} & $3 / 9 / 78$ & 49.3 & 59 & \multicolumn{3}{|c|}{$\mathrm{NA}^{1}$} \\
\hline \multicolumn{3}{|c|}{$\mathrm{NA}^{1}$} & \multicolumn{3}{|c|}{$\mathrm{NA}^{1}$} & \multicolumn{3}{|c|}{$\mathrm{NA}^{1}$} & $9 / 14 / 78$ & 47.8 & 59 & \multicolumn{3}{|c|}{$\mathrm{NA}^{1}$} \\
\hline $3 / 7 / 79$ & 2.9 & 24 & \multicolumn{3}{|c|}{$\mathrm{NA}^{1}$} & & $\mathrm{NA}^{1}$ & & $3 / 7 / 79$ & 42.2 & 58 & & $\mathrm{NA}^{1}$ & \\
\hline $3 / 18 / 80$ & 2.9 & 23 & & $\mathrm{NA}^{1}$ & & & $\mathrm{NA}^{1}$ & & $3 / 20 / 80$ & 34.8 & 61 & & $\mathrm{NA}^{1}$ & \\
\hline $3 / 24 / 81$ & 2.8 & 25 & & $\mathrm{NA}^{1}$ & & & $\mathrm{NA}^{1}$ & & $3 / 18 / 81$ & 29.2 & 60 & & $\mathrm{NA}^{1}$ & \\
\hline $3 / 9 / 82$ & 2.8 & 24 & & $\mathrm{NA}^{1}$ & & & $\mathrm{NA}^{1}$ & & $1 / 12 / 82$ & 27.2 & 59 & & $\mathrm{NA}^{1}$ & \\
\hline $3 / 2 / 83$ & 3.7 & 26 & & $\mathrm{NA}^{1}$ & & & $\mathrm{NA}^{1}$ & & $3 / 9 / 83$ & 22.1 & 61 & & $\mathrm{NA}^{1}$ & \\
\hline & $\mathrm{NA}^{1}$ & & & $\mathrm{NA}^{1}$ & & & $\mathrm{NA}^{1}$ & & $6 / 23 / 83$ & 20.3 & 74 & & $\mathrm{NA}^{1}$ & \\
\hline $2 / 23 / 84$ & 3.3 & 27 & & $\mathrm{NA}^{1}$ & & & $\mathrm{NA}^{1}$ & & $6 / 12 / 84$ & 18.2 & 59 & & $\mathrm{NA}^{1}$ & \\
\hline $3 / 5 / 85$ & 2.9 & 27 & & $\mathrm{NA}^{1}$ & & & $\mathrm{NA}^{1}$ & & $6 / 12 / 85$ & 17.2 & 62 & & $\mathrm{NA}^{1}$ & \\
\hline $3 / 17 / 86$ & 3.0 & 28 & & $\mathrm{NA}^{1}$ & & & $\mathrm{NA}^{1}$ & & $6 / 18 / 86$ & 14.1 & 74 & & $\mathrm{NA}^{1}$ & \\
\hline $3 / 11 / 87$ & 3.1 & 27 & & $\mathrm{NA}^{1}$ & & & $\mathrm{NA}^{1}$ & & & $\mathrm{NA}^{1}$ & & & $\mathrm{NA}^{1}$ & \\
\hline
\end{tabular}

Note: ${ }^{1}$ NA: Data not available

*Drywell data sheets located in the Historical Records folder in IDMS/Managed Information 


\subsection{TANK TY-103 SEGMENT}

\section{TABLE OF CONTENTS}

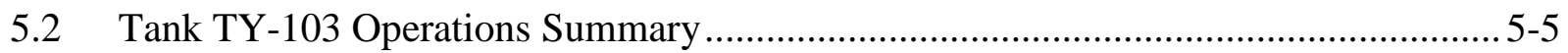

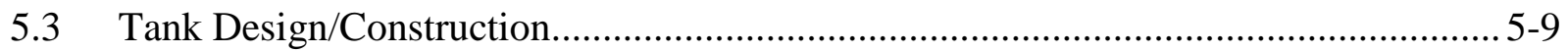

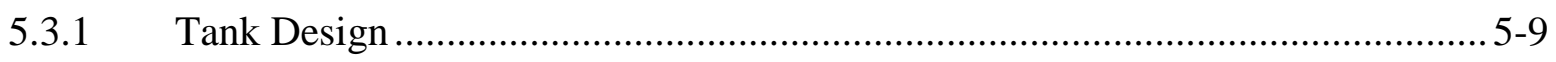

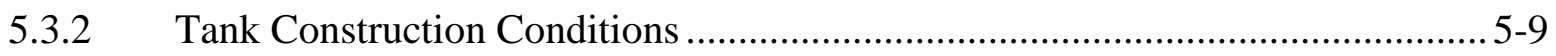

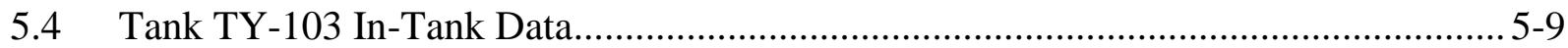

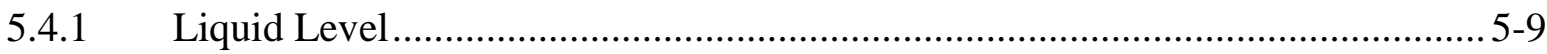

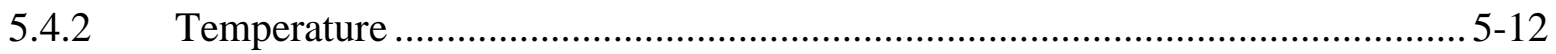

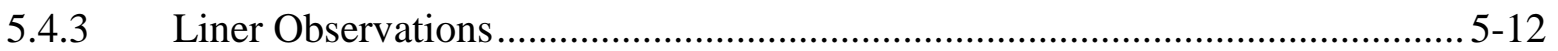

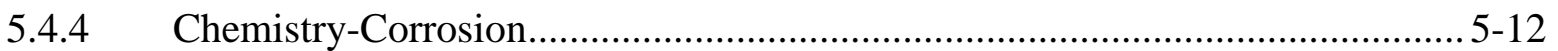

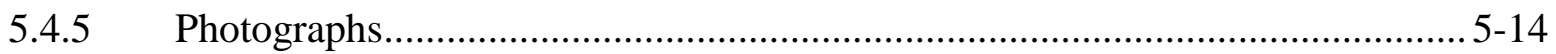

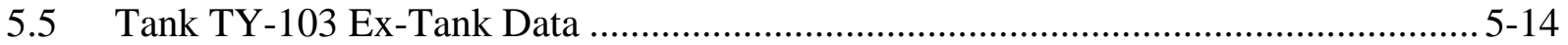

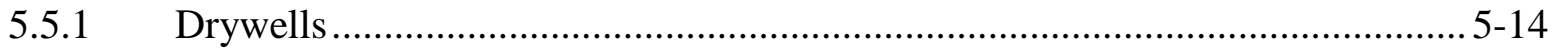

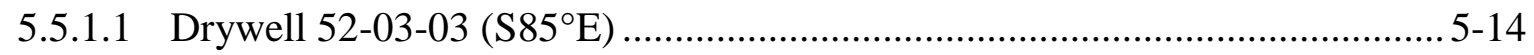

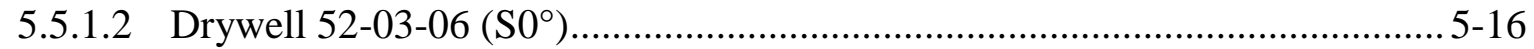

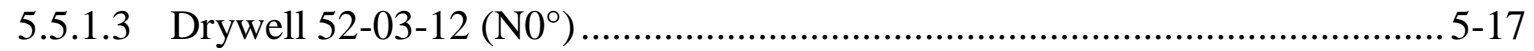

5.5.2 Tank TY-103 Drywell Summary ............................................................. 5-18

5.5.3 2005 Direct Push Logging ..................................................................... 5-18

5.6 Possible Tank TY-103 Liner Leak Location(s)....................................................... 5-19

5.6.1 Leak Detected in 1972 \& 2005 - Site A..................................................... 5-20

5.6.2 Leak Detected in 1973 - Site B ............................................................. 5-21

5.6.3 Leak Detected in 1973 - Site C ............................................................... 5-22

5.6.4 Leak Location Summary …………………............................................... 5-23

5.7 Possible Tank TY-103 Liner Leak Cause(s) ………........................................... 5-25

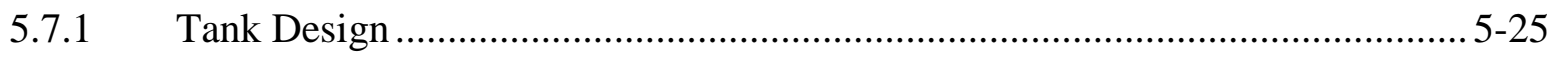

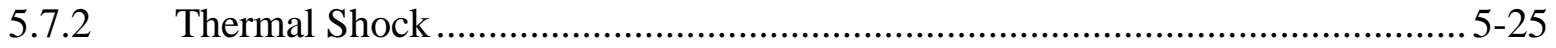

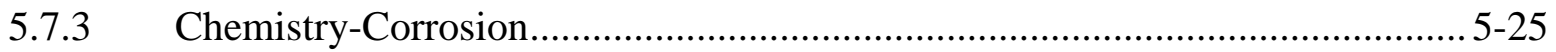

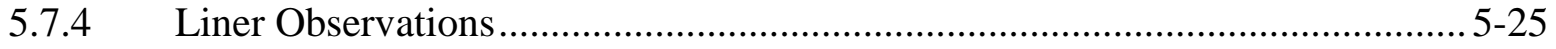

5.7.5 Tank Construction Temperature ………………....................................... 5-26 


\section{APPENDICES}

Appendix A2 Tank TY-103 Gross Gamma Drywell Data .................................................... A2-1

Appendix B2 Tank TY-103 Gamma Surveys of the 2005 Direct Pushes .............................. B2-1

\section{LIST OF FIGURES}

Figure 5-1. Tank TY-103 Associated Drywells................................................................. 5-4

Figure 5-2. Operational Leak History of Tank TY-103 ……….............................................. 5-8

Figure 5-3. Tank TY-103 End of Quarter Surface Level......................................................... 5-10

Figure 5-4. Tank TY-103 Liquid Level August 1971 to August 1973 ...................................... 5-11

Figure 5-5. Tank TY-103 Liquid Level June to September 1973.......................................... 5-12

Figure 5-6. Tank TY-103 Drywell 52-03-03 (HNF-3831) .............................................. 5-15

Figure 5-7. Tank TY-103 Drywell 52-03-06 (HNF-3831) .................................................... 5-16

Figure 5-8. Tank TY-103 Drywell 52-03-12 (HNF-3831) ....................................................... 5-17

Figure 5-10. Tank TY-103 Possible Leak Location (August $1972 \&$ 2005) ............................. 5-20

Figure 5-11. Tank TY-103 Possible Leak Location (July 1973) ............................................. 5-21

Figure 5-12. Tank TY-103 Possible Leak Location (July 1973) ............................................ 5-22

Figure 5-13. Tank TY-103 Possible Radial Leak Locations .................................................... 5-24

Figure B2-1. 2005 Gamma Survey Direct Push Hole C4629 ………………………............ B2-2

Figure B2-2. 2005 Gamma Survey Direct Push Hole C4631 ………………………............ B2-3

Figure B2-3. 2005 Gamma Survey Direct Push Hole C4633 …………………………......... B2-4

Figure B2-4. 2005 Gamma Survey Direct Push Hole C4635 ................................................ B2-5

Figure B2-5. 2005 Gamma Survey Direct Push Hole C5008 .................................................. B2-6

Figure B2-6. 2005 Gamma Survey Direct Push Hole C5010 ……………………………...... B2-7

Figure B2-7. 2005 Gamma Survey Direct Push Hole C5012 ……………………................. B2-8 
RPP-RPT-54911, Rev. 0

\section{LIST OF TABLES}

Table 5-1. Tank TY-103 Waste Storage Chronology …………………………….............. 5-13

Table 5-2. Waste Chemistries for Waste Types Stored in Tank TY-103 ................................ 5-13

Table 5-3. 2005 Direct Push Gross Gamma Results (RPP-RPT-34870).................................... 5-18

Table A2-1. Tank TY-103 Drywell Radioactivity (K counts per minute) .............................. A2-2 


\subsection{TANK TY-103 BACKGROUND HISTORY}

This section provides information on the historical waste loss event associated with SST 241TY-103 (TY-103). There are three drywells located around tank TY-103 with specified distances from the drywell to the tank footing shown in Figure 5-1: 52-03-12 installed in November 1971 and 52-03-03 and 52-03-06 installed in December 1971. Seven direct pushes were installed in 2005: C4629, C4631, C4633, C4635, C5010, and C5012.

The bottom of the tank footing is $\sim 45$-ft 10 1/2-in Below Grade Surface (BGS) with $\sim 6$-ft 10 1/2-in soil cover over the dome (WHC-SD-WM-TI-665, Soil Load above Hanford Waste Storage Tanks; H-2-2244, 75 Foot Composite Storage Tank Sections).

Figure 5-1. Tank TY-103 Associated Drywells

Tank inner ring is steel liner, outer ring is outer edge of tank footing

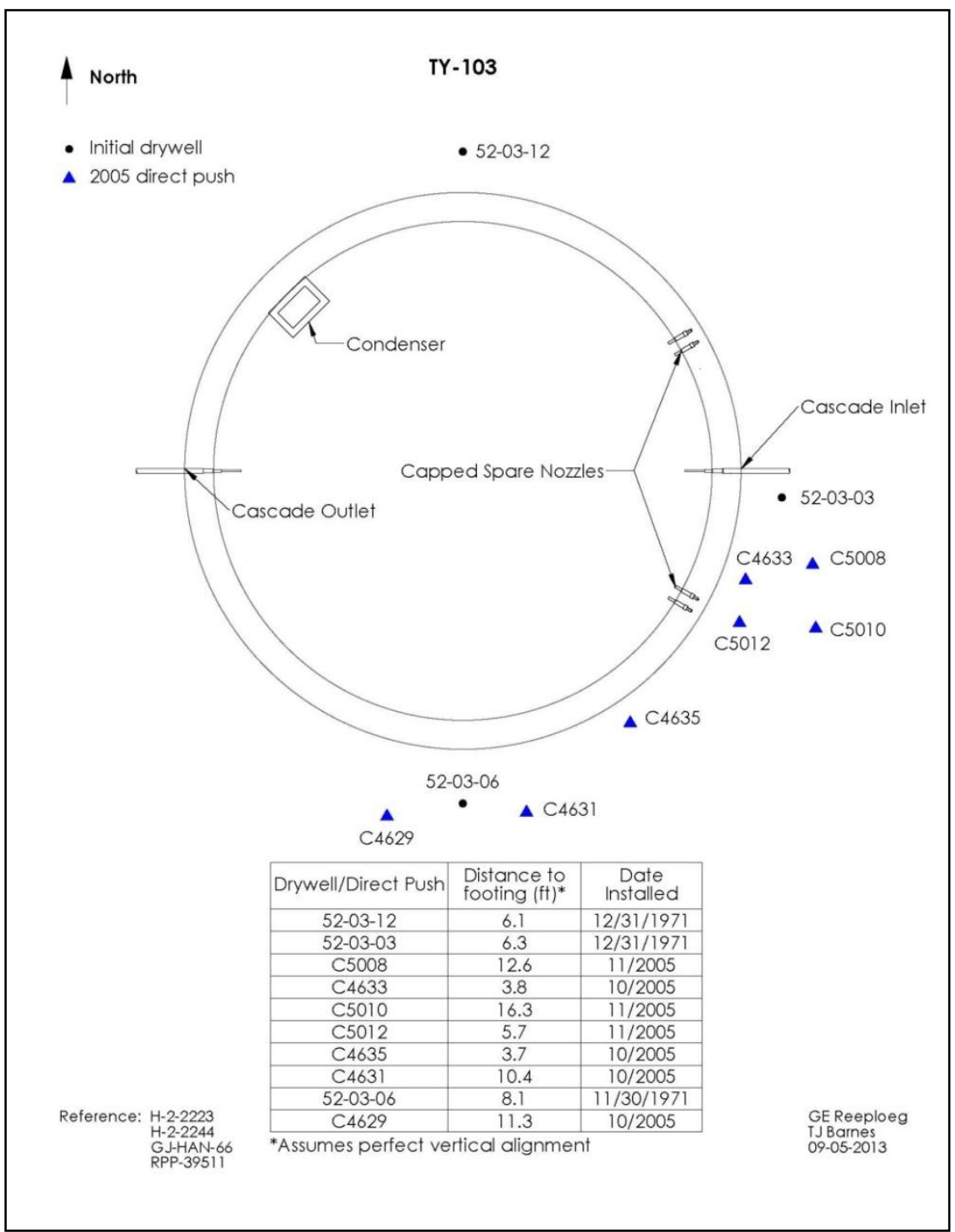

Note: Outer edge of the tank footing is between 42 -ft $10^{1 / 2}$-in to 45 -ft $10^{1 / 2}$-in BGS 
RPP-RPT-54911, Rev. 0

\subsection{TANK TY-103 OPERATIONS SUMMARY}

Tank TY-103 was constructed from 1951 to 1952 and started receiving Tri-Butyl Phosphate (TBP) waste from the 221-U Plant on July 16, 1953 (HW-29054, Separations Section WasteStatus Summary July 31, 1953, page 7). Tank TY-103 continued receiving TBP waste and was filled to $758 \mathrm{kgal}$ with TBP waste by the end of August 1953 (HW-29242, Separations Section Waste-Status Summary August 31, 1953, page 7). In November to December 1954, approximately $624 \mathrm{kgal}$ of TBP waste was transferred out of tank TY-103 to tank TX-111 leaving $131 \mathrm{kgal}$ of TBP waste in tank TY-103 (HW-33904, Separations Section Waste- Status Summary for November 1954; HW-34412, Separations Section Waste-Status Summary for December 1954).

Beginning in January 1955, tank TY-103 received first cycle waste (1C) waste from the bismuth phosphate process conducted in the 221-B and 221-T-Plant (HW-35022, Separations Section Waste-Status Summary for January 1955, page 7). The 1C waste was treated in 221-T Plant with potassium ferrocyanide $(\mathrm{FeCN})$, nickel sulfate, and sodium hydroxide to precipitate nickel ferrocyanide, which scavenged Cs-137 and Sr-90 from the supernatant (HW-33184, $\mathrm{BiPO}_{4}$ Plant Nickel Ferrocyanide Scavenging Flowsheet for First-Cycle Waste Containing No CoatingRemoval Waste; HW-33499, Justification for T Plant First Cycle Waste Scavenging). Tank TY103 was filled and emptied of $1 \mathrm{C}$ waste (i.e., $1 \mathrm{C}-\mathrm{FeCN}$ ) several times in 1955 and 1956. In November 1956, most of the 1C-FeCN supernatant was removed from tank TY-103 and tank TY-103 was reported to contain $\sim 188 \mathrm{kgal}$ of 1C-FeCN sludge (HW-47052, Chemical Processing Department Waste-Status Summary November 1, 1956-November 30, 1956, page 7).

No further waste transactions occurred in tank TY-103 from December 1956 through August 1959. The estimated supernatant and sludge volumes in tank TY-103 based on electrode readings were revised from $13 \mathrm{kgal}$ and $220 \mathrm{kgal}$, respectively, in February 1957 to $70 \mathrm{kgal}$ and $220 \mathrm{kgal}$ in June 1957, and again to $73 \mathrm{kgal}$ and $220 \mathrm{kgal}$ in July 1957 (HW-48846, Chemical Processing Department Waste Status Summary February 1, 1957- February 28, 1957; HW51348, Chemical Processing Department Waste Status Summary June 1, 1957-June 30, 1957; HW-51858, Chemical Processing Department Waste Status Summary July 1, 1957-July 31, 1957 Planning and Scheduling- Production Operation). Volumes were again revised and in July 1959 were reported as $70 \mathrm{kgal}$ of supernatant and $220 \mathrm{kgal}$ of sludge (HW-61582, Chemical Processing Department Waste Status Summary July 1, 1959 - July 31, 1959). No explanation was reported for these discrepancies.

In September and October 1959, tank TY-103 received $226 \mathrm{kgal}$ of TBP supernatant waste from tank TY-106, which was suspected to be leaking (HW-62421, Chemical Processing Department Waste Status Summary September 1, 1959- September 30, 1959, page 7; HW-62723, Chemical Processing Department Waste Status Summary October 1-31, 1959). No further waste transactions occurred in tank TY-103 from November 1959 through June 1961.

In July 1961, tank TY-103 received $\sim 16.5 \mathrm{kgal}$ of waste from the 241-TY-153 catch tank (HW83906 E RD, Chemical Processing Department 200 West Area Tank Farm Inventory and Waste Reports July 1, 1961 through 1965, page 4). In November and December 1961, 215.9 kgal of decontamination waste (DW) were transferred from tank TX-118 to tank TY-103 (HW-83906 E $\mathrm{RD}$, page 5). No further waste transactions occurred in tank TY-103 until 1967. The sludge 
volume in tank TY-103 was reported to be $101 \mathrm{kgal}$ on June 30, 1965 which was $109 \mathrm{kgal}$ less than what was reported in July 1957 (RL-SEP-659, Chemical Processing Department- Waste Status Summary January 1, 1965 through June 30, 1965, page 7). The decrease in sludge volume could have been due to settling and/or dissolution of components from the $1 \mathrm{C}-\mathrm{FeCN}$ present in tank TY-103.

In the third and fourth quarter of 1967, 546 kgal of supernatant were transferred from tank TY103 to tank TY-118 for processing in the 242-T Evaporator (ARH-95, Chemical Processing Division Waste Status Summary July 1, 1967 through September 30, 1967, page 8; ARH-326, Chemical Processing Division Waste Status Summary October 1, 1967 through December 31, 1967, page 8).

Tank TY-103 was used as a staging tank for accumulating waste for transfer to tank TX-118 and processing in the 242-T Evaporator from January 1968 through March 1971. During this period, tank TY-103 received the following waste types from various SSTs (see RPP-RPT-42296, Hanford TY-Farm Leak Assessments Report, for transfer details):

- OWW

- PUREX CW

- DW

- 221-T Plant $1 \mathrm{C} / \mathrm{CW}$ supernatant

- 242-T EB

- 202-S REDOX Plant supernatant waste

- R-EB

- RIX

- BL

- $\mathrm{B}-\mathrm{EB}$

From April 1971 to June 1973, no waste transactions occurred in tank TY-103 and the volume of waste was $\sim 720 \mathrm{kgal}$ (ARH-2074 B, Chemical Processing Division Waste Status Summary April 1, 1971 Through June 30, 1971) consisting of BL, OWW, and RIX. Although tank TY-103 was nearly filled multiple times, transfer records indicate that the waste level did not overflow the cascade level (285-in or $761 \mathrm{kgal})$ to tank TY-104.

Tank TY-103 was first suspected to be leaking in April 1973 when a liquid level loss of 0.4-in occurred and when radioactivity increased in drywell 52-03-03 starting in August 1972 (Draft memo Compton, Tank 241-TY-103 Review). However, tank TY-103 may have leaked before 1973 as liquid levels were decreasing as early as August 1971. Due to decreasing liquid levels and subsequent radioactivity in the drywells, $200 \mathrm{kgal}$ of supernatant was transferred out of tank TY-103 from June 11-13, 1973. After this transfer, radiation levels in the drywells appeared to have stabilized (LET-ARHCO-TY-103, "Status of tank 241-TY-103," October 1973). However, from June 21 through July 5, 1973 the tank developed a slow leak and lost 0.5in with subsequent increased radioactivity in the drywells.

On Monday, July 9, 1973, the Atomic Energy Commission-Richland Operations was verbally informed by the Atlantic Richfield Hanford Company (ARHCO) that the suspect leaker, tank 
TY-103, was classified as a confirmed leaker (RPP-RPT-42296). ARHCO stated in the July 1973 monthly report "The tank contained a blend of non-boiling wastes, including B Plant lowlevel waste, PUREX organic wash waste, and ion exchange loading waste" (ARH-2416 RD, page 85$)$. Tank TY-103 was pumped down to a minimum heel ( 184 kgal pumped) on July 8 , 1973, but the tank continued to leak, losing an additional 0.5-in until a salt well was fabricated and installed on August 14, 1973.

Tank TY-103 was declared stabilized on December 18, 1982 after a jet pump failed. An estimated 5,000 gal of drainable liquid remained. Tank TY-103 is estimated to contain $103 \mathrm{kgal}$ of sludge, $51 \mathrm{kgal}$ of saltcake, and no supernatant (HNF-EP-0182, Rev. 301, Waste Tank Summary Report for Month Ending April 30, 2013).

The operational history of tank TY-103 leak related details including liquid level is charted in Figure 5-2. 


\section{Figure 5-2. Operational Leak History of Tank TY-103}

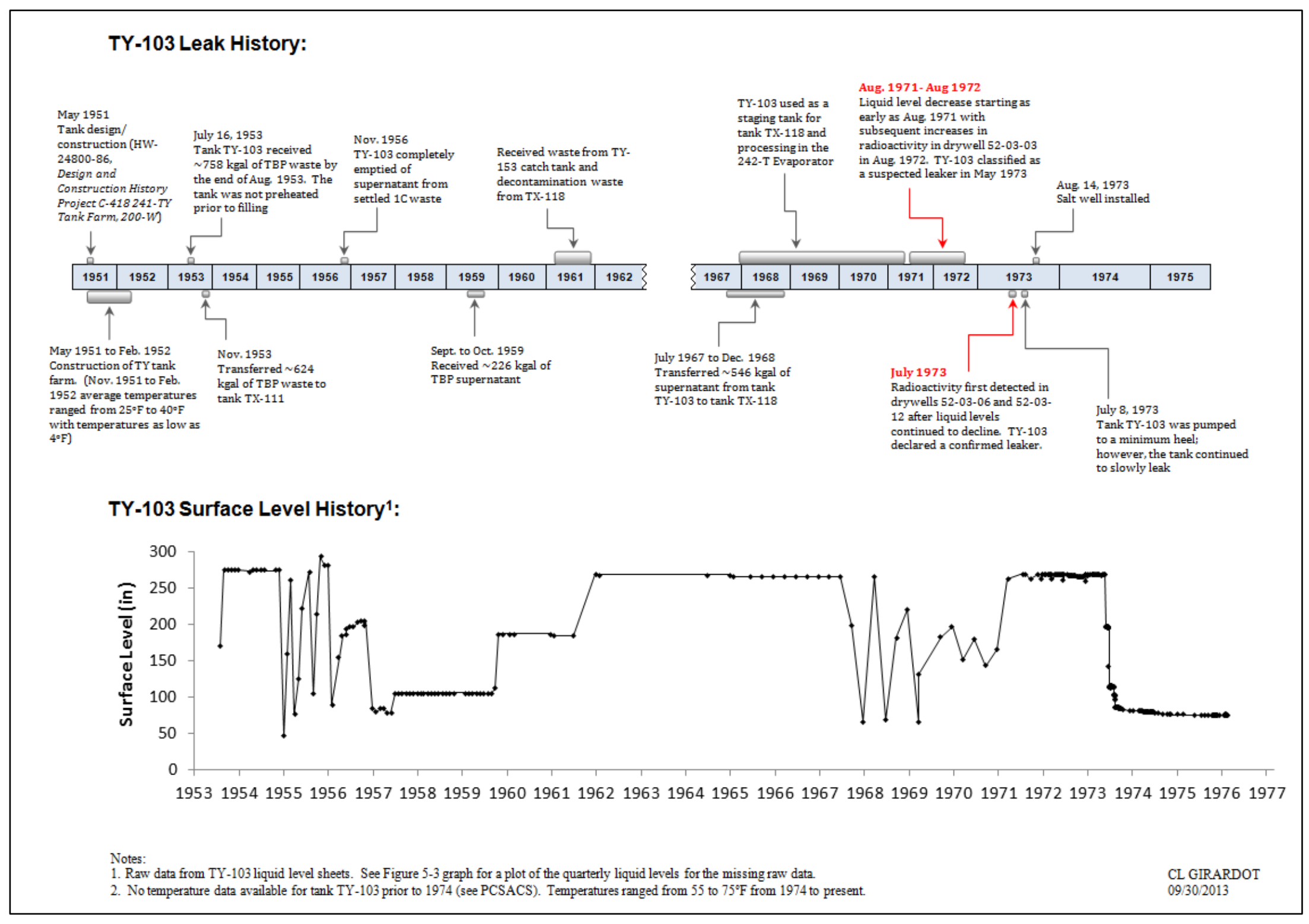


RPP-RPT-54911, Rev. 0

\subsection{TANK DESIGN/CONSTRUCTION}

\subsubsection{Tank Design}

The TY Farm tank design continued important features of the earlier 241-BCTU tanks (BPF73550). The steel bottom intersects the sidewall on a 4-ft radius. Full penetration butt welds with $\mathrm{x}$-ray inspection and three ply asphaltic membrane waterproofing between the wall liner and the concrete shell continued those design features found to be important for liner integrity during the 241-SX Farm leak assessment (RPP-RPT-54910). The top of the tank footing is 42 -ft 101/2-in BGS and is 3 -ft thick with the bottom of the footing at $45-\mathrm{ft} 10^{1 / 2}$-in BGS.

\subsubsection{Tank Construction Conditions}

The TY Farm was constructed from May 1951 to February 1952. Cold weather could have affected the steel during construction in December and January. Temperatures for December 1951 and January 1952 averaged $27.4^{\circ} \mathrm{F}$ and $25.2^{\circ} \mathrm{F}$, respectively, with temperatures as low as $4^{\circ} \mathrm{F}$ (PNNL-15160). Weather precautions for welding and concrete were specified in HW-4696 (see Section 3.1.2).

As described in Section 3.1.2, cold weather affects the ductile-to-brittle steel transition temperature, with $18^{\circ} \mathrm{F}$ being the assumed unrestricted low temperature construction limitation for the carbon steel liner, which could result in a fracture upon impact. Low temperatures experienced during construction at or less than the $18^{\circ} \mathrm{F}$ allowable temperature could cause impact loading (e.g. a dropped tool or piece of equipment from scaffolding) and result in the potential for creating micro-fissures weakening the steel liner.

\subsection{TANK TY-103 IN-TANK DATA}

\subsubsection{Liquid Level}

The liquid level plot in Figure 5-3 indicates the transfer activity into and out of tank TY-103. The liquid levels are end of quarter levels so this figure may not reflect all transfers into and out of the tank that occurred during the operational history of the tank. See Figure 5-2 for historical monthly liquid level readings. 
Figure 5-3. Tank TY-103 End of Quarter Surface Level

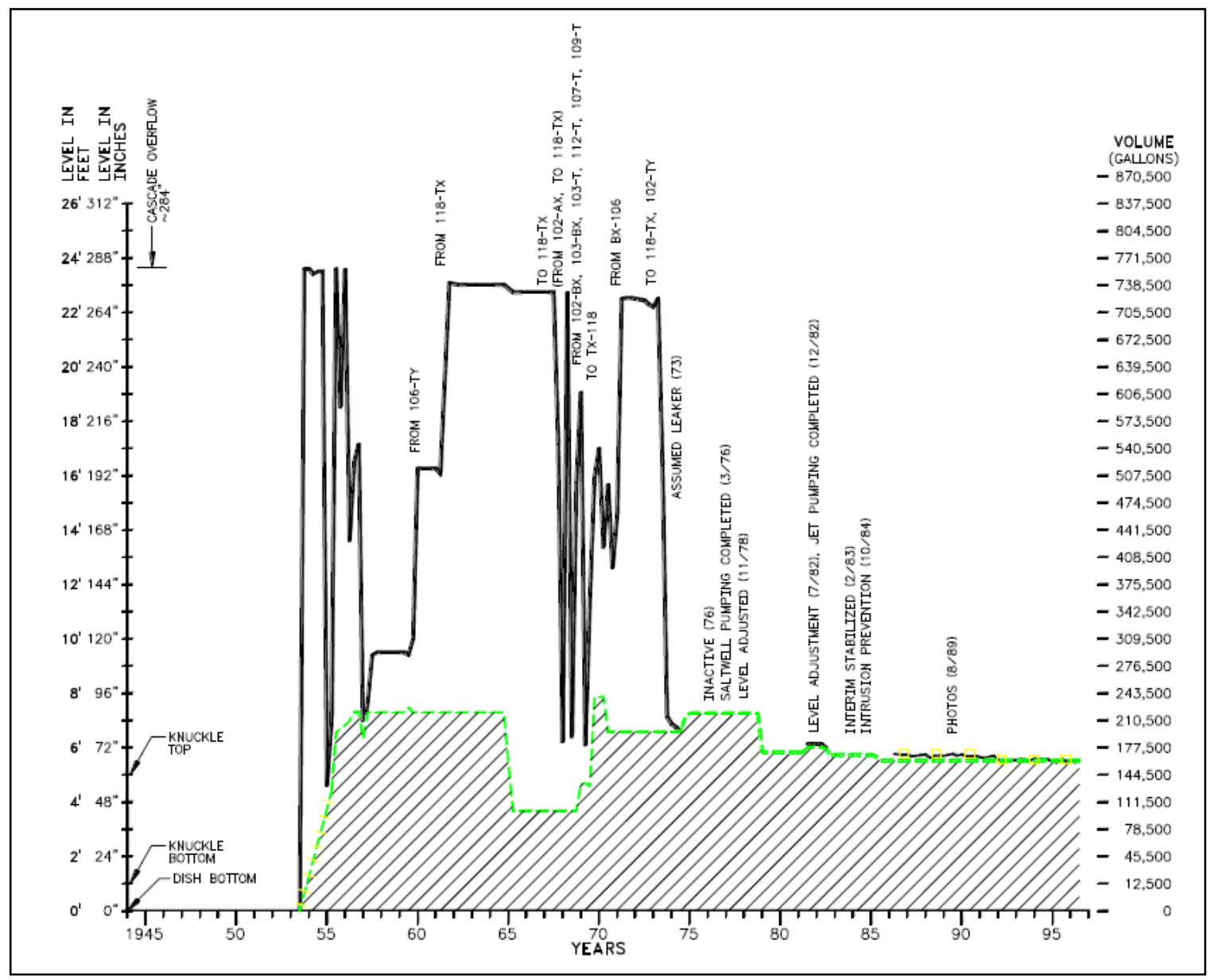

WHC-SD-WM-ER-351, Rev 0, March 1, 1995, Historical Tank Content Estimate for the Northwest Quadrant of the Hanford 200 West Area

The first indication of a leak from tank TY-103 was increased radioactivity detected in drywell 52-03-03 in August 1972 (Internal letter to L.W. Roddy from G.L. Borsheim, Integrity of Tanks 241-BY-103 and 241-TY-103, June 1, 1973). However, decreasing liquid levels from August 6, 1971 to February 19, 1973 were also reported in this letter and were further stated to have varied most likely because of operator and measurement techniques (see Figure 5-4). It was estimated that tank TY-103 had a liquid level loss of 3,000 to 7,000 gal (Internal letter to L.W. Roddy from G.L. Borsheim). 
Figure 5-4. Tank TY-103 Liquid Level August 1971 to August 1973

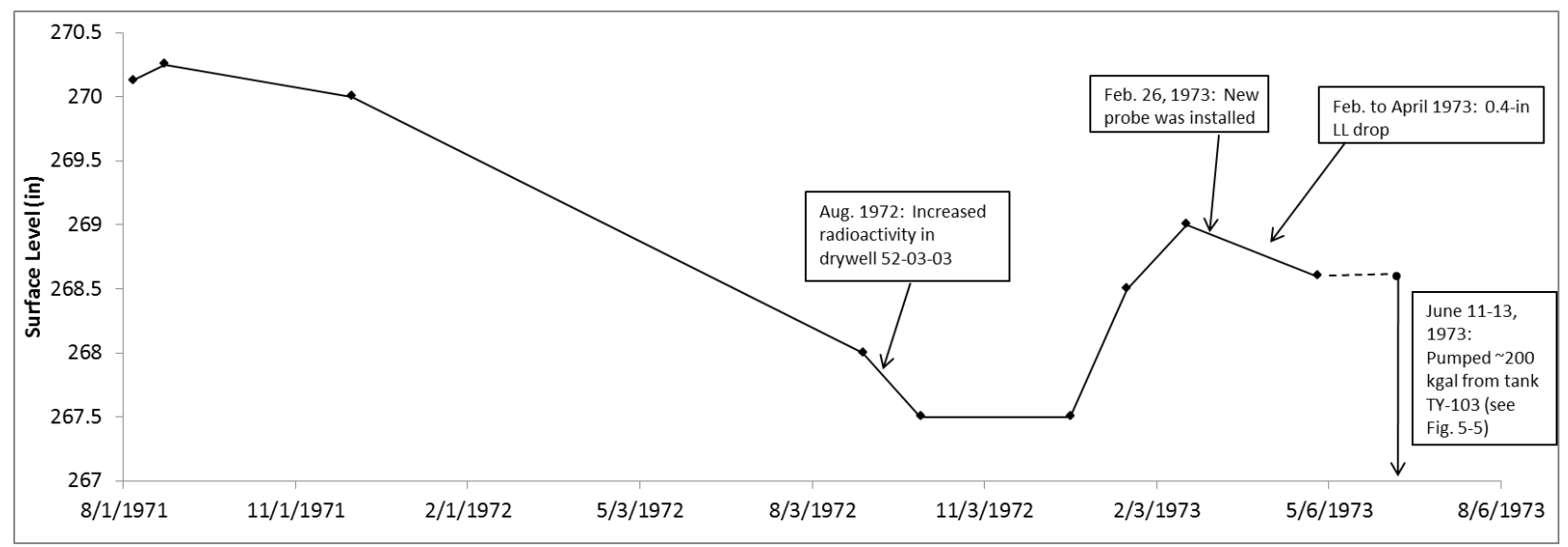

On October 26, 1973 a review was performed on tank TY-103 leak events. The review states that liquid level readings from January through December 1972 indicate a loss of 31/2-in (9,600 gal) by the end of 1972 with subsequent increases in radioactivity in the drywells (Draft memo Compton 1973, Tank 241-TY-103 Review). However, it was reported in this draft memo that liquid levels also had an unexplained increase in January 1973 of >2-in. Drywell 52-03-03 radioactivity continued to increase indicating a continuing leak. A new probe was installed February 26, 1973 (LET-ARHCO-TY-103, Status of Tank 241-TY-103). The next reported liquid level drop was two months later in April 1973 when a 0.4-in liquid level drop was reported (see Figure 5-4) with increased radiation readings in the drywell through May 28, 1973 when the tank was declared a suspect leaker (Draft memo Compton 1973).

During June 11-13, 1973, $200 \mathrm{kgal}$ was pumped from tank TY-103 leaving the liquid level at 196.9-in (see Figure 5-5). Tank TY-103 liquid levels dropped $~ 0.5$-in over a period of 14 days with subsequent increases in radioactivity in the drywells (LET-ARHCO-TY-103).

Approximately $184 \mathrm{kgal}$ was pumped from tank TY-103 on July 6, 1973 leaving the liquid level at 114-in. Tank TY-103 had a 0.5-in liquid level drop from July 15 to August 6, 1973. Due to liquid level decreases a saltwell pump was installed on August 14, 1973, and tank TY-103 was pumped to the sludge level on August 14, 22 and 25, 1973 leaving the liquid level at 86.9 to 87in $(\sim 150 \mathrm{kgal})$. Liquid levels remained relatively constant until September 1973 with saltwell pumping of the tank. Additional pumping of waste out of tank TY-103 occurred from October 1973 to October 1978. 
Figure 5-5. Tank TY-103 Liquid Level June to September 1973

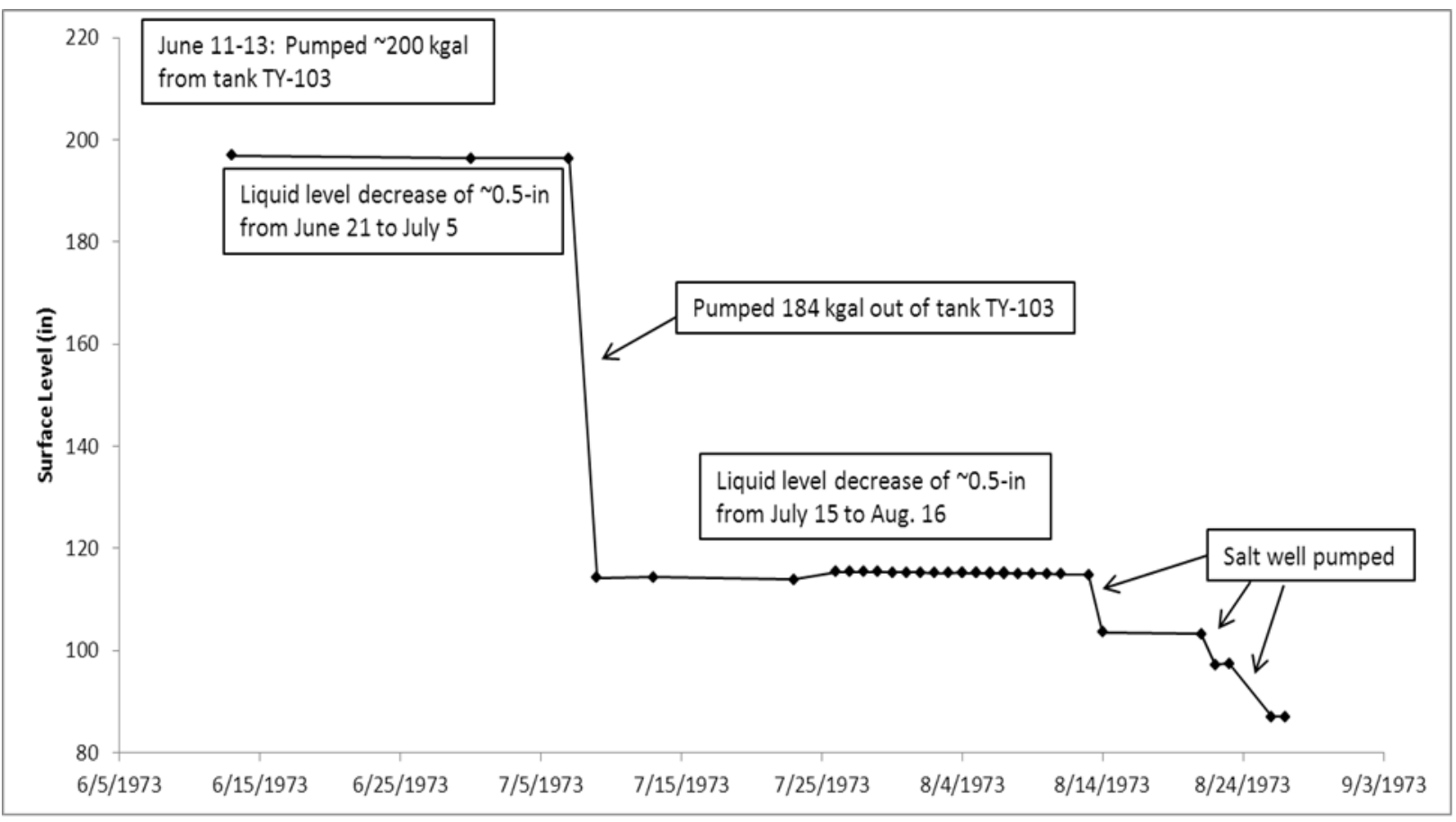

\subsubsection{Temperature}

No temperature data were recovered for tank TY-103 from July 1953 when the tank was first put into service until 1974 . Temperatures ranged from $55^{\circ} \mathrm{F}$ to $75^{\circ} \mathrm{F}$ from 1974 to the present (see PCSACS).

Tank TY-103 received TBP waste from 221-U Plant from July to August 1953. TBP wastes were concentrated and cooled to $\sim 180^{\circ} \mathrm{F}$ within the plant and were estimated to be $110-180^{\circ} \mathrm{F}$ after routing to the storage tanks. Rate of rise temperatures during initial tank filling are unknown.

\subsubsection{Liner Observations}

No liner observations relating to a tank TY-103 leak have been found.

\subsubsection{Chemistry-Corrosion}

Tank TY-103 began receiving waste in July 1953 and received various waste types throughout operation as shown in Table 5-1. The typical concentrations for nitrite, nitrate, and hydroxide for these waste types are shown in Table 5-2. Nitrite and hydroxide are known as nitrate-induced SCC inhibitors. One key characteristic for inhibiting SCC is to maintain a high nitrite concentration to nitrate concentration ratio (see Section 3.2.4). 
RPP-RPT-54911, Rev. 0

Table 5-1. Tank TY-103 Waste Storage Chronology

\begin{tabular}{|c|c|c|}
\hline Date & Waste Type & Length of Storage \\
\hline July 1953 to December 1955 & TBP & $\sim 1.5$ years \\
\hline January 1955 to August 1959 & 1C-FeCN & $\sim 4.5$ years \\
\hline September 1959 to June 1961 & TBP & $\sim 2$ years \\
\hline July 1961 to 1967 & TBP/DW & $\sim 6.5$ years \\
\hline January 1968 to April 1971 & $\begin{array}{c}\text { OWW/CWP/DW/1C- } \\
\text { CW/EB/REDOX HLW } \\
\text { supernatant/R-EB/RIX/BL/B-EB }\end{array}$ & $\sim 2.5$ years \\
\hline April 1971 to June 1973 & BL/OWW/RIX & $\sim 2$ years \\
\hline
\end{tabular}

Table 5-2. Waste Chemistries for Waste Types Stored in Tank TY-103

\begin{tabular}{|c|c|c|c|c|}
\hline Waste Type & {$\left[\mathrm{NO}_{3}{ }^{-}\right]$} & {$\left[\mathrm{NO}_{2}{ }^{-}\right]$} & {$\left[\mathbf{O H}^{-}\right]$} & $\begin{array}{c}\text { Meets Current } \\
\text { DST Specification }\end{array}$ \\
\hline $\mathrm{TBP}^{1}$ & 7.35 & Not reported & 0.09 & No $^{4}$ \\
\hline $\mathrm{CW}^{1}$ & 0.6 & 0.9 & 1.0 & Yes \\
\hline $1 \mathrm{C}^{1}$ & 1.54 & 0.26 & 0.28 & No $^{7}$ \\
\hline $\mathrm{RI}^{1}$ & 1.97 & 0.27 & 0.69 & Yes $^{1}$ \\
\hline $\mathrm{R}^{3}$ & 4.83 & \multicolumn{2}{|c|}{0.74} & No $^{6}$ \\
\hline $\mathrm{OWW}^{1}$ & 0.06 & Not reported & Not reported & Yes $^{5}$ \\
\hline $\mathrm{B}^{1}$ & 1.27 & Not reported & Not reported & Not available \\
\hline $\mathrm{BL}^{1}$ & 5.28 & Not reported & Not reported & Not available \\
\hline
\end{tabular}

1. Reference WHC-EP-0449, 1991, The Sort on Radioactive Waste Type Model: A Method to Sort Single-Shell Tanks into Characteristic Groups.

2. Reference OSD-T-151-00007, Rev. 12, 2013, Operating Specifications for the Double-Shell Storage Tanks.

3. Reference WHC-EP-0772, 1994, Characterization of the Corrosion Behavior of the Carbon Steel Liner in Hanford Site Single-Shell Tanks.

4. To be within the current DST specification limit, [NO3- $]<5.5 \mathrm{M}$

5. According to the assumption from reference WHC-EP-0772, Characterization of the Corrosion Behavior of the Carbon Steel Liner in Hanford Site Single-Shell Tanks.

6. Does not meet the current DST specification since the hydroxide and nitrite concentrations are not greater than or equal to $1.2 \mathrm{M}$.

7. Waste type 1C does not meet the current DST specification for waste chemistry; however, 1C was treated with FeCN, nickel sulfate, and $\mathrm{NaOH}$ to scavenge Cs-137 and Sr-90 prior to being added to tank TY-103. Depending on these ratios, the resulting waste may have met the DST specification.

The first waste tank TY-103 received in July 1953 was TBP waste from 221-U Plant and the tank continued to store only TBP waste for approximately 1.5 years. Tank TY-103 also stored TBP waste from September 1959 to June 1961 which was diluted with DW from 1961 to 1967. Thus, TBP waste was stored for a total of approximately 10 years in tank TY-103. Samples of TBP waste indicate hydroxide concentrations below $0.1 \mathrm{M}$ and nitrate concentrations above $6 \mathrm{M}$. The high nitrate concentration violates the current DST specification for waste chemistry as nitrate must be below $5.5 \mathrm{M}$. These conditions of the TBP waste would likely create an environment conducive to SCC and/or pitting (see Section 3.2.4). 
Tank TY-103 stored 1C waste treated in 221-T Plant with FeCN, nickel sulfate, and sodium hydroxide for approximately 4.5 years. Waste type $1 \mathrm{C}$ does not meet the current DST

specification for waste chemistry; however, after being treated in 221-T Plant the resulting waste may have met the DST specification depending on the ratios mixed with 1C. Tank TY-103 also stored REDOX HLW from January 1968 to April 1971 which does not meet the current DST specification for waste chemistry. However, during this time the tank also stored various waste types which would likely dilute the REDOX HLW. Thus, it can be assumed that $1 \mathrm{C}$ and REDOX HLW had little effect on waste chemistry during this time.

The other waste types stored in tank TY-103 should not have resulted in pitting or SCC. However, information for waste types B and BL are not available and it remains uncertain whether these waste types would increase the propensity for corrosion.

\subsubsection{Photographs}

Earliest photographs taken December 10, 1973 of tank TY-103 were reviewed and no evidence of bulging was found. No other documentation was found indicating a liner bulge in tank TY103. Photographs indicated that a beachline existed up to the tank TY-103 cascade line which carried waste to tank TY-104. No evidence from minimal liquid level data, drywell radioactivity, or a review of documents indicates that the cascade line leaked.

\subsection{TANK TY-103 EX-TANK DATA}

\subsubsection{Drywells}

There are three drywells located around the eastern side of tank TY-103: 52-03-12 installed in November 1971 and 52-03-03 and 52-03-06 installed in December 1971. All of the radiation readings in drywells are assumed to be maximum or peak readings unless otherwise noted (see Sections 3.3 and 3.4). The following subsections report the available drywell information and the drywell summary section provides the analyses of the associated drywells with tank TY-103.

\subsubsection{Drywell 52-03-03 $\left(\mathrm{S85}^{\circ} \mathrm{E}\right)$}

Drywell 52-03-03 was drilled December 31, 1971 with radiation levels recorded as less than values prior to August 1972 (see Appendix A2). Radioactivity was first detected in drywell 5203-03 on August 30, 1972 with a reported value of $>1,000 \mathrm{~K}$ cpm with the scintillation probe (SP) at 46-ft BGS. The next reading with a GM probe was recorded on November 21, 1972 at $3.7 \mathrm{~K}$ cpm and radioactivity continued to increase to $76 \mathrm{~K}$ cpm by March 1973. Radioactivity remained relatively stable at the 45-ft BGS level through June 1986.

In May 1997, Cs-137 and Co-60 were the only man-made radionuclides detected in drywell 5203-03 (GJ-HAN-66). From the ground surface to 22-ft BGS and from 42.5 to 59.5-ft BGS, Cs137 contamination was detected. It was reported in GJ-HAN-66 that extremely high gamma-ray activity saturated the detector between 45.5 and 49.5-ft BGS. Outside of this region, the maximum concentration of $400 \mathrm{pCi} / \mathrm{g}$ was reported at 50-ft BGS. Co-60 contamination was detected intermittently between 51 and 59.5-ft BGS with the maximum concentration of 0.4 pCi/g detected at 58.5-ft BGS. Document GJ-HAN-66 states, "Tank TY-103 probably leaked at 
RPP-RPT-54911, Rev. 0

the knuckle near borehole 52-03-03." Figure 5-6 shows the depth of radioactivity from 1980 to 1990 (HNF-3831).

Figure 5-6. Tank TY-103 Drywell 52-03-03 (HNF-3831)

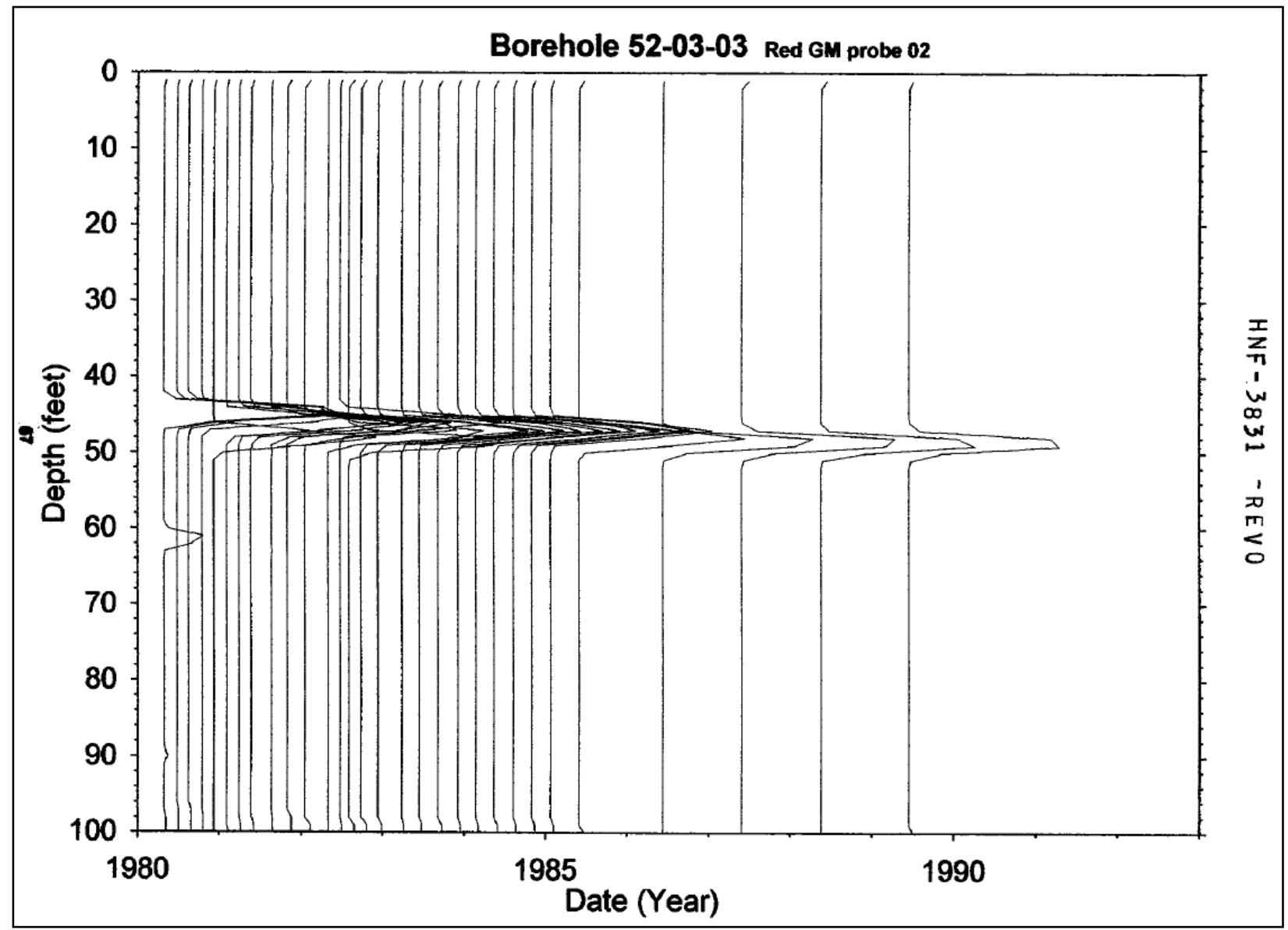

Note: Bottom of the tank footing is $\sim 45$-ft 10-in BGS 


\subsubsection{Drywell 52-03-06 $\left(\mathrm{S0}^{\circ}\right)$}

Drywell 52-03-06 was drilled December 31, 1971 with radiation levels recorded as less than values prior to August 1972 (see Appendix A2). No further drywell records were recovered until July 16, 1973 when readings were reported at 780K cpm with the SP at 55-ft BGS.

Radioactivity continued to remain relatively stable until February 8, 1974 when an additional peak appeared at $64-\mathrm{ft}$ BGS at $204.6 \mathrm{~K}$ cpm with the SP. Radioactivity remained relatively stable through June 1986.

In May 1997, Cs-137 and Co-60 were the only man-made radionuclides detected in this drywell (GJ-HAN-66). Cs-137 contamination was detected from the ground surface to 5-ft BGS, from 8.5 to 14.5 -ft BGS, and from 56 to $61-\mathrm{ft}$ BGS with the maximum concentration of $1.5 \mathrm{pCi} / \mathrm{g}$ reported at the ground surface. A zone of relatively high concentrations of Co-60 was detected continuously from 54 to 100-ft BGS demonstrating that Co-60 is relatively mobile and has migrated a relatively long distance from the contaminant source (GJ-HAN-66).

Figure 5-7 shows the continuing radiation depth to 1995 (HNF-3831). Drywell 52-03-06 is located between tanks TY-103 and TY-105, and it is possible contamination in this drywell could be associated with the leak from tank TY-103 and/or tank TY-105 (see Tank TY-105 segment). However, it seems unlikely contamination detected in this drywell is associated with tank TY-105 since tank TY-105 leaked in 1960 and radioactivity was reported as less than values prior to August 1972.

Figure 5-7. Tank TY-103 Drywell 52-03-06 (HNF-3831)

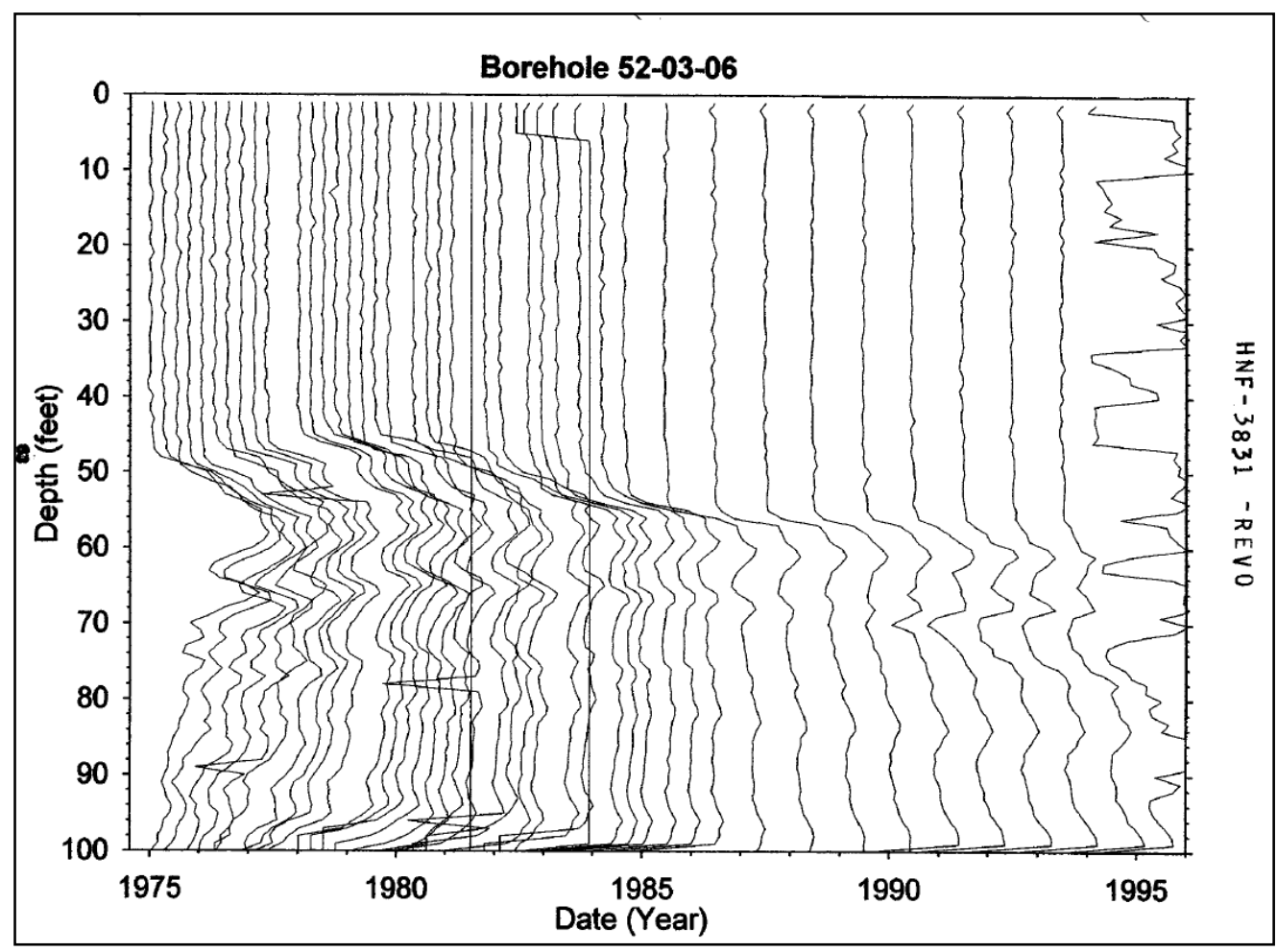

Note: Bottom of the tank footing is $\sim 45$-ft 10 -in BGS 


\subsubsection{Drywell 52-03-12 $\left(\mathrm{NO}^{\circ}\right)$}

Drywell 52-03-12 was drilled November 30, 1971 with the first reported reading on July 1, 1973 at $12 \mathrm{~K} \mathrm{cpm}$ with the SP; however, the BGS level was not specified (see Appendix A2). The next reading was recorded September 9,1973 at $14.4 \mathrm{~K} \mathrm{cpm}$ and $13.8 \mathrm{~K} \mathrm{cpm}$ at $44-\mathrm{ft}$ BGS and $51-\mathrm{ft}$ BGS, respectively. The probe used at this time was unspecified; however, the SP was likely used as readings were recorded at similar radiation levels with the SP in January 1974. Readings remained relatively stable through June 1974 and then slowly decreased to $\sim 3 \mathrm{~K}$ cpm by June 1986.

In May 1997, Cs-137 and Co-60 were the only man-made radionuclides detected in drywell 5203-12 (GJ-HAN-66). From the ground surface to 3.5-ft BGS, Cs-137 was detected continuously with a maximum concentration of $3.6 \mathrm{pCi} / \mathrm{g}$ reported at 1-ft BGS. From 65 to $70-\mathrm{ft}$ BGS and from 71 to $87.5-\mathrm{ft}$ BGS, Co-60 was detected with the maximum concentration of $0.31 \mathrm{pCi} / \mathrm{g}$ reported at 66.5-ft BGS. Document GJ-HAN-66 states, "The SGLS detected relatively low concentrations of Co-60 from 65 to $87.5 \mathrm{ft}$. This contamination probably originated from a leak in tank TY-101 or TY-103." Figure 5-8 shows the depth of radioactivity from 1975 to 1995 (HNF-3831).

Figure 5-8. Tank TY-103 Drywell 52-03-12 (HNF-3831)

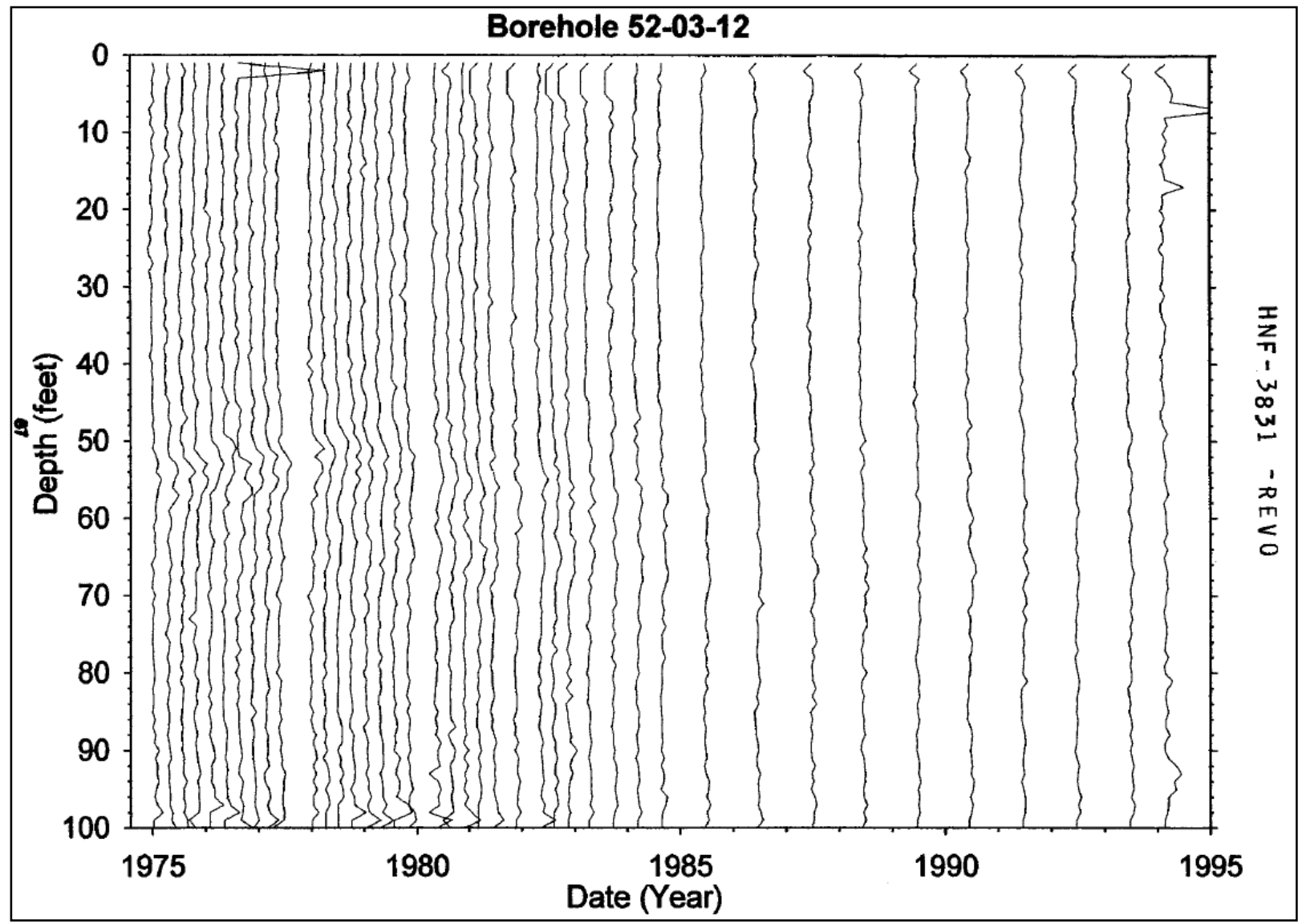

Note: Bottom of the tank footing is $\sim 45$-ft 10 -in BGS 
RPP-RPT-54911, Rev. 0

\subsubsection{Tank TY-103 Drywell Summary}

Tank TY-103 was first suspected of leaking as early as August 1971 due to liquid level decreases and subsequent radioactivity detected in the drywells in August 1972.

Radioactivity was first reported in drywell 52-03-03 in August 1972 at $~ 46-\mathrm{ft}$ BGS and radioactivity levels were significantly higher compared to drywells 52-03-06 and 52-03-12. Radioactivity was first reported in drywells 52-03-06 and 52-03-12 in July 1973. Radioactivity reported in drywell 52-03-06 was reported at a lower BGS level compared to the other two drywells. These three drywells were the only ones located near tank TY-103. No drywells are located near the western portion of the tank so it remains unclear whether radioactivity is present in this area (see Section 5.5.1.2). All three drywells indicate tank TY-103 leaked at or near the tank footing. Outer edge of the tank footing is between 42-ft 101/2-in and 45-ft 101/2-in BGS. Additional direct pushes were installed in 2005 as follows.

\subsubsection{Direct Push Logging}

Direct push logging to further characterize the tank TY-103 leak was conducted in October 2005 with the locations and maximum activity of the direct pushes shown in Figure 5-1 and Table 5-3 (RPP-RPT-34870, Small Diameter Geophysical Logging in the 241-TY Tank farm). Direct pushes were only logged once and only represent one profile in time. Compared to the drywells, direct pushes use a smaller diameter logging tool with different detection capability and results cannot be directly compared to drywell results. All of these direct pushes are vertical or near vertical.

Table 5-3. 2005 Direct Push Gross Gamma Results (RPP-RPT-34870)

\begin{tabular}{|l|c|c|c|c|l|}
\hline $\begin{array}{c}\text { Direct } \\
\text { Push }\end{array}$ & $\begin{array}{c}\text { Initial } \\
\text { Hole } \\
\text { Depth (ft) }\end{array}$ & $\begin{array}{c}\text { Extend } \\
\text { Hole } \\
\text { Depth (ft) }\end{array}$ & $\begin{array}{c}\text { Depth Max. } \\
\text { Activity } \\
\text { (ft BGS) }\end{array}$ & $\begin{array}{c}\text { Max eCs } \\
\text { (pCi/g) }\end{array}$ & Comment \\
\hline C4629 & 47.3 & - & Surface & 17 & $\begin{array}{l}\text { Minor count rate increase at } \\
\text { surface }\end{array}$ \\
\hline C4631 & 47.2 & - & - & Background & \\
\hline C4633 & 61.5 & 69.5 & 48.6 & $22,000,000$ & $\begin{array}{l}\text { NaI Detector Saturated 47-51.5 and } \\
53.5-56 \mathrm{ft}\end{array}$ \\
\hline C4635 & 47.2 & - & Surface & 16 & $\begin{array}{l}\text { Minor count rate increase at } \\
\text { surface }\end{array}$ \\
\hline C5008 & 65 & - & 48.5 & $6,700,000$ & \\
\hline C5010 & 65 & - & - & Background & \\
\hline C5012 & 65 & - & 49 & 280,000 & \\
\hline
\end{tabular}

Note: Direct pushes that did not indicate radioactivity at a lower level were not deepened.

Radioactivity was the highest in direct push C4633 with $2.2 \times 10^{7} \mathrm{pCi} / \mathrm{g}$ at $48.6-\mathrm{ft} \mathrm{BGS}$ indicating tank TY-103 likely leaked near this direct push. Direct pushes C5008 reported $6.7 \mathrm{x}$ $10^{6} \mathrm{pCi} / \mathrm{g}$ at $48.5-\mathrm{ft}$ BGS and C5012 at $2.8 \times 10^{5} \mathrm{pCi} / \mathrm{g}$ at $49-\mathrm{ft}$ BGS. It appears tank TY-103 leaked in one location near the tank footing close to direct push $\mathrm{C} 4633$ with migration to nearby pushes C5008 and C5012 which is supported by radioactivity detected in drywell 52-03-03. 
Direct pushes C4629, C4631, C4635, and C5010 do not report any current radioactivity that could be associated with a tank leak.

Direct pushes C4629 and C4631, located south of tank TY-103, do not report any radioactivity associated with a tank leak despite radioactivity being detected in drywell 52-03-06 at $\sim 55-\mathrm{ft}$ BGS (see Section 5.5.1.2). However, direct pushes C4629 and C4631 were only installed to a depth of 47.3 and 47.4-ft BGS, respectively, and did not intercept the Co-60 detected in 1995.

See Appendix B2 for the gamma surveys for the direct pushes surrounding tank TY-103. See document RPP-RPT-34870 for additional gamma surveys for the direct pushes for TY Farm.

\subsection{POSSIBLE TANK TY-103 LINER LEAK LOCATION(S)}

A liner leak may have penetrated the waterproof membrane at any location or pooled on the waterproof membrane and followed concrete cracks or construction joints to a different location for egress to the soil, including the top of the tank footing. It is likely that the tank TY-103 leak(s) did not occur far above the tank footing because all of the initial indicators, three drywells and three direct pushes, were located at or near the tank footing and the drywells ranged from 6.1-ft to 8.3-ft from the tank footing. Activity was not detected at the BGS level of pipelines. The close proximity of the drywells to the tank footing would tend to indicate a leak was not far up the sidewall; however, a sidewall leak cannot be ruled out near the drywell locations.

There were no drywells or direct pushes located on the western side of tank TY-103. Therefore, it is possible leaks could have occurred and not been detected on the western side of tank TY103. Also, no leak detection laterals were installed in TY Farm. 


\subsubsection{Leak Detected in 1972 \& 2005 - Site A}

Tank TY-103 was first suspected of leaking due to liquid level decreases starting as early as August 1971 with increased radioactivity reported in August 1972 in drywell 52-03-03 at or near the tank footing (see site A in Figure 5-9). In 2005, direct pushes were drilled to further characterize the tank TY-103 leak. Direct pushes C4633, C5008, and C5012 report radioactivity at $49-\mathrm{ft}$ BGS. It is likely the tank leak occurred near drywell 52-03-03 and direct pushes C4633, C5008, and C5012 as radiation levels were the highest compared to the other drywells and pushes. Liquid levels continued to slowly decline and tank TY-103 was classified as a suspected leaker in May 1973.

Figure 5-9. Tank TY-103 Possible Leak Location (August 1972 \& 2005)

Tank inner ring is steel liner, outer ring is outer edge of tank footing

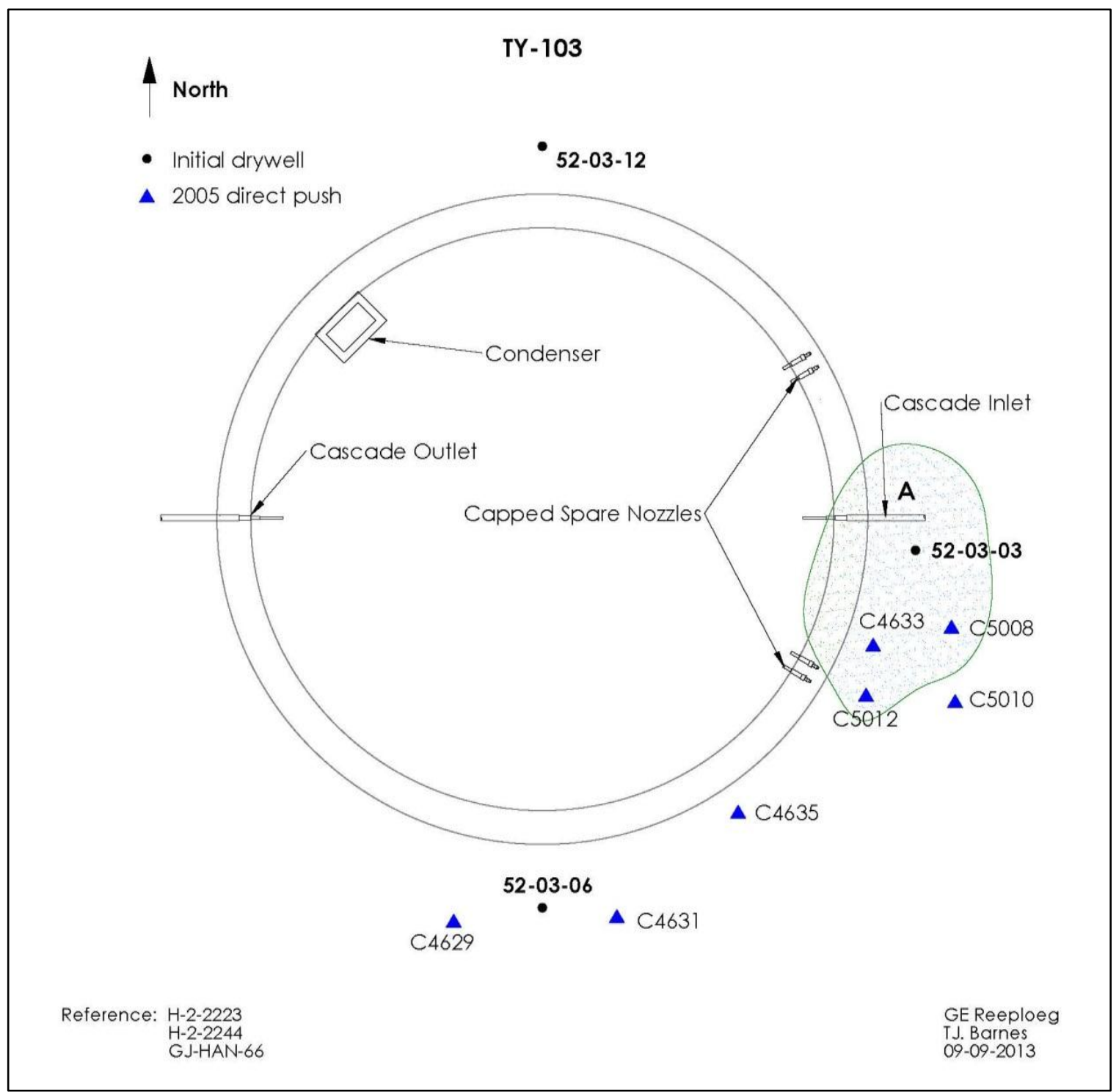

The leak from tank TY-103 was first detected in August 1971 due to a liquid level decrease and subsequent radioactivity detected in drywell 52-03-03 


\subsubsection{Leak Detected in 1973 - Site B}

Radioactivity was first detected July 1973 in drywell 52-03-06 at 55-ft BGS. The radioactivity was detected two years after liquid levels started to decline in 1971 and one year after radioactivity was detected in drywell 52-03-03. It is likely site B (see Figure 5-10) is a separate leak site from site A (see Figure 5-9) since no radioactivity was detected in direct push C4635.

Figure 5-10. Tank TY-103 Possible Leak Location (July 1973)

Tank inner ring is steel liner, outer ring is outer edge of tank footing

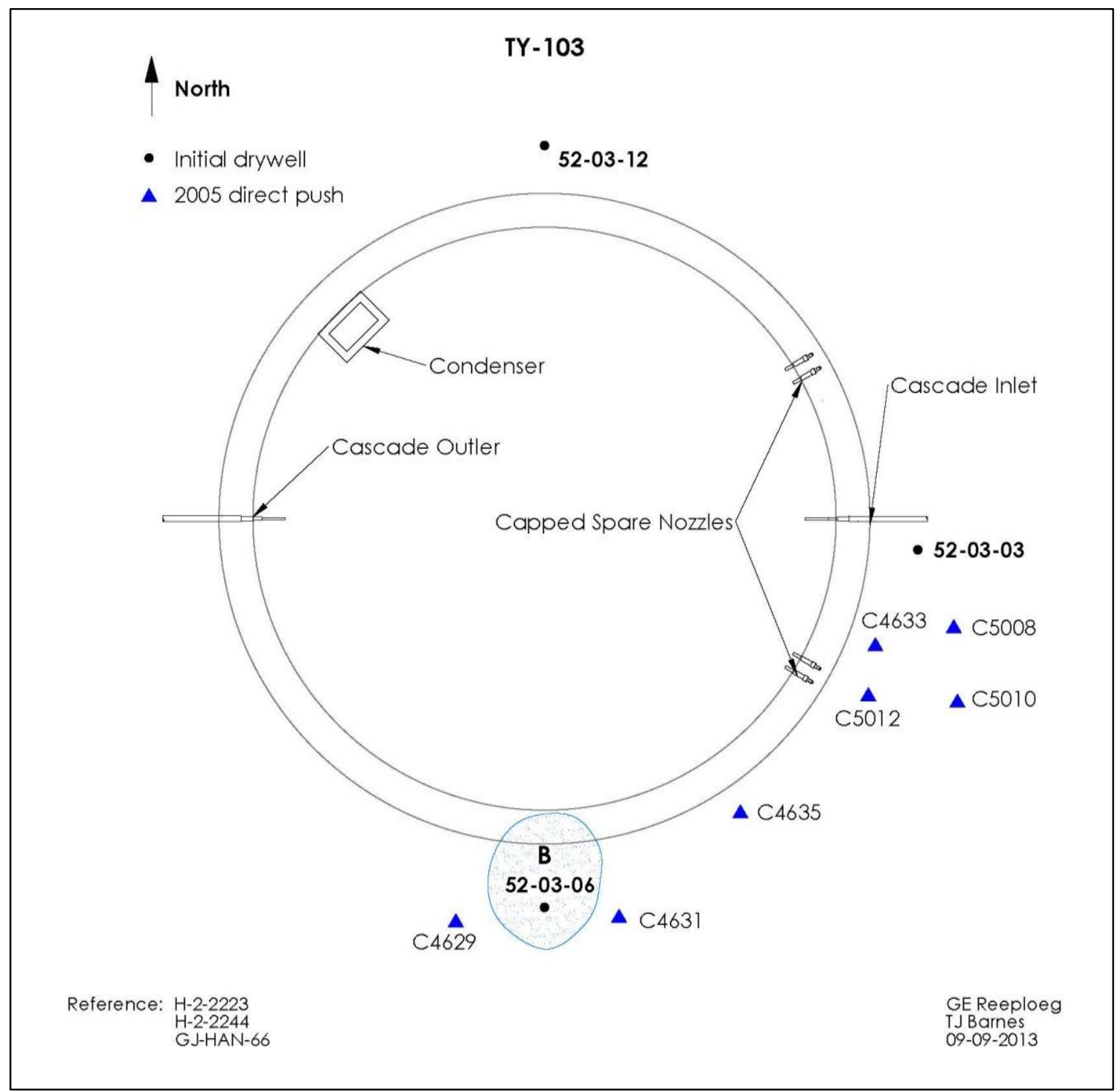

The leak from tank TY-103 was first detected in August 1971 due to a liquid level decrease and subsequent radioactivity detected in drywell 52-03-03 


\subsubsection{Leak Detected in 1973 - Site C}

Radioactivity was first detected July 1973 in drywell 52-03-12 at 44-ft BGS. The radioactivity was detected two years after liquid levels started to decline in 1971 and one year after radioactivity was detected in drywell 52-03-03. It is likely radioactivity at site $\mathrm{C}$ (see Figure 5-11) is a separate leak site from sites A and B as radioactivity was detected at a higher BGS level compared to drywells 52-03-03 and 52-03-06.

It is unknown if contamination is present underneath the tank or in the western portion of the tank as no drywells or laterals are present in these areas.

Figure 5-11. Tank TY-103 Possible Leak Location (July 1973)

Tank inner ring is steel liner, outer ring is outer edge of tank footing

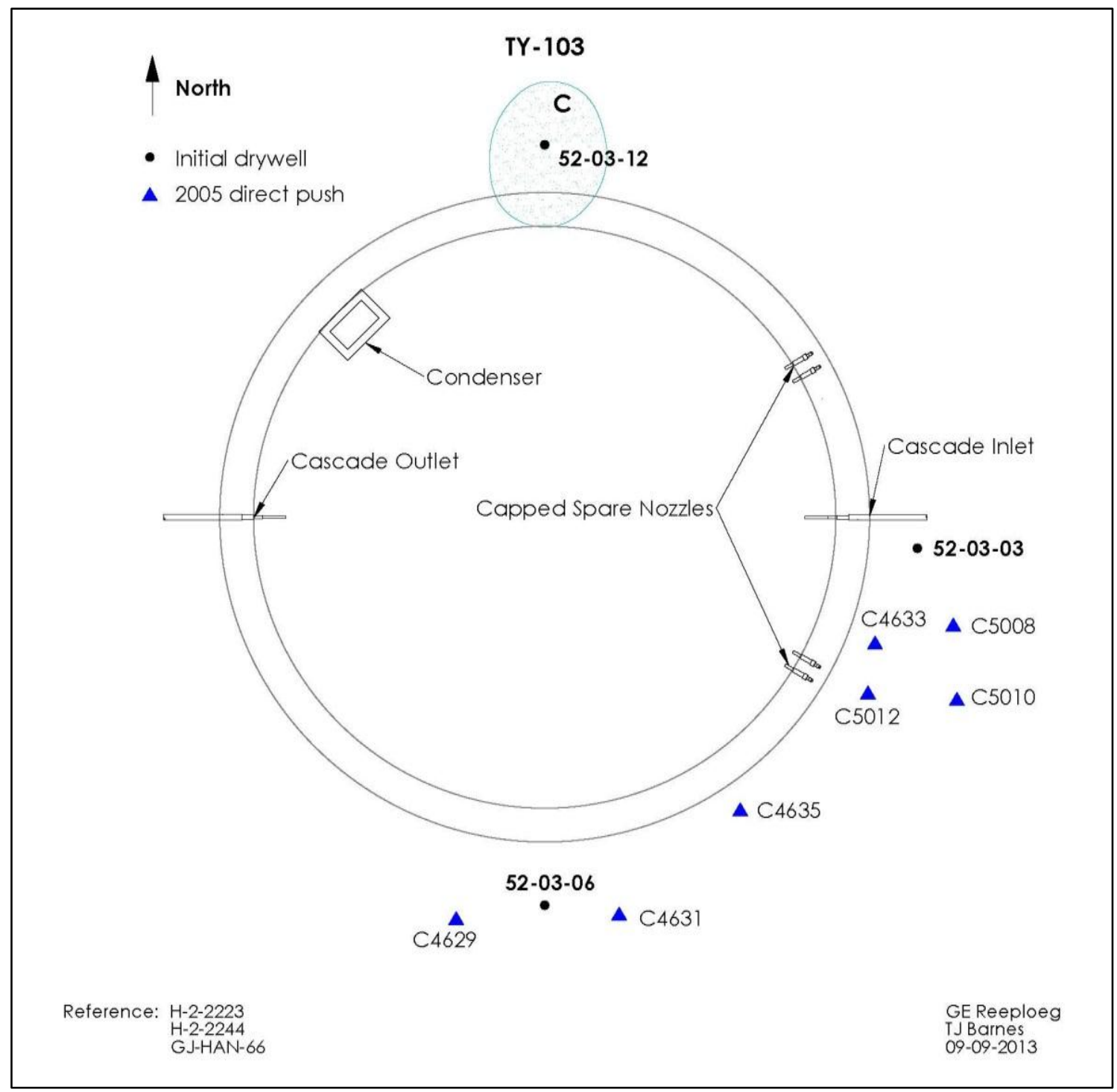

The leak from tank TY-103 was first detected in August 1971 due to a liquid level decrease and subsequent radioactivity detected in drywell 52-03-03 


\subsubsection{Leak Location Summary}

Tank TY-103 was first suspected of leaking as early as August 1971 due to liquid level decreases with subsequent increases in radioactivity in drywell 52-03-03 in August 1972 at or near the tank footing as shown in site A (see Figure 5-12). It is likely the tank first leaked near drywell 52-0303 based on the significant amount of radiation detected in this drywell and as stated in GJHAN-66, "The zone of highly concentrated Cs-137 contamination in borehole 52 03-03 is close to the depth of the knuckle radius of the tank liner; it is likely that the tank leaked along the seam of the knuckle radius near this borehole." In 2005, direct pushes were installed to further characterize the tank TY-103 leak and radioactivity was detected in direct pushes C4633, C5008, and C5012 which would have likely been present when the tank was first suspected of leaking.

Radioactivity was first detected concurrently in drywells 52-03-06 and 52-03-12 at or below the tank footing in July 1973 after liquid levels continued to decline. It is likely that contamination detected in drywell 52-03-06 (site B in Figure 5-12) is a separate leak site since radioactivity was not present in nearby drywells. Site $C$ appears to be a separate leak site from sites A and B, as radioactivity detected in drywell 52-03-12 was at a higher BGS level compared to the other drywells.

The tank TY-103 leak(s) did not occur far above the tank footing because all of the initial indicators, three drywells and three direct pushes, were located at or near the bottom of the tank. It is possible contamination exists underneath the tank and in the western portion of the tank; however, no drywells or laterals are present in this area so it remains inconclusive.

Leak locations in Figure 5-12 are based on peak readings and are a representation of possible initial boundaries of radioactivity.

No evidence was found for a liner bulge occurring in tank TY-103, and it remains unclear if a liner bulge once existed in the tank during its operation. However, tank TY-103 non-boiling temperatures are not likely to be a factor in causing a liner bulge. 
Figure 5-12. Tank TY-103 Possible Radial Leak Locations

Tank inner ring is steel liner, outer ring is outer edge of tank footing

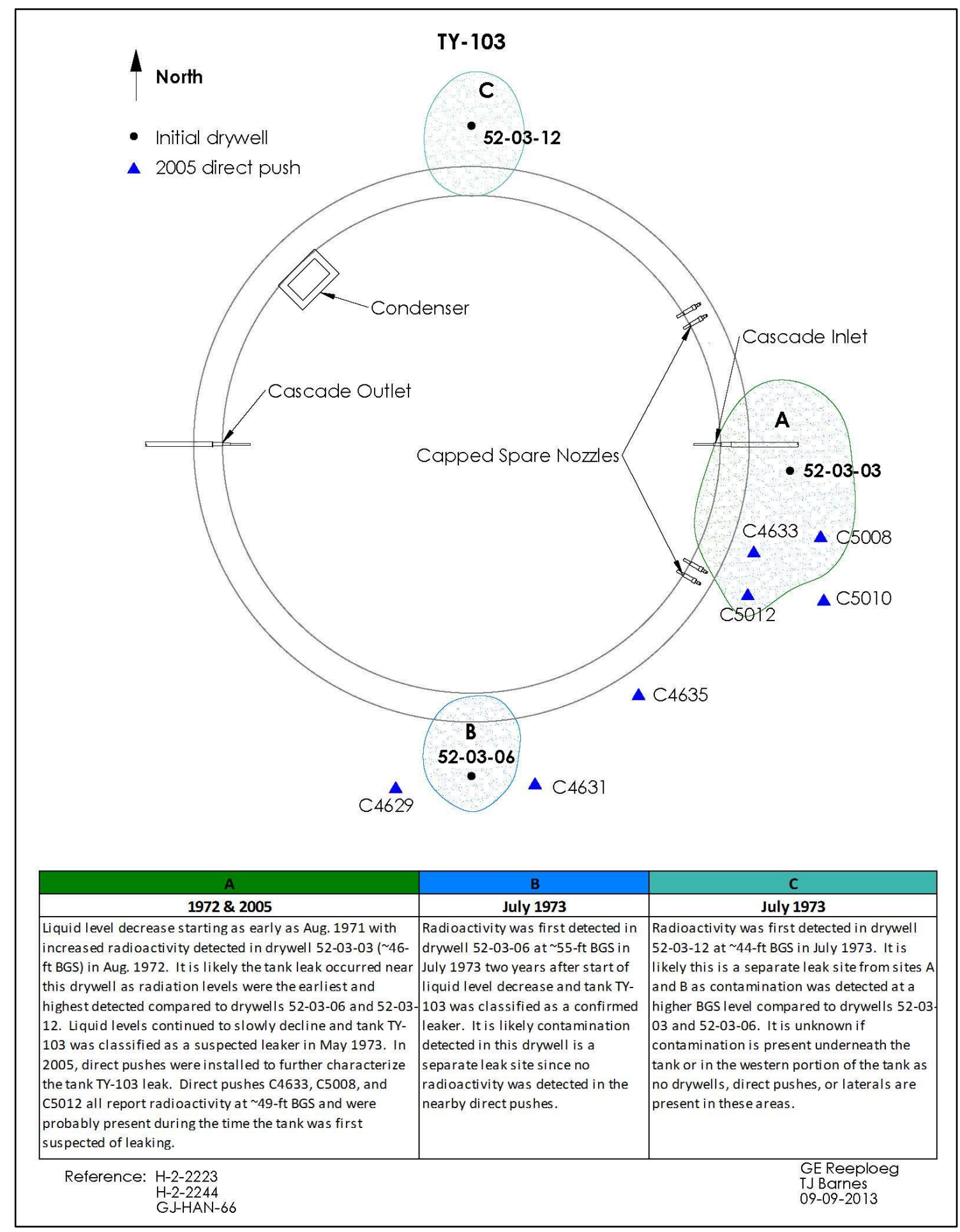


RPP-RPT-54911, Rev. 0

\subsection{POSSIBLE TANK TY-103 LINER LEAK CAUSE(S)}

Tank TY-103 was examined against five conditions that could contribute to a failed liner.

\subsubsection{Tank Design}

The TY Farm tank design does not appear to be a factor contributing to a failed liner (see Section 3.1.1).

\subsubsection{Thermal Shock}

No temperature data are available for tank TY-103 prior to 1974 although tank TY-103 held nonboiling waste. Since no records are available, it is uncertain what the maximum temperature was in tank TY-103 during operation as well as the rate of temperature rise when waste was initially added. However, the thermal attributes of the waste would indicate that thermal stresses were minimal.

Thermal shock creates stress both from rapid temperature rise as well as waste-induced high temperatures.

Temperature requirements in ARH-951 (Limitations for Use of Underground Waste Tanks) issued December 18, 1969 indicated that tank temperatures should be held below $230^{\circ} \mathrm{F}$.

\subsubsection{Chemistry-Corrosion}

Tank TY-103 was first filled with TBP waste and stored a variety of waste types during operation. TBP waste consists of low hydroxide and high nitrate concentrations, likely with low nitrite concentrations, which would create an environment conducive to pitting and SCC.

When the tank was first filled, TBP waste was the only waste added to tank TY-103 for over two years. Additional TBP supernatant waste from tank TY-106 was later added to tank TY-103; however, the added waste was diluted with the waste already present inside the tank. A tank with TBP waste type present would likely increase SCC in the tank liner (see Section 5.4.3).

Waste types $\mathrm{R}$ and $1 \mathrm{C}-\mathrm{FeCN}$ could create an environment conducive to SCC; however, this would be to a lesser degree than TBP waste. Waste types $\mathrm{R}$ and $1 \mathrm{C}-\mathrm{FeCN}$ probably did not increase the propensity for corrosion in tank TY-103 since R was diluted with other waste types and $1 \mathrm{C}$ was treated in 221-T Plant prior to being added to the tank (see Section 5.4.3). Other waste types stored in tank TY-103 should not have resulted in pitting or SCC.

\subsubsection{Liner Observations}

A review of the available photographs taken December 10, 1973 does not contain any evidence of a tank bottom liner bulge. There is no additional documentation available indicating a liner bulge was present in tank TY-103. 
RPP-RPT-54911, Rev. 0

\subsubsection{Tank Construction Temperature}

Average winter temperatures were $27.4^{\circ} \mathrm{F}$ and $25.2^{\circ} \mathrm{F}$ in December 1951 and January 1952, respectively, with temperatures as low as $4^{\circ} \mathrm{F}$ during the period when the TY Farm tank liners were being constructed. The low temperatures experienced during construction were less than the current allowable $18^{\circ} \mathrm{F}$ allowable temperature where impact loading had the potential for creating micro-fissures in the steel liner. The TY Farm construction specifications (HW-4696, Specifications Waste Disposal Facilities 241-BZ and TY Tank Farm) contained specific detailed requirements for cold weather construction and it appears these low temperatures had less of an impact during the construction of TY Farm compared to 241-SX Farm construction (see Section 4.3.2).

\section{$5.8 \quad$ TANK TY-103 CONCLUSIONS}

Evidence indicates that the tank TY-103 liner leaked at or near the tank footing in either two or three locations. Based on engineering judgment and available information the following conditions listed in the order of importance lead to the tank TY-103 failed liner.

1. TBP waste storage chemistry-corrosion - stress corrosion cracking

2. Thermal conditions

3. Construction conditions - ductile-to-brittle transition temperature

There are several liner leak cause conditions that were examined but the most likely cause of the tank TY-103 leak is TBP waste storage chemistry-corrosion. TBP waste storage could have created a chemical environment susceptible to nitrate-induced SCC attack on a potentially stressed metal liner.

Thermal and construction conditions could have also contributed to the liner failure but to a lesser degree. Tank design doesn't seem to have contributed to liner failure and there is no evidence of liner bulging. Some or all of the factors can act serially or together to contribute to tank liner failure. 
RPP-RPT-54911, Rev. 0

\section{APPENDIX A2}

TANK TY-103 GROSS GAMMA DRYWELL DATA 
RPP-RPT-54911, Rev. 0

Table A2-1. Tank TY-103 Drywell Radioactivity (K counts per minute)

(August 1972 to June 1986)

(Drywell Data Sheets* Retrieved on July 20, 2011 and SD-WM-TI-356) (1 of 2 sheets)

\begin{tabular}{|c|c|c|c|c|c|c|c|c|c|c|c|}
\hline \multicolumn{4}{|c|}{ 52-03-03 } & \multicolumn{4}{|c|}{ 52-03-06 } & \multicolumn{4}{|c|}{ 52-03-12 } \\
\hline \multicolumn{4}{|c|}{ Drilled 12/31/71 } & \multicolumn{4}{|c|}{ Drilled 12/31/71 } & \multicolumn{4}{|c|}{ Drilled 11/30/71 } \\
\hline Date & Probe & $\begin{array}{c}\text { Peak } \\
(\mathrm{K} \text { cpm })\end{array}$ & $\begin{array}{c}\text { Depth } \\
\text { (ft BGS) }\end{array}$ & Date & Probe & $\begin{array}{c}\text { Peak } \\
(\text { K cpm })\end{array}$ & $\begin{array}{c}\text { Depth } \\
\text { (ft BGS) }\end{array}$ & Date & Probe & $\begin{array}{c}\text { Peak } \\
(\mathrm{K} \text { cpm })\end{array}$ & $\begin{array}{c}\text { Depth } \\
\text { (ft BGS) }\end{array}$ \\
\hline $\begin{array}{c}\text { Before } \\
8 / 72\end{array}$ & $\mathrm{~N} / \mathrm{A}^{3}$ & $<50^{1}$ & $\begin{array}{l}\text { Entire } \\
\text { length }\end{array}$ & $\begin{array}{c}\text { Before } \\
8 / 72\end{array}$ & $\mathrm{~N} / \mathrm{A}^{3}$ & $<50^{1}$ & $\begin{array}{l}\text { Entire } \\
\text { length }\end{array}$ & & & $A^{3}$ & \\
\hline $8 / 30 / 72$ & SP & $>1000^{1}$ & 46 & \multicolumn{4}{|c|}{$\mathrm{N} / \mathrm{A}^{3}$} & \multicolumn{4}{|c|}{$\mathrm{N} / \mathrm{A}^{3}$} \\
\hline $11 / 21 / 72$ & GMP & $3.7^{1}$ & $\mathrm{~N} / \mathrm{A}^{3}$ & \multicolumn{4}{|c|}{$\mathrm{N} / \mathrm{A}^{3}$} & \multicolumn{4}{|c|}{$\mathrm{N} / \mathrm{A}^{3}$} \\
\hline $3 / 26 / 73$ & GMP & $76^{1}$ & $\mathrm{~N} / \mathrm{A}^{3}$ & \multicolumn{4}{|c|}{$\mathrm{N} / \mathrm{A}^{3}$} & \multicolumn{4}{|c|}{$\mathrm{N} / \mathrm{A}^{3}$} \\
\hline $4 / 30 / 73$ & $\mathrm{~N} / \mathrm{A}^{3}$ & $89.5^{2}$ & $\mathrm{~N} / \mathrm{A}^{3}$ & \multicolumn{4}{|c|}{$\mathrm{N} / \mathrm{A}^{3}$} & \multicolumn{4}{|c|}{$\mathrm{N} / \mathrm{A}^{3}$} \\
\hline $5 / 14 / 73$ & GMP & 89 & $\mathrm{~N} / \mathrm{A}^{3}$ & \multicolumn{4}{|c|}{$\mathrm{N} / \mathrm{A}^{3}$} & \multicolumn{4}{|c|}{$\mathrm{N} / \mathrm{A}^{3}$} \\
\hline $7 / 10 / 73$ & GMP & 111.6 & 45 & $7 / 16 / 73$ & SP & 780 & 55 & $7 / 1 / 73$ & SP & 12 & $\mathrm{~N} / \mathrm{A}^{3}$ \\
\hline $8 / 27 / 73$ & GMP & 107.5 & 44 & $8 / 28 / 73$ & $\mathrm{~N} / \mathrm{A}^{3}$ & 960 & 52 & \multicolumn{4}{|c|}{$\mathrm{N} / \mathrm{A}^{3}$} \\
\hline $9 / 5 / 73$ & GMP & 175 & 40 & \multirow{2}{*}{$9 / 11 / 73$} & \multirow{2}{*}{$\mathrm{N} / \mathrm{A}^{3}$} & \multirow{2}{*}{955} & \multirow{2}{*}{55} & \multirow{2}{*}{$9 / 9 / 73$} & \multirow{2}{*}{$\mathrm{N} / \mathrm{A}^{3}$} & 14.4 & 44 \\
\hline $9 / 27 / 73$ & GMP & 81.5 & 40 & & & & & & & 13.8 & 51 \\
\hline $10 / 11 / 73$ & GMP & 125 & 46 & $10 / 25 / 73$ & GMP & 0.18 & 55 & $10 / 18 / 73$ & $\mathrm{~N} / \mathrm{A}^{3}$ & 14.5 & 52 \\
\hline $11 / 9 / 73$ & GMP & 160.2 & 46 & $11 / 1 / 73$ & SP & 900 & $\mathrm{~N} / \mathrm{A}^{3}$ & $11 / 26 / 73$ & $\mathrm{~N} / \mathrm{A}^{3}$ & 14.5 & 52 \\
\hline $12 / 4 / 73$ & $\mathrm{~N} / \mathrm{A}^{3}$ & 152.5 & 47 & $12 / 18 / 73$ & $\mathrm{~N} / \mathrm{A}^{3}$ & 918 & 57 & $12 / 22 / 73$ & $\mathrm{~N} / \mathrm{A}^{3}$ & 15 & 52 \\
\hline \multirow{2}{*}{$1 / 1 / 74$} & \multirow{2}{*}{ GMP } & \multirow{2}{*}{165} & 47 & 15174 & GMP & 01 & 55 & $1 / 10 / 74$ & $\mathrm{SP}$ & 138 & 44 \\
\hline & & & (7t & 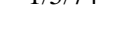 & Gin & 0.1 & (J) & 171914 & JT & 15.0 & 51 \\
\hline & & & & & & 858 & 54 & & 3 & 12 & 41 \\
\hline 270179 & U17 & 101.2 & $7 \mathrm{~J}$ & 27017 & SI & 204.6 & 64 & 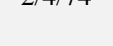 & & 14.75 & 49 \\
\hline נרו202 & CDD & 100 & 4 & 212017 & $\mathrm{CD}$ & 1000.2 & 53 & 2 & $\mathrm{CD}$ & 15 & 46 \\
\hline & & & & & & 300 & 65 & & & & 52 \\
\hline & & & & & & 960 & 58 & & & & \\
\hline $4 / 17 / 74$ & GMP & 247.8 & 46 & $4 / 1 / 74$ & SP & 900 & 61 & $4 / 23 / 74$ & SP & 18.6 & 53 \\
\hline & & & & & & 210 & 69 & & & & \\
\hline $5 / 1717$ & CMD & $240 ?$ & 17 & $5 / 1 / 74$ & $\mathrm{SD}$ & 930 & 56 & $5 / 1 / 74$ & $\mathrm{CD}$ & 10 & $\mathrm{~N}^{3}{ }^{3}$ \\
\hline & & & 41 & 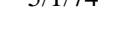 & 31 & 300 & 67 & $3 / 1 / / 4$ & $\mathrm{SP}$ & 10 & N/A \\
\hline $61 / 74$ & GMP & 282 & 16 & $6 / 18 / 74$ & $\mathrm{SD}$ & 910.2 & 55 & 61174 & $\mathrm{CD}$ & 18 & $\mathrm{~N}^{3}$ \\
\hline $0 / 1 / 74$ & UNIT & 202 & 40 & $0 / 10 / 14$ & $\mathrm{Sr}$ & 354 & 68 & $0 / 17 / 4$ & SF & 10 & N/A \\
\hline $7 / 2677$ & CMD & ? חרק & 47 & $7118 / 74$ & $\mathrm{~N}^{3} \mathrm{~A}^{3}$ & 273.6 & 63 & $7 / 1874$ & $\mathrm{~N}^{3}{ }^{3}$ & 75 & 50 \\
\hline 112017 & Uin & 220.2 & 41 & 110014 & & 300 & 66 & 1110814 & & 1.0 & $J 2$ \\
\hline ०/०/74 & CMD & 1225 & 15 & 018174 & 3 & 294 & 54 & 018174 & 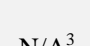 & $6^{4}$ & $\mathrm{~N}^{3} \mathrm{~S}^{3}$ \\
\hline (1) & UIVIT & 152.0 & 45 & $8 / 8 / / 4$ & N/A & 318 & 57 & $8 / 8 / 14$ & N/A & $<6$ & N/A \\
\hline & & & & & 3 & 258 & 55 & $0<17$ & 3 & 79 & \\
\hline 9112714 & UVIT & 219.4 & 40 & 91018 & N/A & 288 & 58 & $9101 / 4$ & N/A & 1.2 & $J 2$ \\
\hline
\end{tabular}


RPP-RPT-54911, Rev. 0

Table A2-1. Tank TY-103 Drywell Radioactivity (K counts per minute)

(August 1972 to June 1986)

(Drywell Data Sheets* Retrieved on July 20, 2011 and SD-WM-TI-356) (2 of 2 sheets)

\begin{tabular}{|c|c|c|c|c|c|c|c|c|c|c|c|}
\hline \multicolumn{4}{|c|}{$52-03-03$} & \multicolumn{4}{|c|}{ 52-03-06 } & \multicolumn{4}{|c|}{ 52-03-12 } \\
\hline Date & Probe & $\begin{array}{c}\begin{array}{c}\text { Peak } \\
(\text { K cpm })\end{array} \\
\end{array}$ & $\begin{array}{c}\text { Depth } \\
\text { (ft BGS) }\end{array}$ & Date & Probe & $\begin{array}{c}\begin{array}{c}\text { Peak } \\
(\text { K cpm })\end{array} \\
\end{array}$ & $\begin{array}{c}\text { Depth } \\
\text { (ft BGS) }\end{array}$ & Date & Probe & $\begin{array}{c}\begin{array}{c}\text { Peak } \\
(\mathrm{K} \text { cpm })\end{array} \\
\end{array}$ & $\begin{array}{c}\text { Depth } \\
\text { (ft BGS) }\end{array}$ \\
\hline \multirow{3}{*}{$10 / 11 / 74$} & \multirow{3}{*}{ GMP } & \multirow{3}{*}{275.2} & \multirow{3}{*}{49} & \multirow{3}{*}{$10 / 4 / 74$} & \multirow{3}{*}{$\mathrm{N} / \mathrm{A}^{3}$} & 96 & 55 & \multirow{3}{*}{$10 / 24 / 74$} & \multirow{3}{*}{$\mathrm{N} / \mathrm{A}^{3}$} & \multirow{3}{*}{6} & \multirow{3}{*}{$\mathrm{N} / \mathrm{A}^{3}$} \\
\hline & & & & & & 108 & 58 & & & & \\
\hline & & & & & & 48 & 67 & & & & \\
\hline \multirow{2}{*}{$11 / 8 / 74$} & \multirow{2}{*}{ SP } & \multirow{2}{*}{4671.4} & \multirow{2}{*}{50} & \multirow{2}{*}{$11 / 8 / 74$} & \multirow{2}{*}{$\mathrm{N} / \mathrm{A}^{3}$} & 277.5 & 62 & \multirow{2}{*}{$11 / 14 / 74$} & \multirow{2}{*}{$\mathrm{N} / \mathrm{A}^{3}$} & \multirow{2}{*}{2.9} & \multirow{2}{*}{54} \\
\hline & & & & & & 157.5 & 70 & & & & \\
\hline \multirow{2}{*}{$12 / 17 / 74$} & \multirow{2}{*}{ SP } & \multirow{2}{*}{4037} & & & 35 & 282 & 57 & & $3=3$ & & \\
\hline & & & 40 & 12/J1/4 & D & 165 & 65 & 1213174 & 1V/A & 0.5 & Jo \\
\hline $1 / 2175$ & $\mathrm{SP}$ & 41463 & 18 & $1 / 2 / 75$ & $N / A^{3}$ & 180 & 58 & 12175 & $N / A^{3}$ & 36 & 57 \\
\hline $1 / 217$ & ST & $4140 . J$ & 40 & (17 & N/A & 96 & 62 & $1 / 21 / 3$ & 1V/A & 3.0 & 31 \\
\hline $2 / 12 / 75$ & CMD & 2709 & 47 & $2 / 8175$ & $N_{13}^{3}$ & 261.2 & 60 & $2 / 8 / 75$ & ${\mathrm{~N} / \mathrm{A}^{3}}^{3}$ & 36 & 57 \\
\hline (210 & Uivi & 217.0 & 41 & 21017 & & 168.0 & 68 & 21017 & 1V/A & 3.0 & (IT \\
\hline $7 / 23 / 75$ & GMP & 285.8 & 47 & $7 / 24 / 75$ & $\mathrm{~N} / \mathrm{A}^{3}$ & 270.7 & 60 & $7 / 24 / 75$ & $\mathrm{~N} / \mathrm{A}^{3}$ & 4.2 & 54 \\
\hline $12 / 30 / 75$ & GMP & 297.1 & 47 & $12 / 24 / 75$ & $\mathrm{~N} / \mathrm{A}^{3}$ & 265.8 & 60 & & & & \\
\hline $5 / 11 / 76$ & GMP & 281.0 & 45 & $3 / 8 / 76$ & $\mathrm{~N} / \mathrm{A}^{3}$ & 260.3 & 59 & $12 / 31 / 75$ & N/A & 4.2 & 53 \\
\hline $8 / 3 / 76$ & GMP & 324.5 & 45 & $7 / 23 / 76$ & $\mathrm{~N} / \mathrm{A}^{3}$ & 232.7 & 59 & & & & \\
\hline $11 / 9 / 76$ & GMP & 259.6 & 45 & & $3 y^{3}$ & & & & 1V/ & & \\
\hline $1 / 11 / 77$ & GMP & 265.3 & 45 & ס & $\mathrm{N} / \mathrm{A}$ & 225.5 & 39 & $1 / 20 / 77$ & $\mathrm{~N} / \mathrm{A}^{3}$ & 3.9 & 53 \\
\hline $5 / 17 / 77$ & GMP & 233.5 & 45 & & & & & & & & \\
\hline $8 / 23 / 77$ & GMP & 244.9 & 45 & $6 / 30 / / 1 /$ & N/A & 225.3 & 58 & $6 / 24 / 71$ & $\mathrm{~N} / \mathrm{A}^{-}$ & 3.1 & 54 \\
\hline $10 / 11 / 77$ & GMP & 339.5 & 45 & $10 / 13 / 77$ & $\mathrm{~N} / \mathrm{A}^{3}$ & 218.7 & 59 & $10 / 14 / 77$ & $\mathrm{~N} / \mathrm{A}^{3}$ & 4.3 & 54 \\
\hline $2 / 14 / 78$ & GMP & 357.3 & 45 & & 3 & $010-$ & & & & & \\
\hline $2 / 21 / 78$ & GMP & $84.5^{4}$ & 45 & (1/15// & $\mathrm{N} / \mathrm{A}$ & 212.5 & סJ & & & & \\
\hline $6 / 6 / 78$ & GMP & 100.6 & 45 & $5 / 5 / 78$ & $\mathrm{~N} / \mathrm{A}^{3}$ & 219.2 & 65 & $4 / 20 / 78$ & $\mathrm{~N} / \mathrm{A}^{3}$ & 4.0 & 52 \\
\hline $10 / 10 / 78$ & GMP & 114.6 & 45 & $10 / 5 / 78$ & $\mathrm{~N} / \mathrm{A}^{3}$ & 213.7 & 65 & $10 / 12 / 78$ & $\mathrm{~N} / \mathrm{A}^{3}$ & 4.3 & 51 \\
\hline $10 / 22 / 79$ & GMP & 109.9 & 45 & $10 / 31 / 79$ & $\mathrm{~N} / \mathrm{A}^{3}$ & 178.9 & 66 & $10 / 16 / 79$ & $\mathrm{~N} / \mathrm{A}^{3}$ & 3.2 & 53 \\
\hline $11 / 5 / 80$ & GMP & 86.2 & 45 & $11 / 12 / 80$ & $\mathrm{~N} / \mathrm{A}^{3}$ & 163.6 & 66 & $11 / 12 / 80$ & N/A ${ }^{3}$ & 2.8 & 56 \\
\hline $11 / 16 / 81$ & GMP & 111.1 & 46 & $12 / 29 / 81$ & $\mathrm{~N} / \mathrm{A}^{3}$ & 146.4 & 66 & $11 / 18 / 81$ & $\mathrm{~N} / \mathrm{A}^{3}$ & 2.9 & 61 \\
\hline $10 / 20 / 82$ & GMP & 116.0 & 47 & $10 / 19 / 82$ & $\mathrm{~N} / \mathrm{A}^{3}$ & 128.2 & 67 & $10 / 19 / 82$ & $\mathrm{~N} / \mathrm{A}^{3}$ & 2.8 & 66 \\
\hline $9 / 20 / 83$ & GMP & 115.4 & 47 & $9 / 11 / 83$ & $\mathrm{~N} / \mathrm{A}^{3}$ & 102 & 67 & $9 / 20 / 83$ & $\mathrm{~N} / \mathrm{A}^{3}$ & 2.5 & 57 \\
\hline $8 / 20 / 84$ & GMP & 120.5 & 47 & $8 / 22 / 84$ & $\mathrm{~N} / \mathrm{A}^{3}$ & 90 & 67 & $8 / 22 / 84$ & $\mathrm{~N} / \mathrm{A}^{3}$ & 2.9 & 67 \\
\hline $6 / 19 / 85$ & GMP & 114.2 & 48 & $6 / 18 / 85$ & $\mathrm{~N} / \mathrm{A}^{3}$ & 81 & 68 & $6 / 18 / 85$ & $N / A^{3}$ & 2.6 & 68 \\
\hline $6 / 11 / 86$ & GMP & 82.5 & 48 & $6 / 11 / 86$ & $\mathrm{~N} / \mathrm{A}^{3}$ & 48.6 & 68 & $6 / 11 / 86$ & $\mathrm{~N} / \mathrm{A}^{3}$ & 2.8 & 71 \\
\hline Note: & $\begin{array}{l}{ }^{1} \text { Refere } \\
{ }^{2} \text { Refer } \\
{ }^{3} \text { N/A: } \\
{ }^{4} \text { New } \\
{ }^{*} \text { Dryw }\end{array}$ & $\begin{array}{l}\text { ed from Int } \\
\text { ced from } M\end{array}$ & $\begin{array}{l}\text { al letter fro } \\
\text { thly Repor } \\
\text { le } \\
\text { ment } \\
\text { cated in th }\end{array}$ & $\begin{array}{l}\text { G.L. Bors } \\
\text { ay } 1973 \text { an }\end{array}$ & $\begin{array}{l}\mathrm{m} \text { to } \mathrm{L} . \\
\text { assumed }\end{array}$ & $\begin{array}{l}\text { Roddy, “ } \\
\text { o be drywel }\end{array}$ & $\begin{array}{l}\text { tegrity of } t \\
2-03-03\end{array}$ & $s 241-B Y-1$ & & $T Y-103 ” \mathrm{Jl}$ & \\
\hline
\end{tabular}


RPP-RPT-54911, Rev. 0

\section{APPENDIX B2}

TANK TY-103 GAMMA SURVEYS OF THE 2005 DIRECT PUSHES

B2-1 
RPP-RPT-54911, Rev. 0

Figure B2-1. 2005 Gamma Survey Direct Push Hole C4629 (RPP-RPT-34870)

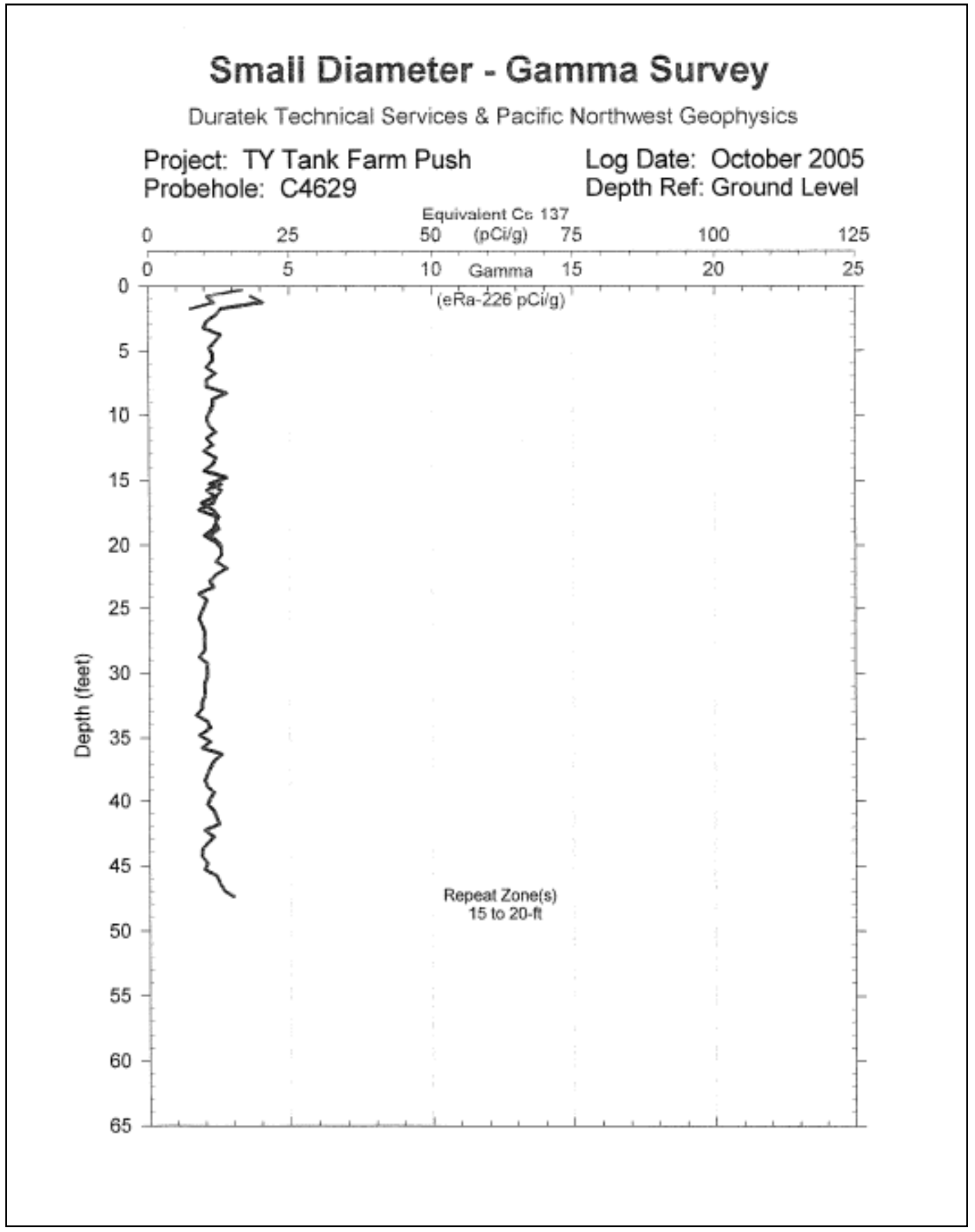


RPP-RPT-54911, Rev. 0

Figure B2-2. 2005 Gamma Survey Direct Push Hole C4631 (RPP-RPT-34870)

\section{Small Diameter - Gamma Survey}

Duratek Technical Services \& Pacific Northwest Geophysics

Project: TY Tank Farm Push

Log Date: October 2005

Probehole: C4631

Depth Ref: Ground Level

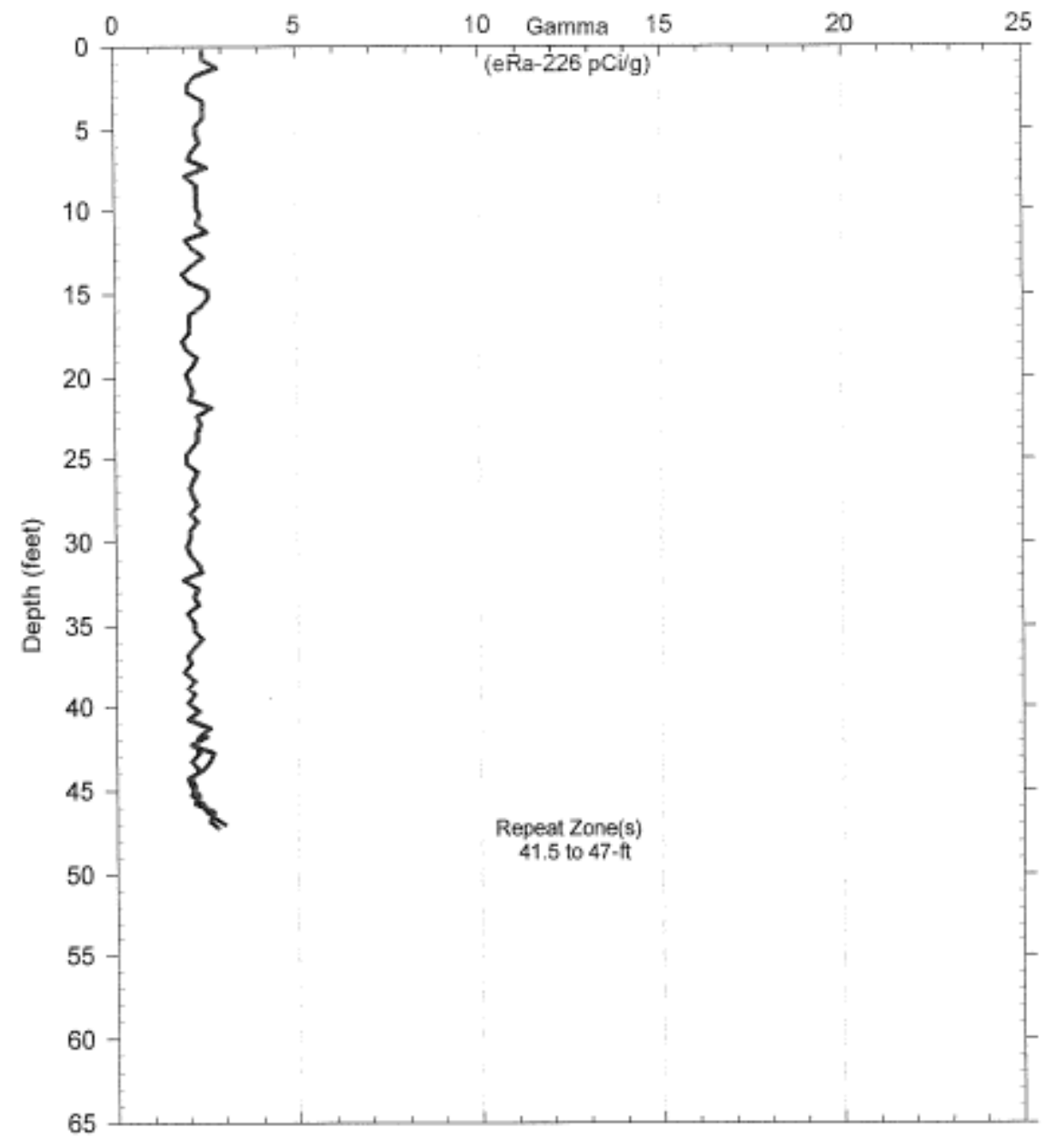


RPP-RPT-54911, Rev. 0

Figure B2-3. 2005 Gamma Survey Direct Push Hole C4633 (RPP-RPT-34870)

\section{Small Diameter - Gamma Survey \\ Duratek Technical Services \& Pacific Northwest Geophysics}

Project: TY Tank Farm Push

Log Date: October 2005

Probehole: C4633

Depth Ref: Ground Level

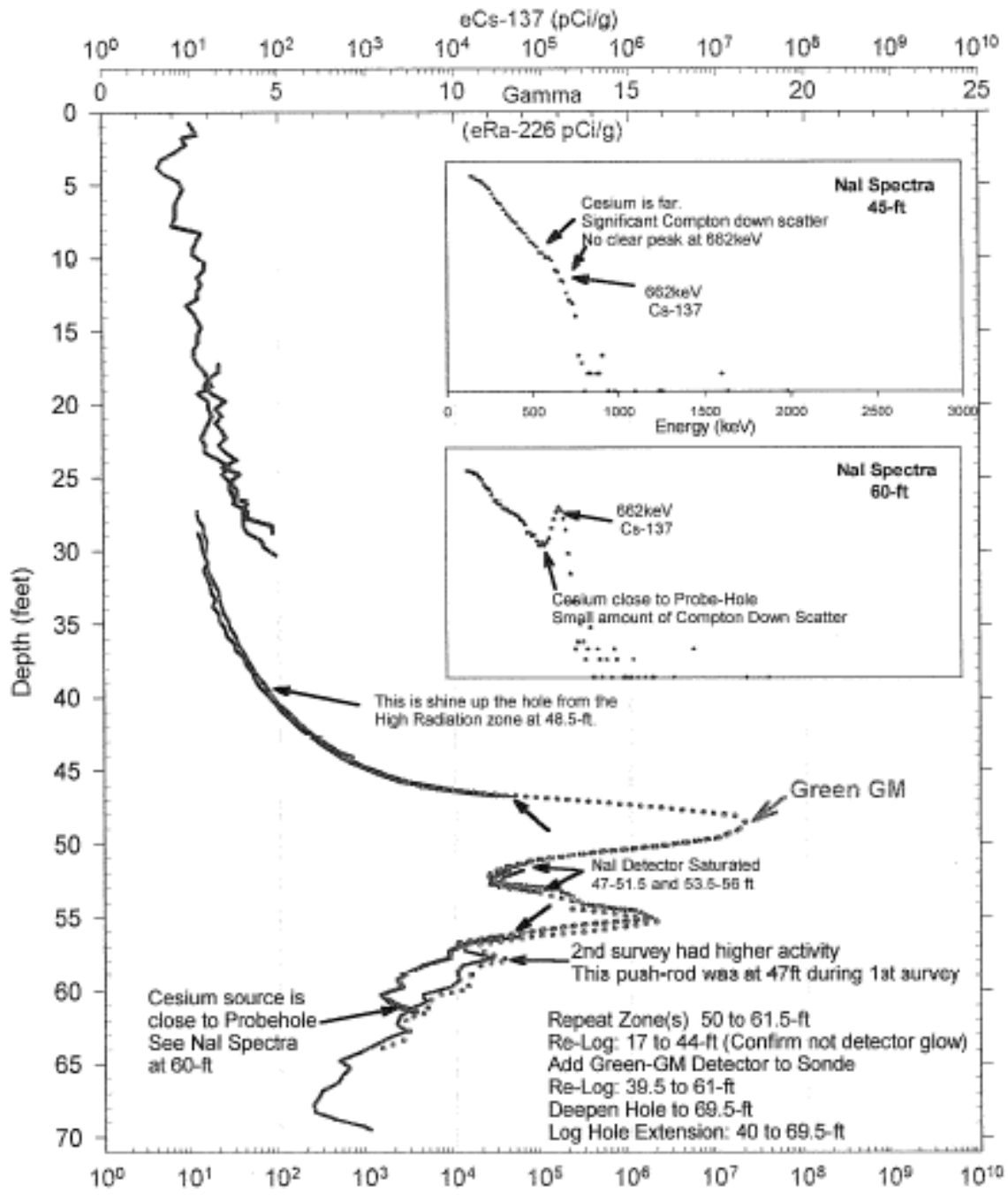


RPP-RPT-54911, Rev. 0

Figure B2-4. 2005 Gamma Survey Direct Push Hole C4635 (RPP-RPT-34870)

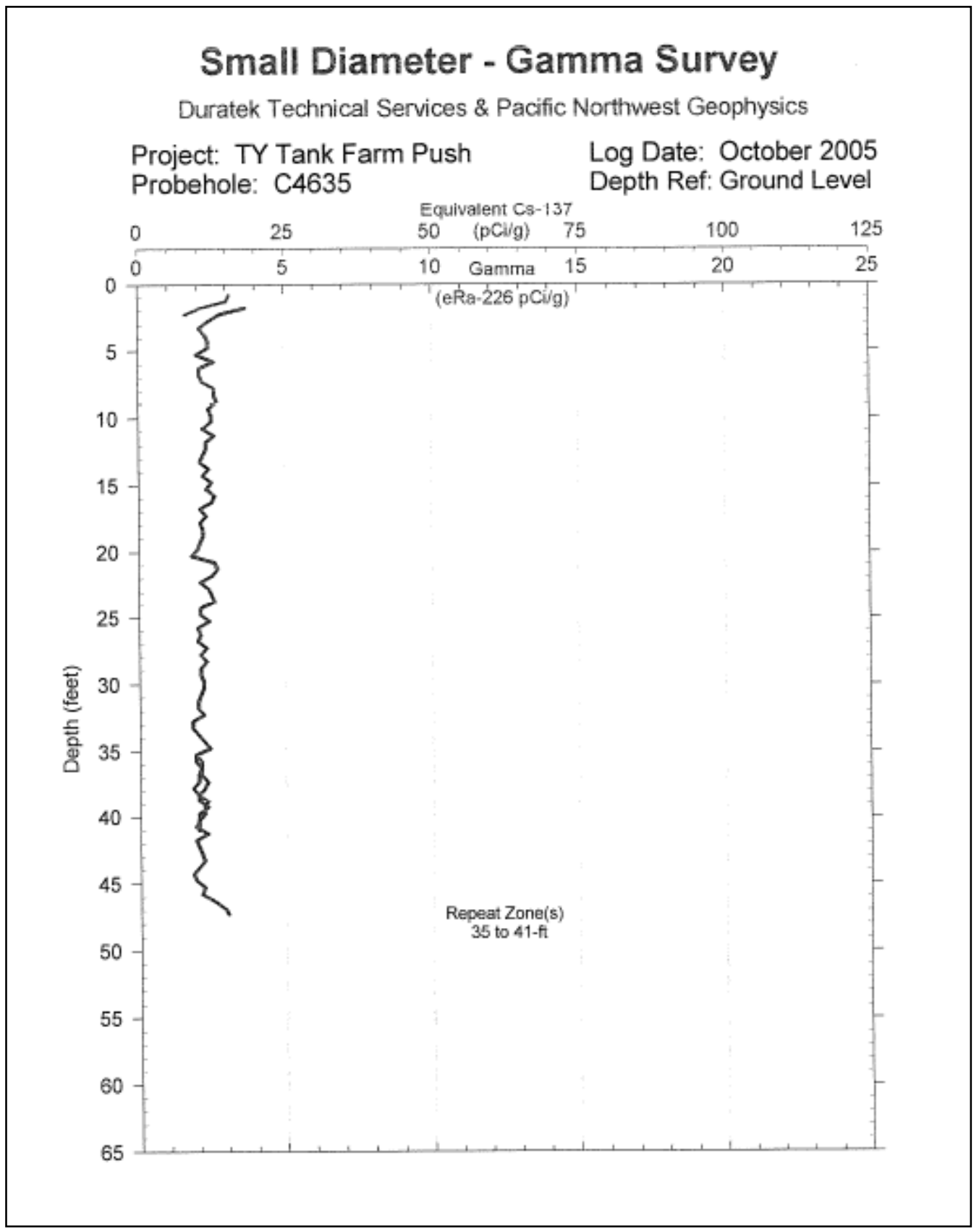


RPP-RPT-54911, Rev. 0

Figure B2-5. 2005 Gamma Survey Direct Push Hole C5008 (RPP-RPT-34870)

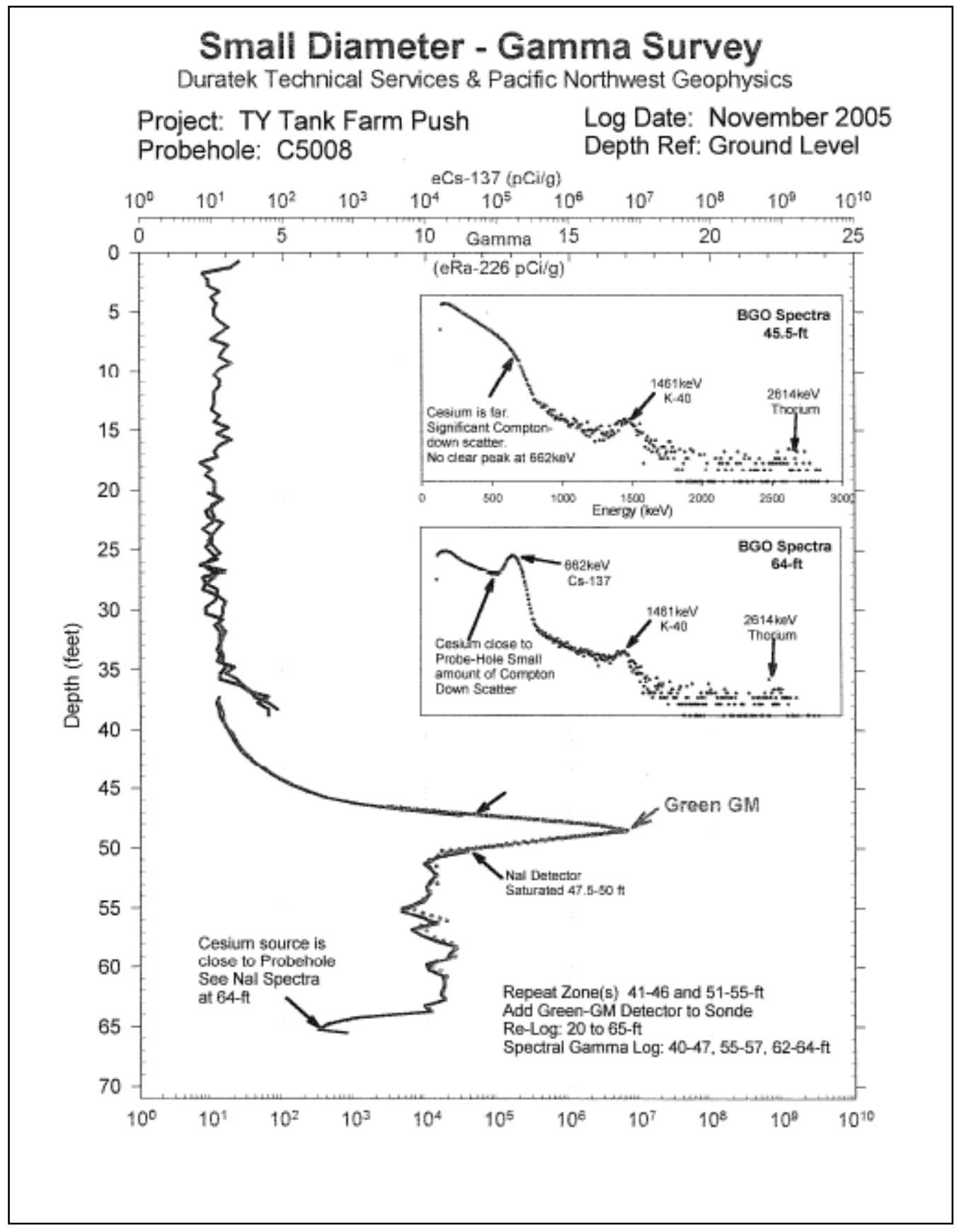


RPP-RPT-54911, Rev. 0

Figure B2-6. 2005 Gamma Survey Direct Push Hole C5010 (RPP-RPT-34870)

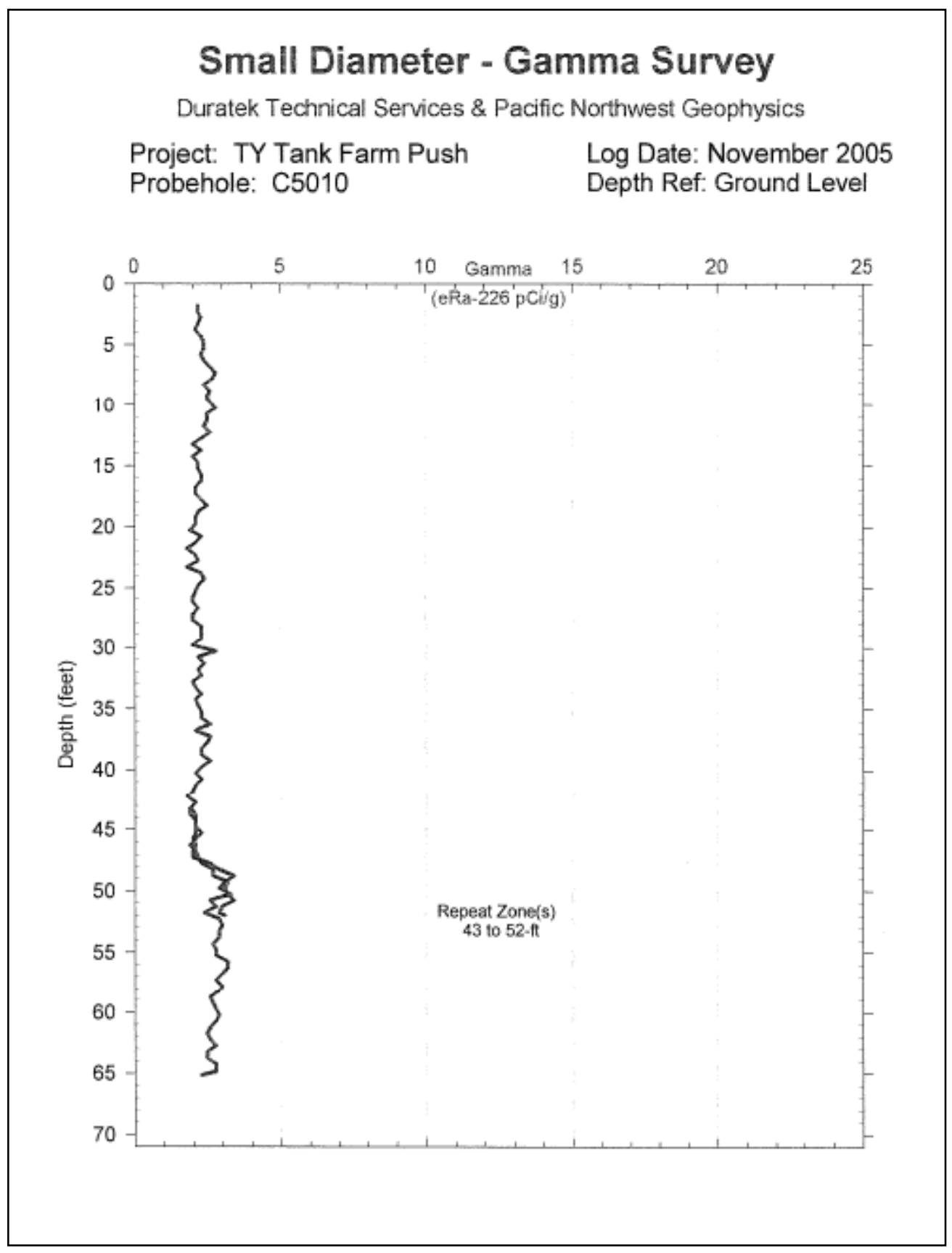


RPP-RPT-54911, Rev. 0

Figure B2-7. 2005 Gamma Survey Direct Push Hole C5012 (RPP-RPT-34870)

\section{Small Diameter - Gamma Survey}

Duratek Technical Services \& Pacific Northwest Geophysics

Project: TY Tank Farm Push

Log Date: November 2005

Probehole: C5012

Depth Ref: Ground Level

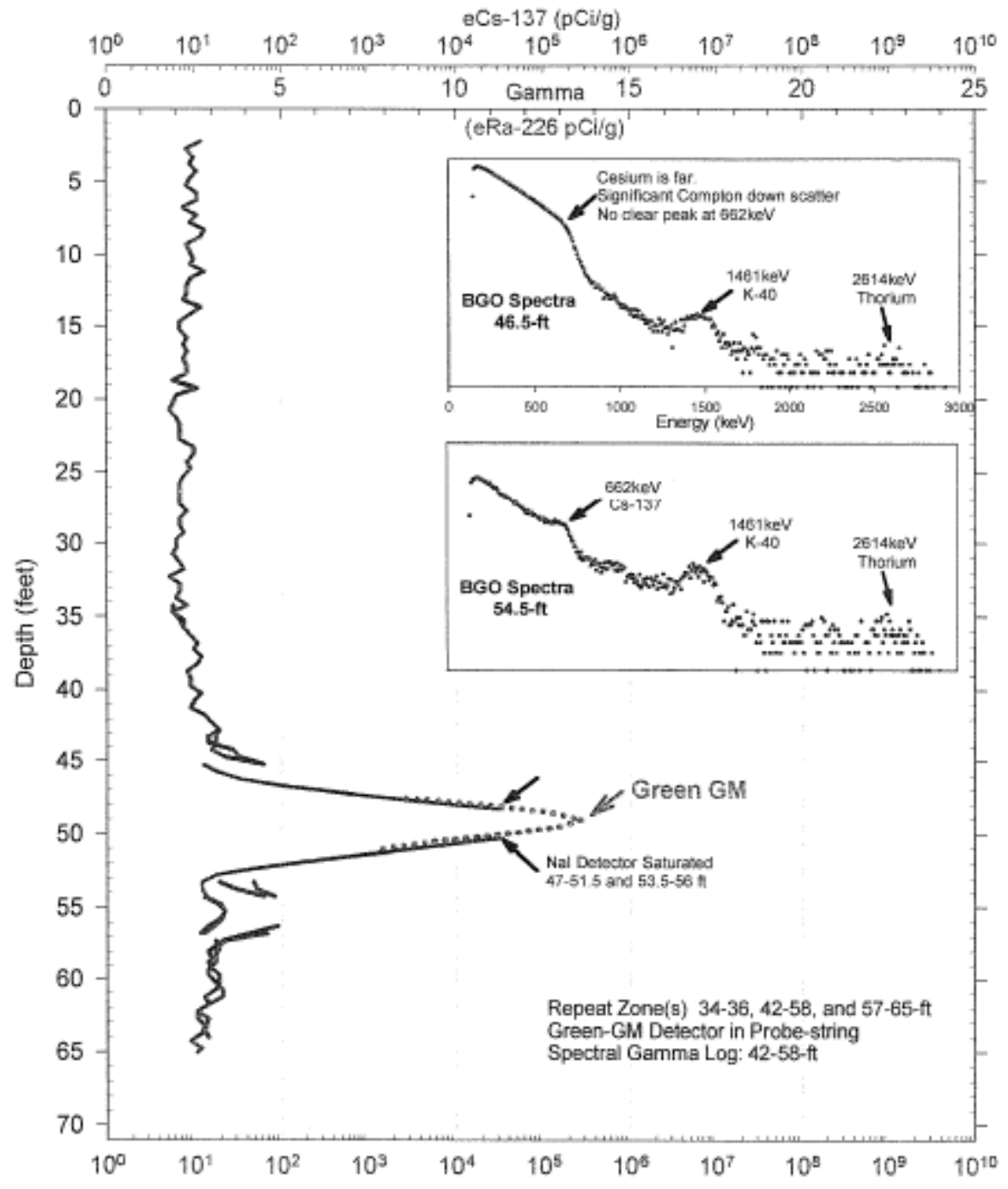




\subsection{TANK TY-104 SEGMENT}

\section{TABLE OF CONTENTS}

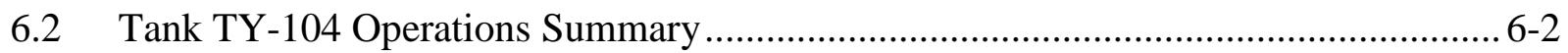

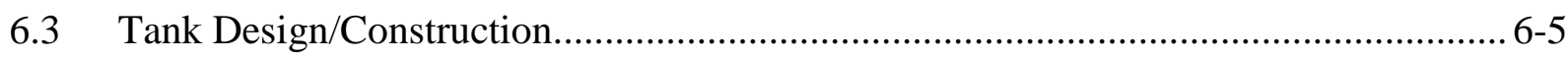

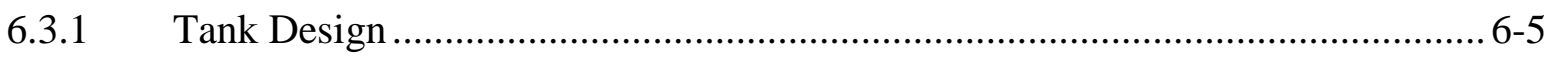

6.3.2 Tank Construction Conditions .................................................................... 6-5

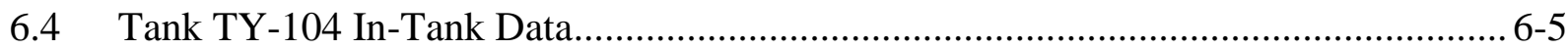

6.4.1 Liquid Level.................................................................................... 6-5

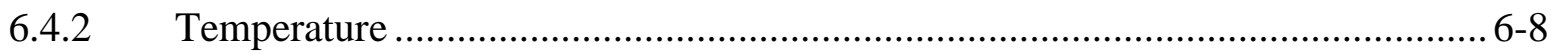

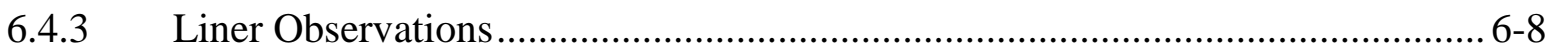

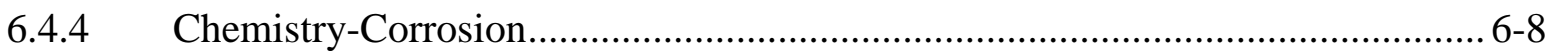

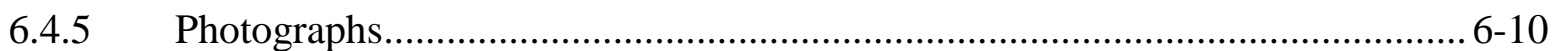

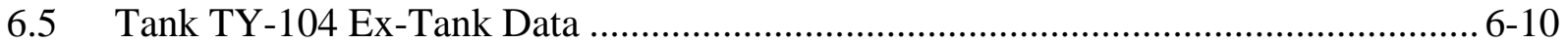

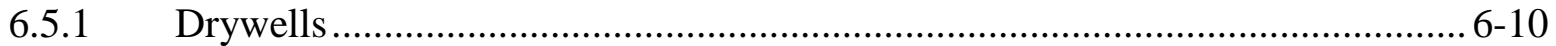

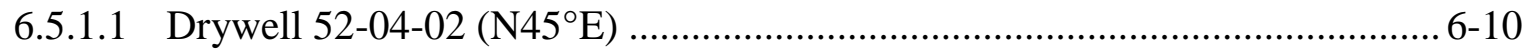

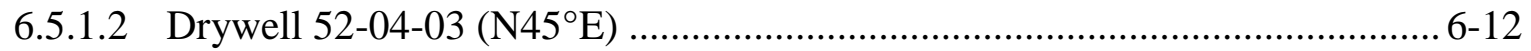

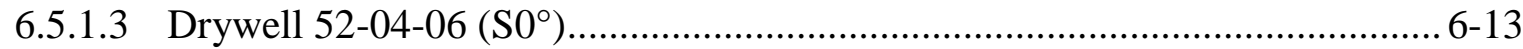

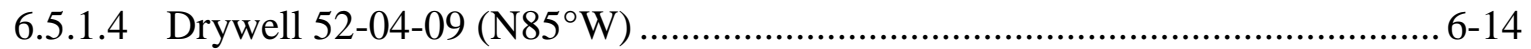

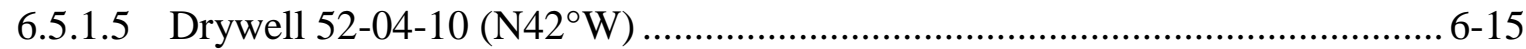

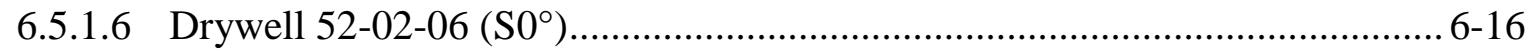

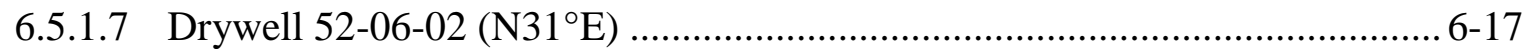

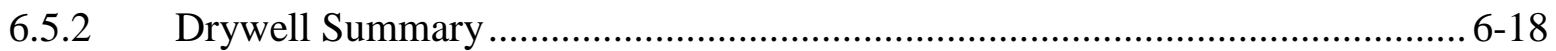

6.6 Cascade Tie Line ........................................................................................... 6-18

6.6.1 Pipeline Cathodic Protection.......................................................................... 6-21

6.7 Possible Tank TY-104 Leak Location(s) …………............................................... 6-22

6.8 Possible Tank TY-104 Liner Leak Cause(s) ……………....................................... 6-22

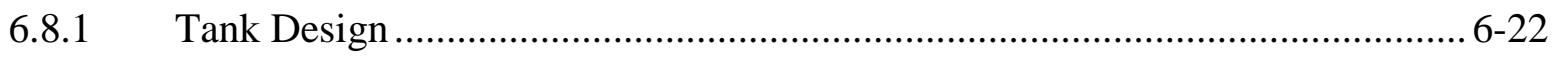

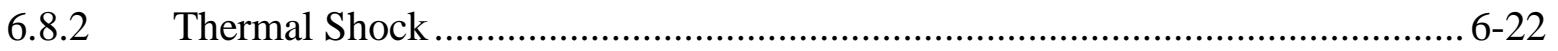

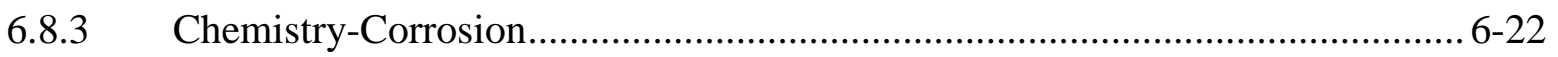

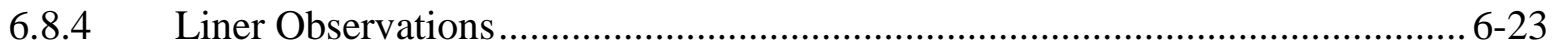

6.8.5 Tank Construction Temperatures.............................................................. 6-23 
RPP-RPT-54911, Rev. 0

6.9 Possible Tank TY-104 Cascade Tie Line Leak Cause(s)....................................... 6-23

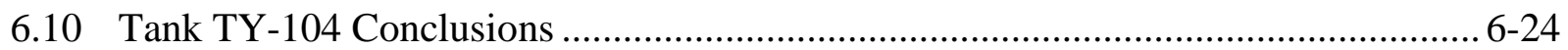

\section{APPENDICES}

Appendix A 3 Tank TY-104 Gross Gamma Drywell Data

A3-1

\section{LIST OF FIGURES}

Figure 6-1. Tank TY-104 Associated Drywells........................................................... 6-1

Figure 6-2. Operational Leak History of Tank TY-104.................................................. 6-4

Figure 6-3. Tank TY-104 End of Quarter Surface Level................................................ 6-6

Figure 6-4. Tank TY-104 Liquid Level June 13, 1973 to March 27, 1974 ........................... 6-7

Figure 6-5. Tank TY-104 Drywell 52-04-02 (HNF-3831) ............................................ 6-11

Figure 6-6. Tank TY-104 Drywell 52-04-03 (HNF-3831) ............................................. 6-12

Figure 6-7. Tank TY-104 Drywell 52-04-06 (HNF-3831) .............................................. 6-13

Figure 6-8. Tank TY-104 Drywell 52-04-09 (HNF-3831) ................................................ 6-14

Figure 6-9. Tank TY-104 Drywell 52-04-10 (HNF-3831) ............................................ 6-15

Figure 6-10. Tank TY-104 Associated Drywell 52-02-06 (HNF-3831) ............................. 6-16

Figure 6-11. Tank TY-104 Associated Drywell 52-06-02 (HNF-3831) ............................. 6-17

Figure 6-12. Tank TY-104 Associated Drywells and Cascade Lines................................... 6-19

Figure 6-13. Tank TY-104 Outlet Cascade Tie Line Details............................................ 6-20

Figure 6-14. BX Farm Cascade Tie Line .................................................................... 6-21

\section{LIST OF TABLES}

Table 6-1. Tank TY-104 Waste Storage Chronology ........................................................ 6-8

Table 6-2. Waste Chemistries for Waste Types Stored in Tank TY-104 .............................. 6-9

Table 6-3. Tank TY-104 Supernatant Sample \#T-3069 May 6, 1974 ................................. 6-10 


\section{RPP-RPT-54911, Rev. 0}

Table A3-1. Tank TY-104 Drywell Radioactivity (K counts per minute). A3-2 


\subsection{TANK TY-104 BACKGROUND HISTORY}

This section provides information on the historical waste loss event associated with SST 241TY-104 (TY-104). There are seven drywells located around tank TY-104 with specified distances from the drywell to the tank footing shown in Figure 6-1: 52-04-03 and 52-04-10 installed in 1952; 52-04-02, 52-04-06, 52-04-09, and 52-06-02 installed in December 1971; 5202-06 installed in 1977.

The bottom of the tank footing is $\sim 46-\mathrm{ft}$ Below Grade Surface (BGS) with $\sim 6$-ft 10-in soil cover over the dome (WHC-SD-WM-TI-665, Soil Load above Hanford Waste Storage Tanks; H-22244, 75 Foot Composite Storage Tank Sections).

Figure 6-1. Tank TY-104 Associated Drywells

Tank inner ring is steel liner, outer ring is outer edge of tank footing

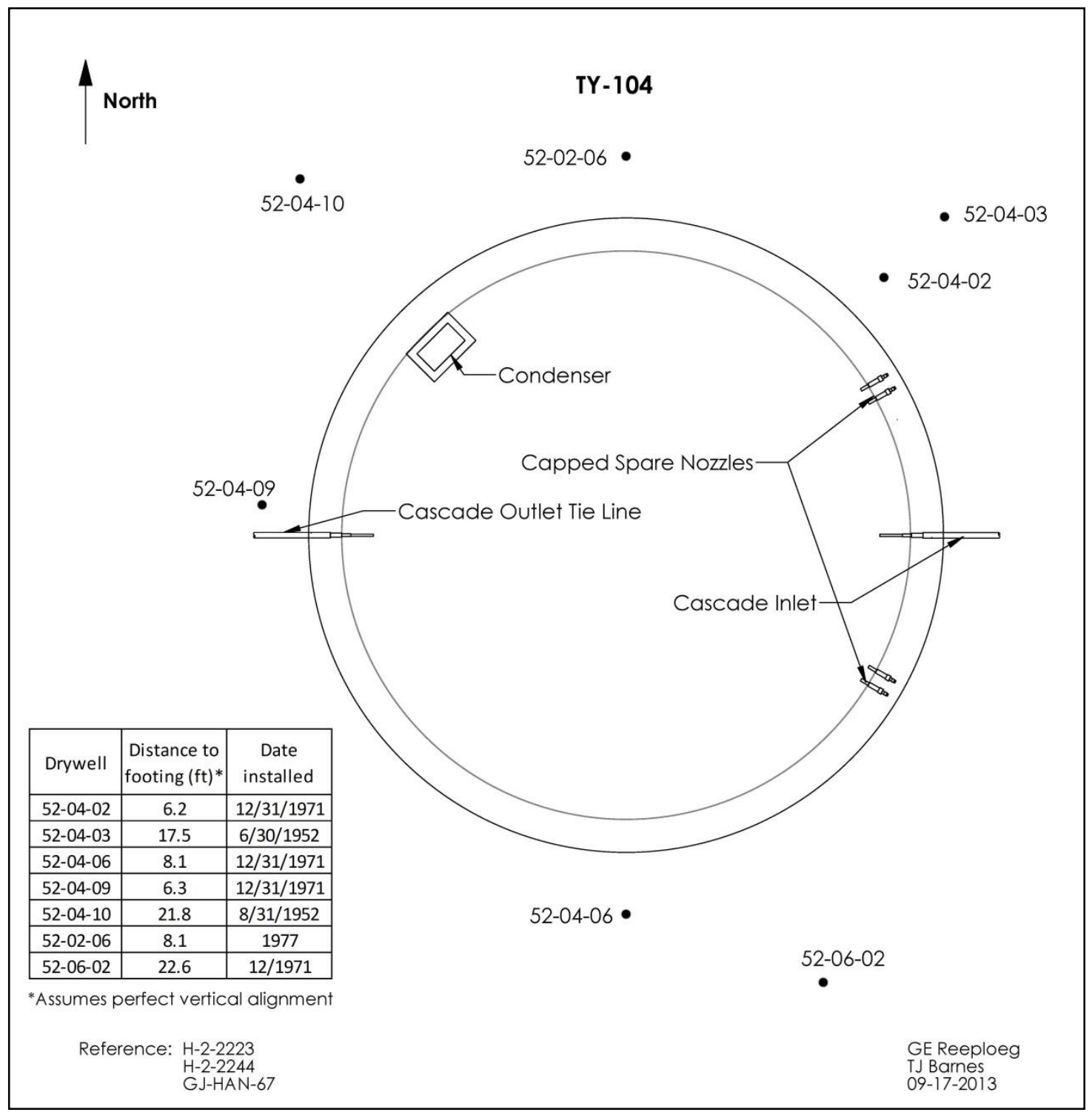


RPP-RPT-54911, Rev. 0

\subsection{TANK TY-104 OPERATIONS SUMMARY}

Tank TY-104 was constructed from 1951 to 1952 and started receiving Tri-Butyl Phosphate (TBP) waste from the cascade of tank TY-103 in August 1953 to November 1953 (HW-29242, Separations Section Waste - Status Summary August 31, 1953). By the end of November 1953, tank TY-104 contained $\sim 758$ kgal of TBP waste (HW-29624, Separations Section Waste - Status Summary September 30, 1953). Tank TY-104 was used as a staging and distributing tank for TBP waste from August 1953 to December 1969. Beginning in December 1953, 617 kgal of TBP waste from tank TY-104 was transferred into tank TX-118 leaving 141 kgal of TBP waste in tank TY-104 (HW-30498, Separations Section Waste - Status Summary December 31, 1953). No further waste transactions occurred until March 1954.

Beginning in March to May 1954, tank TY-104 received 629 kgal of TBP waste from tank TY103 with the volume recorded at $770 \mathrm{kgal}$, which was filled above normal capacity ( $\sim$-ft) (HW32110, Separations Section Waste - Status Summary May 31, 1954). A capped cascade outlet tie line located on the west side of tank TY-104 at a waste height of 272-in which would have been filled during this period (see Section 6.6). No further waste transactions occurred until March 1955 when $709 \mathrm{kgal}$ was transferred from tank TY-104 to tank TX-109 (LA-UR-97-311, Waste Status and Transaction Record Summary (WSTRS Rev. 4)). Approximately $700 \mathrm{kgal}$ of first cycle waste (1C) treated in 221-T Plant with FeCN, nickel sulfate, and sodium hydroxide, waste type 1C-FeCN (see Section 5.2), and TBP waste from tank TY-103 was transferred to tank TY104 in March 1955 (HW-36001, Separations Section Waste - Status Summary for March 1955). In August 1955, $675 \mathrm{kgal}$ of waste was transferred out of tank TY-104 leaving the waste volume at 83 kgal (HW-38926, Separations Section Waste - Status Summary for August 1955). No further waste transactions occurred from September 1955 until January 1957 when water was periodically added to tank TY-104 (HW-47640, Chemical Processing Department WasteStatus Summary December 1, 1956 -December 31, 1956; HW-48144, Chemical Processing Department Waste - Status Summary January 1, 1957 -January 31, 1957). No further transfers occurred from February 1957 through July 1959.

Tank TY-104 began receiving TBP, 1C, and decontamination waste (DW) from tank TX-118 in August 1959 to June 1966 with the volume reported at $736 \mathrm{kgal}$ at the end of June 1966 (HW83906 E RD, Chemical Processing Department 200 West Area Tank Farm Inventory and Waste Reports, July 1, 1961 through September 1966).

From July 1966 to March 1967, approximately 674 kgal of waste was transferred out of tank TY104 to tank TX-118 leaving the volume at $62 \mathrm{kgal}$ at the end of March 1967 (ISO-806, Chemical Processing Division Waste Status Summary January 1, 1967 Through March 31, 1967). Additional TBP and DW were added from tank TX-115 to tank TY-104 in April 1967 to March 1968 (ISO-967, Chemical Processing Division Waste Status Summary April 1, 1967, Through June 30, 1967; ARH-534, Chemical Processing Division Waste Status Summary, January 1, 1968 Through March 31, 1968). The volume in tank TY-104 was reported at $714 \mathrm{kgal}$ at the end of December 1969 (ARH-1200 D, Chemical Processing Division Waste Status Summary, October 1, 1969 Through December 31, 1969). From January to March 1970, 367 kgal of waste was transferred from tank TY-104 to tank TX-118 and $\sim 333 \mathrm{kgal}$ of waste from tank TY103 was transferred to tank TY-104 (ARH-1666 A, Chemical Processing Division Waste Status Summary, January 1, 1970 Through March 31, 1970). An additional 498 kgal was transferred 
out of tank TY-104 leaving the volume in tank TY-104 at $182 \mathrm{kgal}$ by September 1970 (ARH1666 C, Chemical Processing Division Waste Status Summary, July 1, 1970 Through September 30, 1970). From October 1970 to December 1970, $542 \mathrm{kgal}$ of B Plant low level waste, PUREX OWW, and REDOX waste from tank TY-103 was transferred into tank TY-104 (ARH-1666 D, Chemical Processing Division Waste Status Summary, October 1, 1970 Through December 31, 1970).

No further waste transactions occurred from December 1970 to December 1973. At the end of December 1973, tank TY-104 contained $730 \mathrm{kgal}$ of waste (ARH-2794 D, Manufacturing and Waste Management Division Waste Status Summary October 1, 1973 Through December 31, 1973). Tank TY-104 was first suspected of leaking between December 30, 1973 and March 16, 1974 when a 0.6-in liquid level decrease was observed (RHO-CD-1193, Review of Classification of Hanford Single-Shell Tanks 110-B, 111-C, 103-T, 107-TX, 104-TY, and 106-U). Tank TY104 was pumped to a minimum heel (approximately 26-in) when $678 \mathrm{kgal}$ of supernatant was pumped to tank S-110 March 16-25, 1974 and the tank was removed from service. From March to October 1974, a salt well system was installed (P-10 type deep well turbine pump) and pumping started to tank TY-102 reducing the heel to 22-in by October 1974 (RHO-CD-1193).

Tank TY-104 was administratively interim stabilized on November 1983 with a supernatant pool $\sim 45$-ft in diameter in the center of the tank with an estimated volume of $3 \mathrm{kgal}$ (RPP-RPT42296). Tank TY-104 is estimated to contain $43 \mathrm{kgal}$ of sludge, $5 \mathrm{kgal}$ of drainable interstitial liquid, and $1 \mathrm{kgal}$ of supernatant (HNF-EP-0182, Rev. 301, Waste Tank Summary Report for Month Ending April 30, 2013).

The operational history of tank TY-104 leak related details including liquid level is charted in Figure 6-2. 


\section{Figure 6-2. Operational Leak History of Tank TY-104}

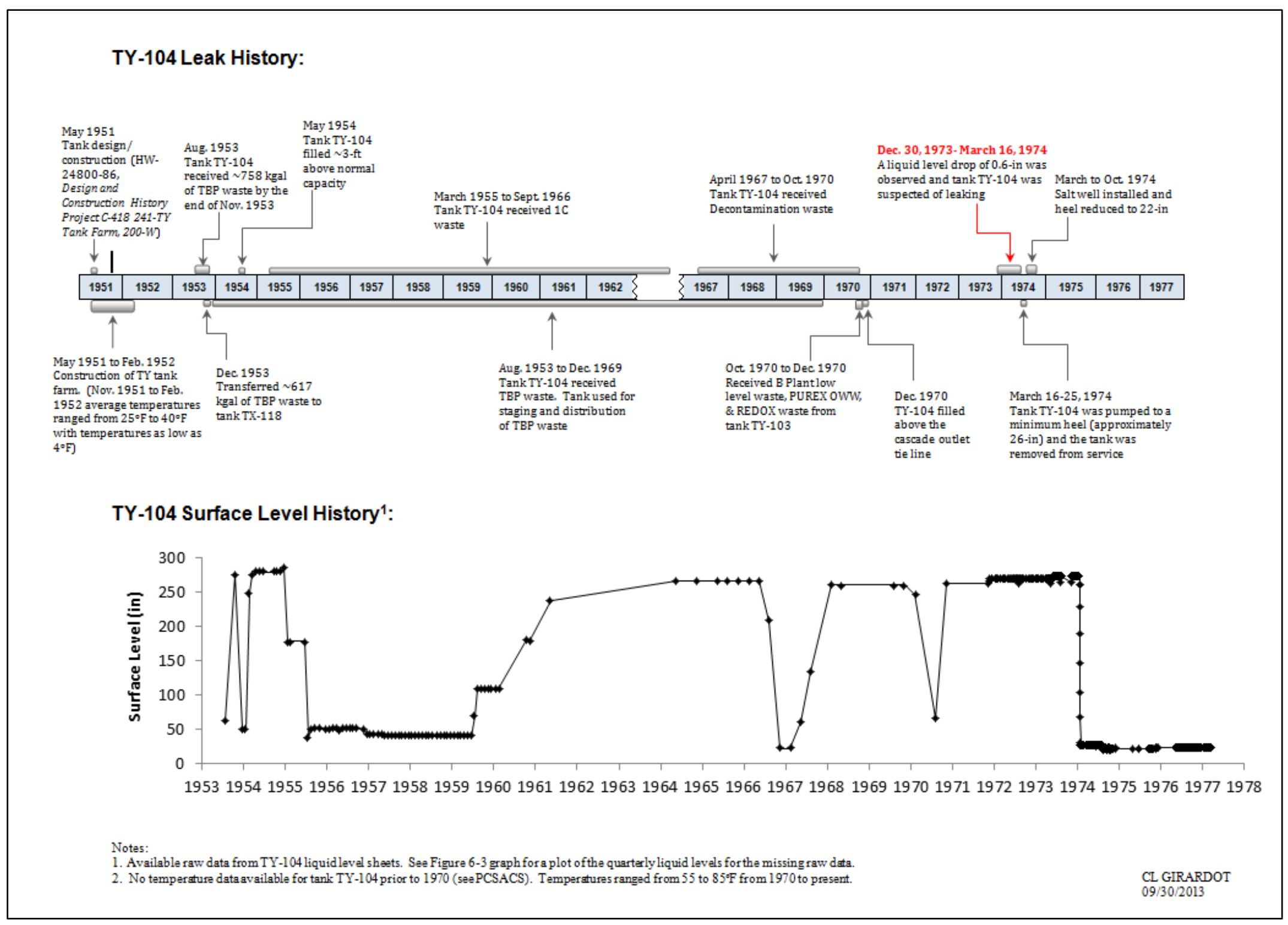


RPP-RPT-54911, Rev. 0

\subsection{TANK DESIGN/CONSTRUCTION}

\subsubsection{Tank Design}

The TY Farm tank design continued important features of the earlier 241-BCTU tanks (BPF73550). The steel bottom intersects the sidewall on a 4-ft radius. Full penetration butt welds with $\mathrm{x}$-ray inspection and three ply asphaltic membrane waterproofing between the wall liner and the concrete shell continued those design features found to be important for liner integrity during the SX Farm leak assessment (RPP-RPT-54910). The top of the tank footing is 43 - $\mathrm{ft}$ BGS and is 3 - $\mathrm{ft}$ thick with the bottom of the footing at $\sim 46-\mathrm{ft}$ BGS.

\subsubsection{Tank Construction Conditions}

The TY Farm was constructed from May 1951 to February 1952. Cold weather could have affected the steel during construction in December and January. Temperatures for December 1951 and January 1952 averaged $27.4^{\circ} \mathrm{F}$ and $25.2^{\circ} \mathrm{F}$, respectively, with temperatures as low as $4^{\circ} \mathrm{F}$ (PNNL-15160). Weather precautions for welding and concrete were specified in HW-4696 (see Section 3.1.2).

As described in Section 3.1.2, cold weather affects the ductile-to-brittle steel transition temperature, with $18^{\circ} \mathrm{F}$ being the assumed unrestricted low temperature construction limitation for the carbon steel liner, which could result in a fracture upon impact. Low temperatures experienced during construction at or less than the $18^{\circ} \mathrm{F}$ allowable temperature could cause impact loading (e.g. a dropped tool or piece of equipment from scaffolding) and result in the potential for creating micro-fissures weakening the steel liner.

\subsection{TANK TY-104 IN-TANK DATA}

\subsubsection{Liquid Level}

The liquid level plot in Figure 6-3 indicates the transfer activity into and out of tank TY-104. The liquid levels are end of quarter levels so this figure may not reflect all transfers into and out of the tank that occurred during the operational history of the tank. See Figure 6-2 for historical monthly liquid level readings.

Tank TY-104 was first suspected of leaking based on a liquid level decrease of 0.6-in between December 30, 1973 and March 16, 1974 (RHO-CD-1193) as shown in Figure 6-4. It was reported in Letter 72730-80-097 that the liquid level in tank TY-104 before and during the observed decrease was very consistent with no anomalies with the exception of the increase in liquid level in August 1973 that was attributed to instrument (FIC gauge) recalibration (Letter 72730-80-097, "Review of Classification of Six Hanford Single-Shell "Questionable Integrity (QI)" Tanks"). Also stated in this letter, the observed 0.6-in liquid level drop "far exceeds the intank repeatability limits $( \pm 0.25$-in) for FIC liquid level gauges." 
Figure 6-3. Tank TY-104 End of Quarter Surface Level

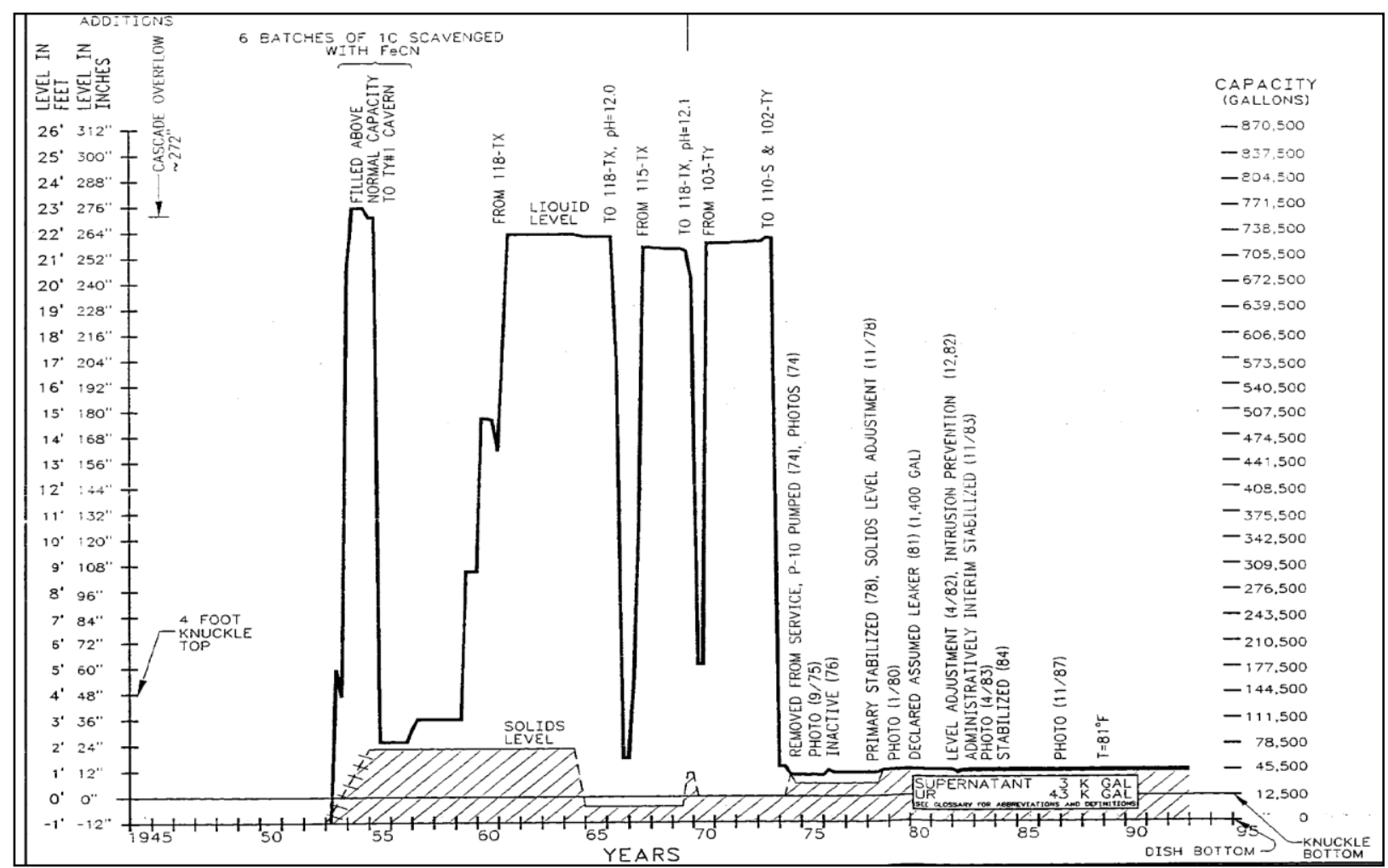

WHC-SD-WM-ER-351, Rev 0, March 1, 1995, Historical Tank Content Estimate for the Northwest Quadrant of the Hanford 200 West Area

Liquid level data for tank TY-104 is unavailable from the last transfer into the tank (December 1970) to June 1, 1973. After the August 1973 recalibration of the FIC gauge, liquid levels were holding at approximately 273.1-in (see Figure 6-4) until December 30, 1973. If this recalibration was correct, then the last transfer into tank TY-104 would have filled the tank above the cascade outlet tie line (located at 272-in) (H-2-2244). The liquid level dropped 0.6-in from December 30, 1973 to March 16, 1974 ( 1-gal/hr) and liquid levels were reported to be 272.5-in on March 16, 1974 (RHO-CD-1193; SD-WM-TI-356). Due to this liquid level drop, tank TY-104 was removed from service and pumped to a minimum heel to tank S-110 from March 16-25, 1974 leaving the liquid level at 26-in. A salt well pump was installed in tank TY-104 and the heel was pumped to tank TY-102 by October 1974 leaving a heel of 22-in. The leak volume from tank TY-104 was estimated to be 1,400 gal based on a 0.5-in liquid level decrease (RHO-CD-1193). 
Figure 6-4. Tank TY-104 Liquid Level June 13, 1973 to March 27, 1974

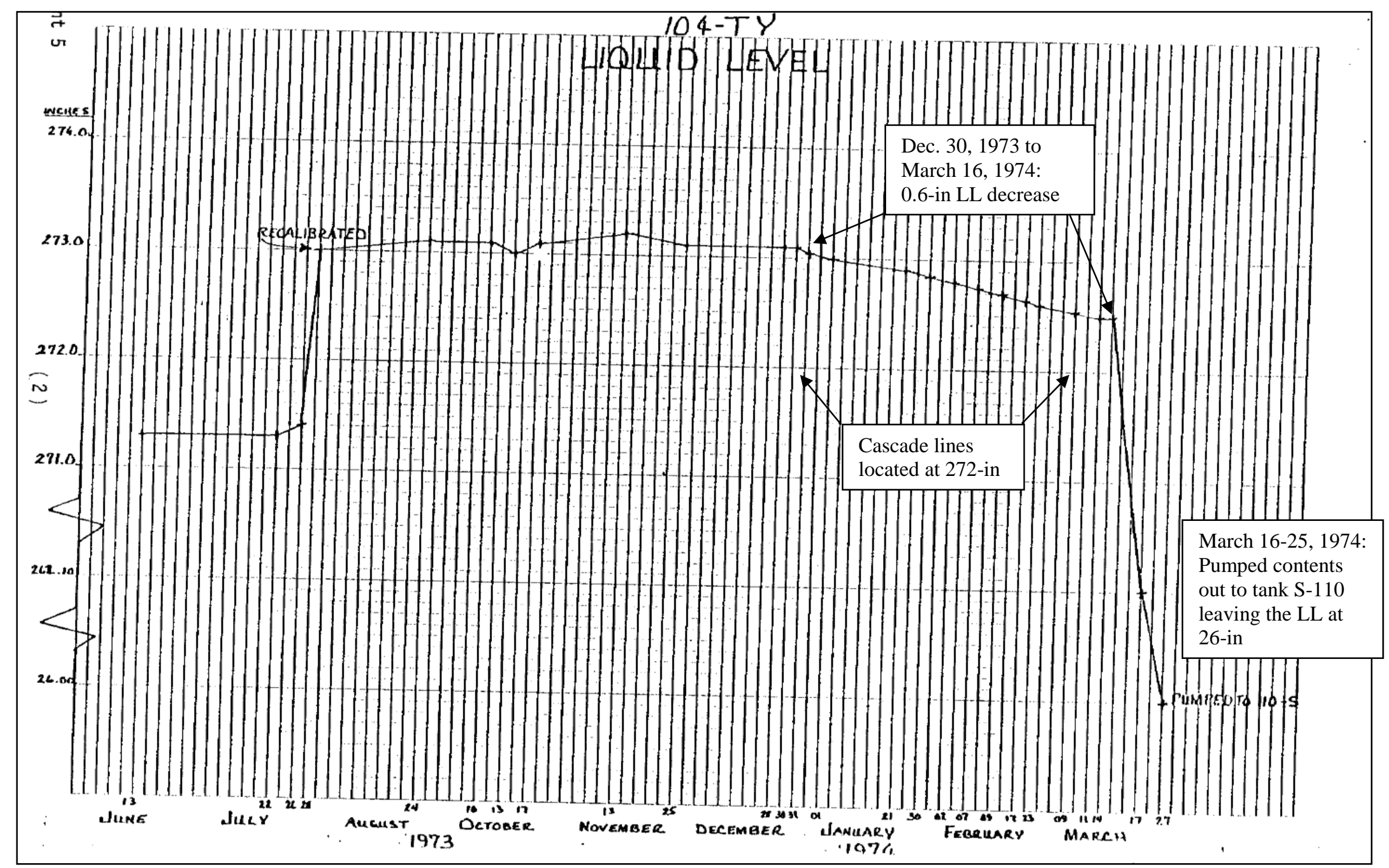

Referenced from Letter 72730-80-097, "Review of Classification of Six Hanford Single-Shell “Questionable Integrity (QI)” Tanks, September 16, 1980 


\subsubsection{Temperature}

No temperature data were recovered for tank TY-104 from August 1953 when the tank was first put into service until 1970. Temperatures ranged from $55^{\circ} \mathrm{F}$ to $85^{\circ} \mathrm{F}$ from 1970 to the present (see PCSACS).

Tank TY-104 received TBP waste from tank TY-103 beginning in August 1953. TBP wastes were concentrated and cooled to $\sim 180^{\circ} \mathrm{F}$ within the plant and were estimated to be $110-180^{\circ} \mathrm{F}$ after routing to the storage tanks. Thus, the TBP waste would likely be a lower temperature as this waste was cascaded from tank TY-103. Rate of rise temperatures during initial tank filling are unknown.

\subsubsection{Liner Observations}

No liner observations relating to a tank TY-104 leak have been found.

\subsubsection{Chemistry-Corrosion}

Tank TY-104 began receiving waste in August 1953 and received various waste types throughout operation as shown in Table 6-1. The typical concentrations for nitrite, nitrate, and hydroxide for these waste types are shown in Table 6-2. Nitrite and hydroxide are known as nitrate-induced SCC inhibitors. One key characteristic for inhibiting SCC is to maintain a high nitrite concentration to nitrate concentration ratio (see Section 3.2.4).

Table 6-1. Tank TY-104 Waste Storage Chronology

\begin{tabular}{|c|c|c|}
\hline Date & Waste Type & Length of Storage \\
\hline August 1953 to March 1955 & TBP & $\sim 1.5$ years \\
\hline March 1955 to July 1959 & 1C-FeCN/TBP ${ }^{1}$ & $\sim 4$ years \\
\hline August 1959 to September 1970 & TBP/1C/DW & $\sim 14$ years \\
\hline October 1970 to March 1974 & BL/OWW/R & $\sim 3.5$ years \\
\hline
\end{tabular}

1. Approximately $700 \mathrm{kgal}$ of $1 \mathrm{C}-\mathrm{FeCN}$ was added on top of a $61 \mathrm{kgal}$ TBP heel 
RPP-RPT-54911, Rev. 0

Table 6-2. Waste Chemistries for Waste Types Stored in Tank TY-104

\begin{tabular}{|c|c|c|c|c|}
\hline Waste Type & {$\left[\mathbf{N O}_{3}{ }^{-}\right]$} & {$\left[\mathbf{N O}_{2}{ }^{-}\right]$} & {$\left[\mathbf{O H}^{-}\right]$} & $\begin{array}{c}\text { Meets Current } \\
\text { DST Specification }\end{array}$ \\
\hline $1 C^{1}$ & 1.54 & 0.26 & 0.28 & $\mathrm{No}^{3}$ \\
\hline $\mathrm{TBP}^{1}$ & 7.35 & Not reported & 0.09 & $\mathrm{No}^{4}$ \\
\hline $\mathrm{R}^{5}$ & 4.83 & \multicolumn{2}{|c|}{0.74} & $\mathrm{No}^{6}$ \\
\hline $\mathrm{OWW}^{1}$ & 0.06 & Not reported & Not reported & Yes $^{7}$ \\
\hline $\mathrm{BL}^{1}$ & 5.28 & Not reported & Not reported & Not available \\
\hline
\end{tabular}

1. Reference WHC-EP-0449, 1991, The Sort on Radioactive Waste Type Model: A Method to Sort Single-Shell Tanks into Characteristic Groups.

2. Reference OSD-T-151-00007, Rev. 12, (2013), Operating Specifications for the Double-Shell Storage Tanks.

3. Waste type 1C does not meet the current DST specification for waste chemistry; however, 1C was treated with FeCN, nickel sulfate, and $\mathrm{NaOH}$ to scavenge Cs-137 and Sr-90 prior to being added to tank TY-104. Depending on these ratios, the resulting waste may have met the DST specification.

4. To be within the current DST specification limit, [NO3-] $<5.5 \mathrm{M}$

5. Reference WHC-EP-0772, 1994, Characterization of the Corrosion Behavior of the Carbon Steel Liner in Hanford Site Single-Shell Tanks.

6. Does not meet the current DST specification since the hydroxide and nitrite concentrations are not greater than or equal to $1.2 \mathrm{M}$.

7. According to the assumption from reference WHC-EP-0772, Characterization of the Corrosion Behavior of the Carbon Steel Liner in Hanford Site Single-Shell Tanks.

The first waste tank TY-104 received in August 1953 was TBP waste cascaded from tank TY103 and the tank continued to store only TBP waste for approximately 1.5 years. Tank TY-104 also stored TBP waste ( 61 kgal) from March 1955 to July 1959 which was diluted with 700 kgal of 1C-FeCN waste and from August 1959 to September 1970 which was diluted with unknown ratios of waste types $1 \mathrm{C}$ and DW. Thus, TBP waste of varying concentrations was stored for a total of approximately 19.5 years in tank TY-104. Samples of TBP waste indicate hydroxide concentrations below $0.1 \mathrm{M}$ and nitrate concentrations above $6 \mathrm{M}$. The high nitrate concentration violates the current DST specification for waste chemistry as nitrate must be below $5.5 \mathrm{M}$. These conditions of the TBP waste would likely create an environment conducive to SCC and/or pitting (see Section 3.2.4).

Tank TY-104 stored 1C waste treated in 221-T Plant with FeCN, nickel sulfate, and sodium hydroxide for approximately four years, which was cascaded from tank TY-103. Waste type 1C does not meet the current DST specification for waste chemistry; however, after being treated in 221-T Plant the resulting waste may have met the DST specification depending on the ratios of hydroxide mixed with 1C. Tank TY-104 also stored REDOX HLW from tank TY-103 from October 1970 through March 1974 which does not meet the current DST specification for waste chemistry. However, during this time the tank also stored various waste types which would likely dilute the REDOX HLW. Thus, it can be assumed that $1 \mathrm{C}$ and REDOX HLW had little effect on waste chemistry during this time.

The other waste types stored in tank TY-104 should not have resulted in pitting or SCC. However, information for waste type BL is not available and it remains uncertain whether this waste type would increase the propensity for corrosion. 
Tank TY-104 was first suspected of leaking due to a 0.6-in liquid level drop between the end of December 1973 and March 1974, and supernatant was pumped out of the tank at the end of March 1974. A memo dated May 6, 1974 (Internal memo MEM-010274 “Analysis of Tank Farm Samples 01/02/74 thru 12/26/74") shows analysis results for tank TY-104 samples (\#T3069) that are likely representative of supernatant in the tank at the time of the leak (see Table 6-3). This sample result indicates the supernatant during this time did not violate the current DST specifications for waste chemistry.

Table 6-3. Tank TY-104 Supernatant Sample \#T-3069 May 6, 1974

\begin{tabular}{|c|c|c|c|c|c|}
\hline Source & Layer & {$\left[\mathbf{O H}^{-}\right] \mathbf{M}$} & {$\left[\mathbf{N O}_{2}^{-}\right] \mathbf{M}$} & {$\left[\mathbf{N O}_{3}^{-}\right] \mathbf{M}$} & {$\left[\mathbf{N O}_{2}^{-}\right] /\left[\mathbf{N O}_{3}{ }^{-}\right]$} \\
\hline TY-104 & Supernatant & 0.468 & 0.384 & 1.62 & 0.24 \\
\hline
\end{tabular}

\subsubsection{Photographs}

Earliest photographs taken April 15 and 16, 1974 of tank TY-104 were reviewed and no evidence of bulging was found. No other documentation was found indicating a liner bulge in tank TY-104.

\subsection{TANK TY-104 EX-TANK DATA}

\subsubsection{Drywells}

There are seven drywells located around tank TY-104: 52-04-03 and 52-04-10 installed in 1952; 52-04-02, 52-04-06, 52-04-09, and 52-06-02 installed in December 1971; 52-02-06 installed in 1977. All of the radiation readings in drywells are assumed to be maximum or peak readings unless otherwise noted (see Sections 3.3 and 3.4). The following subsections report the available drywell information and the drywell summary section provides the analyses of the associated drywells with tank TY-104.

\subsubsection{Drywell 52-04-02 $\left(\mathrm{N}^{\circ} 5^{\circ} \mathrm{E}\right)$}

Drywell 52-04-02 was drilled in December 1971 with the first recoverable reading on July 11, 1973 as less than $12 \mathrm{~K}$ cpm with the scintillation probe (SP). Radiation readings were reported as less than values from July 1973 to June 1986 (see Appendix A3). Document HNF-3831 states that no significant levels of radioactivity are present about the survey probe detection threshold between 1975 and 1994 in this drywell.

In May 1997, Cs-137 was the only man-made radionuclide detected in drywell 52-04-02 (GJHAN-67). Cs-137 was detected intermittently between 7.5 and 24-ft, at 89-ft, 91.5-ft, and at 97.5-ft BGS with concentrations less than $0.2 \mathrm{pCi} / \mathrm{g}$ below 1-ft BGS. Document GJ-HAN-67 states, "The Cs-137 contamination in this borehole is probably the result of surface spills that have migrated down into the backfill surrounding the borehole or surface contamination that adhered to the drill string and was carried downward during the drilling of this borehole." Since historical records report little radioactivity in this drywell, drywell 52-04-02 is not included as part of the leak location for tank TY-104. Figure 6-5 shows the depths of radioactivity from 1975 to 1995 (HNF-3831). 
RPP-RPT-54911, Rev. 0

Figure 6-5. Tank TY-104 Drywell 52-04-02 (HNF-3831)

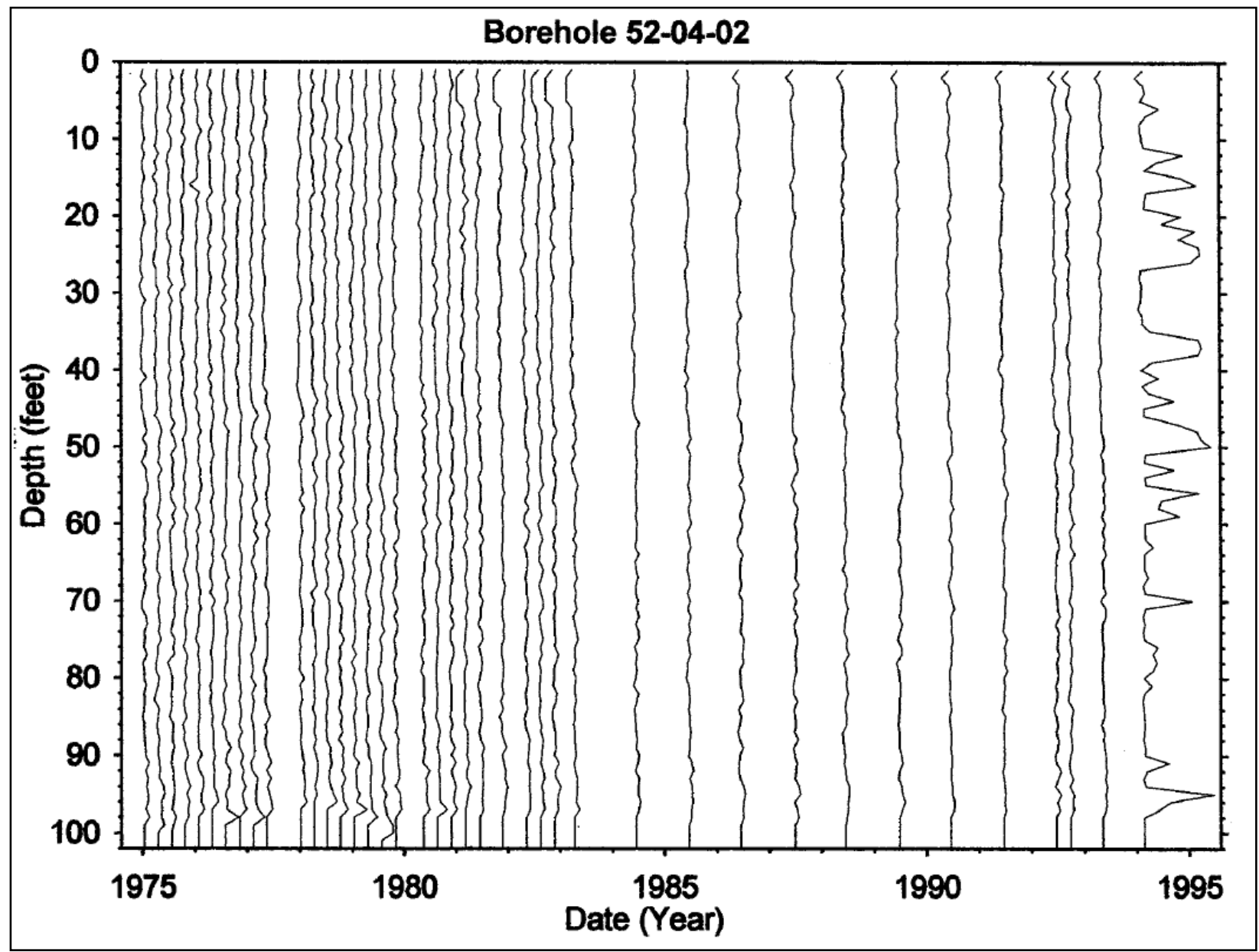

Note: Bottom of the tank footing is $\sim 46$-ft BGS 


\subsubsection{Drywell 52-04-03 $\left(\mathrm{N}^{\circ} 5^{\circ} \mathrm{E}\right)$}

Drywell 52-04-03 was drilled in June 1952 with the first recoverable reading on July 11, 1973 as less than $12 \mathrm{~K}$ cpm with the SP. Radiation readings were reported as less than values from July 1973 to June 1986 (see Appendix A3). Document HNF-3831 states that Cs-137 is only present at the surface at 0 to 8 -ft BGS.

In May 1997, Cs-137 and Eu-154 were the only man-made radionuclides detected in drywell 5204-03 (GJ-HAN-67). Cs-137 was detected from the ground surface to 5.5-ft BGS with concentrations as high as $2,200 \mathrm{pCi} / \mathrm{g}$. Cs-137 was also detected from 16.5 to 18.5 -ft BGS with concentrations up to $1 \mathrm{pCi} / \mathrm{g}$, and at $45-\mathrm{ft}, 51-\mathrm{ft}, 55.5-\mathrm{ft}, 65.5-\mathrm{ft}$, between 80.5 and $81-\mathrm{ft}$, at $92-\mathrm{ft}$, between 98 and 98.5-ft, and at 108-ft BGS at concentrations ranging from 0.2 to $0.5 \mathrm{pCi} / \mathrm{g}$. Eu154 was detected from 2 to 5-ft BGS with a maximum concentration of about $30 \mathrm{pCi} / \mathrm{g}$. Document GJ-HAN-67 states that Cs-137 and Eu-154 contamination are probably related to a transfer line located on the northeast side of the tank. Figure 6-6 shows surface contamination from 1975 to 1995 with no radioactivity detected below the surface (HNF-3831). Surface contamination appeared to not be a concern as radiation readings were reported as less than values (see Appendix A3). Therefore, drywell 52-04-03 is not included as part of the leak location for tank TY-104.

Figure 6-6. Tank TY-104 Drywell 52-04-03 (HNF-3831)

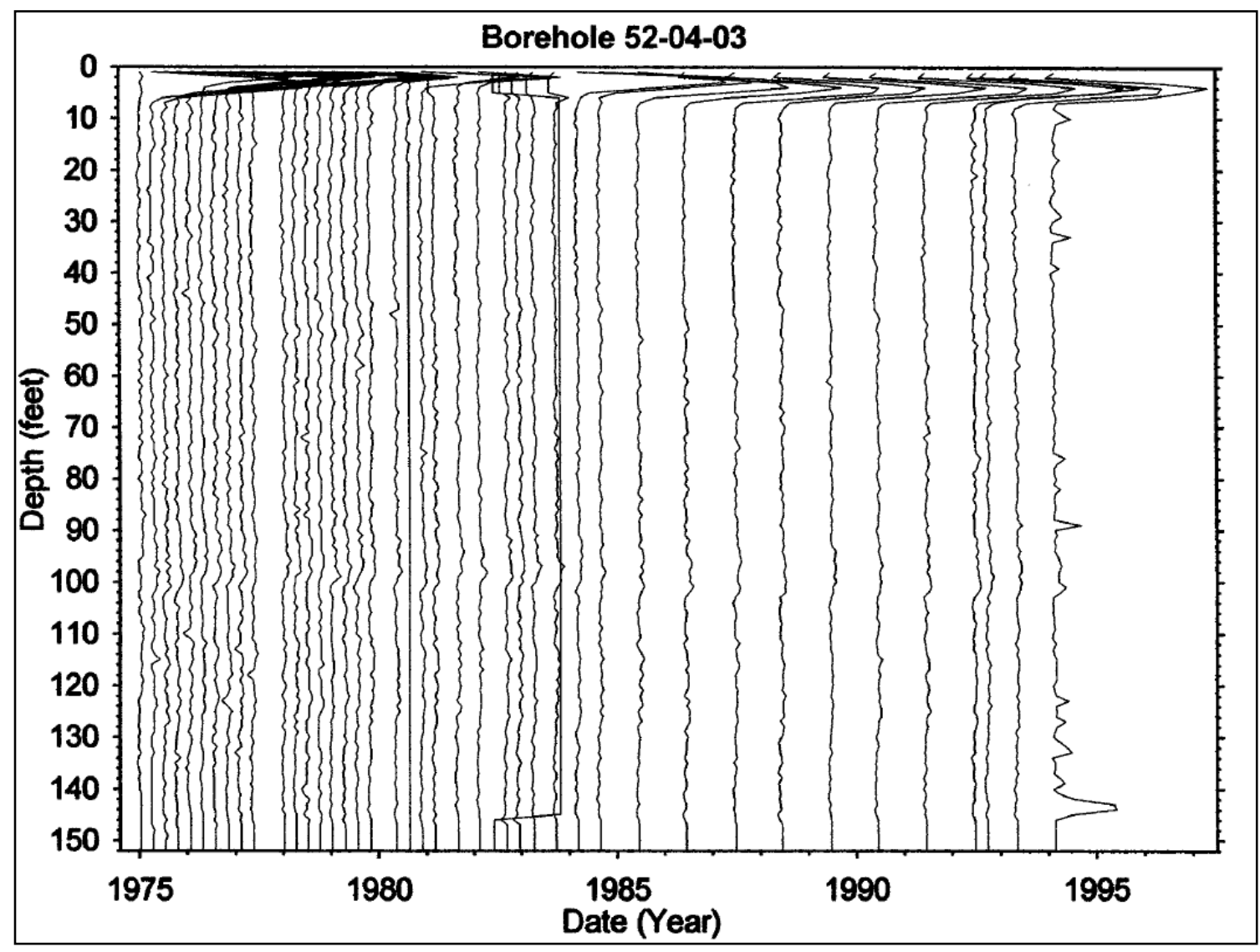

Note: Bottom of the tank footing is 46- $\mathrm{ft}$ BGS 


\subsubsection{Drywell 52-04-06 $\left(\mathrm{S0}^{\circ}\right)$}

Drywell 52-04-06 was drilled in December 1971 with the first recoverable reading on July 11, 1973 as less than $12 \mathrm{~K}$ cpm with the SP. Radiation readings were reported as less than values from July 1973 to June 1986 (see Appendix A3). Document HNF-3831 states that no significant levels of radioactivity are present about the survey probe detection threshold between 1975 and 1994 in this drywell. However, it was also stated in this document that Cs-137 was detected at the surface at 0 to $12-\mathrm{ft}$ BGS and concentrations were less than $1 \mathrm{pCi} / \mathrm{g}$.

In May 1997, Cs-137 was the only radionuclide detected in drywell 52-04-06 (GJ-HAN-67). Cs137 was detected continuously from the ground surface to $12-\mathrm{ft}$ BGS and at the bottom of the drywell with concentrations ranging from 0.2 to $1 \mathrm{pCi} / \mathrm{g}$ at the ground surface and at $0.2 \mathrm{pCi} / \mathrm{g}$ at the bottom of the drywell. Document GJ-HAN-67 states, "There is no indication of subsurface contamination at or below the operating level of the tanks." Therefore, drywell 52-04-06 is not included as part of the leak location for tank TY-104. Figure 6-7 shows depths of radioactivity from 1975 to 1995 (HNF-3831).

Figure 6-7. Tank TY-104 Drywell 52-04-06 (HNF-3831)

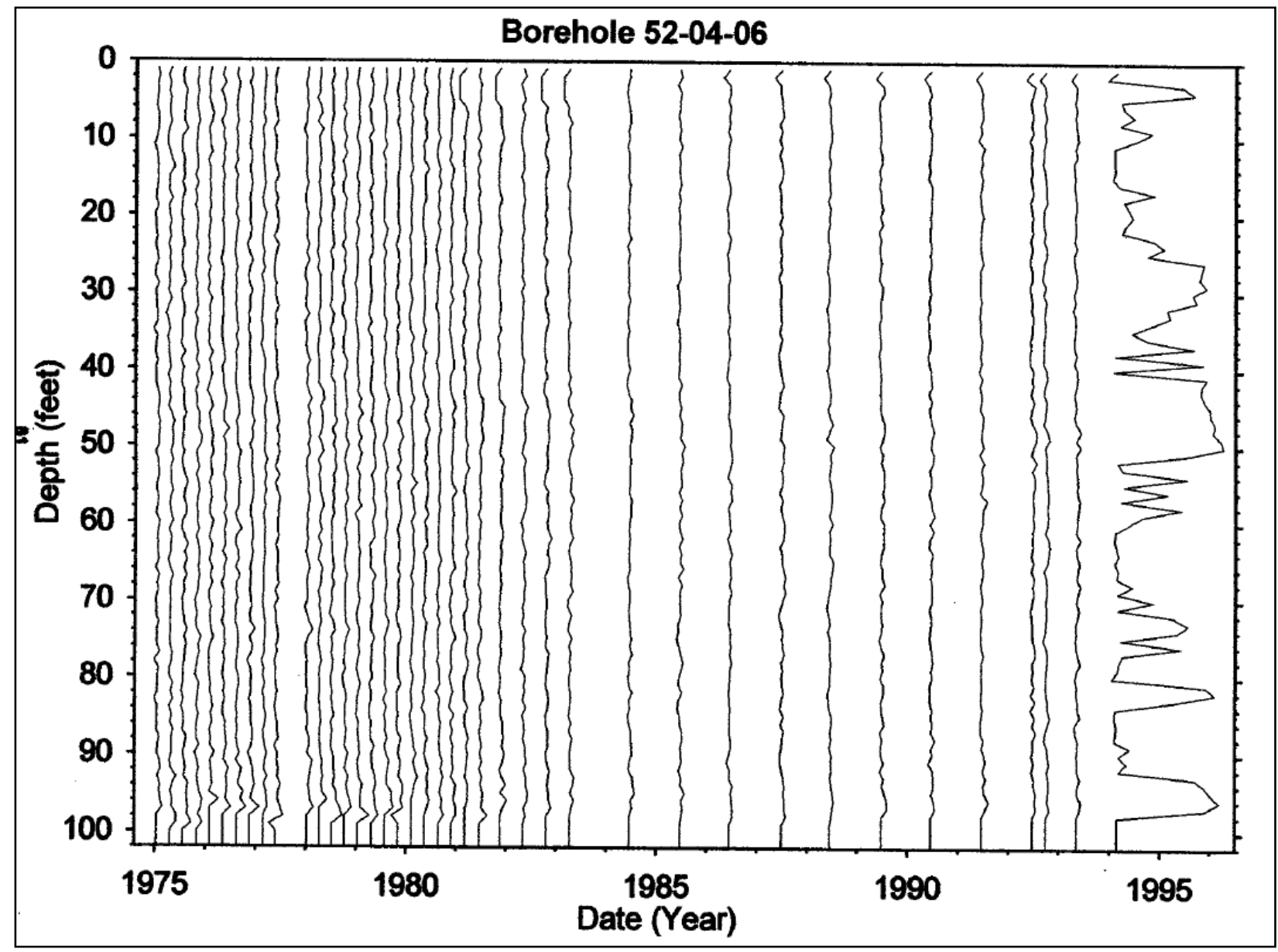

Note: Bottom of the tank footing is $\sim 46-\mathrm{ft}$ BGS 


\subsubsection{Drywell 52-04-09 $\left(\mathrm{N85}^{\circ} \mathrm{W}\right)$}

Drywell 52-04-09 was drilled in December 1971 with the first recoverable reading on July 11, 1973 as less than $12 \mathrm{~K}$ cpm with the SP. Radiation readings were reported as less than values from July 1973 to June 1986 (see Appendix A3). Document HNF-3831 states that low levels of Cs-137 (less than $1 \mathrm{pCi} / \mathrm{g}$ ) are stable from 85 to $100-\mathrm{ft}$ BGS.

In May 1997, Cs-137 was the only man-made radionuclide detected in drywell 52-04-09 (GJHAN-67). Measurable Cs- 137 was detected at the ground surface (about $0.2 \mathrm{pCi} / \mathrm{g}$ ) and from 83.5-ft BGS to the bottom of the drywell (at or below $1 \mathrm{pCi} / \mathrm{g}$ ). Document GJ-HAN-67 states, "The Cs-137 contamination detected by the SGLS between the depths of $83.5 \mathrm{ft}$ and the bottom of the borehole probably originated from a subsurface source such as a leak from one of the tanks within this tank farm, but probably not from tank TY-104. A contaminant plume may have migrated downward from the source and intercepted borehole 52-04-09 at about the 84-ft depth." The conclusion was stated "The low-level contamination in boreholes 52-06-02 and 52-04-09 is related to surface spills or subsurface leaks, but the contamination is not associated with the operation of tank TY-104." Therefore, drywell 52-04-09 is not included as part of the leak location for tank TY-104. Figure 6-8 shows depths of radioactivity from 1975 to 1995 (HNF$3831)$.

Figure 6-8. Tank TY-104 Drywell 52-04-09 (HNF-3831)

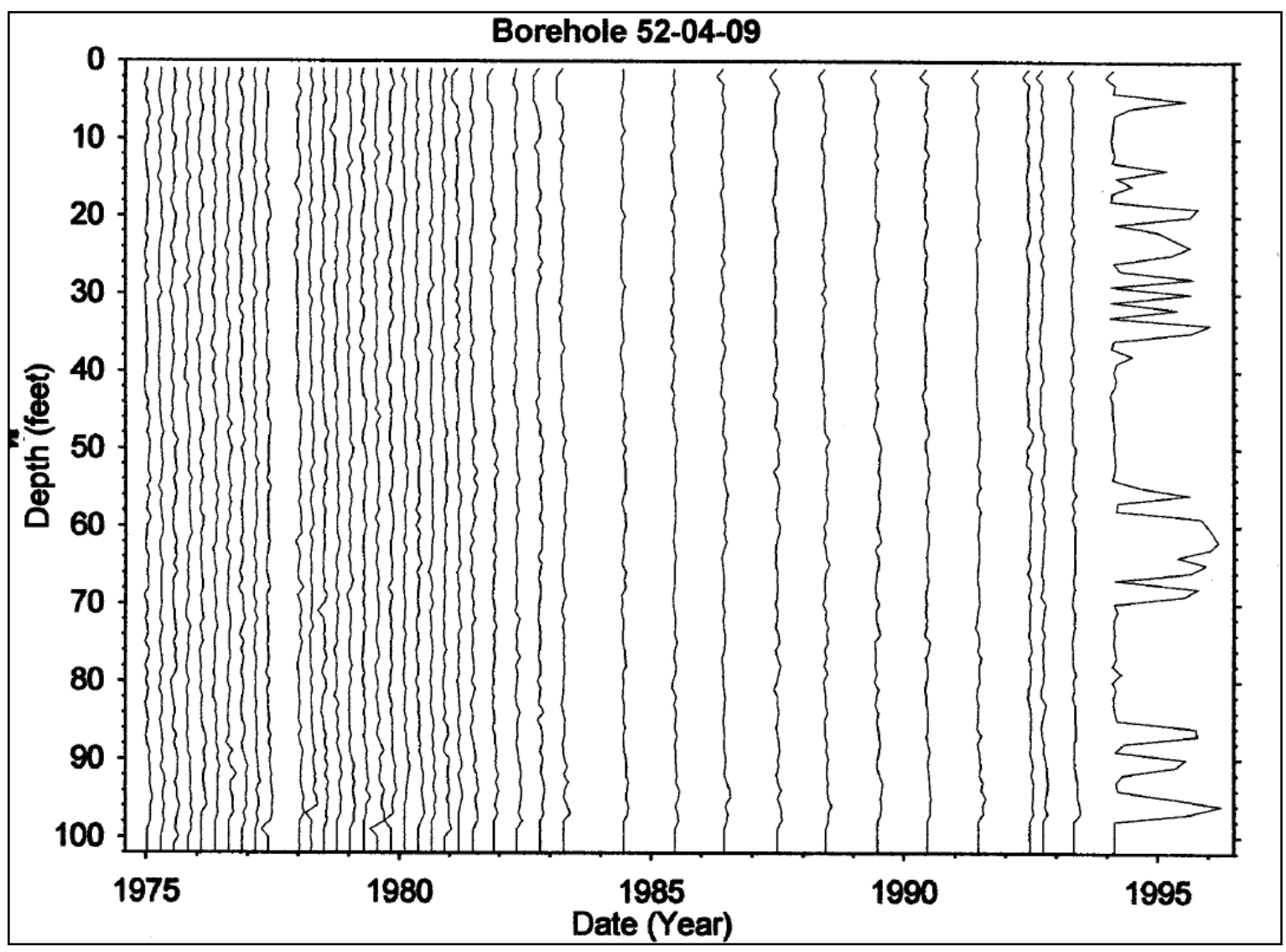

Note: Bottom of the tank footing is $\sim 46-\mathrm{ft}$ BGS 


\subsubsection{Drywell 52-04-10 $\left(\mathrm{N} 42^{\circ} \mathrm{W}\right)$}

Drywell 52-04-10 was drilled in August 1952 with the first recoverable reading on July 11, 1973 as less than $12 \mathrm{~K}$ cpm with the SP. Radiation readings were reported as less than values from July 1973 to June 1986 (see Appendix A3). Document HNF-3831 states that no significant levels of radioactivity are present about the survey probe detection threshold between 1975 and 1994 in this drywell. However, it was also stated in this document that Cs-137 was identified at the surface (0 to $1-\mathrm{ft} \mathrm{BGS}$ ) and concentrations were less than $1 \mathrm{pCi} / \mathrm{g}$.

In May 1997, Cs-137 was the only man-made radionuclide detected in drywell 52-04-10 (GJHAN-67). Cs-137 was detected from the ground surface to 1-ft, at 67-ft, between 87 and $87.5-\mathrm{ft}$, at 99-ft, and at 129.5-ft BGS. The maximum concentration was about $1 \mathrm{pCi} / \mathrm{g}$ at $1-\mathrm{ft} \mathrm{BGS}$. Document GJ-HAN-67 states that radioactivity detected was "probably from surface contamination that migrated along the outside of the casing by the action of surface water." Therefore, drywell 52-04-10 is not included as part of the leak location for tank TY-104. Figure 6-9 shows depths of radioactivity from 1975 to 1995 (HNF-3831).

Figure 6-9. Tank TY-104 Drywell 52-04-10 (HNF-3831)

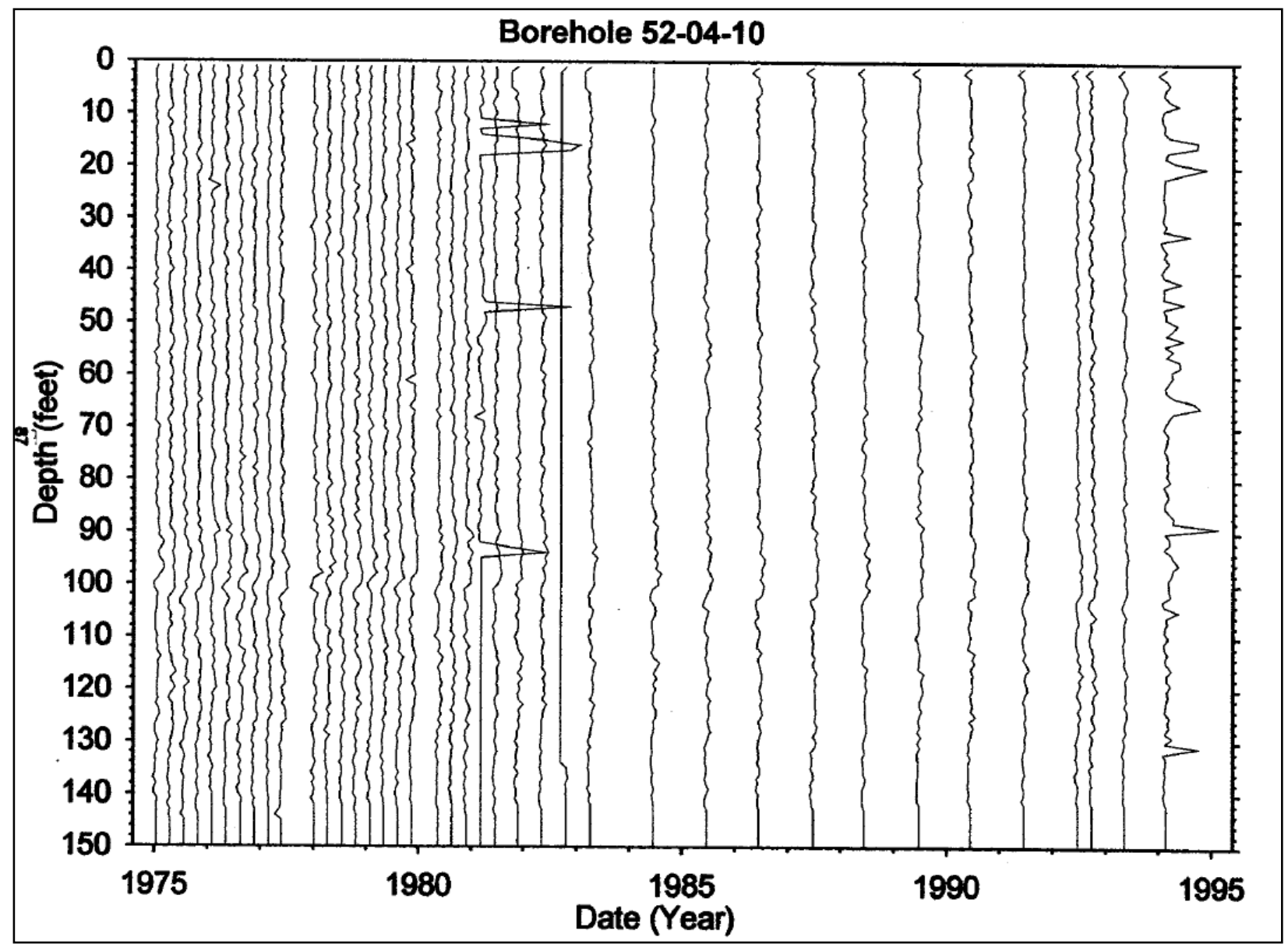

Note: Bottom of the tank footing is $~ 46-\mathrm{ft}$ BGS 


\subsubsection{Drywell 52-02-06 $\left(\mathrm{S0}^{\circ}\right)$}

Drywell 52-02-06 was drilled in 1977 with the first recoverable reading on September 23, 1977 as less than $3 \mathrm{~K}$ cpm with the SP. Radiation readings were reported as less than values from September 1977 to June 1986 (see Appendix A3). Document HNF-3831 states that no significant levels of radioactivity are present about the survey probe detection threshold between 1975 and 1994 in this drywell.

In May 1997, Cs-137 was the only man-made radionuclide detected in drywell 52-02-06 (GJHAN-67). Cs-137 was detected continuously from the ground surface to 3-ft, 12.5 to $15-\mathrm{ft}$, and 19.5 to $20-\mathrm{ft}$ BGS with the highest concentration of $1 \mathrm{pCi} / \mathrm{g}$ reported at 1 -ft BGS. Document GJHAN-67 states the Cs-137 contamination probably originated from a surface spill and was carried downward either during drilling operation or by the action of surface water. It was also stated that, "There is no indication in the SGLS logs of contamination originating from subsurface leaks from underground tanks or their associated facilities." Therefore, drywell 5202-06 is not included as part of the leak location for tank TY-104. Figure 6-10 shows depths of radioactivity from 1978 to 1995 (HNF-3831).

Figure 6-10. Tank TY-104 Associated Drywell 52-02-06 (HNF-3831)

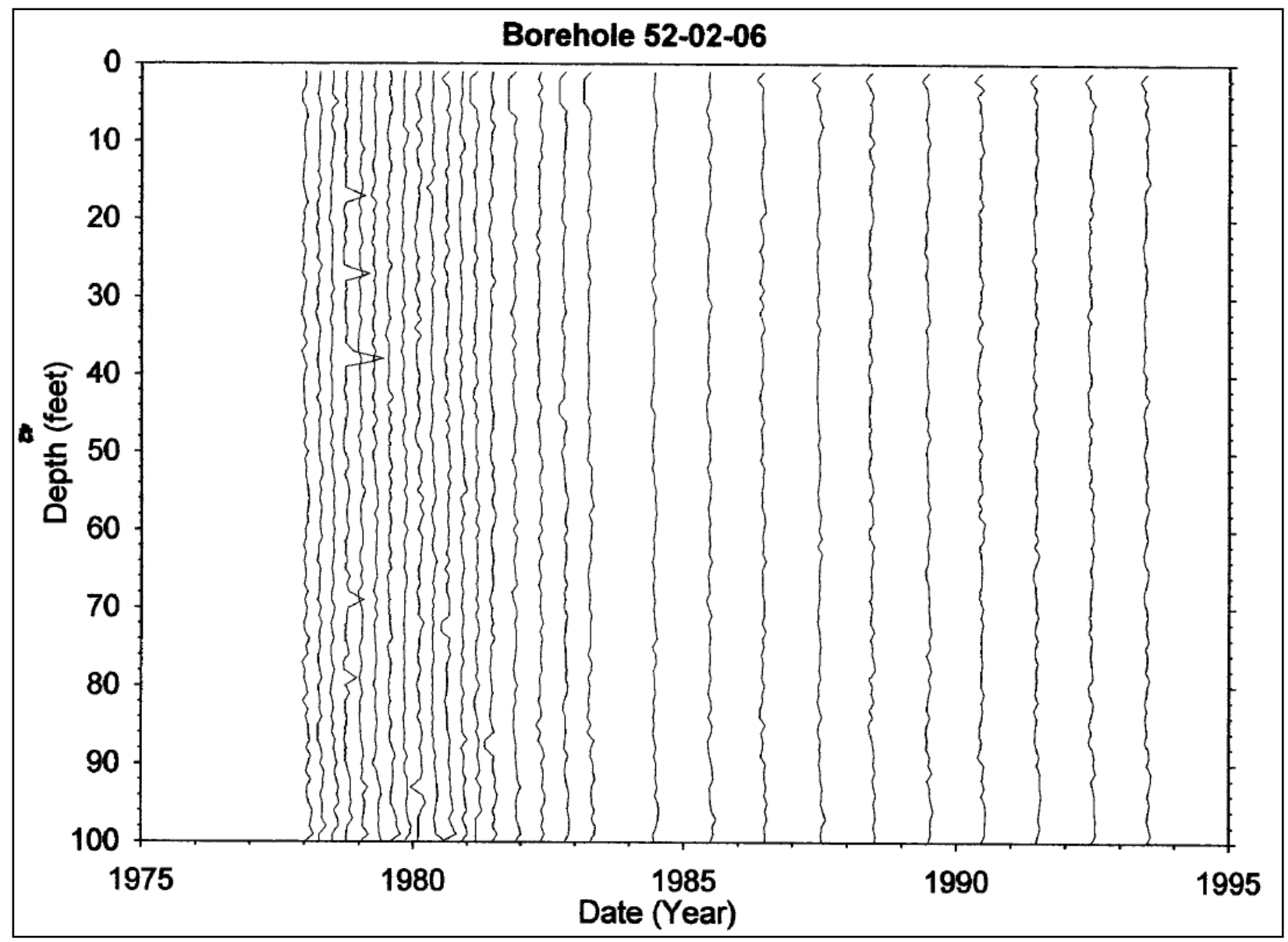

Note: Bottom of the tank footing is 46-ft BGS 


\subsubsection{Drywell 52-06-02 $\left(\mathrm{N} 31^{\circ} \mathrm{E}\right)$}

Drywell 52-04-06 was drilled in December 1971 with the first recoverable reading on April 4, 1974 as less than $12 \mathrm{~K}$ cpm with the SP. Radiation readings were reported as less than values from April 1974 to June 1986 (see Appendix A3). Document HNF-3831 states that Cs-137 contamination is only present at the surface from 0 to $10-\mathrm{ft}$ BGS.

In May 1997, Cs-137 was the only man-made radionuclide detected in drywell 52-06-02 which was detected continuously from the ground surface to 64-ft BGS (GJ-HAN-67). Measured Cs137 concentrations between 3 and 6-ft BGS ranged from about 5 to $13 \mathrm{pCi} / \mathrm{g}$, between 6 and 40$\mathrm{ft}$ BGS concentrations ranged from 1 to $3 \mathrm{pCi} / \mathrm{g}$, and the concentration was less than $1 \mathrm{pCi} / \mathrm{g}$ below 40-ft BGS. Document GJ-HAN-67 states, "Reasons for the relative continuity of the contaminated zone compared to other boreholes associated with tank TY-104 are unknown. However, the contamination in borehole 52-06-02 may be related to the much more pervasive surface contamination that occurs in the vicinity of tank TY-105." Drywell 52-04-03 is therefore not included as part of the leak location for tank TY-104. Figure 6-11 shows depths of radioactivity from 1975 to 1985 (HNF-3831).

Figure 6-11. Tank TY-104 Associated Drywell 52-06-02 (HNF-3831)

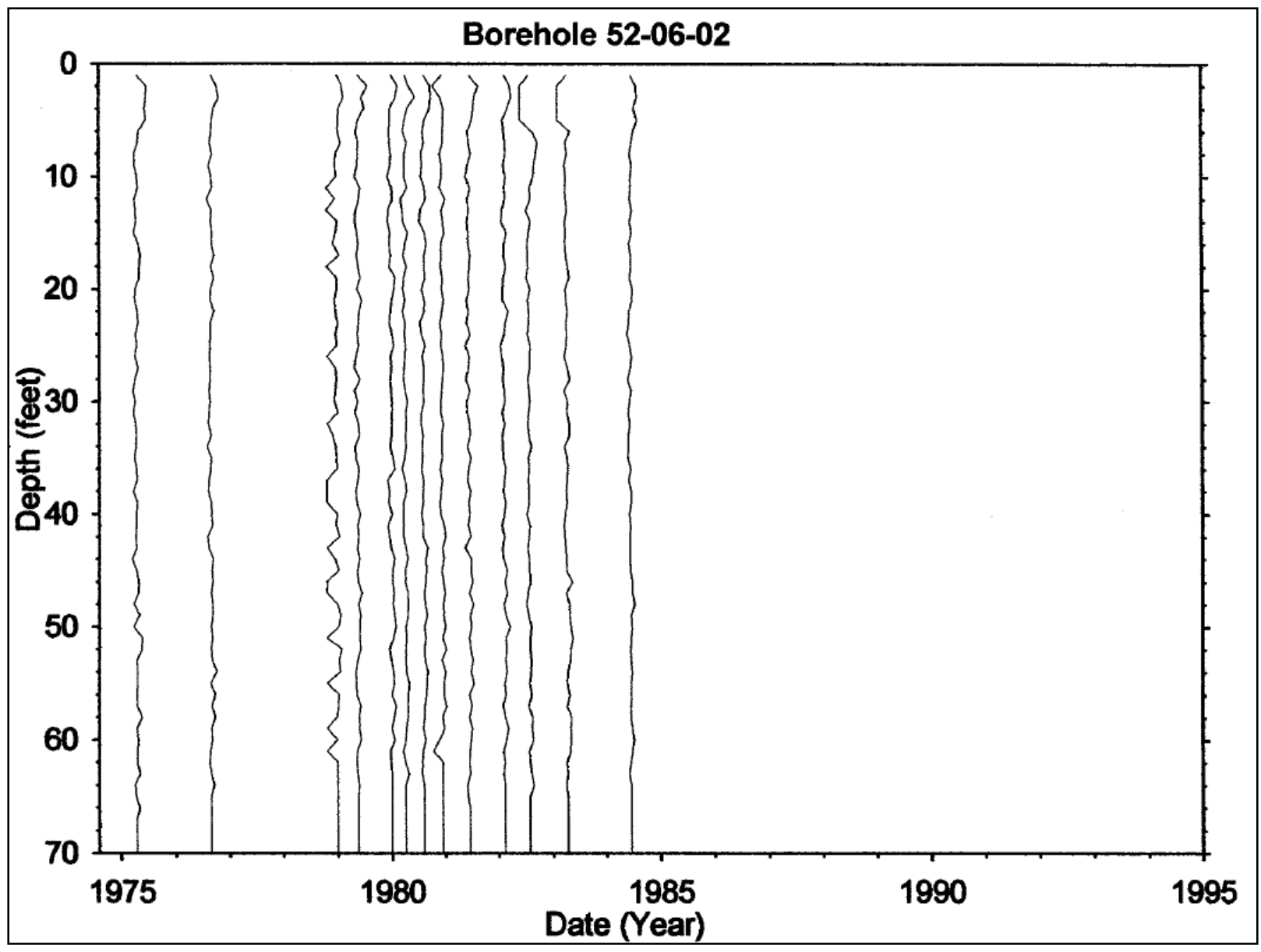

Note: Bottom of the tank footing is 46-ft BGS 
RPP-RPT-54911, Rev. 0

\subsubsection{Drywell Summary}

Tank TY-104 drywells 52-04-02, 52-04-03, 52-04-06, 52-04-09, 52-04-10, 52-02-06, and 52-0602 do not indicate any contamination associated with a tank leak. The SGLS data and historical gross gamma logs show no evidence of contamination that may have come from a tank TY-104 leak (GJ-HAN-67). Tank TY-104 was first assumed to be leaking from the end of December 1973 to March 1974 when an observed liquid level drop of 0.6-in occurred. The estimated leak volume of this tank is approximately 1,400 gal (RPP-RPT-42296), and this small leak would probably not be detected considering the distance to and low density of drywells surrounding this tank (GJ-HAN-67).

\subsection{CASCADE TIE LINE}

Waste from tank TY-103 cascades into tank TY-104 at the 3:00 position (see Figure 6-12) and the tank TY-104 cascade outlet tie line (tie line) is located at the 9:00 position (H-2-2253, 75Foot Tank Nozzle and Piping Details). A cascade inlet pipe is located on tank TY-104 at the 276.5-in waste height. The tie line is located at the 272-in waste height and the capped line extends $\sim 55$-ft 3 -in from the tank liner at a $-2 \%$ gradient (see Figure $6-13$ ). The tie line and sleeve were both capped and welded at $\sim 55$-ft 3 -in from this tank liner. A valve is located along the tie line at 26-ft 2-in from the tank liner. The valve may have been closed. The 4-in Sch. 80 steel pipe was inside an 8-in Sch. 40 pipe sleeve from the tank wall to a slip fit steel connection at the valve end. The 4-in Sch. 80 steel pipe was inside a 6-in Sch. 40 with a slip fit steel pipe sleeve from the valve to the capped end (see Figure 6-13). Note that the tie line slip fit sleeve appears to be mainly for support and some protection from the soil as opposed to leak containment. 
RPP-RPT-54911, Rev. 0

Figure 6-12. Tank TY-104 Associated Drywells and Cascade Lines

Tank inner ring is steel liner, outer ring is outer edge of tank footing

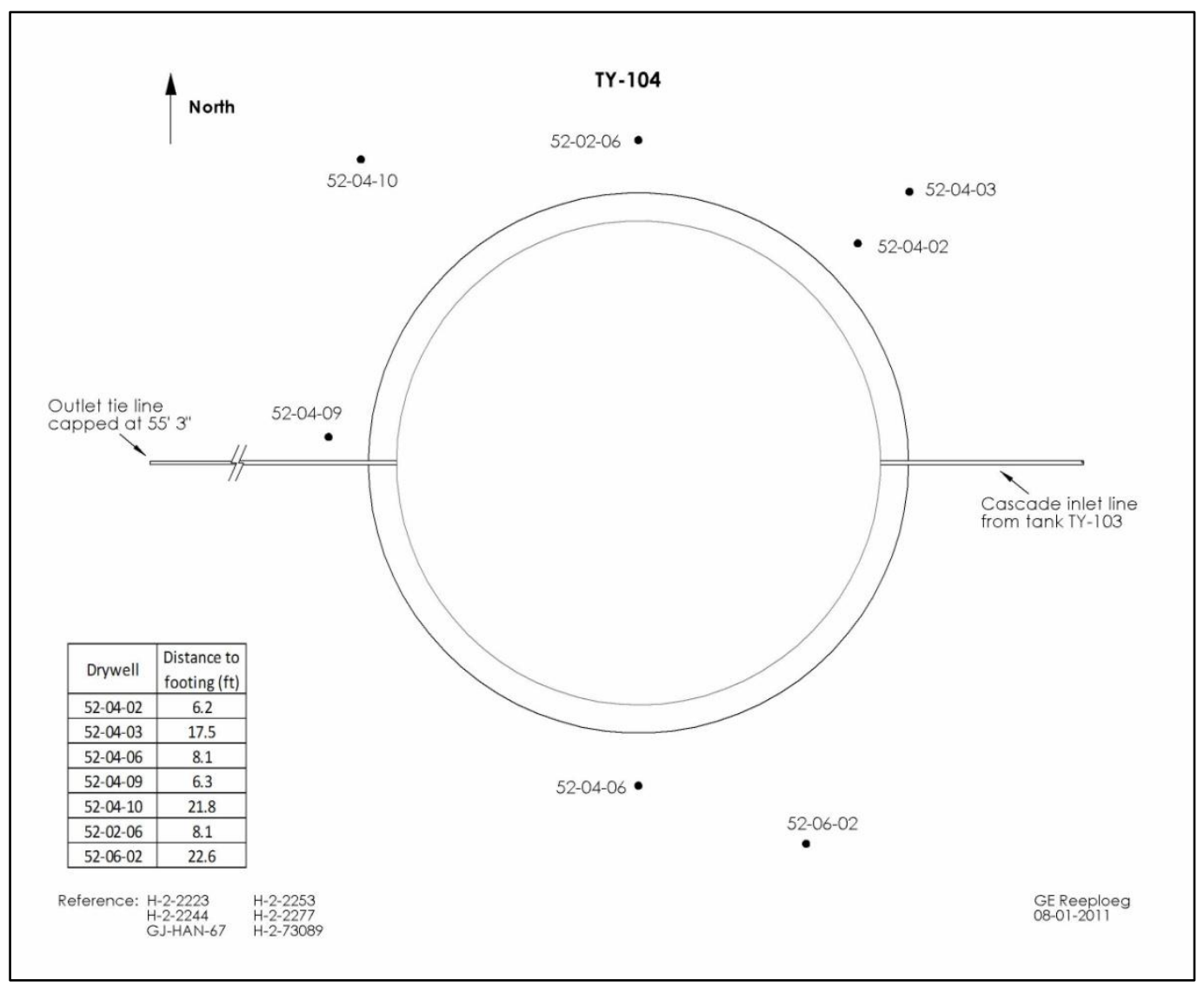

Note: A valve is located 26-ft from the tank liner on the TY-104 outlet tie line.

Tank TY-104 was first overfilled in May 1954 with TBP waste ( 770 kgal or 280 -in) which would have filled the tie line. The tank remained overfilled at $\sim 770 \mathrm{kgal}$ for approximately one year. In March 1955, $709 \mathrm{kgal}$ of TBP waste was transferred out of tank TY-104; however, most of the waste in the tie line would remain in this line as there was a $-2 \%$ gradient along the tie line.

Tank TY-104 was filled above the tie line again in December 1970 (ARH-2074 D, Chemical Processing Division Waste Status Summary October 1, 1971 through December 31, 1971). After a recalibration in July 1973 and no further waste transactions since December 1970, the liquid level was reported to be at $\sim 273$-in in July 1973. If the recalibration was correct, tank TY-104 was overfilled once again above the tie line in December 1970 and remained above the tie line until the supernatant was pumped out of tank TY-104 after the liquid level started decreasing December 30, 1973 (see Section 6.4.1). Therefore, it is possible the tank TY-104 tie line leaked as opposed to a tank liner leak as there was waste in portions of the tie line since 1954; however, it remains inconclusive whether one or both may be leaking. Note that drywell 52-04-09 did not indicate any radioactivity; therefore, it is unlikely the tie line leaked at the tank wall penetration. 
RPP-RPT-54911, Rev. 0

Figure 6-13. Tank TY-104 Outlet Cascade Tie Line Details

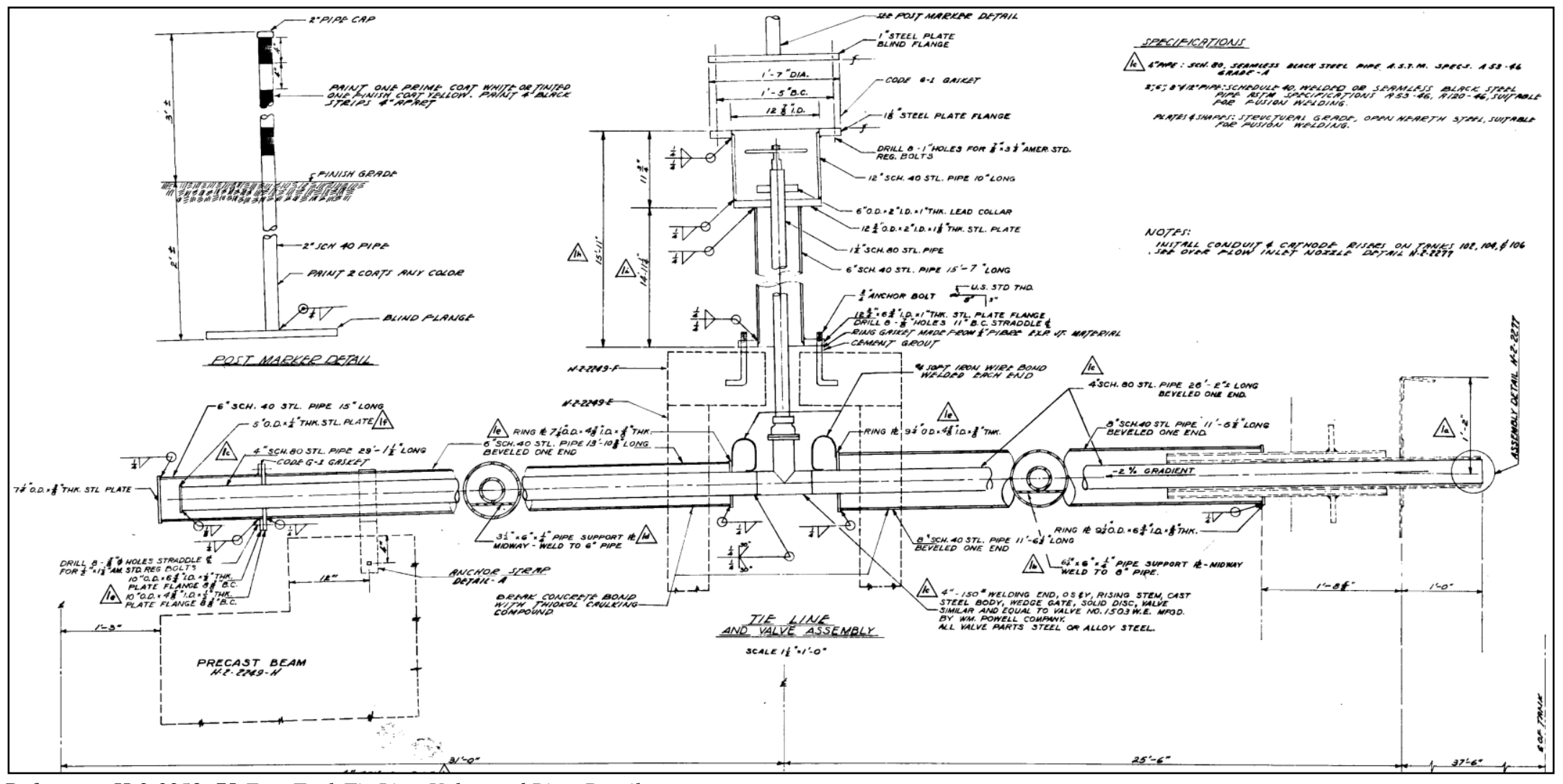

Reference: H-2-2253, 75-Foot Tank Tie Line, Valve, and Riser Details 


\subsubsection{Pipeline Cathodic Protection}

Pipeline cathodic protection systems were first implemented in 1947 at 221-B and 222-T $\mathrm{BiPO}_{4}$ plants. The system was expanded into the tank farm including the construction of TY Farm and its transfer systems which included an impressed current cathodic protection system $(\mathrm{H}-2-3602$, 200 West Area Cathodic Protection). The design features of the system included 30-ft long railroad rails which served as anodes, trench-lay connecting anodes and cathodes, rectifier and bonding.

Bonding was used as indicated in Figure 6-13 to provide an electrical connection of the sleeve to the portion of the line at the valve. This indicates a purposeful effort was made to protect the exposed pipe from galvanic corrosion. The valve and slip fit ends of the pipe sleeve were protected in an enclosure similar to the 241-BX Farm photograph in Figure 6-14. However, this area is the most likely to leak or accumulate leakage which would provide moisture and possible electrical connection corrosion.

Figure 6-14. BX Farm Cascade Tie Line

Photograph N1D0001321 October 6, 1942

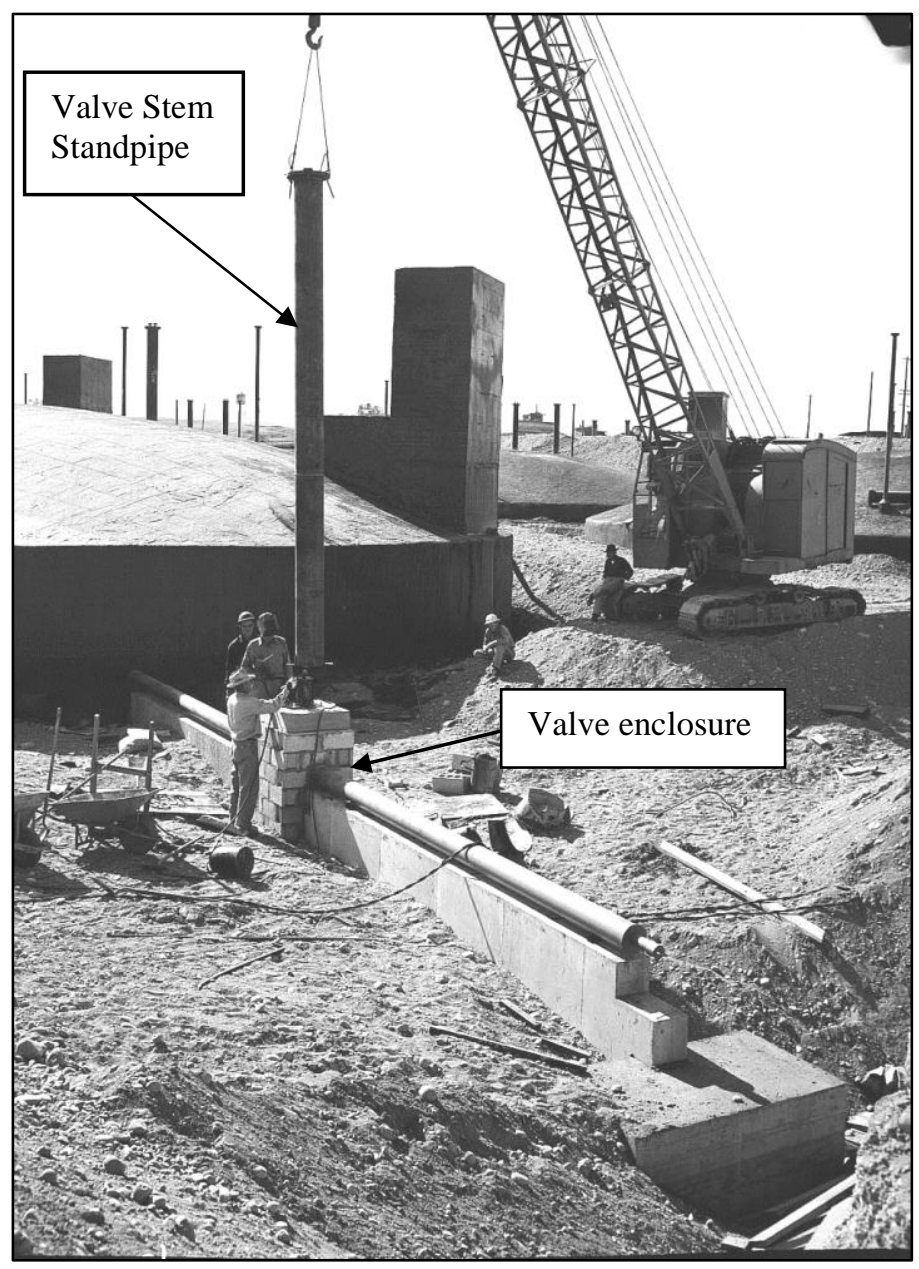


RPP-RPT-54911, Rev. 0

Additional piping was installed in the TY Farm in 1955 which would have required cathodic protection adjustments and increased the demand on the anodes including the anode connections. By 1970, the anodes and other parts of the systems had been in place for nearly 20 years.

\subsection{POSSIBLE TANK TY-104 LEAK LOCATION(S)}

The seven drywells surrounding tank TY-104 did not show radiation that indicated a possible tank liner leak from tank TY-104. There is deeper contamination at $\sim 80-\mathrm{ft}$ BGS in a couple of drywells, but this activity may have resulted from lateral transport of a leak from another source. However, the liquid level decrease in 1973 of 1,400 gal indicated possible leakage from the tank. The volume of leakage from tank TY-104 is probably not large enough to be detected by any of the seven drywells depending on the exact location of any liner leak with the closest drywell at 6.2-ft from the tank outer foundation. A tank liner leak location is therefore not able to be predicted.

A liner leak may not be the only cause for the tank TY-104 liquid level decrease. It is possible a leak may have occurred from the tie line which would probably not be detected by any tank TY104 drywells due to the distance and location of the tie line from the tank coupled with the volume of the leak (see Figure 6-4 and Section 6.6).

Tank TY-104 data is insufficient to identify a leak location and no laterals or direct pushes were installed near tank TY-104.

\subsection{POSSIBLE TANK TY-104 LINER LEAK CAUSE(S)}

\subsubsection{Tank Design}

The TY Farm tank design does not appear to be a factor contributing to a failed liner (see Section 3.1.1).

\subsubsection{Thermal Shock}

No temperature data are available for tank TY-104 prior to 1974 although tank TY-104 held nonboiling waste. Since no records are available, it is uncertain what the maximum temperature was in tank TY-104 during operation as well as the rate of temperature rise when waste was initially added. However, the thermal attributes of the waste would indicate that thermal stresses were minimal.

Thermal shock creates stress both from rapid temperature rise as well as waste-induced high temperatures.

Temperature requirements in ARH-951 (Limitations for Use of Underground Waste Tanks) issued December 18, 1969 indicated that tank temperatures should be held below $230^{\circ} \mathrm{F}$.

\subsubsection{Chemistry-Corrosion}

Tank TY-104 was first filled with TBP waste cascaded from tank TY-103 and stored a variety of waste types during operation. TBP waste consists of low hydroxide and high nitrate 
RPP-RPT-54911, Rev. 0

concentrations, likely with low nitrite concentrations, which would create an environment conducive to pitting and SCC. When the tank was first filled, TBP waste was the only waste present in the tank for $\sim 19$ months. With the addition of $1 \mathrm{C}-\mathrm{FeCN}$ and DW waste types, the hydroxide and nitrite would likely be increased and nitrate decreased making the environment less corrosive than storing only TBP waste (see Section 6.4.3). A tank with TBP waste type present would likely increase the propensity for corrosion in the tank liner and the cascade tie line.

Waste types $\mathrm{R}$ and $1 \mathrm{C}-\mathrm{FeCN}$ could create an environment conducive to SCC; however, this would be to a lesser degree than TBP waste. Waste types $\mathrm{R}$ and $1 \mathrm{C}-\mathrm{FeCN}$ probably did not increase the propensity for corrosion in tank TY-104 since R was diluted with other waste types and $1 \mathrm{C}$ was treated in 221-T Plant prior to being added to the tank (see Section 6.4.3). Other waste types stored in tank TY-104 should not have resulted in pitting or SCC.

\subsubsection{Liner Observations}

A review of the available photographs taken April 15 and 16, 1974 does not contain any evidence of a tank bottom liner bulge. There is no additional documentation available indicating a liner bulge was present in tank TY-104. Photographs also indicate a beachline above the cascade tie line outlet nozzle confirming the liquid level was above the nozzle (see Figure 6-4).

\subsubsection{Tank Construction Temperatures}

Average winter temperatures were $27.4^{\circ} \mathrm{F}$ and $25.2^{\circ} \mathrm{F}$ in December 1951 and January 1952, respectively, with temperatures as low as $4^{\circ} \mathrm{F}$ during the period when the TY Farm tank liners were being constructed. The low temperatures experienced during construction were less than the current allowable $18^{\circ} \mathrm{F}$ allowable temperature where impact loading had the potential for creating micro-fissures in the steel liner. The TY Farm construction specifications (HW-4696, Specifications Waste Disposal Facilities 241-BZ and TY Tank Farm) contained specific detailed requirements for cold weather construction and it appears these low temperatures had less of an impact during the construction of TY Farm (see Section 6.3.2).

\subsection{POSSIBLE TANK TY-104 CASCADE TIE LINE LEAK CAUSE(S)}

Tank TY-104 was overfilled in 1954 above the cascade tie line filling the tie line with TBP waste that would have remained in the tie line for 20 years until the leak was detected in December 1973. Tank TY-104 was overfilled above the cascade tie line for the second time in 1970. Corrosion could have occurred from storing TBP waste initially up to $180^{\circ} \mathrm{F}$ in 1954 leading to failure; however, temperatures likely cooled in the tie line after filling as ambient ground temperature was less than $55^{\circ} \mathrm{F}$. The tie line could have corroded and started leaking through the slip fittings by December 30, 1973 causing the liquid level decrease. Another possible leak site could have occurred at the valve located 26 -ft 2 -in from the tank liner along the tie line. The valve welds, seat, and/or packing could have eventually failed (see Section 6.6). Drywell 52-0409 did not pick up any radioactivity which indicated the tie line penetration through the tank wall was probably not a leak location. All other leaks would have entered the soil at least near the tie line valve because of the upper tie line pipe sleeve or further along the tie line which did not have any adjacent drywells. 
In 1970, after being in place for nearly 20 years, many of the anodes of the pipeline cathodic protection systems may have depleted to the point that they did not provide adequate protection to their respective pipelines. Similar findings were found in a 1980 document on the cathodic protection of the pipes from 242-S Evaporator (Payer, "Evaluation of a Risk Assessment Document for Restarting the 242-S Evaporator). Maintenance and adjustments to the system can also adversely affect the operation of the cathodic protection system. In the absence of cathodic protection and effective corrosion mitigation devices such as coatings, the chance of pipeline failure due to galvanic corrosion increases. The locations in the tie line of greatest susceptibility to galvanic corrosion consist of the tie line sleeve bonding and the exposed valve. Coating was not indicated around the valve area on the tank TY-104 tie line (see Section 6.6.1) but this area was in an enclosure. Diminished or lost cathodic protection would lead to galvanic corrosion.

Either of these conditions or a combination could have caused the 0.6-in liquid level decrease from December 30, 1973 to March 16, 1974.

\subsection{TANK TY-104 CONCLUSIONS}

The tank TY-104 liquid level decreased in 1973. Evidence suggests that there are two possible leak scenarios. One is that the tank TY-104 liner leaked in an unidentified location. The other is that the tank TY-104 tie line leaked also at an unidentified location. Based on engineering judgment and available information the following conditions listed in the order of importance might lead to either one or both of the failures.

1. TBP waste storage chemistry-corrosion- stress corrosion cracking

2. Construction conditions- ductile-to-brittle transition temperature

3. Thermal conditions

The most likely cause of the tank or tie line TY-104 leak is TBP waste storage chemistrycorrosion. TBP waste storage could have created a chemical environment susceptible to nitrateinduced SCC attack on a potentially stressed metal liner. Galvanic corrosion could also have affected the tank TY-104 tie line; therefore, an additional cause of the possible leak from the tank TY-104 tie line is failure of the cathodic protection system leading to increased corrosion of the pipe. Some or all of the factors can act serially or together to contribute to tank liner failure. 
RPP-RPT-54911, Rev. 0

APPENDIX A3

TANK TY-104 GROSS GAMMA DRYWELL DATA

A3-1 
RPP-RPT-54911, Rev. 0

Table A3-1. Tank TY-104 Drywell Radioactivity (K counts per minute)

(July 1973 to June 1986)

(Drywell Data Sheets* Retrieved on July 20, 2011 and SD-WM-TI-356)

\begin{tabular}{|c|c|c|c|c|c|c|c|c|c|c|c|c|c|}
\hline \\
\hline \multicolumn{2}{|c|}{ 52-04-02 } & \multicolumn{2}{|c|}{ 52-04-03 } & \multicolumn{2}{|c|}{ 52-04-06 } & \multicolumn{2}{|c|}{ 52-04-09 } & \multicolumn{2}{|c|}{ 52-04-10 } & \multicolumn{2}{|c|}{ 52-02-06 } & \multicolumn{2}{|c|}{$52-06-02$} \\
\hline \multicolumn{2}{|c|}{ Drilled 12/31/71 } & \multicolumn{2}{|c|}{ Drilled 6/30/52 } & \multicolumn{2}{|c|}{ Drilled 12/31/71 } & \multicolumn{2}{|c|}{ Drilled 12/31/71 } & \multicolumn{2}{|c|}{ Drilled 8/31/52 } & \multicolumn{2}{|c|}{ Drilled 1977} & \multicolumn{2}{|c|}{ Drilled 12/1971 } \\
\hline Date & $\begin{array}{c}\text { Peak } \\
(\text { K cpm) }\end{array}$ & Date & $\begin{array}{c}\text { Peak } \\
(\text { K cpm })\end{array}$ & Date & $\begin{array}{c}\text { Peak } \\
(\text { K cpm) }\end{array}$ & Date & $\begin{array}{c}\text { Peak } \\
(\text { K cpm) }\end{array}$ & Date & $\begin{array}{c}\text { Peak } \\
(\text { K cpm) }\end{array}$ & Date & $\begin{array}{c}\text { Peak } \\
(\mathrm{K} \text { cpm })\end{array}$ & Date & $\begin{array}{c}\text { Peak } \\
(\text { K cpm) }\end{array}$ \\
\hline $7 / 11 / 73$ & $<12$ & $7 / 11 / 73$ & $<12$ & $7 / 11 / 73$ & $<12$ & $7 / 11 / 73$ & $<12$ & $7 / 11 / 73$ & $<12$ & \multirow{9}{*}{\multicolumn{2}{|c|}{$\begin{array}{c}\text { New Well installed } \\
1977\end{array}$}} & \multirow{2}{*}{\multicolumn{2}{|c|}{ New Well ${ }^{2}$}} \\
\hline $9 / 21 / 73$ & $<12$ & $12 / 22 / 73$ & $<12$ & $3 / 19 / 73$ & $<12$ & $3 / 9 / 74$ & $<12$ & $3 / 19 / 73$ & $<12$ & & & & \\
\hline $4 / 16 / 74$ & $<12$ & $4 / 30 / 74$ & $<12$ & $4 / 16 / 74$ & $<12$ & $4 / 16 / 74$ & $<12$ & $4 / 23 / 74$ & $<12$ & & & $4 / 30 / 74$ & $<12$ \\
\hline $8 / 8 / 74$ & $<6^{1}$ & $8 / 8 / 74$ & $<6^{1}$ & $8 / 8 / 74$ & $<6^{1}$ & $8 / 8 / 74$ & $<6^{1}$ & $8 / 8 / 74$ & $<6^{1}$ & & & $8 / 8 / 74$ & $<6^{1}$ \\
\hline $2 / 20 / 75$ & $<3$ & $2 / 20 / 75$ & $<3$ & $2 / 20 / 75$ & $<3$ & $2 / 20 / 75$ & $<3$ & $2 / 20 / 75$ & $<3$ & & & $2 / 20 / 75$ & $<3$ \\
\hline $7 / 24 / 75$ & $<3$ & $7 / 24 / 75$ & $<3$ & $7 / 24 / 75$ & $<3$ & $7 / 24 / 75$ & $<3$ & $7 / 24 / 75$ & $<3$ & & & $7 / 24 / 75$ & $<3$ \\
\hline $12 / 31 / 75$ & $<3$ & $12 / 31 / 75$ & $<3$ & $12 / 31 / 75$ & $<3$ & $12 / 31 / 75$ & $<3$ & $12 / 31 / 75$ & $<3$ & & & $12 / 31 / 75$ & $<3$ \\
\hline $5 / 13 / 76$ & $<3$ & $5 / 13 / 76$ & $<3$ & $5 / 14 / 76$ & $<3$ & $5 / 14 / 76$ & $<3$ & $5 / 14 / 76$ & $<3$ & & & $5 / 16 / 76$ & $<3$ \\
\hline $9 / 25 / 76$ & $<3$ & $9 / 23 / 76$ & $<3$ & 9/23/76 & $<3$ & $9 / 23 / 76$ & $<3$ & $9 / 23 / 76$ & $<3$ & & & $9 / 18 / 76$ & $<3$ \\
\hline $5 / 6 / 77$ & $<3$ & $5 / 5 / 77$ & $<3$ & $5 / 5 / 77$ & $<3$ & $5 / 5 / 77$ & $<3$ & $5 / 5 / 77$ & $<3$ & $9 / 23 / 1977$ & $<3$ & $5 / 20 / 77$ & $<3$ \\
\hline $10 / 14 / 77$ & $<3$ & $10 / 14 / 77$ & $<3$ & $10 / 13 / 77$ & $<3$ & $10 / 13 / 77$ & $<3$ & $10 / 13 / 77$ & $<3$ & 10/13/1977 & $<3$ & $10 / 6 / 77$ & $<3$ \\
\hline $10 / 12 / 78$ & $<3$ & $10 / 12 / 78$ & $<3$ & $10 / 12 / 78$ & $<3$ & $10 / 12 / 78$ & $<3$ & $10 / 12 / 78$ & $<3$ & $10 / 12 / 1978$ & $<3$ & $10 / 5 / 78$ & $<3$ \\
\hline $10 / 18 / 79$ & $<3$ & $10 / 16 / 79$ & $<3$ & $10 / 16 / 79$ & $<3$ & $10 / 16 / 79$ & $<3$ & $10 / 16 / 79$ & $<3$ & $10 / 16 / 1979$ & $<3$ & $10 / 16 / 79$ & $<3$ \\
\hline $11 / 12 / 80$ & $<3$ & $11 / 12 / 80$ & $<3$ & $11 / 12 / 80$ & $<3$ & $11 / 12 / 80$ & $<3$ & $11 / 12 / 80$ & $<3$ & $11 / 12 / 1980$ & $<3$ & $11 / 12 / 80$ & $<3$ \\
\hline $11 / 19 / 81$ & $<3$ & $11 / 19 / 81$ & $<3$ & $11 / 19 / 81$ & $<3$ & $11 / 19 / 81$ & $<3$ & $11 / 19 / 81$ & $<3$ & $11 / 18 / 1981$ & $<3$ & $11 / 19 / 81$ & $<3$ \\
\hline $10 / 19 / 82$ & $<3$ & $10 / 19 / 82$ & $<3$ & $10 / 19 / 82$ & $<3$ & $10 / 19 / 82$ & $<3$ & $10 / 19 / 82$ & $<3$ & $10 / 21 / 1982$ & $<3$ & $10 / 19 / 82$ & $<3$ \\
\hline $6 / 22 / 83$ & $<3$ & $6 / 22 / 83$ & $<3$ & $6 / 22 / 83$ & $<3$ & $6 / 22 / 83$ & $<3$ & $6 / 22 / 83$ & $<3$ & $6 / 22 / 1983$ & $<3$ & $6 / 22 / 83$ & $<3$ \\
\hline $6 / 14 / 84$ & $<3$ & $8 / 22 / 84$ & $<3$ & $6 / 14 / 84$ & $<3$ & $6 / 14 / 84$ & $<3$ & $6 / 14 / 84$ & $<3$ & $6 / 14 / 1984$ & $<3$ & $6 / 14 / 84$ & $<3$ \\
\hline $6 / 18 / 85$ & $<3$ & $6 / 18 / 85$ & $<3$ & $6 / 18 / 85$ & $<3$ & $6 / 18 / 85$ & $<3$ & $6 / 18 / 85$ & $<3$ & 6/18/1985 & $<3$ & $6 / 18 / 85$ & $<3$ \\
\hline $6 / 11 / 86$ & $<3$ & $6 / 13 / 86$ & $<3$ & $6 / 11 / 86$ & $<3$ & $6 / 11 / 86$ & $<3$ & $6 / 11 / 86$ & $<3$ & $6 / 11 / 1986$ & $<3$ & $6 / 11 / 86$ & $<3$ \\
\hline
\end{tabular}

Note: ${ }^{1}$ New monitoring equipment

${ }^{2}$ Drywell 52-06-02 was drilled in December 1971; however, reference SD-WM-TI-356 indicates no readings prior to 1974

*Drywell data sheets located in the Historical Records folder in IDMS/Managed Information 


\subsection{TANK TY-105 SEGMENT}

\section{TABLE OF CONTENTS}

7.1 Tank TY-105 Background History ........................................................................

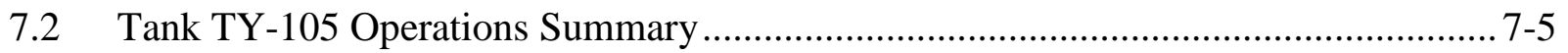

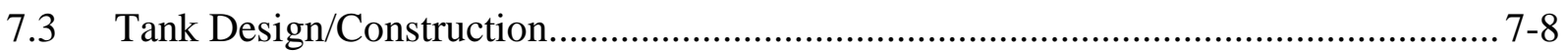

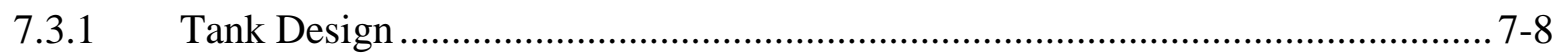

7.3.2 Tank Construction Conditions ........................................................................

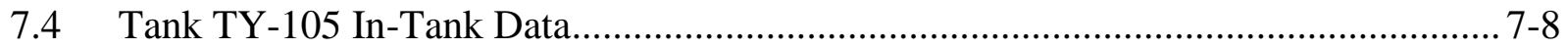

7.4.1 Liquid Level........................................................................................ 7-8

7.4.2 Temperature ..................................................................................

7.4.3 Liner Observations ...............................................................................

7.4.4 Chemistry-Corrosion ..............................................................................

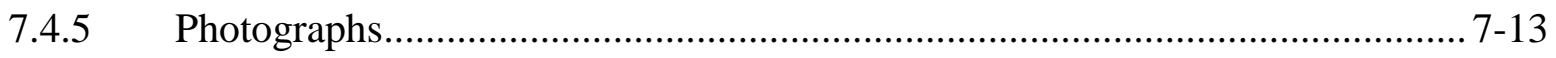

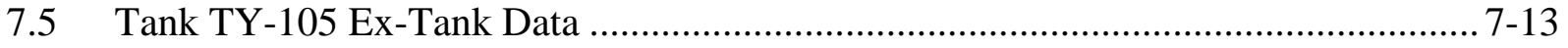

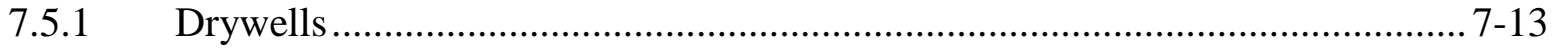

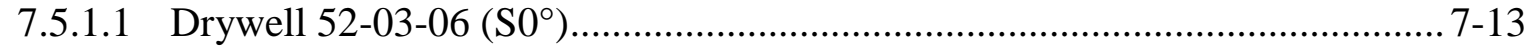

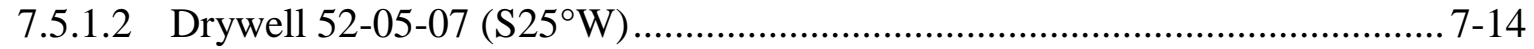

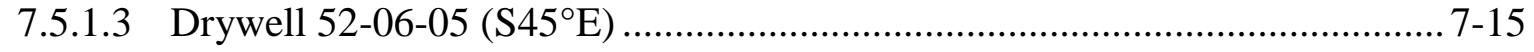

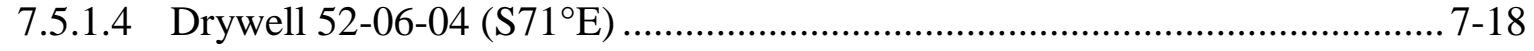

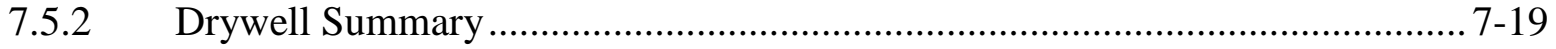

7.5.3 2005 Direct Push Logging ....................................................................... 7-19

7.6 Possible Tank TY-105 Liner Leak Location(s)..................................................... 7-23

7.6.1 Leak Detected in 1973-1974, Site A ........................................................... 7-24

7.6.2 Leak Detected in 2005, Site B .............................................................. 7-25

7.6.3 Leak Location Summary ....................................................................... 7-26

7.7 Possible Tank TY-105 Liner Leak Cause(s) …………………….......................... 7-28

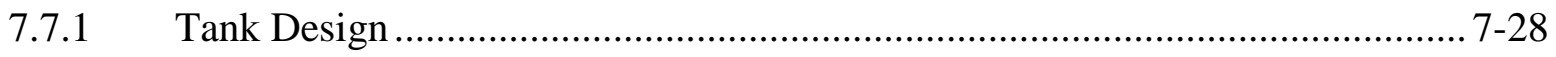

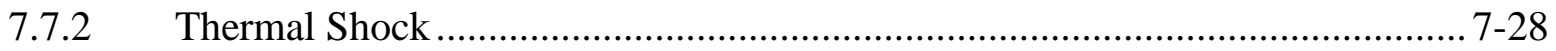

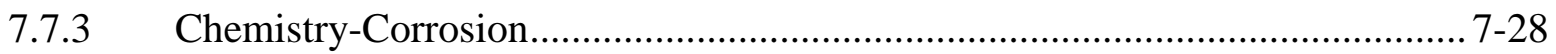

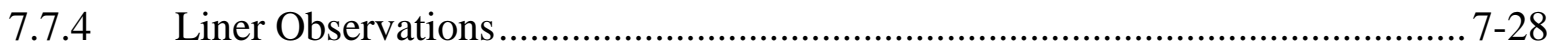

7.7.5 Tank Construction Temperatures............................................................. 


\section{APPENDICES}

Appendix A 4 Tank TY-105 Operational History ........................................................ A4-1

Appendix B 4 Tank TY-105 Gross Gamma Drywell Data............................................... B4-1

Appendix C 4 Tank TY-105 Gamma Surveys of the 2005 Direct Pushes ........................... C4-1

\section{LIST OF FIGURES}

Figure 7-1. Tank TY-105 Associated Drywells........................................................... 7-4

Figure 7-2. Operational Leak History of Tank TY-105.................................................. 7-7

Figure 7-3. Tank TY-105 End of Quarter Surface Level.................................................... 7-9

Figure 7-4. Tank TY-105 Liquid Level April 30, 1958 to October 27, 1960_.................... 7-11

Figure 7-5. Tank TY-105 Associated Drywell 52-03-06 (HNF-3831) ................................ 7-14

Figure 7-6. Tank TY-105 Drywell 52-05-07 (HNF-3831) ............................................ 7-15

Figure 7-7. Tank TY-105 Associated Drywell 52-06-05 Cesium (HNF-3831) .................... 7-16

Figure 7-8. Tank TY-105 Associated Drywell 52-06-05 (HNF-3831) ............................... 7-17

Figure 7-9. Tank TY-105 Associated Drywell 52-06-04 (HNF-3831) ................................ 7-18

Figure 7-11. Tank TY-105 Spare Inlet Nozzles....................................................... 7-21

Figure 7-12. Tank TY-105 Cascade Outlet Relative to Inlet(s)..................................... 7-22

Figure 7-13. Tank TY-105 Spare Inlet Line Asphalt - Gunite Seal................................... 7-22

Figure 7-14. Tank TY-105 Possible Leak Location (1973-1974) ....................................... 7-24

Figure 7-15. Tank TY-105 Possible Leak Location (2005)............................................ 7-25

Figure 7-16. Tank TY-105 Possible Radial Leak Locations .......................................... 7-27

Figure C4-1. 2005 Gamma Survey Direct Push C5016................................................... C4-2

Figure C4-2. 2005 Gamma Survey Direct Push C5018 ................................................... C4-3

Figure C4-3. 2005 Gamma Survey Direct Push C4619.................................................... C4-4 
RPP-RPT-54911, Rev. 0

Figure C4-4. 2005 Gamma Survey Direct Push C4620.................................................... C4-5

Figure C4-5. 2005 Gamma Survey Direct Push C4621 .................................................. C4-6

Figure C4-6. 2005 Gamma Survey Direct Push C4623 .................................................. C4-7

Figure C4-7. 2005 Gamma Survey Direct Push C4625 ....................................................... C4-8

Figure C4-8. 2005 Gamma Survey Direct Push C4637...................................................... C4-9

\section{LIST OF TABLES}

Table 7-1. Tank TY-105 Waste Storage Chronology ...................................................... 7-12

Table 7-2. Waste Chemistries for Waste Types Stored in Tank TY-105 ............................. 7-12

Table 7-2. 2005 Direct Push Gross Gamma Results (RPP-RPT-34870)............................... 7-20

Table A4-1. ARH-R-43 Waste Tank Leak Experience Table (1970) .................................. A4-2

Table B4-1. Tank TY-105 Drywell Radioactivity (K counts per minute)........................... B4-2 


\subsection{TANK TY-105 BACKGROUND HISTORY}

This section provides information on the historical waste loss event associated with SST 241TY-105 (TY-105). There are four drywells located around tank TY-105 with specified distances from the drywell to the tank footing shown in Figure 7-1: 52-06-05 installed in 1952; 52-03-06 installed in December 1971; 52-05-07 and 52-06-04 installed in August 1974. Eight direct pushes were installed in 2005: C5016, C5018, C5020, C4437, C4625, C4623, C4621, and C4619.

The bottom of the tank footing is $~ 46-\mathrm{ft}$ Below Grade Surface (BGS) with $\sim 6$-ft 10-in soil cover over the dome (WHC-SD-WM-TI-665, Soil Load above Hanford Waste Storage Tanks; H-22244, 75 Foot Composite Storage Tank Sections).

Figure 7-1. Tank TY-105 Associated Drywells

Tank inner ring is steel liner, outer ring is outer edge of tank footing

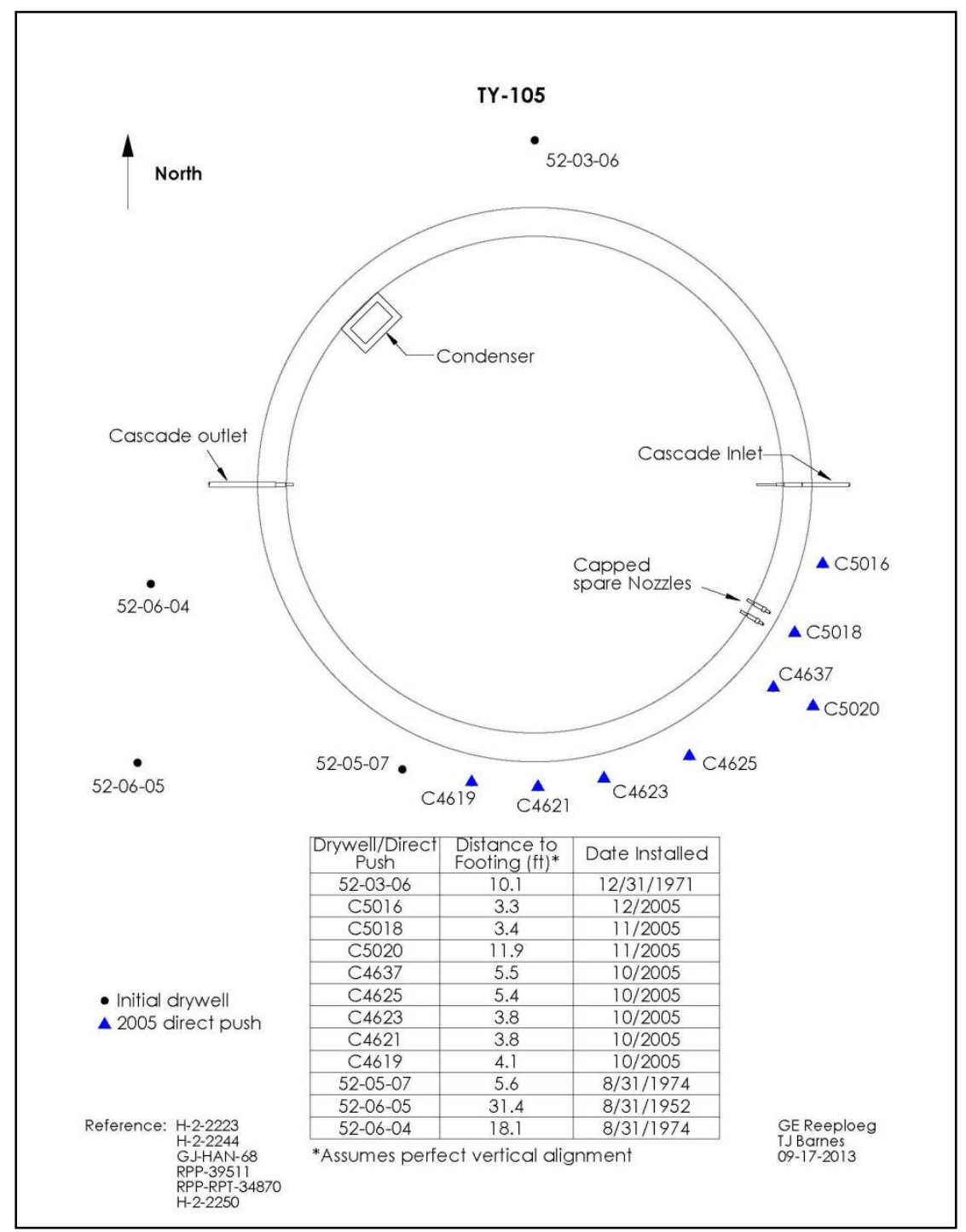


RPP-RPT-54911, Rev. 0

\subsection{TANK TY-105 OPERATIONS SUMMARY}

Tank TY-105 was constructed from 1951 to 1952 and started receiving Tri-Butyl Phosphate (TBP) waste from 221-U Plant in January 1953 (HW-27841, Waste Status Summary Separations Section Period: January 1953). Tank TY-105 continued to receive TBP waste and by August 1953 was filled with $758 \mathrm{kgal}$ of TBP waste (HW-29242, Separations Section Waste - Status Summary August 31, 1953). An additional 1,519 kgal of TBP waste was added to tank TY-105 between September and December 1953, while 1,058 kgal of this waste cascaded into tank TY106 and $461 \mathrm{kgal}$ were transferred out of tank TY-105 to tank TX-118 leaving $517 \mathrm{kgal}$ of TBP waste in the tank in January 1954 (HW-30498, Separations Section Waste - Status Summary December 31, 1953).

In January 1954, approximately 1,199 kgal of TBP waste was added to tank TY-105, of which $958 \mathrm{kgal}$ cascaded to tank TY-106, leaving tank TY-105 full at a volume of $758 \mathrm{kgal}$ (HW30851, Separations Section Waste - Status Summary January 31, 1954). In April 1954 through June $19541,578 \mathrm{kgal}$ of TBP waste was added to tank TY-105 all of which cascaded to tank TY106 (LA-UR-97-311, Waste Status and Transaction Record Summary). From June 1954 through September 1954 1,424 kgal of TBP waste was added to tank TY-105 all of which cascaded to tank TY-106 (LA-UR-97-311). Waste volumes were reported to be $758 \mathrm{kgal}$ in December 1956 (HW-47640, Chemical Processing Department Waste-Status Summary December 1, 1956December 31, 1956). No additional waste was added to tank TY-105 from October 1954 to September 1960. Beginning in January through May 1957, waste volumes were reported at 705 kgal despite no additional transfers into or out of tank TY-105. A new electrode installed in June 1957 resulted in a volume reported at $750 \mathrm{kgal}$ (HW-51348, Chemical Processing Department Waste Status Summary June 1, 1957 -June 30, 1957). Waste volumes continued to be reported between $744 \mathrm{kgal}$ to $752 \mathrm{kgal}$ from July 1957 to August 1960.

In September 1960 a new electrode was installed and tank TY-105 waste volume was reported at 724 kgal (HW-67696, Chemical Processing Department Waste Status Summary September 1, 1960 -September 30, 1960). In October 1960 a 1 1/4-in. (3,400-gal) liquid level drop was observed (HW-67705, Chemical Processing Department Waste Status Summary October 1, 1960 - October 31, 1960). As a result, supernatant was transferred from tank TY-105 to tank TX-108 and tank TX-118 in November and December 1960 leaving approximately $312 \mathrm{kgal}$ of waste in tank TY-105 (HW-68291, Chemical Processing Department Waste Status Summary November 1, 1960 -November 30, 1960; HW-68292, Chemical Processing Department Waste - Status Summary December 1, 1960 - December 31, 1960; HW-71610, Chemical Processing Department Waste Status Summary January 1, 1961 Through June 30, 1961). There is a discrepancy in the records of the amount of supernatant sent to tanks TX-108 and TX-118 in 1960. The waste summary reports (HW-68291 and HW-68292) show 93 kgal was transferred in November and $24 \mathrm{kgal}$ in December. However, HW-83906 records $77 \mathrm{kgal}$ in November and $27.25 \mathrm{kgal}$ in December. It remains unclear which reports were correct.

The liquid level in tank TY-105 continued to slowly decline from February 1961 through August 1974 with saltwell pumping of the tank (SD-WM-TI-356, Waste Storage Tank Status and Leak Detection Criteria). The liquid level decline from February 1961 through August 1974 was 27 kgal. However, the manual tape used to measure liquid level was reported to be contacting solid waste surfaces within the tank, raising uncertainty about the actual liquid level decline from 


\section{RPP-RPT-54911, Rev. 0}

February 1961 through August 1974 (RPP-RPT-42296, Hanford TY-Farm Leak Assessments Report). Also, evaporation may have contributed to the liquid level decrease during this period.

Tank TY-105 was declared a confirmed leaker and removed from service in 1960 and a cumulative leak volume was estimated at $35 \mathrm{kgal}$ (HNF-EP-0182, Waste Tank Summary Report for Month Ending December 31, 2009). Pumping was resumed with the installation of a P-10 pump August 20, 1974 to November 1982. Tank TY-105 continued pumping with a jet pump from November 4, 1982 to December 10, 1982 removing an additional $3.6 \mathrm{kgal}$ of waste and was interim stabilized in February 1983. Tank TY-105 is estimated to contain $231 \mathrm{kgal}$ of sludge, 12 kgal of drainable interstitial liquid, and no supernatant (HNF-EP-0182, Rev. 301, Waste Tank Summary Report for Month Ending April 30, 2013).

The operational history of tank TY-105 leak related details including liquid level is charted in Figure 7-2. 


\section{Figure 7-2. Operational Leak History of Tank TY-105}

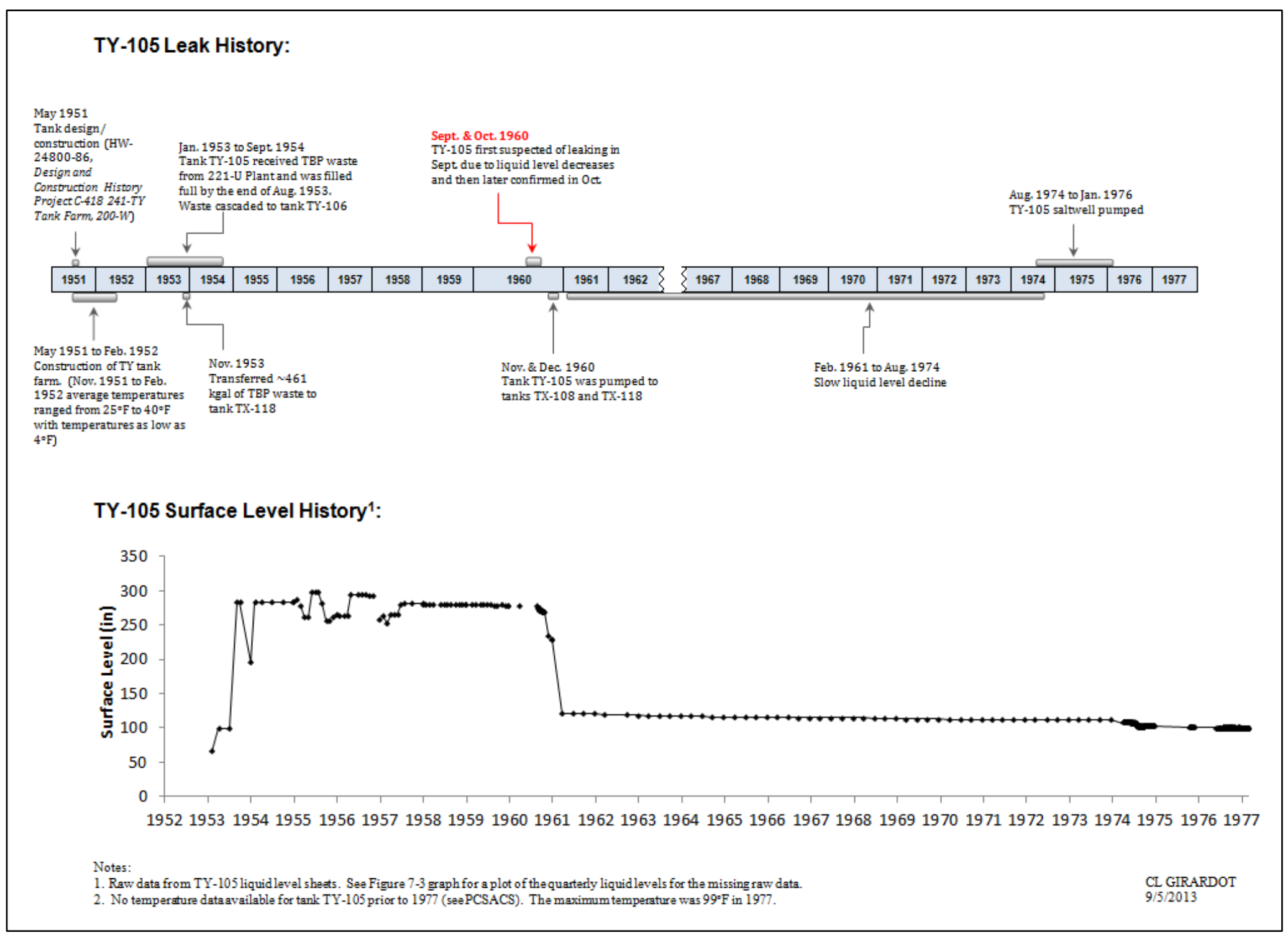


RPP-RPT-54911, Rev. 0

\subsection{TANK DESIGN/CONSTRUCTION}

\subsubsection{Tank Design}

The TY Farm tank design continued important features of the earlier 241-BCTU tanks (BPF73550). The steel bottom intersects the sidewall on a 4-ft radius. Full penetration butt welds with $x$-ray inspection and three ply asphaltic membrane waterproofing between the wall liner and the concrete shell continued those design features found to be important for liner integrity during the 241-SX Farm leak assessment (RPP-RPT-54910). The top of the tank footing is 43 -ft BGS and is 3-ft thick with the bottom of the footing at 4 46-ft BGS.

\subsubsection{Tank Construction Conditions}

The TY Farm was constructed from May 1951 to February 1952. Cold weather could have affected the steel during construction in December and January. Temperatures for December 1951 and January 1952 averaged $27.4^{\circ} \mathrm{F}$ and $25.2^{\circ} \mathrm{F}$, respectively, with temperatures as low as $4^{\circ} \mathrm{F}$ (PNNL-15160). Weather precautions for welding and concrete were specified in HW-4696 (see Section 3.1.2).

As described in Section 3.1.2, cold weather affects the ductile-to-brittle steel transition temperature, with $18^{\circ} \mathrm{F}$ being the assumed unrestricted low temperature construction limitation for the carbon steel liner, which could result in a fracture upon impact. Low temperatures experienced during construction at or less than the $18^{\circ} \mathrm{F}$ allowable temperature could cause impact loading (e.g. a dropped tool or piece of equipment from scaffolding) and result in the potential for creating micro-fissures weakening the steel liner.

\subsection{TANK TY-105 IN-TANK DATA}

\subsubsection{Liquid Level}

The liquid level plot in Figure 7-3 indicates the transfer activity into and out of tank TY-105. The liquid levels are end of quarter levels so this figure may not reflect all transfers into and out of the tank that occurred during the operational history of the tank. Over 5,000 kgal of waste cascaded from tank TY-105 to tank TY-106 over two years in 1953 and 1954 (WHC-SD-WMTI-669, Waste Status and Transaction Record Summary for the Northwest Quadrant of the Hanford 200 Area). See Figure 7-2 for historical monthly liquid level readings. 
Figure 7-3. Tank TY-105 End of Quarter Surface Level

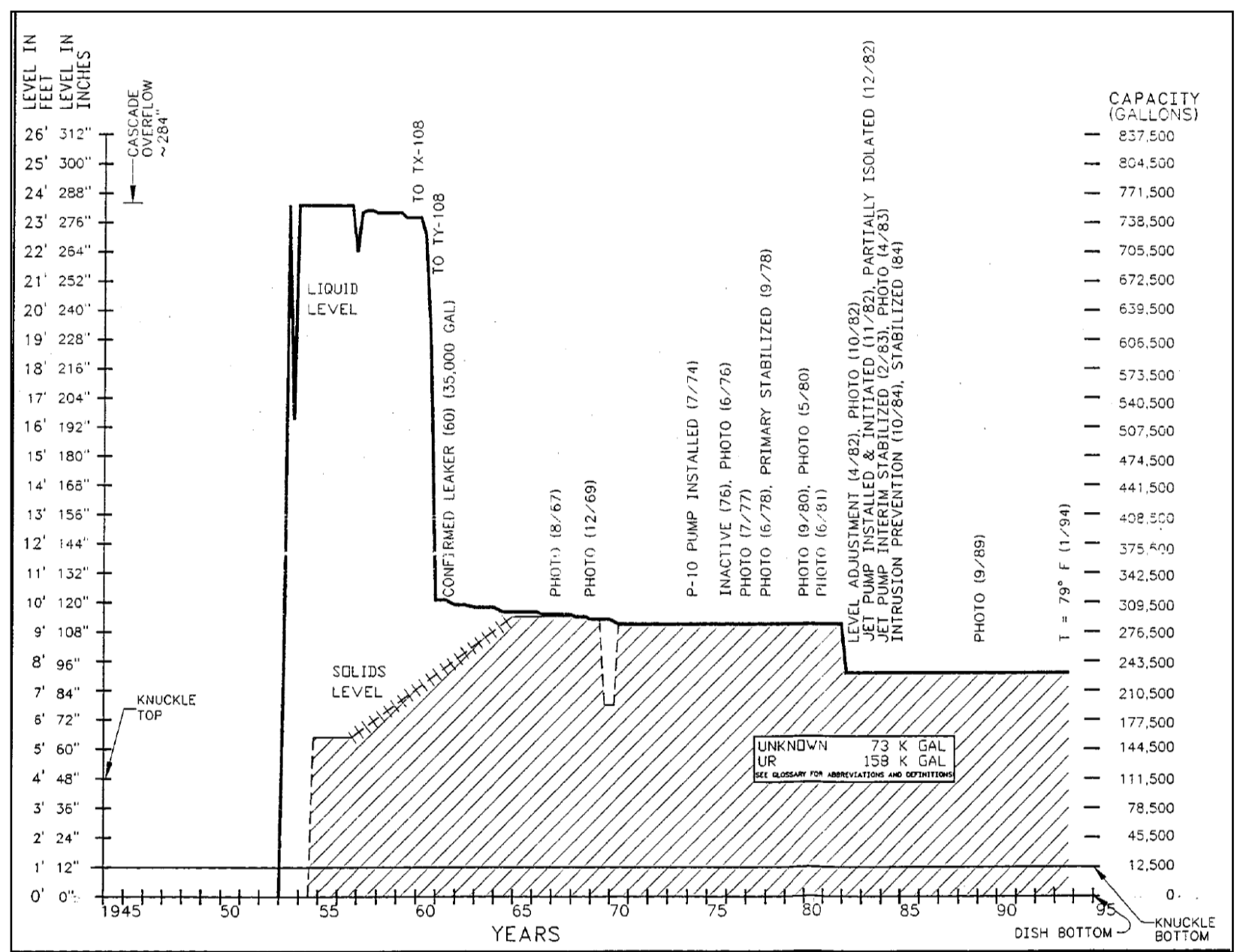

Reference: WHC-SD-WM-ER-351, Rev 0, March 1, 1995, Historical Tank Content Estimate for the Northwest Quadrant of the Hanford 200 West Area

Note: Tank TY-108 does not exist as referred to in this figure, the last transfer out of tank TY-105 was sent to tank TX-118 (HW-68292)

The last transfer into tank TY-105 occurred in January 1954 when the liquid level was reported to be at $758 \mathrm{kgal}$ at a waste height of $~ 283$-in which included the 12-in dished bottom. Liquid levels appeared to be holding steady at this level through December 1956 (HW-47640). There were discrepancies in volumes beginning in January 1957 to June 1957 when volumes were recorded to have dropped to $\sim 700 \mathrm{kgal}(\sim 262$-in). However, a new electrode reading in June 1957 and liquid levels were reported at $750 \mathrm{kgal}(\sim 280$-in) and remained relatively stable through July 1959 (HW-51348).

Liquid levels were recovered with the Waste Handling and Decontamination Operations (WH\&DO) electrode from April 30, 1958 to the end of October 1960 and with the Stiver's recorder in September and October 1960 (see next paragraph), which was placed in service in tank TY-105 on September 14, 1960 (see Figure 7-4). Tank TY-105 was first reported to be a suspected leaker in September 1960 due to liquid level decreases. Internal memo, "105-TY Waste Storage Tank" states, "The electrode has shown a decrease of 2-in (5,500 gal) since the end of August and daily checks, instituted September 15, show a loss of about 1/2-in per week. 


\section{RPP-RPT-54911, Rev. 0}

The electrode readings are being confirmed by an independent measuring device developed by FEO and which is calibrated to 1/16-in. Drywells around the tank have been examined and no evidence of a leak has been found." It appears the liquid level could have started decreasing as early as July 1959 as shown in Figure 7-4. Decreasing liquid levels during this period could have at least partially been the result of evaporation; however, temperatures were not available to confirm the possible extent of evaporation. Document HW-72858, Interim Report Waste Tank Liquid Level Measurement Systems 200-E and 200-W Areas, states, "Reviewing the liquid level records on this tank," [i.e., tank TY-105], "as taken by the manual electrode method, and knowing that a leak existed, a possible trend was indicated that the tank may have been leaking for 6 months or longer."

The independent measuring device referenced in the preceding paragraph (which was assumed to be the Stiver's recorder listed in Internal memo, "105-TY Waste Storage Tank") was an experimental dynamic displacer type instrument that was placed into tank TY-105 in September 1960 (HW-74174, Final Report Liquid Level Gauging System for Radioactive Wastes Storage Facilities 200 Areas). The system utilized a force balance principle to seek the liquid surface and was calibrated to $\pm 1 / 16$-in (HW-74174; Internal memo, "105-TY Waste Storage Tank"). It was reported later in 1963 in document HW-72858 that this liquid level measuring system was found to be "unsuitable for operation at Hanford" (HW-72858).

Internal memo, “105-TY Waste Storage Tank Leak" states daily liquid level checks were "instituted and have confirmed a small (approx. $180 \mathrm{gal} /$ day) but consistent loss from the tank. Readings obtained with the electronic device are in excellent agreement with electrode measurements. Total loss from the tank is indicated to be somewhere between 8,000 and 27,000 gallons." It remains unclear which liquid level probe (WH\&DO electrode or Stiver's Recorder) was more accurate; however, the important thing to note is the rate of liquid level decrease is nearly the same with both the probes indicating a leak from tank TY-105.

It appears that the tank TY-105 liquid level started decreasing at a slow rate, $\sim 26$ gal/day from July 30, 1959 to August 30, 1960, and increased in September 1960 to 175 gal/day (average of both sets of data) as indicated from the data graphed in Figure 7-4. As a result, the supernatant from tank TY-105 was transferred to tanks TX-108 and TX-118 in November and December 1960 leaving the liquid level at 113-in. Liquid levels continued to slowly decline from February 1961 through August 1974 to 27 kgal (RPP-RPT-42296) with saltwell pumping. However, the manual tape used to measure liquid level was reported to be contacting solid waste surfaces within the tank, raising uncertainty about the actual liquid level decline during this time. Evaporation may also have contributed to the liquid level decrease (RPP-RPT-42296). 
RPP-RPT-54911, Rev. 0

Figure 7-4. Tank TY-105 Liquid Level April 30, 1958 to October 27, 1960

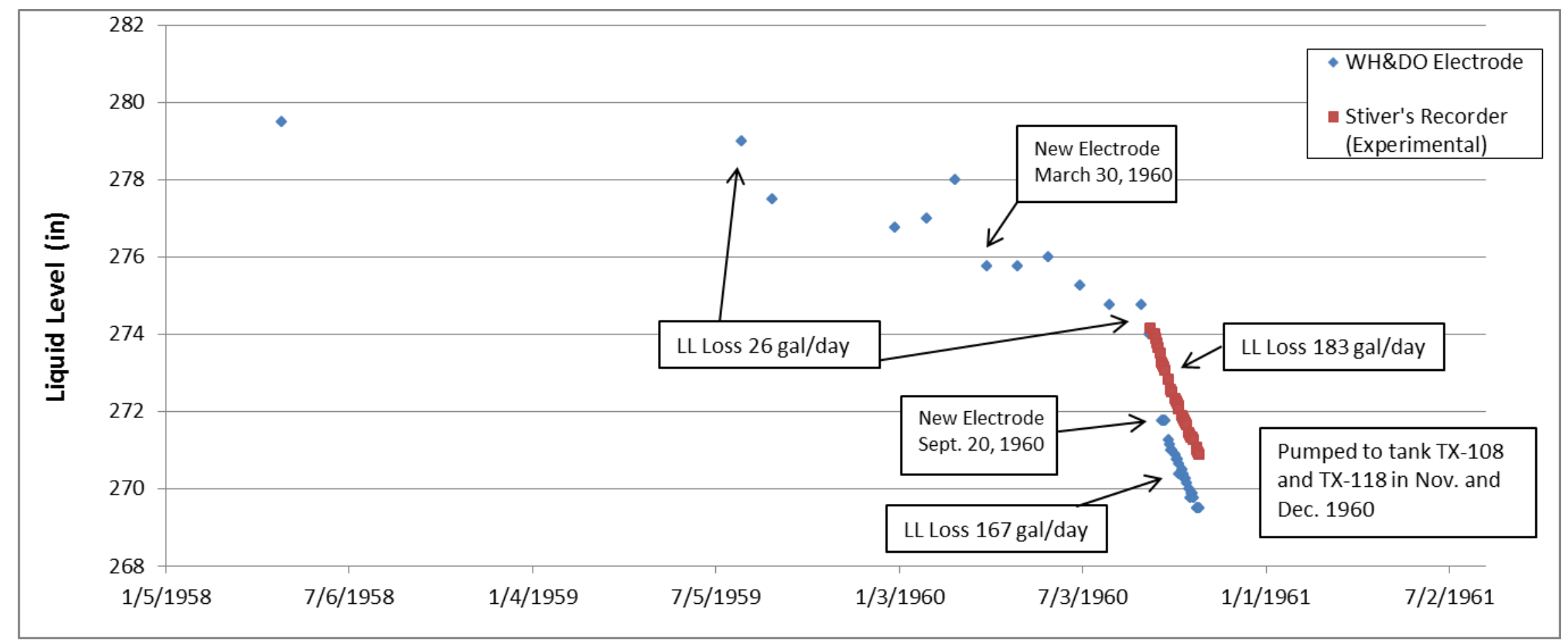

Referenced from Internal memo, "Electrode History on Tank 105-TY" from J.A. Forest to M. L. Short

Note: Liquid level measurements include the 12-in dished bottom 
RPP-RPT-54911, Rev. 0

\subsubsection{Temperature}

No temperature data were recovered for tank TY-105 from January 1953 when the tank was first put into service until 1977 (Historical Tank Content Estimate). The maximum temperature in 1977 was $99^{\circ} \mathrm{F}$ (RPP-RPT-42296).

Tank TY-105 first received TBP waste from 221-U Plant in January 1953. TBP wastes were concentrated and cooled to $\sim 180^{\circ} \mathrm{F}$ within the plant and were estimated to be $110-180^{\circ} \mathrm{F}$ after routing to the storage tanks. Note that $5,000 \mathrm{kgal}$ were routed through the tank to tank TY-106 through the cascade line over a two year period. This would indicate the temperature was near $180^{\circ} \mathrm{F}$ for a significant portion of the two year period. Rate of rise temperatures during initial tank filling are unknown.

\subsubsection{Liner Observations}

No liner observations relating to a tank TY-105 leak have been found.

\subsubsection{Chemistry-Corrosion}

Tank TY-105 began receiving TBP waste from 221-U Plant in January 1953 and only stored TBP waste throughout operation as shown in Table 7-1. The typical concentrations for nitrite, nitrate, and hydroxide for TBP waste is shown in Table 7-2. Nitrite and hydroxide are known as nitrate-induced SCC inhibitors. One key characteristic for inhibiting SCC is to maintain a high nitrite concentration to nitrate concentration ratio (see Section 3.2.4).

Table 7-1. Tank TY-105 Waste Storage Chronology

\begin{tabular}{|c|c|c|}
\hline Date & Waste Type & Length of Storage \\
\hline January 1953 to November 1960 & TBP & $\sim 8$ years \\
\hline
\end{tabular}

Table 7-2. Waste Chemistries for Waste Types Stored in Tank TY-105

\begin{tabular}{|c|c|c|c|c|}
\hline Waste Types & {$\left[\mathrm{NO}_{3}{ }^{-}\right]$} & {$\left[\mathrm{NO}_{2}{ }^{-}\right]$} & {$\left[\mathrm{OH}^{-}\right]$} & $\begin{array}{c}\text { Meets Current } \\
\text { DST Specification }\end{array}$ \\
\hline $\mathrm{TBP}^{1}$ & 7.35 & Not Reported & 0.09 & $\mathrm{No}^{2}$ \\
\hline
\end{tabular}

1. Reference WHC-EP-0449, 1991, The Sort on Radioactive Waste Type Model: A Method to Sort Single-Shell Tanks into Characteristic Groups.

2. Even with no reported value for nitrite, the ratio of nitrate to nitrite and hydroxide would still be less than 2.5 as stated in the current DST specification.

3. Reference OSD-T-151-00007, Rev. 12, (2013), Operating Specifications for the Double-Shell Storage Tanks.

Tank TY-105 was first suspected of leaking in September and October 1960 due to liquid level decreases; however, the tank leak could have begun as early as July 1959. The first waste tank TY-105 received in January 1953 was TBP waste from 221-U Plant and the tank only stored this waste type for approximately 8 years until the tank began leaking.

Flowsheets indicate TBP waste hydroxide concentrations were below $0.1 \mathrm{M}$ and nitrate concentrations above 6M. The high nitrate concentration violates the current DST specification 
for waste chemistry as nitrate must be below 5.5M. These conditions of the TBP waste would likely create an environment conducive to SCC and/or pitting (see Section 3.2.4).

\subsubsection{Photographs}

Earliest available photographs taken December 22, 1969 of tank TY-105 were reviewed and no evidence of bulging was found. No other documentation was found indicating a liner bulge in tank TY-105.

\subsection{TANK TY-105 EX-TANK DATA}

\subsubsection{Drywells}

There are four drywells located around tank TY-105: 52-06-05 installed in 1952; 52-03-06 installed in December 1971; 52-05-07 and 52-06-04 installed in August 1974. All of the radiation readings in drywells are assumed to be maximum or peak readings unless otherwise noted (see Sections 3.3 and 3.4). The following subsections report the available drywell information and the drywell summary section provides the analyses of the associated drywells with tank TY-105.

\subsubsection{Drywell 52-03-06 $\left(\mathrm{S0}^{\circ}\right)$}

Drywell 52-03-06 was drilled December 31, 1971, 11 years after the tank TY-105 leak was detected, with radiation levels recorded as less than values prior to August 1972 (see Appendix B4). No drywell records were recovered from August 1972 until July 16, 1973 when readings were reported at $780 \mathrm{~K}$ cpm with the SP at 55-ft BGS. Radioactivity continued to remain relatively stable until February 8, 1974 when an additional peak appeared at 64-ft BGS at 204.6K cpm with the SP. Radioactivity remained relatively stable through June 1986.

In April 1997, Cs-137 and Co-60 were the only man-made radionuclides detected in drywell 5203-06 (GJ-HAN-68). Cs-137 was detected from the ground surface to 5-ft BGS, from 8.5 to 14.5-ft BGS, and from 56 to 61-ft BGS with the maximum concentration of $1.5 \mathrm{pCi} / \mathrm{g}$ detected at the ground surface. Co-60 was detected continuously from 54 to $100-\mathrm{ft}$ BGS with the maximum concentration of $36.8 \mathrm{pCi} / \mathrm{g}$ detected at $99.5-\mathrm{ft}$ BGS.

Drywell 52-03-06 is located in between tanks TY-103 and TY-105. It is likely radioactivity in this drywell is associated with the leak from tank TY-103 due to similar timing and depth (see tank TY-103 Segment). Also, radioactivity was reported as less than values prior to August 1972, and tank TY-105 was first suspected of leaking in September 1960 due to liquid level decreases (see Tank TY-103 segment). Therefore, drywell 52-03-06 is not included in the leak location for tank TY-105. Figure 7-5 shows the depths of radioactivity from 1975 to 1995 (HNF-3831). 
RPP-RPT-54911, Rev. 0

Figure 7-5. Tank TY-105 Associated Drywell 52-03-06 (HNF-3831)

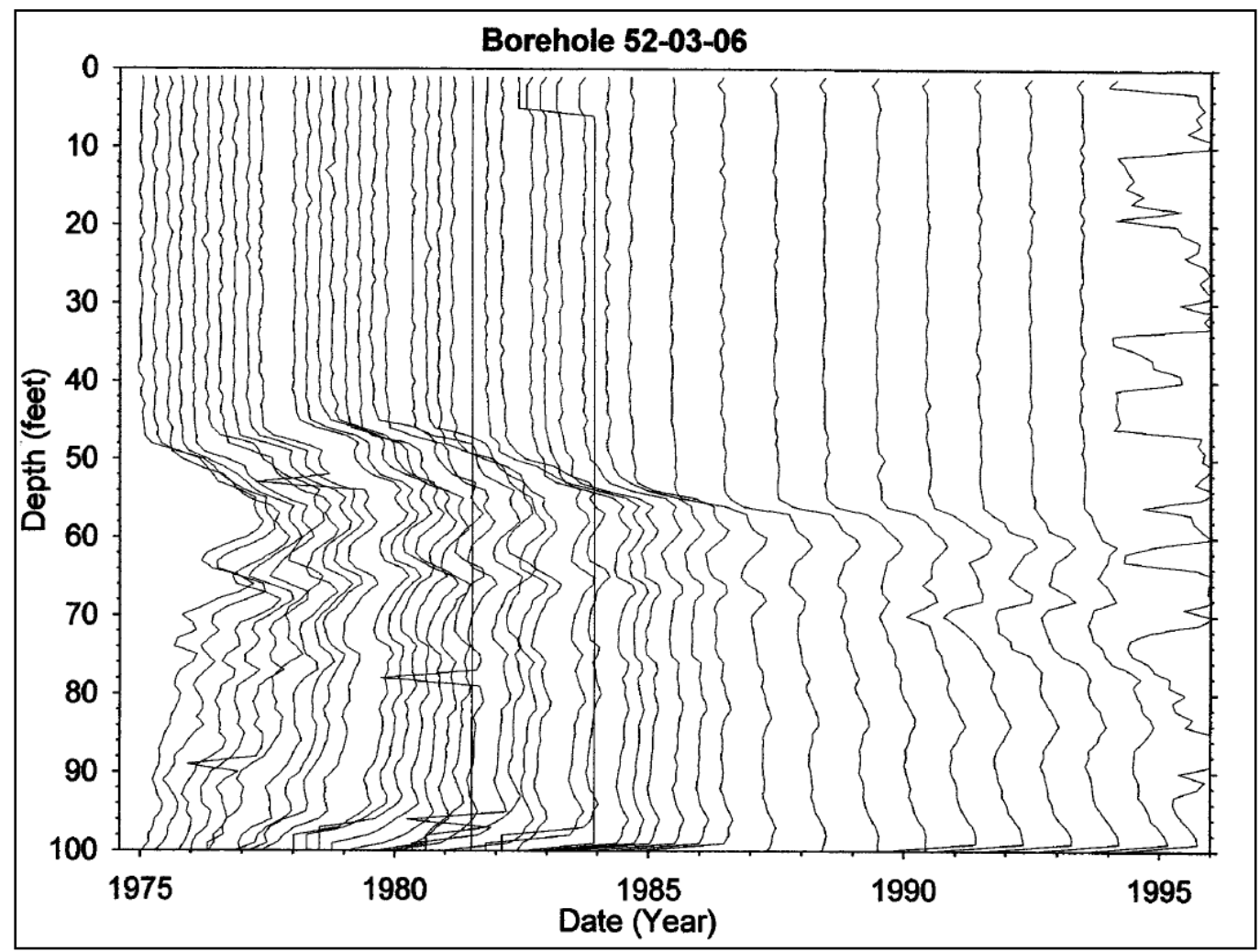

Note: Bottom of the tank footing is 46-ft BGS

\subsubsection{Drywell 52-05-07 $\left({\left.\mathrm{S} 25^{\circ} \mathrm{W}\right)}^{\circ}\right.$}

Drywell 52-05-07 was drilled in August 1974, approximately 14 years after the tank TY-105 leak was first detected. The first recoverable reading for drywell 52-05-07 was reported on September 13, 1974 at $103.4 \mathrm{~K} \mathrm{cpm}$ at $62-\mathrm{ft}$ BGS. Radiation readings remained relatively stable at depths ranging from 58-ft to 71-ft BGS through January 1975. Beginning in February 1975 radiation readings began to slowly decline through June 1986 at depths around 57-ft BGS (see Appendix B4).

In April 1997, Cs-137 and Co-60 were the only man-made radionuclides detected in drywell 5205-07 (GJ-HAN-68). Cs-137 was detected from the ground surface to 76.5-ft BGS and from 87 to 88 -ft BGS with the maximum concentration of $32.4 \mathrm{pCi} / \mathrm{g}$ detected at $52.5-\mathrm{ft}$ BGS. Co-60 was detected from 1 to 2.5 -ft BGS, 5.5 to 7-ft BGS, and from 51.5 to 97-ft BGS with the maximum concentration of $7.1 \mathrm{pCi} / \mathrm{g}$ reported at $62-\mathrm{ft}$ BGS. The historical gross gamma logs show three peaks between 55 and 70-ft (HNF-3831). The SGLS measured Cs-137 and Co-60 contamination between 51 and 97-ft BGS correlates with the historical gross gamma logs. As stated in GJHAN-68, "the contamination detected around borehole 52-05-07 probably originated from a leak from tank TY-105." Figure 7-6 shows the depths of radioactivity from 1975 to 1995 (HNF$3831)$. 
RPP-RPT-54911, Rev. 0

Figure 7-6. Tank TY-105 Drywell 52-05-07 (HNF-3831)

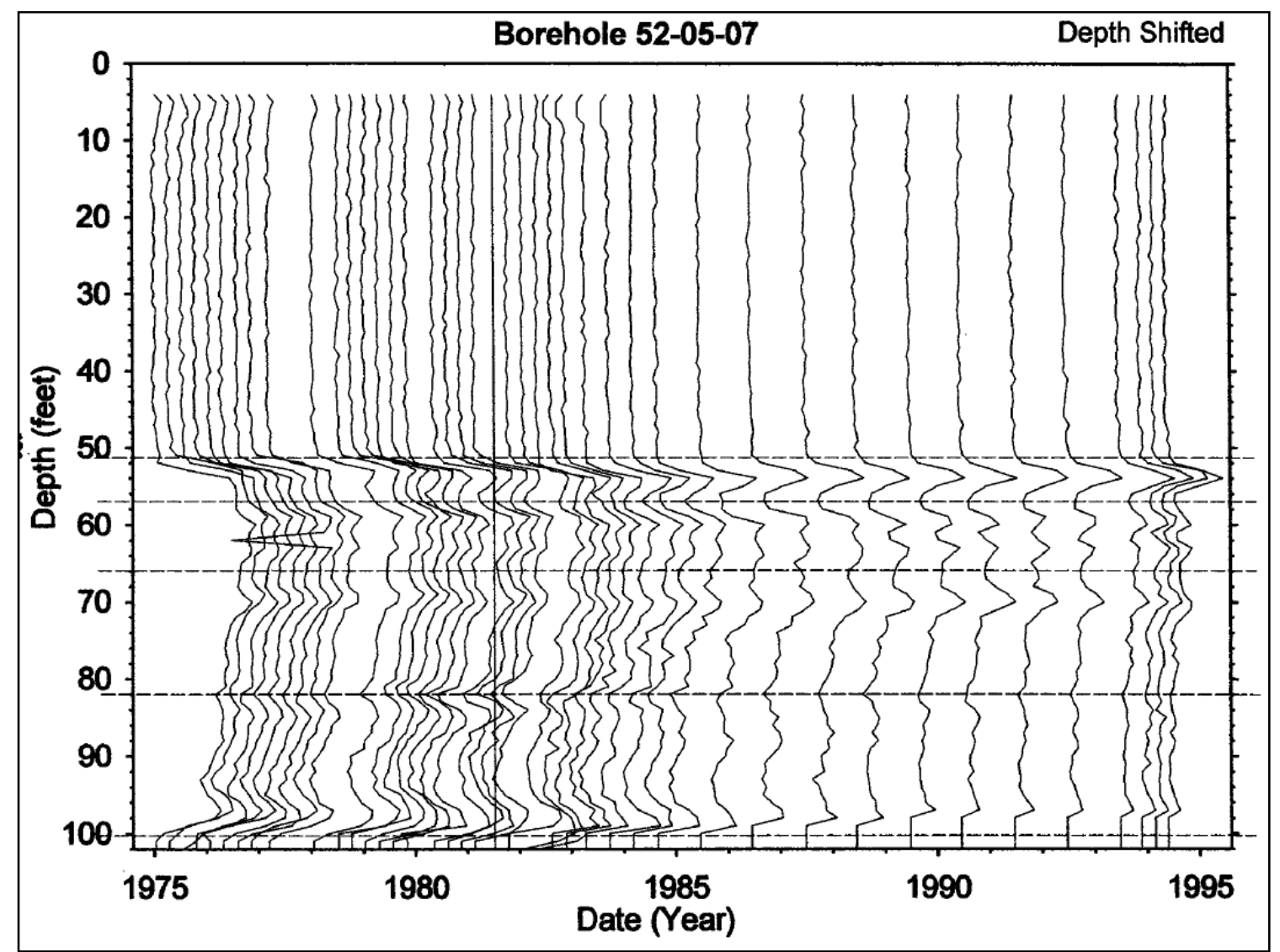

Note: Bottom of the tank footing is $\sim 46-\mathrm{ft}$ BGS. Depth shifting is a method to align the zero point due to inconsistent data collection.

\subsubsection{Drywell 52-06-05 $\left(\mathrm{S} 45^{\circ} \mathrm{E}\right)$}

Drywell 52-06-05 was drilled in August 1952 to a depth of 150-ft 31-ft from tank TY-105 and was the only drywell present at the time the tank TY-105 leak was first detected in 1960. The first recoverable reading was reported on July 16, 1973 at $67.2 \mathrm{~K} \mathrm{cpm}$ at $62-\mathrm{ft}$ BGS. Radiation readings were relatively stable at this depth with an additional peak detected December 18, 1973 at $67.8 \mathrm{~K} \mathrm{cpm}$ at 52-ft BGS. Radioactivity appeared to remain stable until July 1974 and then slowly decreased through June 1986 (see Appendix B4).

There have been previous indications that activity was present in drywell 52-06-05 as early as September 1959; however, after reviewing Internal memo, "105-TY Waste Storage Tank Leak," it seems likely early radioactivity was referring to drywell 52-06-07 which is located near tank TY-106 (see Tank TY-106 Segment).

Two occurrence reports were written for increased activity in drywell 52-06-05 (OR-74-102, Leakage Symptoms as Indicated by Dry Well Activity at Waste Tank 105-TY and OR-74-88, Symptoms Of Leakage As Indicated By Activity In Dry Well 52-06-05 At 106-TY

Waste Tank). Occurrence report OR-74-88 identifies the source of the drywell 52-06-05 contamination as tank TY-105. 
In April 1997, Cs-137 and Co-60 were the only man-made radionuclides detected in drywell 5206-05 (GJ-HAN-68). Cs-137 was detected from the ground surface to 133.5-ft BGS with the maximum concentration of $1.2 \mathrm{pCi} / \mathrm{g}$ reported at $111.5-\mathrm{ft}$ BGS. Co-60 was detected at $2.5-\mathrm{ft}$ BGS, from 52 to $90-\mathrm{ft}$ BGS, from 92.5 to 98.5 -ft BGS, and from 130 to 148-ft BGS with the maximum concentration of $3.2 \mathrm{pCi} / \mathrm{g}$ reported at $144.5-\mathrm{ft} \mathrm{BGS}$.

Radioactivity detected in drywell 52-06-05 appears to be the fairly mobile radioisotopes- short lived Ru-106 and also Co-60 both of which decayed away (see Figure 7-7). The tank TY-106 direct pushes near drywell 52-06-05 did not indicate radioactivity down to $47-\mathrm{ft}$ BGS and were not deepened as there was no indication of further radioactivity (RPP-RPT-39511). Since there was no Cs-137 detected near tank TY-106, indications are that the radioactivity detected in drywell 52-06-05 was the leading edge (mobile radioisotopes) from a tank TY-105 leak. Figure 7-8 shows depths of radioactivity from 1975 to 1994 (HNF-3831).

Figure 7-7. Tank TY-105 Associated Drywell 52-06-05 Cesium (HNF-3831)

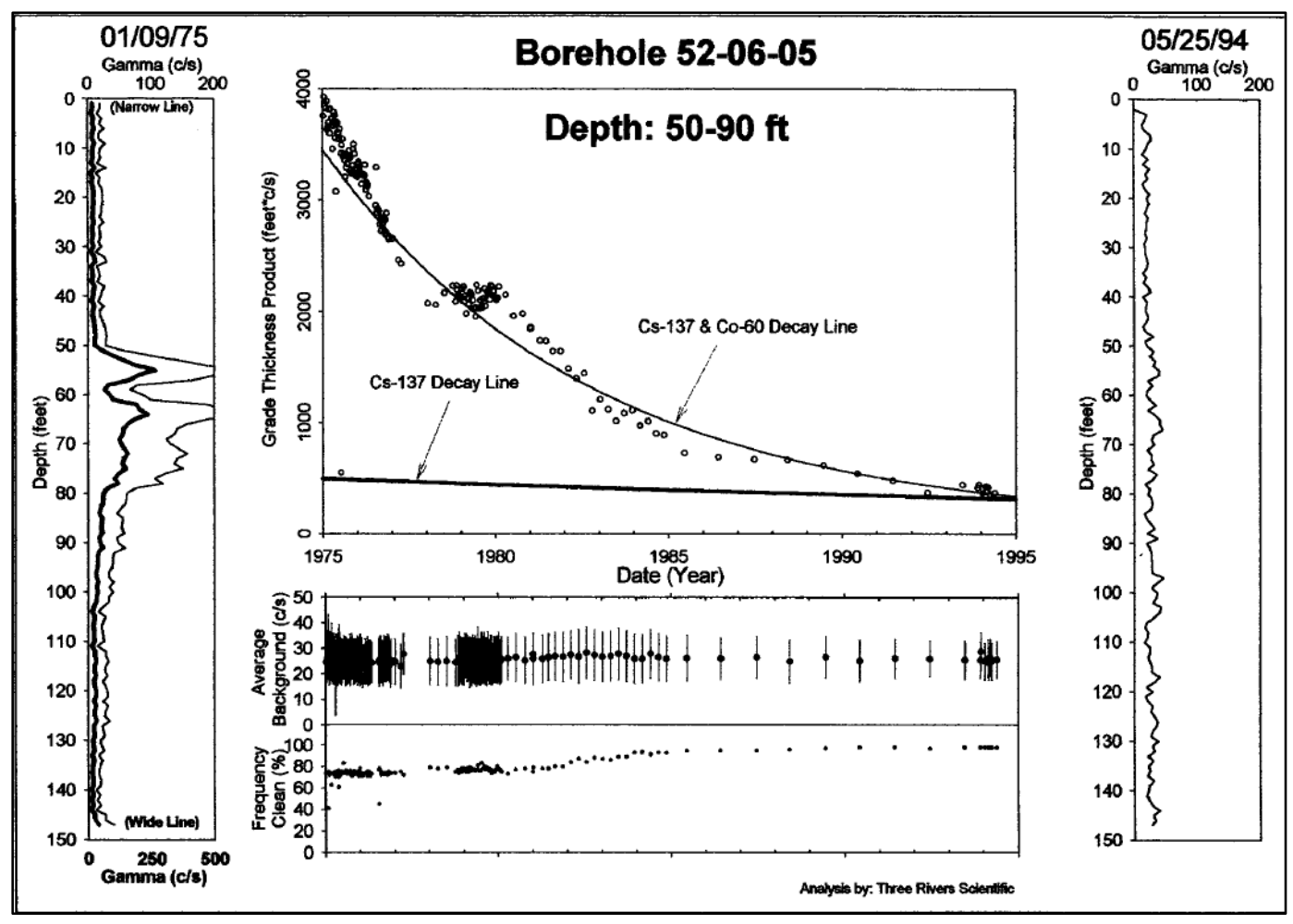


RPP-RPT-54911, Rev. 0

Figure 7-8. Tank TY-105 Associated Drywell 52-06-05 (HNF-3831)

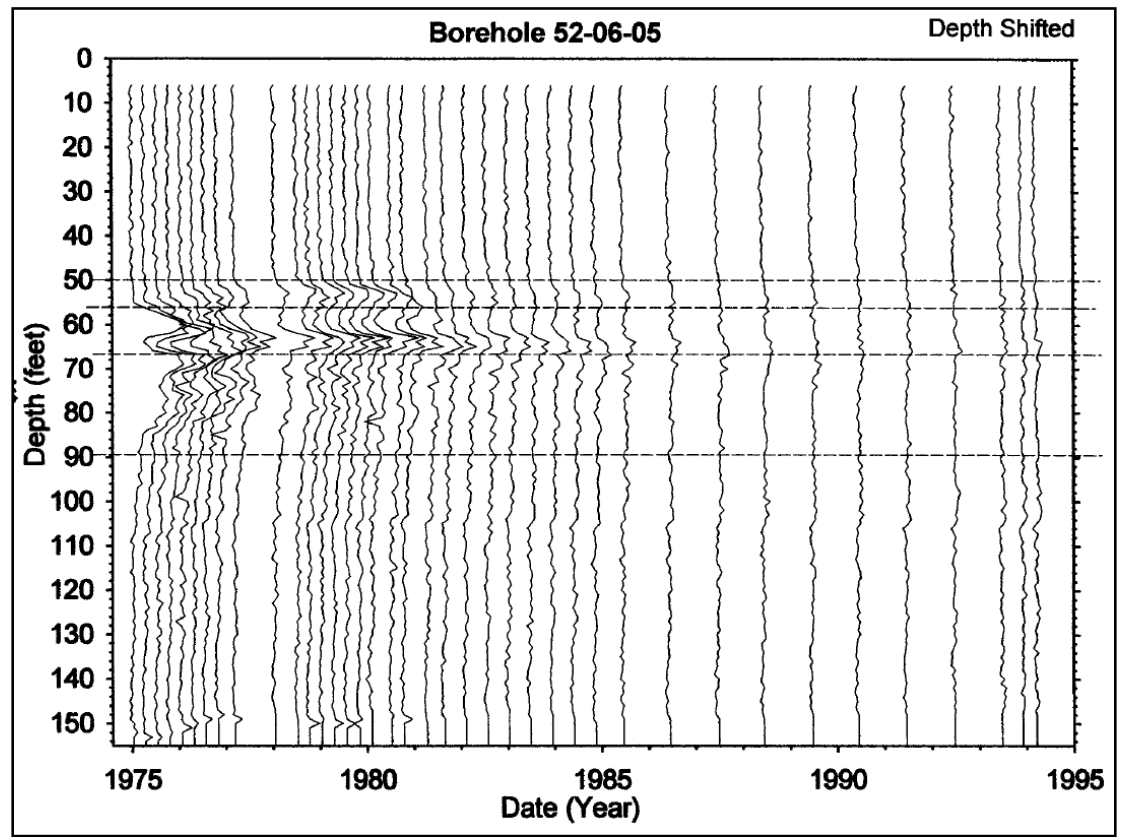

Note: Bottom of the tank footing is $\sim 46-\mathrm{ft}$ BGS. Depth shifting is a method to align the zero point due to inconsistent data collection. 


\subsubsection{Drywell 52-06-04 $\left(\mathrm{S}^{\circ} 1^{\circ} \mathrm{E}\right)$}

Drywell 52-06-04 was drilled in August 1974, approximately 14 years after the tank TY-105 leak was first detected with the first recoverable reading on September 13, 1974 at $8.3 \mathrm{~K} \mathrm{cpm}$ at 53 -ft BGS. Radiation readings gradually declined to $2.5 \mathrm{~K}$ cpm by June 1986 with depths reported at 50-ft BGS from 1974 to 1986 (see Appendix B4).

In April 1997, Cs-137 was the only man-made radionuclide detected in drywell 52-06-04 (GJHAN-68). Cs-137 was detected from the ground surface to 53.5-ft and at 57-ft BGS with the maximum concentration of $1.2 \mathrm{pCi} / \mathrm{g}$ reported at $52-\mathrm{ft}$ BGS. Document GJ-HAN-68 states, "it is possible that the Cs-137 contamination originated from a breach in either tank TY-105 or TY106." However, it seems likely radioactivity detected in this drywell was the result of the tank TY-105 leak (see Section 7.5.3). Radioactivity from 47-56-ft BGS was reported to be stable in document HNF-3831. Figure 7-9 shows depths of radioactivity from 1975 to 1994 (HNF-3831).

Figure 7-9. Tank TY-105 Associated Drywell 52-06-04 (HNF-3831)

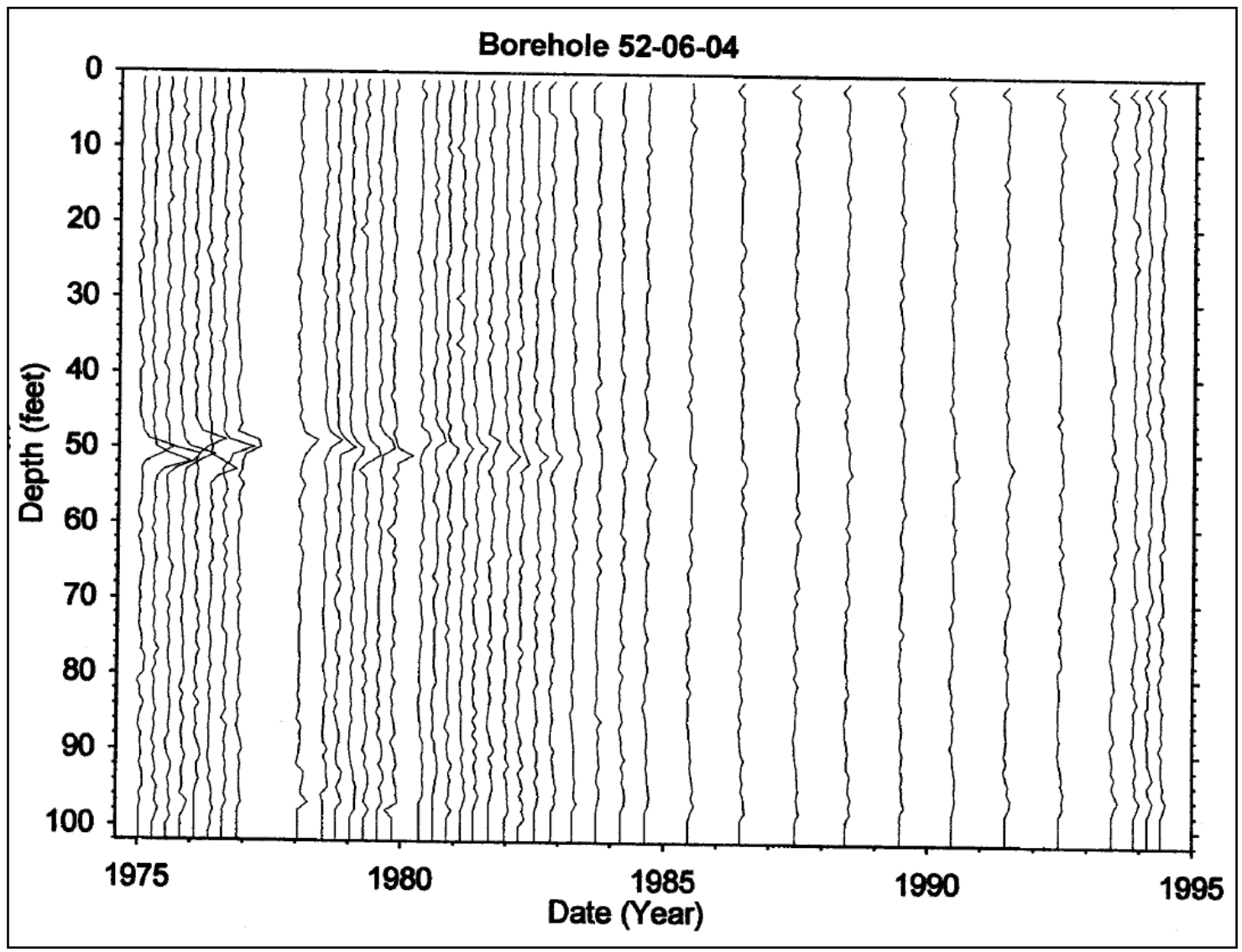

Note: Bottom of the tank footing is 46-ft BGS 


\subsubsection{Drywell Summary}

Tank TY-105 was first suspected of leaking in September 1960 due to liquid level decreases; however, the leak may have started as early as July 1959.

Radioactivity detected in drywell 52-03-06 is likely the result of the tank TY-103 leak due to similar timing and depth (see tank TY-103 Segment). Therefore, drywell 52-03-06 is not included in the leak location for tank TY-105.

The remaining three drywells all detect radioactivity that could be associated with a tank leak. However, data were recovered for these drywells 13-14 years after the tank TY-105 leak was first detected. Only one drywell, 52-06-05, was located near tank TY-105 at the time when the leak was first detected. The first recoverable reading for drywell 52-06-05 reported radioactivity July 1973 at 62-ft BGS with an additional peak detected five months later at 52-ft BGS. The first recoverable reading for drywell 52-05-07 reported radioactivity in September 1974 at 62-ft BGS, and the first recoverable readings for drywell 52-06-04 reported lower levels of radioactivity in September 1974 at 53-ft BGS. The direct pushes near tank TY-106 indicate very little radioactivity was detected on the southeast portion of tank TY-106 (see Tank TY-106 Segment). Therefore, it appears earlier radioactivity detected in drywells 52-06-05, 52-06-04, and 52-05-07 (see Section 7.5.1), located between tanks TY-105 and TY-106, were likely due to the tank TY-105 leak with the possibility of migration from the leak from tank TY-106.

There are no drywells present on the northeastern or northwestern edge of tank TY-105, so it remains unclear whether radioactivity is present in this area. Additional direct pushes were installed in 2005 as follows.

\subsubsection{Direct Push Logging}

Direct push logging to further characterize the tank TY-105 leak was conducted in October 2005 with the locations and maximum activity of the direct pushes shown in Figure 7-1 and Table 7-3 (RPP-RPT-34870, Small Diameter Geophysical Logging in the 241-TY Tank farm). Direct pushes were only logged once and only represent one profile in time. Compared to the drywells, direct pushes use a smaller diameter logging tool and results cannot be directly compared to drywell results. 
RPP-RPT-54911, Rev. 0

Table 7-3. 2005 Direct Push Gross Gamma Results (RPP-RPT-34870)

\begin{tabular}{|l|c|c|c|c|l|}
\hline $\begin{array}{c}\text { Direct } \\
\text { Push }\end{array}$ & $\begin{array}{c}\text { Initial } \\
\text { Hole } \\
\text { Depth (ft) }\end{array}$ & $\begin{array}{c}\text { Extend } \\
\text { Hole } \\
\text { Depth (ft) }\end{array}$ & $\begin{array}{c}\text { Depth Max. } \\
\text { Activity (ft } \\
\text { BGS) }\end{array}$ & $\begin{array}{c}\text { Max eCs } \\
\text { (pCi/g) }\end{array}$ & Comment \\
\hline C4619 & 47.2 & - & - & Background & \\
\hline C4621 & 47.1 & - & Surface & 16 & Minor count rate increase at surface \\
\hline C4623 & 47.1 & - & 43.5 & 90 & \\
\hline C4625 & 47.5 & - & - & Background & \\
\hline C4637 & 47.5 & 61.5 & 52 & $4,800,000$ & NaI Detector Saturated 51.5-53 ft \\
\hline C5016 & 66 & - & 57 & 14 & \\
\hline C5018 & 65 & - & $24.5 \& 52$ & $\begin{array}{c}790,000 ~ \& ~ \\
2,000,000\end{array}$ & $\begin{array}{l}\text { NaI Detector Saturated 23-26 and 51- } \\
53 \mathrm{ft}\end{array}$ \\
\hline C5020 & 65 & - & 53 & 16 & \\
\hline
\end{tabular}

Note: Direct pushes that did not indicate radioactivity at a lower level were not deepened.

Radioactivity was the highest in direct push $\mathrm{C} 4637$ with $4,800 \mathrm{~K} \mathrm{pCi} / \mathrm{g}$ detected at $52-\mathrm{ft}$ BGS. Direct push C5018 had two peaks at 790K and 2,000K, 24.5-ft and 52-ft BGS, respectively.

The peak radioactivity at $\sim 24.5$-ft detected in direct push C5018 (see Figure C4-2) could be from a liner leak or a leak out of one or both of the slip fit capped spare inlet lines (see Figure 7-10). The orientation of two of the spare inlet lines puts them directly in line with direct push C5018 which makes leakage from these lines a possible candidate for the peak at $\sim 24.5$-ft BGS. The lowest point (base) of the capped end of the spare inlet lines is at 11.2-in from the top of the liner ( 21-ft 21/2-in BGS). 


\section{Figure 7-10. Tank TY-105 Spare Inlet Nozzles}

(H-2-2250)

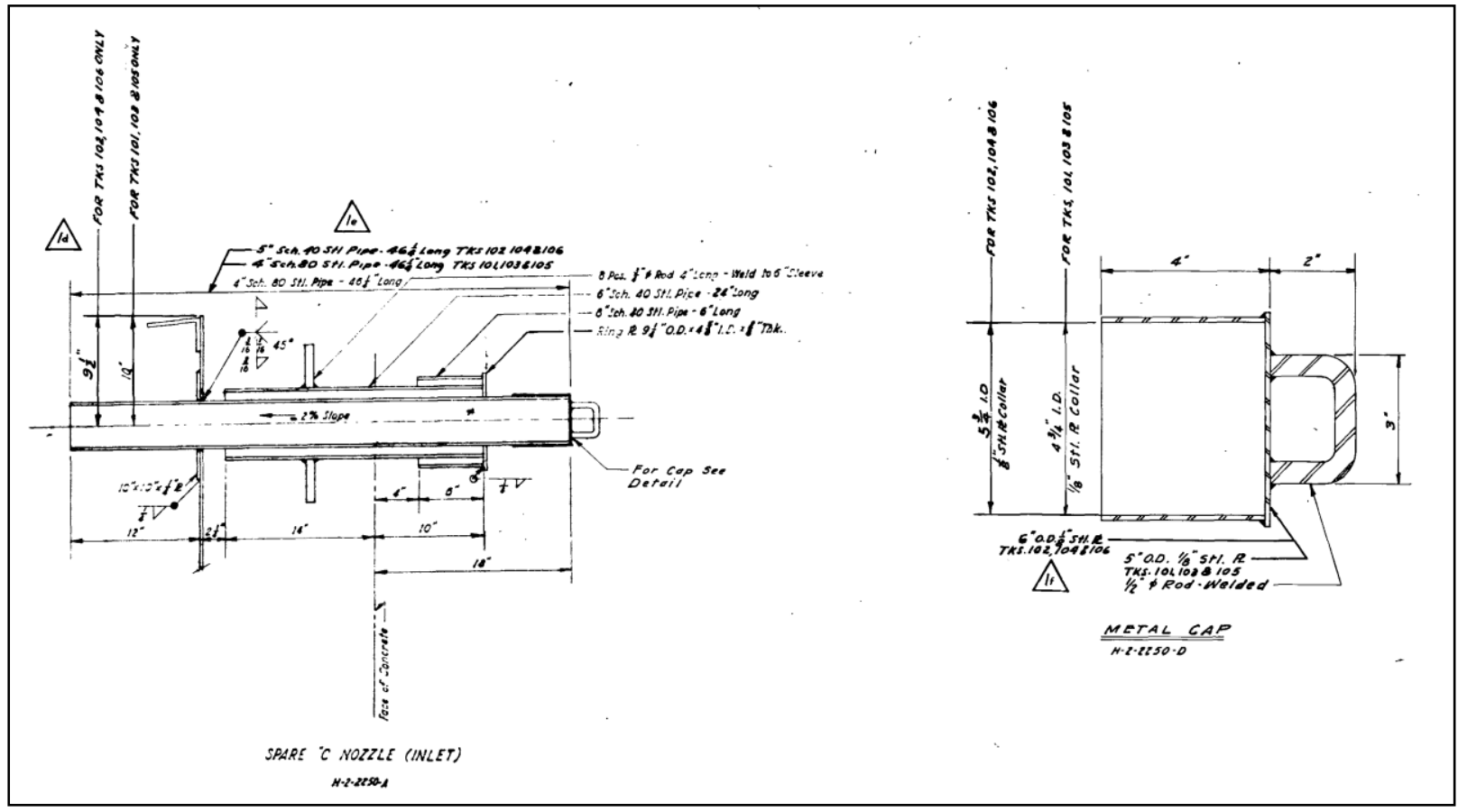

The TBP waste was batch transferred from 221-U Plant to the WR Vault and pumped at a nominal $50 \mathrm{gpm}$ to tank TY-105 which cascaded to tank TY-106. There is no record of overfilling tank TY-105 or plugging of the overflow cascade to tank TY-106 using the recovered quarterly liquid level data. No other liquid level data relative to the two year cascade period has been found. Over 5,000 kgal of TBP waste was transferred to tank TY-105 in batches which cascaded to tank TY-106. The possibility of backing up the liquid level $\sim 4$.4-in to the base of the spare inlet lines may exist (see Figure 7-11). 
RPP-RPT-54911, Rev. 0

Figure 7-11. Tank TY-105 Cascade Outlet Relative to Inlet(s)

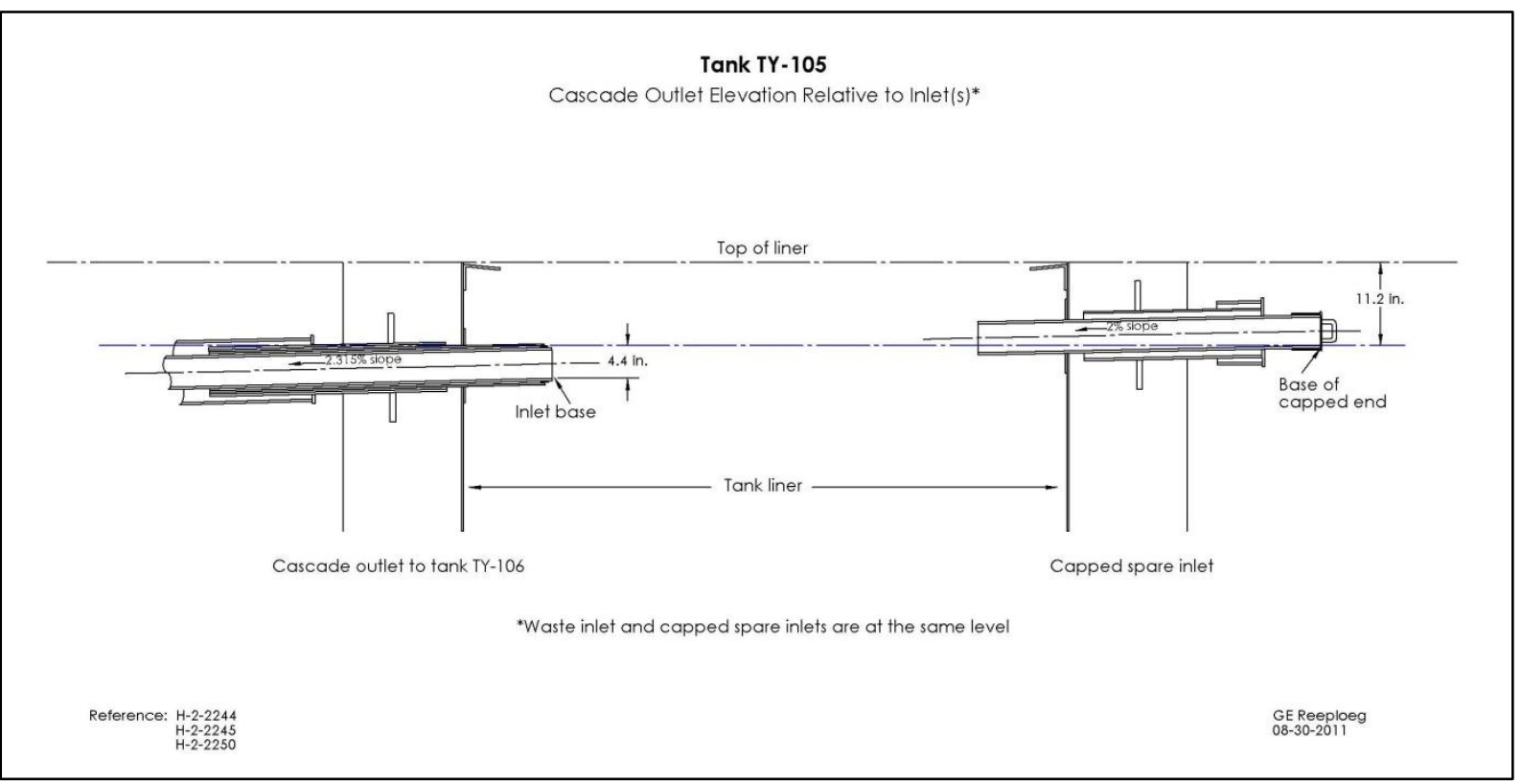

The slip fit capped spare inlet lines are sealed with a coating of asphalt which is covered with gunite (see Figure 7-12). Poor quality of the capped spare inlet seal system or damage during construction/backfilling could have compromised the seal resulting in the possibility of a leak should waste be backed up into the spare inlet at least to the base of the capped end.

\section{Figure 7-12. Tank TY-105 Spare Inlet Line Asphalt - Gunite Seal} (H-2-2277)

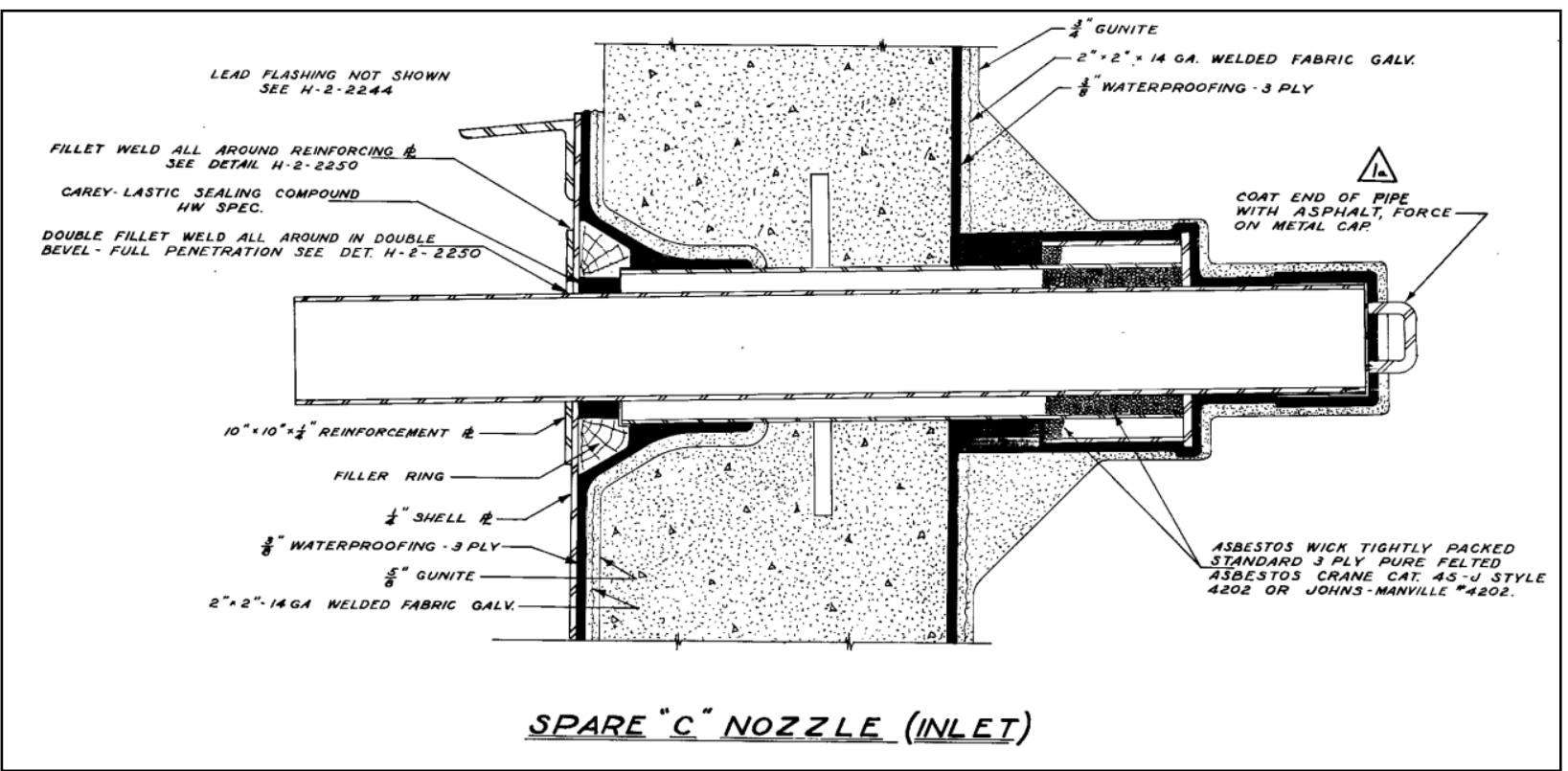

The leakage of the spare inlet lines seems possible; however, a number of conditions as indicated need to take place for this to happen. The other possibility is that the liner leaked above the 
24.5-ft level resulting in the peak at $\sim 24.5$-ft BGS. The evidence to date remains inconclusive; however, capped spare inlet lines have been suspected to leak in other tanks (HW-20742, Loss of depleted Metal Waste Supernatant to Soil).

With the exception of the peak located at 24.5-ft BGS, it appears the 2005 direct push logs indicate the tank TY-105 leaked near the tank footing close to direct pushes C4637 and C5018 as radioactivity detected in these pushes were the highest with possibly some migration to the other nearby drywells.

See Appendix C4 for the gamma surveys for the direct pushes near tank TY-105. See document RPP-RPT-34870 for additional gamma surveys for the direct pushes for TY Farm.

\subsection{POSSIBLE TANK TY-105 LINER LEAK LOCATION(S)}

A liner leak may have penetrated the waterproof membrane at any location and followed concrete cracks or breaks to a different location including the top of the tank footing. It is likely that the tank TY-105 leak(s) did not occur far above the tank footing because all of the initial indicators, three drywells, were located at or near the tank footing. However, only one drywell was present near tank TY-105 when the tank was first suspected of leaking and no data were recovered for this drywell until 13 years after the tank leaked. Also, there are no drywells located on the northwest and eastern side of tank TY-105. Therefore, it is possible early leaks could have occurred in these areas and not have been detected, such as leaks from the capped spare inlet lines at the northeast side of the tank. The 2005 direct pushes were installed on the southeast side of the tank to further characterize the leak(s) from tank TY-105 which identified additional leakage sites.

A leak from the spare inlet nozzles or a tank liner sidewall leak could have resulted in the direct push radioactivity detected at $\sim 24.5$-ft BGS in 2005 (see Section 7.5.3). 


\subsubsection{Leak Detected in 1973-1974, Site A}

Tank TY-105 was first suspected of leaking in September 1960 due to liquid level decreases; however, the leak may have started as early as July 1959. Liquid level decreases were confirmed in October 1960 and supernatant was removed beginning in November 1960. Only drywell 5206-05 was present at the time the leak was detected and no data were recovered for this drywell until 1973. Drywells 52-06-05, 52-05-07, and 52-06-04 all report radioactivity in 1973-1974 likely associated with the tank TY-105 leak (see site A in Figure 7-13). No drywells were present on the eastern and northwestern edge of tank TY-105. In 2005 direct pushes were installed in the south and southwest side of tank TY-105 to further characterize the leak(s). No radioactivity was detected in direct pushes C4619 and C4621.

Figure 7-13. Tank TY-105 Possible Leak Location (1973-1974)

Tank inner ring is steel liner, outer ring is outer edge of tank footing

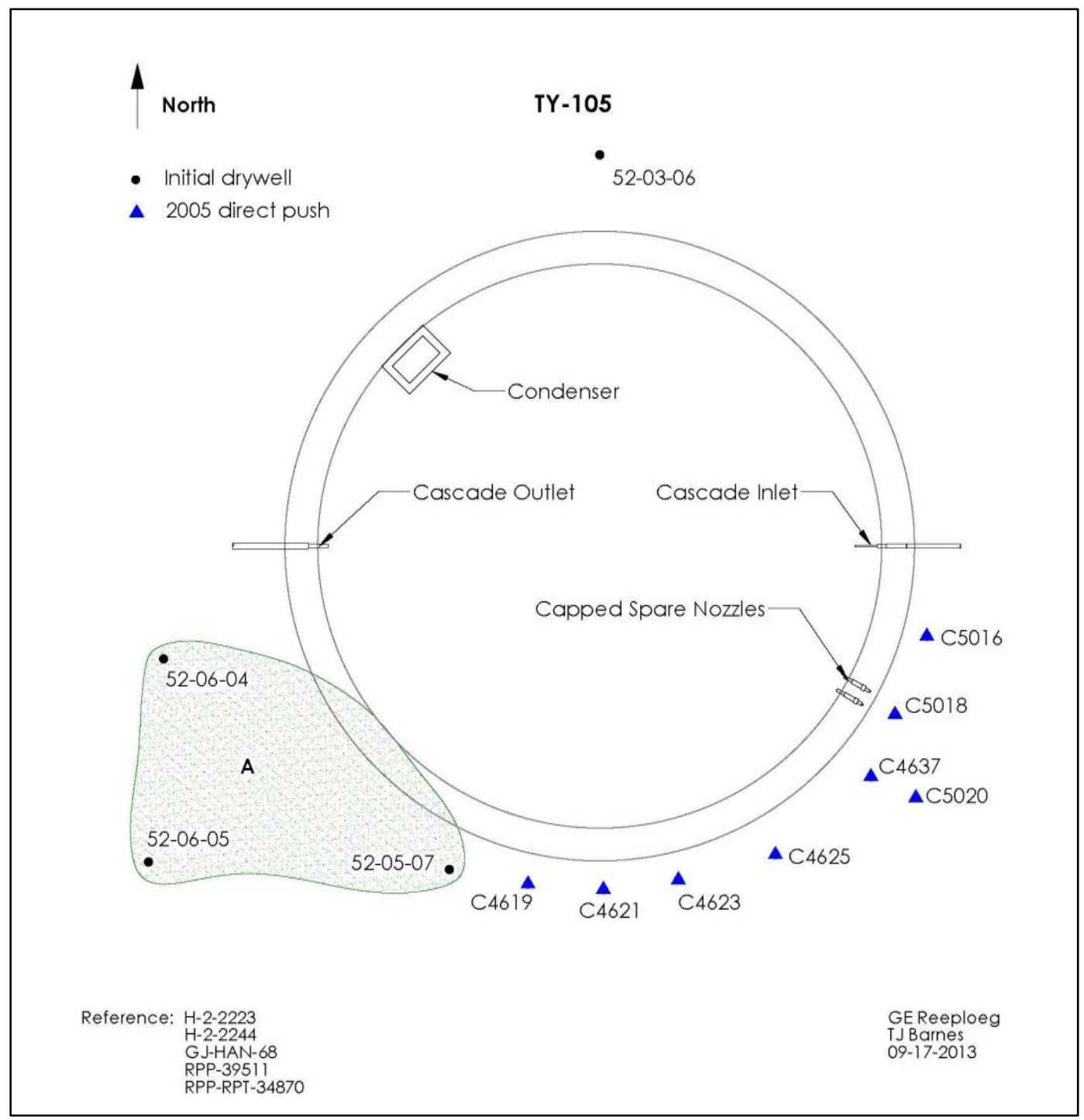

The leak from tank TY-105 was first detected in September 1960 due to liquid level decreases; however, the leak may have started as early as July 1959. 


\subsubsection{Leak Detected in 2005, Site B}

Direct pushes were installed in 2005 along the southeastern portion of tank TY-105 to further characterize the leak. Radioactivity was the highest in direct pushes C4637 and C5018 indicating the tank leaked near these pushes (see Site B in Figure 7-14). Lower levels of radioactivity were detected in the nearby direct pushes possibly indicating migration as the tank leaked 45 years earlier. It appears site B is a separate leak site from site A as no radioactivity were detected in direct pushes C4619 and C4621 (see Figure 7-13). A liner leak above 24.5-ft BGS near direct push C5018 is possible.

Figure 7-14. Tank TY-105 Possible Leak Location (2005)

Tank inner ring is steel liner, outer ring is outer edge of tank footing

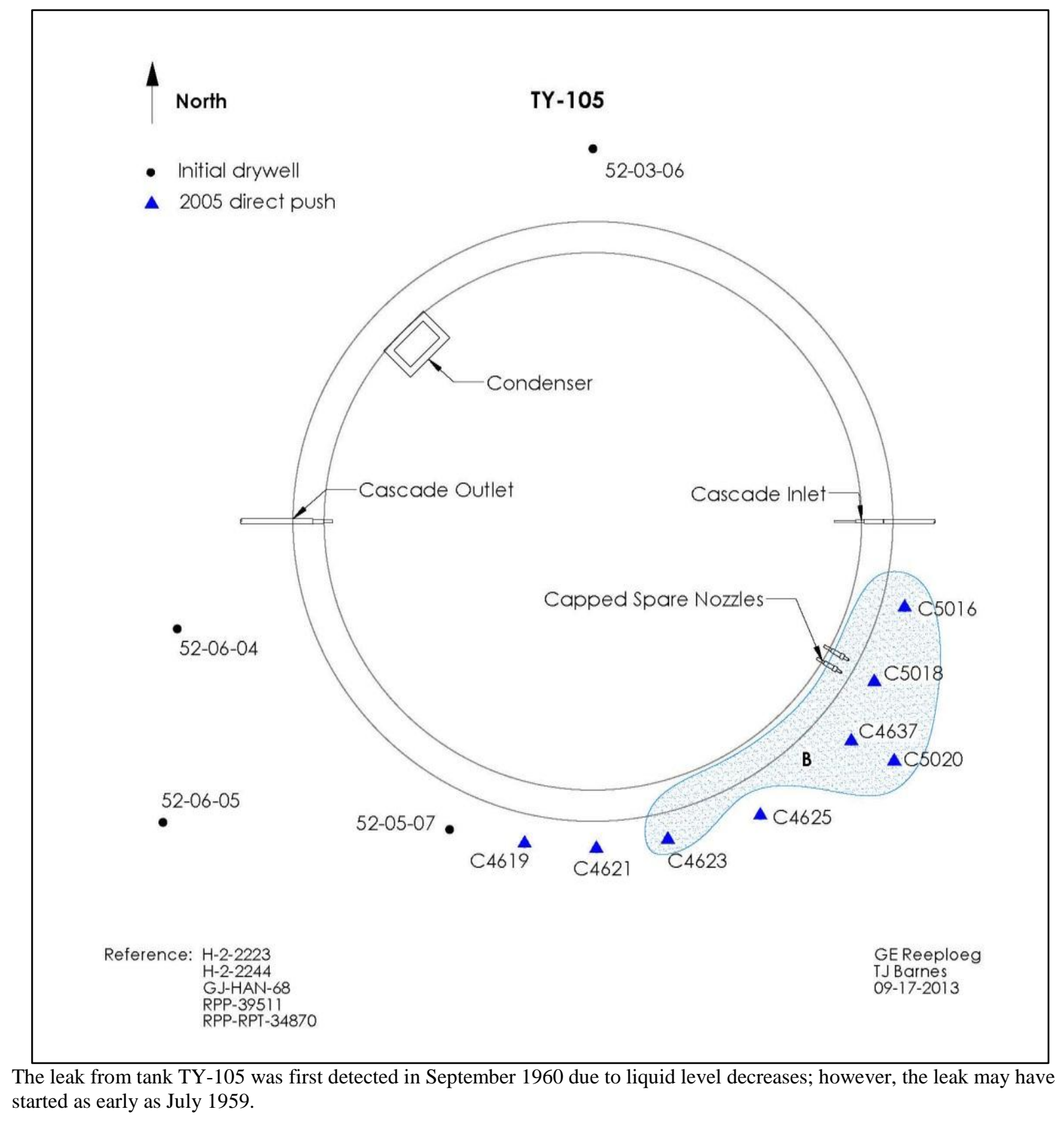




\subsubsection{Leak Location Summary}

Tank TY-105 was first suspected of leaking due to liquid level decreases in September 1960; however, the leak may have begun as early as July 1959. Liquid level decreases were confirmed in October 1960, and contents were pumped out of the tank beginning in November 1960.

Drywell data were not available during this time, and the first recoverable readings were in 19731974 when drywells 52-06-05, 52-05-07, and 52-06-04 all reported radioactivity at or near the tank footing as shown in Site A (see Figure 7-15). These drywells are located between tanks TY-105 and TY-106, and radioactivity detected in these drywells are likely the result of a leak from tank TY-105 (see Section 7.5.2).

In 2005, direct pushes were installed along the southeast edge of tank TY-105. High levels of radioactivity were detected in direct pushes $\mathrm{C} 4637$ and $\mathrm{C} 5018$ with possible migration to the nearby direct pushes as shown in site B (see Figure 7-15). Site B appears to be a separate leak site from site A, as direct pushes located on the southern edge of tank TY-105 do not indicate any radioactivity associated with a tank leak or migration from leak site A.

The tank TY-105 leak(s) likely did not occur far above the tank footing because all of the indicators, three drywells and the 2005 direct pushes, were located at or near the bottom of the tank. A sidewall leak cannot be ruled out as radioactivity was detected in direct push C5018 at a higher BGS level. This radioactivity could be due to a leak from the spare inlet nozzles located in this region. It is possible contamination exists underneath the tank and in the northeast and northwest portion of the tank; however, no drywells, direct pushes, or laterals are present in this area so it remains inconclusive.

Leak locations in Figure 7-15 are based on peak readings and are a representation of possible initial boundaries of radioactivity.

No evidence was found for a liner bulge occurring in tank TY-105, and it remains unclear if a liner bulge once existed in the tank during its operation. However, tank TY-105 non-boiling temperatures are not likely to be a factor in causing a liner bulge. 
Figure 7-15. Tank TY-105 Possible Radial Leak Locations

Tank inner ring is steel liner, outer ring is outer edge of tank footing

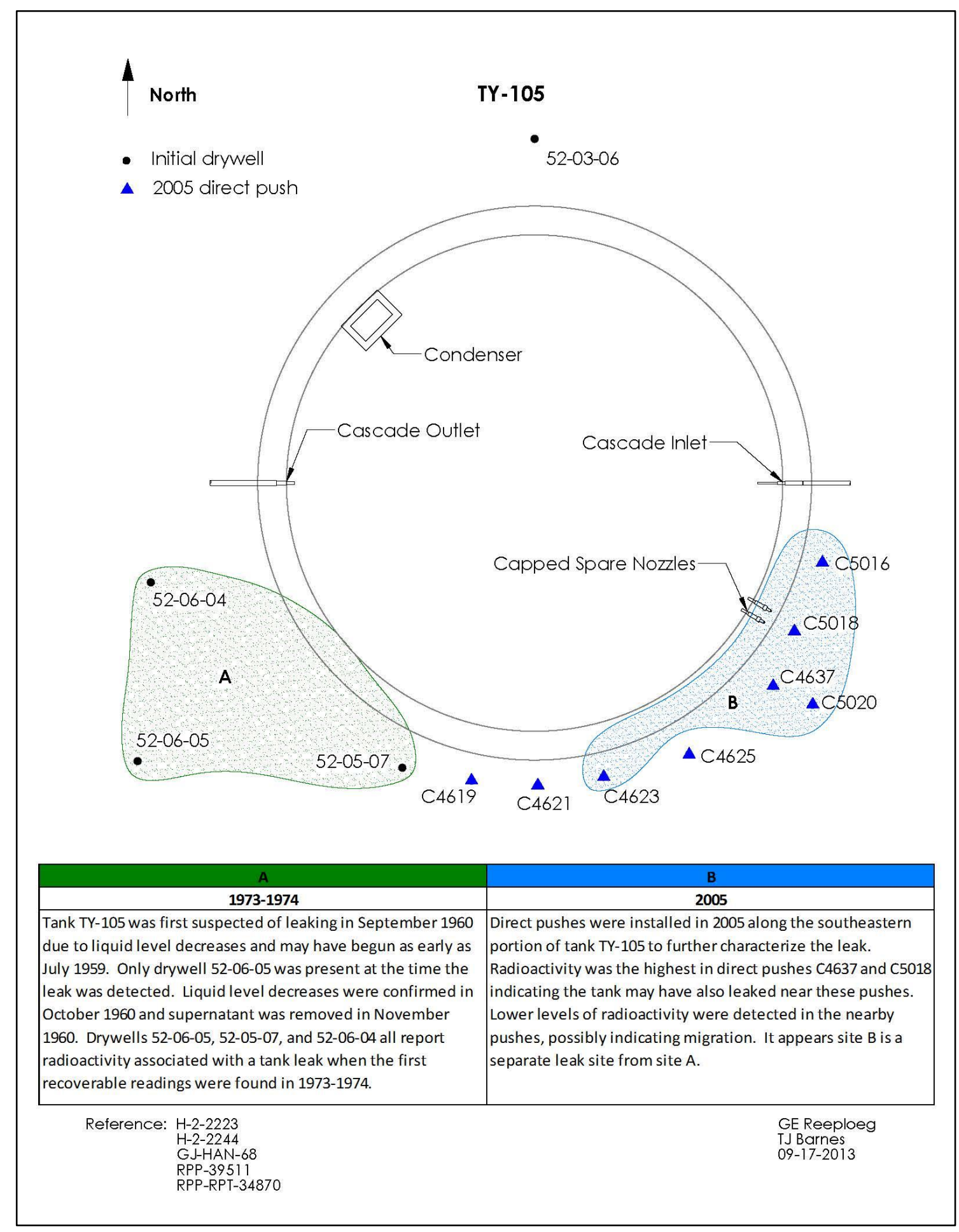


RPP-RPT-54911, Rev. 0

\subsection{POSSIBLE TANK TY-105 LINER LEAK CAUSE(S)}

Tank TY-105 was examined against five conditions that could contribute to a failed liner.

\subsubsection{Tank Design}

The TY Farm tank design does not appear to be a factor contributing to a failed liner (see Section 3.1.1).

\subsubsection{Thermal Shock}

No temperature data are available for tank TY-105 prior to 1977 although tank TY-105 held nonboiling waste. Since no records are available, it is uncertain what the maximum temperature was in tank TY-105 during operation as well as the rate of temperature rise when waste was initially added. However, TBP wastes were estimated to be $110-180^{\circ} \mathrm{F}$ after routing to the storage tanks (see Section 3.1.6). The volume of transfers $(5,000 \mathrm{kgal})$ in a two year period would tend to raise the temperature toward $180^{\circ} \mathrm{F}$.

Thermal shock creates stress both from rapid temperature rise as well as waste-induced high temperatures.

Temperature requirements in ARH-951 (Limitations for Use of Underground Waste Tanks) issued December 18, 1969 indicated that tank temperatures should be held below $230^{\circ} \mathrm{F}$.

\subsubsection{Chemistry-Corrosion}

Tank TY-105 was first filled with TBP waste and stored only TBP waste during operation for approximately eight years until the leak was detected. TBP waste consists of low hydroxide and high nitrate concentrations, with probably low nitrite concentration, which would create an environment conducive to pitting and SCC. A tank with only the TBP waste type present would likely increase SCC in the tank liner (see Section 7.4.3).

\subsubsection{Liner Observations}

A review of the available photographs taken December 22, 1969 does not contain any evidence of a tank bottom liner bulge. There is no additional documentation available indicating a liner bulge was present in tank TY-105.

\subsubsection{Tank Construction Temperatures}

Average winter temperatures were $27.4^{\circ} \mathrm{F}$ and $25.2^{\circ} \mathrm{F}$ in December 1951 and January 1952, respectively, with temperatures as low as $4^{\circ} \mathrm{F}$ during the period when the TY Farm tank liners were being constructed. The low temperatures experienced during construction were less than the current allowable $18^{\circ} \mathrm{F}$ allowable temperature where impact loading had the potential for creating micro-fissures in the steel liner. The TY Farm construction specifications (HW-4696, Specifications Waste Disposal Facilities 241-BZ and TY Tank Farm) contained specific detailed requirements for cold weather construction and it appears these low temperatures had less of an 
impact during the construction of TY Farm compared to 241-SX Farm construction (see Section 3.1.2).

\subsection{TANK TY-105 CONCLUSIONS}

Evidence indicates that the tank TY-105 liner leaked at or near the tank footing in at least two locations. Based on engineering judgment and available information the following conditions listed in the order of importance lead to the tank TY-105 failed liner.

1. TBP waste storage chemistry-corrosion -stress corrosion cracking

2. Thermal conditions

3. Construction conditions-ductile-to-brittle transition temperature

There are several liner leak cause conditions that were examined but the most likely cause of the tank TY-105 leak is TBP waste storage chemistry-corrosion. TBP waste storage could have created a chemical environment susceptible to nitrate-induced SCC attack on a potentially stressed metal liner.

Thermal and construction conditions could have also contributed to the liner failure but to a lesser degree. Tank design doesn't seem to have contributed to liner failure and there is no evidence of liner bulging. Some or all of the factors can act serially or together to contribute to tank liner failure. 
RPP-RPT-54911, Rev. 0

APPENDIX A4

TANK TY-105 OPERATIONAL HISTORY

A4-1 
RPP-RPT-54911, Rev. 0

Table A4-1. ARH-R-43 Waste Tank Leak Experience Table (1970)

\begin{tabular}{|c|c|c|c|c|c|c|c|c|c|c|}
\hline TABLE & $\begin{array}{l}\text { Under } \\
\text { Built }\end{array}$ & $\begin{array}{l}\text { rourd } \\
\text { First } \\
\text { Uscd }\end{array}$ & fazte Storage Sank teal? E. & $x+2 \operatorname{sen} x \in$ & $\begin{array}{l}\text { Leak First } \\
\text { Suspected } \\
\end{array}$ & $\begin{array}{l}\text { Reason for } \\
\text { Suspicion }\end{array}$ & $\begin{array}{l}\text { Leak Confirmed } \\
\text { by } \\
\end{array}$ & $\begin{array}{l}\text { Bulge } \\
\text { Found } \\
\end{array}$ & $\begin{array}{l}\text { Estimsted } \\
\text { Volume of } \\
\text { Leak, gal }\end{array}$ & $\begin{array}{l}\text { Associated } \\
\mathrm{Cs} .137, \mathrm{KCi}\end{array}$ \\
\hline $104-U$ & 1943.44 & 1947 & $\begin{array}{l}\text { Bismuth phosphate waste } \\
\text { Uranium recovery waste }\end{array}$ & Xonboiling & 1956 & Bulged 1 iner & $\begin{array}{l}\text { Refill with } \\
\text { water (1961) }\end{array}$ & Yes & $55,000^{*}$ & 0.09 \\
\hline $11.5-5 x$ & $1953-54$ & 1958 & Redox waste & Bot 1 tng & 1958 & Bulged liner & $\begin{array}{l}\text { Refill with sa1t } \\
\text { solution }(1962)\end{array}$ & Yes & $15,000 * *$ & 8 \\
\hline $106-\mathrm{TV}$ & 1951.52 & 1953 & THP waste & Nonboiling & 1959 & $\begin{array}{l}\text { Liquid leve } 1 \\
\text { measurethents }\end{array}$ & $\begin{array}{l}\text { Soil radiation } \\
\text { readings (1959) }\end{array}$ & & 20,000 & 2 \\
\hline $101 \cdot U$ & $1943 \cdot 44$ & 1946 & $\begin{array}{l}\text { Bismuth phosphate waste } \\
\text { Urani un recovery waste } \\
\text { Redox kaste }\end{array}$ & Nonboiling & 1959 & $\begin{array}{l}\text { Liquid leve } 1 \\
\text { measurements }\end{array}$ & $\begin{array}{l}\text { Liquid leve } 1 \\
\text { measurements }\end{array}$ & & 30,000 & 23 \\
\hline $105 \cdot \mathrm{rY}$ & $1951-52$ & $19 \$ 3$ & TBP vaste & Nonboiling & 1960 & $\begin{array}{l}\text { 1. iquid leve } 1 \\
\text { measurements }\end{array}$ & $\begin{array}{l}\text { Liquid leve } 1 \\
\text { ineasurements }\end{array}$ & & 35,000 & 4 \\
\hline $108-5 x$ & $1953-54$ & 1955 & Redox waste & Boiling & 1962 & $\begin{array}{l}\text { Soil radi- } \\
\text { ation } \\
\text { readings }\end{array}$ & $\begin{array}{l}\text { Liquid level } \\
\text { measurenents }\end{array}$ & Yes & 3,400 & 17 \\
\hline $105=A$ & $1954-55$ & 1963 & Purex waste & Boiling & 1963 & $\begin{array}{l}\text { Soil radi- } \\
\text { ation } \\
\text { readings }\end{array}$ & $\begin{array}{l}\text { Soil radiation } \\
\text { readings }\end{array}$ & Yes & Sma11 & \\
\hline $107 \cdot 5 x$ & $1953-54$ & 1956 & Redox waste & Boiling & 1964 & $\begin{array}{l}\text { Soil radi - } \\
\text { ation } \\
\text { readings }\end{array}$ & $\begin{array}{l}\text { Soil radiation } \\
\text { readings }\end{array}$ & Yes & Smal1 & \\
\hline $109-5 x$ & $1953-54$ & 1955 & Redox waste & Boiling & 1965 & $\begin{array}{l}\text { Soil radi= } \\
\text { ation } \\
\text { readings }\end{array}$ & $\begin{array}{l}\text { No further } \\
\text { evidence }\end{array}$ & No & Sma11 & \\
\hline $115=5 x$ & $1953 \cdot 54$ & 1958 & Redox waste & Boiling & 1965 & $\begin{array}{l}\text { Liquid level } \\
\text { measurements }\end{array}$ & $\begin{array}{l}\text { Soil radiation } \\
\text { readings }\end{array}$ & & $50,000 * \approx *$ & 40 \\
\hline $112-5 x$ & $1953-54$ & 1956 & Redox wastc & Boiling & 1969 & $\begin{array}{l}\text { Liquid level } \\
\text { measurements }\end{array}$ & $\begin{array}{l}\text { Soil radiation } \\
\text { readings }\end{array}$ & Yes & 30,000 & 45 \\
\hline$\because \quad c o$ & $\begin{array}{l}\text { ntatred } \\
\text { rtained } \\
\text { rtatned }\end{array}$ & 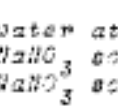 & $\begin{array}{l}\text { tire lakt. } \\
\text { intion at tire of loak. } \\
\text { intion at time of leak. }\end{array}$ & Ias oose: $:$ & Anat ahpert & atant entution. & & & & \\
\hline
\end{tabular}

Note: See tank farm segments for information on individual specific tanks 
RPP-RPT-54911, Rev. 0

APPENDIX B4

TANK TY-105 GROSS GAMMA DRYWELL DATA

B4-1 
RPP-RPT-54911, Rev. 0

Table B4-1. Tank TY-105 Drywell Radioactivity (K counts per minute)

(August 1972 to June 1986) (1 of 2 sheets)

(Drywell Data Sheets* Retrieved on July 20, 2011 and SD-WM-TI-356)

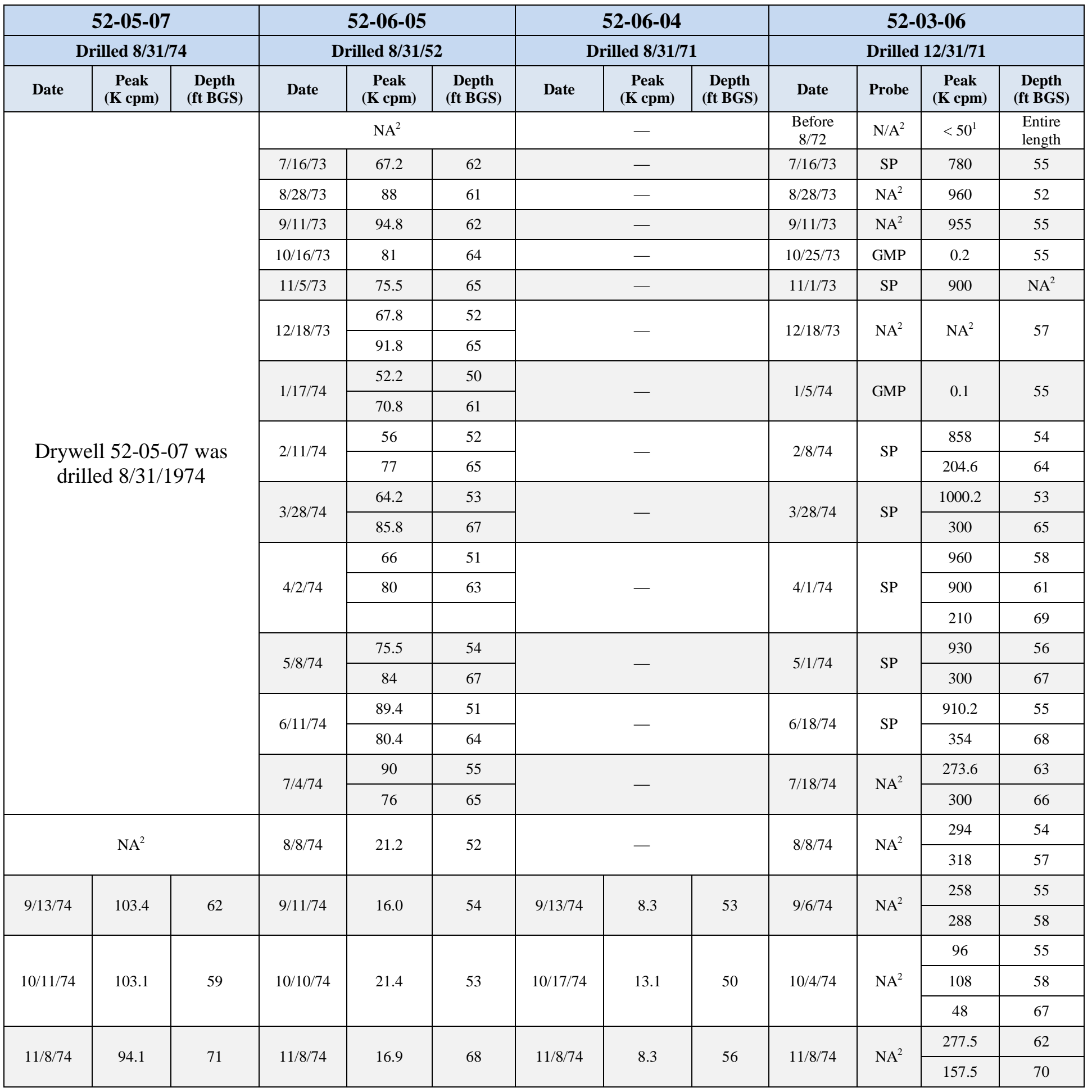


RPP-RPT-54911, Rev. 0

Table B4-1. Tank TY-105 Drywell Radioactivity (K counts per minute)

(August 1972 to June 1986) (2 of 2 sheets)

(Drywell Data Sheets* Retrieved on July 20, 2011 and SD-WM-TI-356)

\begin{tabular}{|c|c|c|c|c|c|c|c|c|c|c|c|c|}
\hline \multicolumn{3}{|c|}{ 52-05-07 } & \multicolumn{3}{|c|}{$52-06-05$} & \multicolumn{3}{|c|}{$52-06-04$} & \multicolumn{4}{|c|}{$52-03-06$} \\
\hline Date & $\begin{array}{c}\text { Peak } \\
\text { (K cpm) }\end{array}$ & $\begin{array}{c}\text { Depth } \\
\text { (ft BGS) }\end{array}$ & Date & $\begin{array}{c}\text { Peak } \\
(\mathrm{K} \text { cpm })\end{array}$ & $\begin{array}{c}\text { Depth } \\
\text { (ft BGS) }\end{array}$ & Date & $\begin{array}{c}\text { Peak } \\
(\mathrm{K} \text { cpm) }\end{array}$ & $\begin{array}{c}\text { Depth } \\
\text { (ft BGS) }\end{array}$ & Date & Probe & $\begin{array}{c}\text { Peak } \\
\text { (K cpm) }\end{array}$ & $\begin{array}{c}\text { Depth } \\
\text { (ft BGS) }\end{array}$ \\
\hline \multirow{2}{*}{$12 / 4 / 74$} & \multirow{2}{*}{98.6} & \multirow{2}{*}{59} & \multirow{2}{*}{$12 / 4 / 74$} & \multirow{2}{*}{16.4} & \multirow{2}{*}{56} & \multirow{2}{*}{$12 / 4 / 74$} & \multirow{2}{*}{8.9} & \multirow{2}{*}{53} & \multirow{2}{*}{$12 / 3 / 74$} & \multirow{2}{*}{$\mathrm{NA}^{2}$} & 282 & 57 \\
\hline & & & & & & & & & & & 165 & 65 \\
\hline \multirow{2}{*}{$1 / 2 / 75$} & \multirow{2}{*}{100.2} & \multirow{2}{*}{59} & \multirow{2}{*}{$1 / 2 / 75$} & \multirow{2}{*}{16.3} & \multirow{2}{*}{56} & \multirow{2}{*}{$1 / 2 / 75$} & \multirow{2}{*}{9.4} & \multirow{2}{*}{52} & \multirow{2}{*}{$1 / 2 / 75$} & \multirow{2}{*}{$\mathrm{NA}^{2}$} & 180 & 58 \\
\hline & & & & & & & & & & & 96 & 62 \\
\hline $2 / 20 / 75$ & 94.0 & 58 & \multirow{2}{*}{$2 / 20 / 75$} & \multirow{2}{*}{13.7} & \multirow{2}{*}{56} & $2 / 20 / 75$ & 100 & 51 & $2 / 8175$ & $\mathrm{NA}^{2}$ & 261.2 & 60 \\
\hline $5 / 1 / 75$ & 90 & 57 & & & & $2 / 20 / 15$ & 10.0 & 31 & $2 / 8 / 13$ & NA & 168 & 68 \\
\hline 7/17/75 & 86.6 & 59 & $7 / 24 / 75$ & 12.8 & 64 & $7 / 24 / 75$ & 8.3 & 51 & $7 / 24 / 75$ & $\mathrm{NA}^{2}$ & 270.7 & 60 \\
\hline $11 / 7 / 75$ & 85.6 & 58 & $12 / 31 / 75$ & 12 & 65 & $12 / 31 / 75$ & 8.5 & 52 & $12 / 24 / 75$ & $\mathrm{NA}^{2}$ & 265.8 & 60 \\
\hline $5 / 14 / 76$ & 79.7 & 56 & $5 / 14 / 76$ & 12.5 & 63 & $5 / 14 / 76$ & 8.1 & 50 & $3 / 8 / 76$ & $\mathrm{NA}^{2}$ & 260.3 & 59 \\
\hline $9 / 23 / 76$ & 77.1 & 56 & & $\mathrm{NA}^{2}$ & & & $\mathrm{NA}^{2}$ & & $7 / 23 / 76$ & $\mathrm{NA}^{2}$ & 232.7 & 59 \\
\hline & $\mathrm{NA}^{2}$ & & $11 / 5 / 76$ & 11.6 & 62 & $11 / 5 / 76$ & 6.9 & 50 & $11 / 25 / 76$ & $\mathrm{NA}^{2}$ & 225.5 & 59 \\
\hline $3 / 10 / 77$ & 73.4 & 56 & $4 / 8 / 77$ & 10.1 & 61 & $7 / 15 / 77$ & 6.2 & 50 & $6 / 30 / 77$ & $\mathrm{NA}^{2}$ & 225.3 & 58 \\
\hline $10 / 6 / 77$ & 67.3 & 56 & $10 / 6 / 77$ & 9.4 & 62 & $10 / 6 / 77$ & 5.7 & 49 & $10 / 13 / 77$ & $\mathrm{NA}^{2}$ & 218.7 & 59 \\
\hline $10 / 5 / 78$ & 57.0 & 57 & $10 / 5 / 78$ & 9.4 & 62 & $10 / 5 / 78$ & 5.0 & 50 & $1 / 13 / 78$ & $\mathrm{NA}^{2}$ & 212.5 & 65 \\
\hline & $\mathrm{NA}^{2}$ & & & $\mathrm{NA}^{2}$ & & & $\mathrm{NA}^{2}$ & & $5 / 5 / 78$ & $\mathrm{NA}^{2}$ & 219.2 & 65 \\
\hline & $\mathrm{NA}^{2}$ & & & $\mathrm{NA}^{2}$ & & & $\mathrm{NA}^{2}$ & & $10 / 5 / 78$ & $\mathrm{NA}^{2}$ & 213.7 & 65 \\
\hline $5 / 16 / 79$ & 52.2 & 55 & $10 / 31 / 79$ & 8.9 & 62 & $10 / 31 / 79$ & 5.1 & 51 & $10 / 31 / 79$ & $\mathrm{NA}^{2}$ & 178.9 & 66 \\
\hline $10 / 16 / 79$ & 47.0 & 57 & & $\mathrm{NA}^{2}$ & & & $\mathrm{NA}^{2}$ & & & & & \\
\hline $11 / 12 / 80$ & 40.9 & 57 & $10 / 14 / 80$ & 10.4 & 62 & $11 / 12 / 80$ & 3.3 & 50 & $11 / 12 / 80$ & $\mathrm{NA}^{2}$ & 163.6 & 66 \\
\hline $12 / 29 / 81$ & 33.3 & 57 & $11 / 19 / 81$ & 7.4 & 64 & $11 / 11 / 81$ & 3.4 & 51 & $12 / 29 / 81$ & $\mathrm{NA}^{2}$ & 146.4 & 66 \\
\hline $10 / 19 / 82$ & 34.6 & 58 & $10 / 19 / 82$ & 5.4 & 64 & $10 / 12 / 82$ & 3.2 & 51 & $10 / 19 / 82$ & $\mathrm{NA}^{2}$ & 128.2 & 67 \\
\hline $9 / 20 / 83$ & 32.0 & 58 & $9 / 20 / 83$ & 5.5 & 65 & $6 / 28 / 83$ & 3.5 & 51 & $9 / 11 / 83$ & $\mathrm{NA}^{2}$ & 102 & 67 \\
\hline $8 / 22 / 84$ & 25.2 & 58 & $8 / 22 / 84$ & 5.0 & 65 & $8 / 22 / 84$ & 3.1 & 51 & $8 / 22 / 84$ & $\mathrm{NA}^{2}$ & 90 & 67 \\
\hline $6 / 18 / 85$ & 21.2 & 59 & $6 / 18 / 85$ & 4.4 & 64 & $6 / 18 / 85$ & 2.5 & 52 & $6 / 18 / 85$ & $\mathrm{NA}^{2}$ & 81 & 68 \\
\hline $6 / 11 / 86$ & 19.2 & 70 & $6 / 11 / 86$ & 4.3 & 64 & $6 / 11 / 86$ & 2.5 & 53 & $6 / 11 / 86$ & $\mathrm{NA}^{2}$ & 48.6 & 68 \\
\hline
\end{tabular}

Note: ${ }^{1}$ Referenced from Internal letter from G.L. Borsheim to L.W. Roddy, “Integrity of tanks 241-BY-103 and 241-TY-103” June 1, 1973

${ }^{2} \mathrm{NA}$ : Data not available

*Drywell data sheets located in the Historical Records folder in IDMS/Managed Information 
RPP-RPT-54911, Rev. 0

APPENDIX C4

TANK TY-105 GAMMA SURVEYS OF THE 2005 DIRECT PUSHES

C4-1 
RPP-RPT-54911, Rev. 0

Figure C4-1. 2005 Gamma Survey Direct Push C5016 (RPP-RPT-34870)

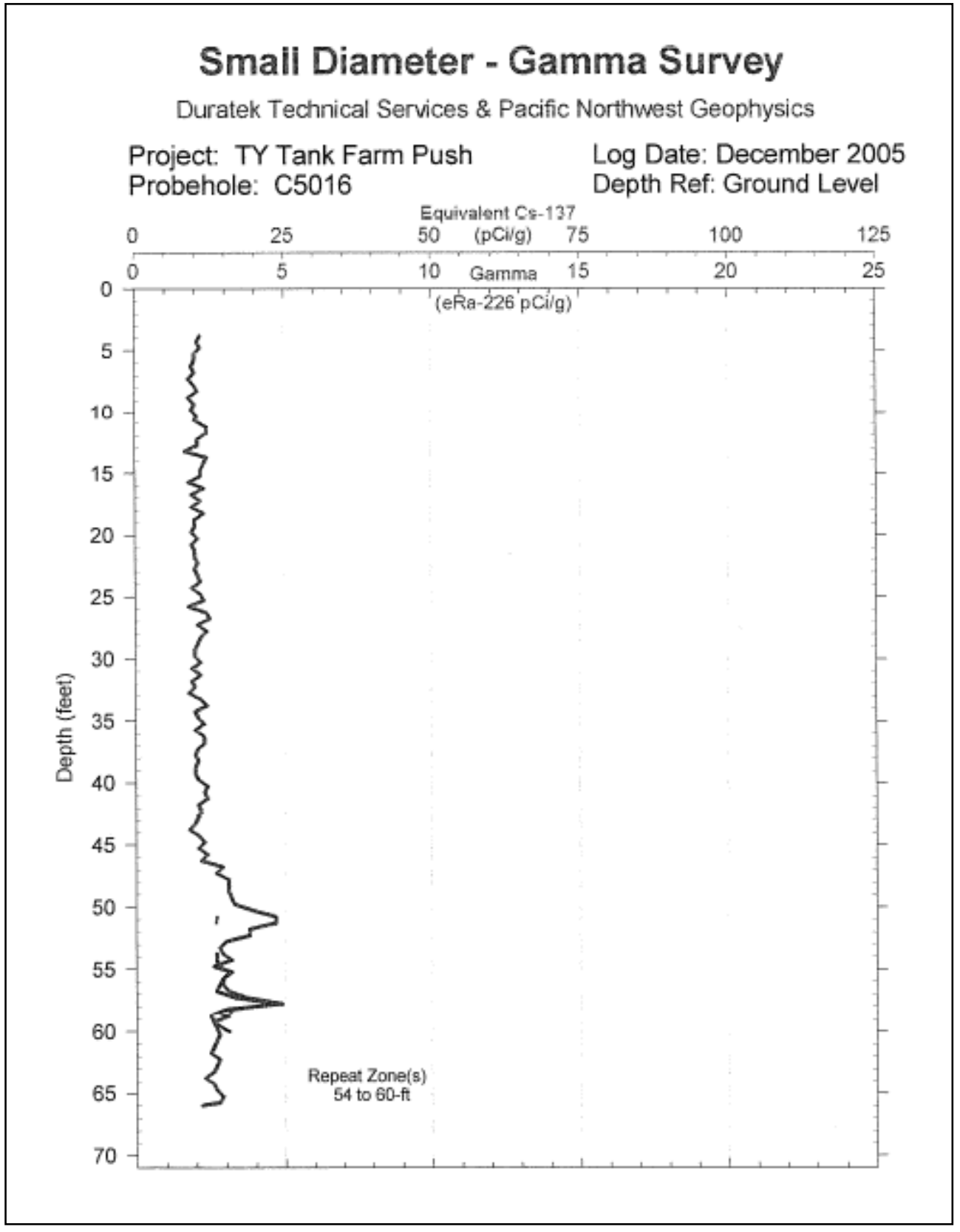


RPP-RPT-54911, Rev. 0

Figure C4-2. 2005 Gamma Survey Direct Push C5018 (RPP-RPT-34870)

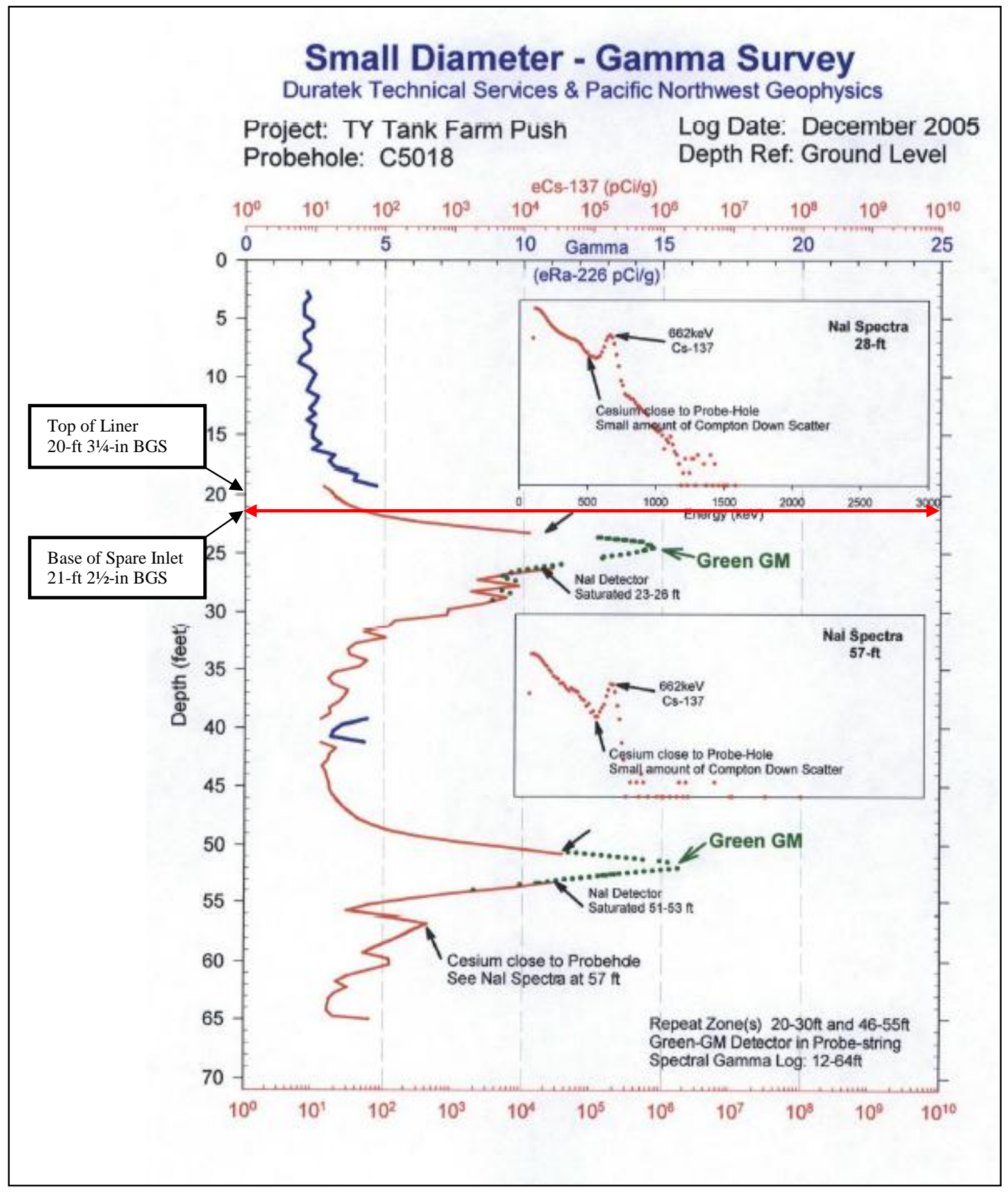


RPP-RPT-54911, Rev. 0

Figure C4-3. 2005 Gamma Survey Direct Push C4619 (RPP-RPT-34870)

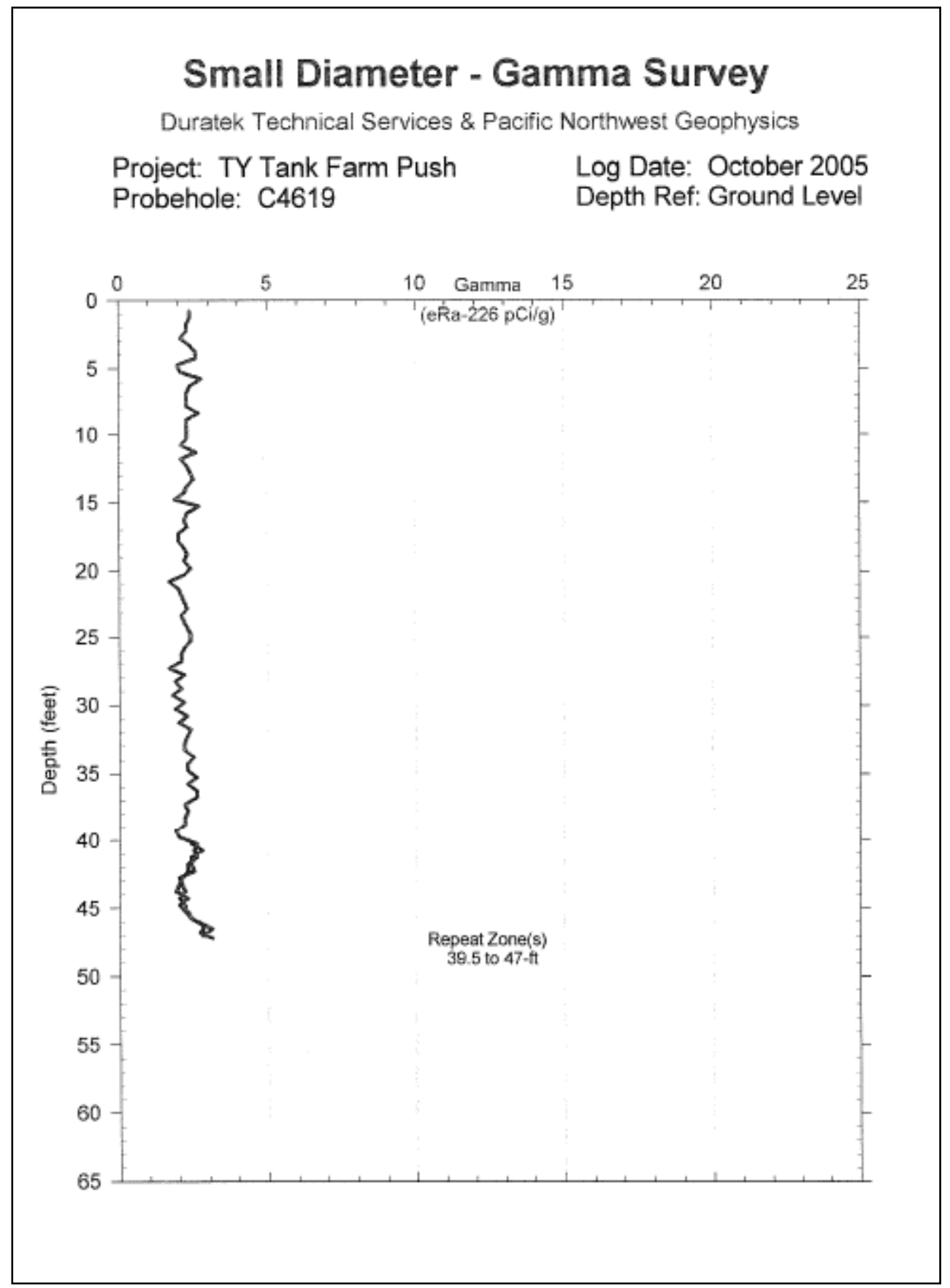


RPP-RPT-54911, Rev. 0

Figure C4-4. 2005 Gamma Survey Direct Push C4620 (RPP-RPT-34870)

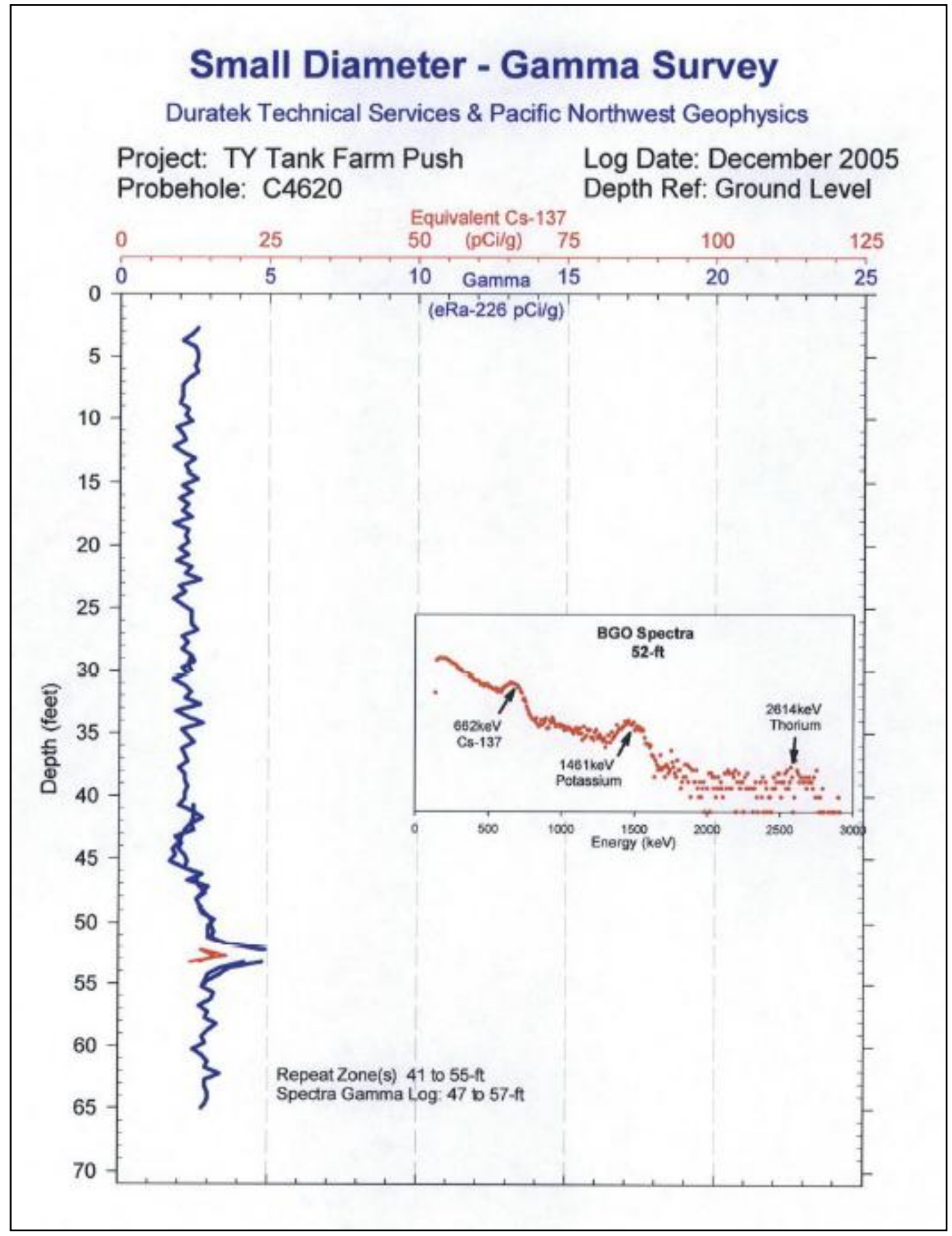


RPP-RPT-54911, Rev. 0

Figure C4-5. 2005 Gamma Survey Direct Push C4621 (RPP-RPT-34870)

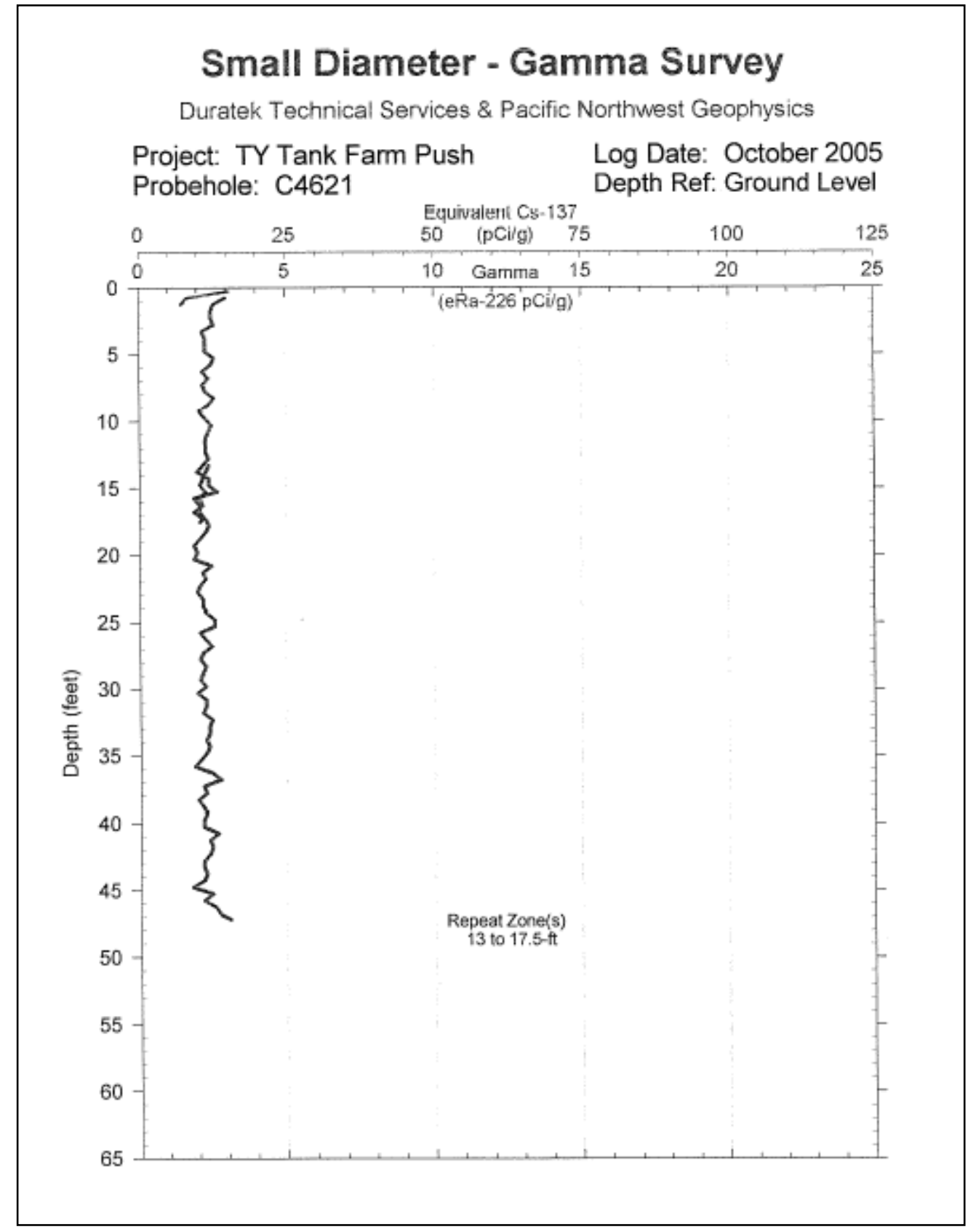


RPP-RPT-54911, Rev. 0

Figure C4-6. 2005 Gamma Survey Direct Push C4623 (RPP-RPT-34870)

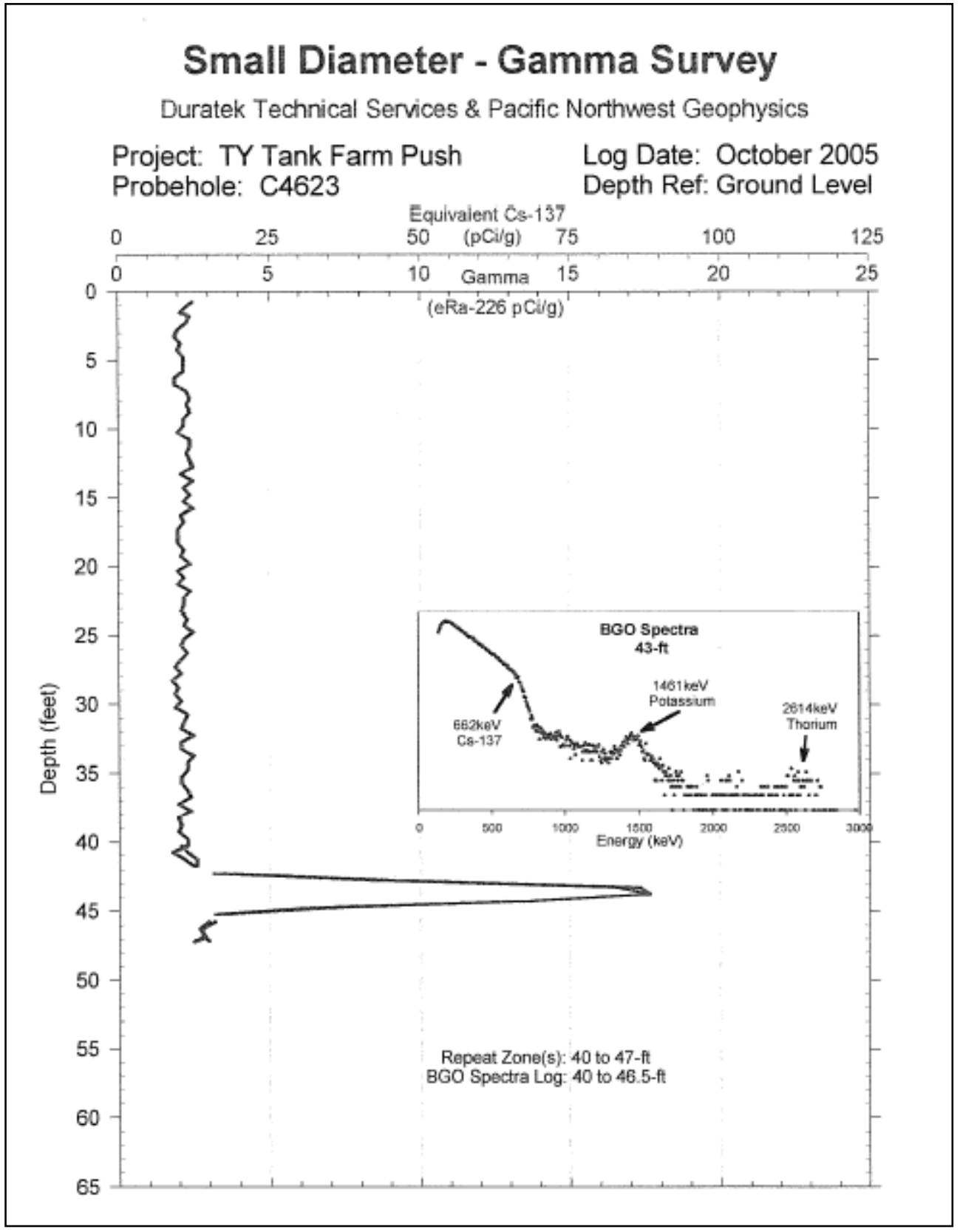


RPP-RPT-54911, Rev. 0

Figure C4-7. 2005 Gamma Survey Direct Push C4625 (RPP-RPT-34870)

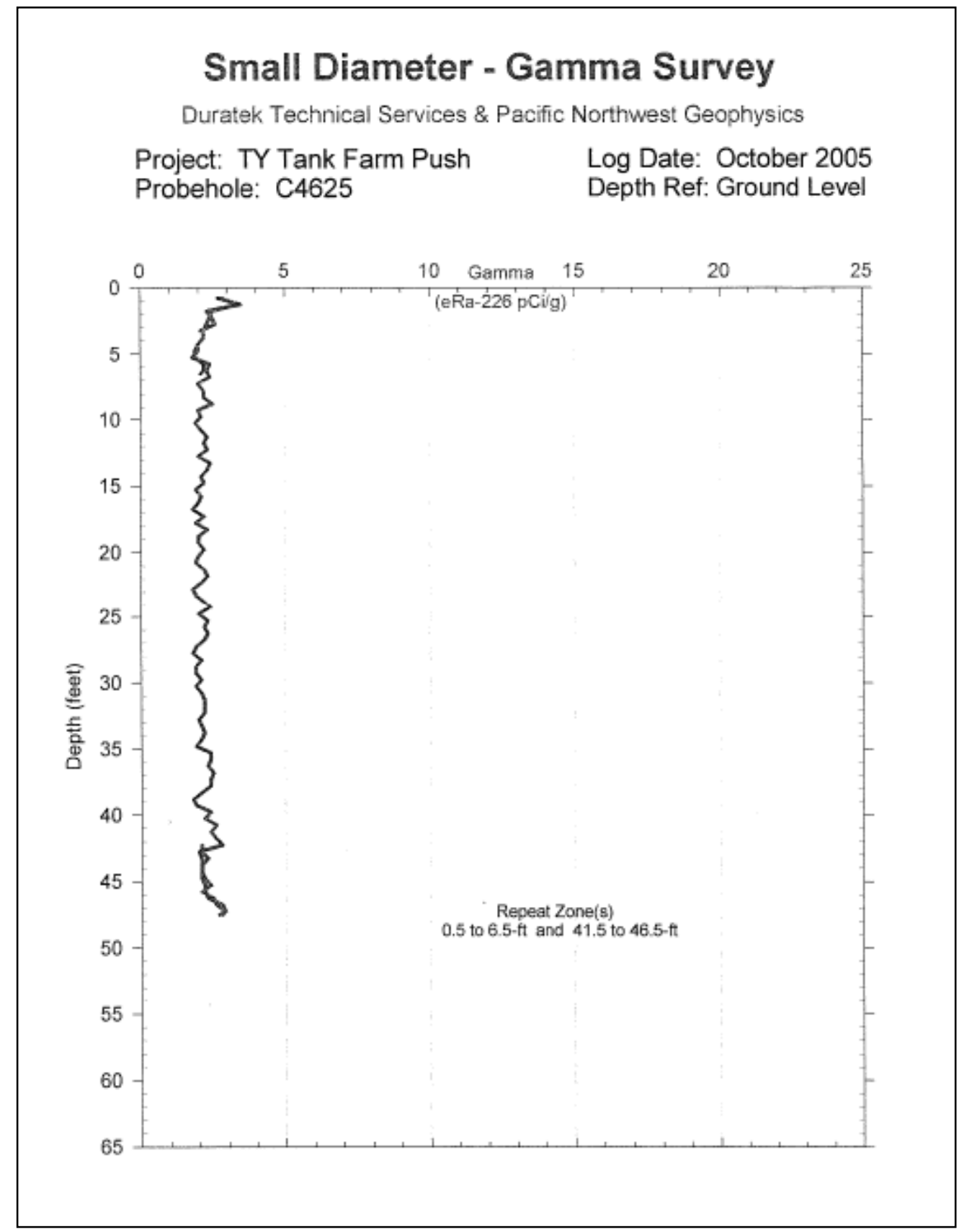


RPP-RPT-54911, Rev. 0

Figure C4-8. 2005 Gamma Survey Direct Push C4637 (RPP-RPT-34870)

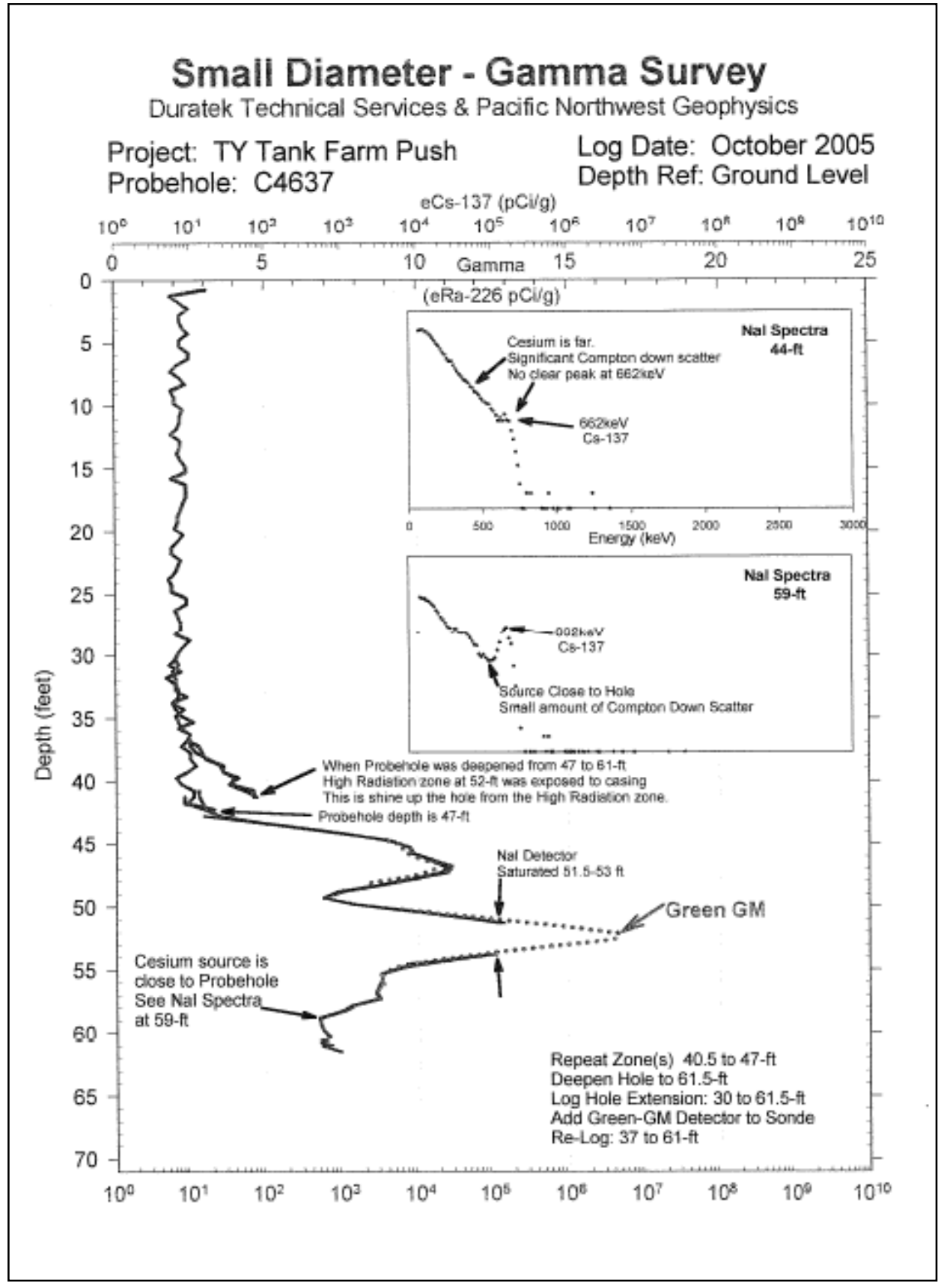




\subsection{TANK TY-106 SEGMENT}

\section{TABLE OF CONTENTS}

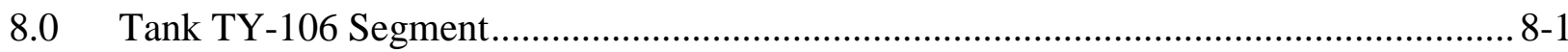

8.1 Tank TY-106 Background History .......................................................................... 8-1

8.2 Tank TY-106 Operations Summary ……………................................................. 8-2

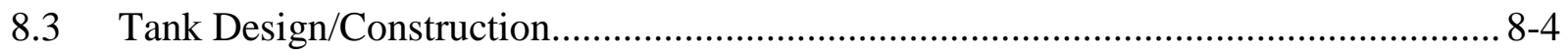

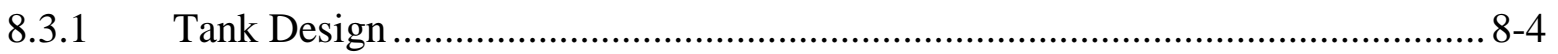

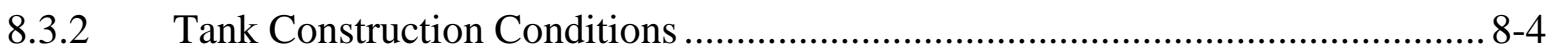

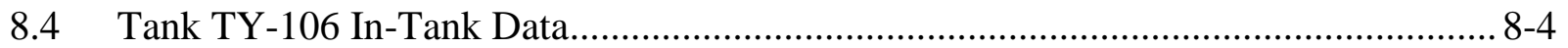

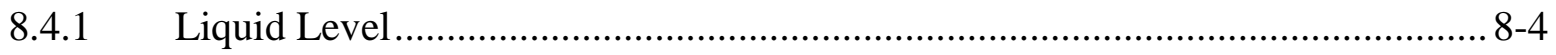

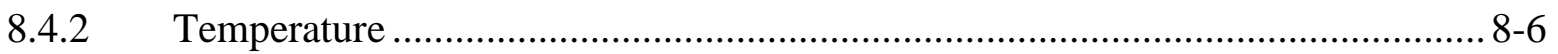

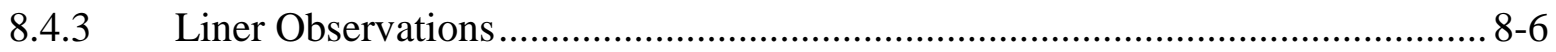

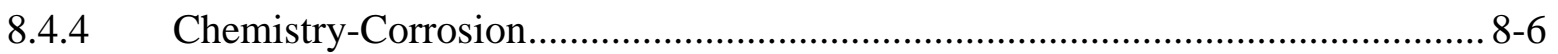

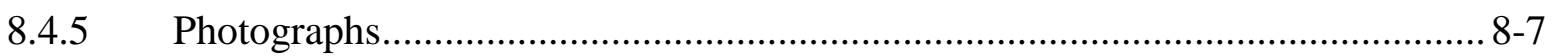

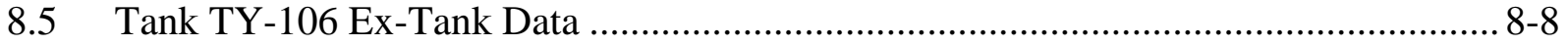

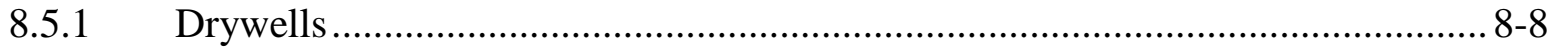

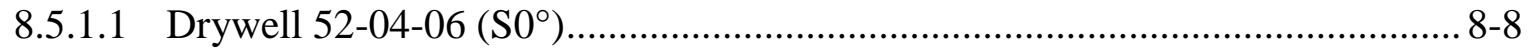

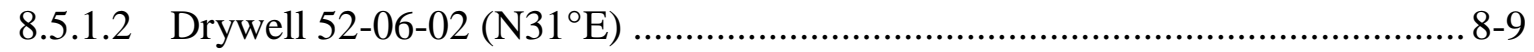

8.5.1.3 Drywell 52-06-04 (S71 $\left.{ }^{\circ} \mathrm{E}\right)$............................................................... 8-10

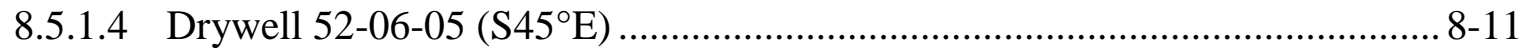

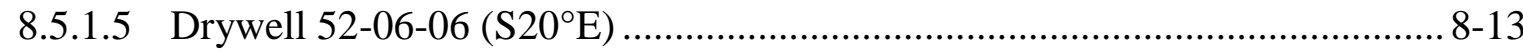

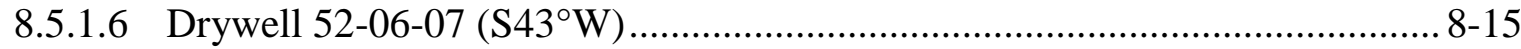

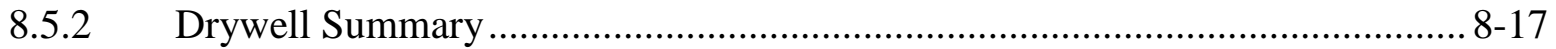

8.5.3 2005 Direct Push Logging ...................................................................... 8-17

8.6 Possible Tank TY-106 Liner Leak Location(s)..................................................... 8-18

8.6.1 Leak Detected in 1974 and 2005, Site A .................................................. 8-19

8.6.2 Leak Detected in 2005, Site B …………………................................ 8-21

8.6.3 Leak Detected in 1973-1974, Site C ........................................................ 8-22

8.6.4 Leak Location Summary ………………................................................... 8-23

8.7 Possible Tank TY-106 Liner Leak Cause(s) ……………………........................ 8-25

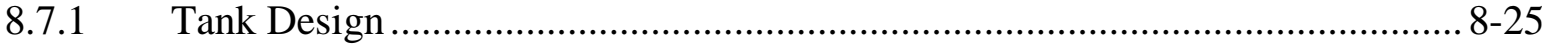




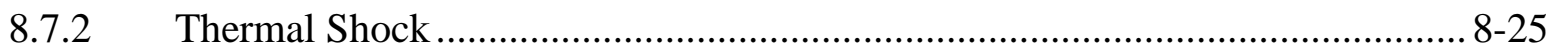

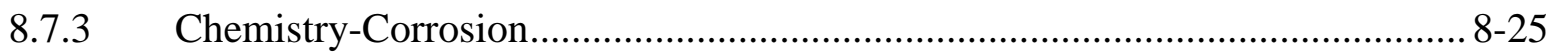

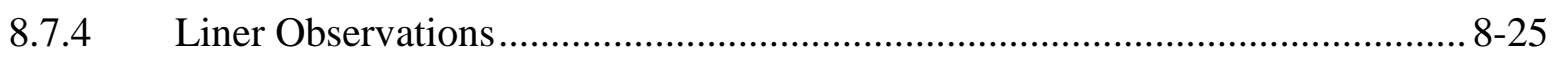

8.7.5 Tank Construction Temperatures.............................................................. 8-25

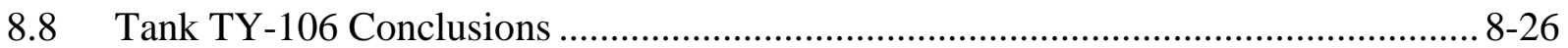

\begin{abstract}
APPENDICES
Appendix A5 Tank TY-106 Operational History ……………………………………............ A5-1

Appendix B5 1960 Memo-Tank 241-TY-105 Waste Storage Leak .......................................... B5-1

Appendix C5 Tank TY-106 Gross Gamma Drywell Data........................................................ C5-1

Appendix D5 Tank TY-106 Gamma Surveys of the 2005 Direct Pushes ................................ D5-1
\end{abstract}

\title{
LIST OF FIGURES
}

Figure 8-1. Tank TY-106 Associated Drywells...................................................................... 8-1

Figure 8-2. Operational Leak History of Tank TY-106........................................................... 8-3

Figure 8-3. Tank TY-106 End of Quarter Surface Level...................................................... 8-5

Figure 8-4. Tank TY-106 Associated Drywell 52-04-06 (HNF-3831) .................................... 8-9

Figure 8-5. Tank TY-106 Drywell 52-06-02 (HNF-3831) ................................................. 8-10

Figure 8-6. Tank TY-106 Drywell 52-06-04 (HNF-3831) ..................................................... 8-11

Figure 8-7. Tank TY-105 Associated Drywell 52-06-05 Cesium (HNF-3831) ........................ 8-12

Figure 8-8. Tank TY-106 Drywell 52-06-05 (HNF-3831) .................................................. 8-13

Figure 8-9. Tank TY-106 Drywell 52-06-06 Cesium (HNF-3831) ........................................ 8-14

Figure 8-10. Tank TY-106 Drywell 52-06-06 (HNF-3831) …………………………......... 8-15

Figure 8-11. Tank TY-106 Drywell 52-06-07 (HNF-3831) ..................................................... 8-16

Figure 8-13. Tank TY-106 Possible Leak Location (1974 \& 2005)........................................ 8-20

Figure 8-14. Tank TY-106 Possible Leak Location (2005).................................................... 8-21

Figure 8-15. Tank TY-106 Possible Leak Location (1973-1974) ……………….................. 8-22 
RPP-RPT-54911, Rev. 0

Figure 8-16. Tank TY-106 Possible Radial Leak Locations .............................................. 8-24

Figure D5-1. 2005 Gamma Survey Direct Push Hole C4603 ............................................. D5-2

Figure D5-2. 2005 Gamma Survey Direct Push Hole C4605 ............................................. D5-3

Figure D5-3. 2005 Gamma Survey Direct Push Hole C4607............................................ D5-4

Figure D5-4. 2005 Gamma Survey Direct Push Hole C4609.............................................. D5-5

Figure D5-5. 2005 Gamma Survey Direct Push Hole C4611 ............................................. D5-6

Figure D5-6. 2005 Gamma Survey Direct Push Hole C4613 ............................................ D5-7

Figure D5-7. 2005 Gamma Survey Direct Push Hole C4615 ............................................ D5-8

Figure D5-8. 2005 Gamma Survey Direct Push Hole C4617 ........................................... D5-9

\section{LIST OF TABLES}

Table 8-1. Tank TY-106 Waste Storage Chronology .................................................... 8-6

Table 8-2. Waste Chemistries for Waste Types Stored in Tank TY-106 ............................. 8-7

Table 8-3. 2005 Direct Push Gross Gamma Results (RPP-RPT-34870).............................. 8-18

Table A5-1. ARH-R-43 Waste Tank Leak Experience Table ............................................ A5-2

Table C5-1. Tank TY-106 Drywell Radioactivity (K counts per minute)............................. C5-2 


\subsection{TANK TY-106 BACKGROUND HISTORY}

This section provides information on the historical waste loss event associated with SST 241TY-106 (TY-106). There are six drywells located around tank TY-106 with specified distances from the drywell to the tank footing shown in Figure 8-1: 52-06-05 and 52-06-07 installed in 1952; 52-04-06 and 52-06-02 installed in December 1971; 52-06-04 and 52-06-06 installed in August 1974. Eight direct pushes were installed in 2005: C4617, C4613, C4615, C4611, C4609, C4607, C4605, and C4603.

The bottom of the tank footing is $~ 46-\mathrm{ft} 4$-in Below Grade Surface (BGS) with $\sim 6$-ft 10-in soil cover over the dome (WHC-SD-WM-TI-665, Soil Load above Hanford Waste Storage Tanks; H2-2244, 75 Foot Composite Storage Tank Sections).

Figure 8-1. Tank TY-106 Associated Drywells

Tank inner ring is steel liner, outer ring is outer edge of tank footing

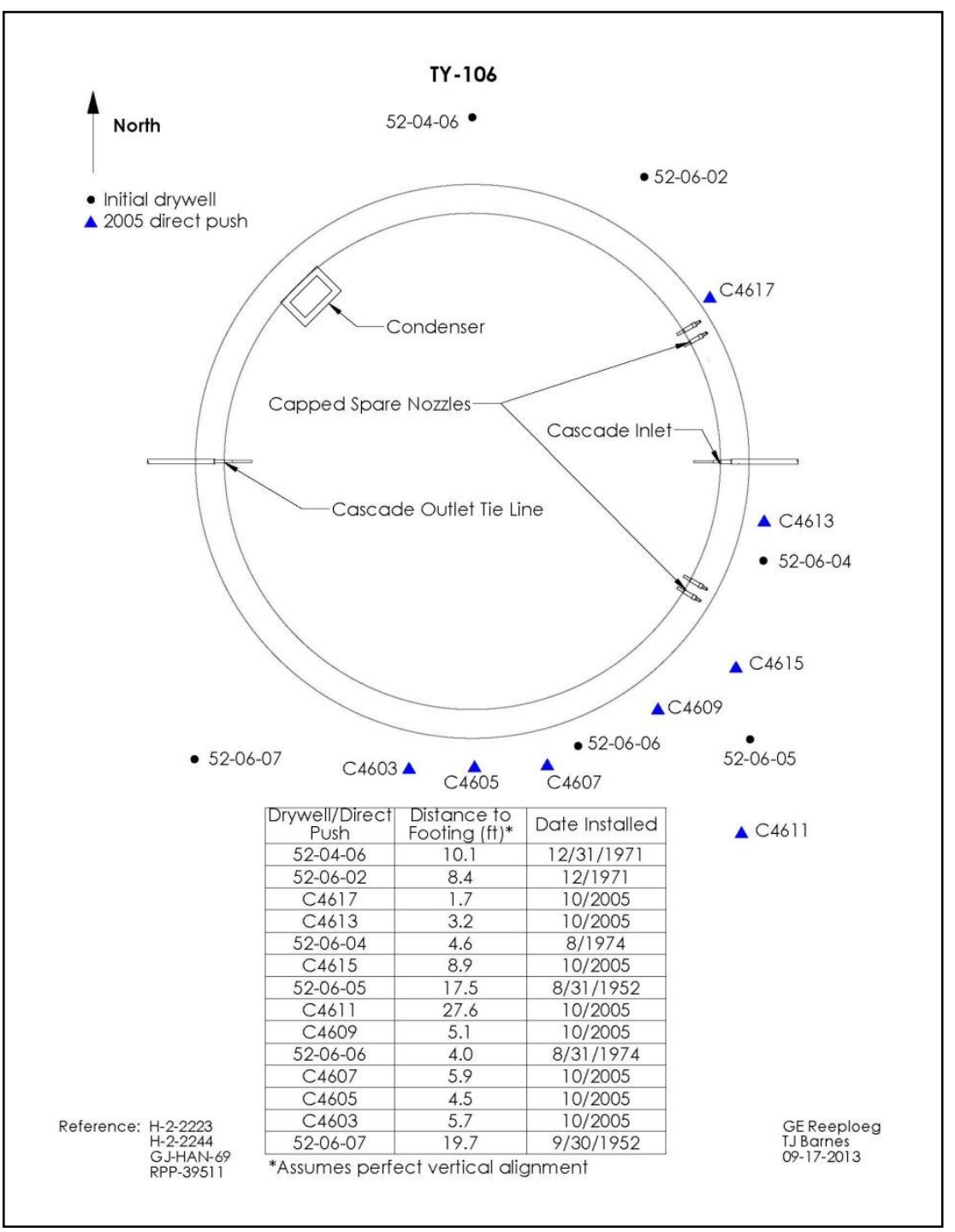


RPP-RPT-54911, Rev. 0

\subsection{TANK TY-106 OPERATIONS SUMMARY}

Tank TY-106 was constructed from 1951 to 1952 and started receiving Tri-Butyl Phosphate (TBP) waste cascaded from tank TY-105 in June 1953 (HW-28712, Separations Section WasteStatus Summary June 1953). By the end of August 1953, tank TY-106 contained $752 \mathrm{kgal}$ of TBP waste (HW-29242, Separations Section Waste- Status Summary August 31, 1953). In September 1953, approximately $714 \mathrm{kgal}$ of TBP waste was transferred out of tank TY-106 to tank TX-118 (HW-29624, Separations Section Waste- Status Summary September 30, 1953). Tank TY-106 continued to receive TBP waste from tank TY-105 and transferred waste to tank TX-118 from 1953 to September 1954 with the volume reported at $758 \mathrm{kgal}$ at the end of September 1954 through December 1956 (RPP-RPT-42296, Hanford TY-Farm Leak Assessments Report). In July 1959, a new electrode was installed in tank TY-106 and the volume was reported to be $722 \mathrm{kgal}$ (HW-61952, Chemical Processing Department Waste Status Summary August 1, 1959 - August 31, 1959).

Tank TY-106 was first suspected of leaking in August 1959 when document HW-61952 indicated a loss of approximately $500 \mathrm{gal} /$ day (see Section 8.4.1) and $74 \mathrm{kgal}$ of waste was pumped to tank TY-101 initiated on August 28, 1959 (HW-61952; HW-61736-DEL, Chemical Processing Department Monthly Report for August, 1959). Tank TY-106 was removed from service and contents from tank TY-106 continued to be pumped to tank TY-101 and TY-103 in September and October 1959 leaving the volume at 20 kgal (RPP-RPT-42296).

Tank TY-106 was administratively interim stabilized in November 1978. Tank TY-106 is estimated to contain $16 \mathrm{kgal}$ of sludge, $1 \mathrm{kgal}$ of drainable interstitial liquid, and no supernatant (HNF-EP-0182, Rev. 301, Waste Tank Summary Report for Month Ending April 30, 2013).

The operational history of tank TY-106 leak related details and liquid level are charted in Figure 8-2. 


\section{Figure 8-2. Operational Leak History of Tank TY-106}

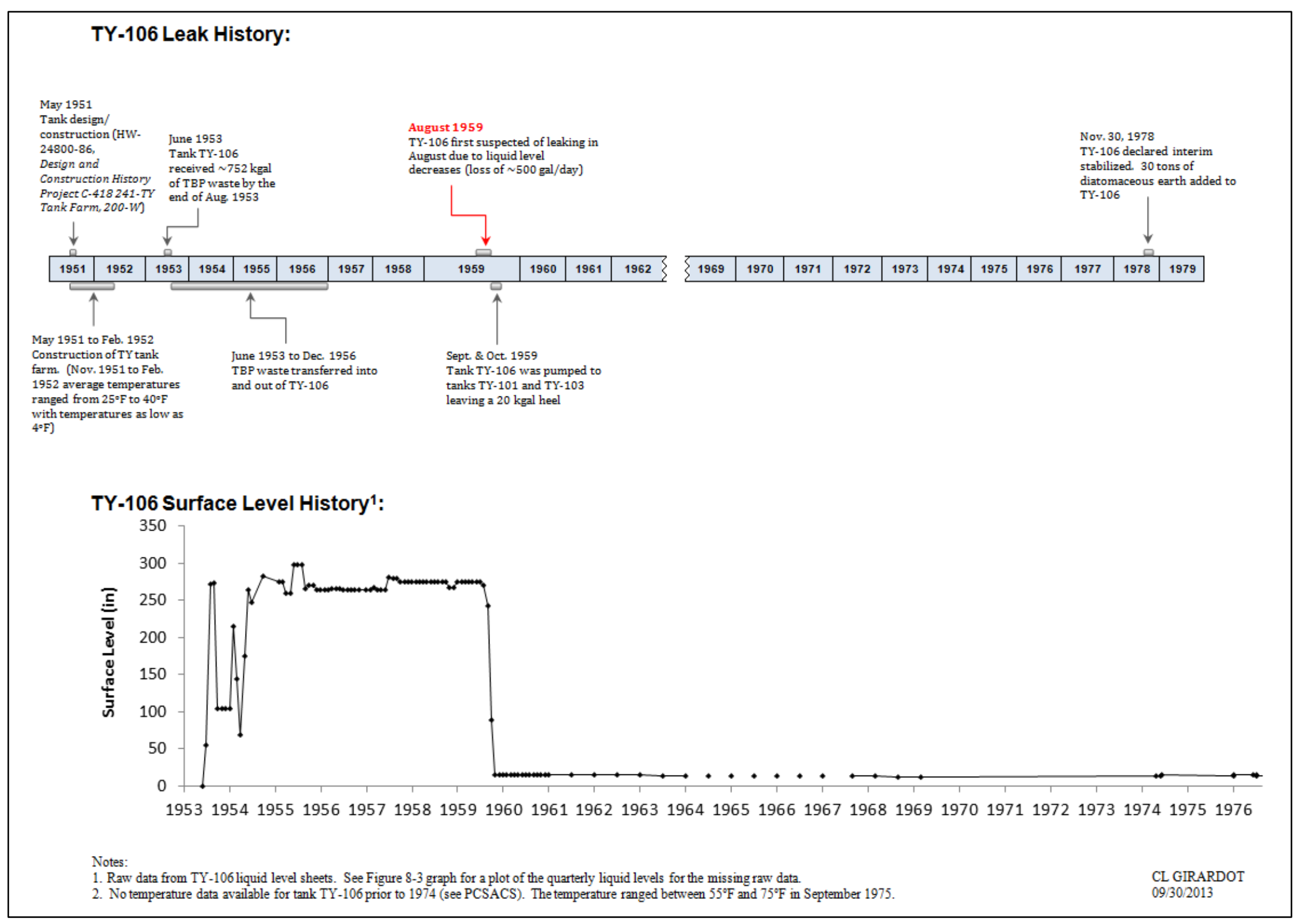


RPP-RPT-54911, Rev. 0

\subsection{TANK DESIGN/CONSTRUCTION}

\subsubsection{Tank Design}

The TY Farm tank design continued important features of the earlier 241-BCTU tanks (BPF73550). The steel bottom intersects the sidewall on a 4-ft radius. Full penetration butt welds with $x$-ray inspection and three ply asphaltic membrane waterproofing between the wall liner and the concrete shell continued those design features found to be important for liner integrity during the 241-SX Farm leak assessment (RPP-RPT-54910). The top of the tank footing is 43 -ft BGS and is 3-ft thick with the bottom of the footing at 4 46-ft BGS.

\subsubsection{Tank Construction Conditions}

The TY Farm was constructed from May 1951 to February 1952. Cold weather could have affected the steel during construction in December and January. Temperatures for December 1951 and January 1952 averaged $27.4^{\circ} \mathrm{F}$ and $25.2^{\circ} \mathrm{F}$, respectively, with temperatures as low as $4^{\circ} \mathrm{F}$ (PNNL-15160). Weather precautions for welding and concrete were specified in $\mathrm{HW}$ 24800-30 (see Section 3.1.2).

As described in Section 3.1.2, cold weather affects the ductile-to-brittle steel transition temperature, with $18^{\circ} \mathrm{F}$ being the assumed unrestricted low temperature construction limitation for the carbon steel liner, which could result in a fracture upon impact. Low temperatures experienced during construction at or less than the $18^{\circ} \mathrm{F}$ allowable temperature could cause impact loading (e.g. a dropped tool or piece of equipment from scaffolding) and result in the potential for creating micro-fissures weakening the steel liner.

\subsection{TANK TY-106 IN-TANK DATA}

\subsubsection{Liquid Level}

The liquid level plot in Figure 8-3 indicates the transfer activity into and out of tank TY-106. The liquid levels are end of quarter levels so this figure may not reflect all transfers into and out of the tank that occurred during the operational history of the tank. See Figure 8-2 for historical monthly liquid level readings. A total of 5,000 kgal was received in tank TY-106 through the cascade line from tank TY-105 over a two year period from 1953 to 1954. 
RPP-RPT-54911, Rev. 0

Figure 8-3. Tank TY-106 End of Quarter Surface Level

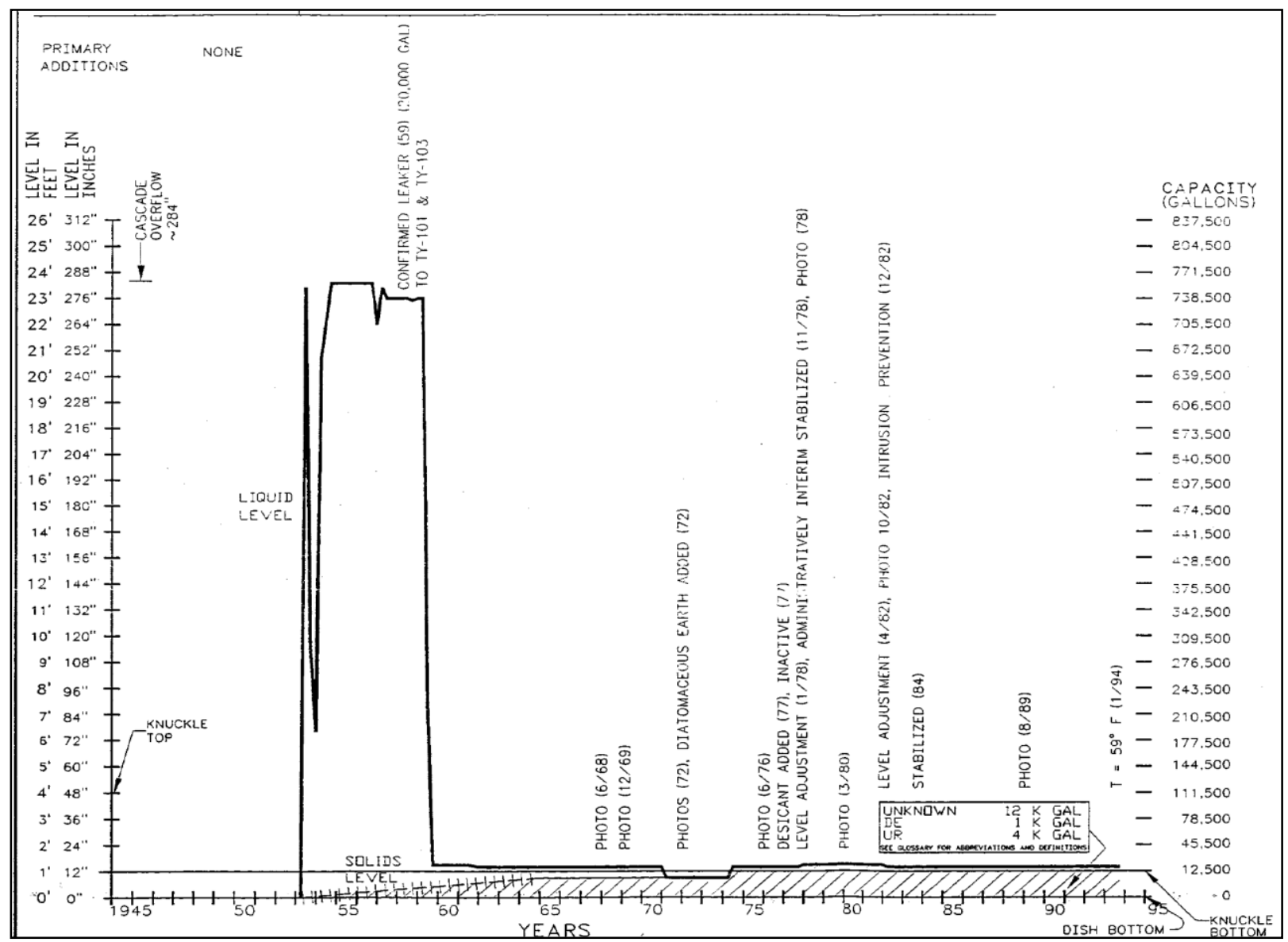

WHC-SD-WM-ER-351, Rev 0, March 1, 1995, Historical Tank Content Estimate for the Northwest Quadrant of the Hanford 200 West Area

The volume of tank TY-106 was reported to be at $758 \mathrm{kgal}$ (full to overflow level) in October 1954, with the last transfer into the tank. The reported waste volumes slowly declined and from January to May 1957, the volume was reported to be 705-706 kgal. Document RPP-RPT-42296 reported these readings to be bad readings as the volume was reported back at $752 \mathrm{kgal}$ in June 1957 indicating intervening readings were incorrect. The waste volume slowly declined and was reported at $739 \mathrm{kgal}$ at the end of June 1959 (RPP-RPT-42296). It was reported in document HW-83906-D-RD, Chemical Processing Department 200 West Area Tank Farm Inventory and Waste Reports January 1959 through June 1961, that, "the 106-TX," [assumed to be 106-TY], "showed a decrease of 6" from the previous months inventory reading. A new electrode has been installed in the 106-TY tank and electrode readings will be obtained daily for any further decrease in liquid level" (HW-83906-D-RD. The volume was reported to be $722 \mathrm{kgal}$ at the end of July 1959 (HW-83906-D-RD).

Daily electrode readings started immediately and indicated a loss of approximately $500 \mathrm{gal} / \mathrm{day}$ (14,000 gal total during the month) for 34 days from tank TY-106 in August 1959 (HW-61736DEL, Chemical Processing Department Monthly Report for August 1959). Daily readings were not found. Beginning August 28, 1959, pumping of tank contents commenced out of tank TY106. Pumping of the TBP waste from tank TY-106 was discontinued at approximately the 12-ft waste level on September 6, 1959 and remained undisturbed through September 14, 1959 (HW- 
62179-DEL, Chemical Processing Department Monthly Report for September 1959). During this period, no further leakage was detected indicating the tank leak was either above the $12-\mathrm{ft}$ level ( 34-ft 10-in BGS) or that the leak had sealed itself. Although no further leakage was apparently detected during the eight day interval, pumping resumed leaving a heel of approximately $20 \mathrm{kgal}$ by the end of October 1959 so that a thorough inspection of the tank liner could be made (see Section 8.4.5) (HW-62179-DEL).

Daily liquid level readings could not be recovered during August 1959 when the tank was reported to be leaking therefore the leak volume remains uncertain as only two data points were recovered, one before the leak was reported and the other after starting the tank transfer.

\subsubsection{Temperature}

No temperature data were recovered for tank TY-106 from June 1953 when the tank was first put into service until 1974 (see PCSACS). The maximum temperature in 1977 was $78^{\circ} \mathrm{F}$ and in September 1975 the temperature ranged between $55^{\circ} \mathrm{F}$ and $75^{\circ} \mathrm{F}$ (RPP-RPT-42296).

Tank TY-106 received TBP waste cascaded from tank TY-105 beginning in June 1953. TBP wastes were concentrated and cooled to $\sim 180^{\circ} \mathrm{F}$ within the plant and were estimated to be 110 $180^{\circ} \mathrm{F}$ after routing to the storage tanks (see Section 3.1.6). Note that $5,000 \mathrm{kgal}$ were routed to tank TY-106 from tank TY-105 through the cascade line over a two year period. This would indicate the temperature was near $180^{\circ} \mathrm{F}$ for a significant portion of the two year period. Rate of rise temperatures during initial tank filling are unknown.

\subsubsection{Liner Observations}

No liner observations relating to a tank TY-106 leak have been found (see Section 8.4.5 for additional details).

\subsubsection{Chemistry-Corrosion}

Tank TY-106 began receiving TBP waste cascaded from tank TY-105 in June 1953 and only stored TBP waste throughout operation as shown in Table 8-1. The typical concentrations for nitrite, nitrate, and hydroxide for TBP waste is shown in Table 8-2. Nitrite and hydroxide are known as nitrate-induced SCC inhibitors. One key characteristic for inhibiting SCC is to maintain a high nitrite concentration to nitrate concentration ratio (see Section 3.2.4).

Table 8-1. Tank TY-106 Waste Storage Chronology

\begin{tabular}{|c|c|c|}
\hline Date & Waste Type & Length of Storage \\
\hline June 1953 to September 1959 & TBP & $\sim 6$ years \\
\hline
\end{tabular}


No photographic evidence of bulging was found. No other documentation was found indicating a liner bulge in tank TY-106.

\subsection{TANK TY-106 EX-TANK DATA}

\subsubsection{Drywells}

There are six drywells located around tank TY-106: 52-06-05 and 52-06-07 installed in 1952; 52-04-06 and 52-06-02 installed in December 1971; 52-06-04 and 52-06-06 installed in August 1974. All of the radiation readings in drywells are assumed to be maximum or peak readings unless otherwise noted (see Sections 3.3 and 3.4). The following subsections report the available drywell information and the drywell summary section provides the analyses of the associated drywells with tank TY-106.

\subsubsection{Drywell 52-04-06 $\left(\mathrm{S0}^{\circ}\right)$}

Drywell 52-04-06 was drilled December 1971, approximately 12 years after the tank TY-106 leak was first detected. The first recoverable reading for drywell 52-04-06 was reported on July 11,1973 at less than $12 \mathrm{~K}$ cpm. Radioactivity was reported as less than values from July 1973 to June 1986 (see Appendix C5). Document HNF-3831 states that no significant levels of radioactivity are present about the survey probe detection threshold between 1975 and 1994 in this drywell.

In May 1997, Cs-137 was the only man-made radionuclide detected in drywell 52-04-06 (GJHAN-69). Cs-137 was detected from the ground surface to 12-ft BGS and at the bottom of this drywell with the highest concentration $(0.79 \mathrm{pCi} / \mathrm{g})$ at $1.5-\mathrm{ft}$ BGS. Document GJ-HAN-69 states, "The near-surface contamination (0 to $12 \mathrm{ft}$ ) probably resulted from surface spills that have migrated down into the backfill surrounding the borehole or contamination that was carried down during the drilling of this borehole." Since historical records report less than values for radioactivity, drywell 52-04-06 is not included as part of the leak location for tank TY-106. Figure 8-4 shows depths of radioactivity from 1975 to 1994 (HNF-3831). 
RPP-RPT-54911, Rev. 0

Figure 8-4. Tank TY-106 Associated Drywell 52-04-06 (HNF-3831)

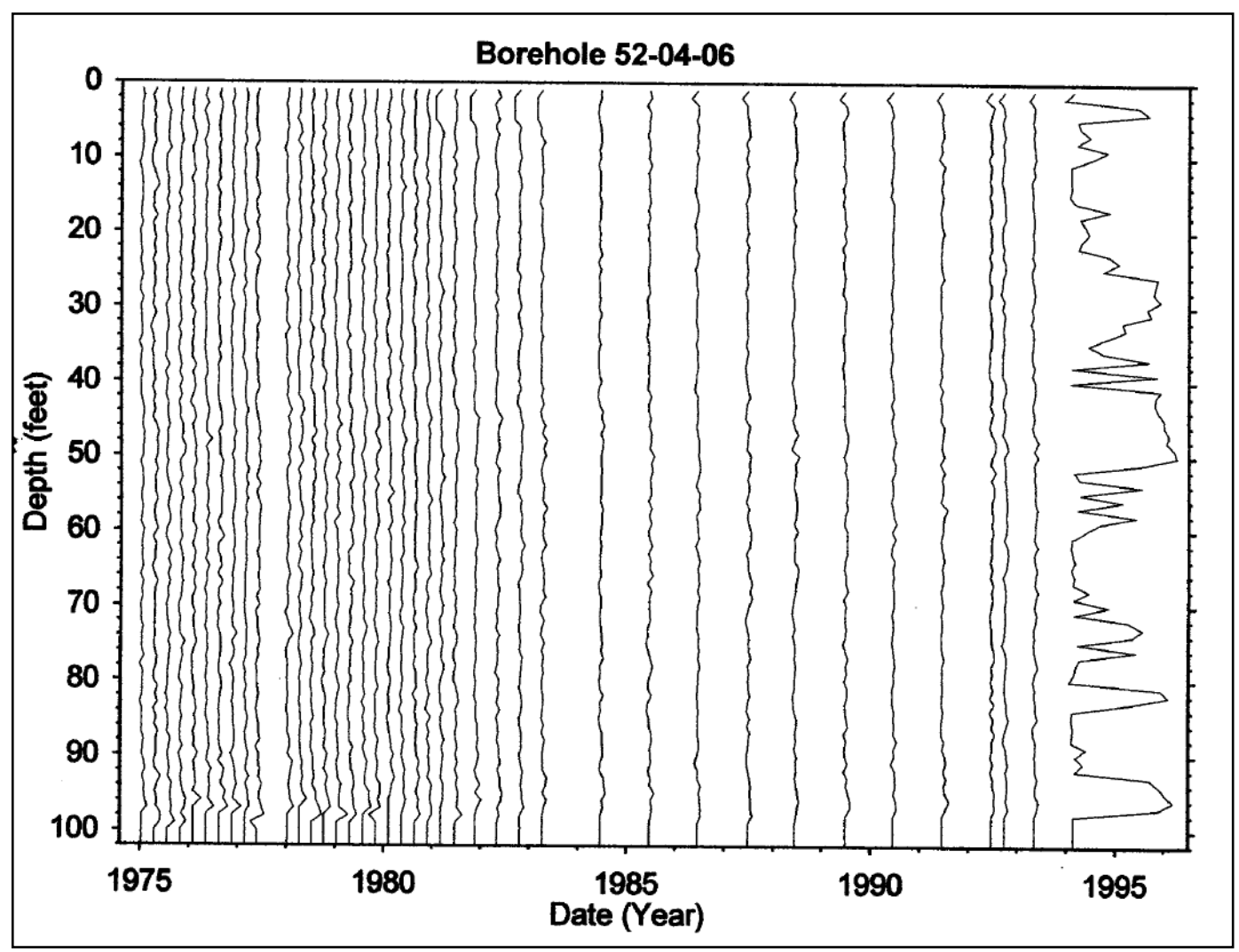

Note: Bottom of the tank footing is $\sim 46-\mathrm{ft}$ BGS

\subsubsection{Drywell 52-06-02 $\left(\mathrm{N} 31^{\circ} \mathrm{E}\right)$}

Drywell 52-06-02 was drilled in December 1971, 12 years after the tank TY-106 leak was first detected, with the first recoverable reading on April 4, 1974 as less than $12 \mathrm{~K}$ cpm with the SP (scintillation probe). Radiation readings were reported as less than values from April 1974 to June 1986 (see Appendix C5). Document HNF-3831 states that Cs-137 contamination is only present at the surface from 0 to $10-\mathrm{ft}$ BGS.

In May 1997, Cs-137 was the only man-made radionuclide detected in drywell 52-06-12 (GJHAN-69). Cs-137 was detected continuously from the ground surface to 65-ft BGS with the maximum concentration of $13.1 \mathrm{pCi} / \mathrm{g}$ reported at 4-ft BGS. As stated in GJ-HAN-67, "Reasons for the relative continuity of the contaminated zone compared to other boreholes associated with tank TY-104 are unknown. However, the contamination in borehole 52-06-02 may be related to the much more pervasive surface contamination that occurs in the vicinity of tank TY-105." Drywell 52-06-02 is therefore not included as part of the leak location for tank TY-106. Figure 8-5 shows depths of radioactivity from 1975 to 1984 (HNF-3831). 
RPP-RPT-54911, Rev. 0

Figure 8-5. Tank TY-106 Drywell 52-06-02 (HNF-3831)

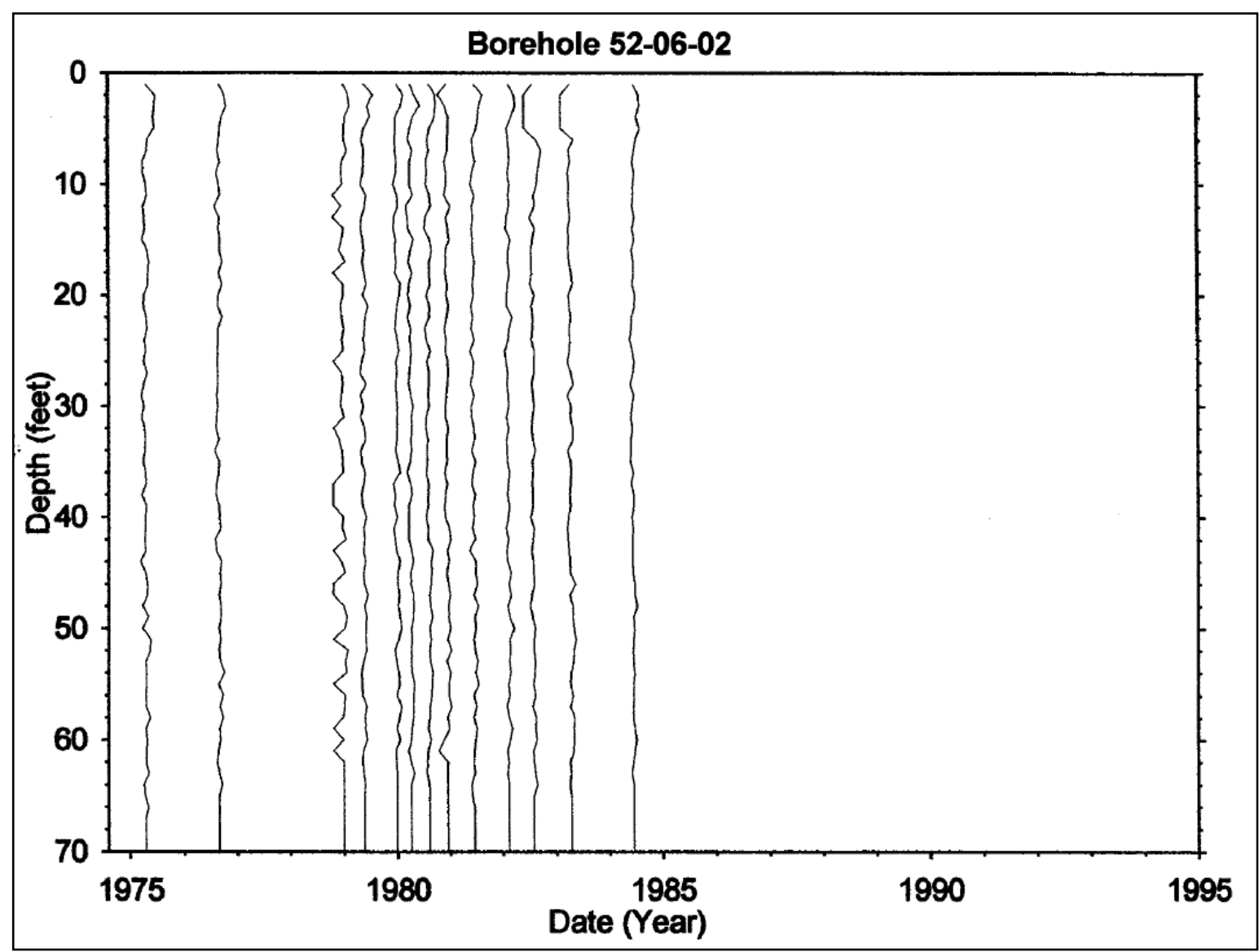

Note: Bottom of the tank footing is 46-ft BGS

\subsubsection{Drywell 52-06-04 $\left(\mathrm{S}^{\circ} 1^{\circ} \mathrm{E}\right)$}

Drywell 52-06-04 was drilled in August 1974, approximately 15 years after the tank TY-106 leak was first detected with the first recoverable reading on September 13, 1974 at $8.3 \mathrm{~K} \mathrm{cpm}$ at 53 -ft BGS. Radiation readings gradually declined to $2.5 \mathrm{~K}$ cpm by June 1986 with depths reported at $\sim 50$-ft BGS from 1974 to 1986 (see Appendix C5).

In May 1997, Cs-137 was the only man-made radionuclide detected in drywell 52-06-04 (GJHAN-69). Cs-137 was detected from the ground surface to 53.5-ft BGS and at 57-ft BGS with the maximum concentration of $1.2 \mathrm{pCi} / \mathrm{g}$ reported at $52-\mathrm{ft}$ BGS. Radioactivity from 47 to $56-\mathrm{ft}$ BGS was reported to be stable in document HNF-3831. Document GJ-HAN-68 states, "it is possible that the Cs-137 contamination originated from a breach in either tank TY-105 or TY106." It appears radioactivity detected in this drywell was the result of the tank TY-105 leak; however, tank TY-106 cannot be entirely ruled out (see tank TY-105 Segment). Figure 8-6 shows depths of radioactivity from 1975 to 1994 (HNF-3831). 
RPP-RPT-54911, Rev. 0

Figure 8-6. Tank TY-106 Drywell 52-06-04 (HNF-3831)

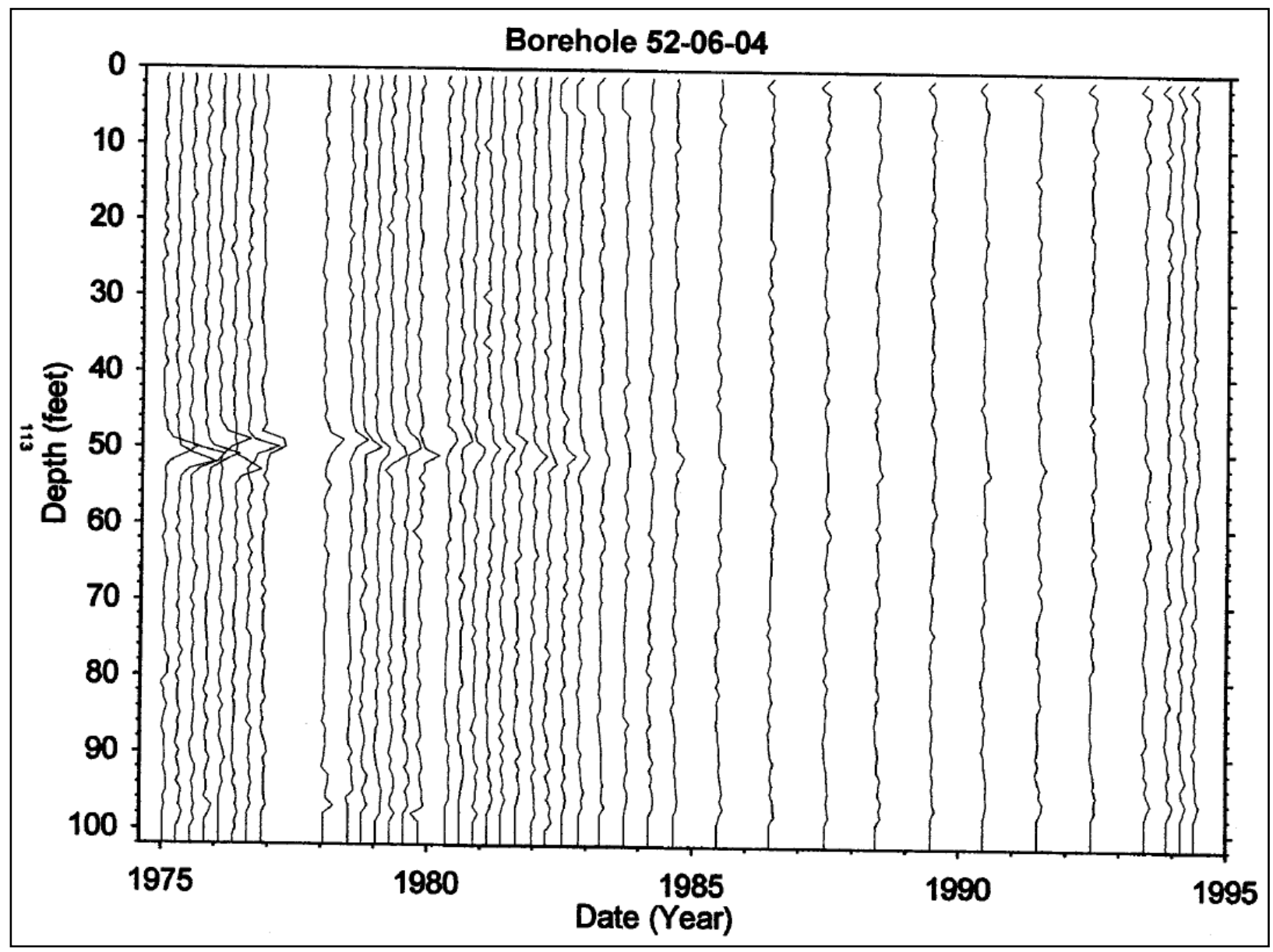

Note: Bottom of the tank footing is $\sim 46-\mathrm{ft} \mathrm{BGS}$

\subsubsection{Drywell 52-06-05 $\left(\mathrm{S45}^{\circ} \mathrm{E}\right)$}

Drywell 52-06-05 was drilled in August 1952 to a depth of 150-ft and was one of the only two drywells present at the time the tank TY-106 leak was first detected in 1959. The first recoverable reading was reported on July 16,1973 at $67.2 \mathrm{~K}$ cpm at $62-\mathrm{ft} \mathrm{BGS.} \mathrm{Radiation}$ readings were relatively stable at this depth with an additional peak detected December 18, 1973 at $67.8 \mathrm{~K} \mathrm{cpm}$ at $52-\mathrm{ft}$ BGS. Radioactivity appeared to remain stable until July 1974 and then slowly decreased through June 1986 (see Appendix C5).

There have been previous indications that activity was present in drywell 52-06-05 as early as September 1959; however, after reviewing Internal memo, "105-TY Waste Storage Tank Leak," it seems likely early radioactivity was referring to drywell 52-06-07 (see Section 8.5.1.6).

Two occurrence reports were written for increased activity in drywell 52-06-05 (OR-74-102, Leakage Symptoms as Indicated by Dry Well Activity at Waste Tank 105-TY and OR-74-88, Symptoms Of Leakage As Indicated By Activity In Dry Well 52-06-05 At 106-TY Waste Tank). Occurrence report OR-74-88 identifies the source of the drywell 52-06-05 contamination as tank TY-105. Figure 8-8 shows depths of radioactivity from 1975 to 1994 (HNF-3831). 
In May 1997, Cs-137 and Co-60 were the only man-made radionuclides detected in drywell 5206-05 (GJ-HAN-69). Cs-137 was detected from the ground surface to 133.5-ft BGS with the maximum concentration of $1.2 \mathrm{pCi} / \mathrm{g}$ reported at $111.5-\mathrm{ft}$ BGS. Co-60 was detected at $2.5-\mathrm{ft}$ BGS, from 52 to 90 -ft BGS, from 92.5 to 98.5 -ft BGS, and from 130 to 148-ft BGS with the maximum concentration of $3.2 \mathrm{pCi} / \mathrm{g}$ reported at $144.5-\mathrm{ft}$ BGS. Radioactivity detected in drywell 52-06-05 appears to be the fairly mobile radioisotopes, short lived $\mathrm{Ru}-106$ and also Co60, both of which decayed away (see Figure 8-7). The tank TY-106 direct pushes near drywell 52-06-05 did not indicate radioactivity down to $\sim 47-\mathrm{ft}$ BGS and were not deepened as there was no indication of further radioactivity (RPP-RPT-39511). Since there was no Cs-137 detected near tank TY-106, indications are that the radioactivity detected in drywell 52-06-05 was the leading edge (mobile radioisotopes) from a tank TY-105 leak.

Figure 8-7. Tank TY-105 Associated Drywell 52-06-05 Cesium (HNF-3831)

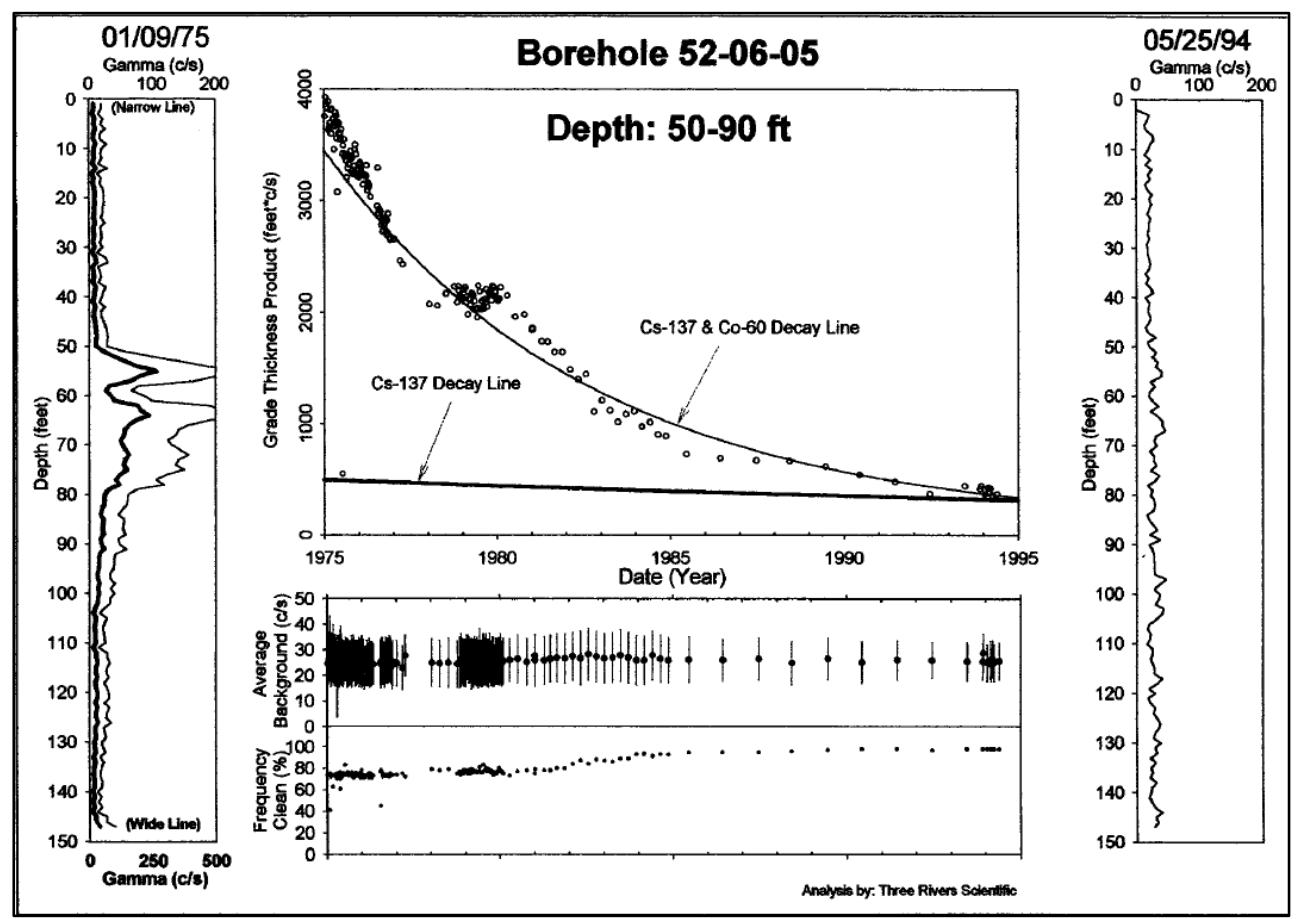


Figure 8-8. Tank TY-106 Drywell 52-06-05 (HNF-3831)

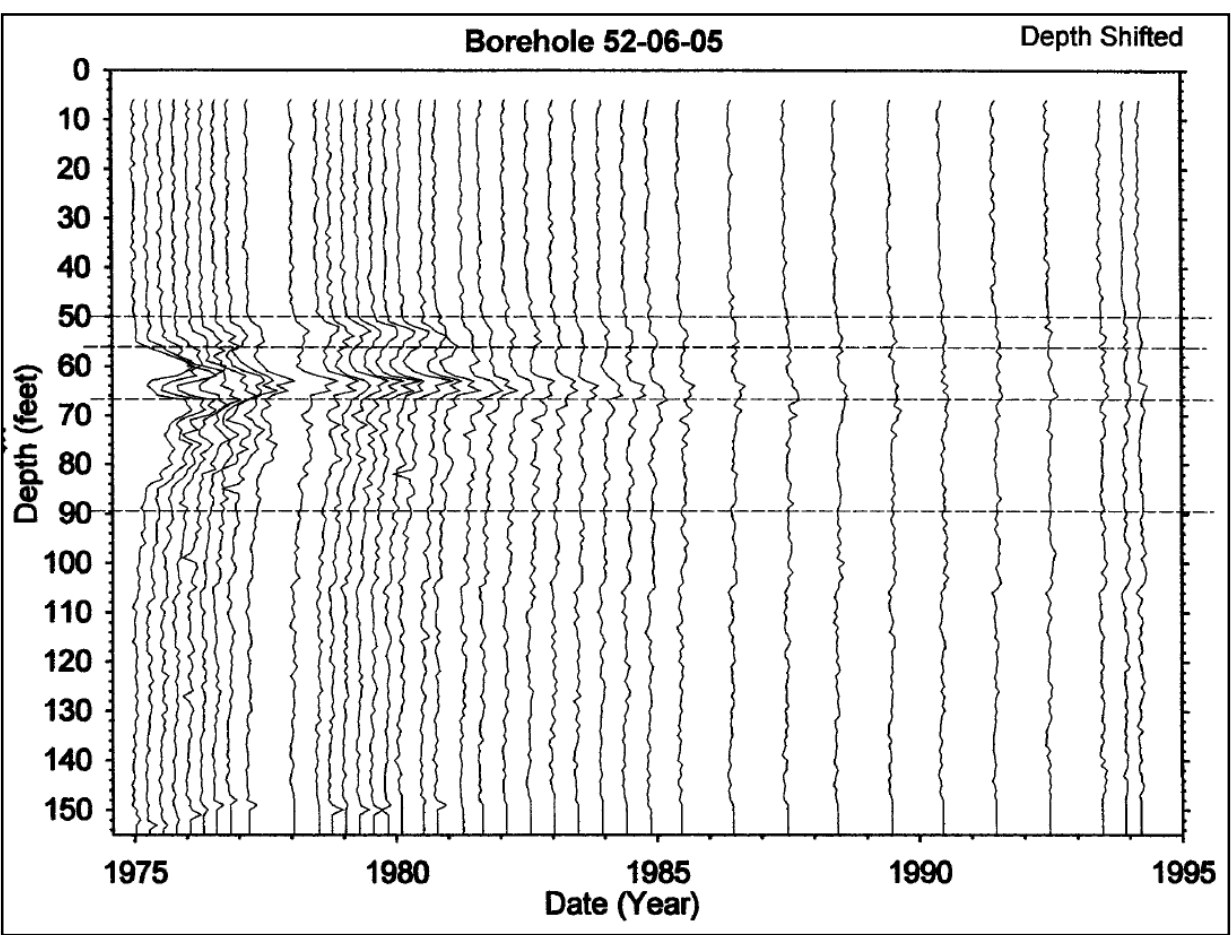

Note: Bottom of the tank footing is $\sim 46-\mathrm{ft}$ BGS. Depth shifting is a method to align the zero point due to inconsistent data collection.

\subsubsection{Drywell 52-06-06 $\left(\mathrm{S20}^{\circ} \mathrm{E}\right)$}

Drywell 52-06-06 was drilled in August 1974, approximately 15 years after the tank TY-106 leak was first detected with the first recoverable reading on September 13, 1974 at $6 \mathrm{~K} \mathrm{cpm}$ at an assumed 77-ft BGS based on subsequent readings. Radiation readings gradually declined to less than values by January 1981 through June 1986 (see Appendix C5).

In May 1997, Cs-137 and Co-60 were the only man-made radionuclides detected in drywell 5206-06 (GJ-HAN-69). Cs-137 was detected from the ground surface to 25-ft BGS and from 29 to $37-\mathrm{ft}$ BGS with the maximum concentration of $6.1 \mathrm{pCi} / \mathrm{g}$ reported at $19-\mathrm{ft}$ BGS. Co-60 was detected at $86-\mathrm{ft}$ BGS and from 95.5 to $100-\mathrm{ft}$ BGS with the maximum concentration of 0.29 pCi/g reported at 100-ft BGS. The GJ-HAN-69 Cs-137 logged in 1996 does not appear to indicate a separate leak of the tank liner at these BGS levels as the tank was pumped to a $20 \mathrm{kgal}$ heel in 1959 and Figure 8-9 shows only Cs-137 decay through 1995 (see Section 8.5.3, 8.6.1, and 8.6.4). 
Figure 8-9. Tank TY-106 Drywell 52-06-06 Cesium (HNF-3831)

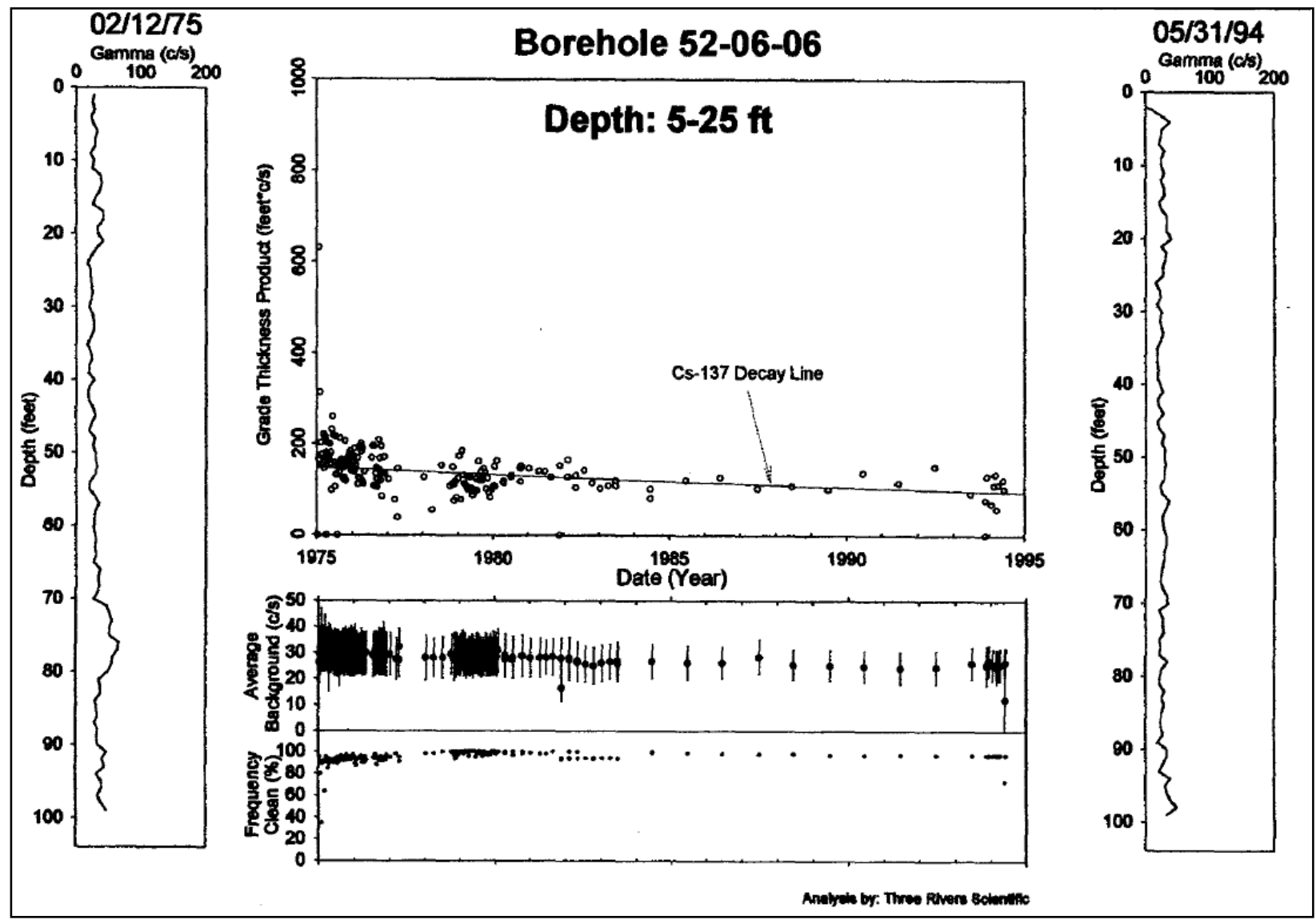

Also stated in GJ-HAN-69, "the Co-60 contamination most likely originated from a leak in either tank TY-103, TY-105, or TY-106..." It seems likely the Co-60 radioactivity detected in this drywell at the lower BGS levels was the result of some of the tank TY-106 leak as supported by the 2005 direct pushes (see Section 8.5.3). Figure 8-10 shows depths of radioactivity from 1975 to 1994 (HNF-3831). 
RPP-RPT-54911, Rev. 0

Figure 8-10. Tank TY-106 Drywell 52-06-06 (HNF-3831)

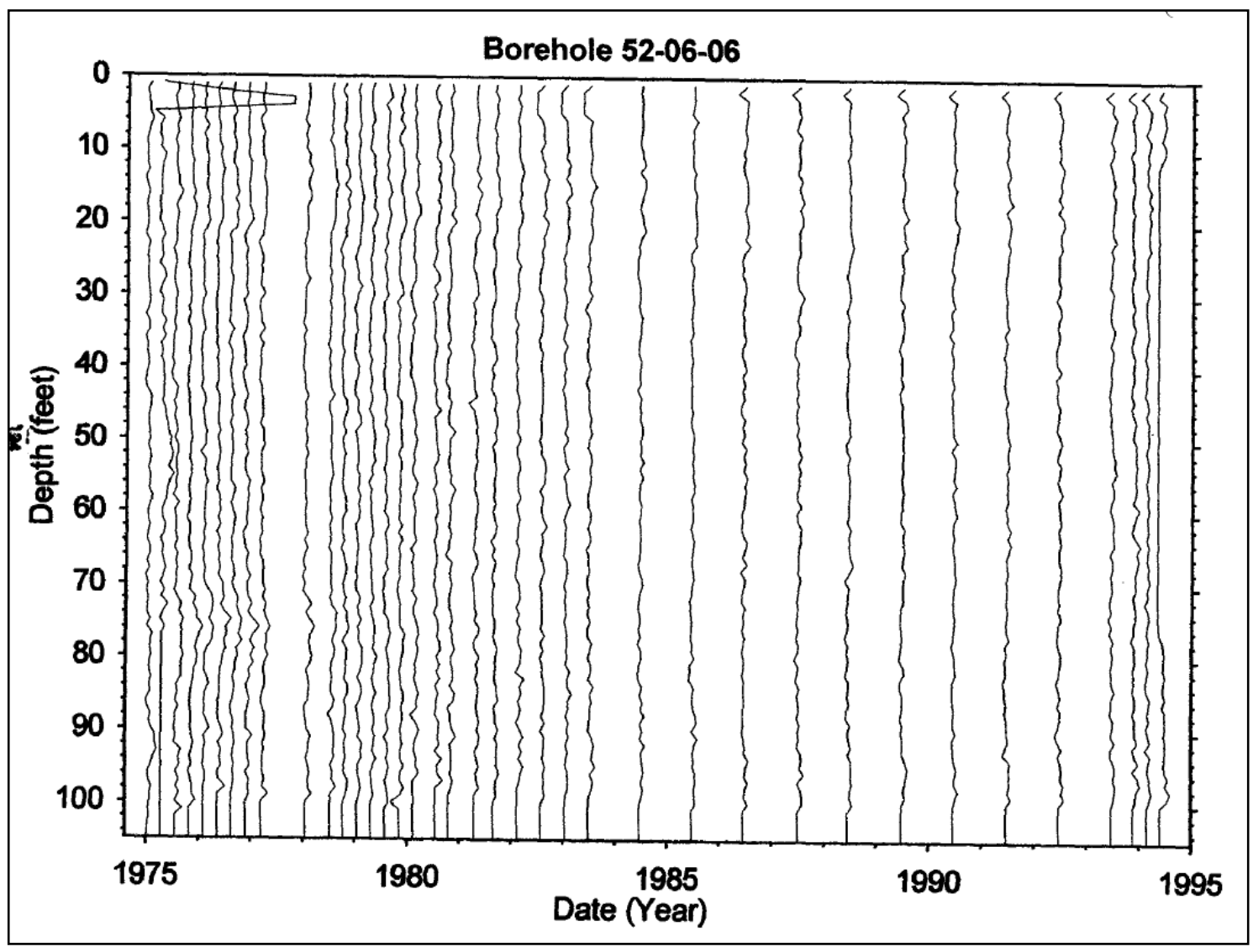

Note: Bottom of the tank footing is $\sim 46-\mathrm{ft}$ BGS

\subsubsection{Drywell 52-06-07 $\left(\mathrm{S43}^{\circ} \mathrm{W}\right)$}

Drywell 52-06-07 was drilled in August 1952 to a depth of 245-ft BGS and was one of the only two drywells present at the time the tank TY-106 leak was first detected in 1959. The first recoverable reading was reported in Internal memo (see Appendix B5) "105-TY Waste Storage Tank Leak" and stated, "Activity (up to 6,000 counts per minute) has been present in one of the wells since September, 1959..." [depth unreported] "...at which time it was concluded that the 106-TY tank was leaking. After transferring the 106-TY waste to another tank, the activity level began to drop and has now decreased to 2500 counts per minute (October 1960)." Also in this memo contains a hand written note stating a person called November 15, 1960 to inform that drywell TY-2 (referring to drywell 52-06-07) had a reading of 3,000 cpm between 191 and 211$\mathrm{ft}$ level. It appears the 191 to $211-\mathrm{ft}$ level refers to the BGS level which was later confirmed in the SGLS plot of drywell 52-06-07 reported in GJ-HAN-69.

The next recoverable reading was reported on July 11,1973 at $11 \mathrm{~K}$ cpm from 75 to 89 -ft BGS. Readings gradually increased to $20 \mathrm{~K}$ cpm through July 1974 between 75-90-ft BGS. Radiation readings then rapidly declined by September 1974 and were reported as less than values by June 1983 (see Appendix C5). 
In May 1997, Cs-137 and Co-60 were the only man-made radionuclides detected in drywell 5206-07 (GJ-HAN-69). Cs-137 was detected from the ground surface to 3-ft BGS and from 195.5 to 198.5 -ft BGS with the maximum concentration of $0.5 \mathrm{pCi} / \mathrm{g}$ reported at $1.5-\mathrm{ft} \mathrm{BGS}$. Co-60 was detected from 200 to $213-\mathrm{ft}$ BGS with the maximum concentration of $0.43 \mathrm{pCi} / \mathrm{g}$ reported at 204.5-ft BGS. Document GJ-HAN-69 states, "The near-surface contamination probably resulted from surface spills migrating down into the backfill surrounding the borehole. The contamination may also have been in the backfill before the borehole was drilled and carried down as the borehole was drilled."

Therefore, drywell 52-06-07 is not considered as part of the leak location for tank TY-106. However, there is a remote possibility that the radioactivity reported in drywell 52-06-07 between 75-ft and 89-ft BGS was a part of the leading edge of a tank TY-106 leak near the southern edge of the tank but the depth seems too low to be a direct link. Also, the BGS level of the 6,000 cpm radioactivity reported in Internal memo "105-TY Waste Storage Tank Leak" remains unknown. The 1973-1974 drywell 52-06-07 radioactivity remains unexplained. Figure 8-11 shows some low level radioactivity in 1975 which decays away (HNF-3831) as is indicated by the Appendix C5 data.

Figure 8-11. Tank TY-106 Drywell 52-06-07 (HNF-3831)

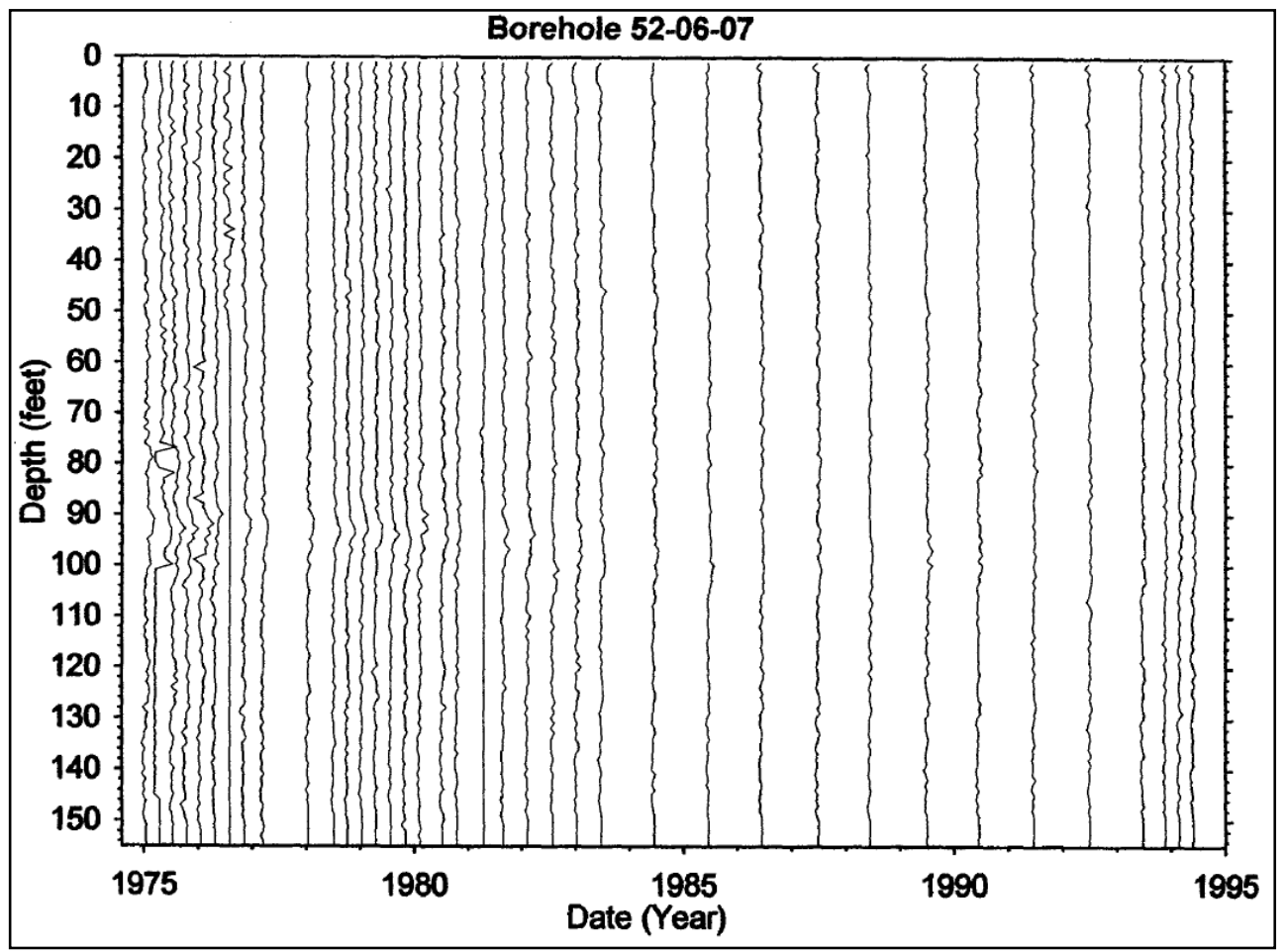

Note: Bottom of the tank footing is $\sim 46-\mathrm{ft} \mathrm{BGS}$ 


\subsubsection{Drywell Summary}

Tank TY-106 was first suspected of leaking in August 1959 due to liquid level decreases after initiating daily liquid level recordings, and contents were removed from tank TY-106 beginning August 28, 1959.

Tank TY-106 associated drywells 52-06-02 and 52-04-06 do not indicate any radioactivity associated with a tank leak. Therefore, drywells 52-06-02 and 52-04-06 are not included in the leak location for tank TY-106. Drywell 52-06-07 is also not considered due to several inconsistencies (see Section 8.5.1.6).

The remaining three drywells all detect radioactivity that could be associated with a tank leak. Drywell 52-06-05 was the only drywell present when tank TY-106 was first suspected of leaking and no data were recovered for this drywell until July 1973. The first recoverable reading for drywell 52-06-05 reported radioactivity July 1973 at 62-ft BGS with an additional peak detected five months later at 52-ft BGS. The first recoverable reading for drywell 52-06-06 reported radioactivity in September 1974 at 77-ft BGS, and the first recoverable readings for drywell 5206-04 reported lower levels of radioactivity in September 1974 at 53-ft BGS. It is likely drywells 52-06-04 and 52-06-05 indicate a leak from tank TY-105 at or near the tank footing (see Tank TY-105 Segment); however, migration from the tank TY-106 leak cannot be ruled out. Radioactivity reported in drywell 52-06-06 is likely the result of a leak from tank TY-106 as supported by the later direct pushes (see Sections 8.5.3 and 8.6.1).

There are no drywells present on the western and part of the eastern edge of tank TY-106, so it remains unclear whether radioactivity is present in this area. Additional drywells were pushed in 2005 as follows.

\subsubsection{Direct Push Logging}

Direct push logging to further characterize the tank TY-106 leak was conducted in October 2005 with the locations and maximum activity of the direct pushes shown in Figure 8-1 and Table 8-3 (RPP-RPT-34870, Small Diameter Geophysical Logging in the 241-TY Tank farm). Direct pushes were only logged once and only represent one profile in time. Compared to the drywells, direct pushes use a smaller diameter logging tool and results cannot be directly compared to drywell results. 
RPP-RPT-54911, Rev. 0

Table 8-3. 2005 Direct Push Gross Gamma Results (RPP-RPT-34870)

\begin{tabular}{|l|c|c|c|c|l|}
\hline $\begin{array}{c}\text { Direct } \\
\text { Push }\end{array}$ & $\begin{array}{c}\text { Initial } \\
\text { Hole } \\
\text { Depth (ft) }\end{array}$ & $\begin{array}{c}\text { Extend } \\
\text { Hole } \\
\text { Depth (ft) }\end{array}$ & $\begin{array}{c}\text { Depth Max. } \\
\text { Activity (ft } \\
\text { BGS) }\end{array}$ & $\begin{array}{c}\text { Max eCs } \\
\text { (pCi/g) }\end{array}$ & \multicolumn{1}{c|}{ Comment } \\
\hline C4603 & 47.1 & 61.3 & 45 & 115 & $\begin{array}{l}\text { Background not reached at bottom } \\
\text { of first survey. Hole Deepened. }\end{array}$ \\
\hline C4605 & 47.5 & 61 & 44.5 & 48 & $\begin{array}{l}\text { Minor count rate increase at bottom } \\
\text { of first survey. Hole Deepened. }\end{array}$ \\
\hline C4607 & 47 & - & Surface & 13 & Minor count rate increase at surface \\
\hline C4609 & 46.5 & - & Surface & 20 & Count rate increase at surface \\
\hline C4611 & 47.2 & - & - & Background & \\
\hline C4613 & 47.5 & - & Surface & 19 & Count rate increase at surface \\
\hline C4615 & 16.6 & - & - & Background & \\
\hline C4617 & 47.5 & 61.4 & 44 & 58 & $\begin{array}{c}\text { Minor count rate increase at bottom } \\
\text { of first survey. Hole Deepened. }\end{array}$ \\
\hline
\end{tabular}

Note: Direct pushes that did not indicate radioactivity at a lower level were not deepened.

Radioactivity was the highest in direct push C4603 with $115 \mathrm{pCi} / \mathrm{g}$ detected at 45 -ft BGS. Direct push C4605, adjacent to direct push C4603, reported $48 \mathrm{pCi} / \mathrm{g}$ at $44.5-\mathrm{ft}$ BGS indicating the tank likely leaked near these two pushes. Direct push $\mathrm{C} 4617$ reported $58 \mathrm{pCi} / \mathrm{g}$ at $44-\mathrm{ft} \mathrm{BGS}$ indicating another possible leak site. Direct pushes C4607, C4609, C4611, C4613, and C4615 do not report any radioactivity that could be associated with a tank leak.

It appears the tank TY-106 leaked near the tank footing in two possible locations close to direct pushes $\mathrm{C} 4603$ and $\mathrm{C} 4617$ as radioactivity detected in these pushes were the highest with possibly some migration to the other nearby drywells and direct pushes. The direct pushes on the southeast side of tank TY-106 indicate very little radioactivity was detected. Therefore, it appears earlier radioactivity detected in drywells 52-06-05 and 52-06-04 (see Section 8.5.2), located between tanks TY-105 and TY-106, were likely due to the tank TY-105 leak with the possibility of some migration from the leak from tank TY-106 (see tank TY-105 Segment).

See Appendix D5 for the gamma surveys for the direct push holes with reported radioactivity: C4603, C4605, and C4617. See document RPP-RPT-34870 for additional gamma surveys for the direct pushes for TY Farm.

\subsection{POSSIBLE TANK TY-106 LINER LEAK LOCATION(S)}

A liner leak may have penetrated the waterproof membrane at any location or pooled on the waterproof membrane and followed concrete cracks or construction joints to a different location for egress to the soil, including the top of the tank footing.

Some liquid level evidence indicates that the tank liner could have leaked above a liquid level of 12-ft (34-ft 10-in BGS). However, drywells and direct push results indicate that the tank TY-106 leak(s) probably did not occur far above the tank footing because all of the initial indicators, three drywells and three direct pushes, were located at or near the tank footing. Only two 
drywells were present near tank TY-106 when the tank was first suspected of leaking and data from only one drywell were recovered at this time. No other drywell data were recovered until approximately 14 years after tank TY-106 was first suspected of leaking. Also, there are no drywells located on the western side of tank TY-106 and no laterals were installed in TY Farm. Therefore, it is possible leaks could have occurred in this area and not have been detected.

\subsubsection{Leak Detected in 1974 and 2005, Site A}

Tank TY-106 was first suspected of leaking in August 1959 due to liquid level decreases. Only drywells 52-06-05 and 52-06-07 were present at the time the leak was detected. Only data for drywell 52-06-07 were recovered at the time tank TY-106 was first suspected of leaking and drywell 52-06-07 is not considered in the leak location for tank TY-106 (see Section 8.5.1.6).

Data for drywell 52-06-06 was first recovered in September 1974 at 77-ft BGS and was reported as less than values by January 1981 probably from more mobile short lived radioisotopes. In 2005, direct pushes were drilled to further characterize the tank TY-106 leak. Direct pushes C4603 and C4605 indicate radioactivity at $\sim 45-\mathrm{ft}$ BGS and radioactivity was the highest in direct push C4603 indicating the tank leaked near this drywell (see site A in Figure 8-12). It is likely the tank leaked near this location in 1959 and short lived mobile radioisotopes migrated to drywell 52-06-06 and decayed away so that no radioactivity was detected in 2005 in direct push C4607, located between direct push C4605 and drywell 52-06-06. No drywells are present on the western edge of tank TY-106. 
RPP-RPT-54911, Rev. 0

Figure 8-12. Tank TY-106 Possible Leak Location (1974 \& 2005)

Tank inner ring is steel liner, outer ring is outer edge of tank footing

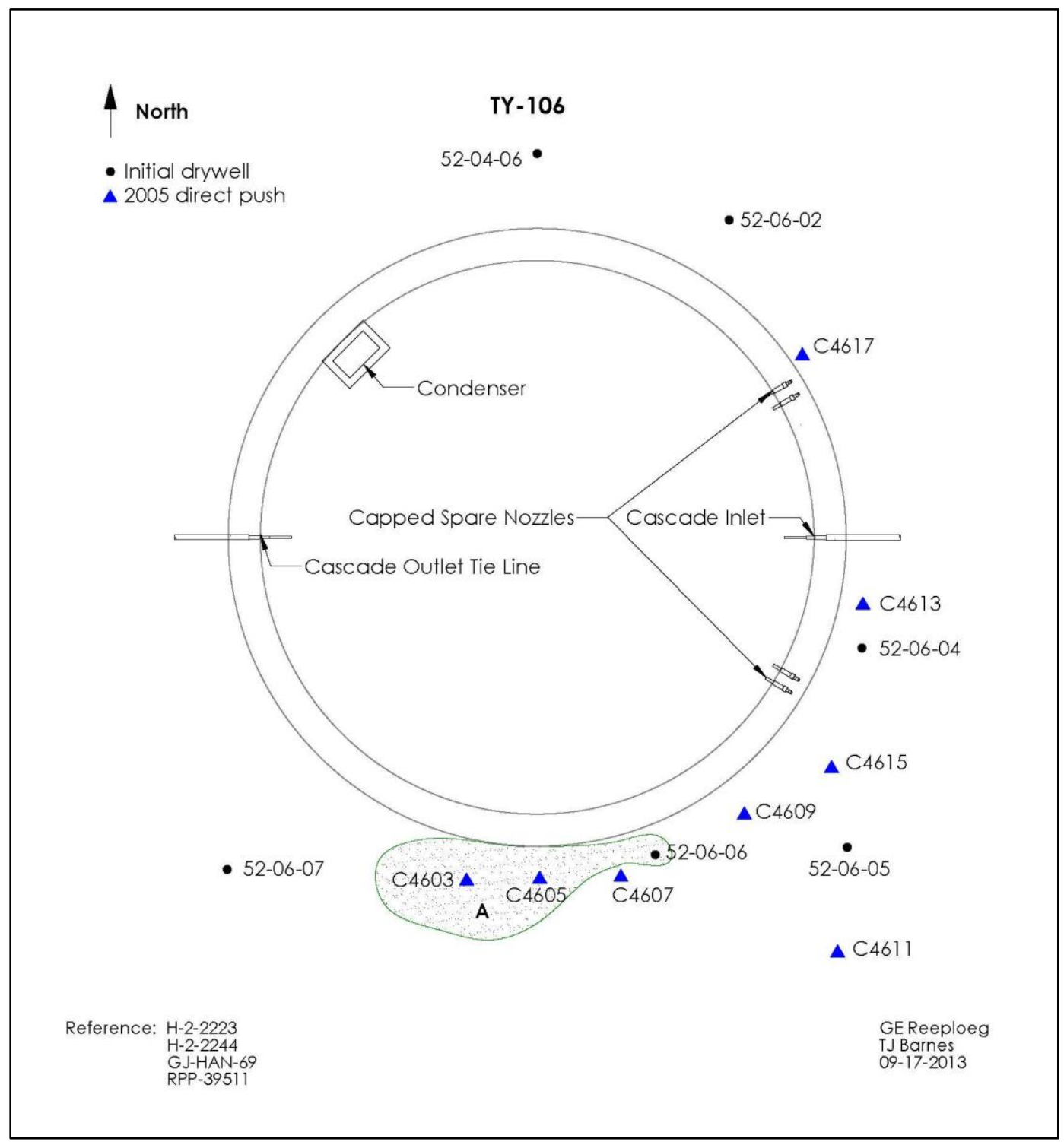

The leak from tank TY-106 was first detected in August 1959 due to liquid level decreases. 


\subsubsection{Leak Detected in 2005, Site B}

In 2005 direct push C4617, located in the northeastern portion of tank TY-106 was constructed. Radioactivity was reported in direct push $\mathrm{C} 4617$ to be $58 \mathrm{pCi} / \mathrm{g}$ at $44-\mathrm{ft} \mathrm{BGS}$. It is possible this is a separate leak location from sites $\mathrm{A}$ and $\mathrm{C}$ as no radioactivity was detected in the nearby drywells or direct pushes (see Figure 8-13).

Figure 8-13. Tank TY-106 Possible Leak Location (2005)

Tank inner ring is steel liner, outer ring is outer edge of tank footing

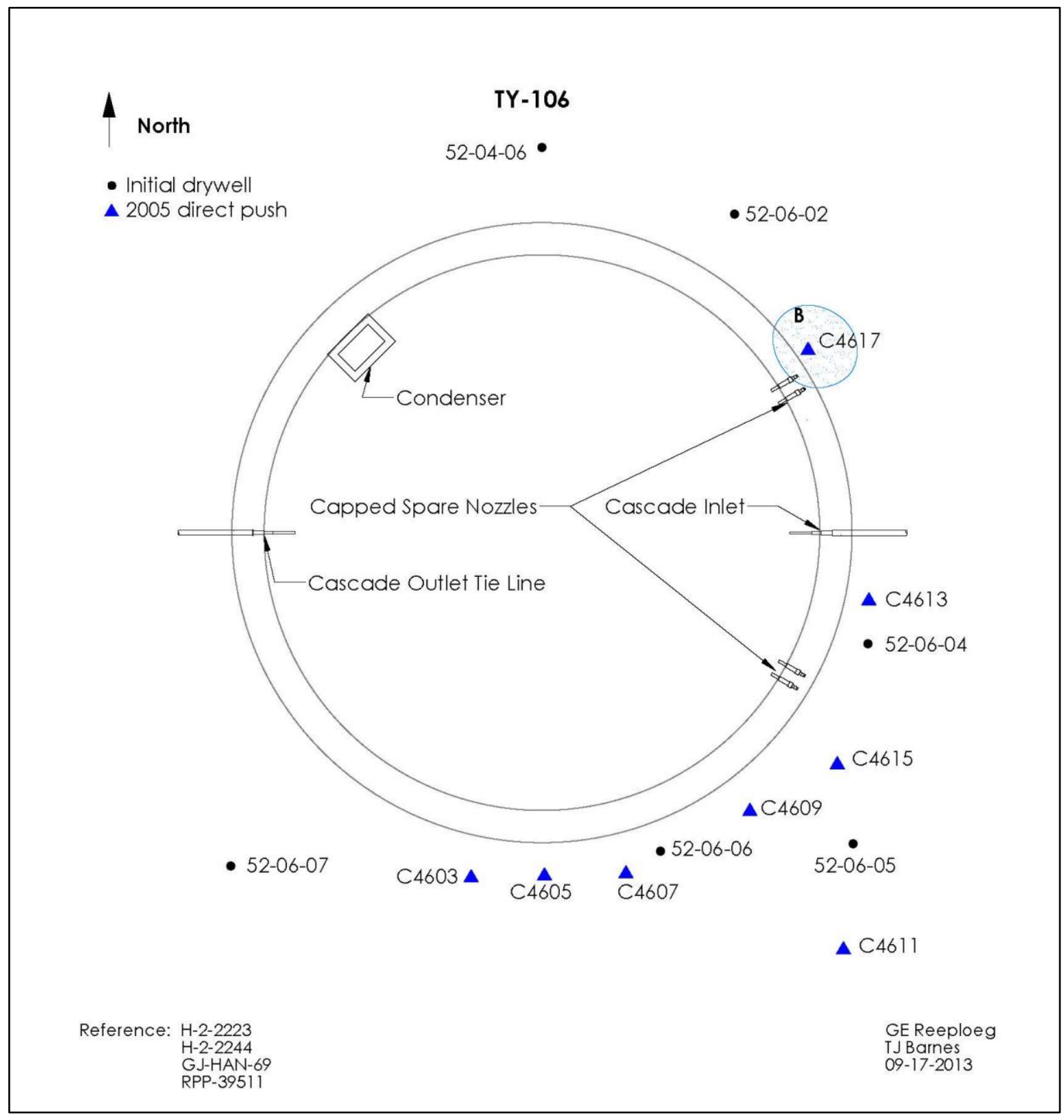

The leak from tank TY-106 was first detected in August 1959 due to liquid level decreases. 


\subsubsection{Leak Detected in 1973-1974, Site C}

The first recoverable data reported radioactivity in drywells 52-06-04 and 52-06-05 between 52$\mathrm{ft}$ and 62-ft BGS in 1973-1974, approximately 14 years after tank TY-106 was first suspected of leaking (see Site C in Figure 8-14). It appears radioactivity detected in these two drywells are from the tank TY-105 leak since the 2005 direct pushes located near these drywells do not report any radioactivity associated with a tank leak. However, there is a possibility that radioactivity detected in these drywells could be due to a leak from tank TY-106 and/or a combination of the tank TY-105 and TY-106 leaks. It is likely this is a separate leak location from sites A and B as no radioactivity was detected in the nearby drywells or direct pushes.

Figure 8-14. Tank TY-106 Possible Leak Location (1973-1974)

Tank inner ring is steel liner, outer ring is outer edge of tank footing

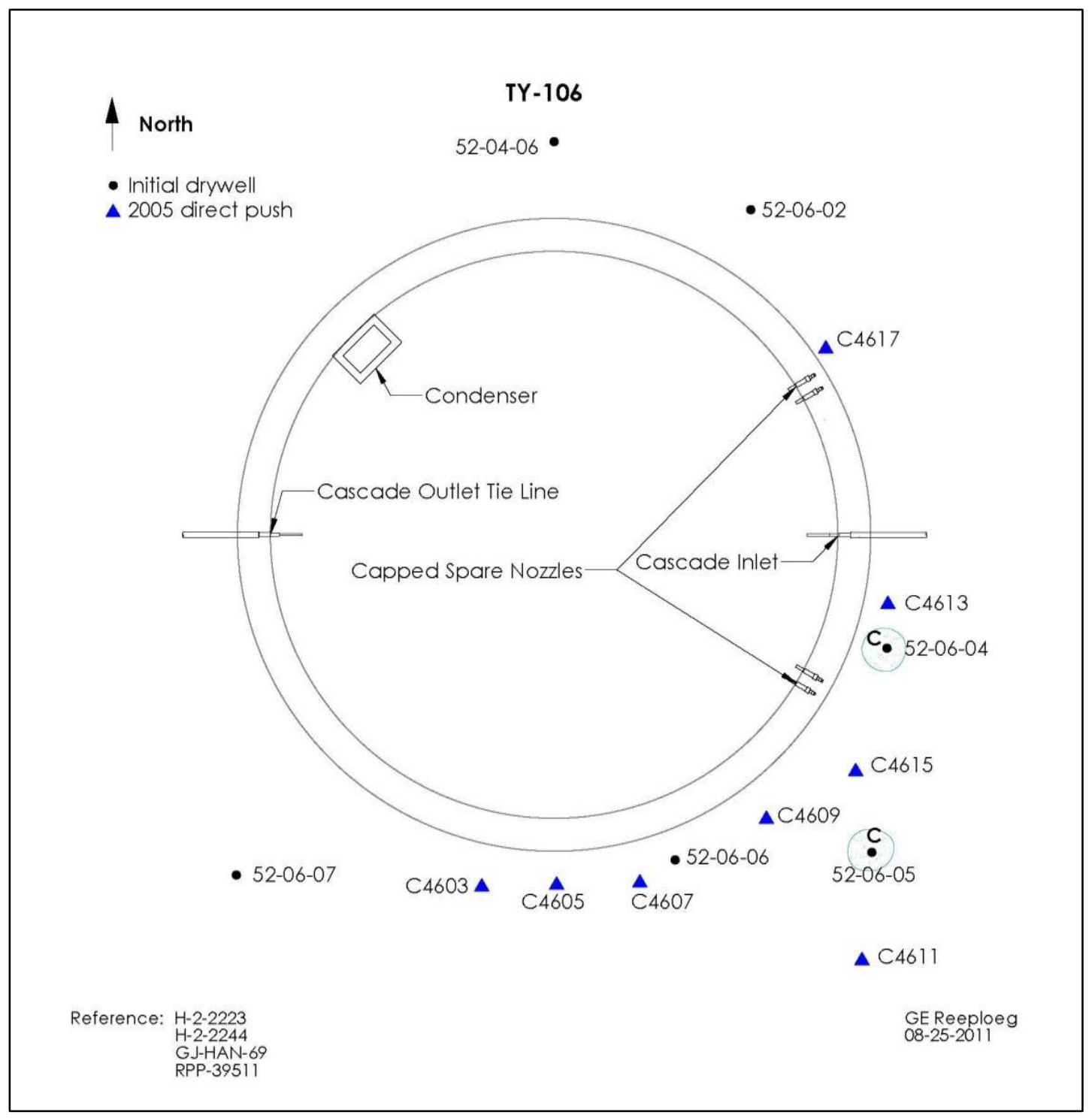

The leak from tank TY-106 was first detected in August 1959 due to liquid level decreases. 


\subsubsection{Leak Location Summary}

Tank TY-106 was first suspected of leaking due to liquid level decreases in August 1959. The first leak site was determined from 1974 drywell 52-06-06 and 2005 direct pushes C4603 and C4605 which reported radioactivity at or near the tank footing as shown in Site A (see Figure 8-15). It appears the leak site is located near direct push C4603 as radioactivity levels were the highest in 2005.

High levels of radioactivity were detected in direct push C4617 in 2005 as shown in site B (see Figure 8-15). Site B appears to be a separate leak site from sites A and C, as the nearby drywells and direct pushes do not detect any radioactivity associated with a tank leak.

Radioactivity was detected in drywells 52-06-04 and 52-06-05 at a lower BGS level in 19731974 as shown in site C (see Figure 8-15). It appears radioactivity detected in these drywells are the result of the tank TY-105 leak; however, it is possible radioactivity in these drywells could be the result of the tank TY-106 leak and/or a combination of the two leaks.

The tank TY-106 leak(s) did not occur far above the tank footing because all of the indicators, one drywell and the 2005 direct pushes, were located below the tank footing. There was some indirect indication from a stable liquid level for eight days that a sidewall leak could have occurred above $~ 34$-ft 10-in BGS but available drywell and direct push evidence does not indicate a sidewall leak. It is possible contamination exists underneath the tank and in the western portion of the tank; however, no drywells or laterals are present in these areas so it remains inconclusive.

Leak locations in Figure 8-15 are based on peak readings and are a representation of possible initial boundaries of radioactivity.

No evidence was found for a liner bulge occurring in tank TY-106, and it remains unclear if a liner bulge once existed in the tank during its operation. However, tank TY-106 non-boiling temperatures are not likely to be a factor in causing a liner bulge. 
Figure 8-15. Tank TY-106 Possible Radial Leak Locations Tank inner ring is steel liner, outer ring is outer edge of tank footing

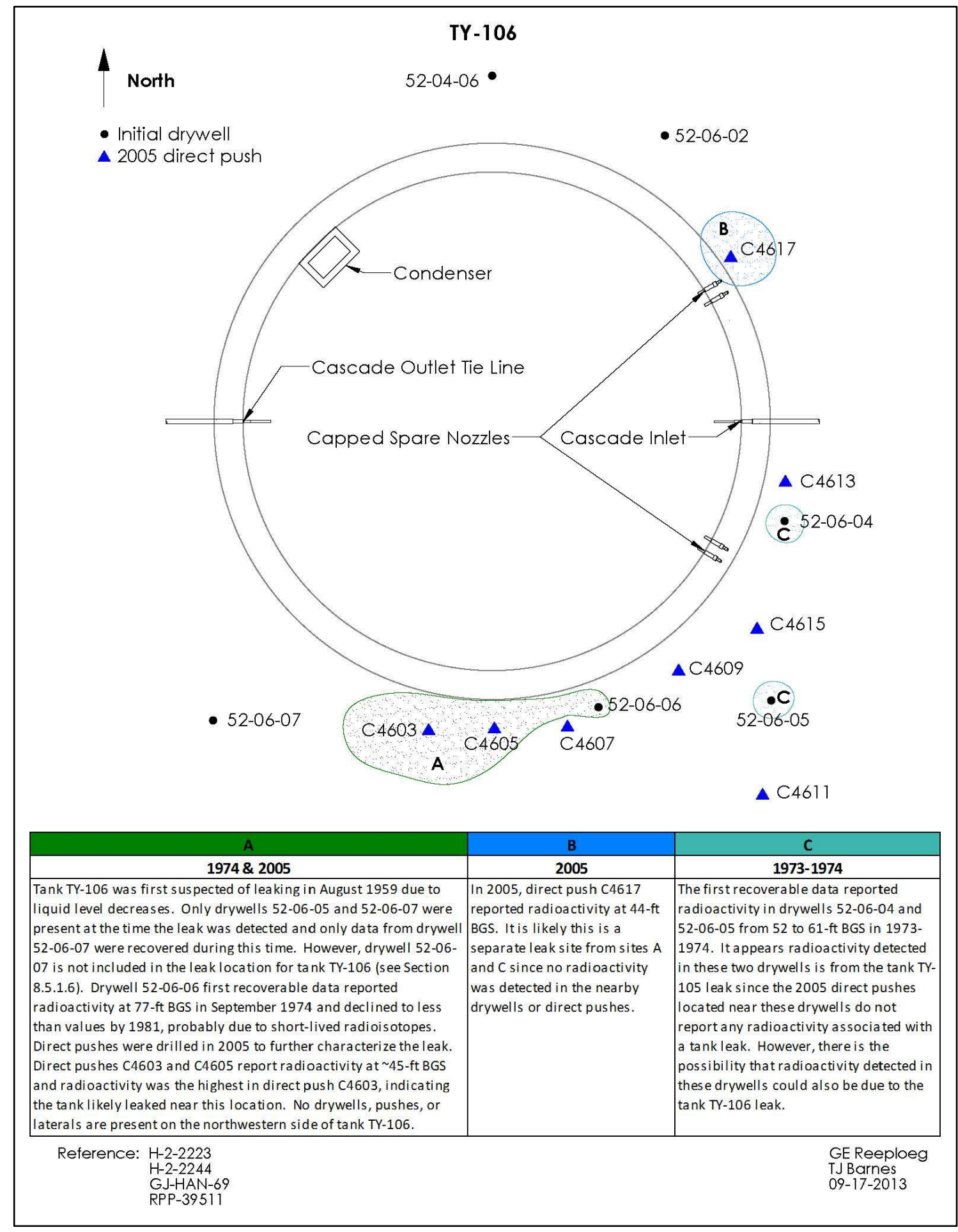


RPP-RPT-54911, Rev. 0

\subsection{POSSIBLE TANK TY-106 LINER LEAK CAUSE(S)}

Tank TY-106 was examined against five conditions that could contribute to a failed liner.

\subsubsection{Tank Design}

The TY Farm tank design does not appear to be a factor contributing to a failed liner (see Section 3.1.1).

\subsubsection{Thermal Shock}

No temperature data are available for tank TY-106 prior to 1974 although tank TY-106 held nonboiling waste. Since no records are available, it is uncertain what the maximum temperature was in tank TY-106 during operation as well as the rate of temperature rise when waste was initially added. However, TBP wastes were estimated to be $110-180^{\circ} \mathrm{F}$ after routing to the storage tanks (see Section 3.2.4). The volume of transfers $(5,000 \mathrm{kgal})$ in a two year period would tend to raise the temperature toward $180^{\circ} \mathrm{F}$.

Thermal shock creates stress both from rapid temperature rise as well as waste-induced high temperatures.

Temperature requirements in ARH-951 (Limitations for Use of Underground Waste Tanks) issued December 18, 1969 indicated that tank temperatures should be held below $230^{\circ} \mathrm{F}$.

\subsubsection{Chemistry-Corrosion}

Tank TY-106 was first filled with TBP waste and stored only TBP waste during operation for approximately six years until the leak was detected. TBP waste consists of low hydroxide and high nitrate concentrations, likely with low nitrite concentration, which would create an environment conducive to pitting and SCC. A tank with only the TBP waste type present would likely increase SCC in the tank liner (see Section 8.4.3).

\subsubsection{Liner Observations}

It was reported that tank TY-106 had portions of the lead flashing missing (HW-67459-DEL). However, after reviewing earliest recoverable photographs taken June 13, 1968 and all available photographs for tank TY-106, it remains inconclusive if portions of the lead flashing were missing. Also, quarterly reports indicated that the liquid level was no higher than the overflow.

No evidence of bulging was found in tank TY-106 and no other documentation was found indicating a liner bulge in tank TY-106.

\subsubsection{Tank Construction Temperatures}

Average winter temperatures were $27.4^{\circ} \mathrm{F}$ and $25.2^{\circ} \mathrm{F}$ in December 1951 and January 1952 , respectively, with temperatures as low as $4^{\circ} \mathrm{F}$ during the period when the TY Farm tank liners were being constructed. The low temperatures experienced during construction were less than the current allowable $18^{\circ} \mathrm{F}$ allowable temperature where impact loading had the potential for 
creating micro-fissures in the steel liner. The TY Farm construction specifications (HW-4696, Specifications Waste Disposal Facilities 241-BZ and TY Tank Farm) contained specific detailed requirements for cold weather construction and it appears these low temperatures had less of an impact during the construction of TY Farm compared to 241-SX Farm construction (see Section 3.1.2).

\subsection{TANK TY-106 CONCLUSIONS}

Evidence indicates that the tank TY-106 liner leaked at or near the tank footing in either two or three locations. Based on engineering judgment and available information the following conditions listed in the order of importance lead to the tank TY-106 failed liner.

1. TBP waste storage chemistry-corrosion -stress corrosion cracking

2. Thermal conditions

3. Construction conditions-ductile-to-brittle transition temperature

There are several liner leak cause conditions that were examined but the most likely cause of the tank TY-106 leak is TBP waste storage chemistry-corrosion. TBP waste storage could have created a chemical environment susceptible to nitrate-induced SCC attack on a potentially stressed metal liner.

Thermal and construction conditions could have also contributed to the liner failure but at a lesser degree. Tank design doesn't seem to have contributed to liner failure and there is no evidence of liner bulging. Some or all of the factors can act serially or together to contribute to tank liner failure. 
RPP-RPT-54911, Rev. 0

\section{APPENDIX A5}

TANK TY-106 OPERATIONAL HISTORY 
RPP-RPT-54911, Rev. 0

Table A5-1. ARH-R-43 Waste Tank Leak Experience Table

\begin{tabular}{|c|c|c|c|c|c|c|c|c|c|c|}
\hline $\begin{array}{l}\text { TABLE } 3 \\
\text { Tank } \\
\end{array}$ & $\begin{array}{l}\text { Whater } \\
\text { Built }\end{array}$ & $\begin{array}{l}\text { rourd } \\
\text { First } \\
\text { Uscd }\end{array}$ & azte Storage Fank Lea? Ea & Service & $\begin{array}{l}\text { Leak First } \\
\text { Suspected } \\
\end{array}$ & $\begin{array}{l}\text { Reason for } \\
\text { Suspicion }\end{array}$ & $\begin{array}{l}\text { Leak Confirmed } \\
\text { by } \\
\end{array}$ & $\begin{array}{l}\text { Bulge } \\
\text { Found } \\
\end{array}$ & $\begin{array}{l}\text { Estimsted } \\
\text { Volume of } \\
\text { Leak, gal } \\
\end{array}$ & $\begin{array}{l}\text { Associated } \\
\mathrm{Cs}=137, \mathrm{kCi}\end{array}$ \\
\hline $104-U$ & $1943 \cdot 44$ & 1947 & $\begin{array}{l}\text { Bismuth phosphate waste } \\
\text { Uranium recovery waste }\end{array}$ & Xonboiling & 1956 & Bulged 1 iner & $\begin{array}{l}\text { Refill with } \\
\text { water (1961) }\end{array}$ & Yes & $55,000^{*}$ & 0.09 \\
\hline $11.3-5 x$ & $1953-54$ & 1958 & Redox waste & Boiling & 1958 & Bulged 1 iner & $\begin{array}{l}\text { Refill with salt } \\
\text { solution }(1962)\end{array}$ & Yes & $15,000^{* *}$ & 8 \\
\hline $106-\mathrm{Tr}$ & 1951.52 & 1953 & THP waste & Nonboiling & 1959 & $\begin{array}{l}\text { Liquid leve } 1 \\
\text { measurentents }\end{array}$ & $\begin{array}{l}\text { Soil radiation } \\
\text { readings (1959) }\end{array}$ & & 20,000 & 2 \\
\hline $101 \cdot U$ & $1943 \cdot 44$ & 1948 & $\begin{array}{l}\text { Bismuth phosphatc waste } \\
\text { Uranitum recovery waste } \\
\text { Redox kaste }\end{array}$ & Nonboiling & 1959 & $\begin{array}{l}\text { Liquid leve } 1 \\
\text { measurements }\end{array}$ & $\begin{array}{l}\text { Liquid leve } 1 \\
\text { measurements }\end{array}$ & & 30,000 & 23 \\
\hline $105 \cdot \mathrm{rY}$ & $1951-52$ & $19 \$ 3$ & TBP waste & Nonboiling & 1960 & $\begin{array}{l}\text { 1.iquid leve } 1 \\
\text { measurements }\end{array}$ & $\begin{array}{l}\text { Liquid leve } 1 \\
\text { neasurements }\end{array}$ & & 35,000 & 4 \\
\hline $108-5 x$ & $1953-54$ & 1955 & Redox waste & Boiling & 1962 & $\begin{array}{l}\text { Soil radi- } \\
\text { ation } \\
\text { readings }\end{array}$ & $\begin{array}{l}\text { Liquid level } \\
\text { measurements }\end{array}$ & Yes & 3,400 & 17 \\
\hline $105=\mathrm{A}$ & $1954-55$ & 1963 & Purcx waste & Boiling & 1963 & $\begin{array}{l}\text { Soil radi- } \\
\text { ation } \\
\text { readings }\end{array}$ & $\begin{array}{l}\text { Soil radiation } \\
\text { readings }\end{array}$ & Yes & Sma11 & \\
\hline $107=5 x$ & $1953-54$ & 1956 & Redox waste & Boiling & 1964 & $\begin{array}{l}\text { Soli radi- } \\
\text { ation } \\
\text { readings }\end{array}$ & $\begin{array}{l}\text { Soil radiation } \\
\text { readings }\end{array}$ & Yes & Smal1 & \\
\hline $109-5 x$ & $1953-54$ & 1955 & Rodox waste & Boiling & 1965 & $\begin{array}{l}\text { Soil radi= } \\
\text { ation } \\
\text { readings }\end{array}$ & $\begin{array}{l}\text { No further } \\
\text { evidence }\end{array}$ & No & Sma11 & \\
\hline $115=\$ X$ & $1953 \cdot 54$ & 1958 & Redox waste & Boiling & 1965 & $\begin{array}{l}\text { Liquid level } \\
\text { measurements }\end{array}$ & $\begin{array}{l}\text { Sold radiation } \\
\text { readings }\end{array}$ & & $50,000 * * \star$ & 40 \\
\hline $11 \geq-5 x$ & $1953-54$ & 1956 & Redox wastc & Boiling & 1969 & $\begin{array}{l}\text { Liquid level } \\
\text { measurements }\end{array}$ & $\begin{array}{l}\text { Soil radiation } \\
\text { readings }\end{array}$ & Yes & 30,000 & 45 \\
\hline$\therefore$ co & $\begin{array}{l}\text { ntained } \\
\text { rtained } \\
\text { rtained }\end{array}$ & 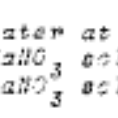 & $\begin{array}{l}\text { tine ast. } \\
\text { lintion at tire of lear. } \\
\text { iution as time of lear. }\end{array}$ & \&\& oos: : & tuat anser & xtant esplution. & & & & \\
\hline
\end{tabular}

Note: See Tank Farm Segments for information on individual specific tanks 
RPP-RPT-54911, Rev. 0

\section{APPENDIX B5}

1960 MEMO-TANK 241-TY-105 WASTE STORAGE LEAK

B5-1 
RPP-RPT-54911, Rev. 0

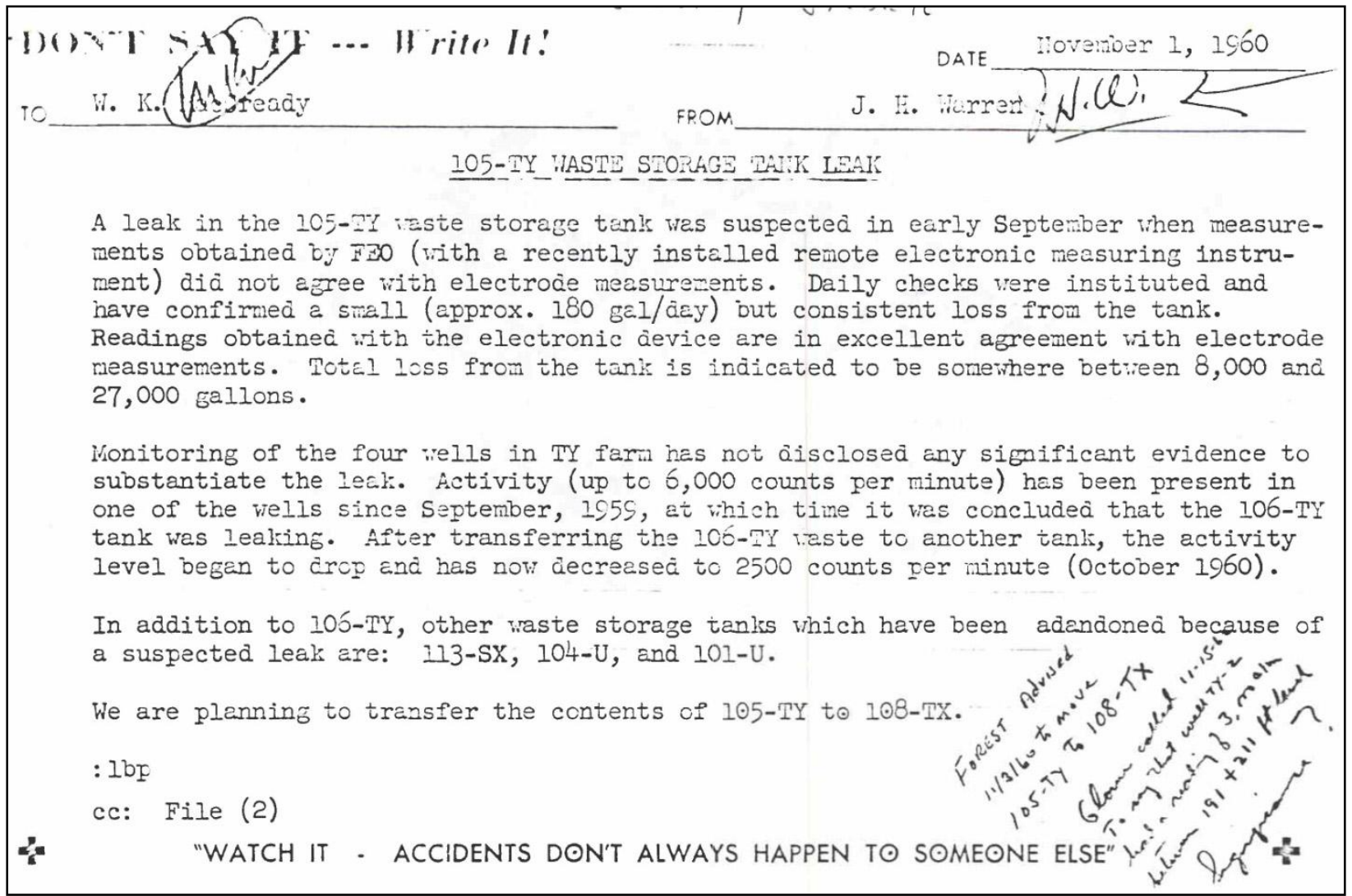

The 6,000 counts per minute is referring to drywell TY-2 (52-06-07). The hand written note refers to TY-2 which is drywell 52-06-07. Therefore, 105-TY appears to be incorrect and should read 106-TY because the following reading at 191 to $211-\mathrm{ft}$ must be for drywell 52-06-07 as no other drywell in TY Farm was drilled to that depth. 
RPP-RPT-54911, Rev. 0

\section{APPENDIX C5}

TANK TY-106 GROSS GAMMA DRYWELL DATA 
Table C5-1. Tank TY-106 Drywell Radioactivity (K counts per minute) (July 1973 to June 1986) (1 of 3 sheets)

(Drywell Data Sheets* Retrieved on August 15, 2011 and SD-WM-TI-356)

\begin{tabular}{|c|c|c|c|c|c|c|c|c|c|c|c|c|c|c|c|}
\hline \multirow{2}{*}{\multicolumn{2}{|c|}{$\begin{array}{c}52-04-06 \\
\text { Drilled 12/31/71 }\end{array}$}} & \multicolumn{2}{|c|}{ 52-06-02 } & \multicolumn{3}{|c|}{ 52-06-04 } & \multicolumn{3}{|c|}{ 52-06-05 } & \multicolumn{3}{|c|}{ 52-06-06 } & \multicolumn{3}{|c|}{ 52-06-07 } \\
\hline & & \multicolumn{2}{|c|}{ Drilled 12/1971 } & \multicolumn{3}{|c|}{ Drilled 8/31/71 } & \multicolumn{3}{|c|}{ Drilled 8/31/52 } & \multicolumn{3}{|c|}{ Drilled 8/31/74 } & \multicolumn{3}{|c|}{ Drilled 9/30/52 } \\
\hline Date & $\begin{array}{c}\text { Peak } \\
(\mathrm{K} \\
\text { cpm })\end{array}$ & Date & $\begin{array}{c}\text { Peak } \\
(\mathbf{K} \text { cpm })\end{array}$ & Date & $\begin{array}{c}\text { Peak } \\
\text { (K } \\
\text { cpm) }\end{array}$ & $\begin{array}{c}\text { Depth } \\
\text { (ft } \\
\text { BGS) }\end{array}$ & Date & $\begin{array}{c}\text { Peak } \\
(\mathrm{K} \\
\text { cpm) }\end{array}$ & $\begin{array}{c}\text { Depth } \\
\text { (ft BGS) }\end{array}$ & Date & $\begin{array}{c}\text { Peak } \\
(\mathrm{K} \\
\text { cpm) }\end{array}$ & $\begin{array}{c}\text { Depth } \\
\text { (ft BGS) }\end{array}$ & Date & $\begin{array}{c}\text { Peak } \\
(\mathrm{K} \\
\text { cpm) }\end{array}$ & $\begin{array}{c}\text { Depth } \\
\text { (ft } \\
\text { BGS) }\end{array}$ \\
\hline \multicolumn{2}{|c|}{ - } & \multicolumn{2}{|c|}{-} & \multicolumn{3}{|c|}{ - } & \multicolumn{3}{|c|}{$\mathrm{NA}^{1}$} & \multicolumn{3}{|c|}{-} & $09 / 1959$ & $6^{2}$ & $\mathrm{NA}^{1}$ \\
\hline \multicolumn{2}{|c|}{ - } & \multicolumn{2}{|c|}{-} & \multicolumn{3}{|c|}{-} & \multicolumn{3}{|c|}{$\mathrm{NA}^{1}$} & \multicolumn{3}{|c|}{-} & $10 / 1959$ & $2.5^{2}$ & $\mathrm{NA}^{1}$ \\
\hline $7 / 11 / 73$ & $<12$ & \multicolumn{2}{|c|}{$\mathrm{NA}^{1}$} & \multicolumn{3}{|c|}{$\mathrm{NA}^{1}$} & $7 / 16 / 73$ & 67.2 & 62 & \multicolumn{3}{|c|}{-} & $7 / 11 / 1973$ & 11 & $75-89$ \\
\hline \multicolumn{2}{|c|}{$\mathrm{NA}^{1}$} & \multicolumn{2}{|c|}{$\mathrm{NA}^{1}$} & \multicolumn{3}{|c|}{$\mathrm{NA}^{1}$} & $8 / 28 / 73$ & 88 & 61 & \multicolumn{3}{|c|}{-} & $8 / 23 / 1973$ & 12 & $68-82$ \\
\hline \multicolumn{2}{|c|}{$\mathrm{NA}^{1}$} & \multicolumn{2}{|c|}{$\mathrm{NA}^{1}$} & & $\mathrm{NA}^{1}$ & & $9 / 11 / 73$ & 94.8 & 62 & & - & & $9 / 21 / 1973$ & 17.4 & 90 \\
\hline & & & & & $\mathrm{NA}^{1}$ & & $10 / 16 / 73$ & 81 & 64 & & - & & $10 / 18 / 1973$ & 15 & 90 \\
\hline & & & & & $\mathrm{NA}^{1}$ & & $11 / 5 / 73$ & 75.5 & 65 & & - & & $11 / 26 / 1973$ & 19.5 & 91 \\
\hline & & & & & 1 & & $1218 / 72$ & 67.8 & 52 & & & & $12 / 1072$ & 160 & 00 \\
\hline & & & & & NA & & $12710 / 7$ & 91.8 & 65 & & - & & 12/281913 & 10.0 & 90 \\
\hline & & & & & $\mathrm{NA}^{1}$ & & $1 / 17 / 74$ & 52.2 & 50 & & - & & $1 / 19 / 1974$ & 14 & $75-87$ \\
\hline & & & & & & & י & 70.8 & 61 & & & & 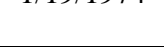 & & \\
\hline & & & & & $\mathrm{NA}^{1}$ & & $2 / 11 / 74$ & 56 & 52 & & - & & & $\mathrm{NA}^{1}$ & \\
\hline & & & & & & & & 77 & 65 & & - & & & & \\
\hline $3 / 10 / 74$ & $<12$ & Nev & Well & & $\mathrm{NA}^{1}$ & & $3 / 28 / 74$ & 64.2 & 53 & & & & $3 / 2 / 1074$ & 15 & $74-04$ \\
\hline & & & & & & & & 85.8 & 67 & & & & & & \\
\hline $4 / 16774$ & $<12$ & $4 / 30 / 74$ & $<12$ & & NA ${ }^{1}$ & & $4 / 2 / 74$ & 66 & 51 & & & & $4 / 10 / 1074$ & 20 & 05 \\
\hline 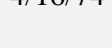 & .12 & & .12 & & & & דורוד & 80 & 63 & & & & דולדולדוד & 20 & (J) \\
\hline & & & & & $N A^{1}$ & & $5 / 8 / 74$ & 75.5 & 54 & & & & $5 / 31 / 1074$ & 18 & 04 \\
\hline & & & & & N & & 4 & 84 & 67 & & - & & 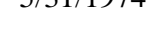 & 10 & 74 \\
\hline
\end{tabular}


RPP-RPT-54911, Rev. 0

Table C5-1. Tank TY-106 Drywell Radioactivity (K counts per minute)

(July 1973 to June 1986) ( 2 of 3 sheets)

(Drywell Data Sheets* Retrieved on August 15, 2011 and SD-WM-TI-356)

\begin{tabular}{|c|c|c|c|c|c|c|c|c|c|c|c|c|c|c|c|}
\hline \multicolumn{2}{|c|}{$52-04-06$} & \multicolumn{2}{|c|}{$52-06-02$} & \multicolumn{3}{|c|}{$52-06-04$} & \multicolumn{3}{|c|}{ 52-06-05 } & \multicolumn{3}{|c|}{$52-06-06$} & \multicolumn{3}{|c|}{ 52-06-07 } \\
\hline Date & $\begin{array}{c}\text { Peak } \\
(\mathrm{K} \text { cpm })\end{array}$ & Date & $\begin{array}{c}\text { Peak } \\
(\text { K cpm) }\end{array}$ & Date & $\begin{array}{c}\text { Peak } \\
(\mathbf{K} \\
\text { cpm) }\end{array}$ & $\begin{array}{c}\text { Depth } \\
\text { (ft BGS) }\end{array}$ & Date & $\begin{array}{c}\text { Peak } \\
(\mathrm{K} \text { cpm })\end{array}$ & $\begin{array}{c}\text { Depth } \\
\text { (ft BGS) }\end{array}$ & Date & $\begin{array}{c}\text { Peak } \\
(\mathrm{K} \text { cpm })\end{array}$ & $\begin{array}{c}\text { Depth } \\
\text { (ft BGS) }\end{array}$ & Date & $\begin{array}{c}\text { Peak } \\
(\mathrm{K} \text { cpm })\end{array}$ & $\begin{array}{c}\text { Depth } \\
\text { (ft BGS) }\end{array}$ \\
\hline \multicolumn{2}{|c|}{$\mathrm{NA}^{1}$} & \multicolumn{2}{|c|}{$\mathrm{NA}^{1}$} & \multicolumn{3}{|c|}{$\mathrm{NA}^{1}$} & $6 / 11 / 74$ & 80.4 & 64 & \multicolumn{3}{|c|}{-} & \multicolumn{3}{|c|}{$\mathrm{NA}^{1}$} \\
\hline \multirow{2}{*}{\multicolumn{2}{|c|}{$\mathrm{NA}^{1}$}} & \multirow{2}{*}{\multicolumn{2}{|c|}{$\mathrm{NA}^{1}$}} & \multirow{2}{*}{\multicolumn{3}{|c|}{$\mathrm{NA}^{1}$}} & \multirow{2}{*}{$7 / 4 / 74$} & 90 & 55 & \multirow{2}{*}{\multicolumn{3}{|c|}{-}} & \multirow{2}{*}{$7 / 4 / 1974$} & \multirow{2}{*}{19} & \multirow{2}{*}{93} \\
\hline & & & & & & & & 76 & 65 & & & & & & \\
\hline $8 / 8 / 74$ & $<6$ & $8 / 8 / 74$ & $<6$ & & $\mathrm{NA}^{1}$ & & $8 / 8 / 74$ & 21.2 & 52 & New Well & - & - & $8 / 8 / 1974$ & 6 & 93 \\
\hline \multicolumn{2}{|c|}{$\mathrm{NA}^{1}$} & \multicolumn{2}{|c|}{$\mathrm{NA}^{1}$} & $9 / 13 / 74$ & 8.3 & 53 & $9 / 11 / 74$ & 16.0 & 54 & $9 / 13 / 1974$ & 6 & $\mathrm{NA}^{1}$ & $9 / 11 / 1974$ & 0.2 & 90 \\
\hline \multicolumn{2}{|c|}{$\mathrm{NA}^{1}$} & \multicolumn{2}{|c|}{$\mathrm{NA}^{1}$} & $10 / 17 / 74$ & 13.1 & 50 & $10 / 10 / 74$ & 21.4 & 53 & \multicolumn{3}{|c|}{$\mathrm{NA}^{1}$} & $10 / 3 / 1974$ & $<0.1$ & $\mathrm{NA}^{1}$ \\
\hline \multicolumn{2}{|c|}{$\mathrm{NA}^{1}$} & \multicolumn{2}{|c|}{$\mathrm{NA}^{1}$} & $11 / 8 / 74$ & 8.3 & 56 & $11 / 8 / 74$ & 16.9 & 68 & \multicolumn{3}{|c|}{$\mathrm{NA}^{1}$} & $11 / 8 / 1974$ & $<0.1$ & $\mathrm{NA}^{1}$ \\
\hline \multicolumn{2}{|c|}{$\mathrm{NA}^{1}$} & \multicolumn{2}{|c|}{$\mathrm{NA}^{1}$} & $12 / 4 / 74$ & 8.9 & 53 & $12 / 4 / 74$ & 16.4 & 56 & $12 / 4 / 1974$ & 6 & $\mathrm{NA}^{1}$ & $12 / 4 / 1974$ & $<0.1$ & $\mathrm{NA}^{1}$ \\
\hline & & & & $1 / 2 / 75$ & 9.4 & 52 & $1 / 2 / 75$ & 16.3 & 56 & & $\mathrm{NA}^{1}$ & & & $\mathrm{NA}^{1}$ & \\
\hline $7 / 24 / 75$ & $<3$ & $7 / 24 / 75$ & $<3$ & $7 / 24 / 75$ & 8.3 & 51 & $7 / 24 / 75$ & 12.8 & 64 & $7 / 24 / 1975$ & 4.4 & 76 & $7 / 24 / 1975$ & 3.4 & 92 \\
\hline $12 / 31 / 75$ & $<3$ & $12 / 31 / 75$ & $<3$ & $12 / 31 / 75$ & 8.5 & 52 & $12 / 31 / 75$ & 12 & 65 & $12 / 31 / 1975$ & 3.7 & 77 & $12 / 24 / 1975$ & 3.4 & 94 \\
\hline $5 / 14 / 76$ & $<3$ & $5 / 16 / 76$ & $<3$ & $5 / 14 / 76$ & 8.1 & 50 & $5 / 14 / 76$ & 12.5 & 63 & $5 / 14 / 1976$ & 3.5 & 76 & $3 / 5 / 1976$ & 3 & 92 \\
\hline $9 / 23 / 76$ & $<3$ & $9 / 18 / 76$ & $<3$ & $11 / 5 / 76$ & 6.9 & 50 & $11 / 5 / 76$ & 11.6 & 62 & $10 / 7 / 1976$ & 3.5 & 75 & $9 / 30 / 1976$ & 2.5 & 92 \\
\hline $5 / 5 / 77$ & $<3$ & $5 / 20 / 77$ & $<3$ & $7 / 15 / 77$ & 6.2 & 50 & $4 / 8 / 77$ & 10.1 & 61 & $3 / 10 / 1977$ & 3.2 & 76 & $3 / 10 / 1977$ & 2.0 & 92 \\
\hline $10 / 13 / 77$ & $<3$ & $10 / 6 / 77$ & $<3$ & $10 / 6 / 77$ & 5.7 & 49 & $10 / 6 / 77$ & 9.4 & 62 & $10 / 6 / 1977$ & 2.9 & 76 & $10 / 6 / 1977$ & 2.5 & 93 \\
\hline $10 / 12 / 78$ & $<3$ & $10 / 5 / 78$ & $<3$ & $10 / 5 / 78$ & 5.0 & 50 & $10 / 5 / 78$ & 9.4 & 62 & $10 / 5 / 1978$ & 2.7 & 76 & $10 / 5 / 1978$ & 2.3 & 94 \\
\hline $10 / 16 / 79$ & $<3$ & $10 / 16 / 79$ & $<3$ & $10 / 31 / 79$ & 5.1 & 51 & $10 / 31 / 79$ & 8.9 & 62 & $10 / 31 / 1979$ & 2.6 & 77 & $10 / 16 / 1979$ & 2.2 & 95 \\
\hline $11 / 12 / 80$ & $<3$ & $11 / 12 / 80$ & $<3$ & $11 / 12 / 80$ & 3.3 & 50 & $10 / 14 / 80$ & 10.4 & 62 & $10 / 16 / 1980$ & 2.9 & 80 & $7 / 8 / 1980$ & 2.0 & 95 \\
\hline & & & & & $\mathrm{NA}^{1}$ & & & $\mathrm{NA}^{1}$ & & $1 / 6 / 1981$ & $<3$ & $\mathrm{NA}^{1}$ & & $\mathrm{NA}^{1}$ & \\
\hline
\end{tabular}


RPP-RPT-54911, Rev. 0

Table C5-1. Tank TY-106 Drywell Radioactivity (K counts per minute)

(July 1973 to June 1986) (3 of 3 sheets)

(Drywell Data Sheets* Retrieved on August 15, 2011 and SD-WM-TI-356)

\begin{tabular}{|c|c|c|c|c|c|c|c|c|c|c|c|c|c|c|c|}
\hline \multicolumn{2}{|c|}{$52-04-06$} & \multicolumn{2}{|c|}{$52-06-02$} & \multicolumn{3}{|c|}{$52-06-04$} & \multicolumn{3}{|c|}{ 52-06-05 } & \multicolumn{3}{|c|}{$52-06-06$} & \multicolumn{3}{|c|}{ 52-06-07 } \\
\hline Date & $\begin{array}{c}\text { Peak } \\
(\mathrm{K} \text { cpm) }\end{array}$ & Date & $\begin{array}{c}\text { Peak } \\
(\mathrm{K} \text { cpm) }\end{array}$ & Date & $\begin{array}{c}\text { Peak } \\
(\mathrm{K} \text { cpm })\end{array}$ & $\begin{array}{c}\text { Depth } \\
\text { (ft BGS) }\end{array}$ & Date & $\begin{array}{c}\text { Peak } \\
(\mathrm{K} \text { cpm) }\end{array}$ & $\begin{array}{c}\text { Depth } \\
\text { (ft BGS) }\end{array}$ & Date & $\begin{array}{c}\text { Peak } \\
(\mathrm{K} \text { cpm })\end{array}$ & $\begin{array}{c}\text { Depth } \\
\text { (ft BGS) }\end{array}$ & Date & $\begin{array}{c}\text { Peak } \\
(\mathrm{K} \text { cpm) }\end{array}$ & $\begin{array}{c}\text { Depth (ft } \\
\text { BGS) }\end{array}$ \\
\hline $11 / 19 / 81$ & $<3$ & $11 / 19 / 81$ & $<3$ & $11 / 11 / 81$ & 3.4 & 51 & $11 / 19 / 81$ & 7.4 & 64 & $11 / 19 / 1981$ & $<3$ & $\mathrm{NA}^{1}$ & $11 / 19 / 1981$ & 2.1 & 92 \\
\hline \multicolumn{2}{|c|}{$\mathrm{NA}^{1}$} & \multicolumn{2}{|c|}{$\mathrm{NA}^{1}$} & \multicolumn{3}{|c|}{$\mathrm{NA}^{1}$} & \multicolumn{3}{|c|}{$\mathrm{NA}^{1}$} & \multicolumn{3}{|c|}{$\mathrm{NA}^{1}$} & $2 / 10 / 1982$ & 2.5 & 94 \\
\hline $10 / 19 / 82$ & $<3$ & $10 / 19 / 82$ & $<3$ & $10 / 12 / 82$ & 3.2 & 51 & $10 / 19 / 82$ & 5.4 & 64 & $10 / 19 / 1982$ & $<3$ & $\mathrm{NA}^{1}$ & $10 / 19 / 1982$ & $<3$ & $\mathrm{NA}^{1}$ \\
\hline $6 / 22 / 83$ & $<3$ & $6 / 22 / 83$ & $<3$ & $6 / 28 / 83$ & 3.5 & 51 & $9 / 20 / 83$ & 5.5 & 65 & $6 / 23 / 1983$ & $<3$ & $\mathrm{NA}^{1}$ & $6 / 22 / 1983$ & $<3$ & $\mathrm{NA}^{1}$ \\
\hline $6 / 14 / 84$ & $<3$ & $6 / 14 / 84$ & $<3$ & $8 / 22 / 84$ & 3.1 & 51 & $8 / 22 / 84$ & 5.0 & 65 & $6 / 14 / 1984$ & $<3$ & $\mathrm{NA}^{1}$ & $6 / 14 / 1984$ & $<3$ & $\mathrm{NA}^{1}$ \\
\hline $6 / 18 / 85$ & $<3$ & $6 / 18 / 85$ & $<3$ & $6 / 18 / 85$ & 2.5 & 52 & $6 / 18 / 85$ & 4.4 & 64 & $6 / 18 / 1985$ & $<3$ & $\mathrm{NA}^{1}$ & $6 / 18 / 1985$ & $<3$ & $\mathrm{NA}^{1}$ \\
\hline $6 / 11 / 86$ & $<3$ & $6 / 11 / 86$ & $<3$ & $6 / 11 / 86$ & 2.5 & 53 & $6 / 11 / 86$ & 4.3 & 64 & $6 / 11 / 1986$ & $<3$ & $\mathrm{NA}^{1}$ & $6 / 11 / 1983$ & $<3$ & $\mathrm{NA}^{1}$ \\
\hline
\end{tabular}

Note: ${ }^{1}$ N/A: Data not available

${ }^{2}$ Referenced from Internal memo "105-TY Waste Storage Tank Leak"

*Drywell data sheets located in the Historical Records folder in IDMS/Managed Information 
RPP-RPT-54911, Rev. 0

APPENDIX D5

TANK TY-106 GAMMA SURVEYS OF THE 2005 DIRECT PUSHES

D5-1 
RPP-RPT-54911, Rev. 0

Figure D5-1. 2005 Gamma Survey Direct Push Hole C4603 (RPP-RPT-34870)

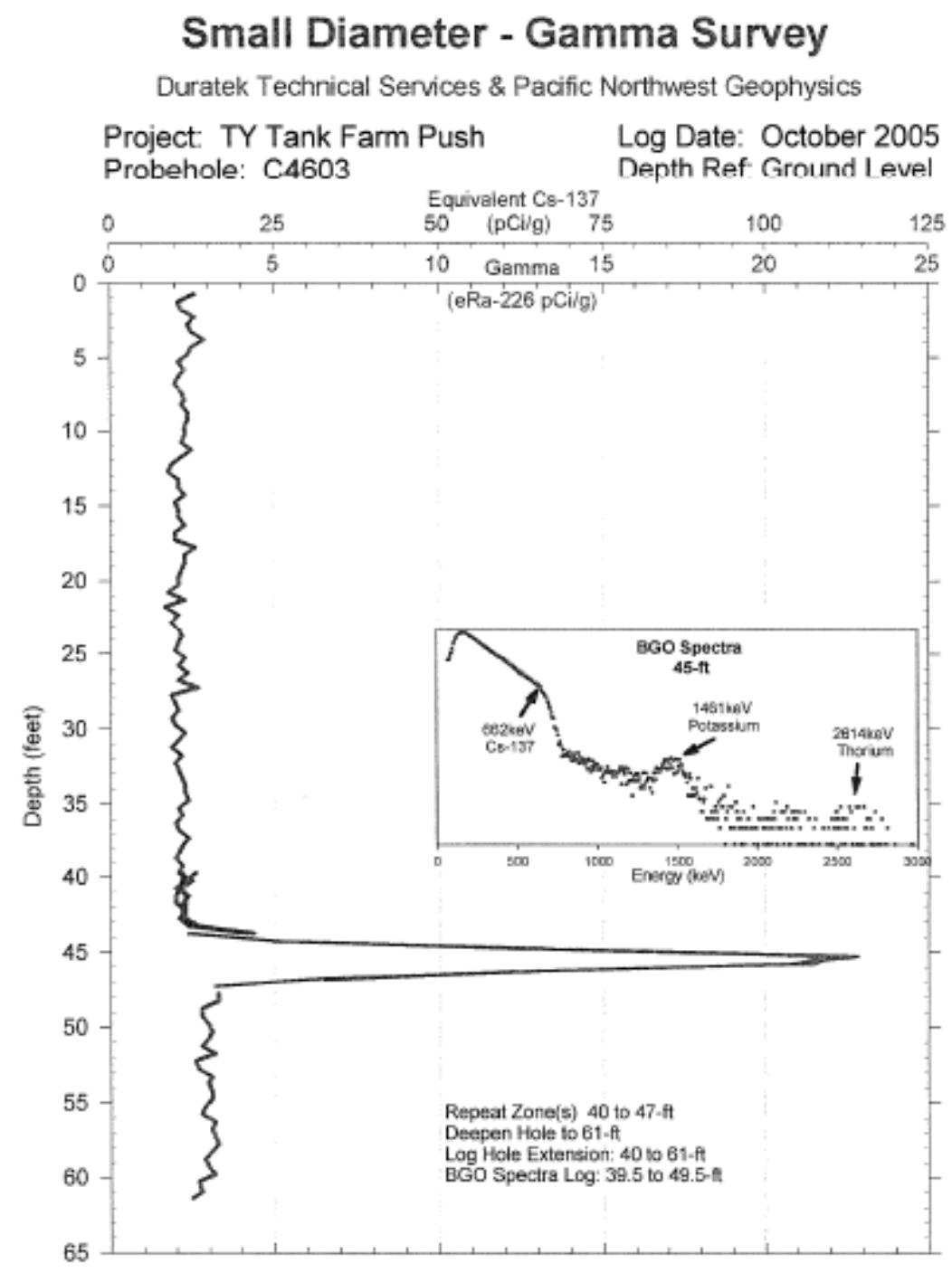


RPP-RPT-54911, Rev. 0

Figure D5-2. 2005 Gamma Survey Direct Push Hole C4605 (RPP-RPT-34870)

\section{Smail Diameter - Gamma Survey}

Duratek Technical Services \& Pacific Northwest Geophysics

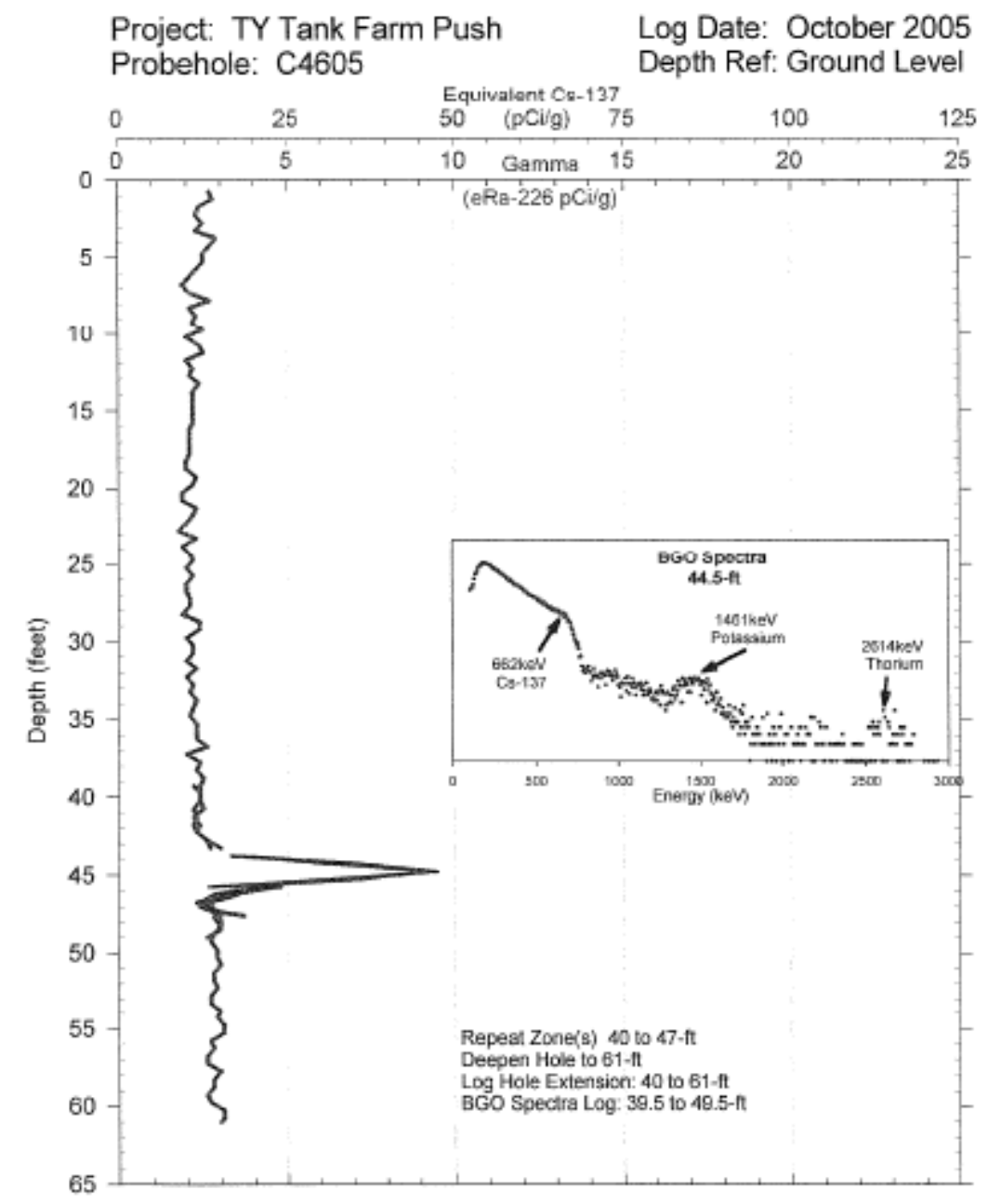


RPP-RPT-54911, Rev. 0

Figure D5-3. 2005 Gamma Survey Direct Push Hole C4607 (RPP-RPT-34870)

\section{Small Diameter - Gamma Survey}

Duratek Technical Services \& Pacific Northwest Geophysics

Project: TY Tank Farm Push

Log Date: October 2005

Probehole: C4607

Depth Ref: Ground Level

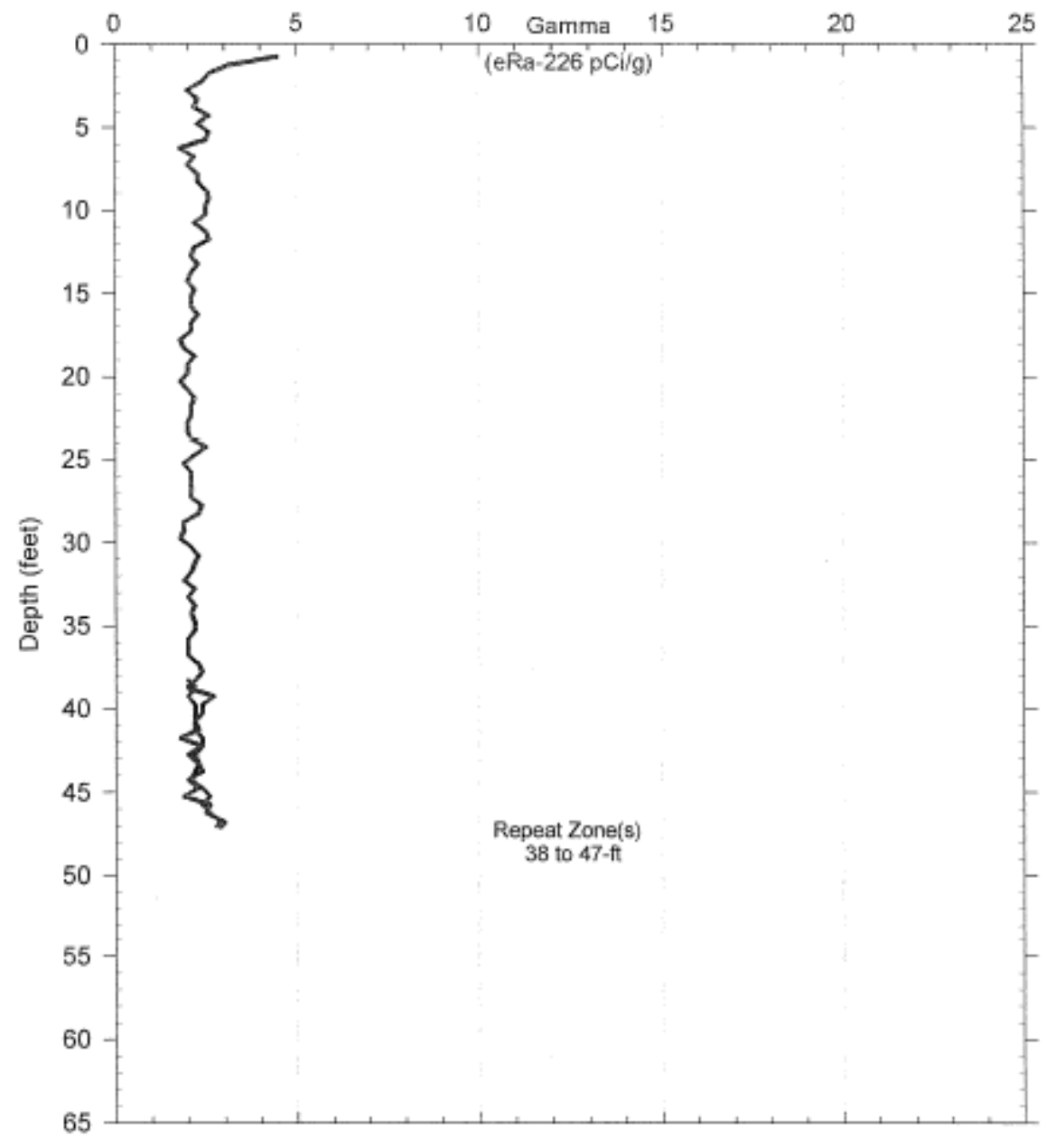


RPP-RPT-54911, Rev. 0

Figure D5-4. 2005 Gamma Survey Direct Push Hole C4609 (RPP-RPT-34870)

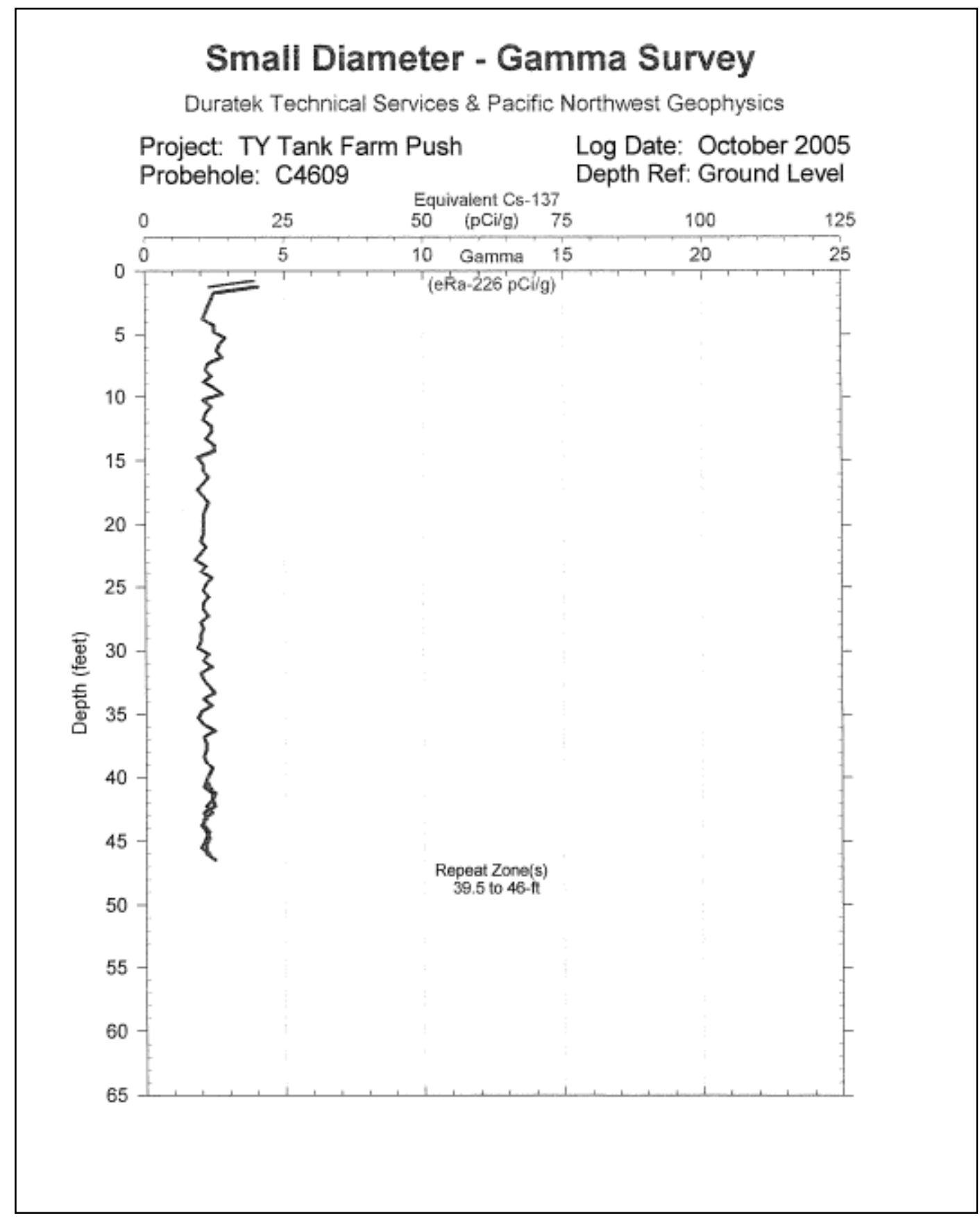


RPP-RPT-54911, Rev. 0

Figure D5-5. 2005 Gamma Survey Direct Push Hole C4611 (RPP-RPT-34870)

\section{Small Diameter - Gamma Survey}

Duratek Technical Services \& Pacific Northwest Geophysics

Project: TY Tank Farm Push Log Date: October 2005

Probehole: C4611 Depth Ref: Ground Level

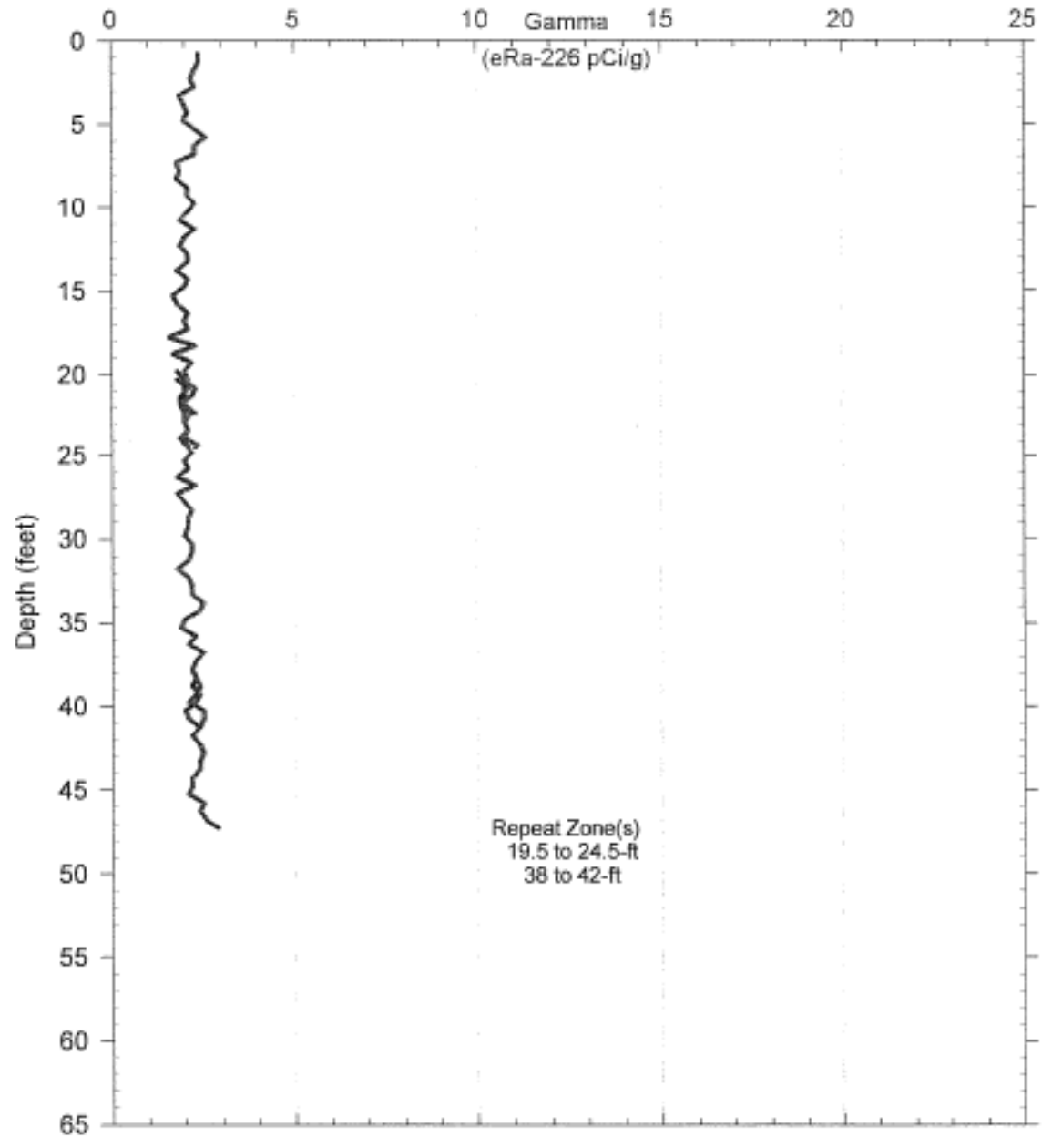


RPP-RPT-54911, Rev. 0

Figure D5-6. 2005 Gamma Survey Direct Push Hole C4613 (RPP-RPT-34870)

\section{Small Diameter - Gamma Survey}

Duratek Technical Services \& Pacific Northwest Geophysics

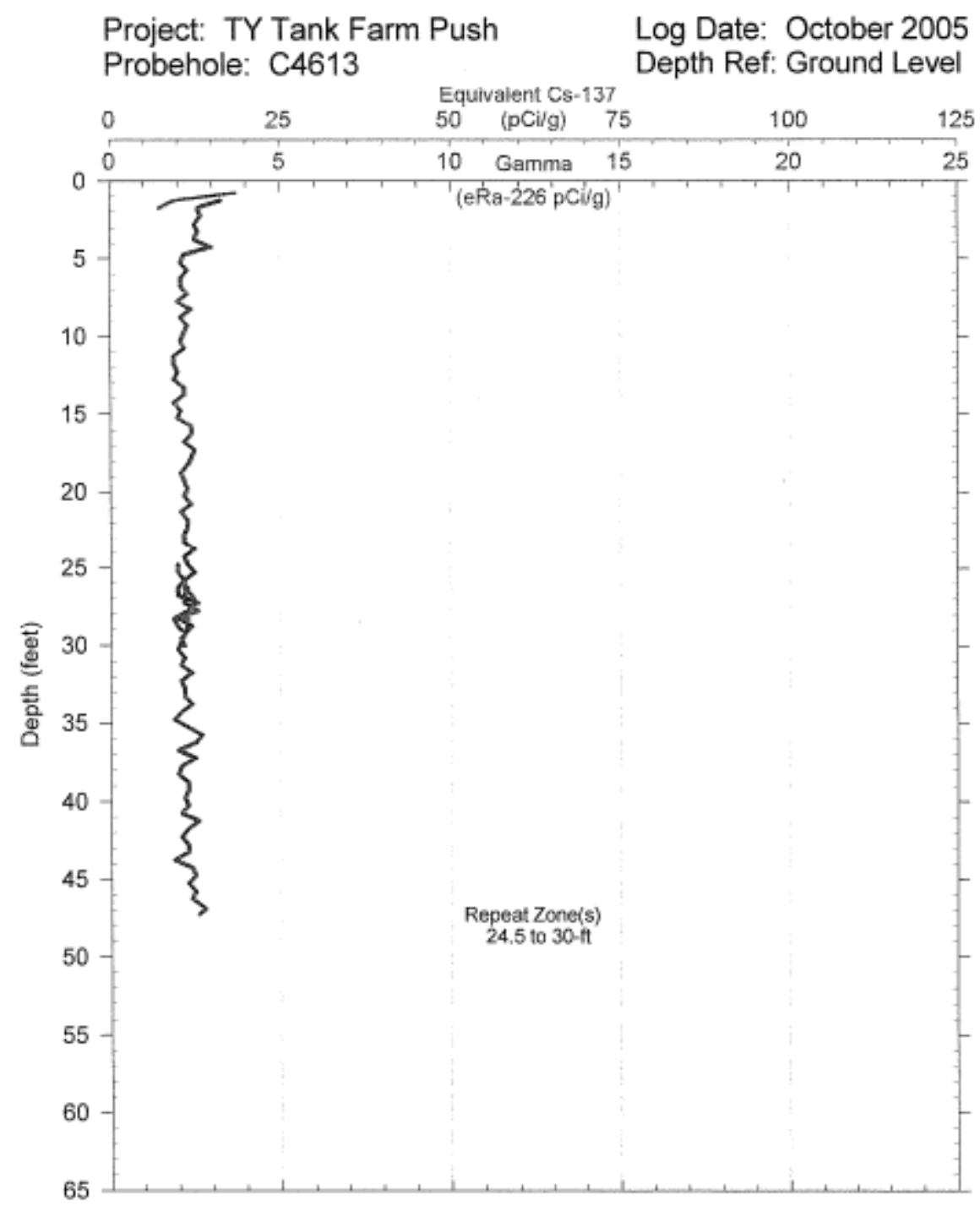


RPP-RPT-54911, Rev. 0

Figure D5-7. 2005 Gamma Survey Direct Push Hole C4615 (RPP-RPT-34870)

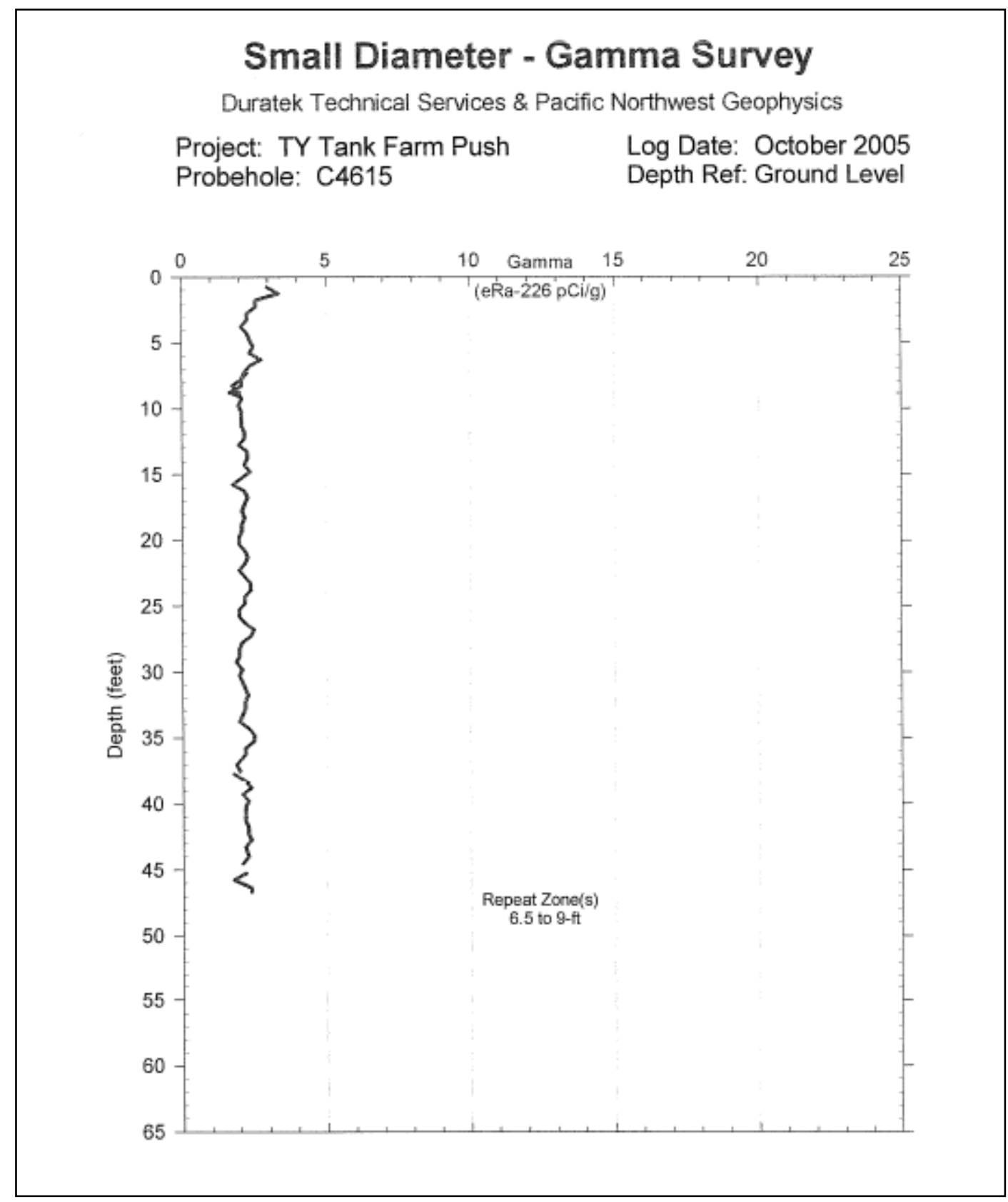


RPP-RPT-54911, Rev. 0

Figure D5-8. 2005 Gamma Survey Direct Push Hole C4617 (RPP-RPT-34870)

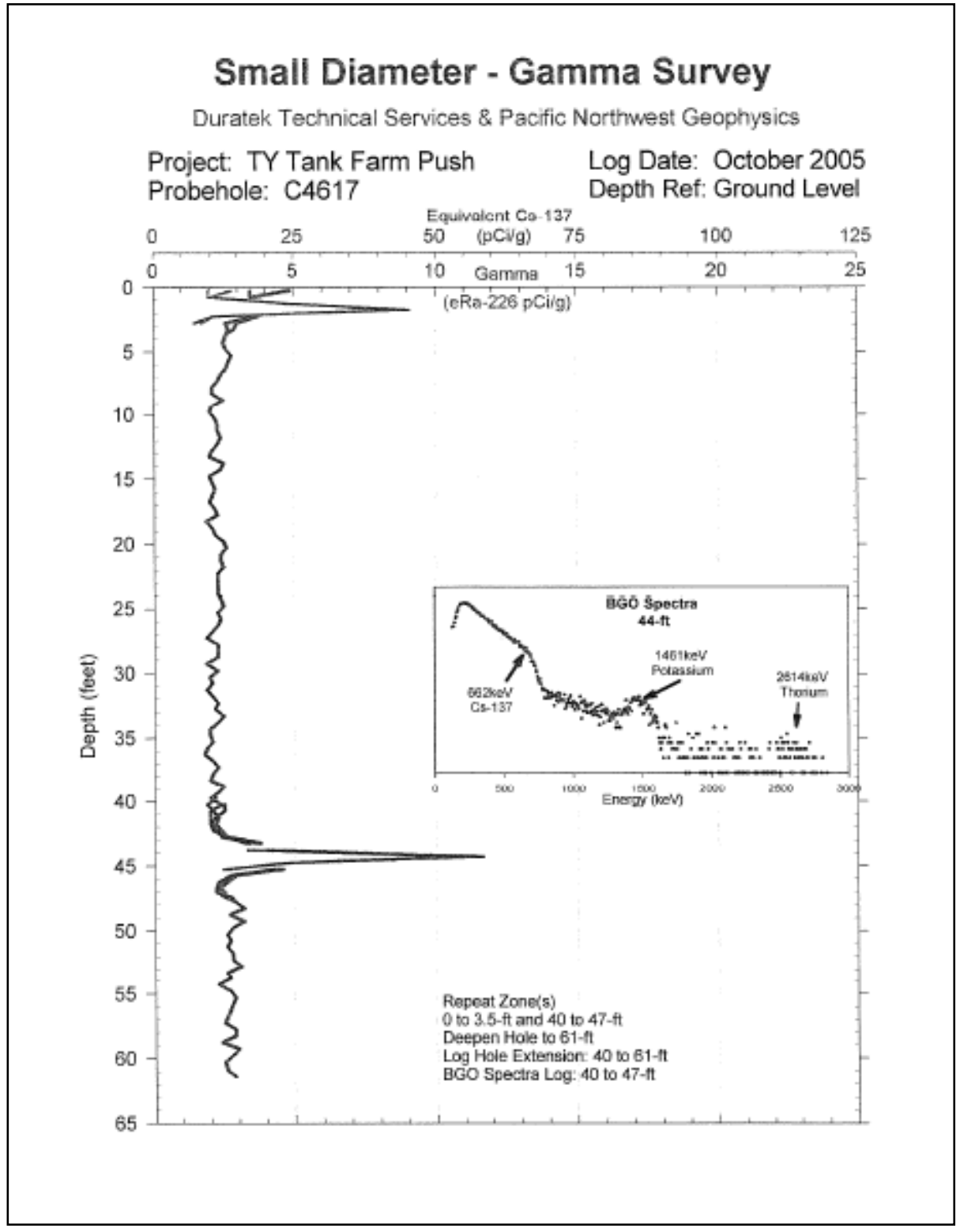


RPP-RPT-54911, Rev. 0

\subsection{CONCLUSIONS}

Several leak locations were identified for tanks BY-103, and TY-103, TY-105, and TY-106. However, the tank TY-104 leak, identified by a liquid level decrease, could not be located as the seven tank TY-104 drywells did not indicate any contamination associated with a tank leak. The tank could have leaked the entire estimated leak volume of $1.4 \mathrm{kgal}$ or the $\sim 55-\mathrm{ft}$ long capped cascade outlet tie line could have leaked as the liquid level was above that point for the liquid level decrease period.

The primary contributor to BY and TY Farm Type III tank liner failures appears to have been chemistry-corrosion with some possible contribution from construction temperature and thermal conditions. Tank design did not appear to be a factor in liner failure as it basically continued the important features of the earlier 241-BCTU tanks; however, there could be many unknown variables present in the quality of materials and quality of construction. No liner observations were found such as bulging that could contribute to or indicate a liner failure (see Table 9-1).

Tank construction occurred in winter under relatively cold conditions which could have affected the ductile-to-brittle transition temperature in some or all of the tanks. The BY Farm construction in December 1948 to January 1949 experienced temperatures as low as $-2^{\circ} \mathrm{F}$ and $\mathrm{TY}$ Farm construction in December 1951 to January 1952 experienced temperatures as low as $4^{\circ} \mathrm{F}$.

Detailed thermal waste storage conditions were not found; however, secondary information from technical manuals and flowsheets indicate storage temperatures were below the tank specification of $230^{\circ} \mathrm{F}$. Temperature rate of rise was not able to be calculated without the detailed temperature data sheets or graphs. It may have been possible under certain conditions to have exceeded the temperature rate of rise specification.

Waste from the TBP process was cooled to $\sim 180^{\circ} \mathrm{F}$ and pumped to the BY and TY Farm tanks in multiple back-to-back batches which could have resulted in storage of the waste at least initially close to $180^{\circ} \mathrm{F}$. The TBP waste was high in the SCC nitrate ion and low in the corrosion inhibiting hydroxide ions which at elevated temperatures could have caused SCC.

There seems to be a relationship between storing undiluted TBP waste and storing diluted TBP waste as all five of the leaking BY and TY tanks examined stored undiluted TBP waste. At least ten of the BY and TY Farm tanks that have not been declared leakers (see Table 9-2) stored diluted TBP waste and possibly all thirteen. All but one of these tanks has been recommended for a formal tank leak assessment (RPP-RPT-43740) in accordance with procedure TFC-ENGCHEM-D-42. A complete analysis of all tanks is compiled in RPP-RPT-55804, Common Factors Relating to Liner Failures in Single-Shell Tanks.

It is possible that no one characteristic of the BY and TY Farm tanks could in isolation from the others have resulted in failure. The storage of undiluted TBP waste seems to be the overriding factor that can lead to tank liner failure possibly coupled with construction conditions and thermal effects. 
RPP-RPT-54911, Rev. 0

Table 9-1. BY and TY Farm Leaking Tanks

\begin{tabular}{|c|c|c|c|c|c|c|c|c|c|}
\hline \multirow{2}{*}{$\begin{array}{l}\text { Leaking } \\
\text { Tank }\end{array}$} & \multicolumn{3}{|c|}{ Initial Waste Details } & \multicolumn{3}{|c|}{ Leak Status } & \multicolumn{2}{|c|}{ TBP Waste Storage } & \multirow{2}{*}{$\begin{array}{c}\text { Thermal Conditions } \\
\begin{array}{c}\text { Estimated } \\
\text { Maximum Temp. }\end{array}\end{array}$} \\
\hline & First Filled & Waste Type & $\begin{array}{l}\text { First Contacted } \\
\text { Waste Type }\end{array}$ & Leak Detected & Indication of leak & Leak Location & $\begin{array}{l}\text { Stored TBP } \\
\text { Waste }\end{array}$ & $\begin{array}{l}\text { TBP Only Storage } \\
\text { Length }\end{array}$ & \\
\hline BY-103 & March 1951 & $\begin{array}{c}\text { MW, TBP, P, CW, } \\
\text { OWW, EB }\end{array}$ & $\begin{array}{l}\text { MW- Emptied June } \\
1954 \text { then TBP added }\end{array}$ & 1959 & Drywell & $\begin{array}{l}\text { Sidewall and near tank } \\
\text { footing }\end{array}$ & Yes & $\sim 2$ years & $180^{\circ} \mathrm{F}$ \\
\hline TY-103 & July 16,1953 & $\begin{array}{c}\text { TBP, 1C-FeCN, DW, } \\
\text { OWW, CWP, } \\
\text { 1C/CW, EB, R, RIX, } \\
\text { BL, B } \\
\end{array}$ & TBP & April 1973 & LL decrease 0.4 -in & Near tank footing & Yes & $\sim 2$ years & $180^{\circ} \mathrm{F}$ \\
\hline TY-104 & August 1953 & $\begin{array}{l}\text { TBP, 1C-FeCN, DW, } \\
\text { OWW, BL }\end{array}$ & $\begin{array}{l}\text { TBP- cascaded from } \\
\text { TY-103 }\end{array}$ & $\begin{array}{l}\text { December 30, } 1973 \text { to } \\
\text { March 16, } 1974\end{array}$ & LL decrease 0.6 -in & $\begin{array}{c}\text { Possible tank liner leak } \\
\text { and/or cascade tie line } \\
\text { leak }\end{array}$ & Yes & $\sim 19$ months & $180^{\circ} \mathrm{F}$ \\
\hline TY-105 & January 1953 & ТВP & TBP & $\begin{array}{l}\text { September } 1960 \text { but may } \\
\text { have started July } 1959\end{array}$ & LL decrease & $\begin{array}{c}\text { Near tank } \\
\text { footing/sidewall/spare } \\
\text { inlet nozzle leak }\end{array}$ & Yes & $\sim 8$ years & $180^{\circ} \mathrm{F}$ \\
\hline TY-106 & June 1953 & TBP & $\begin{array}{l}\text { TBP- cascaded from } \\
\text { TY-105 }\end{array}$ & August 1959 & LL decrease & Near tank footing & Yes & $\sim 6$ years & $180^{\circ} \mathrm{F}$ \\
\hline
\end{tabular}

Note: Construction of BY Farm occurred September 1948 to June 1949. Construction of TY Farm occurred May 1951 to February 1952.

Waste Types: B: B Plant HLW; BL: B Plant low level waste; 1C: first cycle waste; 1C-FeCN: treated 1C (ferrocyanide) waste; CW: coating waste; CWP: PUREX coating waste; DW: decontamination waste; EB: evaporators bottom; MW: metal waste; 
Table 9-2. BY and TY Farm Sound Tanks

\begin{tabular}{|c|c|c|c|c|c|c|c|c|c|}
\hline \multirow{2}{*}{ Tanks } & \multicolumn{3}{|c|}{ Initial Waste Details } & \multicolumn{3}{|c|}{ Leak Status } & \multicolumn{2}{|c|}{ TBP Waste Storage } & \multirow{2}{*}{$\begin{array}{c}\text { Thermal Conditions } \\
\begin{array}{c}\text { Estimated Maximum } \\
\text { Temp. }\end{array}{ }^{4}\end{array}$} \\
\hline & First Filled & Waste Type & $\begin{array}{c}\text { First Contacted } \\
\text { Waste Type }\end{array}$ & Leak Integrity Classification & $\begin{array}{c}\text { Basis for Formal Leak } \\
\text { Assessment }\end{array}$ & Leak Location & $\begin{array}{l}\text { Stored TBP } \\
\text { Waste }\end{array}$ & $\begin{array}{c}\text { TBP Only } \\
\text { Storage Length }\end{array}$ & \\
\hline BY-101 & January 29,1950 & $\begin{array}{l}\text { TBP, EB, CW, Evap., } \\
\text { MW, 1C }\end{array}$ & MW & $\begin{array}{c}\text { Sound but } \\
\text { TFC-ENG-CHEM-D-42 }\end{array}$ & ITS bottoms tank ${ }^{1}$ & - & $\mathrm{Yes}^{5}$ & 5 years & $115^{\circ} \mathrm{F}$ \\
\hline BY-102 & July 23, 1950 & $\begin{array}{c}\text { CW, TBP, EB, MW, } \\
\text { Evap. }\end{array}$ & MW & $\begin{array}{c}\text { Sound but } \\
\text { TFC-ENG-CHEM-D-42 }\end{array}$ & ITS bottoms tank ${ }^{1}$ & - & Yes- diluted & - & $155^{\circ} \mathrm{F}$ \\
\hline BY-104 & February 21, 1951 & $\begin{array}{l}\text { CW, TBP, EB, MW, } \\
\text { NCPLX, IX, Evap. }\end{array}$ & MW & $\begin{array}{c}\text { Sound but } \\
\text { TFC-ENG-CHEM-D-42 } \\
\end{array}$ & ITS bottoms tank ${ }^{1}$ & - & Yes- diluted & - & $237^{\circ} \mathrm{F}$ \\
\hline BY-105 & June 10,1951 & CW, TBP, EB, MW & MW & $\begin{array}{c}\text { Assumed leaker but } \\
\text { TFC-ENG-CHEM-D-42 } \\
\end{array}$ & Drywell $^{1}$ & - & Yes- diluted & - & $180^{\circ} \mathrm{F}$ \\
\hline BY-106 & 1953 & $\begin{array}{l}\text { CW, TBP, EB, 1C, } \\
\text { Evap. }\end{array}$ & $1 \mathrm{C}$ & $\begin{array}{c}\text { Assumed leaker but } \\
\text { TFC-ENG-CHEM-D-42 }\end{array}$ & Drywell $^{1}$ & - & Yes- diluted & - & $199^{\circ} \mathrm{F}$ \\
\hline BY-107 & December 1950 & TBP, CW, 1C, EB & $1 \mathrm{C}$ & $\begin{array}{c}\text { Assumed leaker but } \\
\text { TFC-ENG-CHEM-D-42 }\end{array}$ & Drywell $^{1}$ & - & Yes- diluted & - & $125^{\circ} \mathrm{F}$ \\
\hline BY-108 & April 1951 & $\begin{array}{c}\mathrm{CW}, \mathrm{TBP}, \mathrm{EB}, 1 \mathrm{C}, \\
\text { Evap. }\end{array}$ & $1 \mathrm{C}$ & $\begin{array}{c}\text { Assumed leaker but } \\
\text { TFC-ENG-CHEM-D-42 } \\
\end{array}$ & Drywell $^{1}$ & - & Yes- diluted & - & $154^{\circ} \mathrm{F}$ \\
\hline BY-109 & 1953 & $\begin{array}{c}\text { TBP, EB, CW, } \\
\text { NCPLX, MW, Evap. }\end{array}$ & ТВP & $\begin{array}{c}\text { Sound but } \\
\text { TFC-ENG-CHEM-D-42 }\end{array}$ & ITS bottoms tank ${ }^{1}$ & - & $\mathrm{Yes}^{5}$ & $\sim 7$ years & $138^{\circ} \mathrm{F}$ \\
\hline BY-110 & 1951 & $\begin{array}{c}\text { CW, TBP, EB, } \\
\text { NCPLX, 1C, Evap. }\end{array}$ & $1 \mathrm{C}$ & $\begin{array}{c}\text { Sound but } \\
\text { TFC-ENG-CHEM-D-42 }\end{array}$ & ITS bottoms tank $^{1}$ & - & Yes- diluted & - & $205^{\circ} \mathrm{F}$ \\
\hline BY-111 & 1951 & $\begin{array}{c}\text { CW, OWW, EB, MW, } \\
\text { Evap., TBP }\end{array}$ & MW & $\begin{array}{c}\text { Sound but } \\
\text { TFC-ENG-CHEM-D-42 }\end{array}$ & ITS bottoms tank ${ }^{1}$ & - & $\mathrm{Yes}^{5}$ & $\sim 1$ year & $124^{\circ} \mathrm{F}$ \\
\hline BY-112 & 1951 & $\begin{array}{c}\mathrm{CW}, \mathrm{TBP}, \mathrm{EB}, \mathrm{MW}, \\
\text { Evap. }\end{array}$ & MW & $\begin{array}{c}\text { Sound but } \\
\text { TFC-ENG-CHEM-D-42 }{ }^{1} \\
\end{array}$ & ITS bottoms tank ${ }^{1}$ & - & Yes- diluted & - & $163^{\circ} \mathrm{F}$ \\
\hline TY-101 & 1953 & $\mathrm{R}, 1 \mathrm{C}, \mathrm{TBP}, \mathrm{EB}$ & EB & $\begin{array}{c}\text { Assumed leaker but } \\
\text { TFC-ENG-CHEM-D- } 42^{1}\end{array}$ & LL decrease $0.35-$ in $^{2}$ & - & Yes- diluted & - & $83^{\circ} \mathrm{F}$ \\
\hline TY-102 & 1953 & $\begin{array}{c}\text { EB, 1C, BL, OWW, } \\
\text { RIX, NCPLX, Evap. } \\
\text { Feed } \\
\end{array}$ & EB & Sound $^{3}$ & $\begin{array}{l}\text { Drywell 52-01-09 } \\
\text { increase undefined }\end{array}$ & - & Yes- diluted & - & $82^{\circ} \mathrm{F}$ \\
\hline
\end{tabular}

Note: Construction of BY Farm occurred September 1948 to June 1949. Construction of TY Farm occurred May 1951 to February 1952.

Waste Types: BL: B Plant low level waste; 1C: first cycle waste; CPLX: complexant concentrate; CW: coating waste; DW: decontamination waste; EB: evaporators bottom; Evap: evaporator feed (post 1976); IX: ion exchange waste; MW: metal waste;

2. Reference: RPP-RPT-42296, Rev. 0, Hanford TY-Farm Leak Assessments Report

. Reference: HNF-EP-0182, Rev. 170, Waste Tank Summary Report for Month Ending May 31, 2002

4. Reference: WHC-SD-WM-TI-591, Rev. 0, Maximum Surface Level and Temperature Histories for Hanford Waste Tank 
RPP-RPT-54911, Rev. 0

\subsection{REFERENCES}

ARH-95, 1967, Chemical Processing Division Waste Status Summary July 1, 1967 Through September 30, 1967, Atlantic Richfield Hanford Company Richland, Washington.

ARH-326, 1968, Chemical Processing Division Waste Status Summary October 1, 1967 Through December 31, 1967, Atlantic Richfield Hanford Company, Richland, Washington.

ARH-534, 1968, Chemical Processing Division Waste Status Summary, January 1, 1968 Through March 31, 1968, Atlantic Richfield Hanford Company, Richland, Washington.

ARH-951, 1969, Limitations for Use of Underground Waste Tanks, Atlantic Richfield Hanford Company, Richland, Washington.

ARH-1200 D, 1970, Chemical Processing Division Waste Status Summary, October 1, 1969 Through December 31, 1969, Atlantic Richfield Hanford Company, Richland, Washington.

ARH-1496, Review of Storage Tank Integrity, December 15, 1969, Atlantic Richfield Hanford Company, Richland,Washington.

ARH-1601, 1974, Specifications and Standards for the Operation of Radioactive Waste Tank Farms and Associated Facilities, Atlantic Richfield Hanford Company, Richland, Washington.

ARH-1666 A, 1970, Chemical Processing Division Waste Status Summary, January 1, 1970 Through March 31, 1970, Atlantic Richfield Hanford Company, Richland, Washington.

ARH-1666 C, 1970, Chemical Processing Division Waste Status Summary, July 1, 1970 Through September 30, 1970, Atlantic Richfield Hanford Company, Richland, Washington.

ARH-1666 D, 1971, Chemical Processing Division Waste Status Summary, October 1, 1970 Through December 31, 1970, Atlantic Richfield Hanford Company, Richland, Washington.

ARH-2074 B, 1971, Chemical Processing Division Waste Status Summary April 1, 1971 Through June 30, 1971, Atlantic Richfield Hanford Company, Richland, Washington.

ARH-2074 D, 1971, Chemical Processing Division Waste Status Summary October 1, 1971 Through December 31, 1971, Atlantic Richfield Hanford Company, Richland, Washington. 
ARH-2416 RD DEL, 1972, Chemical Processing Division Monthly Report Summaries January 1972 - December 1973, Atlantic Richfield Hanford Company Richland, Washington.

ARH-2794 D, 1974, Manufacturing and Waste Management Division Waste Status Summary October 1, 1973 Through December 31, 1973, Atlantic Richfield Hanford Company, Richland, Washington.

ARH-R-43, 1970, Management of Radioactive Wastes Stored in Underground Tanks at Hanford, Rev. 2, Atlantic Richfield Hanford Company, Richland, Washington.

ASTM A283, 1946, American Society for Testing and Materials, Standard Specification for Low and Intermediate Tensile Strength Carbon Steel Plates

BPF-73550, 1944, Specification for Construction of Composite Storage Tanks (B, C, T, and U Tank Farms), Hanford Engineer Works, Richland, Washington.

Compton 1973, “Tank 241-TY-103 Review," (draft memo from C. D. Compton to J. R. Rhoades, October 30), Rockwell Hanford Operations, Richland, Washington.

GJ-HAN-19, 1996, Vadose Zone Characterization Project at the Hanford Tank Farms Tank Summary Data Report for Tank BY-102, U.S. Department of Energy, Albuquerque Operations Office/Grand Junction Office, Grand Junction, Colorado.

GJ-HAN-20, 1996, Vadose Zone Characterization Project at the Hanford Tank Farms Tank Summary Data Report for Tank BY-103, U.S. Department of Energy, Albuquerque Operations Office/Grand Junction Office, Grand Junction, Colorado.

GJ-HAN-66, 1997, Vadose Zone Characterization Project at the Hanford Tank Farms Tank Summary Data Report for Tank TY-103, U.S. Department of Energy, Albuquerque Operations Office/Grand Junction Office, Grand Junction, Colorado.

GH-HAN-67, 1997, Vadose Zone Characterization Project at the Hanford Tank Farms Tank Summary Data Report for Tank TY-104, U.S. Department of Energy, Albuquerque Operations Office/Grand Junction Office, Grand Junction, Colorado.

GJ-HAN-68, 1997, Vadose Zone Characterization Project at the Hanford Tank Farms Tank Summary Data Report for Tank TY-105, U.S. Department of Energy, Albuquerque Operations Office/Grand Junction Office, Grand Junction, Colorado.

GJ-HAN-69, 1997, Vadose Zone Characterization Project at the Hanford Tank Farms Tank Summary Data Report for Tank TY-106, U.S. Department of Energy, Albuquerque Operations Office/Grand Junction Office, Grand Junction, Colorado.

GJO-HAN-6, 1997, Vadose Zone Characterization Project at the Hanford Tank Farms BY Tank Farm Report, U.S. Department of Energy, Grand Junction Office, Grand Junction, Colorado. 
GJO-97-30-TAR/GJO-HAN-16, 1998, Hanford Tank Farms Vadose Zone: TY Tank Farm Report, U.S. Department of Energy, Grand Junction Office, Grand Junction, Colorado.

H-2-1312, 1948, 75-Foot Tank Sections, General Electric Company, Richland, Washington.

H-2-1314, 1948, 75-Foot Tank Base Footing and Wall Reinforcing, General Electric Company, Richland, Washington.

H-2-2244, 1951, 75-Foot Composite Storage Tank Sections, General Electric Company, Richland, Washington.

H-2-2246, 1951, 75-Foot Tank Base Footing and Wall Reinforcing, General Electric Company, Richland, Washington.

H-2-2250, 1951, 75-Foot Tank Nozzle and Piping Details, General Electric Company, Richland, Washington.

H-2-2253, 1951, 75-Foot Tank Tie Line, Valve, and Riser Details, General Electric Company, Richland, Washington.

H-2-2277, 1951, 75-Foot Tank Nozzle Assembly Details, General Electric Company, Richland, Washington.

H-2-3602, 1951, 200 West Area Cathodic Protection, General Electric Company, Richland, Washington.

H-2-36933, 2007, Well Information As-Built 200 E Area, Washington River Protection Solutions, Richland, Washington.

H-2-36942, 2011, Well Information As-Built 200 E Area, Washington River Protection Solutions, Richland, Washington.

HNF-3018, 1998, Single-Shell Tank Sluicing History and Failure Frequency, Rev. 0, Vista Research, Inc., Richland, Washington.

HNF-3136, 1999, Analysis Techniques and Monitoring Results, 241-SX Drywell Surveillance Logs, Rev. 0, Lockheed Martin Hanford Corporation, Richland, Washington.

HNF-3532, 1999, Analysis of Historical Gross Gamma Logging Data from BY Tank Farm, Rev. 0, Waste Management Northwest/Three Rivers Scientific, Richland, Washington.

HNF-3831, 1999, Analysis of Historical Gross Gamma Logging Data from TY Tank Farm, Rev. 0, Waste Management Northwest/Three Rivers Scientific, Richland, Washington.

HNF-EP-0182, 2013, Waste Tank Summary Report for Month Ending April 30, 2013, Rev. 301, Washington River Protection Solutions, LLC., Richland, Washington. 
HNF-SD-RE-TI-178, 2005, Single-Shell Tank Interim Stabilization Record, Rev. 9, Babcock Services Inc., Richland Washington.

HW-3783, 1948, Specifications for Construction of Additional Waste Storage Facilities, 200 East Area, Bldg. 241-BY, General Electric Company, Richland, Washington.

HW-4590, 1951, Specification for Non-Destructive Inspection of Welded Joints in Steel Tank Liners for Waste Disposal Facilities, Rev. 1, General Electric Company, Richland, Washington.

HW-4696, 1951, Specifications Waste Disposal Facilities 241-BZ and TY Tank Farm, General Electric Company, Richland, Washington.

HW-19140, 1951, Uranium Recovery Technical Manual, General Electric Company, Richland, Washington.

HW-20742, 1951, Loss of Depleted Metal Waste Supernatant to Soil, General Electric Company, Richland, Washington.

HW-24800-30, 1953, Design and Construction History Project C-271 241-BY Tank Farm, General Electric Company, Richland, Washington.

HW-24800-86, 1953, Design and Construction History Project C-418 241-TY Tank Farm, General Electric Company, Richland, Washington.

HW-27841, 1953, Waste Status Summary Separations Section Period: January 1953, General Electric Company, Richland, Washington.

HW-28712, 1953, Separations Section, Waste - Status Summary June 30, 1953, General Electric Company, Richland, Washington.

HW-29054, 1953, Separations Section Waste - Status Summary July 31, 1953, General Electric Company, Richland, Washington.

HW-29242, 1953, Separations Section Waste - Status Summary August 31, 1953, General Electric Company, Richland, Washington.

HW-29624, 1953, Separations Section Waste - Status Summary September 30, 1953, General Electric Company, Richland, Washington.

HW-30498, 1953, Separations Section Waste - Status Summary December 31, 1953, General Electric Company, Richland, Washington.

HW-30851, 1954, Separations Section Waste - Status Summary January 31, 1954, General Electric Company, Richland, Washington. 
HW-32110, 1954, Separations Section Waste - Status Summary May 31, 1954, General Electric Company, Richland, Washington.

HW-33904, 1954, Separations Section Waste - Status Summary for November 1954, General Electric Company, Richland, Washington.

HW-34412, 1954, Separations Section Waste - Status Summary for December 1954, General Electric Company, Richland, Washington.

HW-35022, 1955, Separations Section Waste - Status Summary for January 1955, General Electric Company, Richland, Washington.

HW-33184, 1954, BiPO4 Plant Nickel Ferrocyanide Scavenging Flowsheet for First-Cycle Waste Containing No Coating-Removal Waste, General Electric Company, Richland, Washington.

HW-33499, 1954, Justification for T Plant First Cycle Waste Scavenging, General Electric Company, Richland, Washington.

HW-36001, 1955, Separations Section Waste - Status Summary for March 1955, General Electric Company, Richland, Washington.

HW-38926, 1955, Separations Section Waste - Status Summary for August 1955, General Electric Company, Richland, Washington.

HW-47052, 1956, Chemical Processing Department Waste - Status Summary November 1, 1956 - November 30, 1956, General Electric Company, Richland, Washington.

HW-47640, 1957, Chemical Processing Department Waste Status Summary December 1, 1956 December 31, 1956, General Electric Company, Richland, Washington.

HW-48144, 1957, Chemical Processing Department Waste - Status Summary January 1, 1957 January 31, 1957, General Electric Company, Richland, Washington.

HW-48846, 1957, Chemical Processing Department Waste - Status Summary February 1, 1957 - February 28, 1957, General Electric Company, Richland, Washington.

HW-51348, 1957, Chemical Processing Department Waste Status Summary June 1, 1957 June 30, 1957, General Electric Company, Richland, Washington.

HW-51858, 1957, Chemical Processing Department Waste Status Summary July 1, 1957 July 31, 1957 Planning and Scheduling - Production Operation, General Electric Company, Richland, Washington.

HW-61582, 1959, Chemical Processing Department Waste Status Summary July 1, 1959July 31, 1959, General Electric Company, Richland, Washington. 
HW-61736-DEL, 1959, Chemical Processing Department Monthly Report for August, 1959, General Electric Company, Richland, Washington.

HW-61952, 1959, Chemical Processing Department Waste Status Summary August 1, 1959 August 31, 1959, General Electric Company, Richland, Washington.

HW-62179-DEL, 1959, Chemical Processing Department Monthly Report for September, 1959, General Electric Company, Richland, Washington.

HW-62421, 1959, Chemical Processing Department Waste Status Summary September 1, 1959 September 30, 1959, General Electric Company, Richland, Washington.

HW-62593-DEL, 1959, Chemical Processing Department Monthly Report for October, 1959, General Electric Company, Richland, Washington.

HW-62723, 1959, Chemical Processing Department Waste Status Summary October 1 - 31, 1959, General Electric Company, Richland, Washington.

HW-62864-DEL, 1959, Chemical Processing Department Monthly Report for November, 1959, General Electric Company, Richland, Washington.

HW-67459-DEL, 1960, Chemical Processing Department Monthly Report for November 1960, General Electric Company, Richland, Washington.

HW-67696, 1960, Chemical Processing Department Waste Status Summary September 1, 1960 September 30, 1960, General Electric Company, Richland, Washington.

HW-67705, 1960, Chemical Processing Department Waste Status Summary October 1, 1960 October 31, 1960, General Electric Company, Richland, Washington.

HW-68291, 1961, Chemical Processing Department Waste Status Summary November 1, 1960 November 30, 1960, General Electric Company, Richland, Washington.

HW-68292, 1961, Chemical Processing Department Waste - Status Summary December 1, 1960 - December 31, 1960, General Electric Company, Richland, Washington.

HW-71610, 1961, Chemical Processing Department Waste Status Summary January 1, 1961 Through June 30, 1961, General Electric Company, Richland, Washington.

HW-72858, 1962, Interim Report Waste Tank Liquid Level Measurement Systems 200-E and 200-W Areas, General Electric Company, Richland, Washington.

HW-74174, 1963, Final Report Liquid Level Gauging System for Radioactive Wastes Storage Facilities 200 Areas, General Electric Company, Richland, Washington.

HW-83906 D RD, 1964, Chemical Processing Department 200 West Area Tank Farm Inventory and Waste Reports January 1959 Through June 1961, General Electric Company, Richland, Washington. 
HW-83906 E RD, 1964, Chemical Processing Department 200 West Area Tank Farm Inventory and Waste Reports July 1, 1961 Through 1965, General Electric Company, Richland, Washington.

Internal memo, 1960, “105-TY Waste Storage Tank” (internal memo from R.E.R. to Hugh-Russ, September 28), Richland, Washington.

Internal letter, 1973, "Integrity of tanks 241-BY-103 and 241-TY-103" (internal letter from G.L. Borsheim to L.W. Roddy, June 1), Richland, Washington.

ISO-806, 1967, Chemical Processing Division Waste Status Summary January 1, 1967 Through March 31, 1967, ISOCHEM Inc., Richland, Washington.

ISO-967, 1967, Chemical Processing Division Waste Status Summary April 1, 1967, Through June 30, 1967, ISOCHEM Inc., Richland, Washington.

LA-UR-97-311, 1997, Waste Status and Transaction Record Summary (WSTRS Rev. 4), Los Alamos National Laboratory, Los Alamos, New Mexico.

LET-ARCHO-TY-103, 1973, "Status of Tank 241-TY-103 Contract AT (45-1)-2130" (letter from W. D. Luening to O. J. Elgert October 19), Atlantic Richfield Hanford Company, Richland, Washington.

LET-082172, 1972, "Maximum Operating Levels and Cascade Levels in 200-West Area Tank Farms" (internal letter from H.N. Raymond to C.J. Francis, August 21).

LET-72730-80-097, 1980, "Review of Classification of Six Hanford Single-Shell "Questionable Integrity (QI)” Tanks” (internal letter from W.M. Lindsay to W.W. Schulz September 16).

MEM-010274, 1974, “Analysis of Tank Farm Samples 01/02/74 Thru 12/26/74” (internal memo from W. H. Sant/R. E. Wheeler to R. L. Walser), Atlantic Richfield Hanford Company, Richland, Washington.

Oblath, S. B. and J. W. Congdon, 1987, "Inhibiting Localized Corrosion during Storage of Dilute SRP Waste", Waste Management '87, Volume 1, Tucson, AZ: Arizona Board of Regents: 599-602.

OSD-T-151-00007, 2012, Operating Specifications for the Double-Shell Storage Tanks, Rev. 10, Washington River Protection Solutions, LLC., Richland, Washington.

OSD-T-151-00017, 1983, Operating Specifications for the Aging Waste Operations in Tank Farms 241-AY and 241-AZ, Rev. B-0, Rockwell Hanford Operations, Richland, Washington.

OR-74-88, 1974, Symptoms Of Leakage As Indicated By Activity In Dry Well 52-06-05 At 106TY Waste Tank, Atlantic Richfield Hanford Company, Richland, Washington. 
OR-74-102, 1974, Leakage Symptoms as Indicated by Drywell Activity at Waste Tank 105-TY, Atlantic Richfield Hanford Company, Richland, Washington.

Payer, J. H., 1980, Evaluation of a Risk Assessment Document for Restarting the 242-S Evaporator, Rockwell Hanford Operations, Richland, Washington.

PNNL-15160, 2005, Hanford Site Climatological Summary 2004 with Historical Data, Pacific Northwest National Laboratory, Richland, Washington.

PNL-5488, 1985, Prediction Equations for Corrosion Rates of A-537 and A-516 Steels in Double Shell Flurry, Pacific Northwest Laboratory, Richland, Washington.

RHO-CD-896, 1980, Review of Classification of Nine Hanford Single-Shell "Questionable Integrity” Tanks, Rockwell Hanford Operations, Richland, Washington.

RHO-CD-1193, 1981, Review of Classification of Hanford Single-Shell Tanks 110-B, 111-C, 103-T, 107-TX, 104-TY, and 106-U, Rockwell Hanford Operations, Richland, Washington.

RHO-RE-EV-4P, 1982, Supporting Information for the Scientific Basis for Establishing Drywell Monitoring Frequencies, Rockwell Hanford Operations, Richland, Washington.

RHO-ST-34, 1981, A Scientific Basis for Establishing Drywell-Monitoring Frequencies, Rockwell Hanford Operations, Richland, Washington.

RL-SEP-659, 1965, Chemical Processing Department - Waste Status Summary, January 1, 1965 Through June 30, 1965, General Electric Company, Richland, Washington.

RPP-32681, 2011, Process to Assess Tank Farm Leaks in Support of Retrieval and Closure Planning, Rev. 1, Washington River Protection Solutions, LLC., Richland, Washington.

RPP-ASMT-53793, 2012, Tank 241-AY-102 Leak Assessment Report, Rev. 0, Washington River Protection Solutions, LLC., Richland, Washington.

RPP-ENV-39658, 2009, Hanford SX-Farm Leak Assessments Report, Rev. 0, CH2M HILL Plateau Remediation Company, Richland, Washington.

RPP-RPT-33306, 2008, IQRPE Integrity Assessment Report for the 242-A Evaporator Tank System, Rev. 0A, CH2M HILL Hanford Group, Inc., Richland, Washington.

RPP-RPT-34870, 2007, Small Diameter Geophysical Logging in the 241-TY Tank farm, Rev.0, CH2M Hill Hanford Group, Richland, Washington.

RPP-RPT-42296, 2010, Hanford TY-Farm Leak Assessments Report, Rev. 0, Washington River Protection Solutions LLC, Richland, Washington.

RPP-RPT-43704, 2011, Hanford BY-Farm Leak Assessments Report, Rev. 0A, CH2M Hill, Richland, Washington. 
RPP-RPT-47337, 2011, Specifications for the Mineralization of the Stress Corrosion Cracking Threat in Double-Shell Tank Wastes, Rev. 0, Washington River Protection Solutions, LLC., Richland, Washington.

RPP-RPT-50840, 2013, 241-BY Tank Farm Construction History, Rev. 0, Washington River Protection Solutions, LLC., Richland, Washington.

RPP-RPT-54909, TBD, Hanford Single-Shell Tank Leak Causes and Locations - Summary, Washington River Protection Solutions, LLC., Richland, Washington.

RPP-RPT-54910, TBD, Hanford Single-Shell Tank Leak Causes and Locations - 241-SX Tank Farm, Washington River Protection Solutions, LLC., Richland, Washington.

RPP-RPT-55804, TBD, Common Factors Relating to Liner Failures in Single-Shell Tanks, Washington River Protection Solutions, Richland, Washington.

SD-WM-TI-356, 1988, Waste Storage Tank Status and Leak Detection Criteria, Rev. 0, Westinghouse Hanford Company, Richland, Washington.

TFC-ENG-CHEM-D-42, 2009, Tank Leak Assessment Process, Rev. B-2, CH2M HILL Hanford Group, Inc., Richland, Washington.

WHC-EP-0449, 1991, The Sort on Radioactive Waste Type Model: A Method to Sort SingleShell Tanks into Characteristic Groups, Westinghouse Hanford Company, Richland, Washington.

WHC-EP-0772, 1994, Characterization of the Corrosion Behavior of the Carbon Steel Liner in Hanford Site Single Shell Tanks, Westinghouse Hanford Company, Richland, Washington.

WHC-MR-0132, 1990, A History of the 200 Area Tank Farms, Westinghouse Hanford Company, Richland, Washington.

WHC-SD-WM-DP-207, 1996, Operating Data to In-Tank Solidification (ITS)-2 for January 1 to October 10, 1974, Rev. 0, Westinghouse Hanford Company, Richland, Washington.

WHC-SD-WM-DP-210, 1996, Operating Data to In-Tank Solidification (ITS)-2 for July 1 to December 31, 1972, Rev. 0, Westinghouse Hanford Company, Richland, Washington.

WHC-SD-WM-DP-231, 1996, Operating Data to In-Tank Solidification (ITS)-2 for January 1 to October 10, 1974, Rev. 0, Westinghouse Hanford Company, Richland, Washington.

WHC-SD-WM-ER-349, 1994, Historical Tank Content Estimate for the Northeast Quadrant of the Hanford 200 East Area, Rev. 0, ICF Kaiser Hanford Company, Richland, Washington. 
WHC-SD-WM-ER-351, 1996, Historical Tank Content Estimate for the Northwest Quadrant of the Hanford 200 West Area, Rev. 0, ICF Kaiser Hanford Company, Richland, Washington.

WHC-SD-WM-ER-556, 1996, Double-Shell Tank Useful Life Analysis, Rev. 0, ICF Kaiser Hanford Company, Richland, Washington.

WHC-SD-WM-TI-665, 2003, Soil Load above Hanford Waste Storage Tanks, Rev. 0C, CH2M Hill Hanford Group, Inc., Richland, Washington.

WHC-SD-WM-TI-669, 1995, Waste Status and Transaction Record Summary for the Northwest Quadrant of the Hanford 200 Area, Rev. 1, Los Alamos National Laboratory, Los Alamos, New Mexico.

WSRC-TR-90-512, 1990, Effect of Temperature on the Nitrite Requirement to Inhibit Washed Sludge, Westinghouse Savannah River Company, Aiken, South Carolina. 
RPP-RPT-54911, Rev. 0

APPENDIX A

\section{MEETING MINUTES}

July 26, 2011

August 9, 2011

August 23, 2011

September 13, 2011

September 27, 2011 


\section{MEETING SUMMARY}

$\begin{array}{ll}\text { From: } & \text { J. G. Field } \\ \text { Phone: } & 376-3753 \\ \text { Location: } & \text { Ecology Office, } \\ \text { Date: } & \text { July 26, 2011 } \\ \text { Subject: } & \text { Tank Farm Leak Inventory and Tank Integrity Assessments } \\ & \\ \text { To: } & \text { Distribution/Attendees }\end{array}$

Attendees:

Joe Caggiano, ECOLOGY

Kelly Elsethagen, CE\&ES/WRPS

Jim Field; WRPS

Les Fort, WRPS

Don Harlow, SC101/WRPS
Andrea Hopkins, WRPS

Brendan Hedel, WRPS Intern

Paul Henwood, S.M. Stoller

Marc Wood, CHPRC

\section{PURPOSE:}

The purpose of this meeting was to:

1) Assess tanks TX-107 and TX-110 (Part 1);

2) Discuss TY-103 leak location and cause (Part 2).

\section{Review Previous Meeting Summaries and Actions:}

The meeting summary for July 12 was reviewed and approved with comments.

\section{Part 1: Tank Farm Leak Inventory Assessments}

Responses to comments from Ecology, S.M. Stoller, and CHPRC on the B tank farm leak inventory assessment report are in progress. The $\mathrm{U}$ tank farm leak assessment report draft is expected to be completed and ready for review in the next two weeks and the C Farm reassessment report in about a month.

The team discussed comments regarding clarifying when a liner leak occurred or other types of releases. The $\mathrm{B}, \mathrm{U}$ and $\mathrm{C}$ farm inventory assessment reports will be revised as needed to distinguish between a liner leak and other types of releases.

\section{TX-107 Assessment}

Tank TX-107 information was presented and discussed by the assessment team.

Information presented will be included in the Assessment Report. Tank TX-107 was categorized as "questionable integrity" in 1977 based on liquid level decreases and increasing drywell activity and declared a confirmed leaker in 1984 with a leak volume estimate of 2,500 gal based on increasing activity in nearby drywells (HNF-EP-0182). SGLS measurements obtained in 1996 show ${ }^{60} \mathrm{Co}$ and ${ }^{154} \mathrm{Eu}$ gamma activity in the zones from 50 to $70 \mathrm{ft}$ bgs in drywells 51-07-18 and 51-07-07 and in other drywells between SSTs TX-103 and TX-107 with a total estimated ${ }^{60} \mathrm{Co}$ activity of $0.075 \mathrm{Ci}$ (using the ${ }^{60} \mathrm{Co}$ contaminated threshold values $>1 \mathrm{pCi} / \mathrm{g}$ from 
GHO-HAN-11). Gamma activity near TX-103 was attributed to a leak from tank TX-107. The ${ }^{60} \mathrm{Co}$ and ${ }^{54} \mathrm{Eu}$ gamma plumes indicate that the plume of the leak (volume) was greater than what would have been seen by a 2,500 gallon release.

SST TX-107 was used as the 242-T Evaporator feed/bottoms recycle tank in 1975, apparently handling B Plant ${ }^{90} \mathrm{Sr}$ recovery waste (SRR waste). A 1976 sample analysis from tank TX-107 showed a ${ }^{90} \mathrm{Sr}$ concentration of $0.37 \mathrm{Ci} / \mathrm{gal}(0.23 \mathrm{Ci} / \mathrm{gal}$ decayed to 1996). This is comparable to a concentration of $0.5 \mathrm{Ci} / \mathrm{gal}$ for SRR waste listed in HDW rev. 4. Therefore a leak volume estimate was calculated using HDW values for SRR waste. Dividing the total ${ }^{60} \mathrm{Co}$ activity measured in TX farm drywells $(0.075 \mathrm{Ci})$ by the SRR concentration estimate for ${ }^{60} \mathrm{Co}$ of $5.7 \mathrm{E}-5$ $\mathrm{Ci} /$ gal, an estimated 1,300 gallons of SRR waste and $\sim 300 \mathrm{Ci}$ of ${ }^{90} \mathrm{Sr}$ (decayed to 1996) could have been released.

The team concluded that a leak in the TX-107 tank liner released an estimated volume of 1,300 gal of SRR waste, with an inventory of $300 \mathrm{Ci}$ of ${ }^{90} \mathrm{Sr}$ (decayed to 1996). Based on the estimated ${ }^{60} \mathrm{Co}$ plume size (for $>1 \mathrm{pCi} / \mathrm{g}$ ) of 3,500 m3 (124,000 ft3) and assuming a 10\% increase in moisture content as a result of the release, the diluted plume may be as large as 100,000 gal. The plume size estimates are based on a limited data set; additional direct push logging is recommended as part of future TX farm investigations to better estimate the distribution and inventory of the plume.

\section{Part 2: Tank Leak Location and Cause}

\section{Tank TY-103}

The draft tank TY-103 integrity assessment was reviewed and discussed; the draft will become part of the report on tank waste leak locations and causes. Storing TBP waste in tank TY-103 was identified as the primary cause for the leak. TBP waste is comprised of low hydroxide and high nitrate concentrations creating an environment conducive to stress corrosion cracking. Other potential conditions that may have affected the tank TY-103 liner failure were elevated temperatures affecting corrosion and conditions during construction.

Several comments and suggestions were made on the tank TY-103 draft report and will be addressed in the final report, including:

- Identify any direct push drywells adjacent to tank TY-103

- Check any other radionuclides detected from drywell 52-03-03 scans in addition to ${ }^{137} \mathrm{Cs}$

- Change the blame buttons for Design to (-) and a deminimus button for Construction

A draft matrix table was presented showing a summary of causal factors contributing to leaks of all tanks assessed thus far and updated to include tank TY-103. This matrix will be revisited as each tank is assessed.

A preliminary draft of the introduction and common information for the BY and TY Farms was made available. This will be updated on a periodic basis as additional BY and TY tanks are assessed. 


\section{ACTIONS:}

\section{Part 1}

1. All: Review meeting summary for discussion in the next meeting.

2. J. Field: Prepare and distribute July 26, 2011 Meeting Summary

3. L. Fort: Prepare TX farm summaries for discussion in the next meeting.

\section{Part 2}

4. All: Review tank TY-103 presented information and provide feedback during the next meeting.

5. D. Harlow: Prepare July 26, 2011, 2011 Meeting Summary

6. D. Washenfelder/ D. Harlow: Prepare to discuss leak location and cause assessment for tank TY-104 and distribute draft information prior to the next meeting.

\section{NEXT MEETING:}

Part 1: Continue TX Farm leak inventory assessments for the other designated "leakers" TX-113, 114, 115, 116, and 117.

Part 2: Discuss leak location and causes for tank 241-TY-104.

Date: $\quad$ August 9, 2011

Time: $\quad$ Part 1: 9:00-10:00, part 2: 10:00-11:00

Location: ECOLOGY Office 

From:
Phone:
Location:
Date:
Subject:

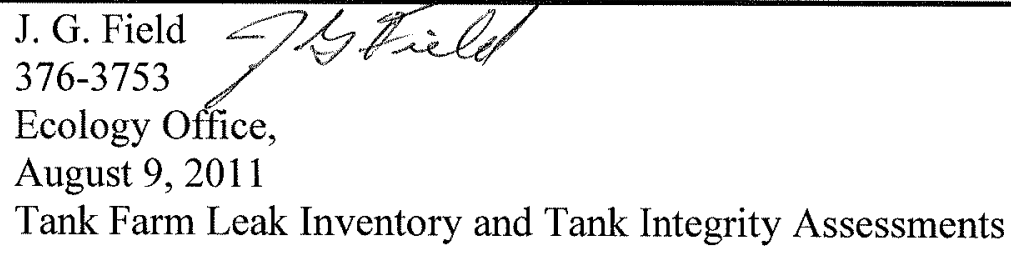

Tank Farm Leak Inventory and Tank Integrity Assessments

To:

Distribution/Attendees

Attendees:

Mike Barnes, ECOLOGY

Joe Caggiano, ECOLOGY

Jim Field; WRPS

Les Fort, WRPS

Crystal Girardot, WRPS

Andrea Hopkins, WRPS

Brendan Hedel, WRPS Intern
Paul Henwood, S.M. Stoller

Jeremy Johnson, ORP

Gretchen Reeploeg, WRPS

Chelsea Rosenkrance, WRPS

Dennis Washenfelder, WRPS

Marc Wood, CHPRC

\section{PURPOSE:}

The purpose of this meeting was to:

1) Assess tanks TX-110, TX-113 and TX-114 (Part 1);

2) Discuss TY-104 leak location and cause (Part 2).

\section{Review Previous Meeting Summaries and Actions:}

The meeting summary for July 26 was reviewed and approved. Comments on the B tank farm leak assessment report are being incorporated. There were no comments on the Tank TY-103 leak location and cause summary; Part 2.

\section{Part 1: Tank Farm Leak Inventory Assessments}

It was noted that the B farm assessment report should attempt to include inventory estimates for overflows and other releases using the same approach as the $\mathrm{C}$ tank farm reassessment. These inventory estimates will be added to the draft B tank farm report and discussed with Ecology along with responses to their comments.

The team discussed the rationale for TX-107 inventory estimates based on SGLS ${ }^{60} \mathrm{Co}$ values $>1$ $\mathrm{pCi} / \mathrm{g}$ when the $\mathrm{SGLS}$ detected ${ }^{60} \mathrm{Co}$ as low as $0.1 \mathrm{pCi} / \mathrm{g}$. The interpretation and rationale for selection of the greater than $1 \mathrm{pCi} / \mathrm{g}$ was that it could account for the majority of the ${ }^{60} \mathrm{Co}$ mass near tank TX-107. Lower levels would include ${ }^{60} \mathrm{Co}$ activity and plume volume estimates for other TX farm tanks. It was agreed that the $1 \mathrm{pCi} / \mathrm{g}$ threshold estimate was reasonable and the logic for selecting the value will need to be explained in the assessment report.

Also discussed were parallels between SX Farm tanks and Tank A-105, showing high gamma activity in the laterals but little activity in drywells. This raises questions about how much 
RPP-RPT-54911, Rev. 0

gamma activity may be in the soils, but not detected in drywells in other farms. A 1979 letter (Stallos report) to the Inspector Generals Office (IGO) regarding tank leaks and the 1980 IGO response "Report on Alleged Cover-Ups of Leaks of Radioactive Materials at Hanford" was discussed and distributed to the group after the meeting. This report may explain why some tanks in TX farm and other farms were later classified "questionable integrity" based on little information.

\section{TX-110 Assessment}

Tank 241-TX-110 (TX-110) information was presented and discussed by the assessment team. Information presented will be included in the Assessment Report. Tank TX-110 was declared "questionable integrity" in 1977 as a result of a low activity radiation peak detected at $54 \mathrm{ft}$ bgs in drywell 51-10-01. The peak rapidly decayed between 1977 and 1985 following a ${ }^{106} \mathrm{Ru}$ decay line and 1996 SGLS data shows less than $1 \mathrm{pCi} / \mathrm{g}$ gamma activity in drywells near tank TX-110 except for small spikes to $<10 \mathrm{pCi} / \mathrm{g}$ near surface and at the bottom of drywell 51-10-12. This indicates the historical gamma peak was likely due to waste migrating from another source, possibly in the area around tanks TX-107 or TX-114. No other assessments have been conducted for tank TX-110 and no leak volume estimate was determined except that it was included as one of the group of 19 tanks assessed by Baumhardt in 1989 with a cumulated leak volume of 8,000 gal. The waste in tank TX-110 has not been sampled. No data or information was found indicating the presence of a liner leak and there is little data to suggest a release occurred near the tank. As a result, no inventory is assigned and it is recommended that the current leak classification for tank TX-110 be reassessed per TFC-ENG-CHEM-D-42.

\section{TX-113 Assessment}

Tank 241-TX-113 (TX-113) information was presented and discussed by the assessment team. Information presented will be included in the Assessment Report. Tank TX-113 was declared "questionable integrity" in 1974 as a result of increasing gamma activity in drywell 51-14-04. No other assessments have been conducted for this tank and no leak volume estimate was determined except that tank TX-113 was also included as one of the group of 19 tanks assessed by Baumhardt in 1989. Historical transfer records show that the tank was filled above the cascade outlet as a result of cascade plugging of the cascade lines and in-tank photographs show the waste level was well above the cascade line, indicating the potential for releases from the cascade lines or spare inlet ports. Drywells around tank TX-113 show $\sim 10 \mathrm{pCi} / \mathrm{g}$ of ${ }^{137} \mathrm{Cs}$ activity between the surface and $20 \mathrm{ft}$ below surface. This activity is attributed to cascade line leaks, near surface transfer line leaks or spills during operations. At about $45 \mathrm{ft}$ bgs (below the tank base) in drywell 51-14-04, 1E5 pCi/g of ${ }^{137} \mathrm{Cs}$ activity was detected. Because there was a sharp ${ }^{137} \mathrm{Cs}$ spike at $45 \mathrm{ft}$ with no path to it, the high ${ }^{137} \mathrm{Cs}$ activity has all indications to be from a liner leak from tank 241-TX-114 (TX-114). TX-114 appears to be the more likely source of the detected contamination. As a result, a waste inventory associated with this plume is assigned to tank TX-114 and the team recommends that the current leak classification for tank TX-113 be reassessed per TFC-ENG-CHEM-D-42.

\section{TX-114 Assessment}

Tank TX-114 information was presented and discussed by the assessment team. Information presented will be included in the Assessment Report. Tank TX-114 was declared "questionable integrity" in 1974, the same time as tank TX-113, as a result of increasing gamma 
activity in drywell 51-14-04, located only $2 \mathrm{ft}$ from the East side of tank TX-114. Like tank TX113, historical transfer records show that tank TX-114 was filled above the cascade outlet as a result of plugging of the cascade lines and in-tank photographs show the waste level was well above the cascade line, indicating the potential for releases from the cascade lines or spare inlets. The drywells show $\sim 100 \mathrm{pCi} / \mathrm{g}$ of ${ }^{137} \mathrm{Cs}$ activity between the surface and $20 \mathrm{ft}$ below surface in drywells 51-14-04 and 51-14-11. This activity was attributed to cascade line leaks, near surface transfer line leaks and spills during operations.

As noted above in the tank TX-113 discussion, a high activity ${ }^{137} \mathrm{Cs}$ plume was detected in drywell 51-14-04. This activity was present when the drywell was installed in 1974. It is assumed that the contamination migrated along the top of the sediments below the tank farm excavation to boreholes 51-14-08 and 51-14-11. However, it cannot be discounted that the source of the contamination being detected in the boreholes associated with tank TX-114 could have originated from tank TX-113. .

Evaporator bottoms (EB) supernate from the 242-T evaporator was in tank TX-114 from 1954 to 1974 and a 1974 TX-114 sample showed a ${ }^{137} \mathrm{Cs}$ concentration of $0.22 \mathrm{Ci} / \mathrm{L}$ in the tank. This is higher than the HDW model T1 saltcake supernatant estimate for ${ }^{137} \mathrm{Cs}$ of $0.018 \mathrm{Ci} / \mathrm{L}$ (LA-UR96-3860). Leak volume and inventory estimates for the plume observed in drywell 51-14-04 will be discussed in the next meeting.

\section{Part 2: Tank Leak Location and Cause}

\section{Tank 241-TY-104 (TY-104)}

The draft tank TY-104 integrity assessment was reviewed and discussed; the draft will become part of the report on tank waste leak locations and causes. Two possible leak scenarios were identified: a tank liner leak and/or cascade discharge tie line leak. Storing TBP waste in tank TY-104 was identified as the primary cause for a liner or cascade line leak. TBP waste is comprised of low hydroxide and high nitrate concentrations creating an environment conducive to stress corrosion cracking. Other potential factors that may have affected the tank TY-104 liner failure were elevated temperatures affecting the rate of corrosion and conditions during construction.

Storing TBP waste in the cascade outlet tie line of tank TY-104 was identified as the primary cause for a tie line leak. Another condition possibly leading to the tie line leak is failure of the cathodic protection on the tie line which may have resulted in galvanic corrosion.

Several suggestions were made on the tank TY-104 draft report and will be investigated including:

- Investigate TBP waste in more detail including:

$\circ$ effect of adding fresh TBP metal recovery waste from U Plant vs. aged TBP waste

$O$ effect of the first waste added to a tank being TBP waste compared with the effect of having a waste heel in the tank when TBP waste was first added (e.g., reducing 
the magnitude of the corrosion reaction by the tank liner being passivated by the waste heel from TBP waste attack)

- length of time holding only TBP metal recovery waste fresh from U Plant

- Investigate the concentration differences of the stress corrosion inhibitors between TBP waste and leachate from the SX Farm tanks that were nitrate leached

- Review the 1994 document (WHC-EP-0772, Characterization of the Corrosion Behavior of the Carbon Steel Liner in Hanford Site Single-Shell Tanks) as a resource to determine corrosivity of TBP waste compared to other wastes; it may contain additional TBP sample data

TBP investigation results will be reported in individual tank segments and tank farm sections as well as the Common factors of Liner Failures report, as appropriate.

A draft matrix table was presented showing a summary of causal factors contributing to leaks of all tanks assessed thus far and updated to include tank TY-104. This matrix will be revisited as each tank is assessed.

Tank TY-105 will be addressed at the next meeting.

\section{ACTIONS:}

\section{Part 1}

7. All: Review meeting summary for discussion in the next meeting.

8. J. Field: Prepare and distribute August 9, 2011 Meeting Summary

9. L. Fort: Prepare TX farm summaries for discussion in the next meeting.

\section{Part 2}

10. All: Review tank TY-104 presented information and provide feedback during the next meeting.

11. D. Harlow/ C Girardot: Prepare August 9, 2011 Meeting Summary

12. D. Washenfelder/ D. Harlow: Prepare to discuss leak location and cause assessment for tank TY-105 and distribute draft information prior to the next meeting.

\section{NEXT MEETING:}

Part 1: Discuss leak inventory estimates for Tank TX-114 and continue TX Farm leak inventory assessments for tanks TX-115, 116, and 117.

Part 2: Discuss leak location and causes for tank 241-TY-105.

Date: $\quad$ August 23, 2011

Time: $\quad$ Part 1: 9:00-10:00, part 2: 10:00-11:00

Location: ECOLOGY Office 


\section{MEETING SUMMARY}

\begin{tabular}{ll}
\hline From: & J. G. Field 74 ielf \\
Phone: & $376-3753$ \\
Location: & Ecology Office, \\
Date: & August 23, 2011 \\
Subject: & Tank Farm Leak Inventory and Tank Integrity Assessments \\
& \\
To: & Distribution/Attendees
\end{tabular}

Attendees:

Mike Barnes, ECOLOGY

Kayle Boomer, WRPS

Joe Caggiano, ECOLOGY

Jim Field; WRPS

Crystal Girardot, WRPS

Don Harlow, SC101/WRPS
Andrea Hopkins, WRPS

Paul Henwood, S.M. Stoller

Jeremy Johnson, ORP

Chelsea Rosenkrance, WRPS

Marc Wood, CHPRC

\section{PURPOSE:}

The purpose of this meeting was to:

1) Discuss waste release estimates for B farm; Tank TX-114 waste release estimates; and assess waste releases for Tanks TX-115, 116 and 117 (Part 1);

2) Discuss TY-105 leak location and cause (Part 2).

\section{Review Previous Meeting Summaries and Actions:}

The August 9 meeting summary was reviewed and approved with comments.

The comments will be incorporated in the final meeting summary.

\section{Part 1: Tank Farm Leak Inventory Assessments}

\section{B Farm UPR Release Estimates}

As discussed in the August 9 meeting, preliminary inventory estimates (volume and waste composition) were presented for releases near B farm tanks identified in assessments as potential cascade line leaks or spills from overfilling of the tank. These estimates will be included in the B Farm Assessment report. Because liquid level decreases were not observed or could be attributed to something other than a release to the soil, liquid level changes were not used to estimate the volume of releases. Release volumes and inventories were estimated based on drywell data showing the presence and location of ${ }^{137} \mathrm{Cs}$ or ${ }^{60} \mathrm{Co}$ activity, and tank waste analytical results from near the assumed time of a release.

SGLS activity levels were low in drywells surrounding tanks 241-B-103, B-111 and B-112 and data was insufficient to estimate a release inventory for these tanks. Inventories were estimated and presented to the assessment team for tanks 241-B-101, B-105, and B-106. 
The data suggests that there may have been two separate releases near tank B-101, one on the north east side of the tank and one on the south side of the tank. Both releases combined appear to have a ${ }^{137} \mathrm{Cs}$ inventory of up to $450 \mathrm{Ci}$. Assuming the waste was B-Plant low level evaporator bottoms waste, based on a 1975 tank B-101 supernate sample (assumed to be representative of the wase leaked because 1975 was the year gamma activity was first detected in drywell 20-0105 ) the waste composition of $0.25 \mathrm{Ci} / \mathrm{gal}$ could result in a 2,000 gal release. This volume estimate would be larger for a dilute waste stream.

If the ${ }^{137} \mathrm{Cs}$ and ${ }^{60} \mathrm{Co}$ activity detected near tanks B-105 and B-106 is from a cascade line leak, ${ }^{137} \mathrm{Cs}$ migrated $30 \mathrm{ft}$ downward and only about $7 \mathrm{ft}$ horizontally (approximate distance to drywell); this seemed unreasonable to some of the assessment team members. If the activity was due to a cascade line leak, an estimated 7,000 $\mathrm{Ci}$ of ${ }^{137} \mathrm{Cs}$ could have been released from each of 3 cascade line locations (the tank B-105 cascade inlet, the tank B-105 outlet, and the B-106 inlet) with a released waste volume of 37,000 gal (based on 1975 tank samples [0.19 Ci/gal]) and a combined release volume of 121,000 gal. If the tanks leaked at the tank bottom, 6,000 $\mathrm{Ci}$ and 32,000 gal of waste could have been released from each location.

These rough inventory estimates are based on data from one drywell near the B-105 and B-106 inlet cascade lines. The waste type and time of releases, the date and source of releases, the size and shape of the ${ }^{137} \mathrm{Cs}$ plume, and the composition of waste released were assumed based on limited data. Based on the measured ${ }^{137} \mathrm{Cs}$ activity in the drywells, it was assumed that: 1) the outer edge of the ${ }^{137} \mathrm{Cs}$ plume was at the drywall, 2) the ${ }^{137} \mathrm{Cs}$ plume would travel about the same horizontal distance in all directions, and 3) the ${ }^{137} \mathrm{Cs}$ concentration for the entire plume from the release source (either about $20 \mathrm{ft}$ bgs near the tank or at $37 \mathrm{ft}$ bgs [the bottom of the tank]) to about $60 \mathrm{ft}$ bgs was at the ${ }^{137} \mathrm{Cs}$ saturation capacity for soil $\left[\sim 5(10)^{7} \mathrm{pCi} / \mathrm{g}\right]$, If better inventory estimates are required, additional data and information is needed.

\section{1-TX-114 Tank Leak Inventory Estimate}

Drywell 510-14-04 shows a ${ }^{137} \mathrm{Cs}$ peak at about $45 \mathrm{ft}$ bgs with nothing above it. Assuming the

${ }^{137} \mathrm{Cs}$ activity is from a leak near the bottom of the tank, based on the size and shape of the plume, if the drywell activity represents the edge of the plume and the entire plume is at ${ }^{137} \mathrm{Cs}$ sorption capacity $\left[5(10)^{7} \mathrm{pCi} / \mathrm{g}\right.$ ], an estimated $6,000 \mathrm{Ci}$ could have been released with a release volume of 7,000 gal based on 1974 tank samples $(0.83 \mathrm{Ci} / \mathrm{gal})$. This sample result is assumed to be representative of the waste leaked because the same waste type (EB) was in the tank from 1954 to 1974 and high gamma activity was present when drywell 51-14-04 was first installed in 1974.

This estimate is based primarily on data from one drywell near the release location. The waste type and time of releases, the date and source of releases, the size and shape of releases, and the composition of waste released were assumed based on limited data. If better inventory estimates are required, additional data is needed.

\section{1-TX-115 Assessment}

Tank information was presented and discussed by the assessment team.

The information presented will be included in the TX Farm Assessment Report. Tank TX-115 was declared "questionable integrity" in 1977 as a result of gamma activity in drywell 51-15-04. 
The tank was included in the group of 19 tanks assessed by Baumhardt in 1989 for which no leak inventory estimate could be determined and a leak volume of 8,000 gal was assumed based on average leak volume estimates for 21 other tanks. Historical transfer records and photos show that TX-115 was filled above the cascade outlet in 1952, indicating the potential for releases from the cascade line. However, there is no evidence of a liquid level decrease until supernate was pumped in the first quarter of 1953. This suggests that little or no liquid was released when the tank was overfilled. SGLS logs in drywells around the tank show low levels of ${ }^{137}$ Cs activity $(<10 \mathrm{pCi} / \mathrm{g})$. Historical gamma peaks were observed in 1977 in drywells 51-15-04. The gamma peak started at about $20 \mathrm{ft}$ bgs and migrated deeper and decayed away by 1994 indicating a mobile short lived radionuclide such as ${ }^{106} \mathrm{Ru}$ that may have migrated from a cascade line leak or spare inlet overflow (spare inlets are near drywell 51-15-04).

Because drywell data shows low activity and there is no occurrence report or indication of a liquid level decrease, no inventory for a release was estimated for this tank and the team recommends that the current leak classification for tank TX-115 be reassessed per TFC-ENGCHEM-D-42.

\section{1-TX-116 and TX-117 Assessment}

Tank information was presented and discussed by the assessment team. The information presented will be included in the TX Farm Assessment Report. Tank TX-116 and TX-117 were both declared "questionable integrity" in 1977 as a result of gamma activity in drywells near the tanks. Like Tank TX-115, these tanks were included in the group of 19 tanks assessed by Baumhardt in 1989. Historical transfer records and photos show that both tanks were filled above the cascade outlet, indicating the potential for releases from the cascade line or spare inlet overflow. SGLS logs in drywells around the tank show low levels of ${ }^{137} \mathrm{Cs}$ activity $(<100 \mathrm{pCi} / \mathrm{g}$ ) below $20 \mathrm{ft}$ bgs. Historical gamma peaks and recommendations for these tanks will be further discussed in the next meeting.

\section{Part 2: Tank Leak Location and Cause}

\section{Tank 241-TY-105}

The draft tank TY-105 integrity assessment was reviewed and discussed; the draft will become part of the report on tank waste leak locations and causes. Storing TBP waste in tank TY-105 was identified as the primary cause for a liner leak. TBP waste is comprised of low hydroxide and high nitrate concentrations creating an environment conducive to stress corrosion cracking. Other potential conditions that may have affected the tank TY-105 liner failure were elevated temperatures affecting corrosion and conditions during construction.

It was suggested to investigate the source of radioactivity detected in the direct push C5018 at 24.5-ft BGS as the inlet spare lines located near drywell C5018 may not be a likely source as there was no direct indication the tank was overfilled. It was also suggested to investigate and identify the drywells that reported radioactivity when they were first drilled. 
Mechanisms for liquid air interface (LAI) corrosion were discussed and it was indicated that corrosion at the LAI is likely less of a concern compared with other types of corrosion such as stress corrosion cracking.

A draft matrix table was presented showing a summary of causal factors contributing to leaks of all tanks assessed thus far and updated to include tank TY-105. This matrix will be revisited as each tank is assessed.

A tracking matrix was distributed to show the status and progress of the Tank Leak Cause and Location assessments and reports for TPA Target M-045-91F-T04.

Tank TY-106 and possibly BY-103 will be addressed at the next meeting.

\section{ACTIONS:}

\section{Part 1}

13. All: Review meeting summary for discussion in the next meeting.

14. J. Field: Prepare and distribute August 23, 2011 Meeting Summary

15. L. Fort: Prepare TX farm summaries for discussion in the next meeting.

\section{Part 2}

16. All: Review tank TY-105 presented information and provide feedback during the next meeting.

17. D. Harlow/ C Girardot: Prepare August 23, 2011 Meeting Summary

18. D. Harlow: Prepare to discuss leak location and cause assessment for tank TY-106 and distribute draft information prior to the next meeting.

\section{NEXT MEETING:}

Part 1: Continue to discuss leak inventory estimates for Tanks TX-116 and 117 and begin to discuss "sound" tanks

Part 2: Discuss leak location and causes for tank 241-TY-106.

Date: $\quad$ September 13, 2011

Time: $\quad$ Part 1: 9:00-10:00, part 2: 10:00-11:00

Location: ECOLOGY Office 


\author{
From: J. G. Field \\ Phone: $\quad 376-3753$ \\ Location: Ecology Office, \\ Date: $\quad$ September 13, 2011 \\ Subject: Tank Farm Leak Inventory and Tank Integrity Assessments
}

To: Distribution/Attendees

Attendees:
Mike Barnes, ECOLOGY
Andrea Hopkins, WRPS
Joe Caggiano, ECOLOGY
Jeremy Johnson, ORP
Jim Field; WRPS
Gretchen, Reeploeg, WRPS
Crystal Girardot, WRPS
Chelsea Rosenkrance, WRPS
Don Harlow, SC101/WRPS
Marc Wood, CHPRC

Paul Henwood, S.M. Stoller

\title{
PURPOSE:
}

The purpose of this meeting was to:

1) Discuss assess waste releases for Tanks TX-116 and 117 (Part 1);

2) Discuss TY-106 and BY-103 leak locations and causes (Part 2).

\section{Review Previous Meeting Summaries and Actions:}

The August 23 meeting summary was reviewed and approved with comments.

The comments will be incorporated in the final meeting summary.

\section{Part 1: Tank Farm Leak Inventory Assessments}

\section{1-TX-116 and TX-117 Assessment}

Tank information was again presented and discussed by the assessment team.

The Historical gamma peaks of 2,000 cps and 3,300 cps were discovered in drywells 51-16-04 and 51-16-11 at about 40 and $50 \mathrm{ft}$ bgs respectively when the wells were first logged in 1973 . The gamma activity quickly decayed away in drywell 51-16-04 and decayed then increased to 6,500 cps in drywell 51-16-11 before it decayed away. Except for low levels of near surface ${ }^{137} \mathrm{Cs}$ activity $(<50 \mathrm{pCi} / \mathrm{g})$ and occasional hits $<1 \mathrm{pCi}$ lower in the drywell, Gamma activity was not present in $1996 \mathrm{SGLS}$ logs. This indicates the presence of mobile and/or quick decaying radionuclides that may have migrated from another source such as TX-110 or TX-114 or may be related to small releases from tank systems releases (overflows) or transfer lines. Because there wasn't an indication of a release from the tank waste surface measurements and because SGLS gamma activity was low, no inventory was estimated for these tanks. It was recommended that the current "assumed leaker" integrity classifications for these tanks be reassessed per TFCENG-CHEM-D-42. 
RPP-RPT-54911, Rev. 0

\section{Part 2: Tank Leak Location and Cause}

\section{Tank TY-106}

The draft tank TY-106 integrity assessment was reviewed and discussed; the draft will become part of the report on tank waste leak locations and causes. The initial storing of TBP waste in tank TY-106 was identified as the most likely cause for a liner leak. TBP waste was high in nitrate ion (causal factor for stress corrosion cracking) and low in the corrosion inhibiting hydroxide and nitrite ions. At elevated temperatures the stress corrosion cracking reaction would be enhanced. Other potential conditions that may have affected the tank TY-106 liner failure were elevated temperatures affecting steel corrosion and conditions during construction. It was suggested to clarify the chemistry conditions for TBP waste in Section 8.4.3 to explain how TBP waste would increase tank liner stress corrosion cracking.

\section{Tank BY-103}

The draft tank BY-103 integrity assessment was reviewed and discussed; the draft will become part of the report on tank waste leak locations and causes. Storing TBP waste in tank BY-103 was identified as the most likely cause for a liner leak. Other potential conditions that may have affected the tank BY-103 liner failure were elevated temperatures affecting steel corrosion and conditions during construction.

A draft matrix table was distributed to the review team showing a summary of causal factors contributing to leaks of all tanks assessed to date including tanks TY-106 and BY-103. An updated matrix will be revisited as each tank is assessed.

The draft BY and TY Farm Report will be discussed in the next meeting.

\section{ACTIONS:}

\section{Part 1}

19. All: Review meeting summary for discussion in the next meeting.

20. J. Field: Prepare and distribute September 13, 2011 Meeting Summary

21. L. Fort: Prepare TX farm summaries for discussion in the next meeting.

\section{Part 2}

22. All: Review tank TY-106 and BY-103 presented information and provide feedback during the next meeting.

23. D. Harlow/ C Girardot: Prepare September 13, 2011 Meeting Summary

24. D. Harlow: Prepare to discuss leak location and cause assessment write up for TY and BY tanks.

\section{NEXT MEETING:}

Part 1: Discuss leak inventory estimates for "sound" tanks, beginning with Tank TX-118.

Part 2: Discuss leak location and causes report for TY and BY tanks.

Date: $\quad$ September 27, 2011

Time: $\quad$ Part 1: 9:00-10:00, part 2: 10:00-11:00

Location: ECOLOGY Office 


\title{
DRAFT MEETING SUMMARY
}

\author{
From: \\ Phone: \\ J. G. Field \\ Location: \\ 376-3753 \\ Date: \\ Ecology Office, \\ Subject: \\ September 27, 2011 \\ Tank Farm Leak Inventory and Tank Integrity Assessments
}

To:

Distribution/Attendees

Attendees:
Joe Caggiano, ECOLOGY
Jim Field; WRPS
Les Fort, WRPS
Crystal Girardot, URS/WRPS
Don Harlow, SC101/WRPS

\author{
Paul Henwood, S.M. Stoller \\ Andrea Hopkins, WRPS \\ Chelsea Rosenkrance, WRPS \\ Dennis Washenfelder, WRPS \\ Marc Wood, CHPRC
}

\section{PURPOSE:}

The purpose of this meeting was to:

1) Discuss further the reassessment of waste releases from Tanks C-101 and C-104;

2) Discuss the TY and BY leak locations and causes report (Part 2).

\section{Review Previous Meeting Summaries and Actions:}

The September 13 meeting summary was reviewed and approved by those in attendance.

\section{Part 1: Tank Farm Leak Inventory Assessments}

The status of tank leak inventory assessment reports was discussed. The Hanford B Farm Leak Inventory Assessments Report (RPP-RPT-49089) was issued last week. Comments from Ecology, S. M. Stoller and CHPRC on the U Farm leak inventory assessments report were accepted or clarifications added to the text. Ecology indicated that they did not need to review or discuss responses further and the report should be released. The $\mathrm{U}$ farm report will be issued this week. A draft of the $\mathrm{C}$ farm reassessment report is completed and ready for editing. Tanks $\mathrm{C}$ 101 and C-104 discussions in today's meeting will be included in the report.

\section{Revised Inventory Estimates for tanks 241-C-101 and 241-C-104}

Additional information for tanks C-101 and C-104 was presented and discussed by the assessment team. It was proposed that the new information and inventory estimates be incorporated into the reassessment report. All participants concurred with the new estimates.

The revised inventory estimates and information presented are included in Attachment A.

Tank C-108 releases and drywell data were also discussed. It was noted that while ${ }^{60} \mathrm{Co}$ was observed near other tanks. The base of tank C-108 appears to be the only place in C Farm that ${ }^{60} \mathrm{Co}$ is still moving. Several possibilities for this movement were discussed. 
RPP-RPT-54911, Rev. 0

\section{Part 2: Tank Leak Location and Cause}

\section{BY and TY Farm Leak Location and Causes Draft Report}

The draft BY and TY Farm Leak Location and Causes report was reviewed and conclusions discussed.

The primary contributor to leaks in Type III tank liners in the BY and TY Farms appears to be chemistry-corrosion with some possible impacts from construction temperature and thermal shock caused by waste additions. Tank design did not appear to be a factor in liner failure because the BY and TY Tanks design continued the features of the earlier 241-BCTU tanks; however, there could be many unknown variables present in the quality of materials and construction. No observed liner abnormalities were found such as deformations (bulging) that could contribute to or indicate a liner failure.

There seems to be a relationship between storing undiluted TBP waste and liner failure. All five of the leaking BY and TY tanks examined stored undiluted TBP waste. At least ten of the BY and TY Farm tanks that have not been declared leakers stored diluted TBP waste.

Resolution of the comments received for the individual BY and TY Farm tank segments and for the SX Farm report will be distributed for review and a follow-on meeting will be scheduled as needed to discuss individual comments, if necessary.

Erratum: A possible cascade tie line leak at the 9:00 position should have been discussed as a potential cause for the TY-104 liquid level decrease as opposed to a liner leak. The cause could have been stress-corrosion cracking (SCC) resulting from the TBP waste in the sloping capped cascade tie line. This will be discussed in the conclusions of the BY and TY Farm Leak Location and Causes report.

This meeting concludes the leak cause and location assessments for the 13 individual tank segments required to be assessed during fiscal year 2011.

\section{ACTIONS:}

25. J. Field: Prepare and distribute September 27, 2011 Meeting Summary.

26. D. Harlow/ C Girardot: Prepare September 27, 2011 Meeting Summary (Part 2).

27. All: Review meeting summary. Send redline comments to J. Field or D. Harlow.

\section{NEXT MEETING:}

Future meetings are on hold due to FY12 budget limitations.

Thanks to all for supporting this process. Best wishes to Marc Wood in his retirement. 
RPP-RPT-54911, Rev. 0

\section{Attachment A}

\section{Tank 241-C-101 and 241-C-102 Revised Leak Assessment Estimates}

\subsection{Tank C-101 Leak Assessment Considerations}

The waste surface level measurements from 1965 through 1969 (when tank C-101 waste was pumped down to a waste depth of $\sim 40$ inches) showed an unaccounted surface level measurement decrease of $\sim 13.5$ inches ( 37,000 gallons) (Figure 3-9). Evaporation calculations reported in RPP-ENV-33418 indicate that given the high heat and high temperature of PSN waste, up to $85 \mathrm{gal} /$ day of supernate could have evaporated from the tank over this time period, potentially accounting for all of the liquid level decrease over 4 years. However, if as expected, a condenser was operating during that time, supernate would have condensed back into the tank and the evaporation rate may have been negligible. Some condensers were reported to have leaked and condensate may have been released from the condenser pit to the soils.

Supernate may also have been released through the cascade or spare inlet line. Liquid level measurements indicate that the tank was filled above the cascade outlet with PSN waste from the CR vault from 1965 to 1969 . High levels of total gamma activity were detected when the drywells were constructed in 1970. Most of the gamma activity decayed away following a ${ }^{106} \mathrm{Ru}$ decay curve and less than $1,000 \mathrm{pCi} / \mathrm{g}$ of ${ }^{137} \mathrm{Cs}$ gamma activity was observed in $1997 \mathrm{SGLS}$ measurements. If 37,000 gallons of PSN waste was released much greater ${ }^{137} \mathrm{Cs}$ activity would be expected compared to what was observed.

It is likely that the waste released was not PSN but was mostly PUREX cladding waste (CW) and or condensate. In March 1965 a 6 inch transfer line (line \#8041) failed and permitted coating waste from the PUREX Plant to leak into the encasement between the 152-CR diversion box and Tank 102-C and drain to Tanks 101-C, 102-C, and 103-C via the tank pump pits. (RLSEP-405, page B-2). As shown in Table 3-2, cladding waste has low ${ }^{137} \mathrm{Cs}$ content and ${ }^{60} \mathrm{Co}$ content. A cladding waste release mixed with condensate would account for the ${ }^{60} \mathrm{Co}$ observed in drywells near tank C-101 and the lower ${ }^{137} \mathrm{Cs}$ levels compared to a PSN release.

In 2011 direct push slant holes under the tank (C8101/2 at site A and C8103/4 at site B) were logged and sampled. Low gamma activity was observed throughout the profile in both holes. Quick-turn-around sample results showed low nitrate and ${ }^{99} \mathrm{Tc}$ was not detected except at low concentrations deep in slant hole C8104. The drywell and direct push logging results indicate that if a large volume of waste was released, mobile contaminants may have been flushed below the depth of the direct push hole $(\sim 180 \mathrm{ft} \mathrm{bgs})$ to the ground water.

The SGE results show a low resistivity anomaly extending deep below tank C-101. The resistivity anomaly results may indicate the presence of elevated salts such as nitrate or sodium (indicating a waste source) or may indicate higher moisture regions below the tank. 
RPP-RPT-54911, Rev. 0

\subsection{CONCLUSIONS AND RECOMMENDATIONS}

An informal assessment of tank C-101 concluded based on liquid level measurements, evaporation calculations, and low gamma activity in drywells that the tank probably did not leak, and if it did leak the release point would have been high on the tank wall (RPP-ENV-33418).

The liquid level decrease is likely due to a cascade and/or spare inlet release and evaporation. A formal tank integrity assessment (TFC-ENG-CHEM-D-42) is planned to review results of slant hole logging and sampling near tank C-101.

\subsubsection{Release Type}

Probably a cascade line and spare inlet release. Currently classified as a tank leak; a reassessment of the leak status is recommended per TFC-ENG-CHEM-D-42. Preliminary assessments concluded that if the tank did leak it was probably on the tank wall $\sim 54$ inches above the bottom of the tank.

\subsubsection{Depth of Release}

Mobile contaminants may have reached groundwater. The 1975 total gamma drywell data showed high activity starting at about $20 \mathrm{ft}$ bgs in drywells 30-01-01 and 30-01-09. Most of the gamma activity decayed away by 1980 and thereafter decay followed a ${ }^{137}$ Cs decay line, Drywells 30-01-01 and 30-01-09 show ${ }^{60} \mathrm{Co}$ activity at about $40 \mathrm{ft}$ bgs. Logging results from 2 slant direct push holes beneath tank C-101show low gamma levels throughout the well profile and samples from the direct push slant holes indicate little or no $\mathrm{NO}_{3}$ and ${ }^{99} \mathrm{Tc}$, but slightly increasing levels with depth, indicating mobile contaminants may have reached groundwater, however, no indication of downward migration was observed in historical drywell total gamma trend plots between 1975 and 1995. SGE data shows lower resistivity that extends from beneath tank C-101 and moves downward approaching groundwater. The resistivity anomaly may be due to high soil moisture spike observed in direct push logs or may indicate the presence of saline plumes not encountered by the direct push holes or samples.

As shown in Table 1, for purposes of estimating the inventory of material released, it was assumed that ${ }^{137} \mathrm{Cs}$ extends from $20 \mathrm{ft}$ bgs (the level of the spare inlets) to $38 \mathrm{ft}$ bgs.

\subsubsection{Timing of the Release}

Based on liquid level decreases and the presence of high levels of ${ }^{106} \mathrm{Ru}$ still in the soil in $1975 \mathrm{a}$ release or releases appear to have occurred beginning in 1965 after the tank was over filled with PSN from the CR vault and after it received cladding waste from a broken line in the transfer encasement. The release continued through 1969 with a decreasing release rate as the liquid level in the tank decreased below the level of the spare inlet and cascade lines.

\subsubsection{Magnitude of Release}

The estimated mass of ${ }^{137} \mathrm{Cs}$ released from tank C-101 was $\sim 800$ curies based on a saturated release model indicated by an attenuated reading at 28 feet bgs from drywell 30-01-09.

Assuming the estimated volume of waste released was 37,000 gallons (based on a 13.5 in liquid level decrease), the release concentration for ${ }^{137} \mathrm{Cs}$ would be $\sim 0.005 \mathrm{Ci} / \mathrm{L}$ (800 Ci / 37,000 gal). If the supernate released was all cladding waste with a ${ }^{137} \mathrm{Cs}$ concentration of $0.000755 \mathrm{Ci} / \mathrm{L}$ (based on the HDW, Rev. 5 composition for PUREX cladding waste (CWP1)), the estimated 
release volume would be $\sim 175,000$ gallons. This is unreasonably high based on the observed liquid level decrease and indicates that the waste was more concentrated than CWP1 supernate.

Before receiving CWP1 waste, tank C-101 received PUREX waste (P1) that has a much higher cesium concentration than PUREX cladding waste. Ratioing the CWP1 supernate ${ }^{137} \mathrm{Cs}$ waste composition with the $\mathrm{P} 1$ supernate ${ }^{137} \mathrm{Cs}$ waste composition results in a means to estimate the mass (concentration) of the other prominent waste constituents contained in the estimated waste release. Table 1 displays the estimated waste inventory for the prominent tank C-101 waste release via the spare inlet. Figure 1 shows the assumed configuration of the release; inventory calculations are further described in RPP-ENV-33418. Based on an estimated release of 37,000 gal and assuming a 5\% increase in moisture content within the wetted volume of the plume as a result of the release, the release may have spread over a soil volume of $\sim 100,000 \mathrm{ft}^{3}(37,000 \mathrm{gal} /$ $\left.0.05 / 7.48 \mathrm{gal} / \mathrm{ft}^{3}\right)$, essentially the size of the tank itself. 
RPP-RPT-54911, Rev. 0

Table 1. Tank 241-C-101 Waste Release Inventory Estimate Calculation

\begin{tabular}{|c|c|c|c|c|c|c|}
\hline \multirow{8}{*}{$\begin{array}{c}\text { CS PLUME } \\
\text { DIMENSIONS \& } \\
\text { SOIL } \\
\text { CHARACTERISTICS }\end{array}$} & Well & $\begin{array}{l}\text { Cs Plume } \\
\text { Cross- } \\
\text { Sectional } \\
\text { Radius } \\
\end{array}$ & & $\begin{array}{l}\text { Cs Plume } \\
\text { Cross- } \\
\text { Sectional } \\
\text { Area } \\
\end{array}$ & & \\
\hline & 30-01-09 & 10 & $\mathrm{ft}$ & 314 & $\mathrm{ft}^{\wedge} 2$ & \\
\hline & & \multicolumn{5}{|c|}{$\begin{array}{l}13 \text { feet from nearest spare inlet }-3 \mathrm{ft} \text { assumed attenuation, since measured }{ }^{137} \mathrm{Cs} \text { drywell } \\
\text { concentration is well below saturation capacity }\end{array}$} \\
\hline & \multicolumn{3}{|c|}{13 feet from nearest spare inlet } & & & \\
\hline & $\begin{array}{l}\text { Depth of } \\
\text { plume }\end{array}$ & \multicolumn{2}{|c|}{$\begin{array}{l}\text { 137Cs (pCi/g) in 30-01-09 } \\
\text { (time corrected) }\end{array}$} & $\mathrm{ft}$ & & \\
\hline & $18-28$ & \multicolumn{2}{|l|}{$1.00 \mathrm{E}+07$} & 10 & & \\
\hline & \multirow[t]{2}{*}{ Volume } & $\mathrm{ft} 3$ & $\begin{array}{l}\text { Grams of } \\
\text { soil }\end{array}$ & Ci: Cs-137 & & \\
\hline & & 1570 & $8.00 \mathrm{E}+07$ & $8.00 \mathrm{E}+02$ & & \\
\hline $\begin{array}{l}\text { Waste } \\
\text { Release } \\
\text { Volume }\end{array}$ & 37,000 & gallons & & & & \\
\hline \multirow[t]{5}{*}{$\begin{array}{l}\text { WASTE TYPE } \\
\text { CONSTANTS }\end{array}$} & $\begin{array}{l}\text { CWP1 Waste } \\
\text { Type } \\
\text { Constants Ci/L } \\
\text { Constants in } \\
\text { Ci/L (RPP- } \\
\text { 19822) }\end{array}$ & $\mathrm{Ci} / \mathrm{L}$ & $\begin{array}{l}\text { P1 Waste } \\
\text { Type } \\
\text { Constants Ci/L } \\
\text { Constants in } \\
\text { Ci/L (RPP. } \\
\text { 19822) } \\
\end{array}$ & $\mathrm{Ci} / \mathrm{L}$ & $\begin{array}{l}\text { Ratio of } \\
\text { CWP1 } \\
\text { Waste Type } \\
\text { to P1 Waste } \\
\text { Type }\end{array}$ & $\begin{array}{l}\text { Calculated total } \\
\text { Ci in the waste } \\
\text { release }\end{array}$ \\
\hline & Co-60 & $1.23 \mathrm{E}-05$ & & $1.23 \mathrm{E}-05$ & $98 \%$ & $1.72 \mathrm{E}+00$ \\
\hline & Eu-154 & $2.73 \mathrm{E}-07$ & & $1.12 \mathrm{E}-04$ & & $3.13 E-01$ \\
\hline & Cs-137 & $7.55 \mathrm{E}-04$ & & 0.283 & & $8.00 \mathrm{E}+02$ \\
\hline & Tc-99 & $2.84 \mathrm{E}-07$ & & $7.46 \mathrm{E}-05$ & & $2.23 \mathrm{E}-01$ \\
\hline \multirow[t]{6}{*}{$\begin{array}{l}\text { Total Wetted } \\
\text { Volume of plume }\end{array}$} & $\begin{array}{l}\text { Release } \\
\text { Volume }\end{array}$ & $\begin{array}{l}\text { r } \\
\text { Assuming 5\% } \\
\text { Soil Moisture } \\
\text { increase } \\
\text { within the } \\
\text { wetted } \\
\text { volume (ft^3 }\end{array}$ & $\begin{array}{l}\text { Shape } \\
\text { dimension } \\
\text { s for } \\
\text { diagonal } \\
\text { cut } \\
\text { cylinder }\end{array}$ & & & \\
\hline & \multirow[t]{5}{*}{37,000 gal } & \multirow{5}{*}{$\begin{array}{l}100,000 \mathrm{ft}^{3} \\
(37,000 / 0.0 \\
5 / 7.48 \\
\left.\mathrm{gal} / \mathrm{ft}^{3}\right)\end{array}$} & Diameter & 40 & $\mathrm{Ft}$ & \\
\hline & & & Depth & Averaged & & \\
\hline & & & $18-28$ & 10 & $\mathrm{Ft}$ & \\
\hline & & & $28-34$ & 6 & $\mathrm{Ft}$ & \\
\hline & & & $34-42$ & 8 & $\mathrm{Ft}$ & \\
\hline
\end{tabular}


Figure 1. Tank 241-C-101 Conceptual Diagram of Postulated Cesium and Moisture Plume

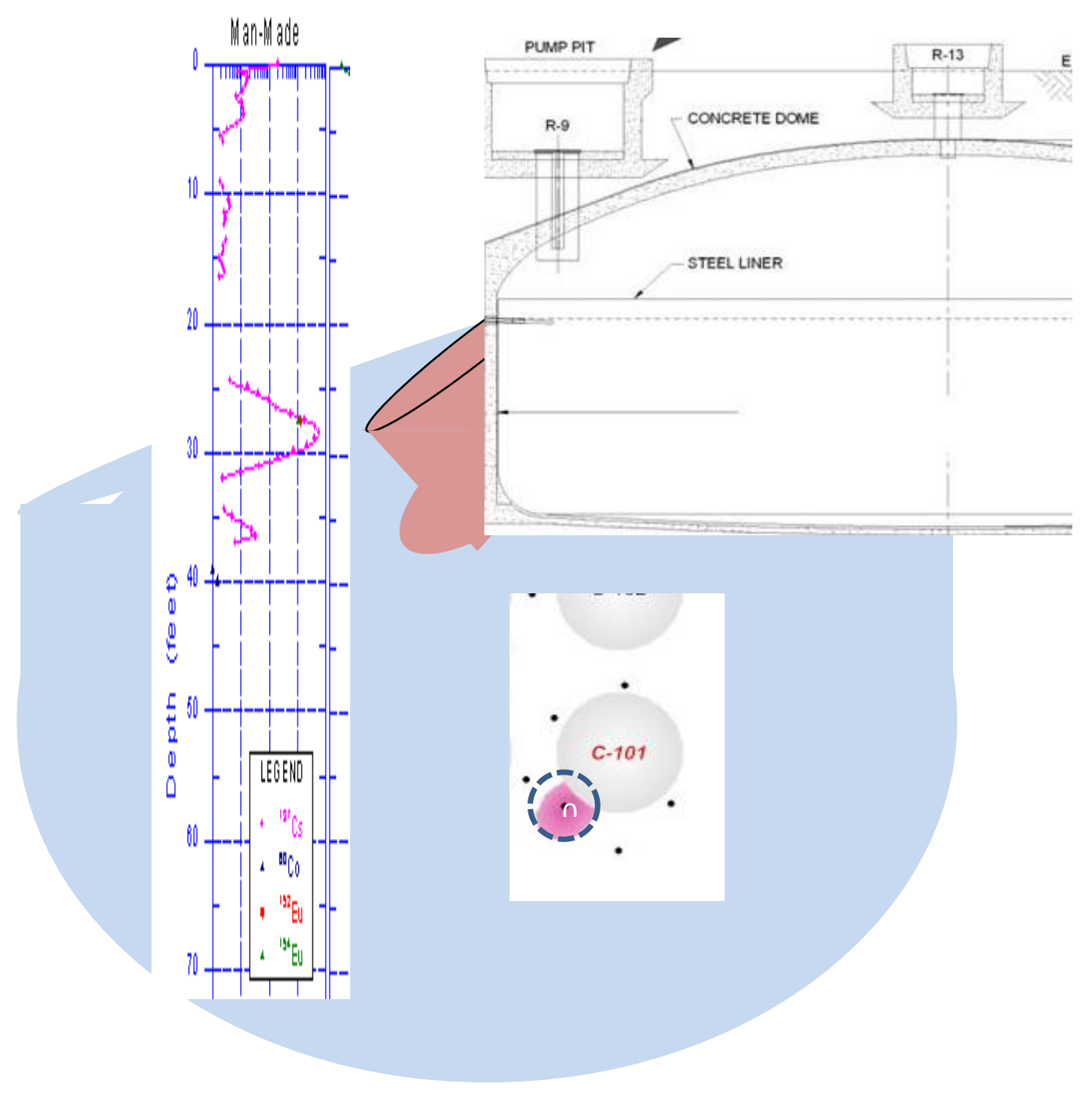

\subsection{TANK 241-C-104 LEAK ASSESSMENT CONSIDERATIONS}

Drywell 30-04-03 shows a ${ }^{137} \mathrm{Cs}$ peak at about $23 \mathrm{ft}$ bgs and ${ }^{60} \mathrm{Co}$ readings from 23 to $50 \mathrm{ft}$ bgs (drywell was only read to 49 feet). This drywell is close to the cascade line between tank C-104 and C-105.

The large plume originating between tanks C-104 and C-105 appears to be from the cascade line connecting these two tanks. It is possible that one or both of these tanks may have been the source of the waste release, but the apparent depth and location of the suspected origin of the plume suggest that the most likely source of the released waste is the cascade line. This plume appears to have migrated downward and eastward of tank C-103 to a depth of at least 125 $\mathrm{ft}$. The maximum depth of this plume in the vicinity of its source is questionable because of the limited depth of boreholes in that area. 
Transfers during the time the tank was overfilled may have masked liquid level decreases. Releases may also be attributed to a V103 pipeline leak.

\subsection{CONCLUSIONS AND RECOMMENDATIONS}

Tank C-104 appears to be sound as previously classified. However drywells show ${ }^{137} \mathrm{Cs}$ near the tank and ${ }^{60} \mathrm{Co}$ plumes that appear to extend from the tank cascade line and migrate outward and downward.

\subsubsection{Release Type}

The observed gamma activity in drywells is attributed primarily to cascade line releases from tank C-104 and may also be attributed to spare inlet overflows from tanks C-104 and C-105 and V103 pipeline leaks.

\subsubsection{Depth of Release}

The ${ }^{137} \mathrm{Cs}$ appears to have migrated from the cascade level to a peak at $23 \mathrm{ft}$ bgs and was assumed to extend downward near the tank to a depth of $50 \mathrm{ft}$ (about $10 \mathrm{ft}$ below the base of the tank). The 60Co plume appears to have migrated downward and eastward of tank C-103 to a depth of at least $125 \mathrm{ft}$.

\subsubsection{Timing of the Release}

The release was assumed to have occurred around 1965 when the tank was overfilled.

\subsubsection{Magnitude of Release}

Since there is no record of a transfer, the volume of the release due to the overfill was determined from the tank waste surface level decrease reported in WHC-MR-0132 in the first quarter of 1966. The volume difference was 28,000 gallons (560,000 gal to 532,000 gal)..

For comparison purposes, if an assumed plume volume was described as an increase in the water (wetted) content from a "background" of $4 \%$ to $9 \%$ (a 5\% increase) would account for approximately 23,000 gallons (see calculations for a modeled Co plume in Table 2). Assuming the waste released was CWP1 the mass of ${ }^{137} \mathrm{Cs}$ was estimated to be slightly over 60 curies at 23,000 gallons and 80 curies for a 28,000 gallon release. This mass could easily be masked next to the tank at the soil cesium saturation concentration of $\sim 1 \mathrm{E} 7 \mathrm{pCi} / \mathrm{g}$. Using CWP1 waste composition would also result in a $\sim 28,000$ gallons release; and $\sim 1.3 \mathrm{Ci}^{60} \mathrm{Co}$ at the time of the release. It was assumed that the ${ }^{60} \mathrm{Co}$ plume extended past the measured depth in the drywell to 80 feet; thereby resulting in a release that could have been $\sim 28,000$ gallons. Figure 2 shows the conceptual plume configurations and drywell data. 
RPP-RPT-54911, Rev. 0

Table 2. C-104 Conceptual Waste Plume Calculations

\begin{tabular}{|c|c|c|c|c|c|c|}
\hline \multirow{6}{*}{$\begin{array}{c}\text { PLUME } \\
\text { DIMENSIONS }\end{array}$} & \multirow{2}{*}{$\begin{array}{r}\text { Well } \\
30-04-03 \\
\end{array}$} & \multicolumn{2}{|c|}{$\begin{array}{c}\text { Plume Cross-Sectional } \\
\text { Radius }\end{array}$} & \multicolumn{2}{|c|}{$\begin{array}{c}\text { Plume Cross-Sectional } \\
\text { Area }\end{array}$} & \\
\hline & & 20 & $\mathrm{ft}$ & 1256 & $\mathrm{ft}^{\wedge} 2$ & \\
\hline & Shape & \multicolumn{4}{|c|}{ Cylinder; Half Sphere Bottom } & \\
\hline & Segment & $\begin{array}{l}\text { Segment } \\
\text { Depth } \\
\text { (ft) }\end{array}$ & \multicolumn{2}{|c|}{$\begin{array}{c}60 \text { Co in } 30-04-03 \text { (time } \\
\text { corrected to release } \\
\text { point) }\end{array}$} & \multicolumn{2}{|c|}{ Segment Height } \\
\hline & 1 & $25-30$ & $3.20 \mathrm{E}+01$ & $\mathrm{pCi} / \mathrm{g}$ & 5 & $\mathrm{ft}$ \\
\hline & $2 \& 3$ & $30-70$ & $3.20 \mathrm{E}+01$ & $\mathrm{pCi} / \mathrm{g}$ & 40 & $\mathrm{ft}$ \\
\hline \multirow{5}{*}{$\begin{array}{l}\text { WASTE TYPE } \\
\text { CONSTANTS }\end{array}$} & \multicolumn{2}{|c|}{$\begin{array}{c}\text { CWP1 Waste Type } \\
\text { Constants Ci/L } \\
\text { Constants in Ci/L (RPP- } \\
\text { 19822) }\end{array}$} & $\begin{array}{c}\text { Calculated } \\
\qquad \mathrm{Ci}\end{array}$ & & & \\
\hline & Co-60 & $1.23 \mathrm{E}-05$ & $1.07 \mathrm{E}+00$ & & & \\
\hline & Eu-154 & $2.73 \mathrm{E}-07$ & $2.38 \mathrm{E}-02$ & & & \\
\hline & Cs-137 & $7.55 \mathrm{E}-04$ & $6.57 \mathrm{E}+01$ & & & \\
\hline & Tc-99 & $2.84 \mathrm{E}-07$ & $2.47 \mathrm{E}-02$ & & & \\
\hline
\end{tabular}

\begin{tabular}{|c|c|c|c|c|}
\hline \multirow{4}{*}{$\begin{array}{c}\text { CURIES IN } \\
\text { MODELED } \\
\text { RELEASE } \\
\text { VOLUME }\end{array}$} & Segment & $\begin{array}{c}\text { Segment Volume (ft^3) } \\
\text { [(Segment Cross- } \\
\text { Sectional } \\
\text { Area)x(Segment } \\
\text { Height)] }\end{array}$ & $\begin{array}{c}\text { Co60 } \\
\text { Mass (g) }\end{array}$ & $\begin{array}{c}\text { Co60 (Ci) [(Segment } \\
\text { Co60 Mass, g)x(Segment } \\
\text { Time-Corrected Co60, } \\
\text { pCi/g)] }\end{array}$ \\
\cline { 2 - 5 } & 1 & 3140 & 160064640 & $5.12 \mathrm{E}-03$ \\
\cline { 2 - 5 } & 2 & 50240 & $2.561 \mathrm{E}+09$ & $8.20 \mathrm{E}-02$ \\
\cline { 2 - 5 } & 3 & 8400 & 428198400 & $1.37 \mathrm{E}-02$ \\
\hline
\end{tabular}

\begin{tabular}{|c|c|c|c|c|c|c|}
\hline \multirow{3}{*}{ OUTPUTS } & \multicolumn{3}{|c|}{$\begin{array}{l}\text { Co60 Volume Estimate based on } \\
\text { CWP1 [(Total Curies)x(CWP1 Cs137 } \\
\text { Waste Type Constant, Ci/L)x(3.785 } \\
\text { gal/L)] }\end{array}$} & 2200 & gal & \\
\hline & $\begin{array}{l}\text { Total } \\
\text { Waste } \\
\text { Plume } \\
\text { Volume } \\
\left(\mathrm{ft}^{\wedge} 3\right) \\
\end{array}$ & $\begin{array}{l}\text { Assumed } \\
\text { Soil } \\
\text { Moisture }\end{array}$ & $\begin{array}{c}\text { Total } \\
\text { Wetted } \\
\text { Volume, } \\
\text { Assuming } \\
\left(\mathrm{ft}^{\wedge} 3\right) \\
\end{array}$ & \multicolumn{2}{|c|}{$\begin{array}{c}\text { Release (gal) [(Wetted } \\
\left.\text { Volume, } \mathrm{ft}^{\wedge} 3\right) \times(7.48 \\
\text { gal/ft^3)] }\end{array}$} & $\begin{array}{l}\text { Rounded } \\
\text { Release } \\
\text { Estimate } \\
\text { (gal) }\end{array}$ \\
\hline & 61780 & $5 \%$ & 3089 & \multicolumn{2}{|c|}{23000} & 23000 \\
\hline
\end{tabular}


RPP-RPT-54911, Rev. 0

Figure 2: C-104 Conceptual Waste Plume Visualizations \& Drywell Logging Data
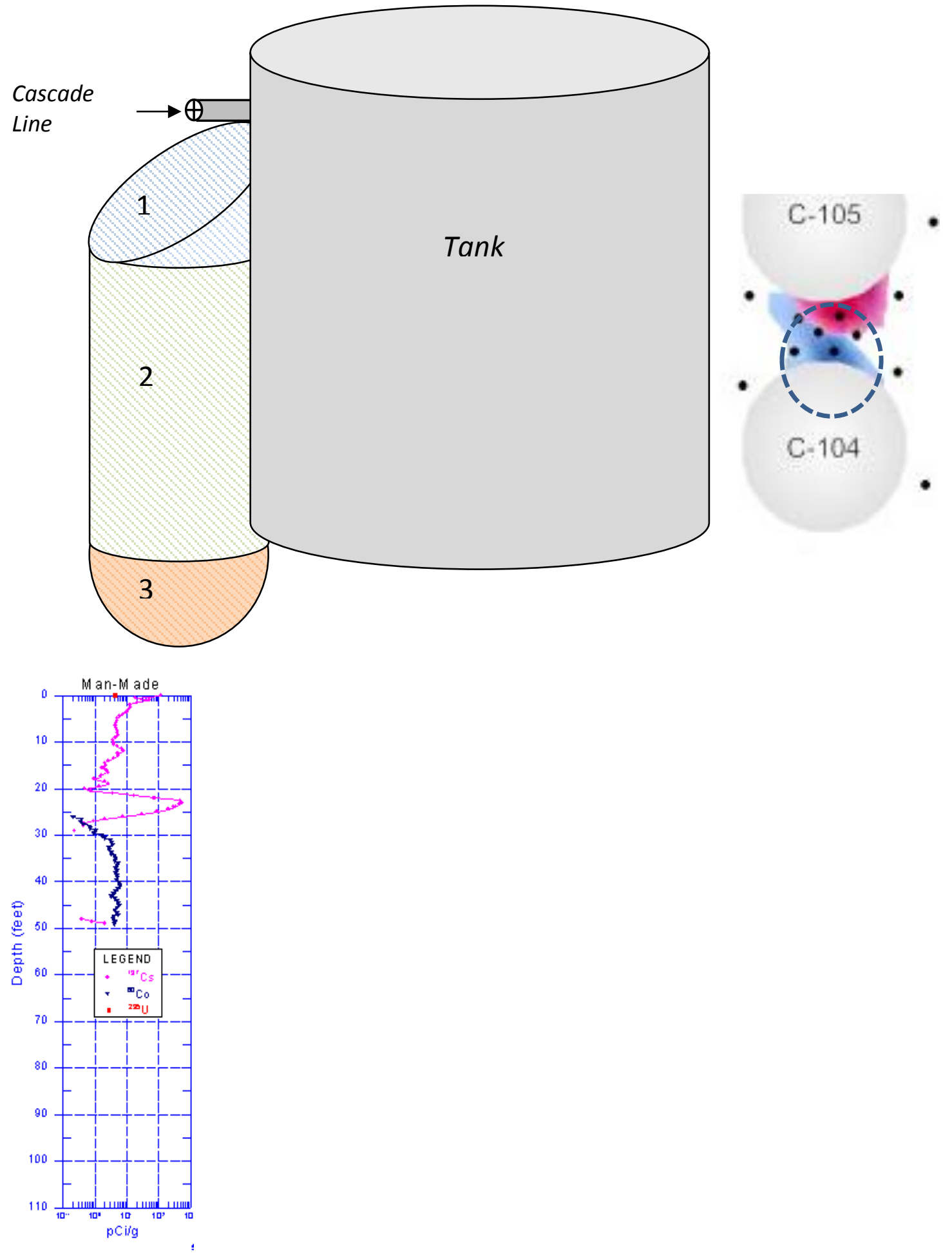
RPP-RPT-54911, Rev. 0

\section{APPENDIX B}

COMMENTS RESOLUTIONS FOR BY AND TY FARM REPORTS 
RPP-RPT-54911, Rev. 0

\section{TANKS TY-104 AND TY-105 SEGMENTS J. A. Caggiano REVIEW COMMENTS 8-17-11 with Responses}

\section{GENERAL COMMENTS:}

1. There are format issues with the TY-104 report that need to be corrected; figure captions appear out of place in relation to the figures.

- This has been corrected in the documents

2. The comment that a particular well was eliminated from consideration during the determination of leak location needs to be corrected. What you mean to say, I think, is that the lack of detections above background at a particular location indicates the likelihood that if there were a liner breach, it likely occurred elsewhere. That assumes that the volume of the leak is sufficient to allow interstitial liquid flow to within the detectable radius for the tools being used in the drywell. When assessing drywell logging data, one needs to assess the entire farm to get the "big picture", as releases from one tank may migrate to intercept drywells surrounding other tanks. The statement needs to be rephrased for this tank as well as for others.

- Added in the following to Section 3.4 and referred to this section in the individual segments: "The lack of radioactivity above background in a drywell indicates that if there was a liner leak it either occurred at another location or the leak flow was insufficient to be detected with the probes used in the drywell. When there is no radioactivity detected in a drywell or no recoverable data for a drywell it is not included as part of the leak location analysis."

3. In this report (and in others), there appears to be a distinction between quarterly liquid levels and routine LLs measured at more frequent intervals. This becomes an issue when no LL data are said to be available, but the figure clearly shows quarterly LL data. Many transfers may have occurred during a quarter that render these figures only approximate. The ambiguity of LLs is clearly illustrated in TY-105 where different levels were obtained using different instruments, leaving one to determine which, if any, are valid. An explanation is in order.

- Added in statement to the time line figures in each segment that says "See Figure xx graph for a plot of the quarterly liquid levels for the missing data."

- Added this to TY-105 segment, "It remains unclear which liquid level probe (WH\&DO electrode or Stiver's Recorder) was more accurate; however, the important thing to note is the rate of liquid level decrease is nearly the same with both the probes indicating a leak from tank TY-105."

4. In discussing drywell logging, it appears that only the historical gross gamma data are mentioned, but the more reliable 1990s spectral gamma data are ignored. Both should be discussed for all tanks, as the historical data indicate the development and growth/decay of peaks, while the spectral data identify the specific isotope and estimated quantity of activity present in the hole. 
- SGLS data was investigated for each drywell; however, if the information did not provide additional information for identifying leak location then was omitted from the write up.

\section{SPECIFIC COMMENTS:}

1. On Figure 6-1, the distance of the drywell to the footing given in the inserted table in the lower left corner of the figure likely assumes that the boreholes are vertical and this is a calculated distance based on the ground surface distance between the tank footing and the drywell. A qualifying footnote or note should be included. Also, check the number of significant figures, as I believe the stated distances on the figure may exceed significance. This comment applies to TY-104 and TY-105.

- Added in footnote to each drywell table to say "Assumes perfect vertical alignment"

2. Pg. 6-4, para 2 refers to the "capped cascade outlet". Please explain, because the meaning is not clear.

- Added in "See section 6.6" which contains more detailed information

3. Pg. 6-6, Fig. 6-2. The dates of likely overfilling would be a good addition to this timeline.

- $\quad$ This will be added to the timeline

4. On pg. 6-7 (and elsewhere), temperature data are given. Are these Hanford specific data or some other regional data (e.g., the Pasco airport)? Please clarify.

- Reference document PNNL-15160 for the temperatures given

5. In discussing chemical corrosion (Sect. 6.4.3), nothing is stated about "beach lines" that might be visible on in-tank photos or the enhanced environment for corrosion at the air/liquid interface - an effect that becomes important when LLs did not change over long periods or the LL data are highly uncertain because of probe changes/alteration. Please address. This appears to be the case for both TY-104 and TY-105.

- Added in the following to Section 3.2.4: "Uniform corrosion rates for single shell tanks are reported to be generally less than 1 mil/year (HNF-3018, Single-Shell Tank Sluicing History and Failure Frequency). This is assumed to be the liquid phase. The average corrosion rate for the liquid/vapor interface is reported to be approximately the same as for the liquid (WHC-SD-WM-ER-556, Double-Shell Tank Useful Life Analysis). The single shell tank failures are therefore not likely due to uniform corrosion."

6. In discussing drywell logs, there is no mention of elevated Cs-137 values in a couple of drywells between 80 and $100 \mathrm{ft}$ that clearly show on the spectral gamma logs. Please discuss the significance/relevance of these detections and what the potential sources may have been.

- A quote was added from GJ-HAN-67 indicating the conclusion was that tank TY-104 was not the source

7. For TY-105, drywells reveal a story that is being overlooked. There's Co- $60<100 \mathrm{pCi} / \mathrm{g}$ in several drywells from 50-100 ft. There must be a source for this contaminant and TY-105 is certainly a candidate. There's both Cs-137 and Co-60 from 50 to $90+\mathrm{ft}$. in drywell 52-05-07 
up to $50 \mathrm{pCi} / \mathrm{g}(\mathrm{Cs}-137)$ and up to $10 \mathrm{pCi} / \mathrm{g}$ Co-60. How do you explain those detections? Looks like tank waste to me. Please address.

- We are saying tank TY-105 leaked near the locations in Figure 7-15. The Co-60 in 1996 more or less confirms the leak location we addressed. From the data sources as well as the GJ-HAN-xx documentation, individual drywells are addressed in separate subsections.

8. Pg. 7-24, Sect. 7.7.1. The design flaw in this tank was the failure to adequately seal the spare inlet line on the SE side of the tank that allowed an overfill to leak through the spare inlet. Please discuss. The combination of the faulty seal and high pressure head in the tank led to a release.

- A leak from the spare inlet lines remains inconclusive and no records were located indicating the tank was overfilled. This was discussed in section 7.5.3.

9. Pg. 7-25. I think a fourth bullet is in order stipulating the overfilling of the tank creating the hydrostatic head that would drive waste through a poorly sealed inlet line.

- No record has been found indicating tank TY-105 was overfilled. Therefore, a fourth bullet point will not be added.

10. Table A-1 lists only the 1963 release from A-105, NOT the release attributed to the steam explosion and liner rupture in January 1965. This should be corrected, or the table should be dated so that it is clear that the table was released before the January 1965 release and liner failure.

- Added note to the figure: "See tank farm segments for information on individual specific tanks" 


\section{TANK TY-106 SEGMENT \\ J. A. Caggiano Review Comments 9-14-11 with Responses}

1. Pg. 8-5, para 2. The estimated average loss per day is 500 gal; however this is essentially unmeasureable using instruments of the time. It would be better to state that a loss of $X$ thousand gallons was noted after a drop in LL of XX in. The average loss over this period is estimated at $500 \mathrm{~g} / \mathrm{d}$. Given what was stated about liquid level measurements, what is the likely uncertainty of these values?

- Added "see Section 8.4.1" which goes into more depth about liquid level decreases. There were no records located indicating the amount of LL decrease over a period of time, only the calculated $500 \mathrm{gal} /$ day from document HW-61952.

2. Pg. 8-7, Sect. 8.3.2. As tank construction is considered one of the leading potential causes of a release, here or somewhere there should be an explanation of why such cold temperatures during construction are a hazard and could have resulted in damage or contributed to possible future damage.

- At the end of this section it refers to Section 3.1.2 which gives in depth explanations.

3. Pg. 8-8, para 1. Why were these "bad readings"? How can one tell a "bad" reading from a good one and what criteria does one use to distinguish the two? Makes one wonder whether any liquid level measurements are reliable, what the uncertainty in LLs is, and LLs are a principal means for detecting releases.

- Rephrased this sentence to the following: "The reported waste volumes slowly declined and from January to May 1957, the volume was reported to be 705-706 kgal. Document RPP-RPT-42296 reported these readings to be bad readings as the volume was reported back at $752 \mathrm{kgal}$ in June 1957 indicating intervening readings were incorrect."

4. Pg. 8-10, Sect. 8.5. The reader would benefit from a reminder that there are no laterals beneath any tanks in TY Farm.

- $\quad$ Added this statement to Section 8.6

5. Pg. 8-11, Sect. 8.5.1.1. Whatever was detected in this drywell in 1973 must have been shortlived, because the 1975 logs show nothing but background - assuming that the logs are reliable.

- All data recovered were reported as less than values and nothing is planned to be added to this section.

6. Pg. 8-13, Sect. 8.5.1.3. The activity shown at $\sim 50 \mathrm{ft}$. in this borehole appears to be a short lived isotope; however, the spectral gamma log shows up to $1 \mathrm{pCi} / \mathrm{g}$ about 30 years later. This leads to two questions: 1) what is the detection capability of the tool(s) used in 1975 ? and 2) does the mid-1990s data reflect a more recent leak at the same location? Please address.

- 1) This was addressed in Section 3.1.8 Drywells

- 2) Will add to Section 3.4. 
7. Pg. 8-14, Sect. 8.5.1.4. A comparison of Fig 8-7 with the spectral gamma log for this borehole leads one to speculate about several things: 1) is the 1970s data all Co-60? 2) what's the detection capability of the historical gross gamma log to Co-60? 3) can one infer Co-60 mobility from this comparison, as it appears to extend deeper than the base of the signature in the 1970s logs? Please address.

- Added more detailed explanation and comparison of historical gross gamma with the 1996 SGLS.

8. Pg. 8-15, Sect. 8.5.1.5. Comparing the historical gross gamma logs with the spectral gamma logs taken some 25 or so years later, again the impression is that there is either a new release of Cs-137 or that the detection capability of the gross gamma logs taken in the 1970s was 10 $\mathrm{pCi} / \mathrm{g}$ or more. The limits of detection of the logging tools really should be addressed. Please do so.

- Added perspective on the $\mathrm{Cs}^{137}$ level between 1975 and 1995 in HNF-3831 and the 1996 SGLS in GJ-HAN-69 to Section 8.5.1.5.

9. Pg. 8-16, Sect. 8.5.1.6. When I compare the text here with the adjoining figure, there seems to be a disagreement unless the scale of the figure is deceiving. The figure also seems to contrast with what's in Table B-1. Please check into and correct these seeming inconsistencies.

- Changed the last sentence in Section 8.5.1.6 to indicate early low level radioactivity decayed away.

10. Pg. 8-20, Sect. 8.6.1. Push holes (such as C-4603, C-4605 etc.) are logged once and are not drywells in the same sense as those numbered 52-06-xx). The tank farm surveillance boreholes/drywells are open and accessible to provide continuous logging, whereas the push holes are logged one time and represent one profile in time. As the push holes use a smaller diameter logging tool, the results are not directly comparable. Please correct this terminology.

- Addressed this comment in the 2005 Direct Push Logging section in the TY Farm segments where it applies

11. Pg. 8-20, Sect. 8.6.1. The text says that only data for drywell 52-06-07 were available in 1959 , but table B-1 in the Appendix shows that the data weren't available. This is a conflicting statement that needs to be resolved. Please correct.

- This comment will be addressed in the report

12. FYI. Push holes are constructed using compression and vibration and are not "drilled" in the conventional sense. Please correct this terminology.

- Terminology was corrected in the report 


\section{TANK BY-103 SEGMENT J. A. Caggiano Review Comments 9-15-11 with Responses}

1. Pg. 4-5, Sect. 4.5.1.1. The spectral gamma log in 22-00-01 in the mid-90s shows Cs-137 above $10 \mathrm{pCi} / \mathrm{g}$. The historical log for this same drywell shows nothing. Again this raises the possibility that either the gross gamma detector was not sufficiently sensitive, or that a leak developed and propagated after 1994.

- It does appear these historical figures do not show any peaks below $10 \mathrm{pCi} / \mathrm{g}$ and we added the following statement in the upfront drywell section (Section 3.3): "Drywell sections contain gross gamma figures taken from HNF-3532 and HNF-3831 showing continuing or new contamination in the drywells based on below grade surface (BGS) depth from 1975 to 1994 (HNF-3532, Analysis of Historical Gross Gamma Logging Data from BY Tank Farm; HNF-3831, Analysis of Historical Gross Gamma Logging Data from TY Tank Farm). Later drywell data can be found in the spectral gamma logs (SGLS) (GJO-HAN-6, Vadose Zone Characterization Project at the Hanford Tank Farms BY Tank Farm Report; GJO-HAN-16, Vadose Zone Characterization Project at the Hanford Tank Farms TY Tank Farm Report). The gross gamma figure detection sensitivity is less than SGLS at $10 \mathrm{pCi} / \mathrm{g}$. Therefore, radioactivity $\leq 10$ $\mathrm{pCi} / \mathrm{g}$ does not appear on the gross gamma figures (GJO-HAN-6; GJO-HAN-16). "

2. Pg. 4-6, Sect. 4.5.1.2. The peaks appearing on the gross gamma logs from 1975 to the early 1980s seem to be Co-60, as judged from the spectral gamma logs. While there is a difference in the sensitivity, the base of the historical plume demonstrated on the gross gamma logs has continued downward movement when compared to the baseline spectral gamma logging. Another example of Co-60 mobility.

- Agree. Conclusion should be the same as it relates to the leak location.

3. Pg. 4-7, Sect. 4.5.1.3. There's a couple of peaks of Co-60 in this borehole: one broad one between $\sim 54$ to $\sim 80 \mathrm{ft}$. and another deeper peak at $\sim 124 \mathrm{ft}$. Co-60 has penetrated deep in this borehole suggesting this to be an old release.

- Indicated that the gross gamma was mostly $\mathrm{Co}^{60}$ and that SGLS later indicated an interval between $\sim 54-\mathrm{ft}$ and $\sim 80$-ft with a peak at $\sim 124-\mathrm{ft}$. Not sure about the older comment as the 1975 gross gamma indicated $\mathrm{Co}^{60}$ down to $\sim 140-\mathrm{ft}$. In any case the leak location would be the same.

4. Pg. 4-8, Sect. 4.5.1.4. Whatever caused the spike shortly before and after 1980 has disappeared and does not appear on the profiles from about 1983 forward. This peak also does not shown in the spectral gamma logs in 199? So it is likely some short-lived isotope, possibly Ru-106.

- No other data were available after 1975 except for Figure 4-7. The spike seen around 1980 at the bottom of the drywell does not seem to provide any additional information to a possible leak location. This will not be addressed in the report.

5. Pg. 4-10, Fig. 4-9. The spectral gamma log for borehole 22-03-04 shows two Co-60 peaks; one at $\sim 45 \mathrm{ft}$. and the other at $\sim 75 \mathrm{ft}$. The shallower one shows on Fig. 4-9, but the deeper 
one does not suggesting that there is a separate event - either a release or migration from some source other than BY-103. There is a distinct break in the spectral gamma log between these two peaks, so Co-60 migration does not seem likely to explain the deeper peak. Please address.

- Tank BY-103 appears to be the explanation of the Co-60 peaks as no other tanks or other sources are located near this drywell. However, the apparent break between the two is unexplained. Will add this statement into the report.

6. Pg. 4-11, Sect. 4.5.1.7. Dates of drywell readings in the 1970s in the text are inconsistent with those reported in Table A-1. Please correct or at least explain why.

- This was re-checked and clarified in the report

7. Pg. 4-11, Sect. 4.5.1.7. Borehole 22-03-05 is only a few feet from the spare inlet lines which were "capped". LLs on Fig. 4-3 do not show this tank as being overfilled, but overfilling and a release through one or both of the spare inlet lines appears a likely cause for this release which seems to originate at approximately the depth of the spare inlet lines. Other release mechanisms are possible, but the geographic proximity leads one to believe that a release from a spare inlet line is very possible. Please address. This borehole also provides another example of the mobility of Co-60.

- The following sentence was added to Section 4.5.1 Drywells: "The following subsections report the available drywell information and the drywell summary section provides the analyses of the associated drywells with tank BY-103."

- Also, data supports that a sidewall leak is likely the explanation of radioactivity detected in this drywell since the peak detected at $~ 36$ - $\mathrm{ft}$ BGS is 14-ft below the spare nozzles and $\sim 10-\mathrm{ft}$ above the top of the tank footing. With the drywell located $2.1-\mathrm{ft}$ from the tank footing, the probability favors a tank sidewall leak. This will also be added to Section 4.6.1.

8. Pg. 4-12, Sect. 4.5.1.8. Dates and readings between the text here and in Table A-1 differ. Please make consistent.

- This was clarified in this section

9. Pg. 4-12, Sect. 4.5.1.8. There may have been some release from a cascade line, but the most significant release occurred at the base of the tank and indicates that BY-103 leaked, possibly because of a loss of integrity of the liner. Please add.

- Added in "see Section 4.5.2" as we talk about this possible scenario in this section

10. Pg. 4-13, Sect. 4.5.1.9. Two points here: 1) With between 10 and $100 \mathrm{pCi} / \mathrm{g}$ shown on the spectral gamma log and nothing on the historical gamma logs in the top $10 \mathrm{ft}$., there has either been a post-1995 surface spill or the detection limit for the historical gross gamma logs is above $10 \mathrm{pCi} / \mathrm{g} \mathrm{Cs}-137,2$ ) another case of Co-60 mobility as shown on the spectral gamma $\log$ for this borehole.

- Addressed with the following sentence added to this section: GJ-HAN-20 reported no evidence exists for a subsurface source of $\mathrm{Cs}^{137}$ at this drywell; however, surface contamination was reported. 
11. Pg. 4-15, Fig. 4-14. Another great example of Co-60 mobility, and this isotope is at least a partial surrogate for other non-gamma emitting contaminants and their spread in the soil.

- This comment appears to be a statement and therefore will not be addressed in the report. The Leak Location and Causes documentation is not addressing the spread of contamination in the soil.

12. Pg. 4-16, Fig. 4-15. Again, a comparison of the spectral gamma logs for Cs-137 and the gross gamma logs indicates a limit of detection for the old gross gamma tool of $10 \mathrm{pCi} / \mathrm{g}$ or greater, because none of the Cs-137 shown on the spectral gamma log for the top $50 \mathrm{ft}$. is shown on the gross gamma historical logs.

- See the response to comment \#1

13. Pg. 4-17, Sect. 4.5.2. There is no question that this tank released waste to the soil; there are questions about the actual point of egress and whether it was a structural failure of the liner or leakage through a spare inlet port during likely overfilling. What might be useful is to plot a time series of detections to see where the release was first detected and how it might have spread. This might help in determining whether the release was just from a spare inlet or whether other release points were present.

- Not sure about the likely overfilling as there was no LL evidence that there was an overfilling that doesn't mean there wasn't any. The level BGS of the primary side wall leak sites was $\sim 14$-ft below the capped spare inlet lines and therefore with a drywell only 2.1 -ft from the tank footing (assuming vertical drywell) the probability seemed to favor a sidewall leak as opposed to a leak through the capped spare inlet. The timing of the primary leak locations seemed to point to subsequent migration to adjoining drywells therefore didn't graph the timing.

14. Pg. 4-20, para 1. I would add the spare inlet lines as another possible release point for this tank.

- This was added to this section

15. Pg. 4-21. The leak location may be from a sidewall, but it may not be from a failure of the liner, as the spare inlet lines are located very close to 22-03-05. The difference in mechanism, liner failure vs. inlet line sealing, are significant.

- Added the following to address this comment: "The peak at 36-ft BGS is 14-ft below the capped spare inlets and $\sim 10-\mathrm{ft}$ above the top of the tank footing with drywell 22 03-05 located 2.1-ft from the tank footing. The probability favors a tank sidewall leak versus a leak from the capped spare inlets."

\section{SOME GENERAL COMMENTS:}

1. Someone with greater familiarity with drywell logging and the tools used to do it through time should evaluate the drywell records, as these seem to be the principal evidence of release for this tank.

- The GJ-HAN-XX documents have been used to add to our data as well as a backup or add to our analysis as a way of augmenting drywell logging analysis. The team was 
not set up to have a drywell logging expert available however we have informally consulted with experts on occasion. The level of analysis to identify potential leak locations did not seem to justify an expert analysis over and above the documents available and the review of ORP and Ecology personnel. The movement of radionuclides through the soil with time is not part of the leak location and cause assessment as the leak location is not changed with increasing radioactivity.

2. The TBP waste not only facilitated corrosion, but it may have contributed to the mobility of Co-60 by forming anionic complexes that give the isotope much greater mobility than that of a typical metal. Thus, TBP waste is detrimental for several reasons.

- The possible increased mobility of anionic $\mathrm{Co}^{60}$ complexes is detrimental in the environment but the report is concerned with the liner failure possibilities.

3. Consistency checks are needed to be sure the text agrees with data shown in the appendices. Where they differ, some explanation is in order.

- Any differences seen were acknowledged

4. When PUREX HLW is added to a tank, the age and temperature of the waste at the time of addition are important information and should be provided when available - considering the design specifications for temperature in these tanks.

- Transfer's leading to the storage of PUREX HLW supernatant were reviewed however no data was recovered. It was thought that the waste would be at or slightly above $180^{\circ} \mathrm{F}$ and below the $230^{\circ} \mathrm{F}$ tank temperature limit. This was stated in the document. 


\section{DISTRIBUTION SHEET}

To

From

Page $\quad 1$ Of 1

Distribution

Technical Integration

Date

$09 / 18 / 2013$

\section{Project Title/Work Order}

EDT No.

$\mathrm{N} / \mathrm{A}$

RPP-RPT-54911, Rev. 0, Hanford Single-Shell Tank Leak Causes and

Locations - 241-BY and 241-TY Tank Farms

ECN No.

$\mathrm{N} / \mathrm{A}$

Name

T.J. Barnes

K.D. Boomer

R.A. Burk

C.A. Burke

A.B. Carlson

K.G. Duncan

J.G. Field

L.A. Fort

C.L. Girardot

R.E. Gregory

D.G. Harlow

D.B. Little

T.J. Venetz

J.A. Voogd

D.J. Washenfelder

T.W. Fletcher

E.M. Mattlin

J.M. Johnson

S.E. Killoy

M.R. Greene

J.J. Luke

\begin{tabular}{|c|c|c|c|c|}
\hline MSIN & $\begin{array}{c}\text { Text } \\
\text { With All } \\
\text { Attach. }\end{array}$ & Text Only & $\begin{array}{c}\text { Attach./ } \\
\text { Appendix } \\
\text { Only }\end{array}$ & $\begin{array}{c}\text { EDT/ECN } \\
\text { Only }\end{array}$ \\
\hline$R 2-53$ & $\mathrm{X}$ & & & \\
\hline$R 2-53$ & $\mathrm{X}$ & & & \\
\hline$S 7-83$ & $\mathrm{X}$ & & & \\
\hline$S 7-75$ & $\mathrm{X}$ & & & \\
\hline R2 -53 & $\mathrm{X}$ & & & \\
\hline R2 -53 & $\mathrm{X}$ & & & \\
\hline$S 7-90$ & $\mathrm{X}$ & & & \\
\hline H6-13 & $\mathrm{X}$ & & & \\
\hline$R 2-53$ & $\mathrm{X}$ & & & \\
\hline$S 7-68$ & $\mathrm{X}$ & & & \\
\hline$R 2-53$ & $\mathrm{X}$ & & & \\
\hline$R 2-58$ & $\mathrm{X}$ & & & \\
\hline$R 2-53$ & $\mathrm{X}$ & & & \\
\hline $\mathrm{R} 1-51$ & $\mathrm{X}$ & & & \\
\hline$R 2-53$ & $\mathrm{X}$ & & & \\
\hline H6-60 & $\mathrm{X}$ & & & \\
\hline $\mathrm{H} 6-6 \mathrm{O}$ & $\mathrm{X}$ & & & \\
\hline$R 6-60$ & $\mathrm{X}$ & & & \\
\hline $\mathrm{H} 6-14$ & $\mathrm{X}$ & & & \\
\hline$R 1-51$ & $\mathrm{x}$ & & & \\
\hline H $6-14$ & $\mathrm{X}$ & & & \\
\hline & & & & \\
\hline & & & & \\
\hline & & & & \\
\hline & & & & \\
\hline & & & & \\
\hline
\end{tabular}

